
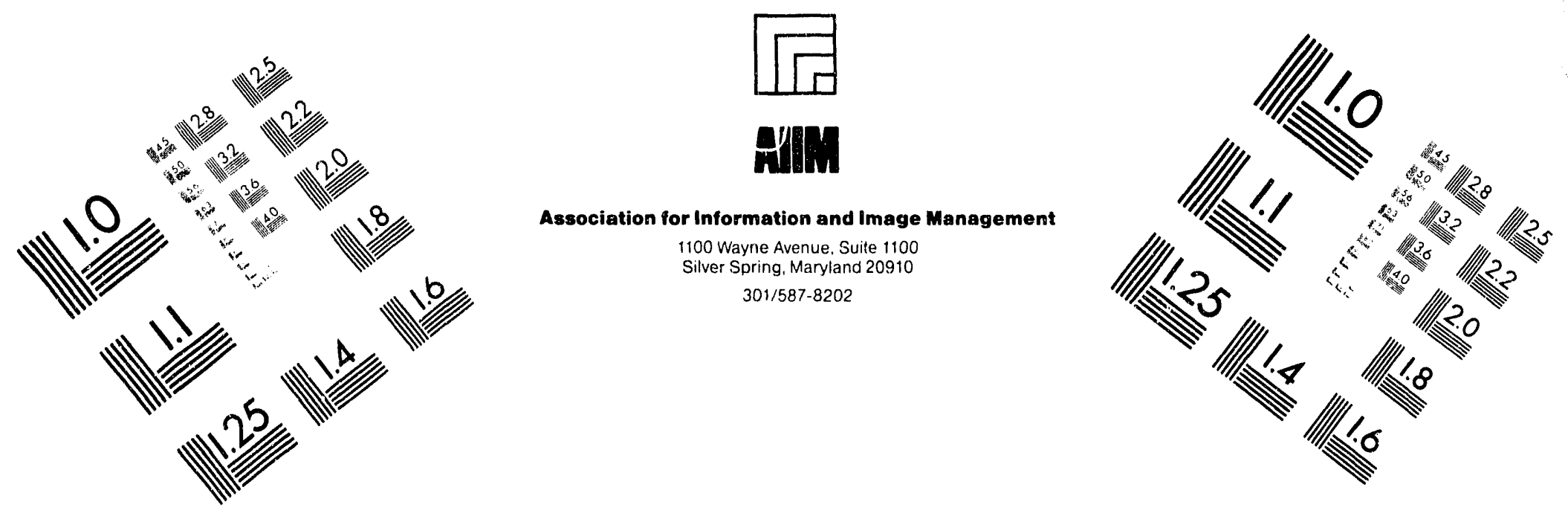

\title{
Centimeter
}

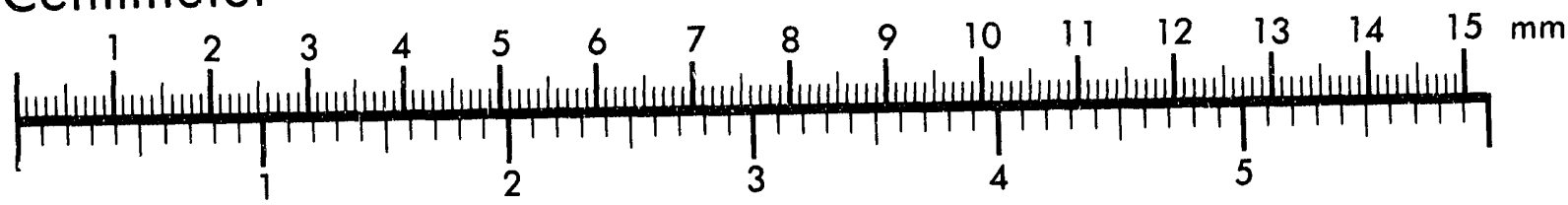
Inches
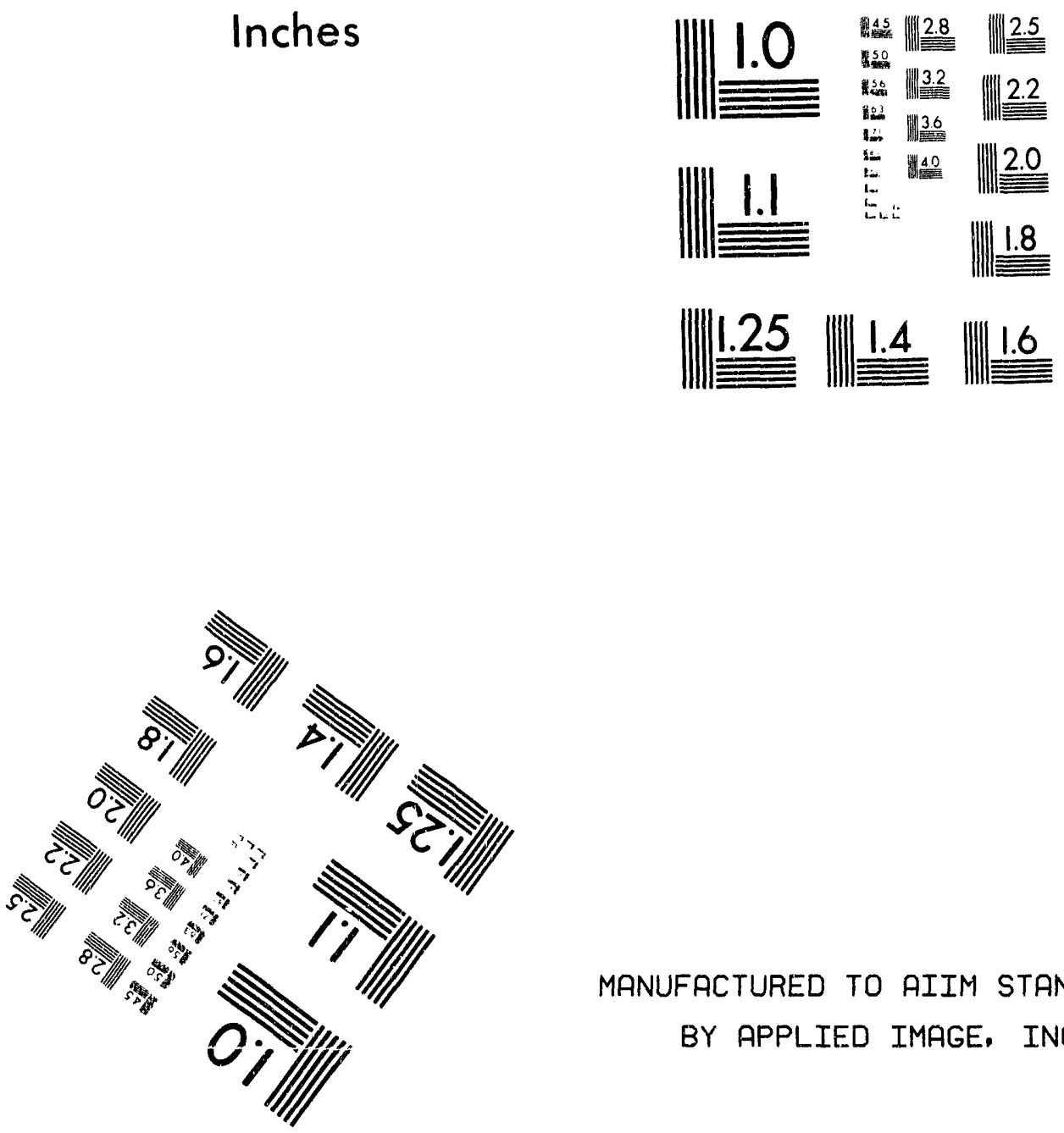

MANUFACTURED TO AIIM STANDARDS

BY APPLIED IMAGE, INC.

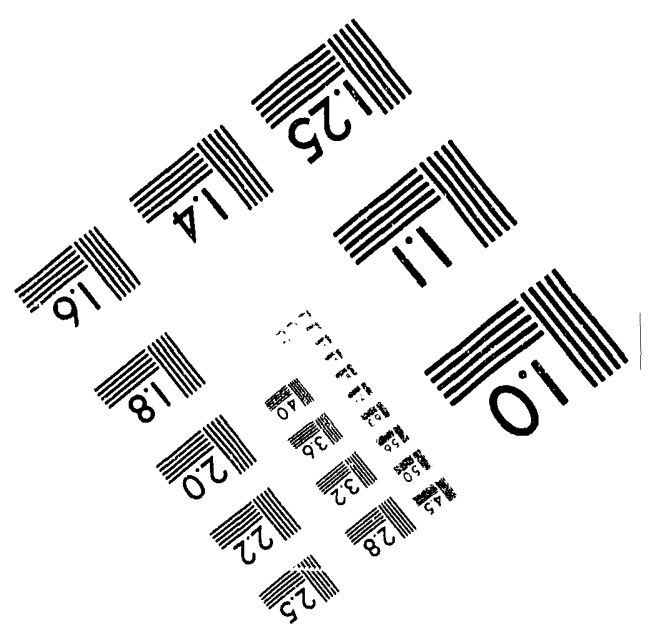



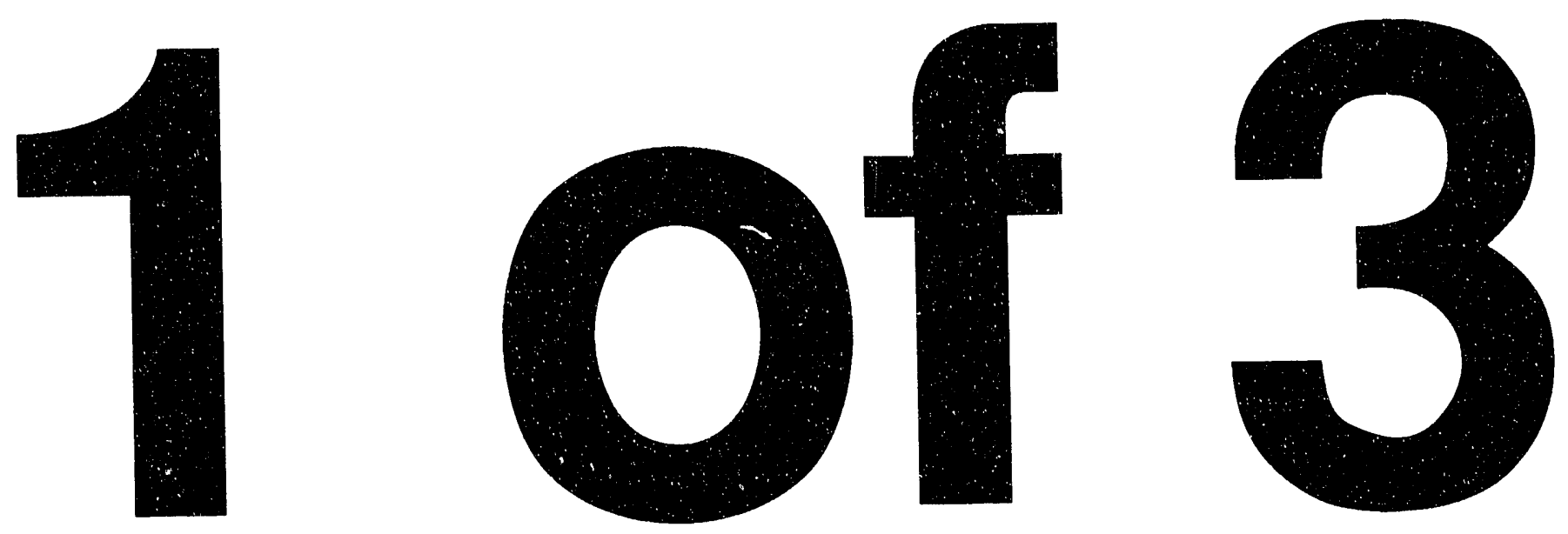


\section{Examination of Forced Unsteady Separated Flow Fields on a Rotating Wind Turbine Blade}

Steven Huyer

University of Colorado

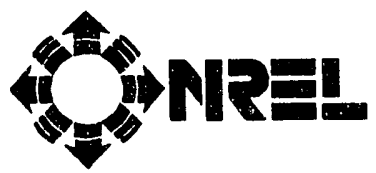

National Renewable Energy Laboratory 1617 Cole Boulevard Golden, Colorado 80401-3393

Operated by Midwest Research Institute for the U.S. Department of Energy under Contract No. DE-AC02-83CH10093 Prepared under task no. WE318120

April 1993 


\section{NOTICE}

NOTICE: This report was prepared as an account of work sponsored by an agency of the United States government. Neither the United States govemmient nor any agency thereof, nor any of their employees, makes any warranty, express or implied, or assumes any legal liability or responsibility for the accuracy, completeness, or usefulness of any information, apparatus, product, or process disclosed, or represents that its use would not infringe privately owned rights. Reference herein to any specific commercial product, process, or service by trade name, trademark, manufacturer, or othenwise does not necessarily constitute or imply its endorsement, recommendation, or favoring by the United States government or any agency thereof. The views and opinions of authors expressed herein do not necessarily state or reflect those of the United States govemment or any agency thereof.

Printed in the United States of America

Available from:

National Technical Information Service

U.S. Department of Commerce

5285 Port Royal Road

Springfield, VA 22161

Price: Microfiche A01

Printed Copy A11

Codes are used for pricing all publications. The code is determined by the number of pages in the publication. Information pertaining to the pricing codes can be found in the current issue of the following publications which are generally available in most libraries: Energy Research Abstracts (ERA); Government Reports Announcements and Index (GRA and I); Scientific and Technical Abstract Reports (STAR); and publication NTIS-PR-360 available from NTIS at the above address. 


\section{Table of Contents}

$\underline{\text { Page }}$

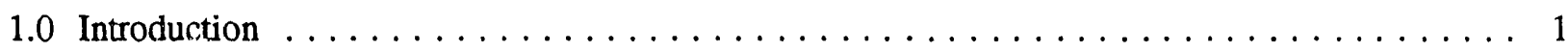

2.0 Methodology $\ldots \ldots \ldots \ldots \ldots \ldots \ldots \ldots \ldots \ldots \ldots \ldots \ldots \ldots \ldots \ldots \ldots$

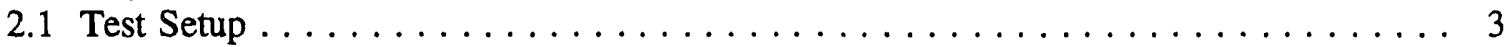

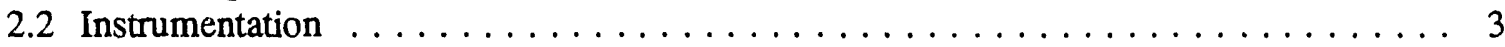

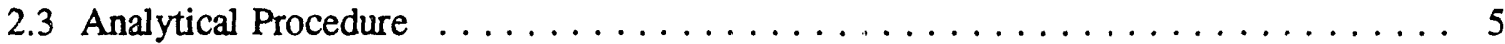

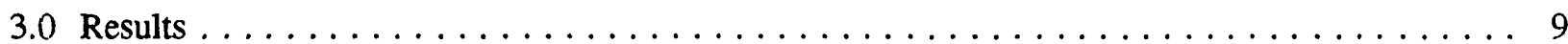

3.1 Theoretical versus Experimental $q$ and $\alpha . \ldots \ldots \ldots \ldots \ldots \ldots \ldots$

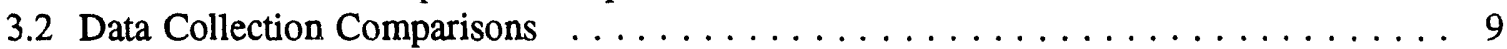

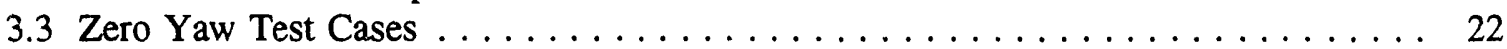

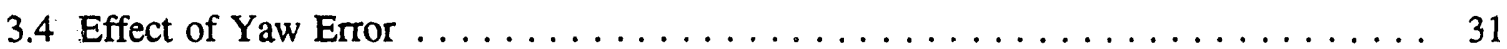

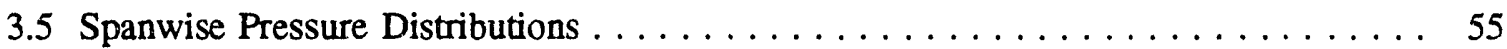

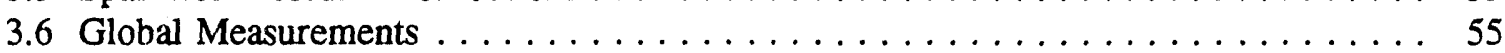

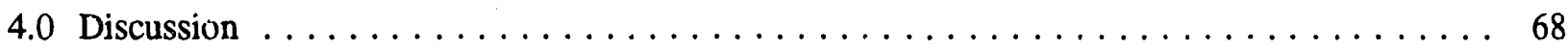

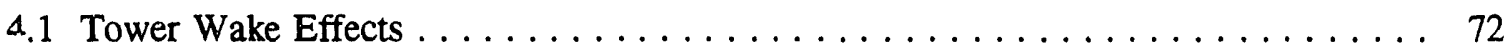

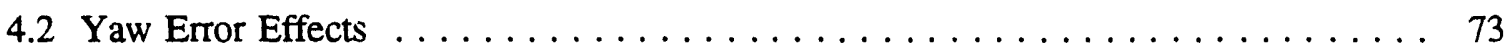

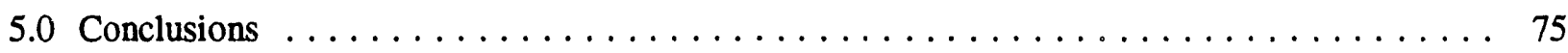

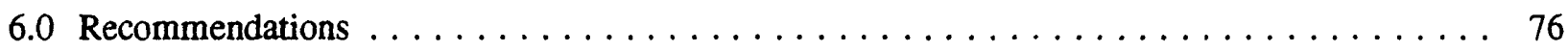

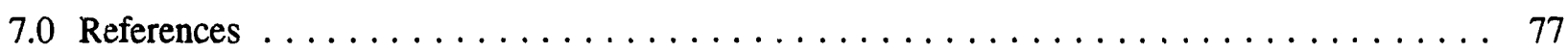

Appendix A: Computer Programs $\ldots \ldots \ldots \ldots \ldots \ldots \ldots \ldots \ldots \ldots \ldots \ldots \ldots \ldots \ldots$

Appendix B: Experimental and Theoretical Motion Histories $\ldots \ldots \ldots \ldots \ldots \ldots \ldots$ B-1

Appendix C: Zero Yaw Test Cases, Cycle Averaged $\ldots \ldots \ldots \ldots \ldots \ldots \ldots \ldots$

Appendix D: Yaw Error Test Cases, Cycle Averaged $\ldots \ldots \ldots \ldots \ldots \ldots \ldots \ldots \ldots$

Appendix E: Yaw Error Test Cases, Single Cycle Data . . . . . . . . . . . E E-1

Appendix F: Chordwise Pressure Distributions, From Single Cycle Data . . . . . . . . F F-1 


\section{List of Figures}

$\underline{\text { Page }}$

Figure 2-1. Grumman Wind Stream 33 downwind horizontal axis

wind turbine

Figure $2-2 . \quad$ Geometry of total velocity relative to blade $\ldots \ldots \ldots \ldots \ldots$

Figure 3-1. Theoretical vs. measured instantaneous dynamic pressure, $\mathrm{q}$

(uncorrected). $\mathrm{V}_{\mathrm{w}}=15 \mathrm{~m} / \mathrm{s}, \gamma=30^{\circ}$

Figure 3-2. Theoretical vs. measured instantaneous dynamic pressure, q (corrected).

$\mathrm{V}_{\mathrm{w}}=15 \mathrm{~m} / \mathrm{s}, \gamma=30^{\circ}$

Figure 3-3. Theoretical vs. measured instantaneous dynamic pressure, $\mathrm{q}$ (corrected).

$\mathrm{V}_{\mathrm{w}}=15 \mathrm{~m} / \mathrm{s}, \gamma=-30^{\circ}$

Figure 3-4. Theoretical vs. measured AOA, a. $V_{w}=15 \mathrm{~m} / \mathrm{s}, \gamma=30^{\circ}$ and $-30^{\circ} \ldots \ldots 13$

Figure 3-5. Upper surface pressure distribution vs. azimuth angle. $V_{w}=14.2 \mathrm{~m} / \mathrm{s}, \gamma=30^{\circ}$,

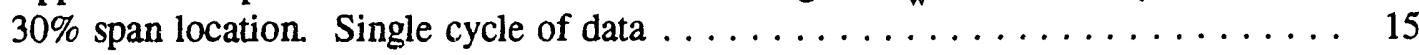

Figure 3-6. Upper surface pressure distribution vs. azimuth angle. $V_{w}=14 \mathrm{~m} / \mathrm{s}, \gamma=30^{\circ}$,

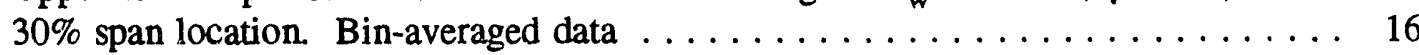

Figure 3-7. Upper surface pressure distribution vs. azimuth angle. $V_{w}=15 \mathrm{~m} / \mathrm{s}, \gamma=30^{\circ}$, $30 \%$ span location. Cycle-averaged data . . . . . . . . . . . . . 17

Figure 3-8. Upper surface pressure distribution vs. azimuth angle. $V_{w}=14 \mathrm{~m} / \mathrm{s}, \gamma=30^{\circ}$, $63 \%$ span location. Bin-averaged data $\ldots \ldots \ldots \ldots \ldots \ldots \ldots \ldots$

Figure 3-9. Upper surface pressure distribution vs. azimuth angle. $V_{w}=15 \mathrm{~m} / \mathrm{s}, \gamma=30^{\circ}$, $63 \%$ span location. Cycle-averaged data . . . . . . . . . . . . . . 19

Figure 3-10. Upper surface pressure standard deviation vs. azimuth angle. $V_{w}=14 \mathrm{~m} / \mathrm{s}, \gamma=$ $30^{\circ}, 63 \%$ span location. Bin-averaged data $\ldots \ldots \ldots \ldots \ldots \ldots \ldots \ldots \ldots \ldots \ldots$

Figure 3-11. Upper surface pressure standard deviation vs. azimuth angle. $V_{w}=15 \mathrm{~m} / \mathrm{s}, \gamma=$ $30^{\circ}, 63 \%$ span location. Cycle-averaged data $\ldots \ldots \ldots \ldots \ldots \ldots \ldots \ldots$

Figure 3 -12. Theoretical AOA vs. wind velocity $\ldots \ldots \ldots \ldots \ldots \ldots \ldots \ldots \ldots$

Figure 3-13. Upper surface pressure distribution vs. azimuth angle. $V_{w}=14.7 \mathrm{~m} / \mathrm{s}, \gamma=0^{\circ}$, $30 \%$ span location. Cycle-averaged data . . . . . . . . . . . . 24

Figure 3-14. Upper surface pressure distribution vs. azimuth angle. $V_{w}=14.7 \mathrm{~m} / \mathrm{s}, \gamma=0^{\circ}$, $47 \%$ span location. Cycle-averaged data 


\section{List of Figures (continued)}

Figure 3-15. Upper surface pressure distribution vs. azimuth angle. $V_{w}=14.7 \mathrm{~m} / \mathrm{s}, \gamma=0^{\circ}$, $63 \%$ span lovation. Cycle-averaged data . . . . . . . . . . . . . . . 26

Figure 3-16. Upper surface pressure distribution vs. azimuth angle. $V_{w}=14.7 \mathrm{~m} / \mathrm{s}, \gamma=0^{\circ}$, $80 \%$ span location. Cycle-averaged data . . . . . . . . . . . . . 27

Figure 3-17. Upper surface pressure distribution vs. azimuth angle. $V_{w}=7.7 \mathrm{~m} / \mathrm{s}, \gamma=0^{\circ}$, $63 \%$ span location. Cycle-averaged data ................. 28

Figure 3-18. Upper surface pressure distribution vs. azimuth angle. $V_{w}=18.7 \mathrm{~m} / \mathrm{s}, \gamma=0^{\circ}$, $63 \%$ span location. Cycle-averaged data . . . . . . . . . . . . . . 29

Figure 3-19. Upper surface pressure distribution vs. azimuth angle. $V_{w}=23.0 \mathrm{~m} / \mathrm{s}, \gamma=0^{\circ}$,

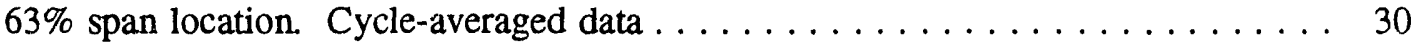

Figure 3-20. Normal force coefficients vs. azimuth angle at $30 \%, 47 \%, 63 \%$, and $80 \%$ span for zero yaw test cases. Cycle-averaged data $\ldots \ldots \ldots \ldots \ldots \ldots \ldots \ldots \ldots \ldots$

Figure 3-21. Static $C_{n}$ vs. AOA from wind tunnel test data $\ldots \ldots \ldots \ldots \ldots \ldots$

Figure 3-22. Upper surface pressure distribution vs. azimuth angle. $V_{w}=15 \mathrm{~m} / \mathrm{s}, \gamma=-30^{\circ}$,

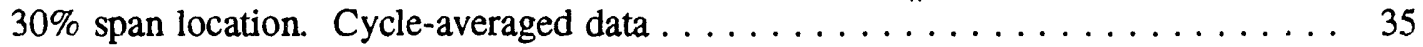

Figure 3-23. Upper surface pressure distribution vs. azimuth angle. $V_{w}=15 \mathrm{~m} / \mathrm{s}, \gamma=-30^{\circ}$,

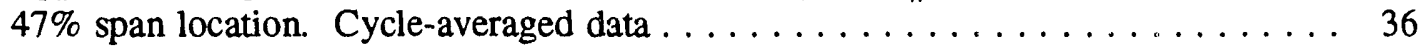

Figure 3-24. Upper surface pressure distribution vs. azimuth angle. $V_{w}=15 \mathrm{~m} / \mathrm{s}, \gamma=-30^{\circ}$,

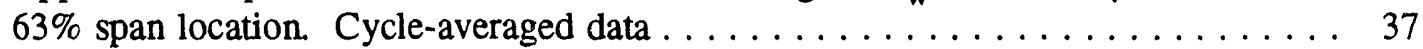

Figure 3-25. Upper surface pressure distribution vs. azimuth angle. $V_{w}=15 \mathrm{~m} / \mathrm{s}, \gamma=30^{\circ}$, $80 \%$ span location. Cycle-averaged data . . . . . . . . . . . . . . . 38

Figure 3-26. Upper surface pressure distribution vs. azimuth angle. $V_{w}=15 \mathrm{~m} / \mathrm{s}, \gamma=15^{\circ}$,

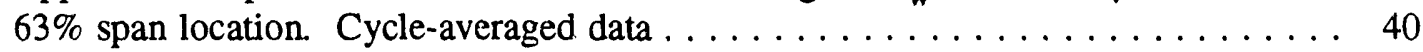

Figure 3-27. Upper surface pressure distribution vs. azimuth angle. $V_{w}=15 \mathrm{~m} / \mathrm{s}, \gamma=-15^{\circ}$, $63 \%$ span location. Cycle-averaged data . . . . . . . . . . . . . 41

Figure 3-28. Suction pressure coefficient vs. chord location. $V_{w}=15 \mathrm{~m} / \mathrm{s}, \gamma=30^{\circ}$, $30 \%$ span location. Single cycle of data. Chordwise pressure distributions taken at specific points in rotational cycle $\ldots \ldots \ldots \ldots \ldots \ldots \ldots$

Figure 3-29. Suction pressure coefficient vs. chord location. $V_{w}=15 \mathrm{~m} / \mathrm{s}, \gamma=30^{\circ}$, 47\% span location. Single cycle of data. Chordwise pressure distributions taken at specific points in rotational cycle $\ldots \ldots \ldots \ldots \ldots \ldots \ldots \ldots \ldots \ldots$ 


\section{List of Figures (continued)}

Page

Figure 3-30. Suction pressure coefficient vs. chord location. $V_{w}=15 \mathrm{~m} / \mathrm{s}, \gamma=30^{\circ}$, $63 \%$ span location. Single cycle of data. Chordwise pressure distributions taken at specific points in rotational cycle $\ldots \ldots \ldots \ldots \ldots \ldots \ldots \ldots$

Figure 3-31. Suction pressure coefficient vs. chord location. $V_{w}=15 \mathrm{~m} / \mathrm{s}, \gamma=30^{\circ}$, $80 \%$ span location. Single cycle of data. Chordwise pressure distributions taken at specific points in rotational cycle

Figure 3-32. Suction pressure peak chord location vs. azimuth angle for $30 \%, 47 \%, 63 \%$, and $80 \%$ span locations. $\mathrm{V}_{\mathrm{w}}=15 \mathrm{~m} / \mathrm{s}, \gamma=30^{\circ}$. Single cycle of data $\ldots \ldots \ldots$

Figure 3-33. Normal force coefficient vs. azimuth angle at $30 \%, 47 \%, 63 \%$, and $80 \%$ span locations for the four yaw cases examined. Cycle-averaged data . . . . . . . . .

Figure 3-34. Theoretical AOA vs. azimuth angle at $30 \%, 47 \%, 63 \%$, and $80 \%$ span locations for the four yaw cases examined $\ldots \ldots \ldots \ldots \ldots \ldots \ldots$

Figure 3-35. Instantaneous dynamic pressure vs. azimuth angle at 30\%, 47\%,63\%, and $80 \%$ span locations for the four yaw cases examined $\ldots \ldots \ldots \ldots \ldots \ldots$

Figure 3-36. Normal force coefficient vs. AOA at 30\%, 47\%, 63\%, and $80 \%$ span locations. $\mathrm{V}_{\mathrm{w}}=15 \mathrm{~m} / \mathrm{s}, \gamma=30^{\circ}$. Cycle-averaged data. $\mathrm{C}_{\mathrm{n}}$ normalized by constant dynamic

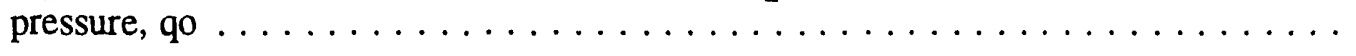

Figure 3-37. Normal force coefficient vs. AOA at 30\%, 47\%, 63\%, and $80 \%$ span locations. $\mathrm{V}_{\mathrm{w}}=15 \mathrm{~m} / \mathrm{s}, \gamma=30^{\circ}$. Cycle-averaged data. $\mathrm{C}_{\mathrm{n}}$ normalized by instantaneous

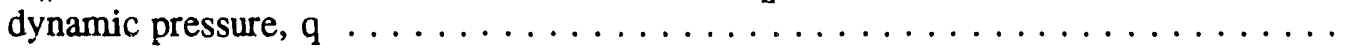

Figure 3-38. Normal force coefficient vs. AOA at 30\%, 47\%, 63\%, and $80 \%$ span locations. $\mathrm{V}_{\mathrm{w}}=15 \mathrm{~m} / \mathrm{s}, \gamma=-30^{\circ}$. Cycle-averaged data. $\mathrm{C}_{\mathrm{n}}$ normalized by instantaneous

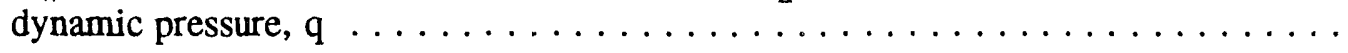

Figure 3-39. Spanwise pressure distribution vs. azimuth angle. $V_{w}=7.7 \mathrm{~m} / \mathrm{s}, \gamma=0^{\circ}, 4 \%$ chord location. Cycle-averaged data $\ldots \ldots \ldots \ldots \ldots \ldots \ldots$

Figure 3-40. Spanwise pressure distribution vs. azimuth angle. $V_{w}=14.7 \mathrm{~m} / \mathrm{s}, \gamma=0^{\circ}, 4 \%$ chord

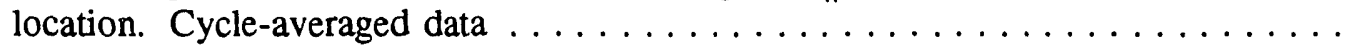

Figure 3-41. Spanwise pressure distribution vs. azimuth angle. $V_{w}=15 \mathrm{~m} / \mathrm{s}, \gamma=30^{\circ}, 4 \%$ chord

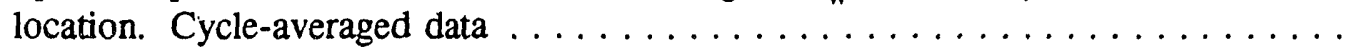

Figure 3-42. Root flap bending moment vs. azimuth angle. $\gamma=0^{\circ}, \mathrm{V}_{\mathrm{w}}=7.7,14.7,18.7$, and

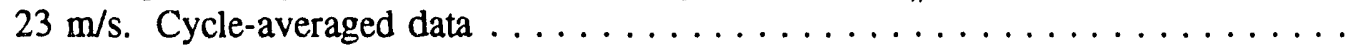

Figure 3-43. Low-speed shaft torque vs. azimuth angle. $\gamma=0^{\circ}, V_{w}=7.7,14.7,18.7$, and $23 \mathrm{~m} / \mathrm{s}$. Cycle-averaged data $\ldots \ldots \ldots \ldots \ldots \ldots \ldots \ldots$ 


\section{List of Figures (concluded)}

$\underline{\text { Page }}$

Figure 3-44. Generator power vs. azimuth angle. $\gamma=0^{\circ}, V_{w}=7.7,14.7,18.7$, and $23 \mathrm{~m} / \mathrm{s}$.

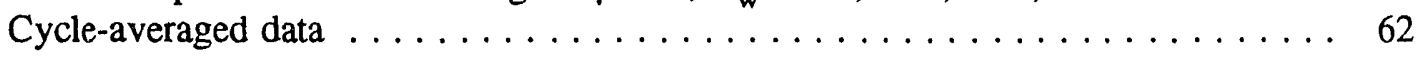

Figure 3-45. Root flap bending moment vs. azimuth angle. $V_{w}=15 \mathrm{~m} / \mathrm{s}, \gamma=30^{\circ},-30^{\circ}, 15^{\circ}$, and

$-15^{\circ}$. Cycle-averaged data $\ldots \ldots \ldots \ldots \ldots \ldots \ldots \ldots \ldots \ldots$

Figure 3-46. Low-speed shaft torque vs. azimuth angle. $V_{w}=15 \mathrm{~m} / \mathrm{s}, \gamma=30^{\circ},-30^{\circ}, 15^{\circ}$, and

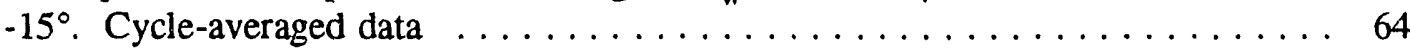

Figure 3-47. Generator power vs. azimuth angle. $V_{w}=15 \mathrm{~m} / \mathrm{s}, \gamma=30^{\circ},-30^{\circ}, 15^{\circ}$, and $-15^{\circ}$.

Cycle-averaged data

Figure 3-48. Yaw moment vs. azimuth angle. $V_{w}=15 \mathrm{~m} / \mathrm{s}, \gamma=30^{\circ},-30^{\circ}, 15^{\circ}$, and $-15^{\circ}$.

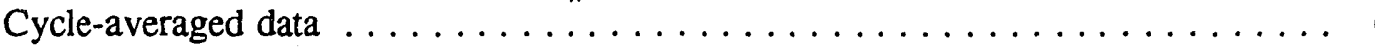

Figure 4-1. Flow visualization sequence depicting dynamic stall vortex formation and a pitching rectangular wing. $\mathrm{V}=3.3 \mathrm{~m} / \mathrm{s}, \alpha+=0.4,0^{\circ}-60^{\circ}$ single pitch. Taken from

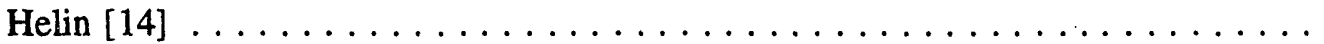

Figure 4-2. Upper surface pressure distribution for a pitching rectangular wing for similar conditions to those shown in Figure $4-1 . V=6.6 \mathrm{~m} / \mathrm{s}$, $a+=0.4,0^{\circ}-60^{\circ}$ single pitch. Taken from Robinson, Walker, and Wissler [16] 


\section{List of Tables}

$\underline{\text { Page }}$

Table 1. Angle of Attack Variation $\ldots \ldots \ldots \ldots \ldots \ldots \ldots \ldots \ldots \ldots \ldots$ 


\section{Nomenclature}

\begin{tabular}{|c|c|}
\hline c & blade chord (m) \\
\hline $\mathrm{C}_{\mathrm{p}}$ & pressure coefficient (normalized by qo) \\
\hline $\mathrm{C}_{\mathrm{n}}$ & normal force coefficient \\
\hline $\mathrm{C}_{\mathrm{t}}$ & tangential force coefficient \\
\hline f & rotational frequency $(1.2 \mathrm{~Hz})$ \\
\hline hshr & horizontal wind shear across disk $(\mathrm{m} / \mathrm{s})$ \\
\hline $\mathbf{K}$ & reduced frequency parameter $\left(\pi \mathrm{fc} / \mathrm{V}_{\text {tot }}\right)$ \\
\hline qo & constant dynamic pressure $=1 / 2 \rho\left(\mathrm{Vw}^{2}+\mathrm{Vr}^{2}\right)\left(\mathrm{N} / \mathrm{m}^{2}\right)$ \\
\hline q & instantaneous local dynamic pressure $=1 / 2 \rho \mathrm{V}_{\text {tot }}^{2}\left(\mathrm{~N} / \mathrm{m}^{2}\right)$ \\
\hline r & local blade radius or span location (m) \\
\hline $\mathbf{R}$ & blade radius or full semispan $(5.05 \mathrm{~m})$ \\
\hline $\operatorname{Re}$ & Reynolds number $\left(\mathrm{c}_{\text {tot }} / v\right)$ \\
\hline $\mathrm{V}_{\mathrm{c}}$ & cross-flow velocity component to disk $(\mathrm{m} / \mathrm{s})$ \\
\hline $\mathrm{V}_{\text {in }}$ & induced velocity component $(\mathrm{m} / \mathrm{s})$ \\
\hline $\mathrm{V}_{\mathrm{n}}$ & normal velocity component to rotor disk $(\mathrm{m} / \mathrm{s})$ \\
\hline $\mathrm{V}_{\mathrm{r}}$ & local radial velocity $=\mathrm{r} \omega(\mathrm{m} / \mathrm{s})$ \\
\hline $\mathrm{V}_{\mathrm{s}}$ & spanwise velocity component $(\mathrm{m} / \mathrm{s})$ \\
\hline vshr & vertical wind shear across disk $(\mathrm{m} / \mathrm{s})$ \\
\hline $\mathrm{V}_{\text {tot }}$ & total velocity (vector sum) $(\mathrm{m} / \mathrm{s})$ \\
\hline $\mathrm{V}_{\mathrm{ws}}$ & disk-averaged wind speed $(\mathrm{m} / \mathrm{s})$ \\
\hline $\mathrm{V}_{\mathrm{w}}$ & local wind velocity $(\mathrm{m} / \mathrm{s})$ \\
\hline
\end{tabular}

\section{Greek}

$\alpha \quad$ local blade angle of attack (deg)

$\alpha^{*} \quad$ effective pitch rate $(\mathrm{rad} / \mathrm{sec})$

$\alpha^{+} \quad$ effective nondimensional pitch rate $\left(\alpha^{*} \mathrm{c} / \mathrm{V}_{\text {tot }}\right)$

$\beta \quad$ blade pitch angle (deg)

$\gamma \quad$ yaw error (yaw angle - wind direction) (deg)

$v \quad$ coefficient of kinematic viscosity $\left(\mathrm{m}^{2} / \mathrm{s}\right)$

$\rho \quad$ air density $\left(\mathrm{kg} / \mathrm{m}^{3}\right)$ 
angle of total velocity vector relative to disk (deg)

$\psi$

azimuth angle (deg)

$\omega$

radial frequency $(2 \pi f)$ 
TP-442-4864

\subsection{Introduction}

The wind turbine industry is currently facing many problems regarding the construction of efficient and predictable wind turbine machines [3-9]. Inadequate prediction of power output and structural loads has led to the failure of power generators, gear boxes, and even the turbine blades themselves. This, in turn, increases operating and maintenance costs, making it difficult for the wind industry to compete with other established nonrenewable energy sources such as coal, oil, and gas. A 20-30 year life for wind turbines is a necessary goal to enable wind power to compete effectively with power generation by nonrenewable energy sources. Because the corresponding loading and power output is caused by aerodynamic forces, it is important to develop an understanding of the underlying fluid dynamics to enable accurate determination of structural and power requirements.

Steady state, two-dimensional wind tunnel data are generally used to predict aerodynamic loads on wind turbine blades. The aerodynamic loading is then used to estimate structural loading and power output. According to Butterfield et al. [7,8], these methods consistently underpredict the actual loading and power output measured in the field. Because there was little existing physical evidence, it was difficult to determine whether the cause of enhanced loading was due to unsteady aerodynamics, three-dimensional considerations, or both. The need for concrete experimental evidence provided the impetus for the "Combined Experiment." This experiment was designed to measure aerodynamic loads acting on a horizontal axis wind turbine (HAWT) blade in a field environment. Surface pressure data were used to determine aerodynamic loading. A sufficient number of pressure transducers and a high sampling rate allowed for exceptional spatial and temporal resolution of the flow fields elicited during normal operation of a wind turbine. These data were then compared with static results. From these data, hypotheses regarding the effects of both unsteady aerodynamics and three-dimensional effects could be established.

Preliminary experimental evidence indicated that some of the underlying fluid dynamic phenomena could be attributed to dynamic stall, or more specifically, to the generation of forced unsteady separated flow fields. The formation of discrete, cohesive vortex structures may be considered part of the unsteady separated flow development process. The formation of dynamic stall vortices has been seen in wind tunnel tests for pitching or oscillating airfoils [10-19]. Although the blades on this particular wind turbine do not pitch or oscillate, yawing of the rotor disk with respect to the wind, sudden wind gusts, or passage through the wake of a cylindrical tower may produce geometrically similar effects. Wind tunnel tests conducted by researchers at the University of Colorado [13-19] have shown that significant and highiy transient loading occurs during the formation of these complex vortical flow fields. Two-dimensional tests conducted on pitching or oscillating airfoils have shown that lift coefficients as great as three to five times maximum static counterparts resulted during nascent formation of this vortex. As the vortex grew and was shed into the wake, significant decreases in lift were observed. Tests on pitching rectangular wings [16] demonstrated similar lift overshoots, but vortex formation and shedding were then inherently more complex as the flow development process became three-dimensional. Three-dimensional considerations are extremely important for a rotating wind turbine because local velocity and local angle attack will vary as a function of iocal radius.

A collaborative research effort between the University of Colorado and the National Renewable Energy Laboratory was conducted to systematically categorize the local and global effects of three-dimensional forced unsteady separated flow fields. A physical understanding of the associated aerodynamics may offer some explanations for component failure to the wind turbine industry. In addition, extensive sets of experimental data will certainly assist in the development of a generic physical model of three-dimensional forced unsteady separated flow fields. This generic model may then provide increased understanding of the flow fields generated by airplane or submarine propellers, helicopter rotors, and unsteady flows in compressors and ducted fans. The following report summarizes the progress that has been made to date 
regarding the analysis of the "Combined Experiment" data. Figures and corresponding computer programs may be found in the appendices at the end of this report. A discussion of the results and a rudimentary physical model based on current experimental evidence is proposed. Recommendations for future investigations and a critique of the results are also provided. 
TP-442-4864

\subsection{Methodology}

\subsection{Test Setup}

A Grumman Wind Stream 33 downstream HAWT with a $10.1-\mathrm{m}$ rotor diameter and a three-bladed hub was used in the Combined Experiment (Figure 2-1). The turbine blades consisted of an S809 airfoil section with a $45.7-\mathrm{cm}$ chord and a 4.3-m span, resulting in an aspect ratio of 9.4 . The blade section begins at approximately $15 \%$ radius $(0.7575 \mathrm{~m})$ from the center of the hub and extends to $100 \% \mathrm{r}$ ( 5.05 $\mathrm{m})$. Spanwise locations are referenced from the center of the hub to the blade tip, and chordwise locations are referenced from the leading edge at a given spanwise location. To eliminate the complicated effects of wing geometry, a turbine blade of constant chord, zero twist, and zero taper was used. This allowed for data analysis for a given wind velo vity, wind direction, and blade pitch angle only. Rotational velocity was held constant at $72 \mathrm{rpm}$ for all cests. Since rotational velocity was constant, instantaneous azimuth angle provided a direct representation of time relative to the rotation cycle. At $\psi=0^{\circ}$, the instrumented turbine blade is pointing straight up at 12:00; and at 180 Also, the turbine blade rotates counter-clockwise relative to an observer behind the turbine looking into the wind. A vertical plane array consisting of 13 prop-vane anemometers was located one rotor diameter upstream of the turbine spaced to characterize the inflow field. Wind direction was provided by the hub-height anemometer, and wind speed was available at 12 other locations. In addition, wind speed was provided as an average of all eight prop-vanes located at $40 \%$ span. More information regarding the specifics of the test setup and measurement uncertainties may be found in Butterfield et al. $[1,2]$.

\subsection{Instrumentation}

Upper and lower surface pressure measurements were made at different chordwise and spanwise locations. Four 32-tap ESP-32 pressure transducers were installed inside the test blade near the chordwise distributed taps. At $30 \%, 47 \%, 63 \%$, and $80 \%$ local semispan, 36 pressure taps, $1.0 \mathrm{~mm}$ in diameter, were aligned along the chord. Pressure transducers were also distributed in approximately $6 \%$ span increments at $4 \%$ and $36 \%$ chord to provide spanwise pressure distributions. Surface pressures were typically less than $480 \mathrm{~N} / \mathrm{m}^{2}(0.0 \% \mathrm{psi})$ and were accurate to $2 \%\left(10 \mathrm{~N} / \mathrm{m}^{2}\right.$, or $\left.0.001 \mathrm{psi}\right)$ of the expected dynamic pressure. The pressure transducers could be calibrated by remote control, allowing for direct calibration while the turbine was in motion. It was necessary to calibrate the transducers every 5 minutes to account for any zero drift common in solid-state transducers. Pressure data were collected at a sample rate of $520.8 \mathrm{~Hz}$ and alias- filtered at $100 \mathrm{~Hz}$. Integration of the pressure data provided normal and tangential force coefficient data. Additional information regarding the pressure measurement system may be found in References 3 and 4.

Additional data were provided by four total pressure probes located at 34\%, 50.3\%, 67.3\%, and $86 \%$ span. These data were accurate (less than $10 \%$ error) for local angles of attack (AOAs) less than $\pm 40^{\circ}$. Total pressure probes extended approximately $61 \mathrm{~cm}$ ahead of the leading edge. This resulted in a phase difference relative to an instantaneous azimuth angle between $25^{\circ}$ at $34 \%$ span to $11^{\circ}$ at $86 \%$ span. Because of the resultant phase shift, the difference in span locations, and physical limitations at inboard span locations because of high AOAs, measured dynamic pressure data could not be correlated directly with pressure data taken at $30 \%, 47 \%, 63 \%$, and $80 \%$ span. AOAs were also measured at $34 \%, 50.3 \%$, $67.3 \%$, and $86 \%$ span locations using a flag sensor described by Butterfield et al. [5]. AOA data were limited to $-20^{\circ}-0^{\circ}$, and the resonant frequency was approximately $10 \mathrm{~Hz}$. Many of the flow field perturbations were greater than $10 \mathrm{~Hz}$, and resonance could be seen in the signal. Also, at inboard span locations, the local angle of attack was greater than $40^{\circ}$. For these reasons and similar reasons mentioned above for measured dynamic pressure, AOA data also could not be directly correlated with measurements taken at $30 \%, 47 \%, 63 \%$, and $80 \%$ span. 


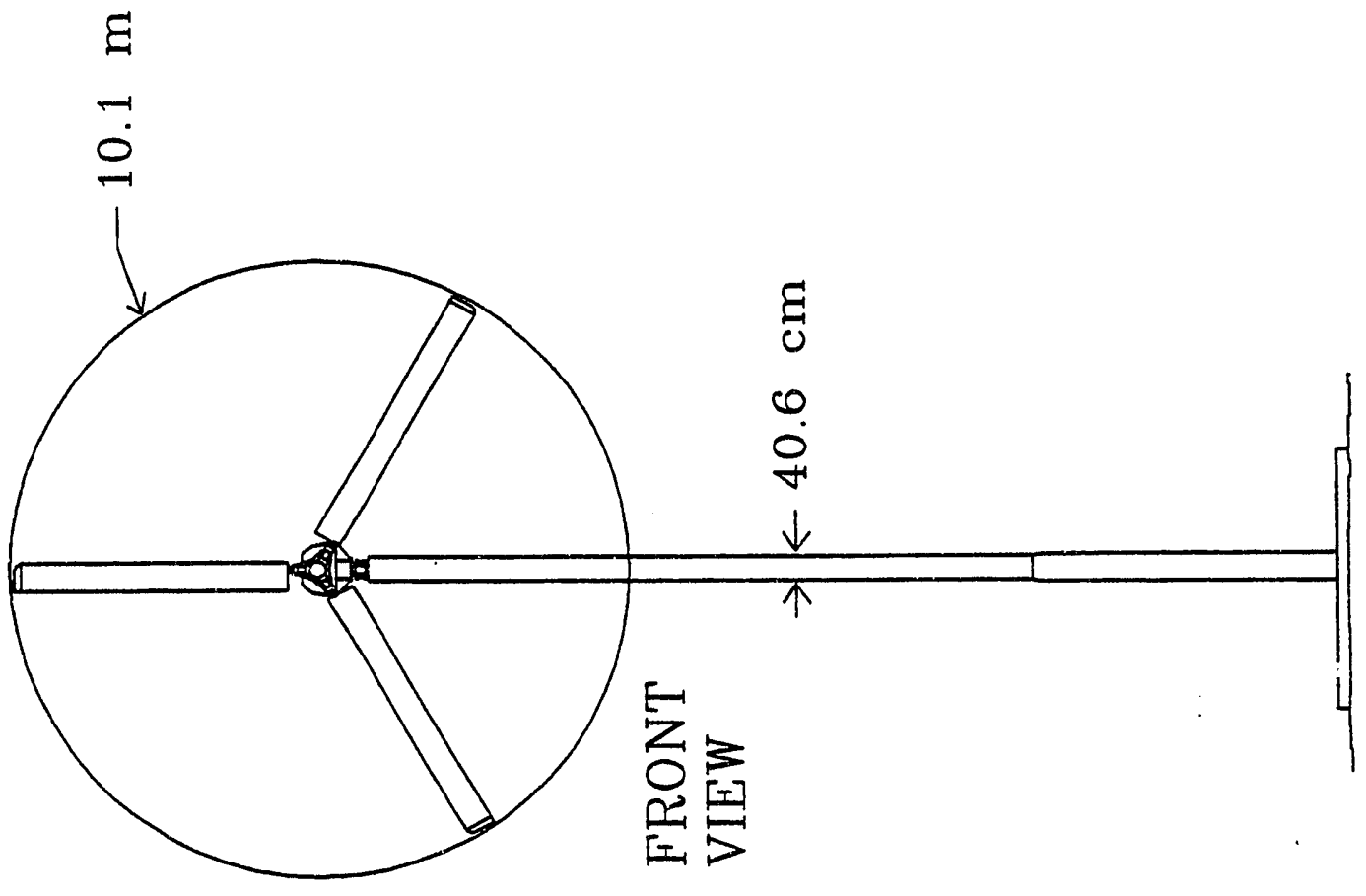

总

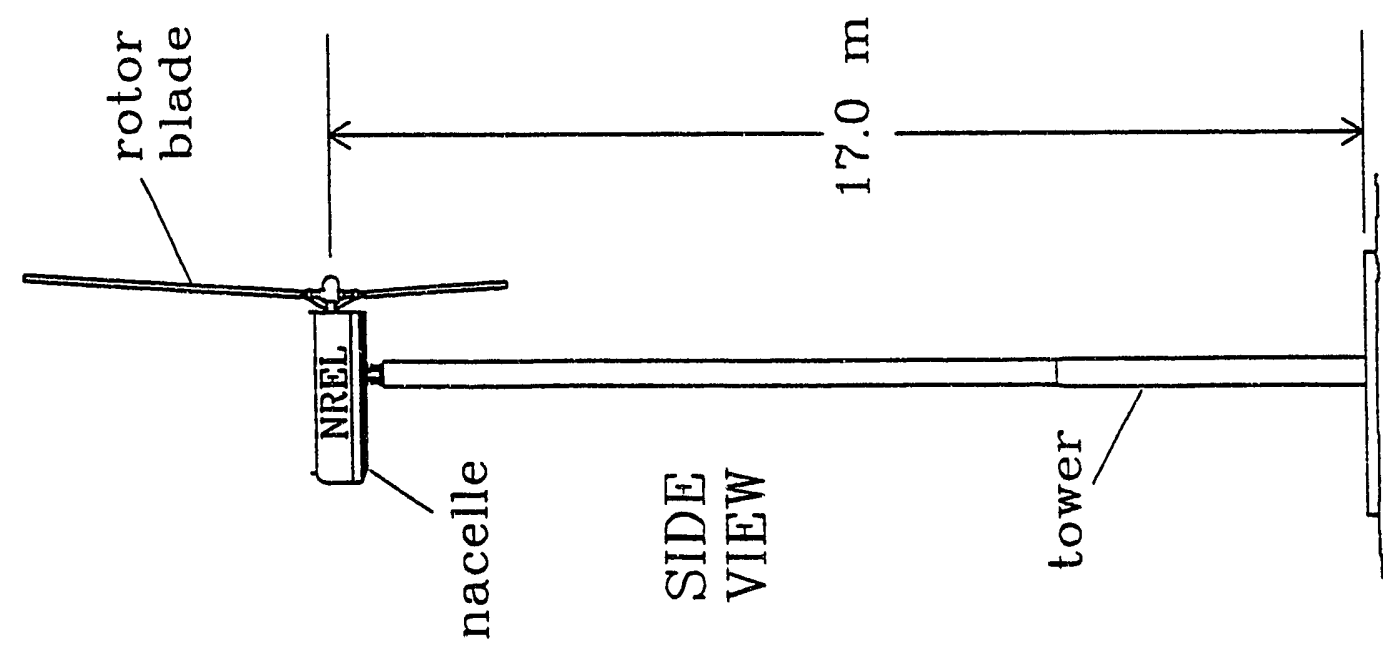




\subsection{Analytical Procedure}

Typical data sets consisted of 239 channels of data sampled at $520.8 \mathrm{~Hz}$ over 5 minutes. This generated enormous amounts of data, with each data set consisting of approximately 150 Mbytes of digital data. For obvious reasons, analysis of entire data sets was considered impractical. Instead, specific subsets were analyzed that conformed to desired input criteria such as wind velocity and wind direction. "Binning" software was written to systematically extract data for test conditions that were similar. For example, if dependent channel data were to be plotted as a function of instantaneous azimuth angle, one could choose to select data for a given disk averaged wind speed and sort these data into $1^{\circ}$ incremental bins ranging from $0^{\circ}$ to $360^{\circ}$ azimuth. Initially, bin-averaged data were used to provide pseudo-time series data for a constant win' velocity, although it was more like block-averaged data. Any dependent variable could be plotted as a function of an independent variable, with a limiting variable as a criterion that selects the desired data sets.

Because time-series data were desired, the binning technique did not prove trustworthy for several reasons. Most important, by design, the bin-averaged technique eliminates most transient disturbances. Also, only one limiting variable could be chosen in this program. For example, a constant wind velocity may he desired, but data will still be collected for relatively high yaw error. As will be shown later, nonzero y'a " results in significantly different pressure distributions and force coefficients than those that result from zelo yaw. A method that extracts continuous strings of time-series data was desired. In other words, complete cycles of data, in which azimuth angle varied from $0^{\circ}$ to $360^{\circ}$, were deemed necessary to determine the temporal dependence of desired data. For this reason, additional software was developed to extract specific cycles of data that conformed to input criteria based on disk-averaged wind speed and yaw direction. In addition, a number of cycles conforming to these criteria could be averaged, providing both mean and standard deviations.

As mentioned earlier, both dynamic pressure and AOA data could not be directly correlated with measurements taken at $30 \%, 47 \%, 63 \%$, and $80 \%$ span. These data were vital in order to determine the equivalent motion of the turbine blade. Only data taken at $67.3 \%$ and $86 \%$ span locations were consistently within the designed limits of the measurement system. Location of the total pressure probes and AOA sensors were approximately $6 \%$ span outboard from the pressure port locations. This resulted in an additional effect caused by increased rotational velocity. In order to correlate dynamic pressure and AOA data with measurements taken at $30 \%, 47 \%, 63 \%$, and $80 \%$ span, two approaches could have been used. One, all data could have been corrected according to the geometry of the turbine relative to the blade. Or, a complete theoretical model of dynamic pressure and AOA based on the geometry of the wind turbine relative to the wind could have been used. Because the first option would have required theoretical corrections anyway, the latter method was chosen. The geometric setup of the wind turbine relative to the wind is shown in Figure 2-2. Local wind velocity was modified by horizontal and vertical wind shear:

$$
\mathrm{V}_{\mathrm{w}}=\mathrm{V}_{\mathrm{ws}}+(\mathrm{r} / \mathrm{R}) \mathrm{vshr} \cos \psi+(\mathrm{r} / \mathrm{R}) \mathrm{hshr} \sin \psi
$$

Induced velocity and skewed wake corrections were added as well (see Hansen et al. [6]) with:

$$
\begin{aligned}
& \text { ao }=\left(1-\left(1-C_{b}\right)^{1 / 2}\right) / 2 \\
& \text { where } C_{b}=\text { head loss coefficient } \\
& =0.414,0.2143,0.2348 \text {, and } 0.2106
\end{aligned}
$$



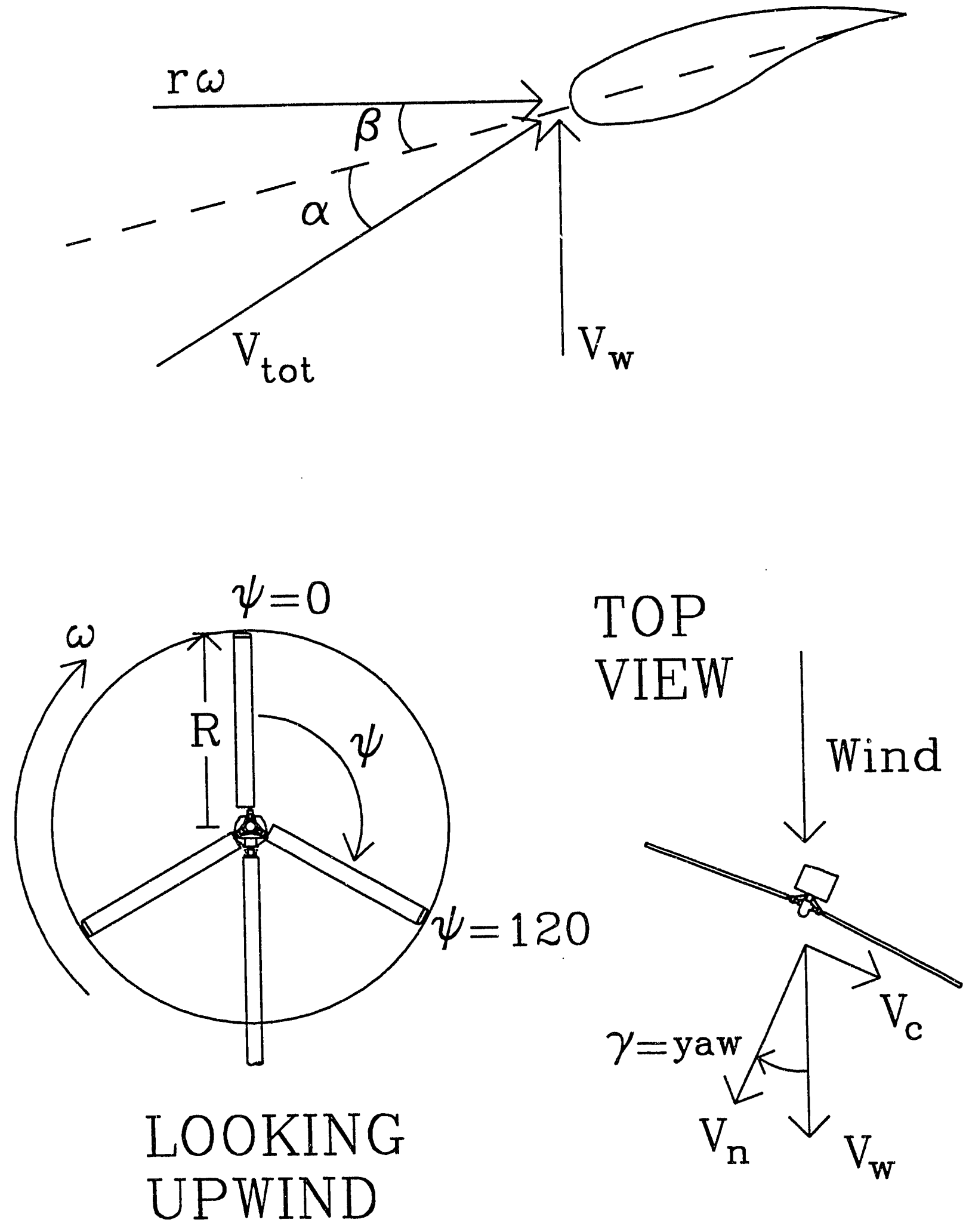

Figure 2-2. Geometry of total velocity relative to blade 
at

$30 \%, 47 \%, 63 \%$, and $80 \%$ span, respectively

skewed wake:

$$
\mathrm{sw}=\left\{1+15 \pi / 32[(1-\cos \gamma) /(1+\cos \gamma)]^{1 / 2}(\mathrm{r} / \mathrm{R}) \sin \psi\right\}
$$

and:

$$
\mathrm{a}=\mathrm{V}_{\mathrm{ind}} / \mathrm{V}_{\mathrm{w}}=\mathrm{ao}^{*} \mathrm{sw} \text {. }
$$

For nonzero yaw, local wind velocity was reduced to normal and cross-flow components relative to the rotor disk and corrected by the induced velocity:

$$
\begin{aligned}
& \mathrm{V}_{\mathrm{n}}=\mathrm{V}_{\mathrm{w}}(1-\mathrm{a}) \cos \gamma \\
& \mathrm{V}_{\mathrm{c}}=-\mathrm{V}_{\mathrm{w}} \sin \gamma
\end{aligned}
$$

Tangential velocity vector is then modified because of cross-flow:

$$
\begin{aligned}
& \mathrm{V}_{\mathrm{t}}=\mathrm{V}_{\mathrm{r}}+\mathrm{V}_{\mathrm{c}} \cos \psi \\
& \mathrm{V}_{\mathrm{s}}=\mathrm{V}_{\mathrm{c}} \sin \psi
\end{aligned}
$$

now: $\quad V_{\text {tot }}=\left(V_{\mathrm{n}}^{2}+\mathrm{V}_{\mathrm{t}}^{2}+\mathrm{V}_{\mathrm{s}}^{2}\right)^{1 / 2}$

local dynamic pressure:

$$
q=1 / 2 \rho V_{\text {tot }}^{2}
$$

relative velocity vector angle:

$$
\phi=\operatorname{atan}\left(\mathrm{V}_{\mathrm{n}} / \mathrm{V}_{\mathrm{t}}\right)
$$

local angle of attack:

$$
\alpha=\phi-B
$$

Finally, a simple model was constructed for the velocity deficit in the wake of the tower. A cosine function was used to estimate the mean velocity profile in the tower wake with a maximum deficit of $30 \%$ of the total wind velocity. This was the weakest part of the model, because vortex shedding occurred off the cylindrical tower; it will not be detailed here but may be found in the programs located in Appendix A.

The data presented in this report will summarize effects of increased wind velocity and yaw angle relative to the incoming flow. Cycle-averaged data will generally be presented, but both binned and single-cycle data will be contrasted with cycle-averaged data to illustrate the differences in data character. Cycleaveraged data consisted of five cycles of data that shared similar input criteria. In general, individual cycles of data were extracted from different points in the test run and were not continuous cycles of data. Wind velocities of $7.7,14.7,18.7$, and $23 \mathrm{~m} / \mathrm{s}$ were used for a $0^{\circ}$ yaw error. Yaw errors of $30^{\circ},-30^{\circ}, 15^{\circ}$, and $-15^{\circ}$ were examined for a constant wind velocity of $15 \mathrm{~m} / \mathrm{s}$. Surface pressure distributions were plotted as a function of azimuth angle (thereby representing a time series of data because the rotation rate 
was constant) at $30 \%, 47 \%, 63 \%$, and $80 \%$ spanwise locations. Surface pressure data were normalized by freestream dynamic pressure that was calculated based on rotational velocity and wind velocity alone. Integrated normal force coefficients were plotted as a function of azimuth angle. In addition, normal force data were plotted as a function of theoretical geometric AOA to allow for direct comparisons between steady-state wind tunnel data. These data were normalized by theoretical dynamic pressure incorporating effects of yaw and tower shadow. This was necessary because dynamic pressure is constant for wind tunnel tests but varies over the rotation cycle because of cross flow for a yawed turbine and inflow variations. 


\subsection{Results}

\subsection{Theoretical versus Experimental $q$ and $\alpha$ :}

Comparisons between theoretical and measured dynamic pressure (q) are shown in Figure 3-1 for the $30^{\circ}$ yaw test case. Measured dynamic pressures were taken for single cycle data and were averaged over five similar cycles for wind velocity $=15+1.5 \mathrm{~m} / \mathrm{s}$ and $30^{\circ} \pm 3^{\circ}$ yaw error. The four spanwise locations examined correspond to pressure probe locations of $34 \%, 50.6 \%, 67.3 \%$, and $86 \%$ span. As can be seen, measured $\mathrm{q}$ is out of phase with theoretical $\mathrm{q}$. This can be explained by the fact that the pressure probes extended $61 \mathrm{~cm}$ ahead of the blade leading edge. Theoretical $\mathrm{q}$, however, underpredicts maximum measured $q$ values and over-predicts minimum measured $q$ values. Here, there are four possible sources for error. First, because the pressure probes are accurate to only $40^{\circ}$, dynamic pressure measurements were not considered accurate at $34 \%$ span and azimuth angles from $0^{\circ}$ to $90^{\circ}$ and from $270^{\circ}$ to $360^{\circ}$. In this region, the local AOA varies between $45^{\circ}$ and $65^{\circ}$. Second, the turbine is generating power, and energy that is extracted from the wind may result in decreased relative velocity (this is termed induced velocity). Third, the turbine is yawed, and a skewed wake effect, which results in increased velocity on one side of the turbine and decreased wind velocity on the other side, may result in differing q values. Fourth, effects of horizontal and vertical wind shear were not accounted for in this model.

A second physical model was then formulated that included the effects of induced velocity, skewed wake, and wind shear. These results may be seen in Figure 3-2 for the $30^{\circ}$ yaw test case. As can be seen, theoretical results compare most favorably with experimental results at $86 \%, 67.3 \%$, and $50.6 \%$ spanwise locations. Errors at $34 \%$ span may be attributed to physical limitations inherent to the total pressure probe. Comparison between data taken at $-30^{\circ}$ yaw error (Figure 3-3) show that theoretical q almost exactly matches the measured $q$ values except for the phase difference. For both yaw cases, however, changes in $\mathrm{q}$ as a result of passage through the tower wake demonstrate the inadequacy of the assumed velocity deficit in the wake or possible response of the probe. It appears clear that shedding of vortex structures occurs, resulting in large, transient fluctuations in dynamic pressure. Overall, the theoretical dynamic pressure model appears to adequately model actual dynamic pressure. This allows for direct correlation of AOA and dynamic pressure with measurements taken at 30\%, 47\%, 63\%, and 80\% span.

Comparisons between theoretical and measured AOA are shown in Figure 3-4 for cycle-averaged data for $30^{\circ}$ and $-30^{\circ}$ yaw error. The test conditions are equivalent to those for the measured q results of Figures 3-2 and 3-3. Span locations of $86 \%$ and $67.3 \%$ alone are examined, because these are the only two AOA probes known to be fully functional in the expected AOA range. All corrections input into the theoretical q model are also incorporated into the AOA model. In both cases, the effect of resonance is clear as the probe passes through the tower wake. The resonance damps out in one-fourth of the rotational cycle, and a resonant frequency of $10 \mathrm{~Hz}$ was observed. This resulted in some phase differences in measured and theoretical AOA because the probe response time was too long to measure the high frequency transient as the blade passed through the tower wake. The theoretical model, however, continues to underestimate the maximum $\mathrm{AOA}$ from $0^{\circ}$ to $45^{\circ}$ and $325^{\circ}$ to $360^{\circ}$ azimuth. The theoretical model predicts AOA variation for the negative yaw test case. Again, the deficit in local AOA as a result of tower shadow passage is evident. Modification of the tower wake incorporating a vortex shedding model may be necessary.

\subsection{Data Collection Comparisons}

As mentioned earlier, two different software programs were written to extract data of interest. The "binning" technique extracted single data points and placed these points in specific bins. To generate binned data, time-series data could be sorted according to azimuth angle using a limiting variable such 

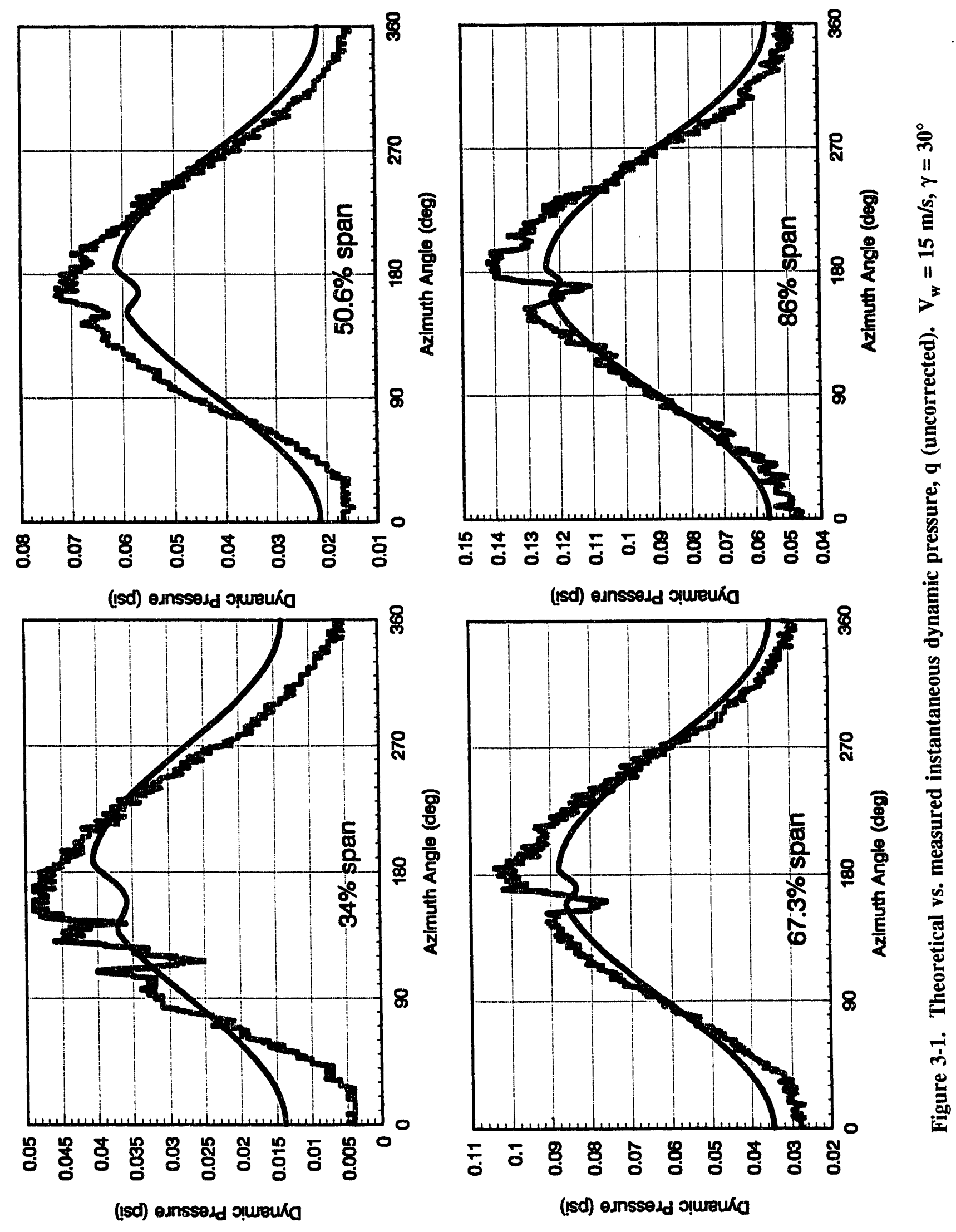

(sd) ednssedd ग!ureuka 

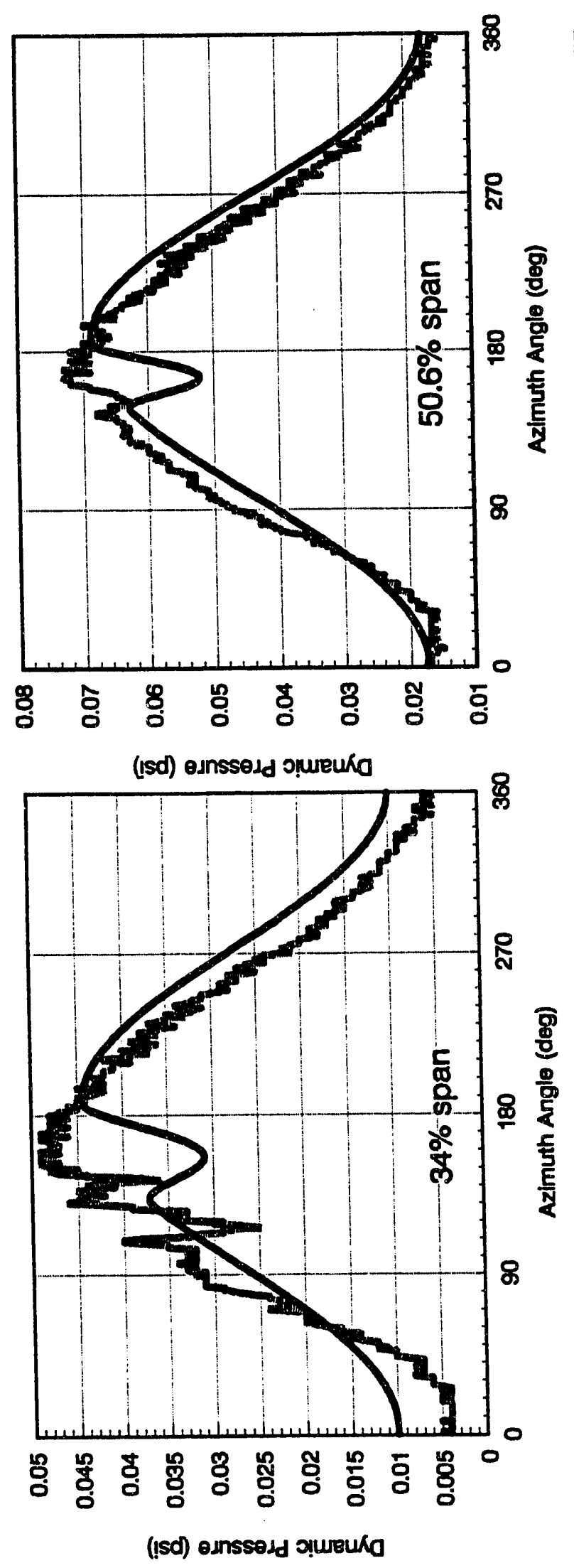

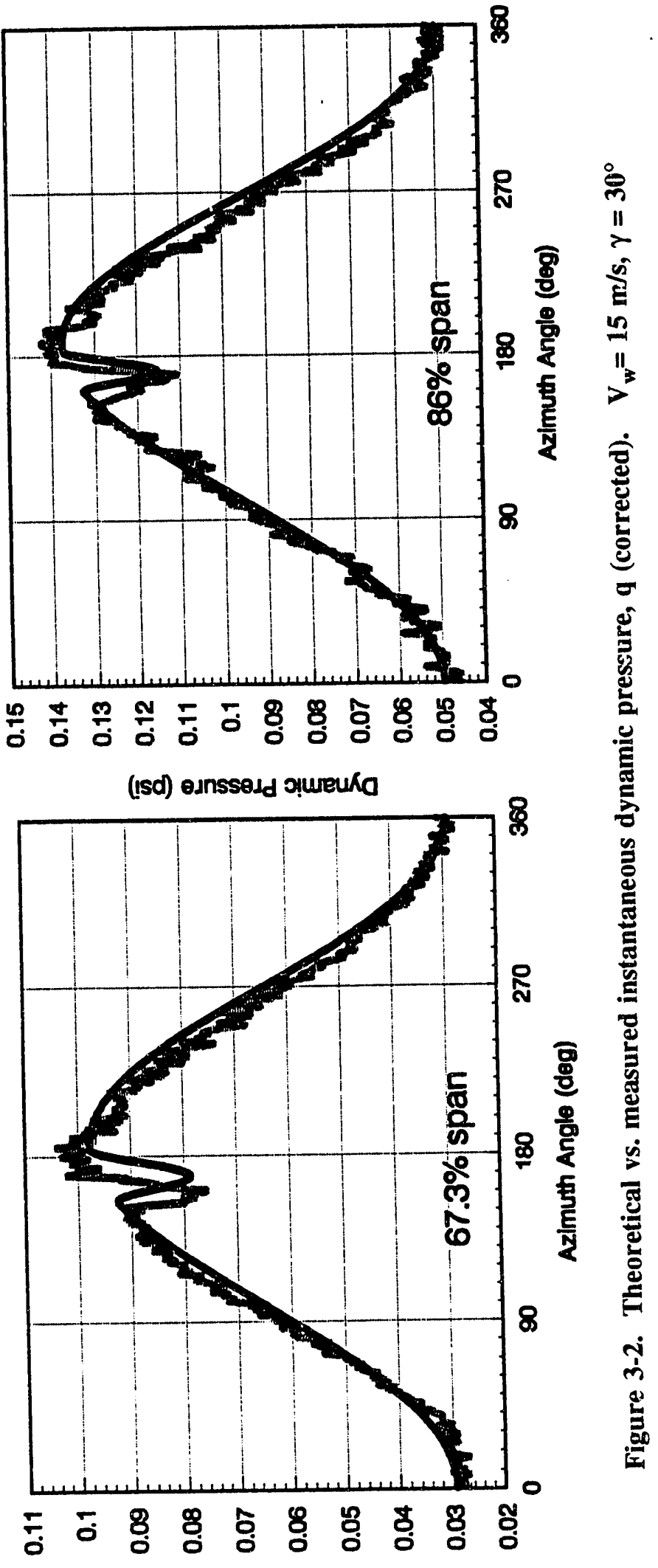

(isd) enssedd pureuka 


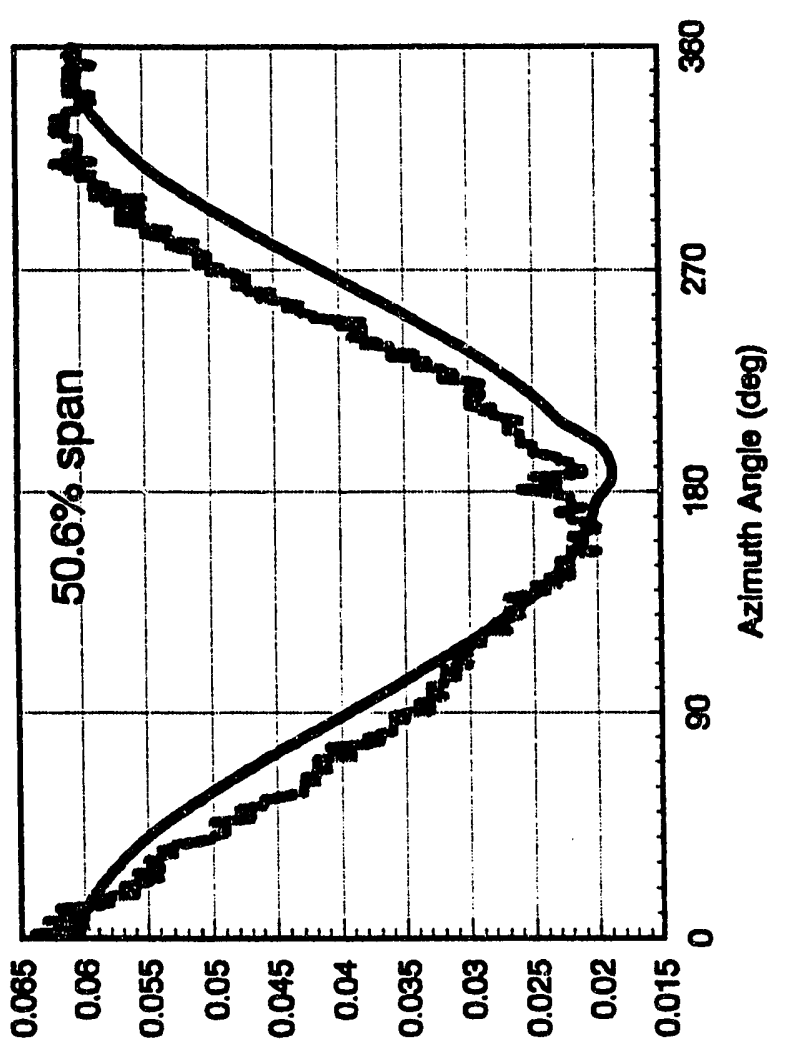

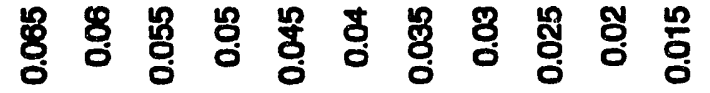

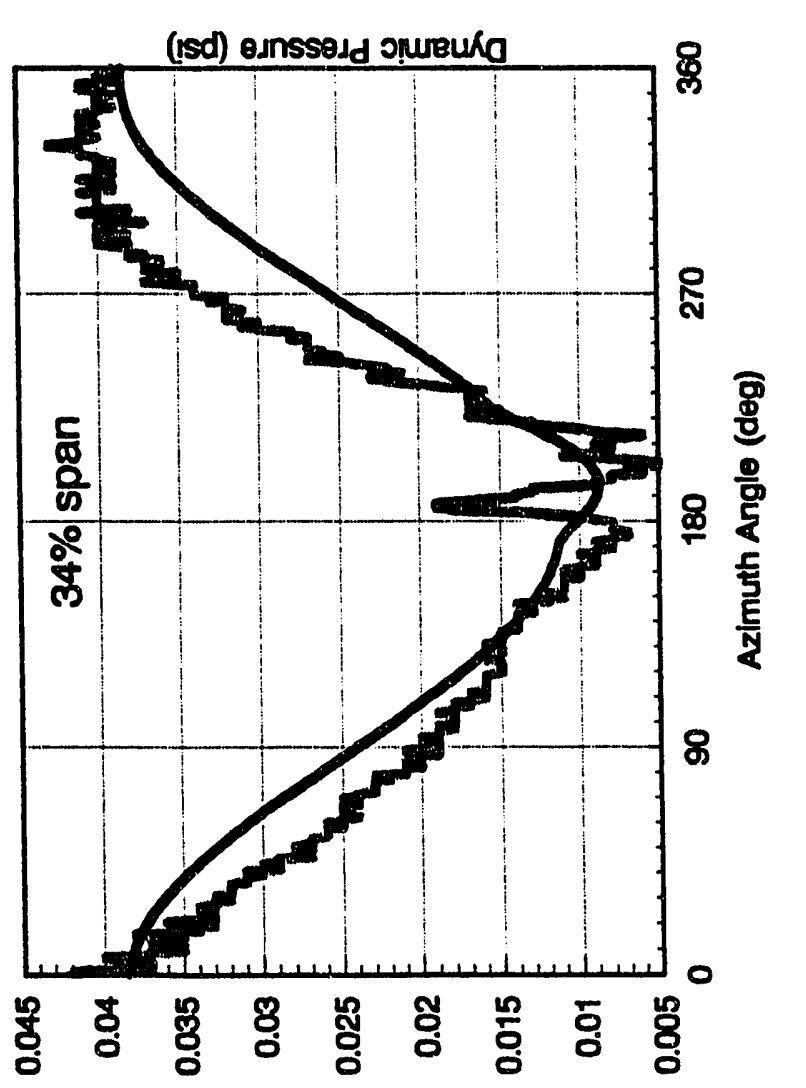

(Isd) inssedd giureuka

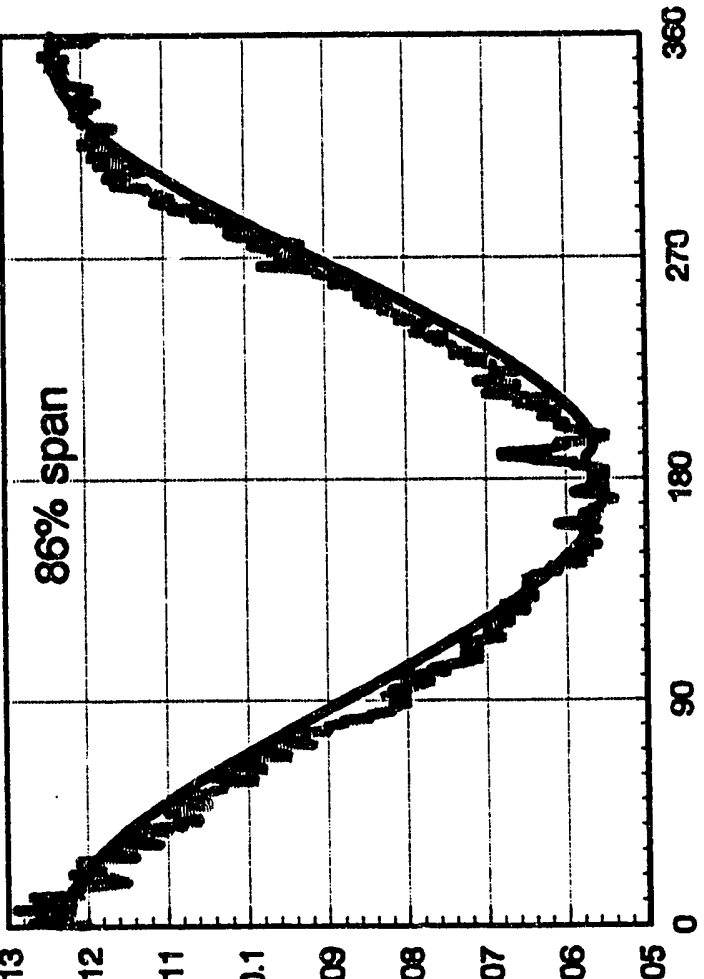

$\frac{m}{0}$ N 둥

(iso) ednssedd pureuka

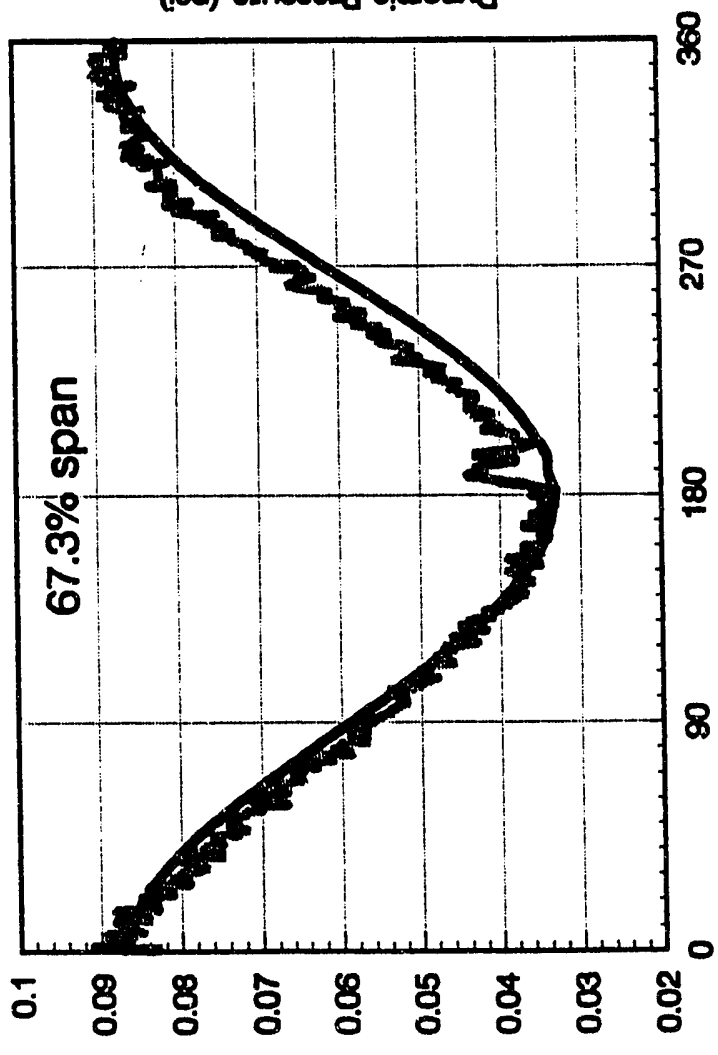

(isd) ednssedd ọureuka

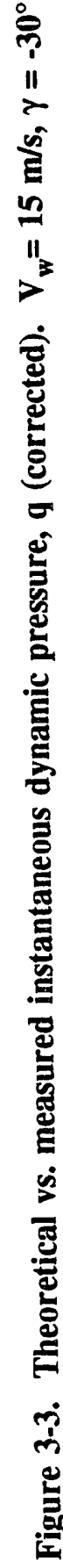



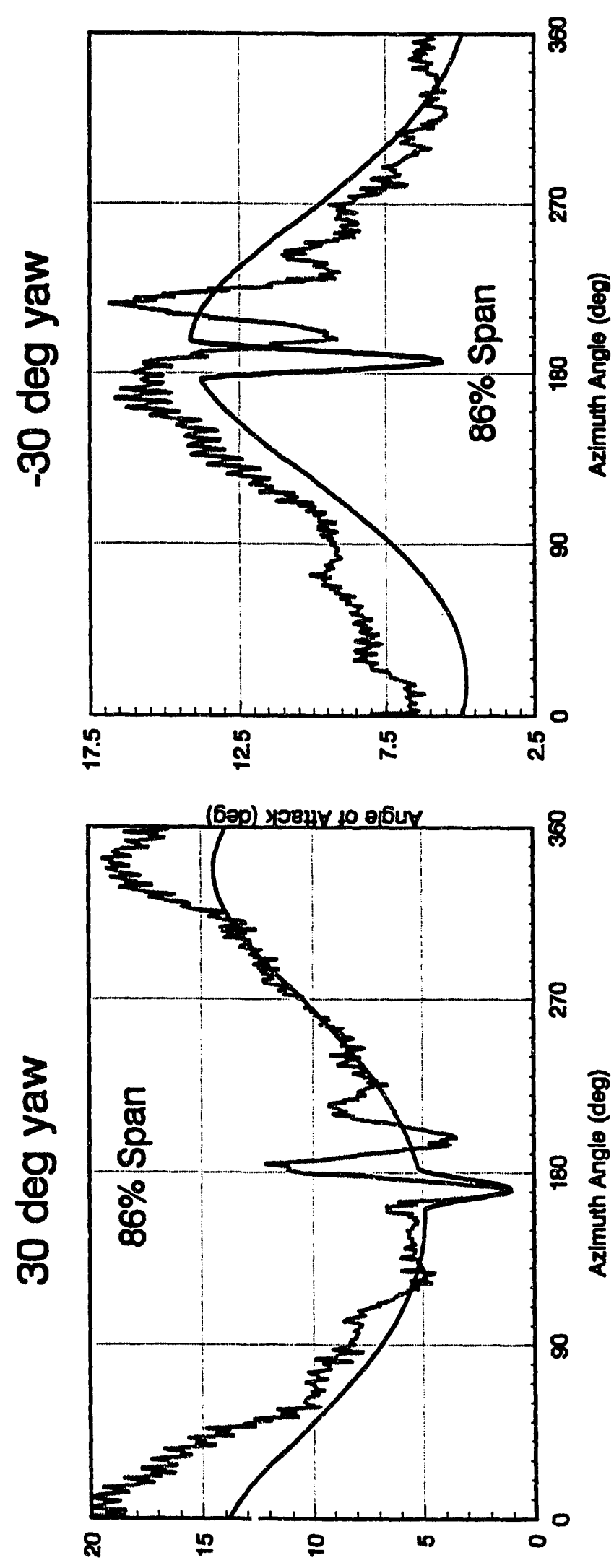

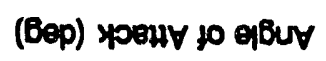

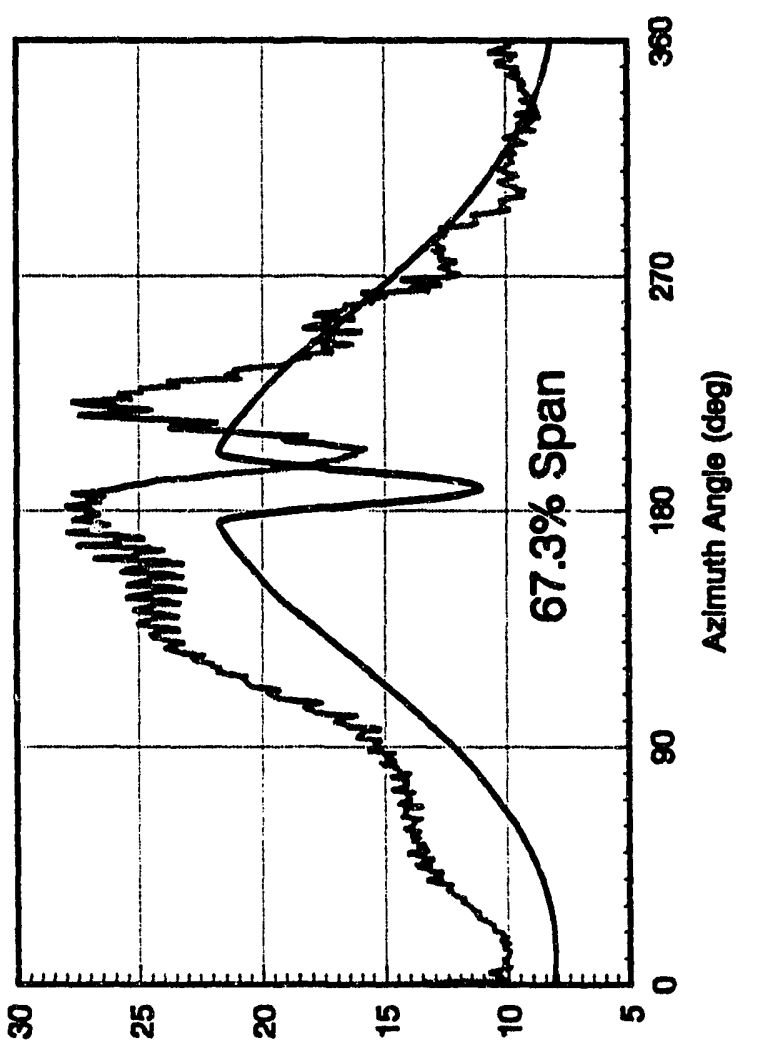

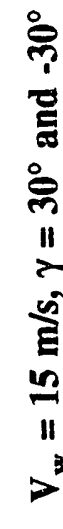

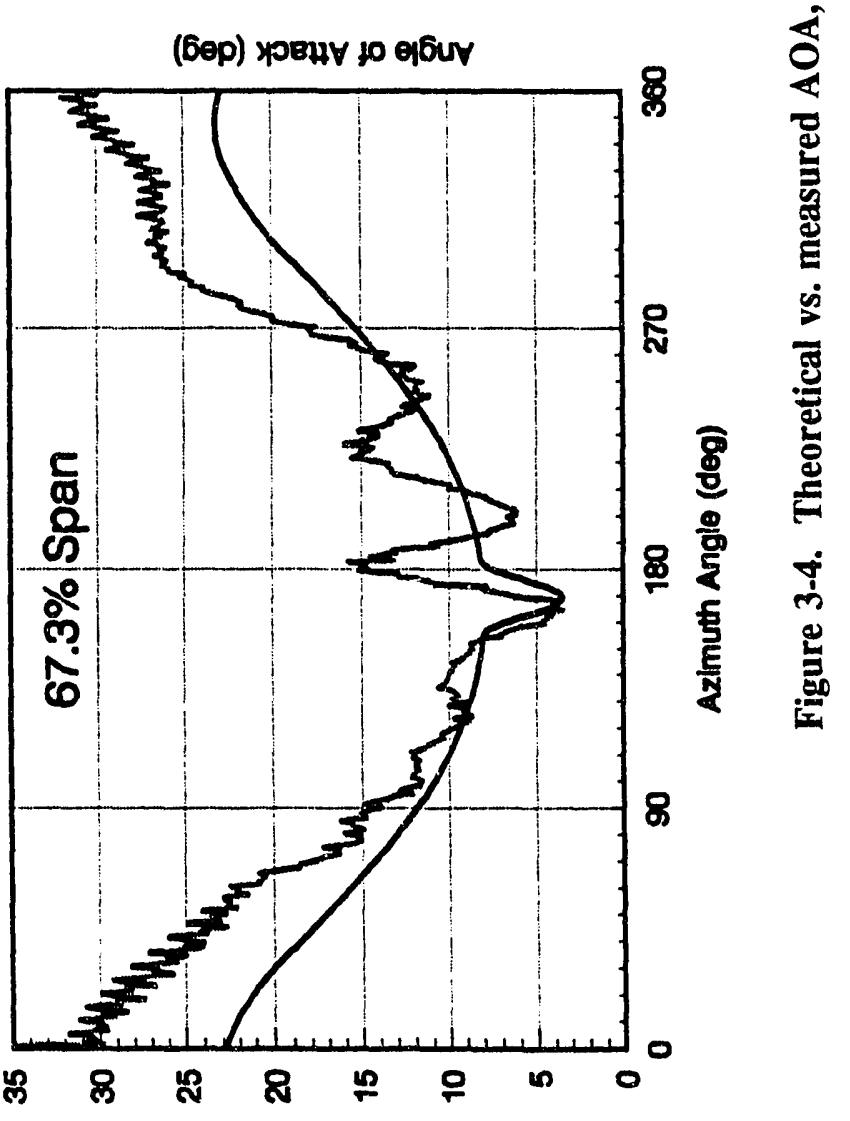

(6ep) ypy to efour 
as wind velocity or wind direction to sort out the desired data. A cycle extraction program, conversely, extracted specific strings of time-series data for one complete blade rotation cycle ( $0^{\circ}$ to $360^{\circ}$ azimuth). The second method therefore extracted a real time series of data, and both wind velocity and wind direction were used to sort out the desired data. In this manner, a single cycle of data could be analyzed, or multiple cycles having similar sorting criteria could be ensemble-averaged to provide mean and standard deviation values. In this section, single-cycle data will be compared with both the binning method and the cycle extraction method, and the differences will be highlighted.

Upper surface pressure distributions are shown in Figures 3-5 to 3-7 at 30\% span for the $15 \mathrm{~m} / \mathrm{s}$ wind velocity and $30^{\circ}$ yaw error test case for single-cycle, bin-averaged and cycle-averaged test cases, respectively. Reduced frequency values, $\mathrm{K}$, are also shown on each plot, and their significance will be discussed later in this report. Suction pressure coefficient (-Cp) was plotted along the chord as a function of azimuth angle, providing a topographical pressure plot. $\mathrm{Cp}$ was normalized by a constant value of dynamic pressure that incorporated constant local rotation velocity and disk-averaged wind speed only. It is stressed that the time-varying $\mathrm{q}$ was not used. Two suction pressure peaks were seen in the singlecycle data taken at $30 \%$ span. The first occurred at approximately $130^{\circ}$ azimuth $(-5)$ and the second occurred at approximately $185^{\circ}(-10.5)$. As the cycle continued, a suction pressure ridge in the direction of increasing chord and azimuth angle was clearly seen.

Bin-averaged data are shown in Figure 3-6 at 30\% span for equivalent test conditions. Some major differences existed between these data and the bin-averaged data. The first suction pressure peak appeared significantly smaller (-3.8) compared with the single-cycle data, and the magnitude of the stall LE suction pressure peak is approximately $20 \%$ less, as well. In addition, the bin-averaged technique "washes out" the suction pressure peak. Instead of a large, transient spike, the bin-averaged suction peak persisted from $175^{\circ}$ to $200^{\circ}$ azimuth. Finally, the suction pressure ridge seen clearly in the single-cycle data did not appear as clear in the bin-averaged data, demonstrating that the bin-averaged technique smooths out most of the unsteadiness.

Cycle-averaged data, averaged over five cycles, are shown in Figure 3-7, and they demonstrated similar phenomena compared with those of bin averaged data. Both LE suction pressure peaks were approximately $20 \%$ smaller than in the single-cycle data. The suction pressure ridge appeared more clearly in the cycle-averaged data, than in the bin-averaged data. The suction pressure ridge was most clear in single-cycle data, however. Differences between bin-averaged and cycle-averaged data may be further illustrated in Figures 3-8 and 3-9 for pressure distributions taken at 63\% span for bin-averaged and cycle-averaged data, respectively. In both cases, suction pressure gradually rose from $180^{\circ}$ to $270^{\circ}$ azimuth. The suction pressure peak was approximately $25 \%$ greater $(-8.5)$ for the cycle-averaged case, however. In addition, the maximum suction pressure rise was followed by a precipitous decrease in suction pressure to -1.0 for the cycle-averaged case, whereas a gradual decline in suction pressure was observed in the bin-averaged data.

One final comparison is shown in Figures 3-10 and 3-11 for suction pressure standard deviation distributions. For better comparison, these data are plotted in fashion similar to that of the surface pressure distributions. Standard deviations were taken at $63 \%$ span. As can be seen in Figure 3-10, a maximum standard deviation of approximately 3 was observed in the bin-averaged data. In addition, relatively high standard deviation persisted throughout a large portion of the rotation cycle ( $90^{\circ}$ to $330^{\circ}$ azimuth). Error seen in the cycle- averaged data (Figure 3-11) were one-third that of bin-averaged results.

These standard deviations were also seen only at approximately $180^{\circ}$ and $270^{\circ}$ azimuth, where large transients existed. A large decrease in standard deviation was seen at $280^{\circ}$ azimuth, corresponding to the precipitous decrease in suction pressure seen in Figure 3-9. In general, the cycle-averaged data were 
TP-442-4864

(do-) 7La!plftoos asnssald woltons

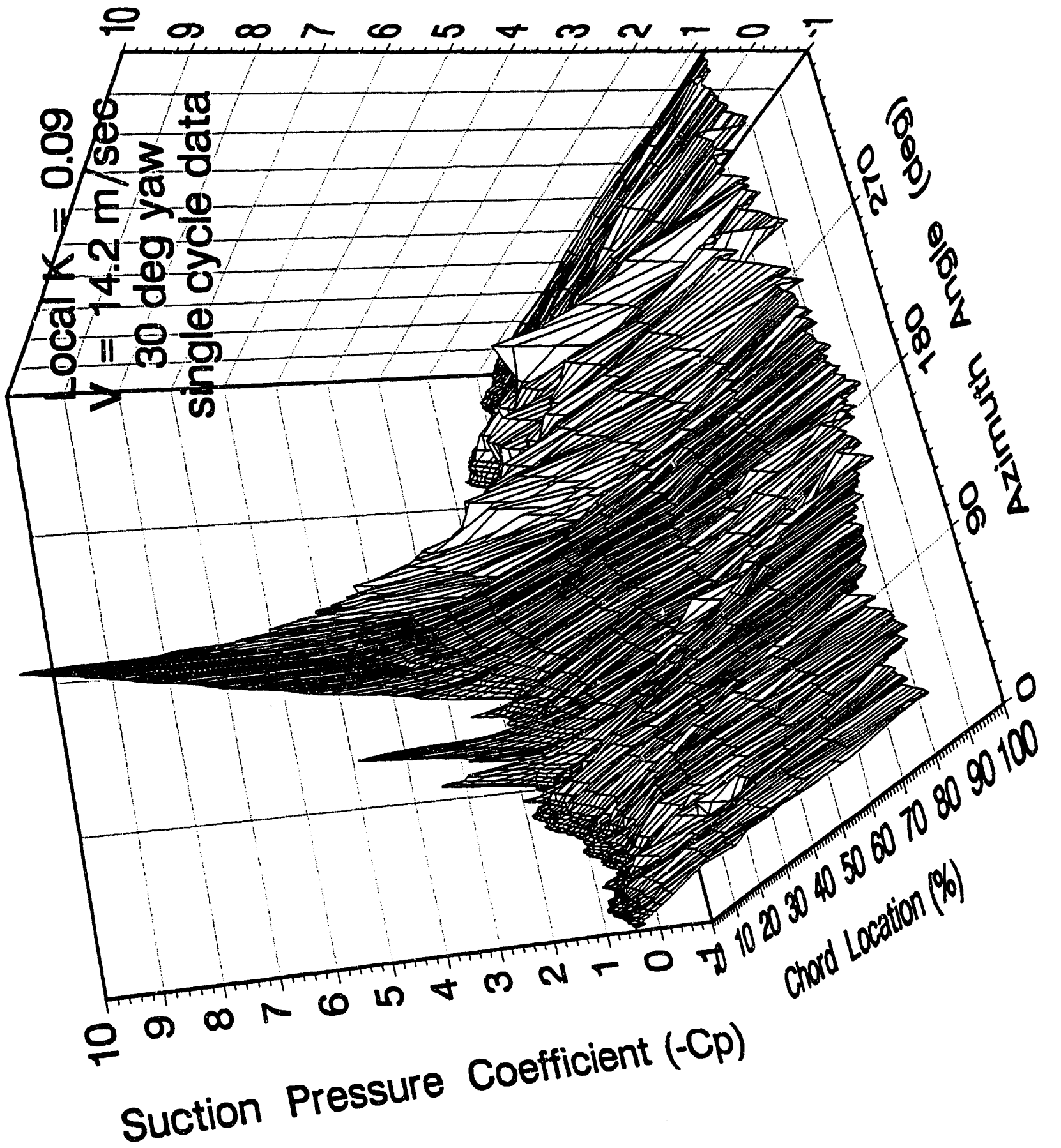

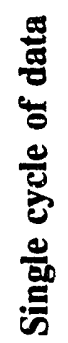

氖

结

$\stackrel{\mathscr{m}^{\circ}}{\circ}$

ले

II

ही

$\stackrel{+}{\sharp}$

$>$

ن

穿

을

巡

苋

น่ं 
(d)-) zuo!o!lyoos esnsseld wolfans

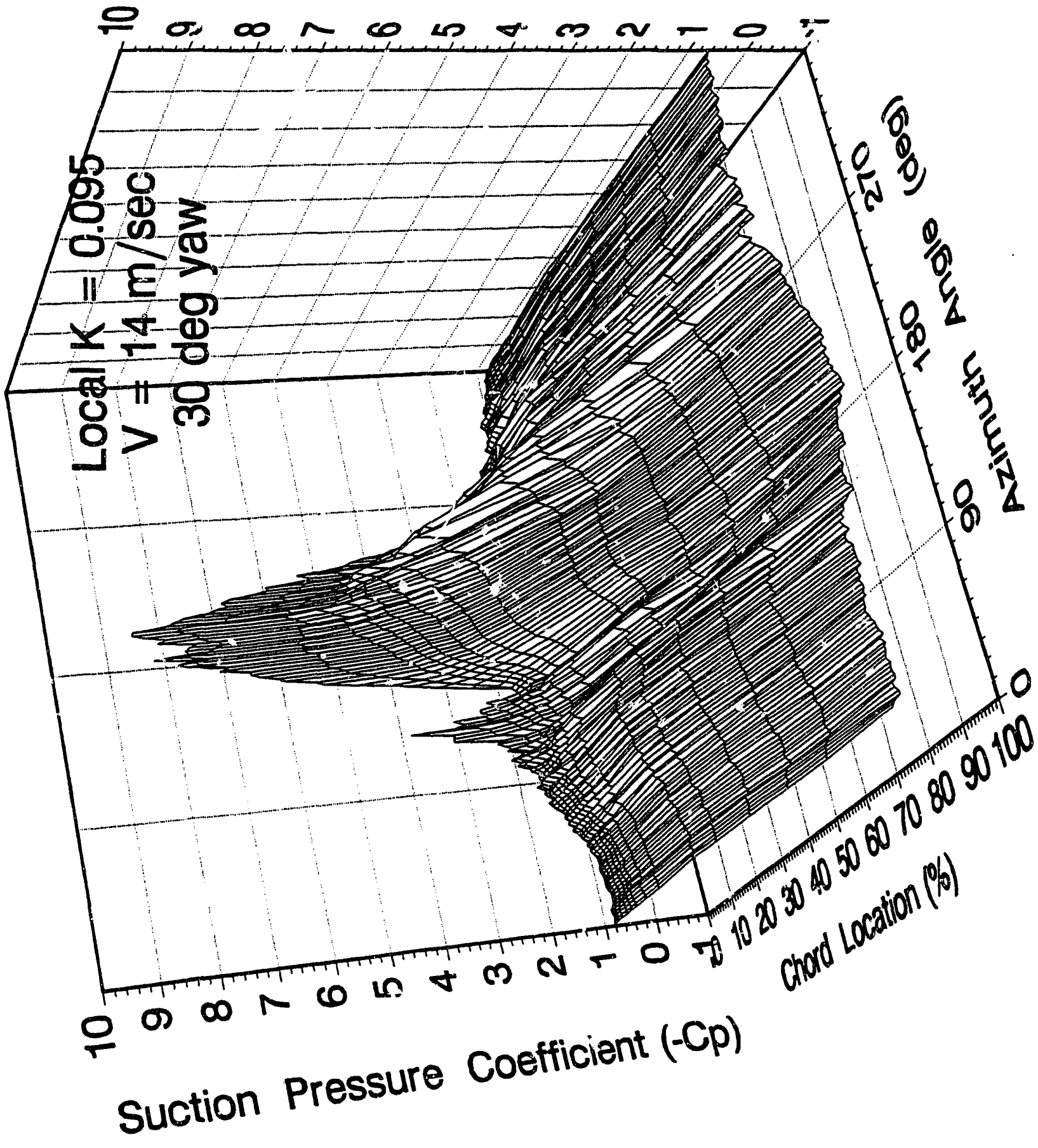

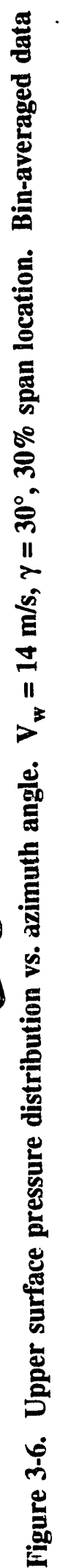




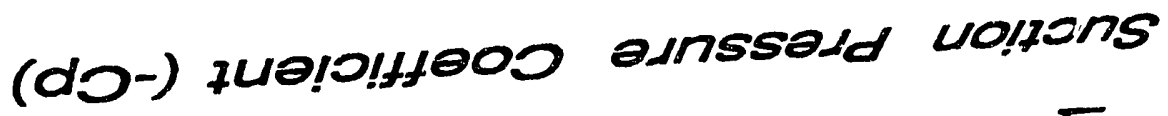

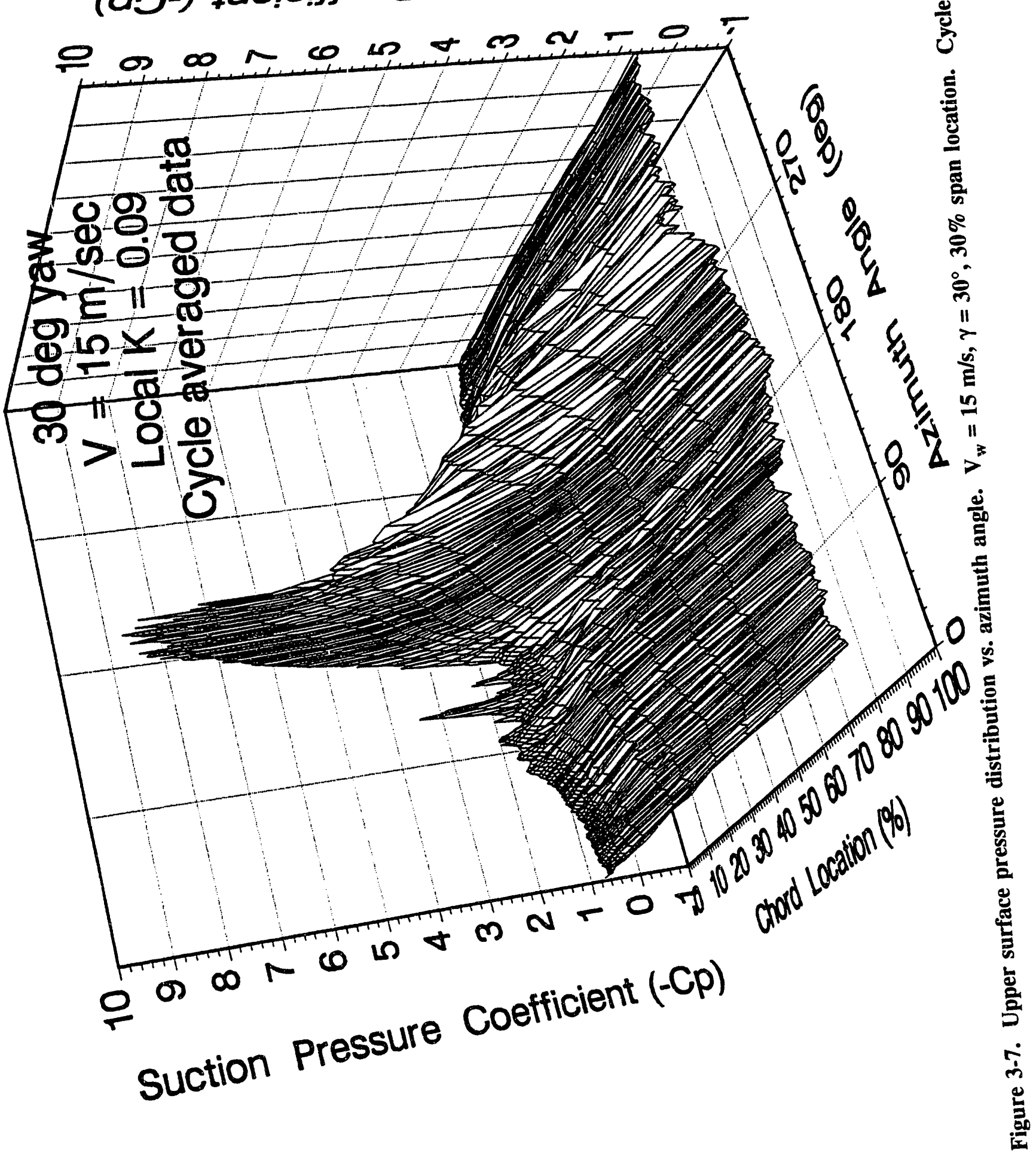


(d)-) 7we!o!fyeo0 esnssedd wolfons

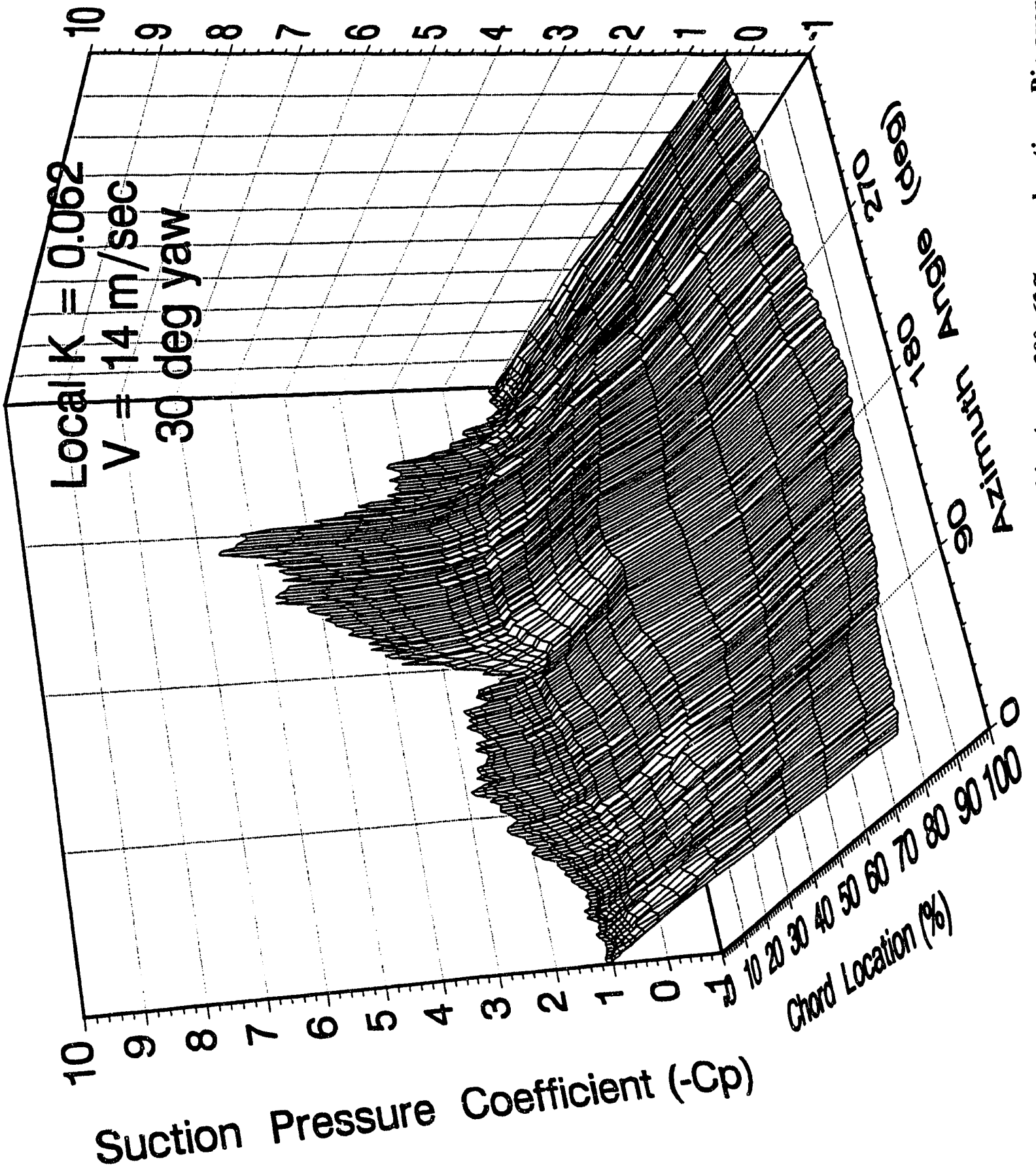

폻



3

递

ํㅗㄹ

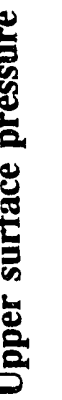

m. 


\section{(dD-) 7ue!o!fleod asmssald wolpons}

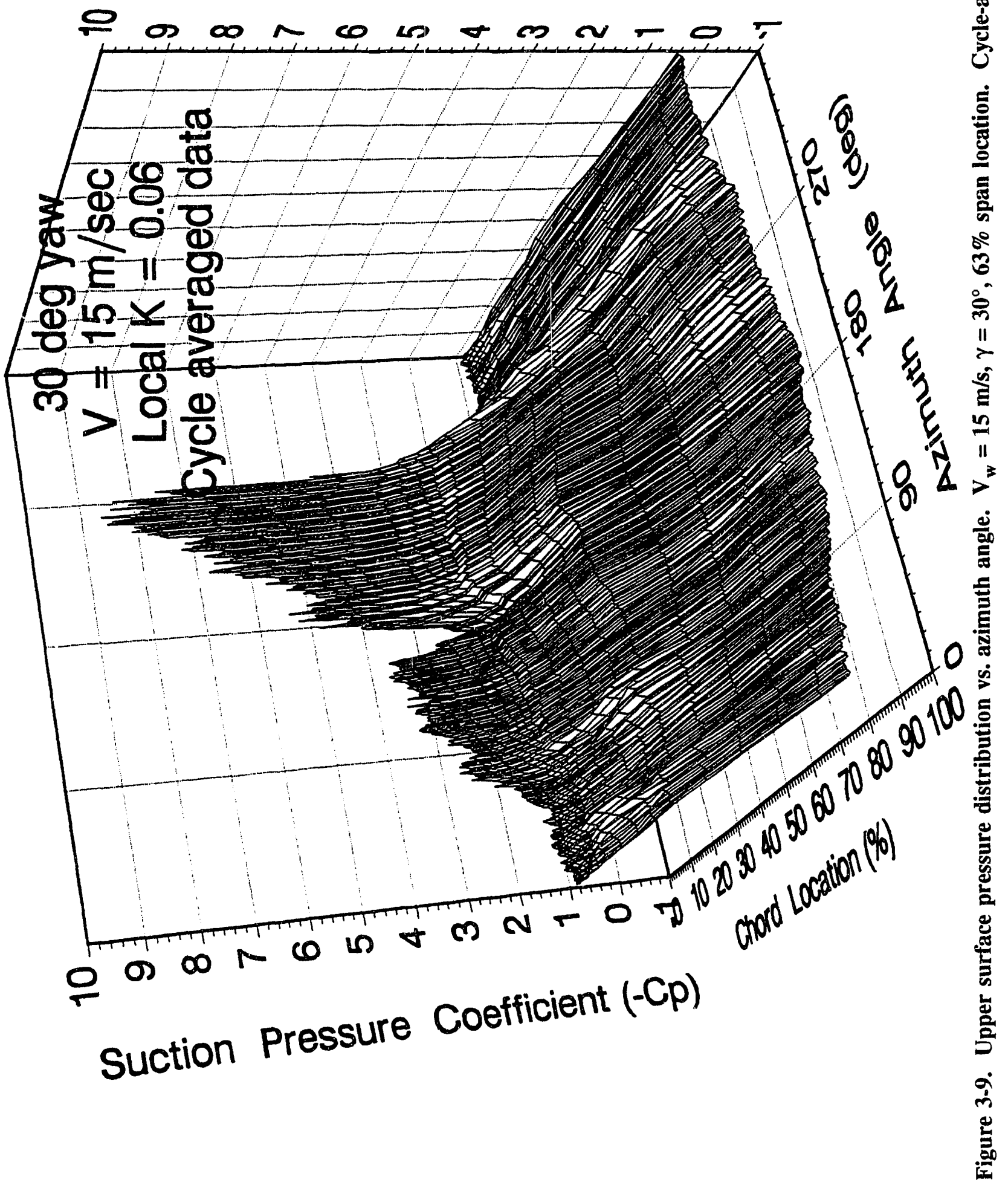




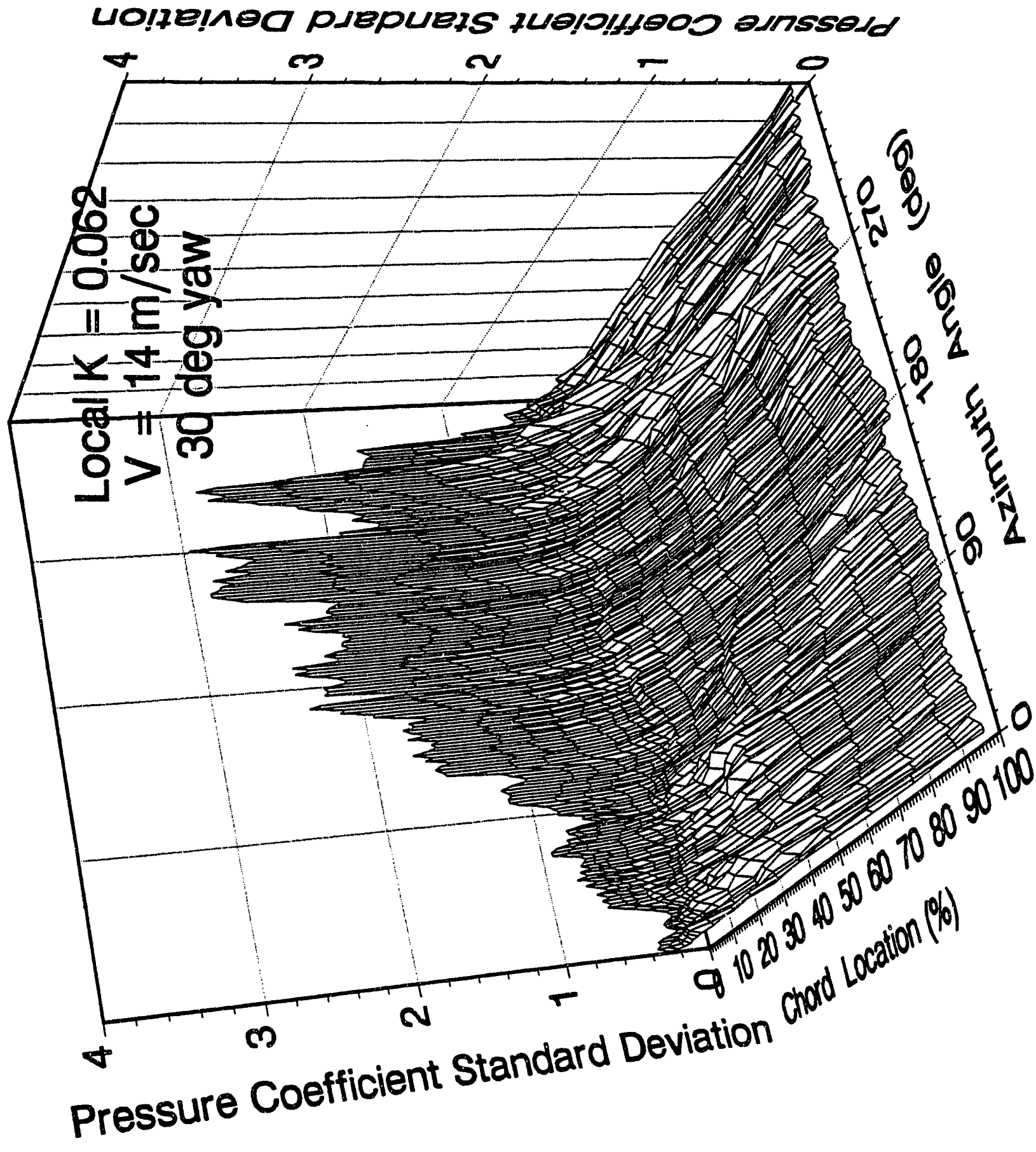


(do) wolfe!noa wets esnssedd wolpons

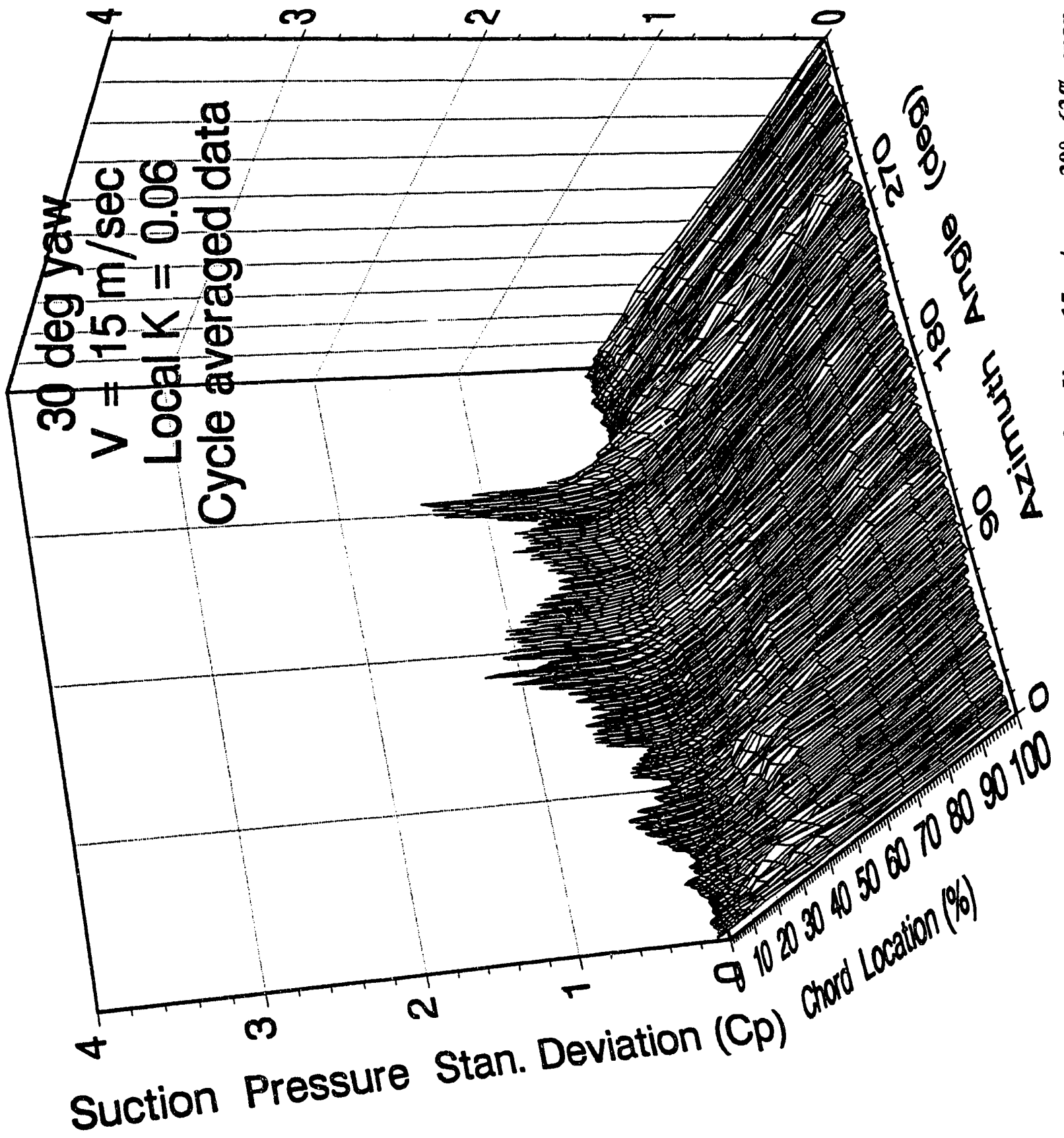

$\stackrel{d}{3}$

芯

ํㅠํ

กิ

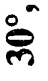

II

है

n

- (1) $10 \Omega$ (1)

$\frac{\dot{s}}{\mathrm{x}}$

递

$\dot{2}$

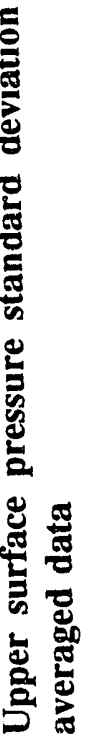

$\dot{m}$

总 
consistently more transient in nature and provided a statistically more accurate account of trends seen in the data, compared with bin-averaged results. Unless otherwise noted, all data presented in this report are cycle-averaged over five cycles.

\subsection{Zero Yaw Test Cases}

Prior to discussion of zero yaw results, a brief summary of static data is in order. Figure 3-12 shows geometric AOA variation as a function of wind speed at $30 \%, 47 \%, 63 \%, 80 \%$, and $100 \%$ span for zero yaw test cases. As can be seen, geometric angle of attack increased with increased wind velocity, and decreased from $30 \%$ to $80 \%$ spanwise locations. Static data revealed that this particular airfoil section stalled at approximately $16^{\circ}$. For stalled conditions, $\mathrm{Cp}$ values of approximately -1.0 were observed from leading to trailing edge, remaining approximately constant over the entire upper surface. $C_{n}$ values of approximately 0.922 were observed at stall.

Upper surface pressure distributions are plotted topologically in Figures 3-13 to 3-16 for 30\%, 47\%, 63\%, and $80 \%$ spanwise locations, respectively for zero yaw and $14.7 \mathrm{~m} / \mathrm{s}$ wind velocity. Nondimensional pitch rate, at, is shown on each plot, and its significance will be discussed later in this report. Again, five cycles of data were averaged. Suction pressure was plotted along the chord as a function of instantaneous azimuth angle. At $\psi=0^{\circ}$, the blade is at 12:00; and at $\psi=180^{\circ}$, the blade is at $6: 00$, directly behind the tower. For Vws $=14.7 \mathrm{~m} / \mathrm{s}$, local geometric AOAs were $40^{\circ}, 27^{\circ}, 19.5^{\circ}$, and $13.75^{\circ}$ at $30 \%, 47 \%, 63 \%$, and $80 \%$ span, respectively. At $30 \%$ span, the spanwise pressure distribution showed a pronounced increase in LE suction pressure at $\psi=190^{\circ}$. This distribution also shows a ridge in suction pressure that formed in the direction of increasing chord and azimuth angle. This ridge did not persist to the trailing edge, however. Because of the high AOA, one would expect stalled flow conditions at $30 \%$ span, but the pressure distribution was not indicative of this.

At $47 \%$ span, a surge in LE suction pressure was observed at $\psi=195^{\circ}$. A ridge of suction pressure was seen to extend to the trailing edge. During most of the cycle, the pressure distribution was chaiacteristic of stalled flow conditions and was similar to that seen for two-dimensional wind tunnel tests at high AOAs.

At $63 \%$ span, there was also a pronounced increase in LE suction pressure at $\psi=195^{\circ}$. LE pressure decreased moderately, however, and did not exhibit a highly transient signature typical at $30 \%$ and $47 \%$ span. Leading edge suction pressures persisted to approximately $\psi=270^{\circ}$, where a significant drop-off was observed. The blade section did not appear to stall, however, even though the local AOA was greater than the static stall angle. The pressure distribution implied attached flow conditions throughout the entire rotation cycle. Finally, a suction pressure ridge was not seen in the pressure distribution.

At $80 \%$ span, there was little variation in the pressure distribution except in the vicinity of the tower wake, where a modest decrease $(-1.0)$ in suction pressure was observed. There was a modest buildup of leading edge suction pressure at $\psi=270^{\circ}$. Overall, the pressure distribution indicated an attached flowfield throughout the entire rotation cycle.

Effects of wind velocity may be demonstrated in Figures $3-17$ to $3-19$ for $\mathrm{V}_{\mathrm{ws}}=7.7 \mathrm{~m} / \mathrm{s}, 18.7 \mathrm{~m} / \mathrm{s}$, and $23 \mathrm{~m} / \mathrm{s}$ at $63 \%$ span. These wind speeds corresponded to effective geometric AOAs of $6^{\circ}, 25.5^{\circ}$, and $31^{\circ}$, respectively. At $7.7 \mathrm{~m} / \mathrm{s}$, the pressure distribution indicated attached flow with a small decrease in $\mathrm{LE}$ suction pressure as the blade passed through the tower wake. This pressure distribution was consistent with that seen for steady-state wind tunnel tests at low AOAs. An increase in wind velocity to $18.7 \mathrm{~m} / \mathrm{s}$ showed extremely different behavior. A large transient spike in LE suction pressure was seen as the blade passed through the tower wake. This particular pressure distribution appeared similar to the pressure 


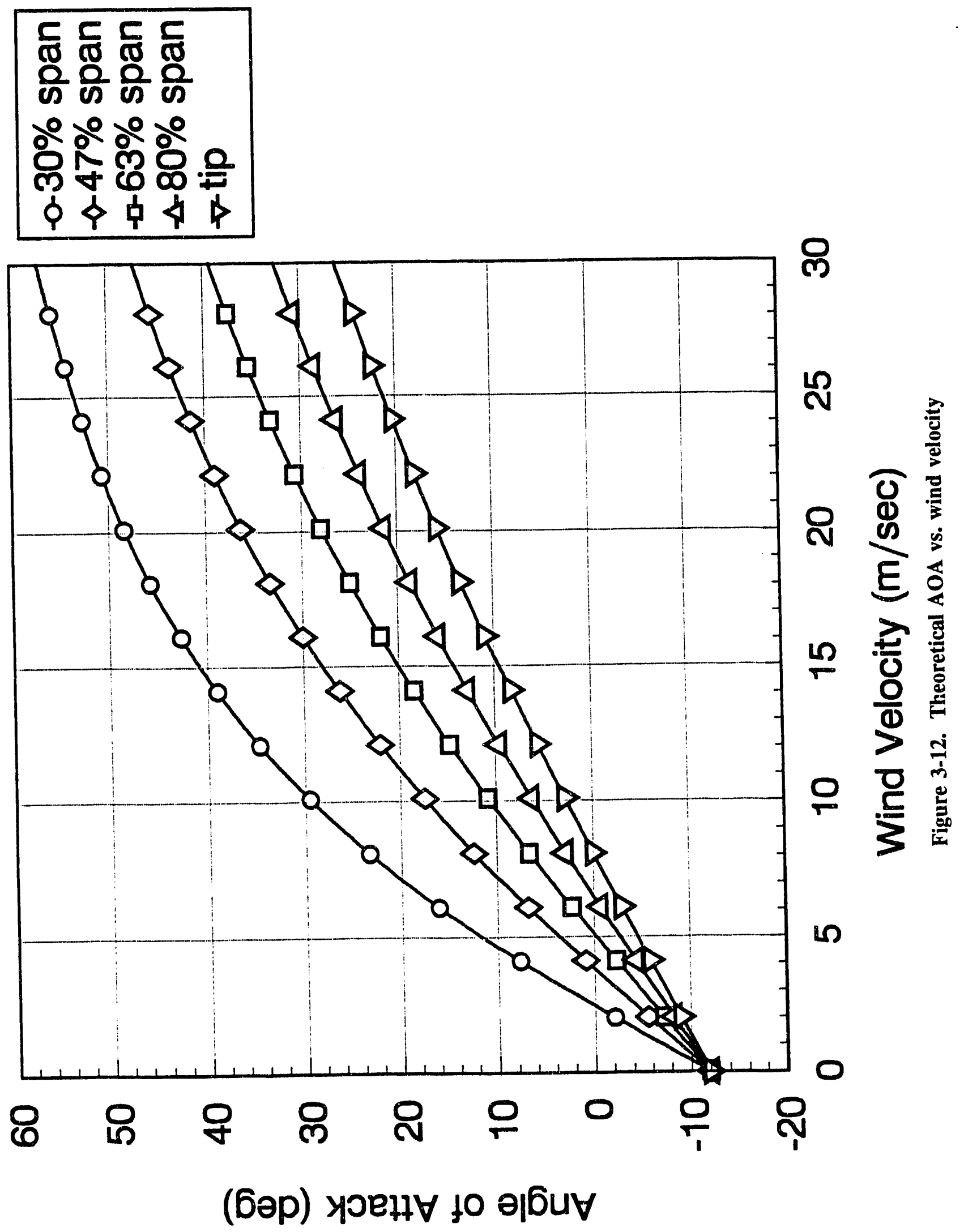




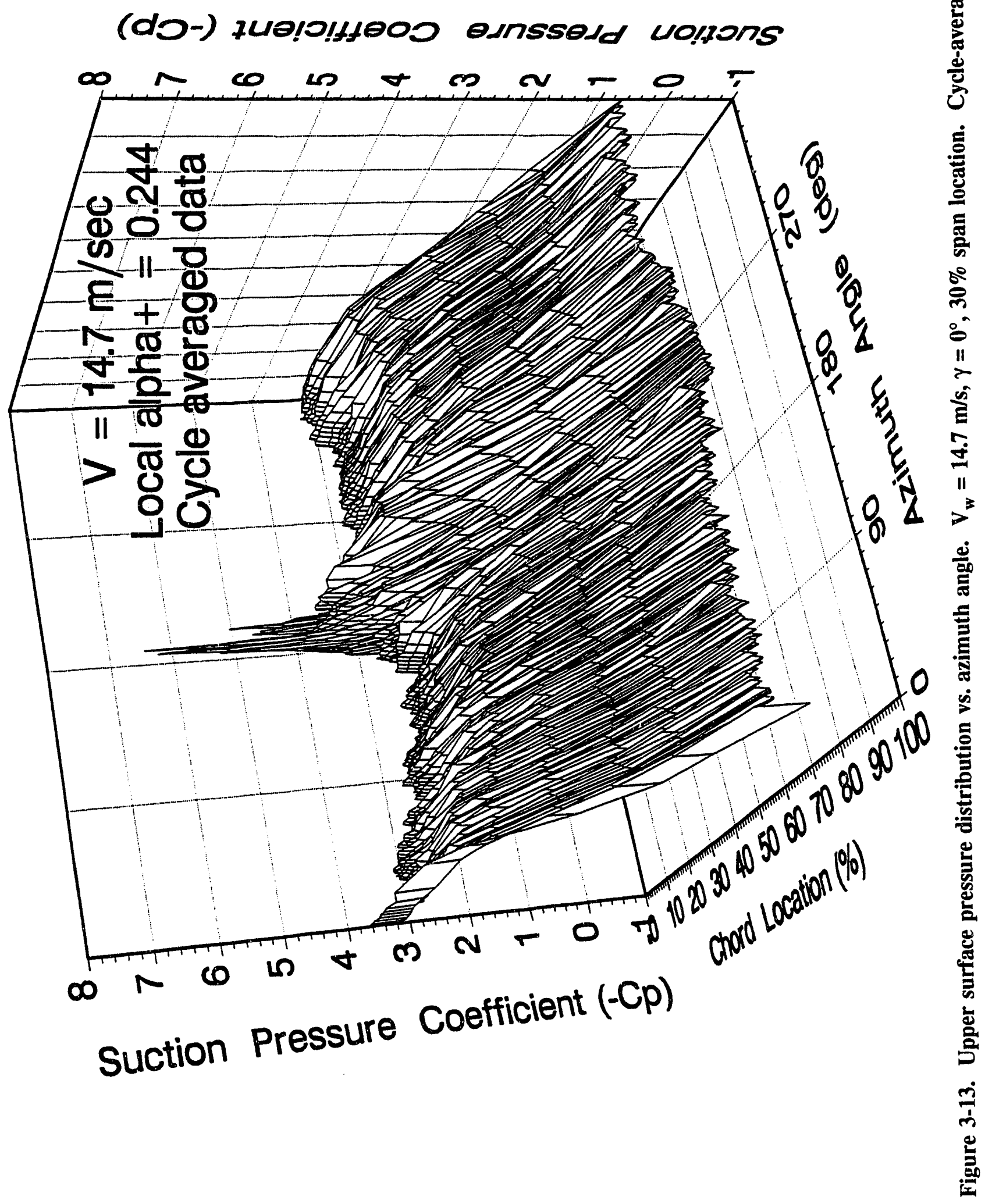


(do-) 7ua!o!ffeos elnssedd wolpans

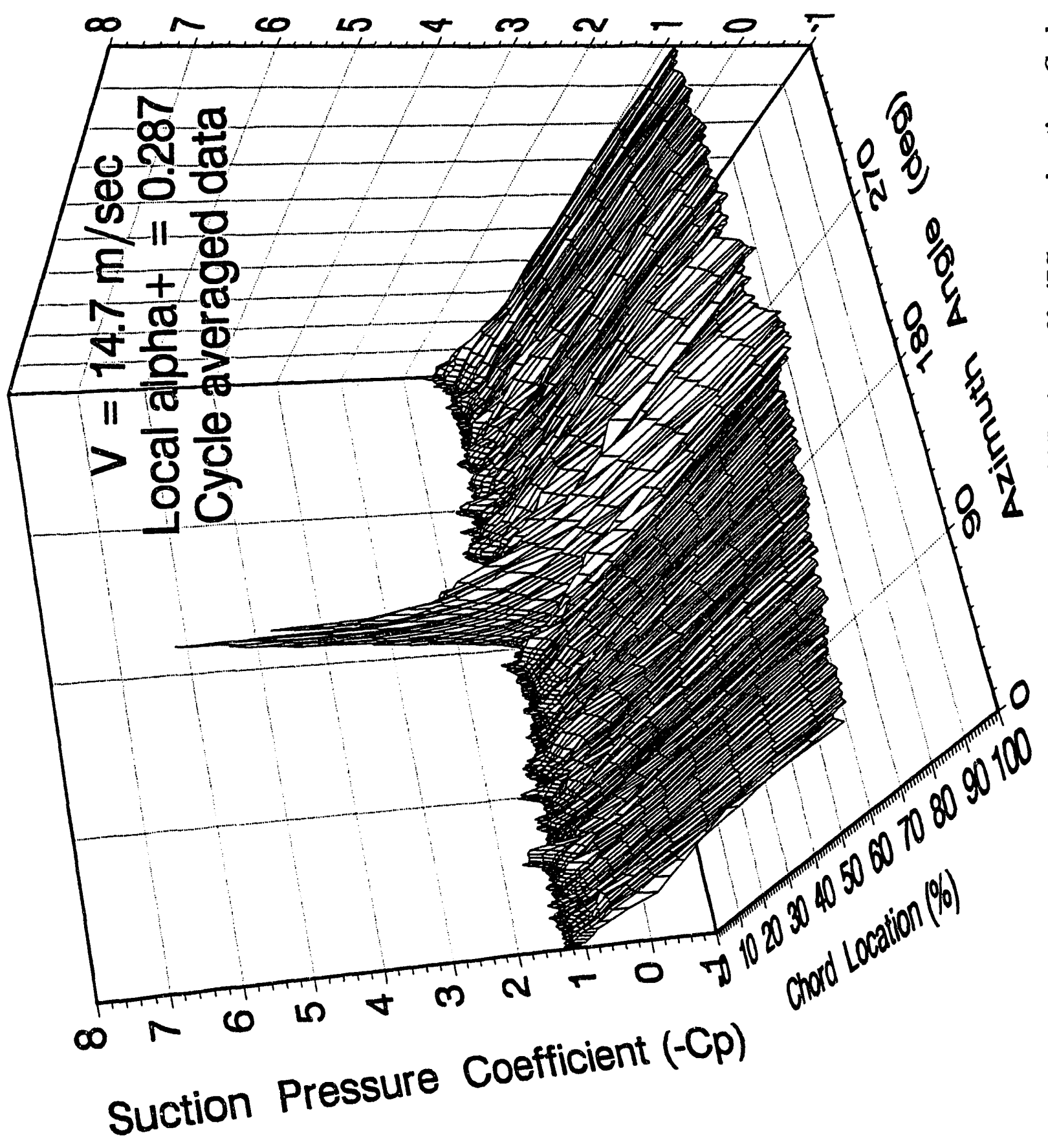

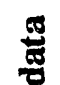

"

氖

2

$\stackrel{8}{8}$

2

i

$>$ 


\section{(d)-) zuololfyeos esnsseld wolfons}

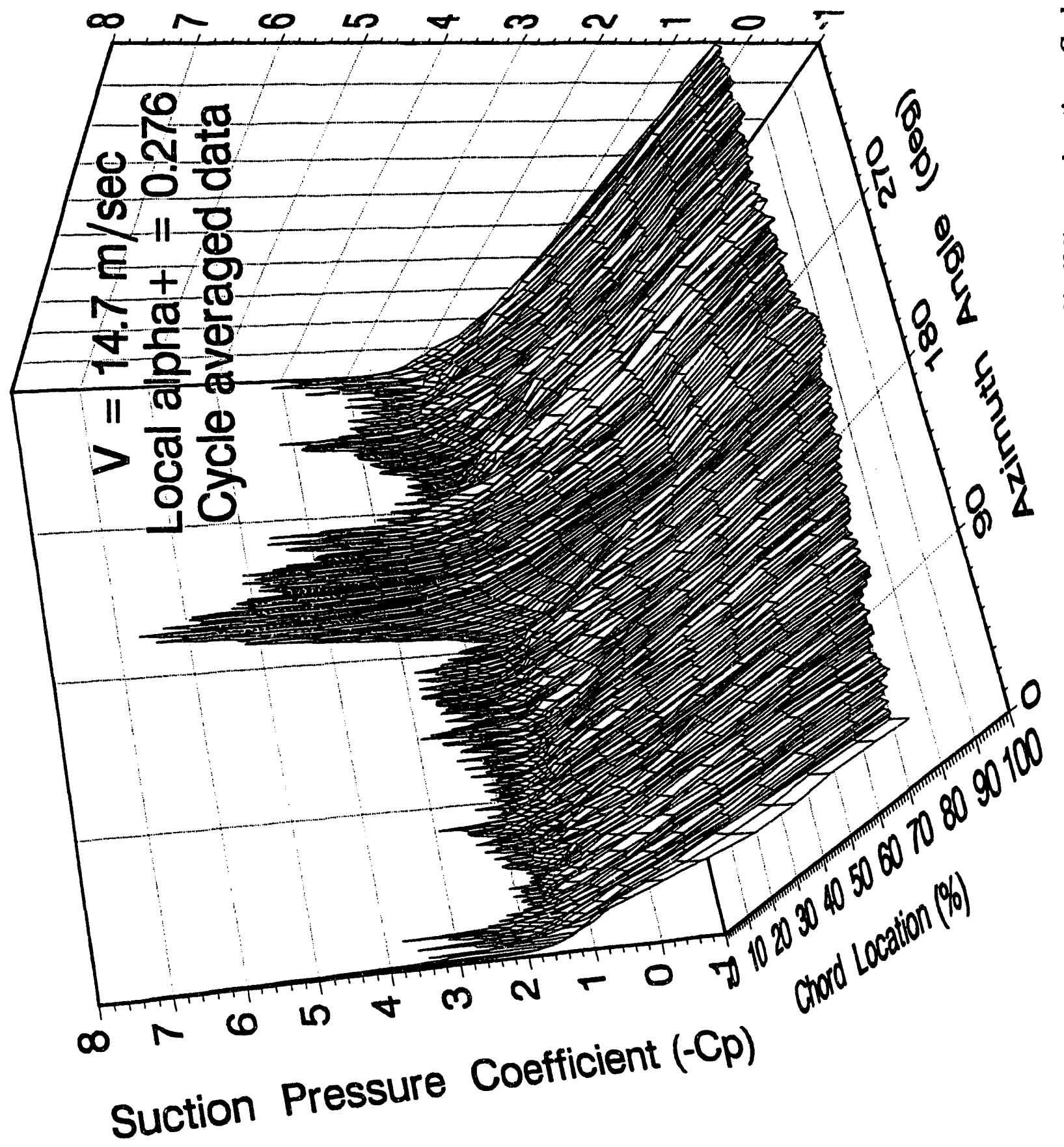

苋

苞

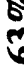

$\stackrel{\circ}{\circ}$

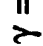

हิ

7

$>^{3}$ 


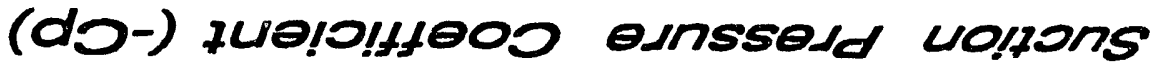

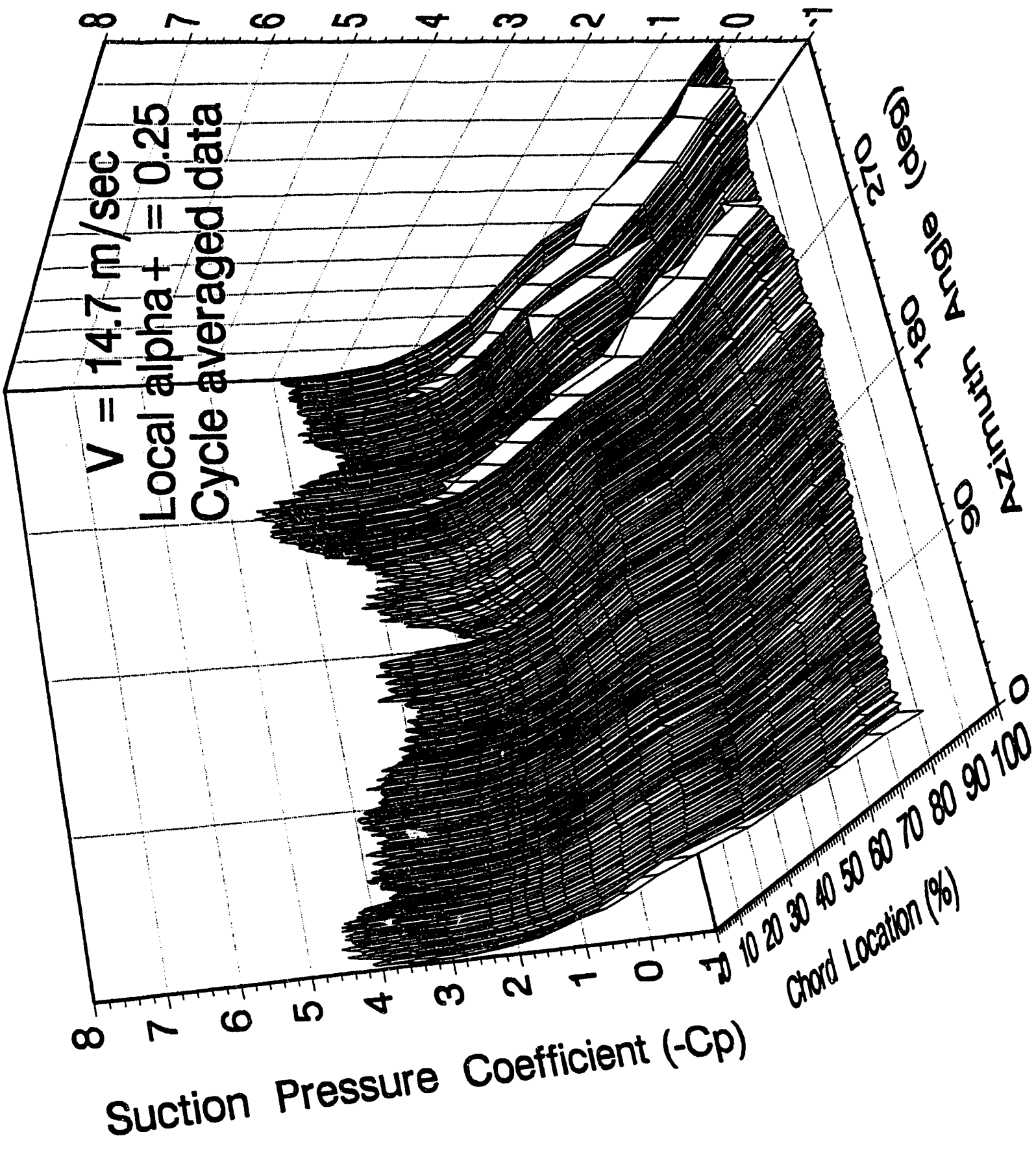

苞

옹

$\overbrace{}^{\circ}$

$\stackrel{8}{\circ}$ 


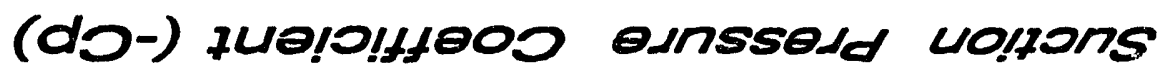

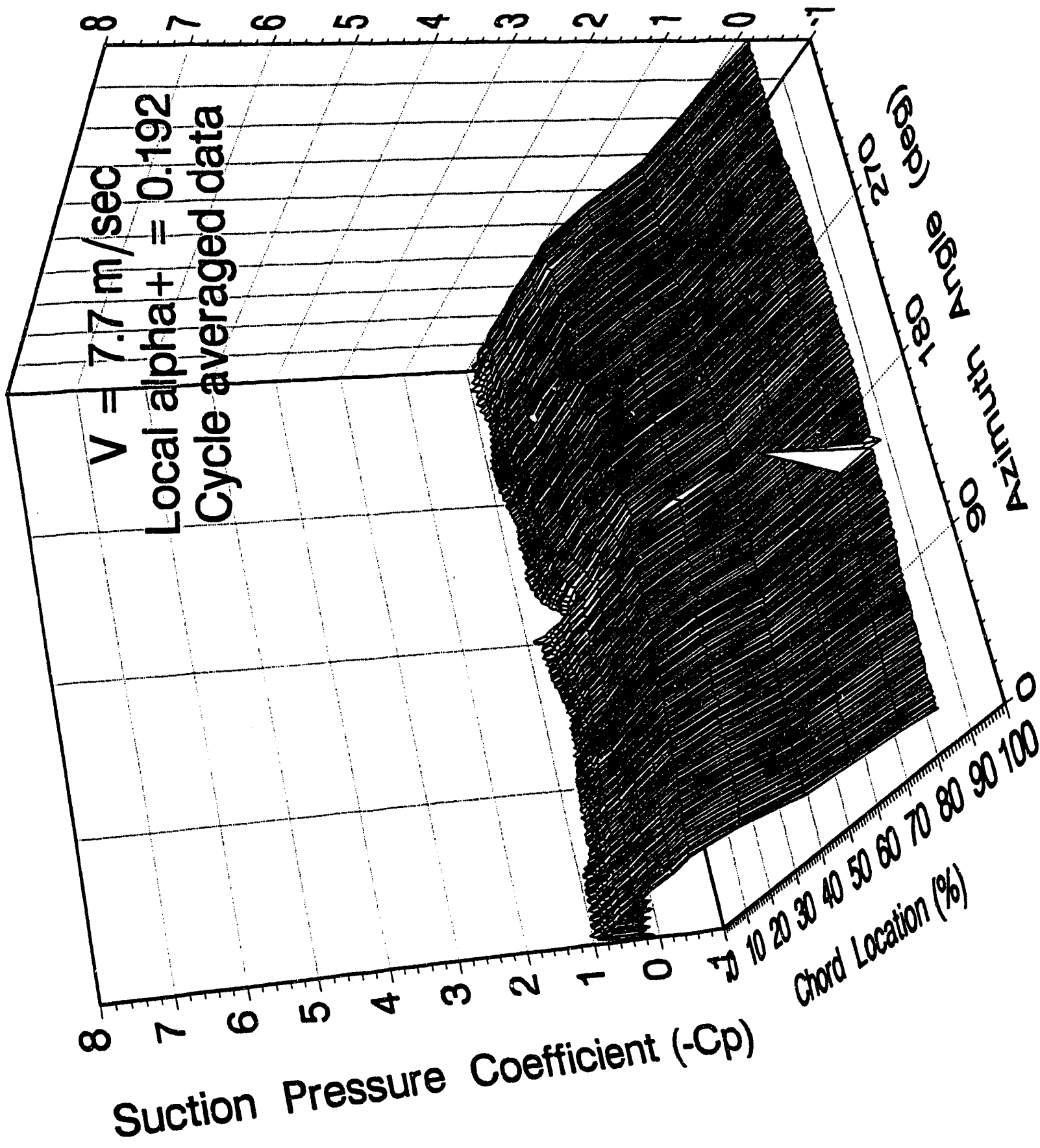

营

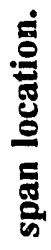

is

8

$>$ 
(do-) 7ualolfteob esnssedd wolfons

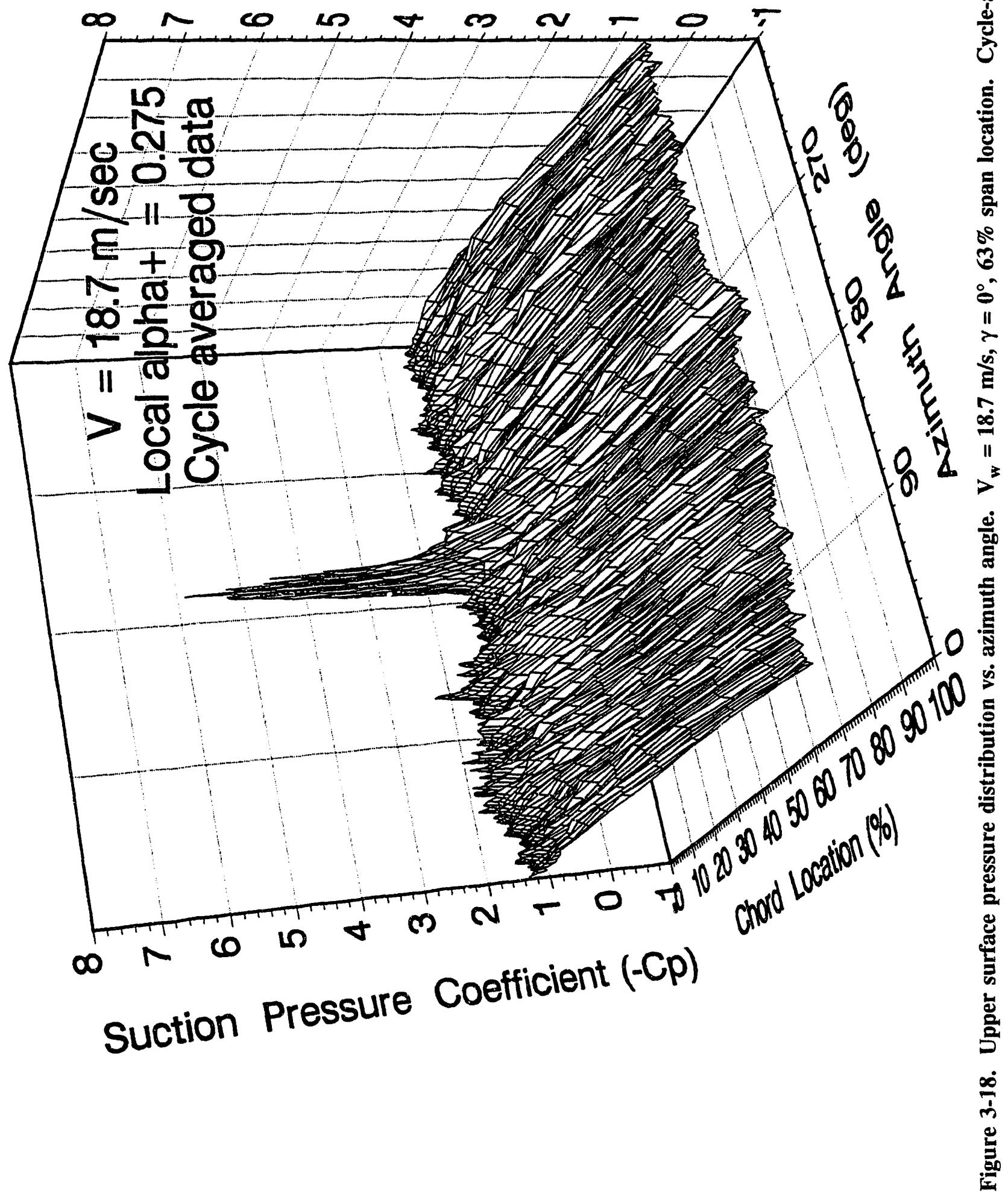




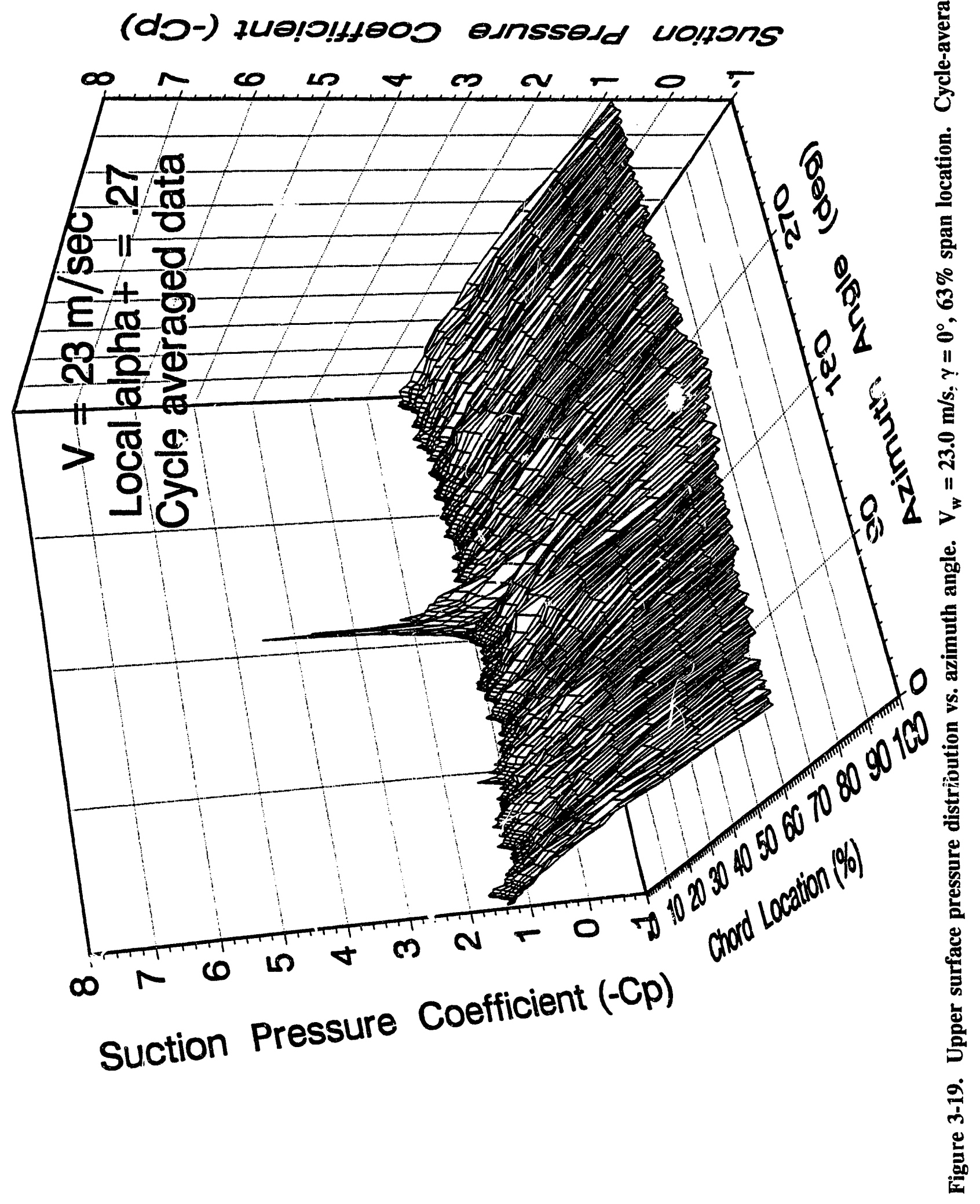


distribution seen at $47 \%$ span for $V_{w s}=14.7 \mathrm{~m} / \mathrm{s}$ (Figure 3-14). There did not appear to be a ridge of suction pressure to the trailing edge, but a ridge did persist to approximately $20 \% \mathrm{c}$. The pressure distribution also indicated stalled flow conditions during the remainder of the rotation cycle.

An increase in wind velocity to $23 \mathrm{~m} / \mathrm{s}$ showed that the blade section remained stalled throughout most of the rotation cycle. The tower wake signature was still seen in the pressure distribution except that the magnitude of the suction pressure spike decreased from -6 at $18.7 \mathrm{~m} / \mathrm{s}$ to -4.5 at $23 \mathrm{~m} / \mathrm{s}$. Similar trends were seen at $47 \%$ span. Although not shown, an increase in wind velocity to $23 \mathrm{~m} / \mathrm{sec}$ completely eliminated the large suction pressure peak at $47 \%$ span. A modest increase in suction pressure was observed along the entire chord as the blade passed through the tower wake.

Normal force coefficients were plotted as a function of azimuth angle to demonstrate the global effects of increased wind velocity at each spanwise location (Figure 3-20). Static values are provided as a reference in Figure 3-21. To provide a reference, maximum and minimum AOA are shown in Table 1, where minimum AOA occurs at $\psi=180^{\circ}$, in the center of the tower wake. Passage through the tower wake had significant effects on $C_{n}$ values for each wind velocity and span location examined. For the low- speed test case $(7.7 \mathrm{~m} / \mathrm{s}), \mathrm{C}_{\mathrm{n}}$ values remained approximately constant until the blade passed through the tower wake. This coulc be seen by a significant decrease in $\mathrm{C}_{\mathrm{n}}$. From $47 \%$ to $80 \%$ span, average $\mathrm{C}_{\mathrm{n}}$ values appeared to be approximately $10 \%$ less than the static counterparts that were obtained during two-dimensional wind tunnel tests. At $30 \%$ span, the average $C_{n}$ of 1.4 was approximately $45 \%$ greater than maximum static counterparts.

As wind velocity was approximately doubled to $14.7 \mathrm{~m} / \mathrm{s}$, significant differences in $\mathrm{C}_{\mathrm{n}}$ character was observed. At $30 \%$ and $47 \%$ span, the decrease in $C_{n}$ as the blade passed through the tower wake was followed by ar overshoot in $C_{n}$. This overshoot was approximately $20 \%$ of average values throughout the rotation cyule. At $30 \%$ span, $C_{n}$ values were typically 2.2 , or more than $100 \%$ greater than maximum static $C_{n}$ values. Even at $47 \%$ and $63 \%$ span, $C_{n}$ values were, on average, $25 \%$ greater than maximum steady values. At $80 \%$ span, $C_{n}$ values were approximately the same as static values.

For the high wind speeds (18.7 and $23 \mathrm{~m} / \mathrm{s}), \mathrm{C}_{\mathrm{n}}$ values increased further, and consistent lift enhancement was seen, compared with static wind tunnel values at all span locations. Lift overshoots $40 \%$ greater than maximum static values were seen at $80 \%$ span even though, geometrically, each spanwise station should experience stalled flow conditions. Significant lift enhancement and significant lift overshoots continued to occur, however.

\subsection{Effect of Yaw Error}

Effects of yaw error (referred to simply as yaw) resulted in even more complex pressure distributions compared with the zero-yaw case. For yawed data, the effect of passage through the tower wake and cyclic variation in $\mathrm{q}$ and local AOA were superimposed, making it very difficult to predict the possible flow fields that were formed. Repeatable trends were found and will be summarized in this section.

Upper surface cycle-averaged pressure distributions for the $30^{\circ}$ case were presented earlier in Figures 3-7 and 3-9 for 30\% and 63\% spanwise locations, respectively. At 30\% span, very large (-8.5) and transient LE suction pressure peaks were formed at approximately $\psi=140^{\circ}$ and $180^{\circ}$. It is likely that the first peak was a result of prssage through the tower wake. Because of the high yaw, the blade would be passing through the wake at this point in the rotation cycle. The formation of the second peak was seen later, and the peak was significantly larger. The formation of a suction pressure ridge in the direction of increasing chord and azimuth angle was also observed. 

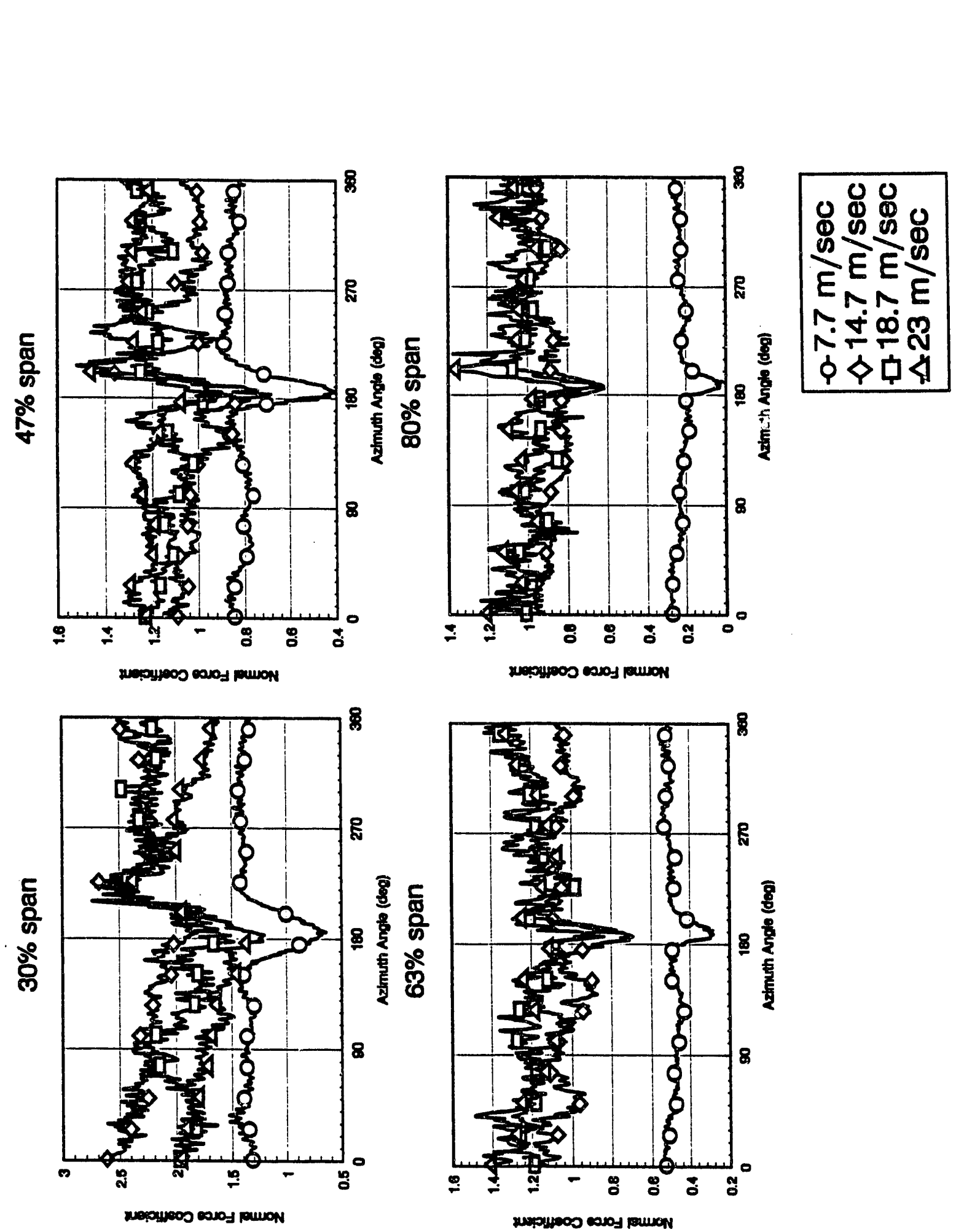


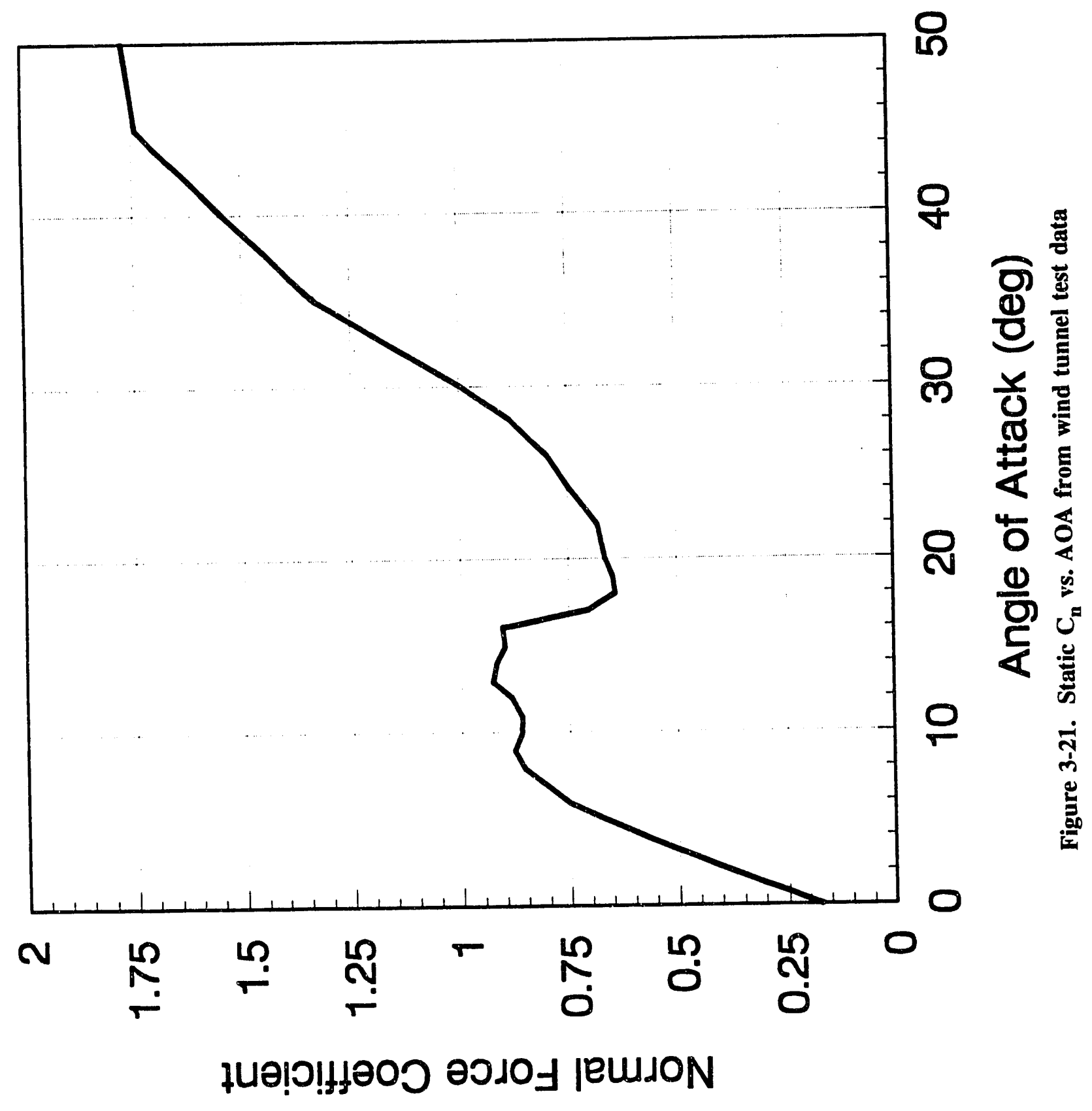


Table 1. Angle of Attack Variation

\begin{tabular}{||c|c|c|c|c||}
\hline \hline $\mathrm{V}_{\mathrm{w}}(\mathrm{m} / \mathrm{s})$ & 7.7 & 14.7 & 18.7 & 23 \\
\hline $\mathrm{Span}$ & $\alpha_{\min }-\alpha_{\max }$ & $\alpha_{\min }-\alpha_{\max }$ & $\alpha_{\min }-\alpha_{\max }$ & $\alpha_{\min }-\alpha_{\max }$ \\
\hline $30 \%$ & $13.3^{\circ}-22.0^{\circ}$ & $30.0^{\circ}-40.1^{\circ}$ & $36.9^{\circ}-46.1^{\circ}$ & $42.6^{\circ}-5.12^{\circ}$ \\
\hline $47 \%$ & $4.76^{\circ}-11.3^{\circ}$ & $17.9^{\circ}-27.4^{\circ}$ & $24.2^{\circ}-33.8^{\circ}$ & $29.5^{\circ}$ \\
\hline $63 \%$ & $0.66^{\circ}-5.8^{\circ}$ & $11.2^{\circ}-19.5^{\circ}$ & $16.6^{\circ}-25.5^{\circ}$ & $21.4^{\circ}-31.3^{\circ}$ \\
\hline $80 \%$ & $-1.97^{\circ}-2.19^{\circ}$ & $6.67^{\circ}-13.7^{\circ}$ & $11.3^{\circ}-19.1^{\circ}$ & $15.4^{\circ}-24.6^{\circ}$ \\
\hline
\end{tabular}

The pressure distribution at $63 \%$ span in Figure 3-9 was significantly different in character. At $\psi=165^{\circ}$, a pronounced decrease in suction pressure (from -3.5 to -1.5 ) was seen as the blade passed through the tower wake. As the blade passed out of the wake, a gradual increase in LE suction pressure was seen from $\psi=180^{\circ}$ to $280^{\circ}$, with a maximum value of -8.5 . This gradual increase in LE suction pressure was immediately followed by a precipitous decrease to -1.0 , and the blade section exhibited stalled flow conditions.

Pressure distributions were also taken for the negative yaw case of $-30^{\circ}$ and are noticeably different from those taken for the positive yaw case. Upper surface pressure distributions are shown in Figures 3-22 to $3-25$ for $30 \%, 47 \%, 63 \%$, and $80 \%$ span locations, respectively. At $30 \%$ span, a single LE suction pressure peak was observed at $w=240^{\circ}$. This is likely caused by passage through the tower wake. At $\psi=270^{\circ}$, LE suction pressure gradually built up and reached a maximum of -8 at approximately $\psi=20^{\circ}$. This is in contrast to the highly transient LE suction pressure peak formed for positive yaw. From $\psi=45^{\circ}$ to $80^{\circ}$, the formation of a suction pressure ridge was seen in the direction of increasing chord and azimuth angle. Gradual decreases in suction pressure were then seen along the entire chord, indicating stalled flow conditions.

At $47 \%$ span, a relatively small $(-2.5)$ LE suction pressure peak was seen at $\psi=225^{\circ}$ as the blade passed through the tower wake. Prior to the formation of this peak, the pressure distribution indicated that the blade section was stalled. As the blade continued to rotate, there was a gradual buildup in LE suction pressure with approximately constant values of -8 seen between $340^{\circ}$ and $45^{\circ}$ azimuth. A barely imperceptible suction pressure ridge was seen at $45^{\circ}$ but persisted only to approximately $50 \%$ chord. This was followed by a sudden loss of suction pressure, indicating that the blade section became stalled.

At $63 \%$ span, a suction pressure peak at $\psi=210^{\circ}$ indicated passage through the tower wake. Again, a gradual buildup of LE suction pressure was observed, although this buildup appeared more gradual compared with $47 \%$ span results. Maximum suction pressures were seen between $45^{\circ}$ and $90^{\circ}$ azimuth, followed by a loss of suction pressure that indicated stalled flow conditions. For this case, a brief suction pressure ridge was seen at $\psi=110^{\circ}$, and this ridge persisted to about $30 \%$ chord.

At $80 \%$ span, a LE suction pressure deficit was seen at $\psi=190^{\circ}$, followed by a modest LE suction pressure overshoot. Suction pressures appeared to remain approximately constant but the pressure distribution did not indicate stalled flow conditions. From $\psi=30^{\circ}$ to $135^{\circ}$, a gradual buildup in LE suction pressure was seen. This was followed by a gradual decrease in LE suction, which was in contrast 
(d)-) 7uololfye00 asnssadd wolzons

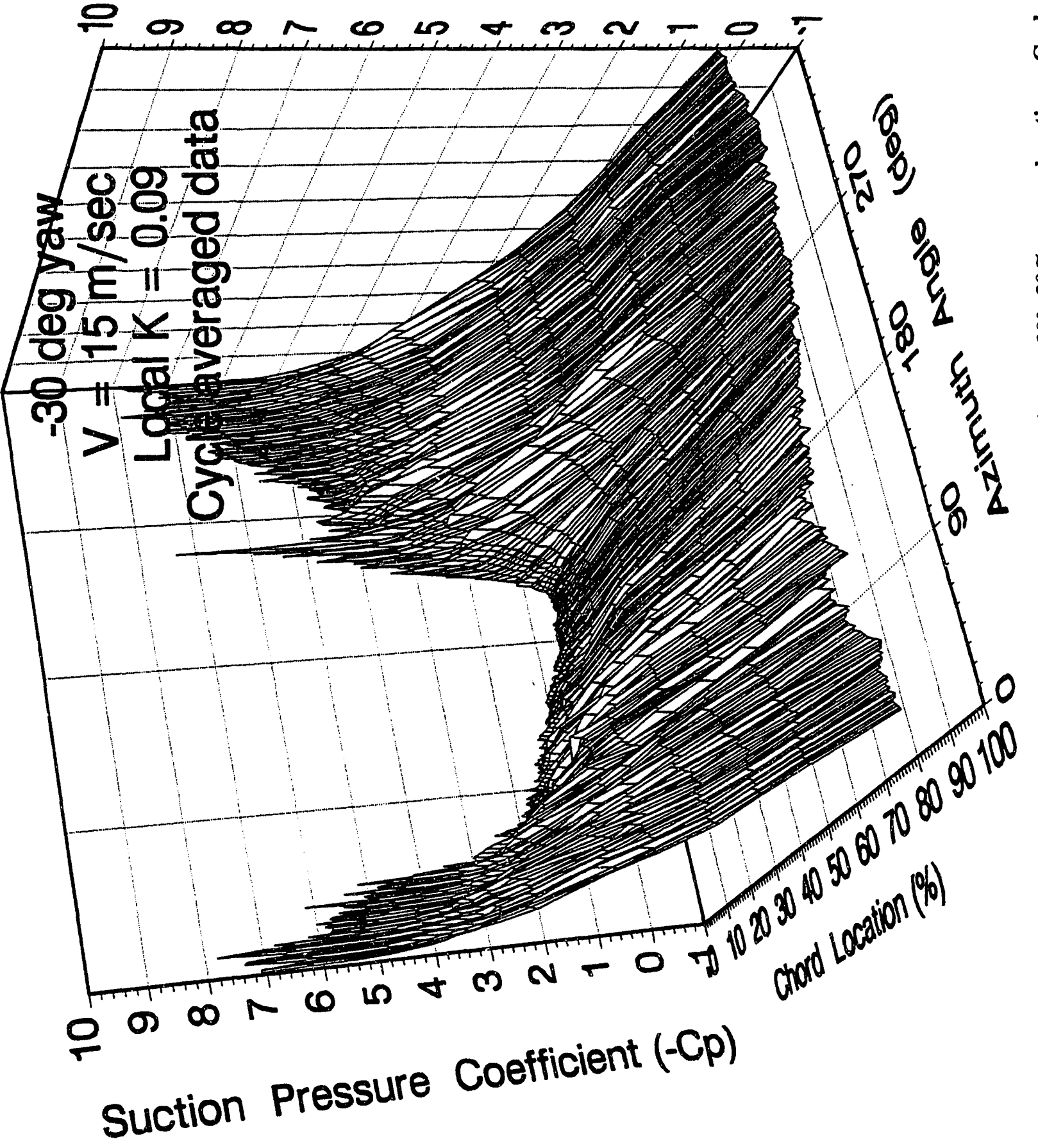

苋

葛

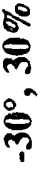

II

है

n

7

$\frac{\dot{0}}{\mathrm{x}}$

总

:



กั่

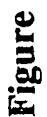


(do-) 7uo!olfHe00 asnssedd wolfons

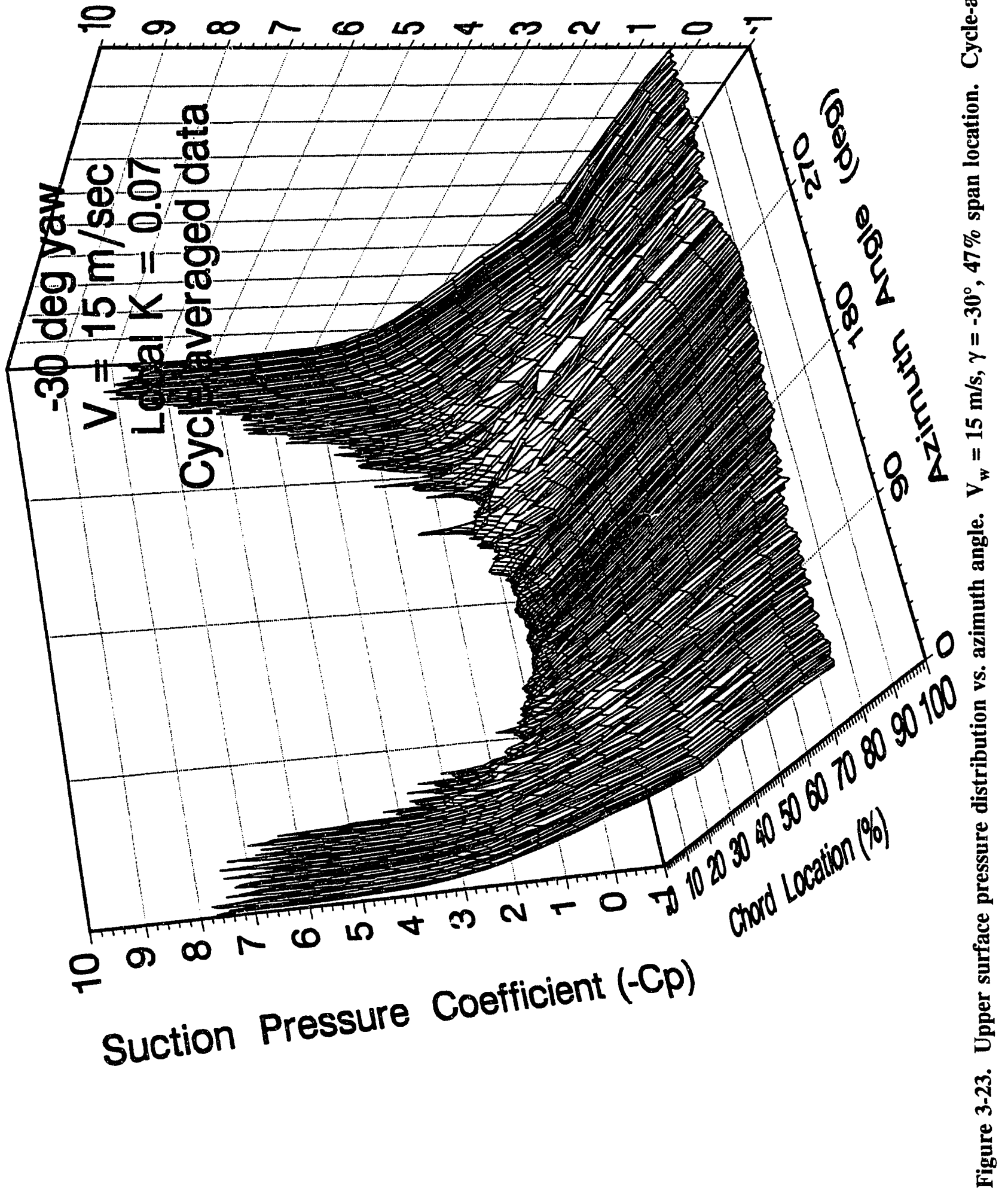


(d)-) 7uolo!ffeos adnssadd wolpons

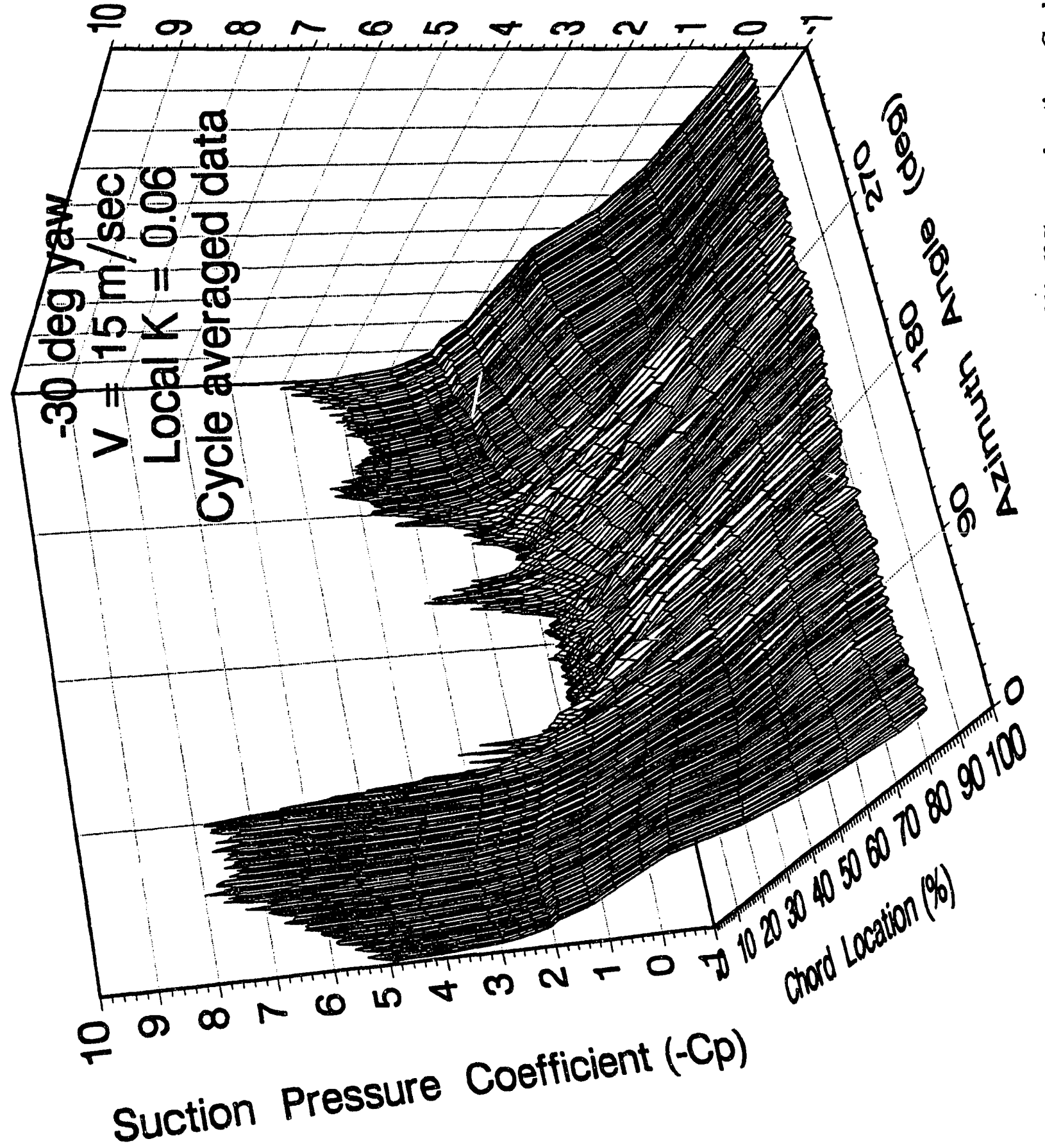

है $\stackrel{\circ}{\%}$

क $10 \times 1$ 
(d)-) 7wololfye00 asnssedd wolpons

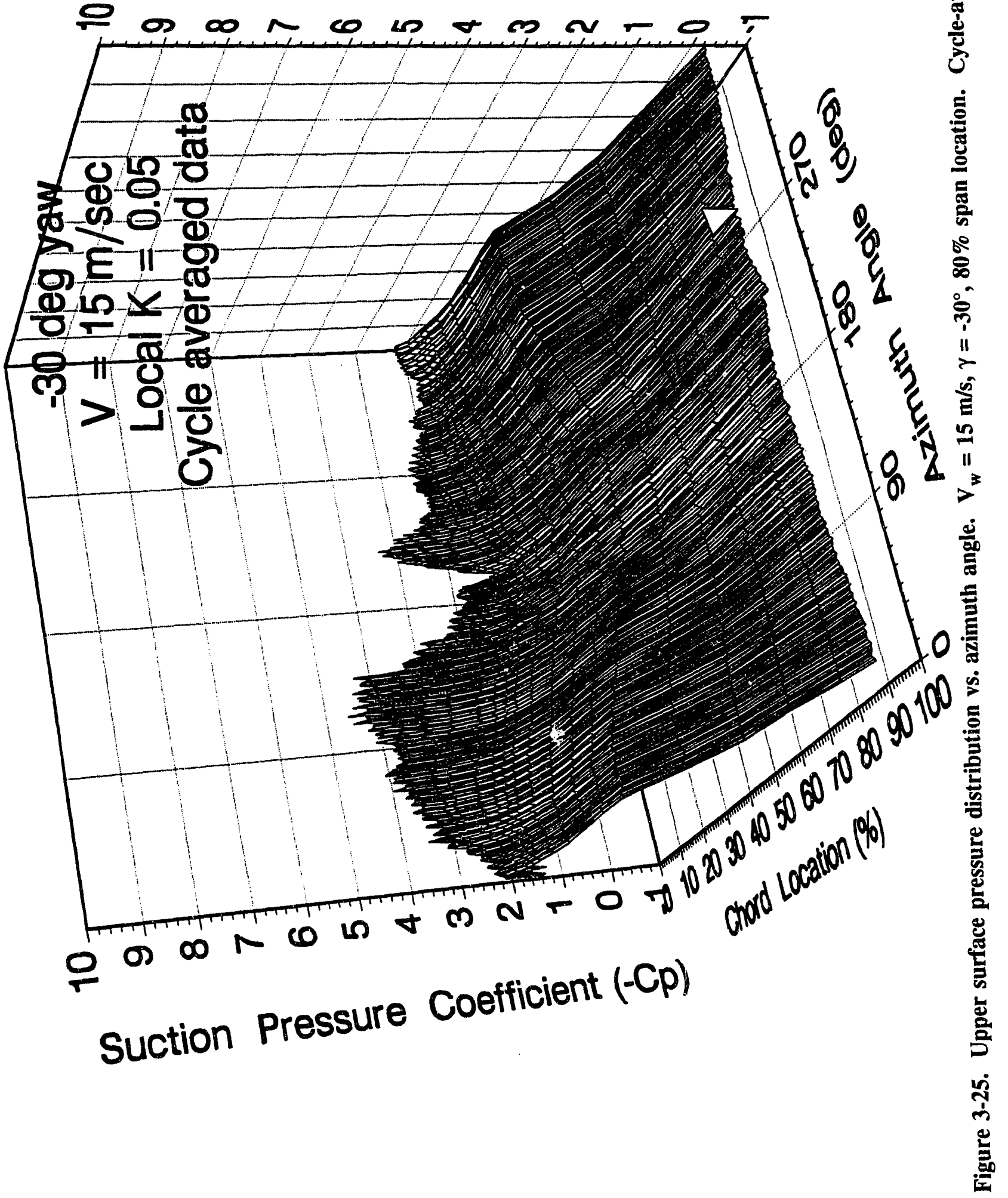


to the precipitous decrease seen at inboard span locations. Also, there did not appear to be a suction pressure ridge. The blade section never did exhibit stalled flow conditions.

Upper surface pressure distributions taken at 63\% span are presented in Figures 3-26 and 3-27 for $15^{\circ}$ and $-15^{\circ}$ yaw and $15 \mathrm{~m} / \mathrm{s}$, respectively. For $15^{\circ}$ yaw, a large and transient LE suction pressure peak was observed at $\psi=200^{\circ}$. This was followed by a decrease in LE suction pressure, but the flow appeared attached up to $\psi=280^{\circ}$. A mild suction pressure ridge was then observed but was only seen up to $25 \%$ chord. After the formation of this ridge, stalled flow conditions appeared to exist up to $\psi=90^{\circ}$. From $90^{\circ}$ to $180^{\circ}$, a small, gradual increase in LE suction pressure was seen prior to the formation of the transient pressure peak.

The similar pressure distribution seen for the $-15^{\circ}$ yaw was much different compared with the $15^{\circ}$ yaw test case. A LE suction peak was seen at $\psi=200^{\circ}$, corresponding to passage through the tower wake. A gradual buildup in LE suction pressure was then observed from $\psi=270^{\circ}$ to $45^{\circ}$. This was followed by a modest decrease in LE suction pressure from $\psi=45^{\circ}$ to $120^{\circ}$, after which stalled flow conditions appeared to persist.

Although upper surface pressure distributions demonstrated highly transient behavior, more definitive evidence of vortex formation was desired. Chordwise pressure distributions were examined to highlight the formation of a suction pressure ridge seen in the three-dimensional pressure distributions. Because the pressure ridges were most obvious in the single-cycle data, these data were examined to follow the suction pressure peaks as the rotational cycle advanced. Chordwise pressure distributions are shown in Figures $3-28$ to $3-31$ at $30 \%, 47 \%, 63 \%$ and $80 \%$ span locations, respectively, for the $30^{\circ}$ yaw test case and $15 \mathrm{~m} / \mathrm{s}$ wind velocity. Multiple plots are shown as the rotational cycle advanced, emphasizing the temporal displacement of the suction pressure ridge. It has been shown that a vortex leaves a clear signature in a pressure distribution because it is a region of low pressure [20]. If a vortex is present, a suction peak in the chordwise pressure distribution would exist, corresponding with the approximate location of the vortex. If no vortex was present, suction pressure would decrease as approximately $1 / \mathrm{x}$ (where $\mathrm{x}$ is chordwise position) similar to static two-dimensional wind tunnel results.

At $30 \%$ span, the pressure distribution taken at $\psi=186^{\circ}$ appeared to decrease as $1 / \mathrm{c}$. Maximum LE suction pressure was observed at this point in the rotation cycle. At $\psi=192^{\circ}$, a suction peak of -6.84 was seen at $2 \%$ c. At $\psi=196^{\wedge}$, the suction peak moved to $6 \% \mathrm{c}$, and at $205^{\circ}$, it moved to $14 \% \mathrm{c}$. Notice that as the pressure peak moved downstream, its magnitude diminished, but the region of influence increased. As the rotation cycle continued, the pressure peak moved downstream and was centered at $36 \%$ c for $\psi=218^{\circ}, 44 \% \mathrm{c}$ for $\psi=227^{\circ}$, and $68 \% \mathrm{c}$ for $\psi=245^{\circ}$.

Suction pressure peaks were also found in the pressure distributions at $47 \%$ span. For $w=219^{\circ}$, suction pressure decreased as $1 / \mathrm{c}$ where LE suction pressure reached a maximum. At $228^{\circ}$, a suction peak was observed at $8 \% \mathrm{c}$. As the cycle continued to $231^{\circ}$, this peak moved to $14 \%$ span, but its magnitude decreased by $75 \%$. By $248^{\circ}$, the suction peak had moved to $36 \% \mathrm{c}$; and by $256^{\circ}$ it had moved to $56 \% \mathrm{c}$, but had become barely noticeable. At $\psi=273^{\circ}$, the chordwise pressure distribution is indicative of stailed flow conditions. It is interesting to note that no further suction peaks were found prior to stall.

At $63 \%$ span, the only suction peaks observed were located upstream of $8 \% \mathrm{c}$. A small pressure peak was seen at $1 \% \mathrm{c}$ for $\psi=278^{\circ}$, and it moved to $8 \% \mathrm{c}$ for $\psi=281^{\circ}$. A small peak may be implied at $14 \%$ c for $\psi=284^{\circ}$, but no further peaks were observed downstream as the cycle advanced. The remaining chordwise pressure distributions were indicative of attached flow. At $80 \%$ span, there was no evidence 
(do-) 7ua!plfte00 asnssald wolpons

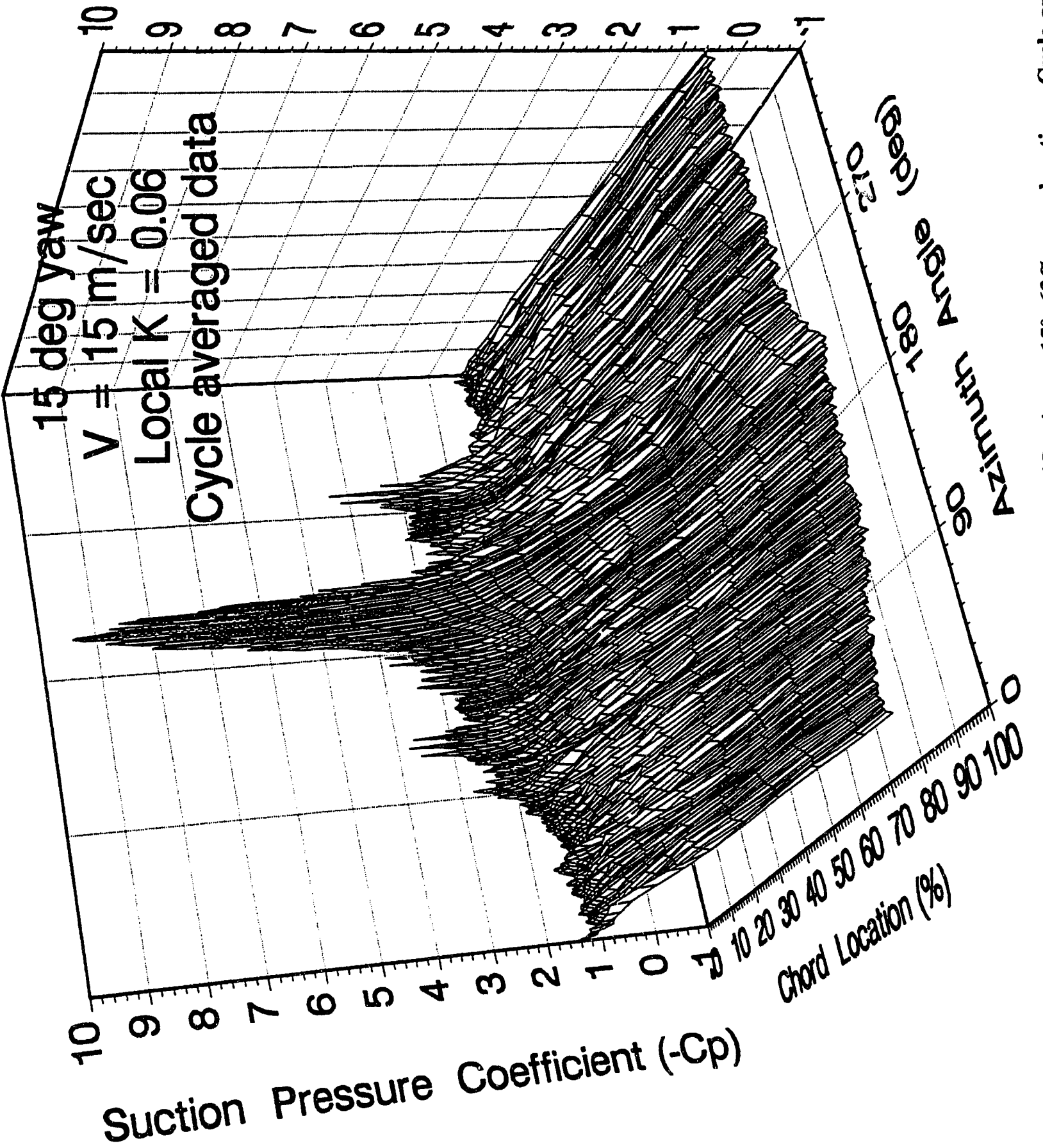

苋

蒫

$\stackrel{8}{3}$

in

(1) -

$+>0 \frac{0}{0}$ 
(d)-) 7ublo!ffeos asnssajd wolfons

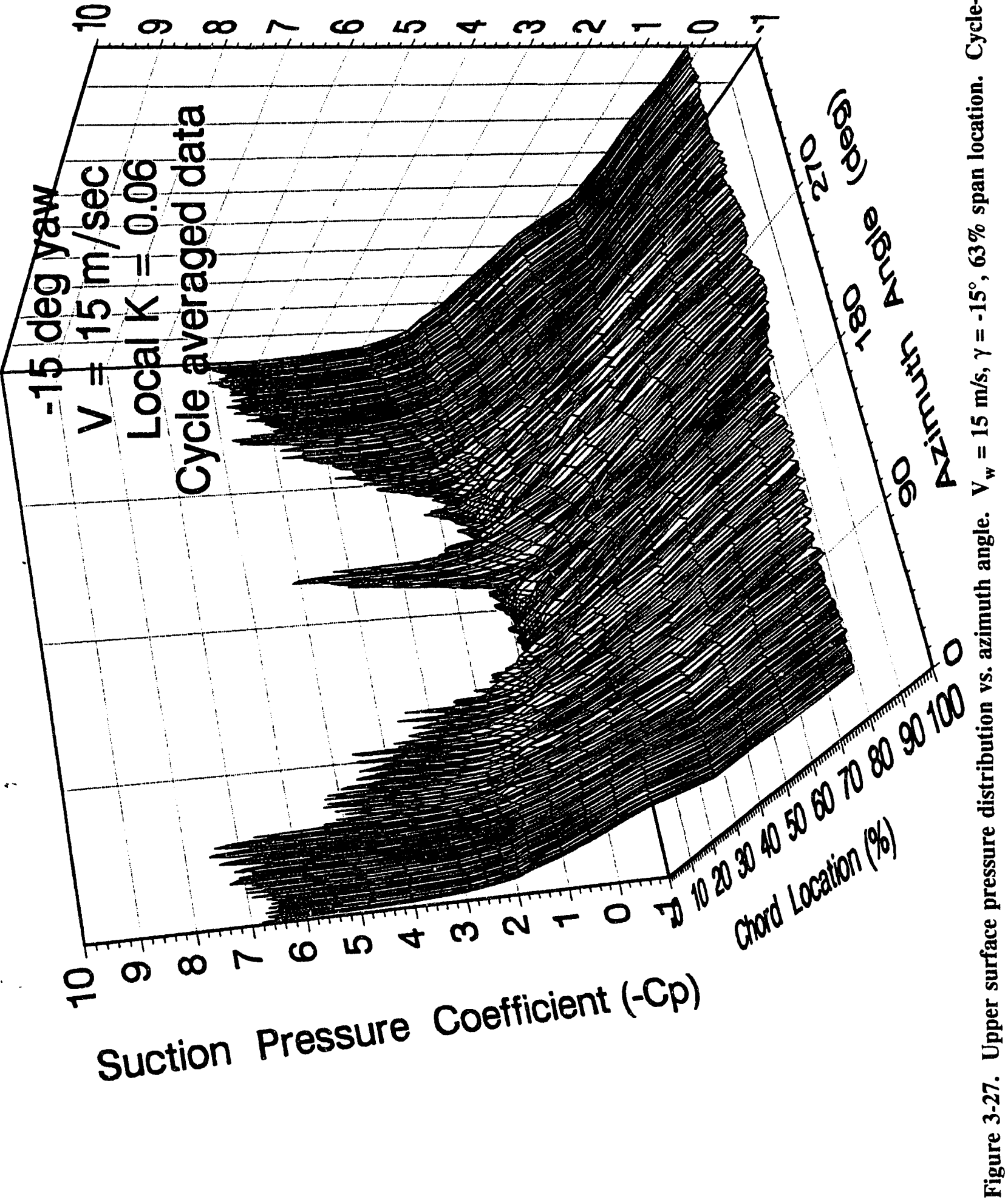



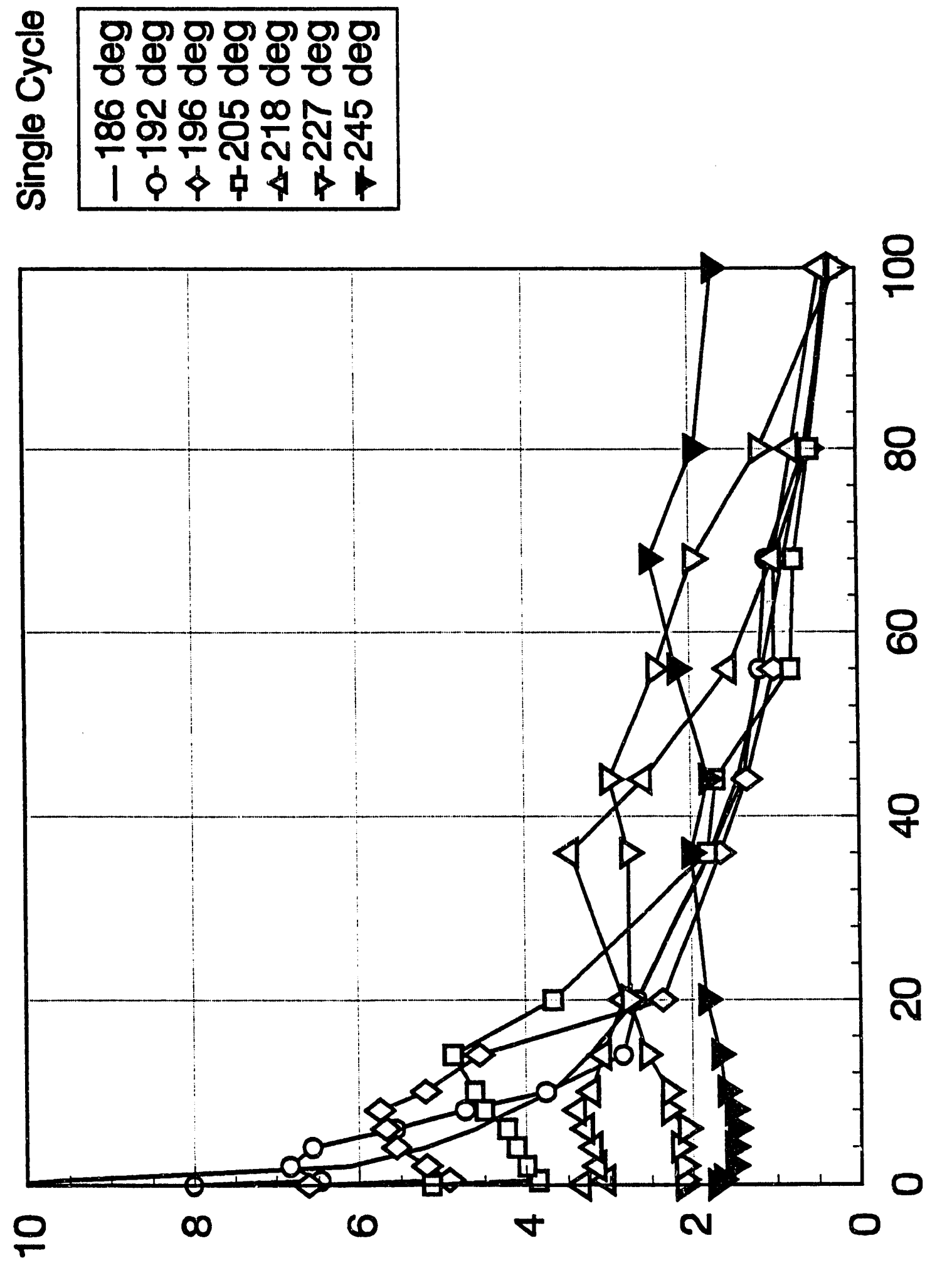

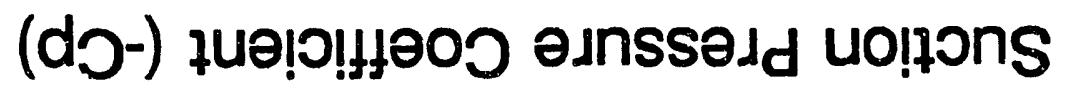

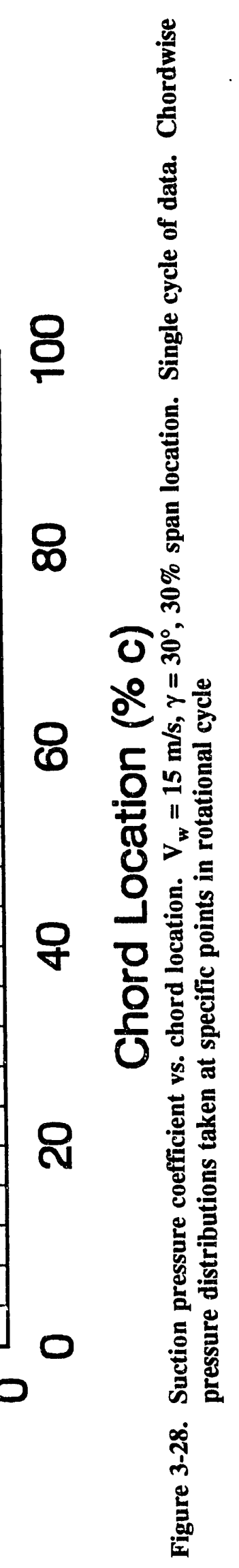



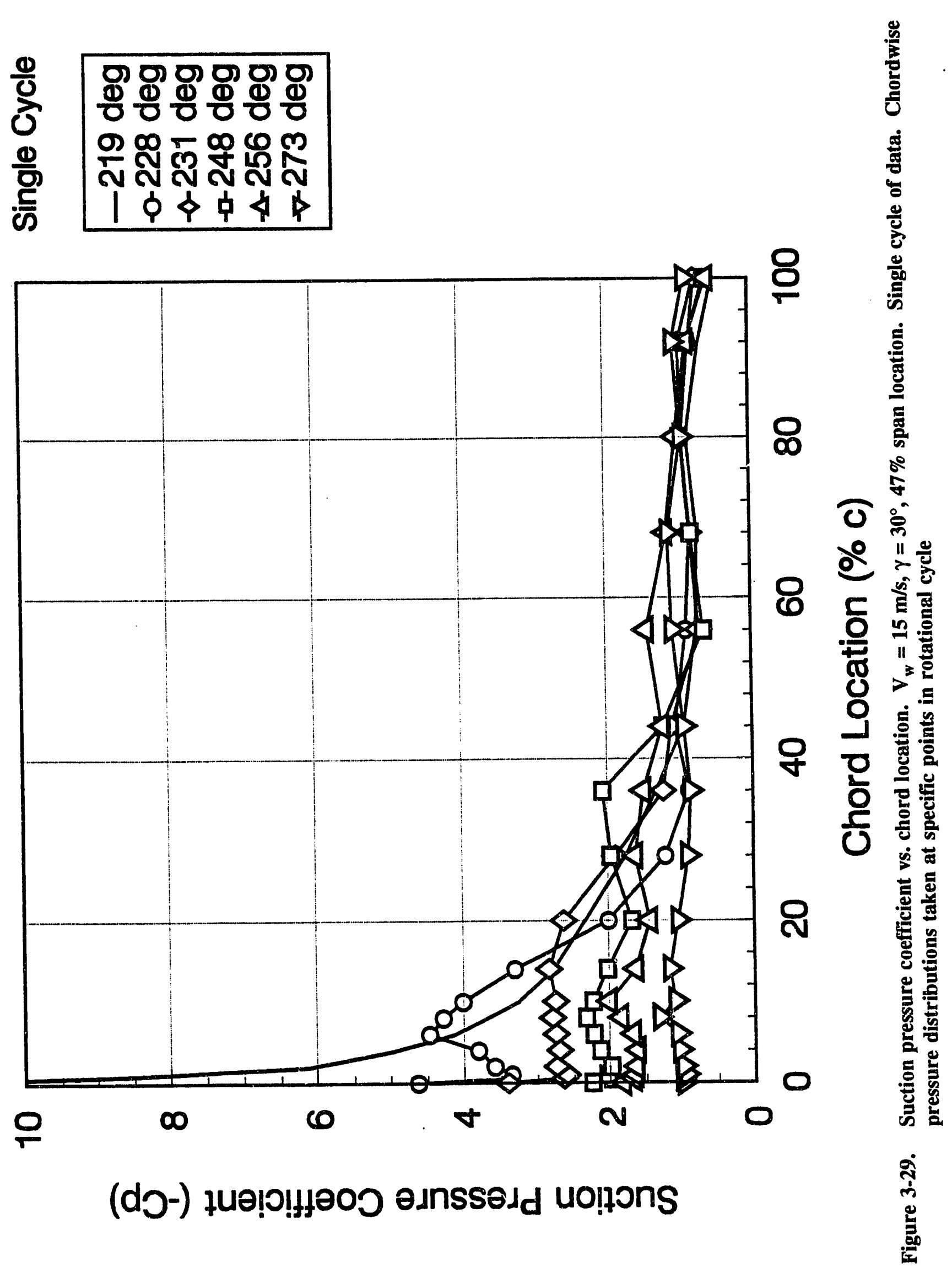

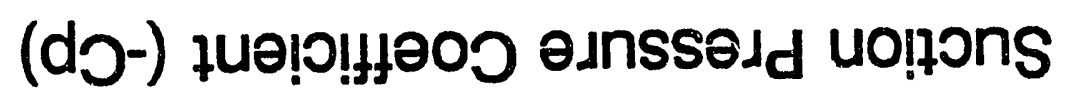




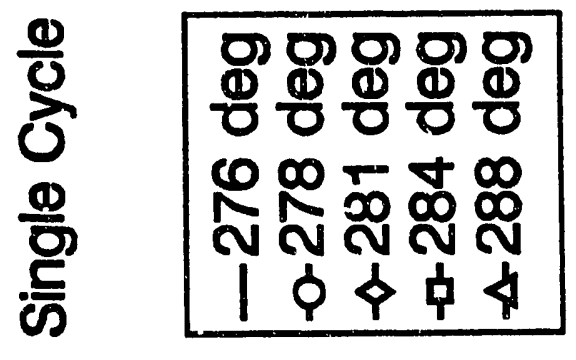

롱
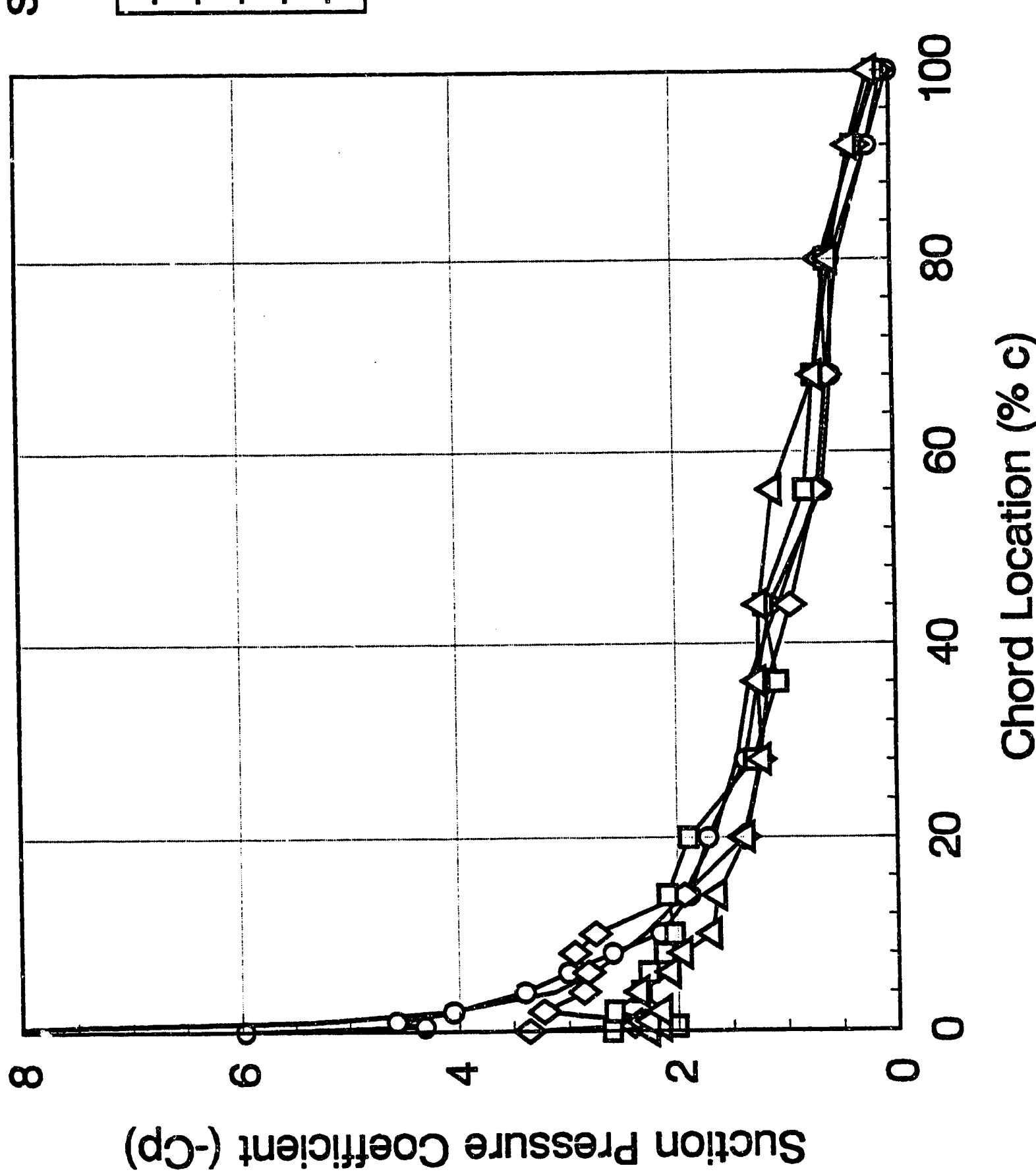

$\frac{9}{8}$

头

है

递

톨

छี

รีำ

8

(1) 6

$0 \stackrel{\circ}{\circ}$

(2) $11 \frac{0}{2}$

욜 뜧

(8)

$0>$ 눈

J

范

(2)

它

를 톨

递

空

흘

完 

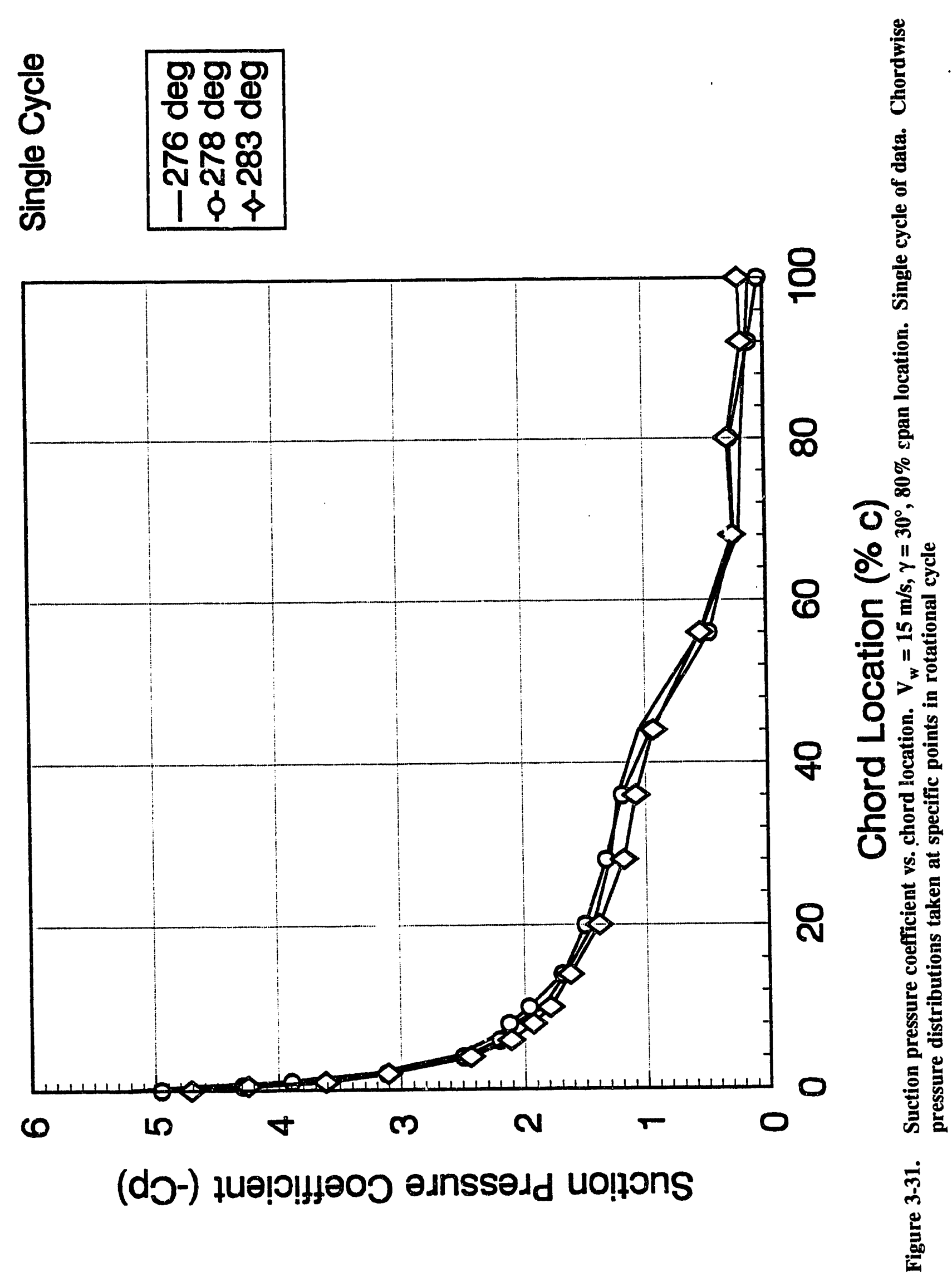
of suction pressure peaks downstream of the leading edge at any time during the rotation cycle following the time when maximum LE suction pressure occurred.

For a given chord location, there was a time in the rotation cycle at which maximum suction pressure occurred. For the formation of a vortex structure, this would occur when the vortex was centered at that given chord location. To approximate probable vortex position, Figure 3-32 shows the suction pressure peak chord location as a function of azimuth angle at the four spanwise locations examined. If these data are assumed to be representative of vortex position as a function of time, this figure would approximate the convective time histories of vortices that formed at these span locations. Figure 3-32 shows that movement of the suction pressure peak took the most time at $30 \%$ span $\left(\psi=187^{\circ}\right.$ to $247^{\circ}$ ), took approximately the same time at $47 \%$ span, but was practically instantaneous at $63 \%$ and $80 \%$ span.

Normal force coefficients were plotted as a function of azimuth angle for the four yaw cases examined at $30 \%, 47 \%, 63 \%$ and $80 \%$ span in Figure 3-33. In general, $C_{n}$ values were approximately $180^{\circ}$ out of phase for positive and negative yaw, as expected. At 30\% span, two $C_{n}$ peaks were seen for the $30^{\circ}$ yaw case, corresponding with the LE suction peaks observed. In addition, two peaks were seen for the $15^{\circ}$ yaw case, although the magnitude of the first $C_{n}$ peak was smaller by comparison. Cyclic $C_{n}$ variations for the negative yaw cases appeared quite similar and were $180^{\circ}$ out of phase with positive yaw cases and only showed one peak. Maximum $C_{n}$ values were approximately 2.5 for all cases, and minimum $C_{n}$ values were approximately 1.0 except for the minimum value of 1.5 seen for the $15^{\circ}$ yaw case. Maximum values were approximately $250 \%$ greater than maximum static $C_{n}$ values at stall.

At $47 \%$ span, considerable cyclic variation in $C_{n}$ continued to be observed. Peak $C_{n}$ values of 1.8 were seen for the positive yaw cases, although the peak seemed more transient for the $15^{\circ}$ yaw case compared with the $30^{\circ}$ yaw case. Maximum $C_{n}$ was 1.6 for $-30^{\circ}$ yaw but was only 1.3 for $-15^{\circ}$. At $63 \%$ span, maximum $C_{n}$ values of 1.5 were seen for all test cases. The $C_{n}$ curves appeared similar in character for the negative yaw cases. This was not the case for the positive yaw cases. Minimum $C_{n}$ values were approximately $20 \%$ less for $30^{\circ}$ yaw compared with $15^{\circ}$ yaw. Maximum values were equivalent, although peak $C_{n}$ values persisted for a greater portion of the cycle for $30^{\circ}$ yaw. Similar trends continued at $80 \%$ span, except that maximum $C_{n}$ values decreased to approximately 1.2. Similar minimum $C_{n}$ values were seen for the negative yaw cases, and minimum $C_{n}$ values diminished an additional $10 \%$ for the positive yaw cases.

Theoretical geometric angle of attack, $\alpha$, and instantaneous dynamic pressure, q, are plotted in Figures 3-34 and 3-35, respectively. These graphs demonstrate the effective temporal motion histories for a yawed turbine with respect to the wind for $30^{\circ},-30^{\circ}, 15^{\circ}$, and $-15^{\circ}$ yaw. Effects of induced velocity, skewed wake, wind shear, and tower wake effects are included. AOA variations are greatest at $30 \%$ span and smallest at $80 \%$ span. At $30 \%, 47 \%$, and $63 \%$ span, the effective AOA ranges from pre- to post-static stall, based on wind tunnel data. At $80 \%$ span, maximum AOA were found to be comparable to the static stall AOA. Temporal variations in q were found to be $180^{\circ}$ out of phase compared with angle of attack variations. In addition, the largest variations in $q$ occurred at $80 \%$ span with the relative amplitude decreasing at inboard spanwise locations.

Normal force ccefficient was plotted as a function of AOA in Figure 3-36 for the $30^{\circ}$ yaw test case and $15 \mathrm{~m} / \mathrm{s}$ wind velocity. These data were normalized by constant dynamic pressure, qo. As a reference, static wind tunnel data were plotted, indicated by the dashed line. $C_{n}$ data were generally different, depending on increasing or decreasing effective AOA forming the observed hysteresis loops. At 30\% span, significant hysteresis was seen between $\alpha=15^{\circ}$ and $34^{\circ}$, and consistent lift enhancement was seen compared with static values. From $40^{\circ}$ to $60^{\circ}$, minimal hysteresis was observed, and lift reduction was seen. 

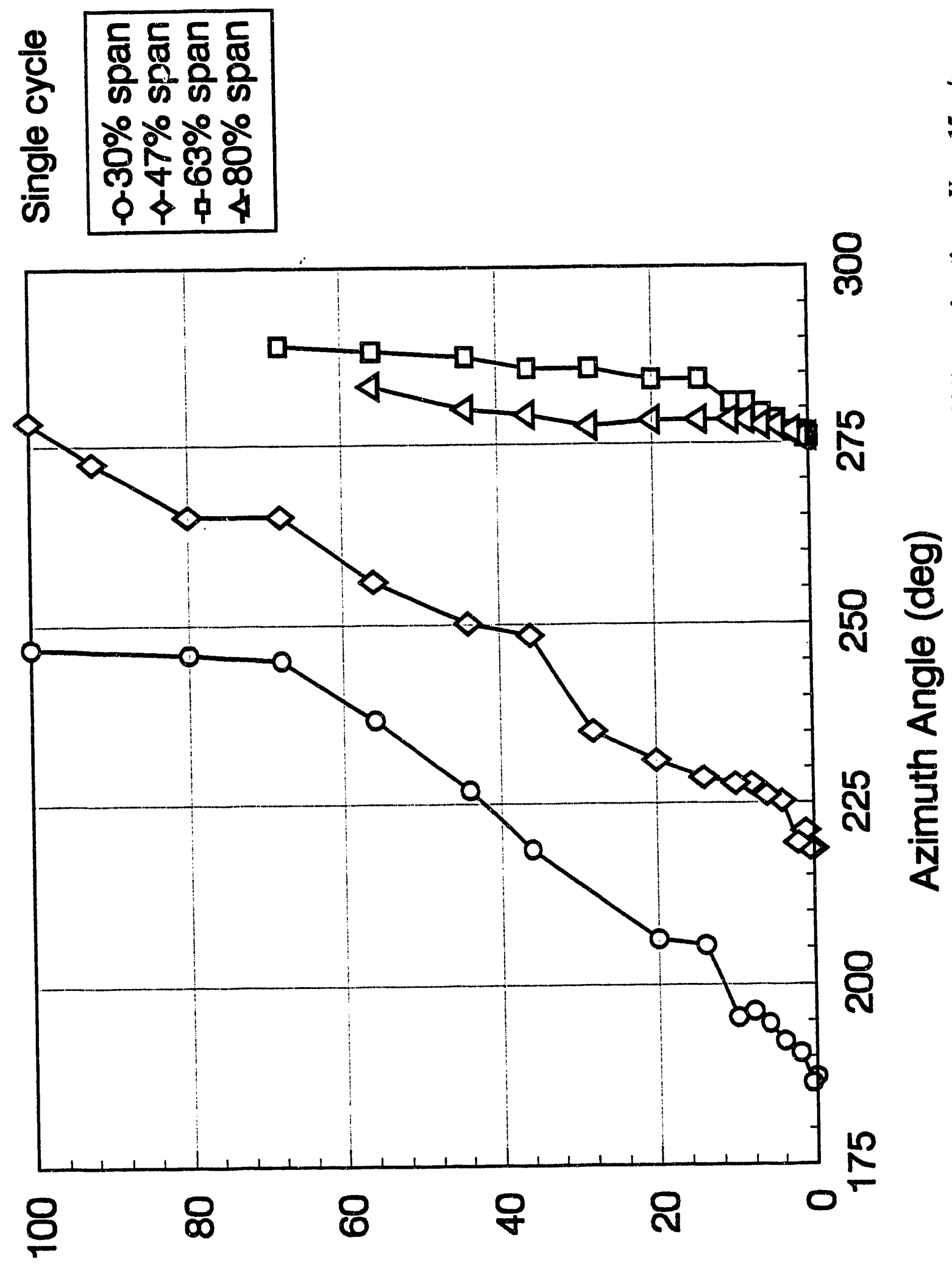

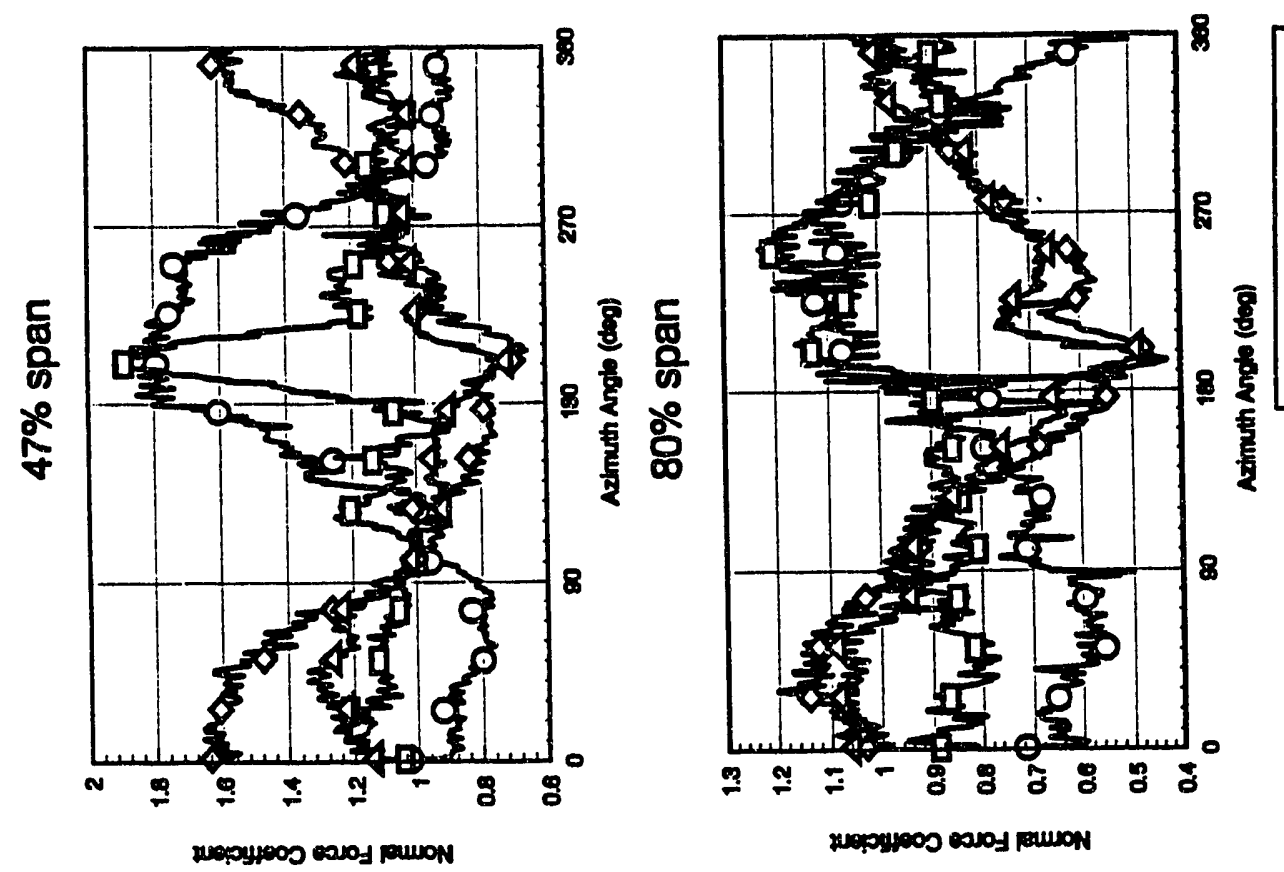

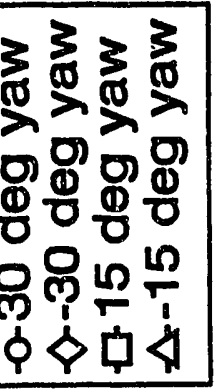


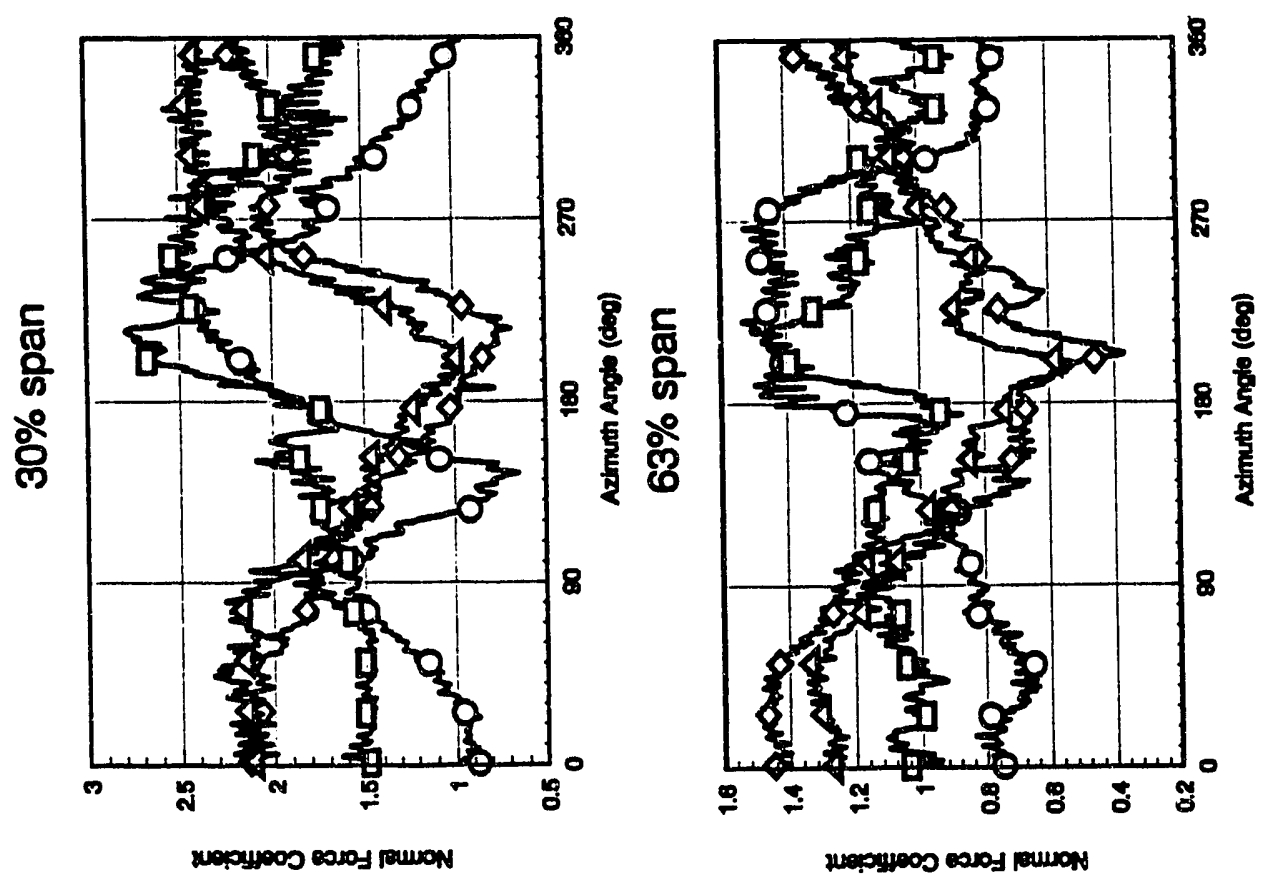

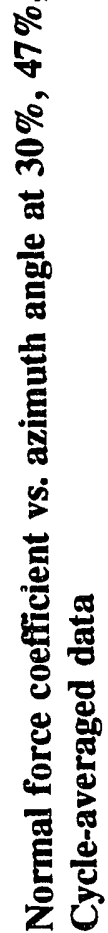

mं 

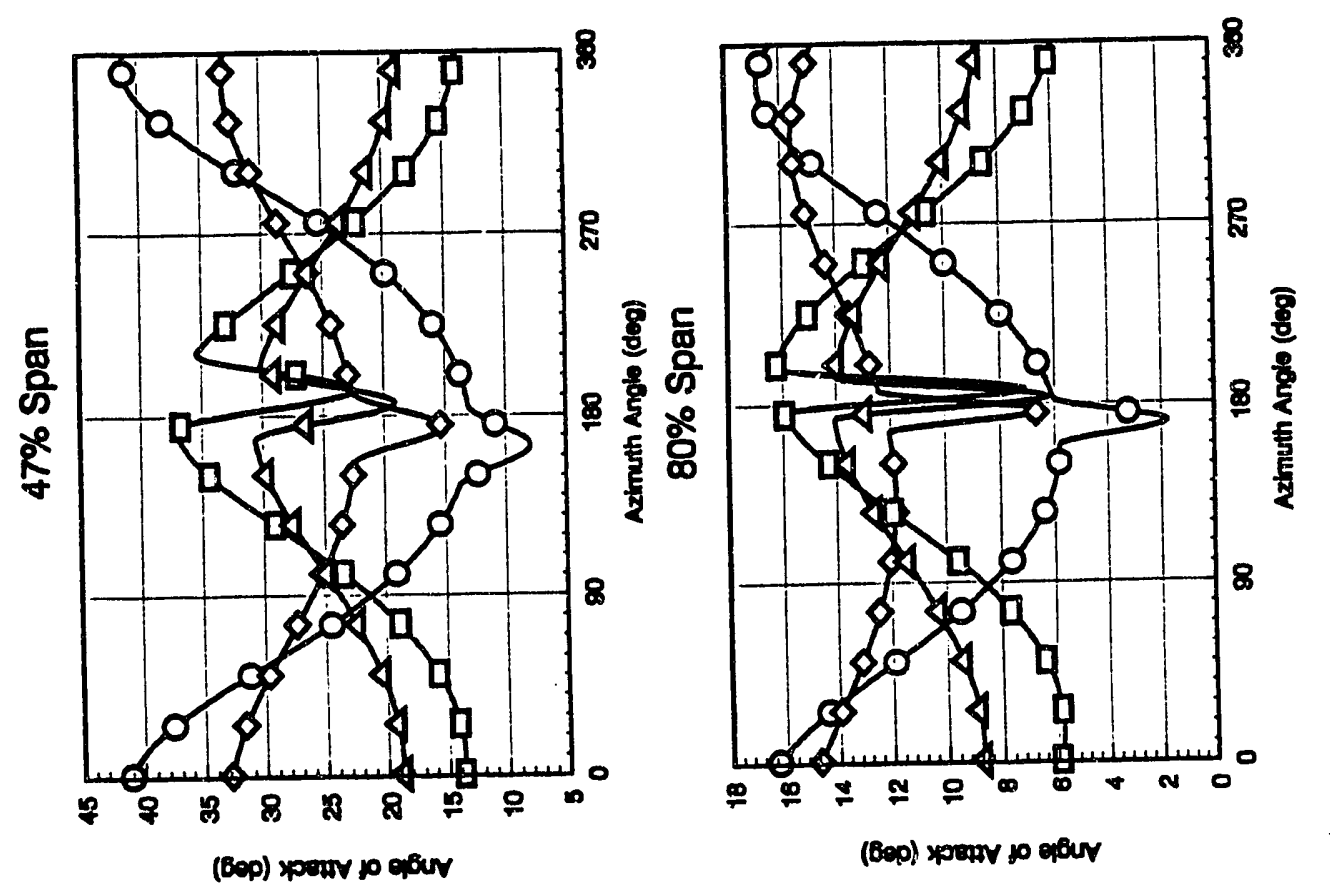

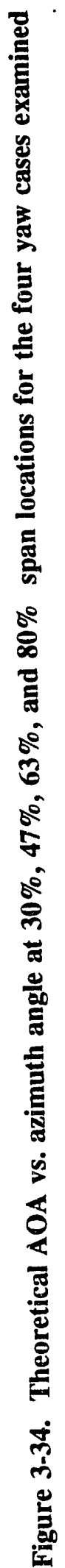
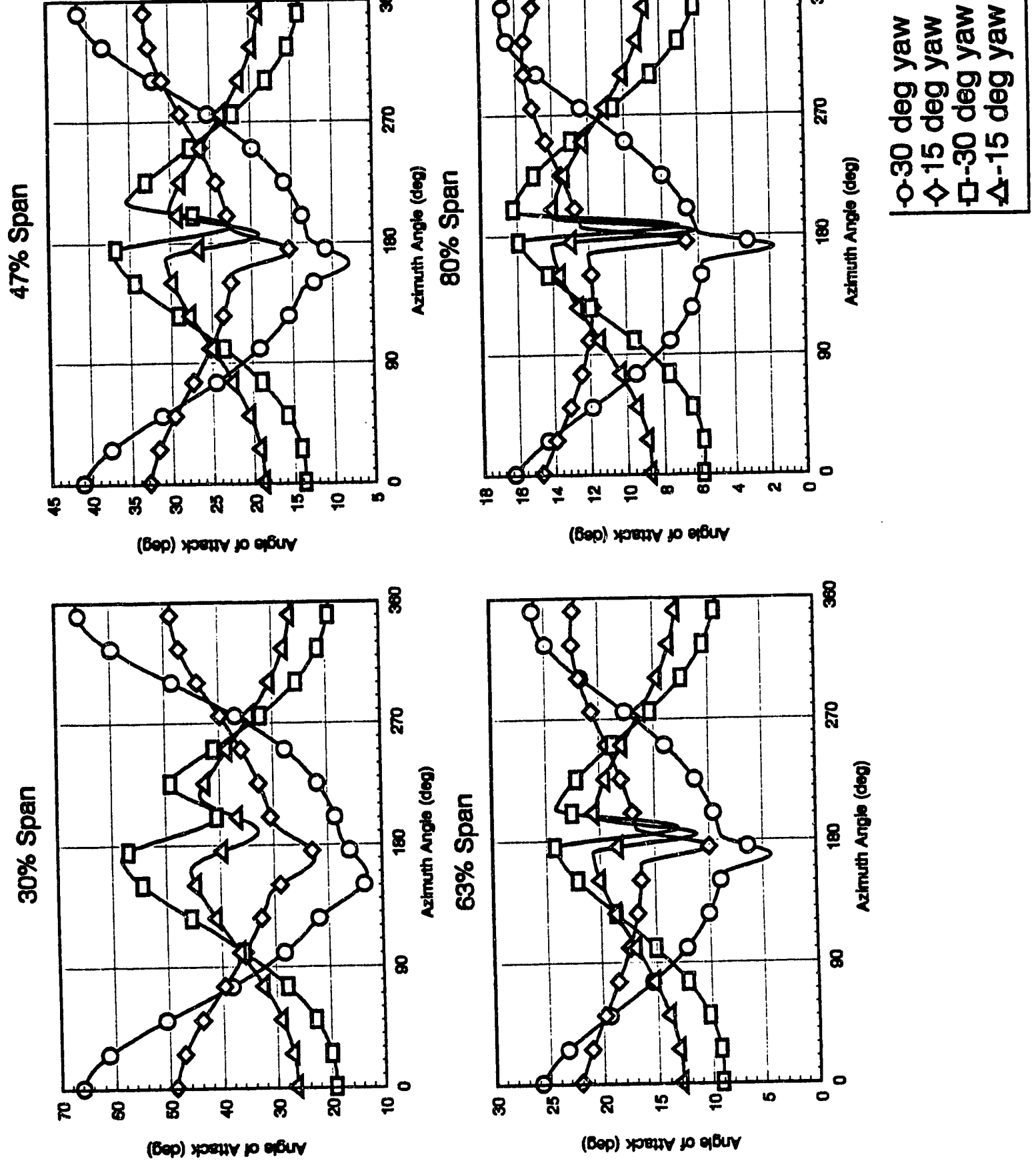

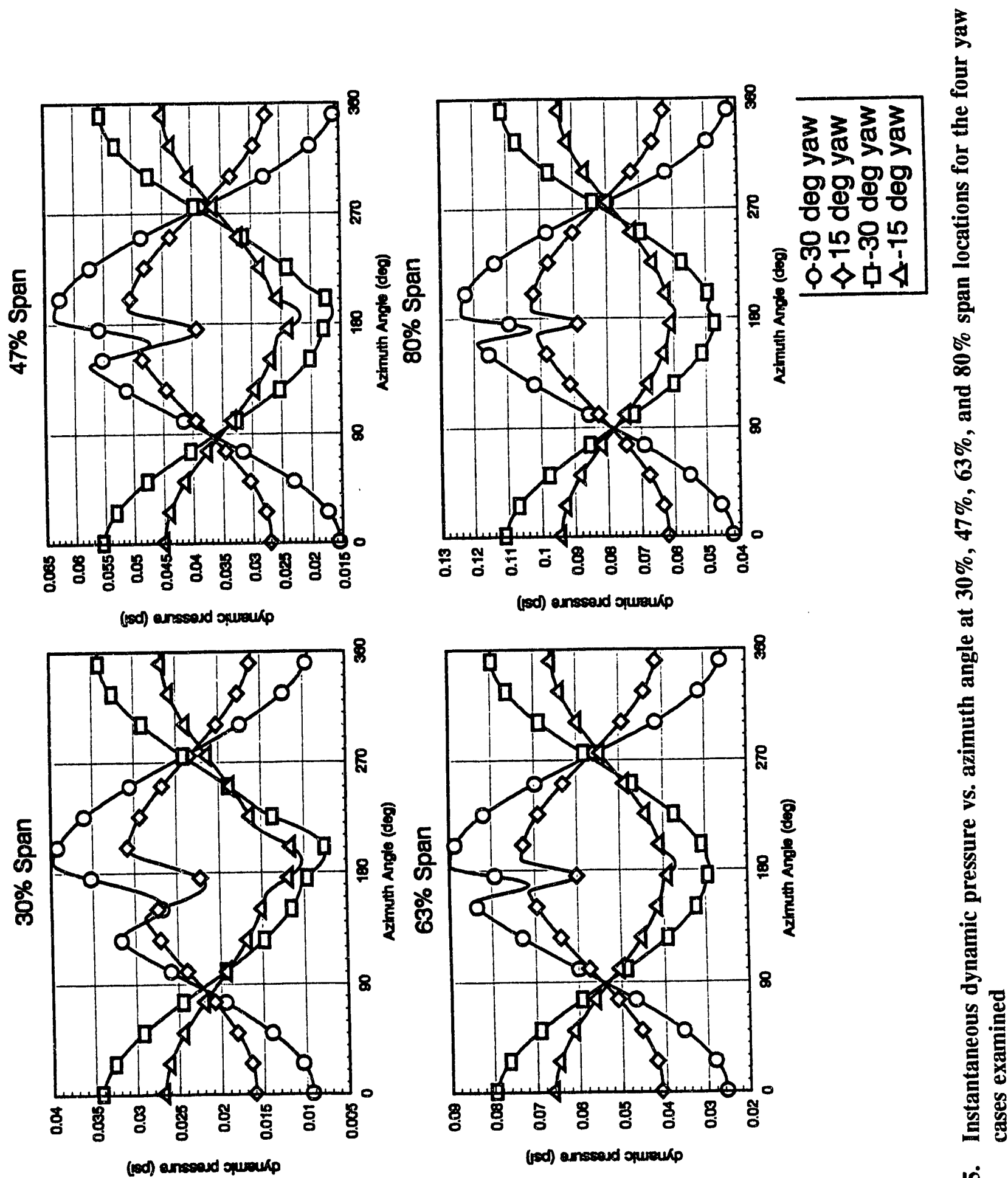

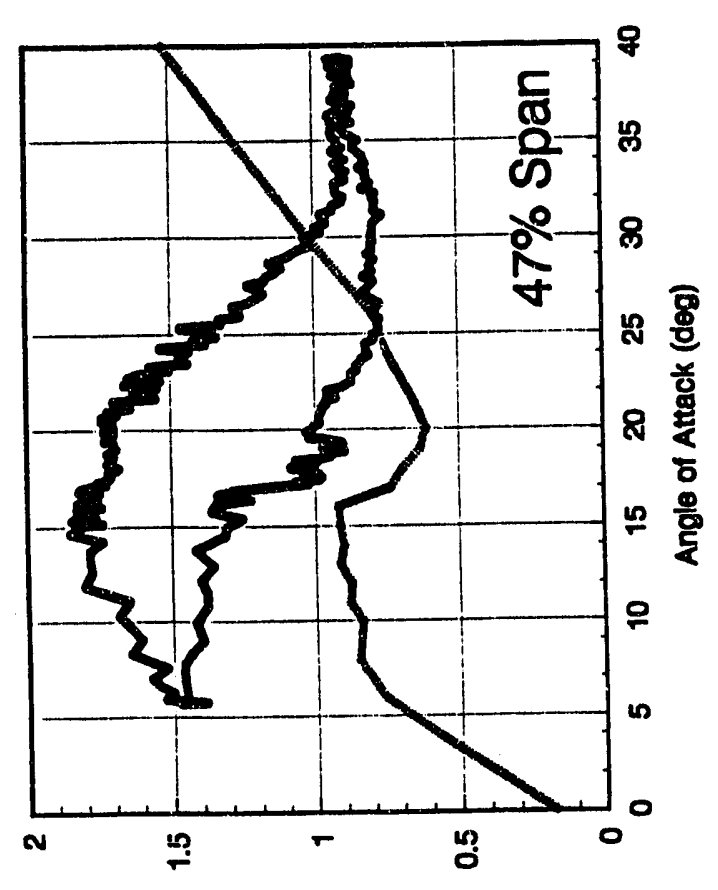

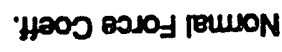

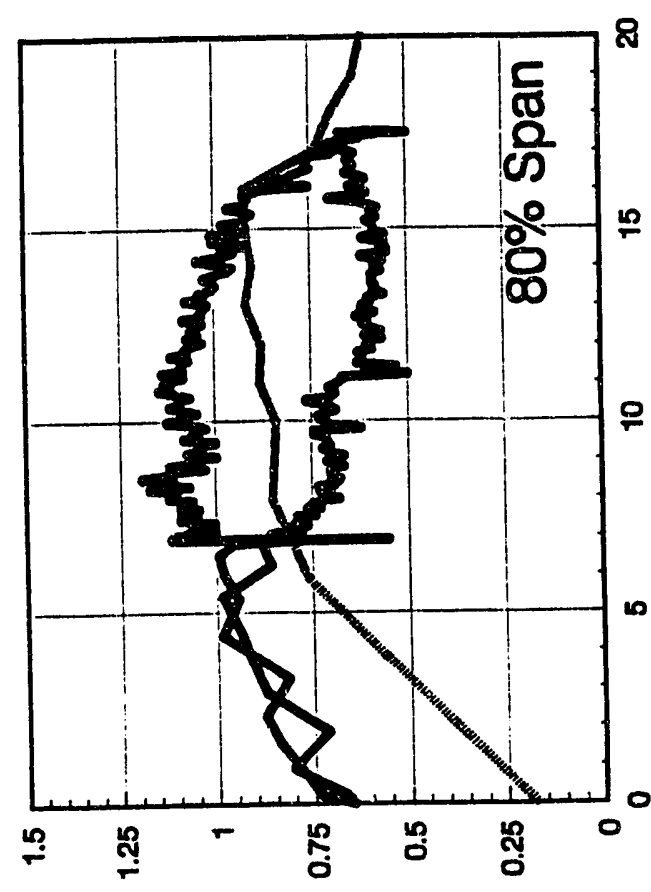

范

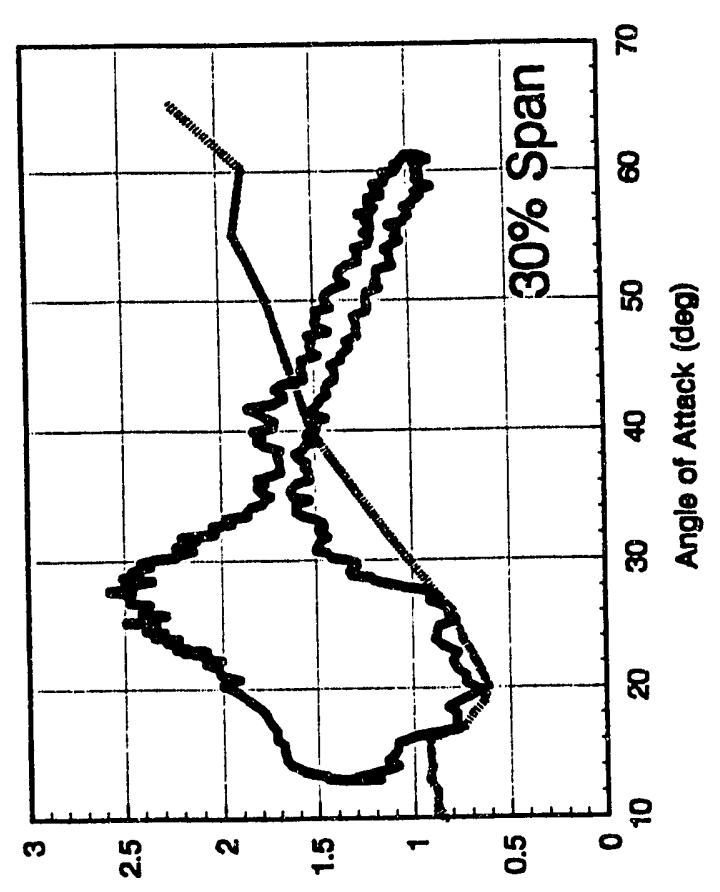

'HOOJ 050] REULN

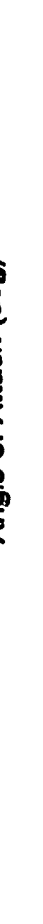

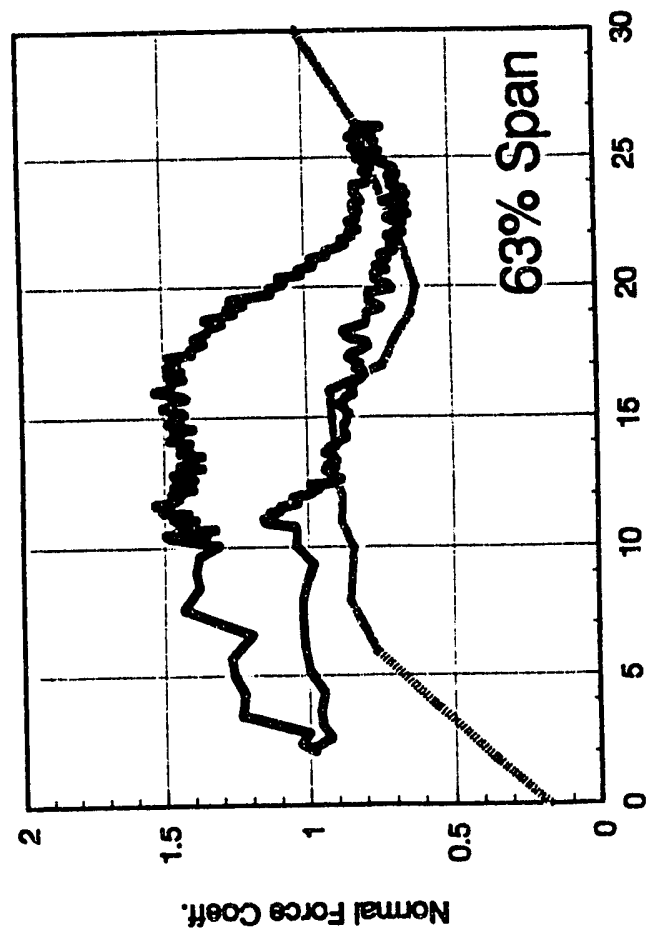

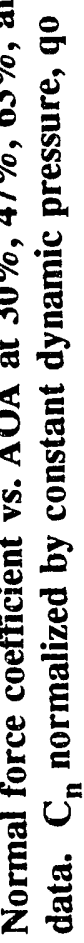

芦 
At $47 \%$ and $63 \%$ span, consistent lift enhancement was generally seen, and significant hysteresis was observed. At $80 \%$ span, a hysteresis loop was formed, and lift enhancement was observed for increasing $a$ and lift reduction was observed for decreasing $\alpha$. At each span location, maximum $C_{n}$ was seen proximal to minimum $A O A$, and minimum $C_{n}$ was seen near maximum AOA. Also, the $C_{n}$ versus $\alpha$ curves appeared to diverge from static values. This can be demonstrated especially well at $80 \%$ span. The characteristics of these $C_{n}$ versus $\alpha$ curves were found to be inconsistent with wind tunnel tests for pitching and oscillating wings [10-12,14,16].

Large, cyclic variations in instantaneous dynamic pressure associated with a yawed turbine were demonstrated in Figure 3-35. To explain the inconsistencies between present observations and wind tunnel tests, $C_{n}$ values were nondimensionalized by instantaneous dynamic pressure, $q$. These results may be seen in Figures 3-37 and 3-38 for $30^{\circ}$ and $-30^{\circ}$ yaw, respectively. Since constant dynamic pressure was used in the wind tunnel tests referenced above, it seems necessary to nondimensionalize the force coefficients by instantaneous $q$ to provide fair comparisons. The $C_{n}$ versus $\alpha$ curves shown in Figure 3-37 for the $30^{\circ}$ yaw case were significantly different from those in Figure 3-36, where qo was used to nondimensionalize the normal forces. At 30\% span, a hysteresis loop was observed between $\alpha=15^{\circ}$ and $30^{\circ}$, and considerable lift enhancement twice that of static values was observed. From $30^{\circ}$ to $65^{\circ}$, little hysteresis was seen, although lift enhancement was still observed. Notice, however, that the lift slope followed that of the static data, with dynamic values offset by approximately 0.5 . Finally, maximum $\mathrm{C}_{\mathrm{n}}$ values were seen at maximum $\alpha$.

At $47 \%$ span, hysteresis was observed between $\alpha=7.5^{\circ}$ and $\alpha=30^{\circ}$, with minimal hysteresis observed for the higher AOAs. Considerable lift enhancement continued to occur and was as great as $50 \%$ of maximum static values. For a greater than $30^{\circ}$, the lift slope approximately followed that of the static data but was offset by 0.1 . This is qualitatively similar to results seen at $30 \%$ span.

At $63 \%$ span, the width of the hysteresis loop appeared to diminish, but lift enhancement was still observed, now reaching $75 \%$ of maximum static values. Minimal lift reduction was seen at the lower effective AOAs for decreasing $\alpha$. At $80 \%$ span, the hysteresis loop appeared minimal. In addition, lift reduction was seen between $6^{\circ}$ and $10^{\circ}$ for increasing or decreasing $\alpha$. Considerable lift enhancement, $60 \%$ greater than maximum static values, occurred near maximum $\alpha$. The dynamic lift slope also appeared equivalent to the linear part of the static lift slope. Finally, at each span location, maximum $C_{n}$ values were observed at maximum AOA and were much more consistent with wind tunnel data provided for oscillating wings.

The $C_{n}$ versus $\alpha$ curves for the $-30^{\circ}$ yaw case appeared quite different compared with the positive yaw case. At 30\% span, minimal hysteresis was observed at the lower AOAs. Between $\alpha=40^{\circ}$ and $\alpha=50^{\circ}$, a significant increase in $C_{n}$ was observed, forming a hysteresis loop. This increase was observed as the blade decreased in AOA, however. As the AOA continued to decrease, the curve diverged greatly, with $C_{n}$ values decreasing to 1.5 between $\alpha=42^{\circ}$ and $\alpha=48^{\circ}$. This occurred as the blade passed out of the tower wake, and the divergence may have been caused by inadequate modeling of the tower wake. Otherwise, the dynamic lift slope followed closely the static lift slope.

At $47 \%$ span, little hysteresis was observed except at lower angles, but consistent lift enhancement did occur. Lift was enhanced by as much as $100 \%$ over static values. Effects of passage through the tower wake may be seen at higher AOAs, as the trace appeared to diverge from the dynamic curve and formed a "zig-zag" in the trace. At $63 \%$ span, increased hysteresis was seen, and consistent lift enhancement was observed, with maximum dynamic values $50 \%$ greater than static counterparts. At $80 \%$ span, lift enhancement was observed as the blade was increasing in $\alpha$ and lift reduction was observed as the blade was decreasing in $\alpha$. Increased hysteresis was observed as well. The trace drawn out followed the static curve as the blade passed through the tower shadow. In general, increased hysteresis was observed at 

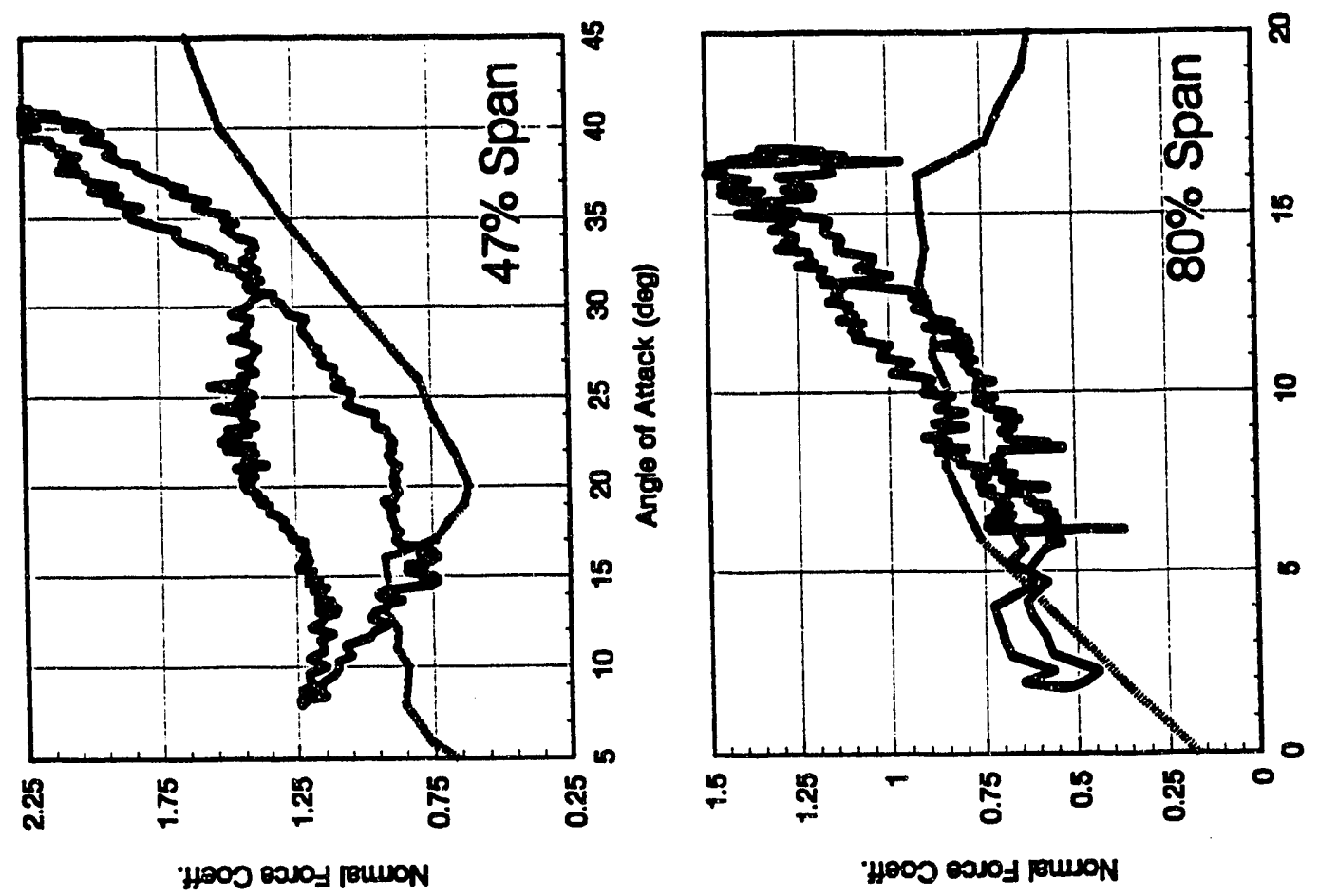

告

'H000 c000] punoN

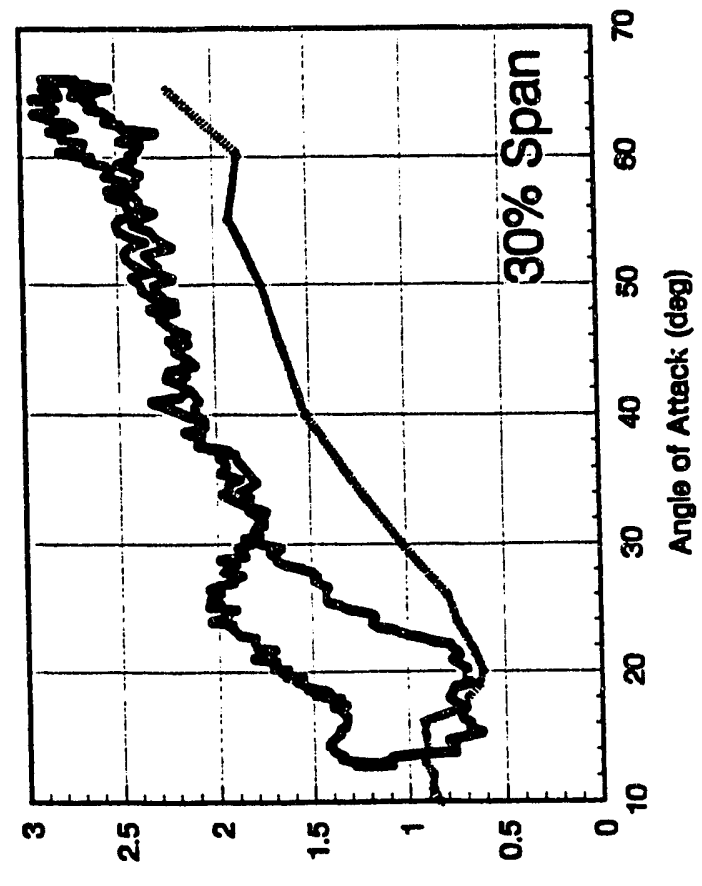

HeOS eavey fRUNON

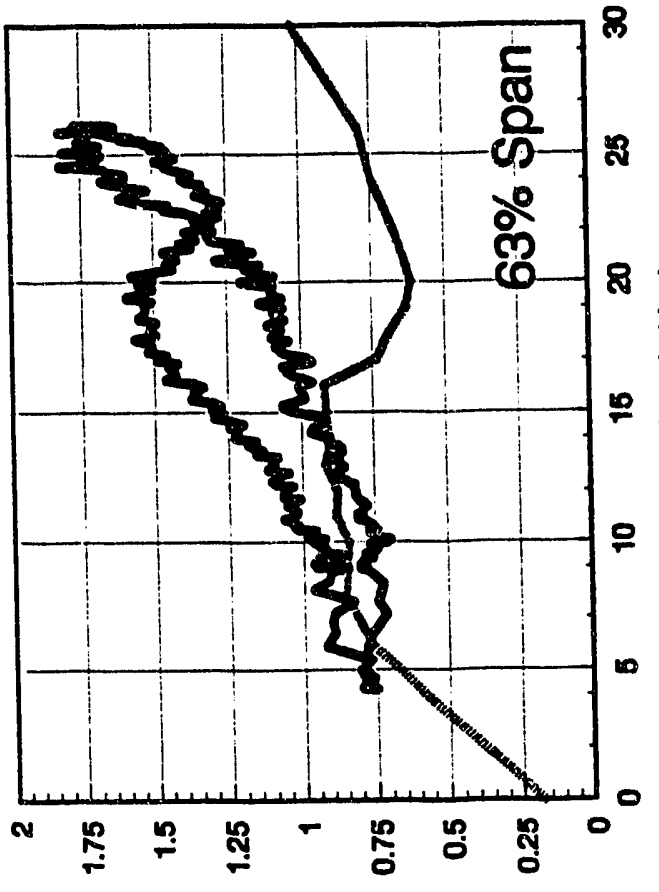

'H800 8010J BuسNN

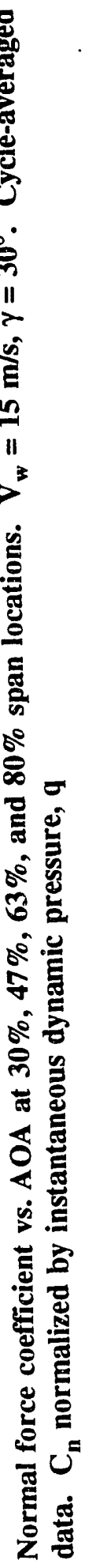

官 
TP-442-4864
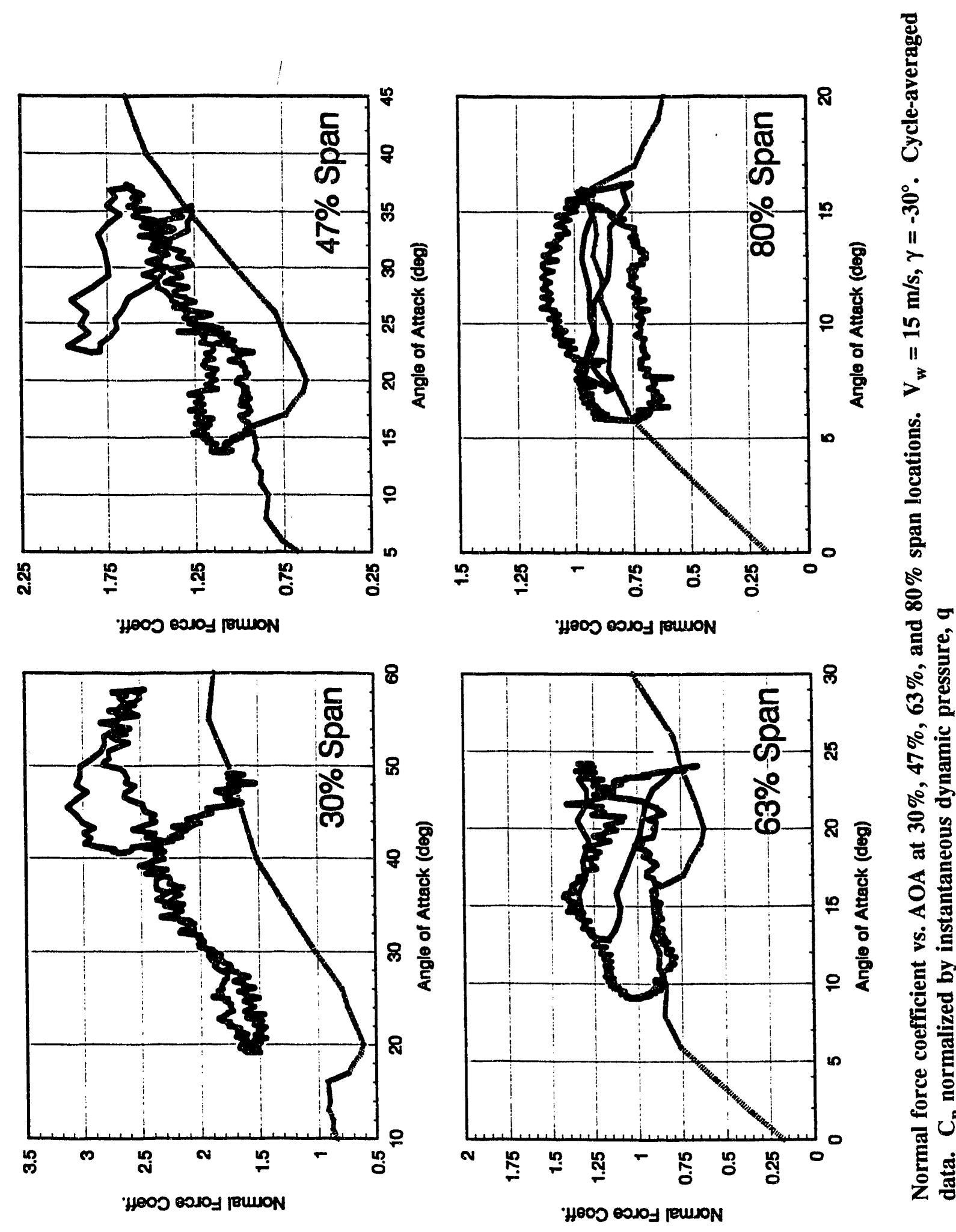

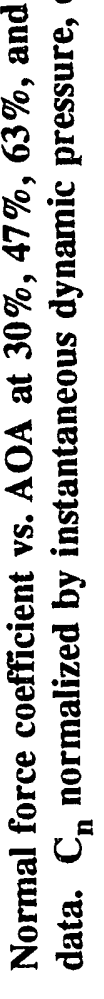

突

54 
outboard span locations. As the blade passed through the tower wake, the curves seemed to diverge at each span location. This is in contrast to the curves seen for positive yaw, in which the traces seemed well-behaved as the blade passed through the tower shadow.

\subsection{Spanwise Pressure Distributions}

Upper surface suction pressure was plotted as a function of span at $4 \%$ chord to provide spanwise pressure distributions. $C_{p}$ values were nondimensionalized by the wind velocity and tip velocity only to reflect the genuine nature of the pressure forces acting on the blade. Figures 3-39 and 3-40 show the spanwise pressure distribution for zero yaw and $V_{w}=7.7 \mathrm{~m} / \mathrm{s}$ and $V_{w}=14.7 \mathrm{~m} / \mathrm{s}$, respectively. At $7.7 \mathrm{~m} / \mathrm{s}$, the spanwise pressure distribution reflects a relatively steady flow field. At $\psi=180^{\circ}$, the effect of passage through the tower wake may be seen by a suction pressure valley in the topographical distribution. Maximum suction pressure was also seen at inboard span locations and decreased from $C_{p}=0.5$ at $30 \%$ span to 0.3 at $80 \%$ span. This indicates that the largest forces acting on the blade occur at $30 \%$ span.

As the wind velocity was doubled to $14.7 \mathrm{~m} / \mathrm{s}$, significant differences in flow field behavior were observed. At $30 \%$ span, average $\mathrm{C}_{\mathrm{p}}$ values are approximately 0.8 . These values decrease to approximately 0.5 at $47 \%$ span and then increase linearly to 1.75 at $80 \%$ span. The effects of passage through the tower wake also appear different. Formation of a suction pressure ridge in the spanwise pressure distribution first appears at $40 \%$ span and gradually builds, reaching a peak at $53 \%$ span. This suction pressure ridge persists out to $80 \%$ span. Overall, this spanwise pressure distribution appears to be quite unsteady and turbulent compared with that of the lower wind speed test case.

Spanwise pressure distribution is also shown in Figure 3-41 for $V_{w}=15 \mathrm{~m} / \mathrm{s}$ and $30^{\circ}$ yaw. This pressure distribution is considerably different than that for the $0^{\circ}$ yaw test case. As can be seen, at $\psi=190^{\circ}$, a suction pressure peak was seen at $30 \%$ span and extended outboard to $80 \%$ span. Interestingly, the magnitude of $\mathrm{C}_{\mathrm{p}}$ was approximately -1.5 and appeared equivalent from $30 \%$ to $80 \%$ span. Although hidden in the figure, suction pressure decreased rapidly to -0.5 at $220^{\circ}$. At $80 \%$ span, however, the $C_{p}$ values of -1.5 continue to persist to $\psi=330^{\circ}$. In fact, at outboard stations along the span, there was a corresponding increase in high suction pressure residence time with respect to the rotation cycle. From $\psi=0^{\circ}$ to $90^{\circ}, \mathrm{C}_{\mathrm{p}}$ values appeared to decrease minimally from $30 \%$ to $80 \%$ span.

\subsection{Global Measurements}

Measurements of bending mornents, yaw moments, torque and power output were investigated to determine the global effects of the resultant aerodynamic forces produced by a rotating wind turbine. Root flap bending moment results from forces that are applied normal to the upper surface of the wind turbine blade and measured at the point where the blade attaches to the hub. Low-speed shaft torque results from the generation of forces in the plane of rotation and is responsible for the generation of power to the wind turbine. Generator power was also measured and is a direct result of low-speed shaft torque. Yaw moment was also measured for yaw cases in which the yaw brake was set. Because the wind turbine was designed as a downwind machine, the turbine will tend to align itself with the wind because the relative amount of lift will be greater on one side of the rotor disk than on the other. The difference in force produced a yaw moment that can be measured with the brake on. It should be noted that low-speed shaft torque, power generation, and yaw moment result as a combined effect of all three blades.

Root flap bending moment was plotted as a function of azimuth angle in Figure 3-42 for zero yaw and the four wind speeds examined. An increase in $\mathrm{V}_{\mathrm{w}}$ resulted in a corresponding increase in flap bending moment. Flap bending moment was not constant throughout the rotation cycle. For each test case, there 


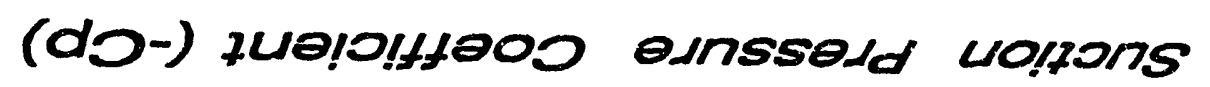

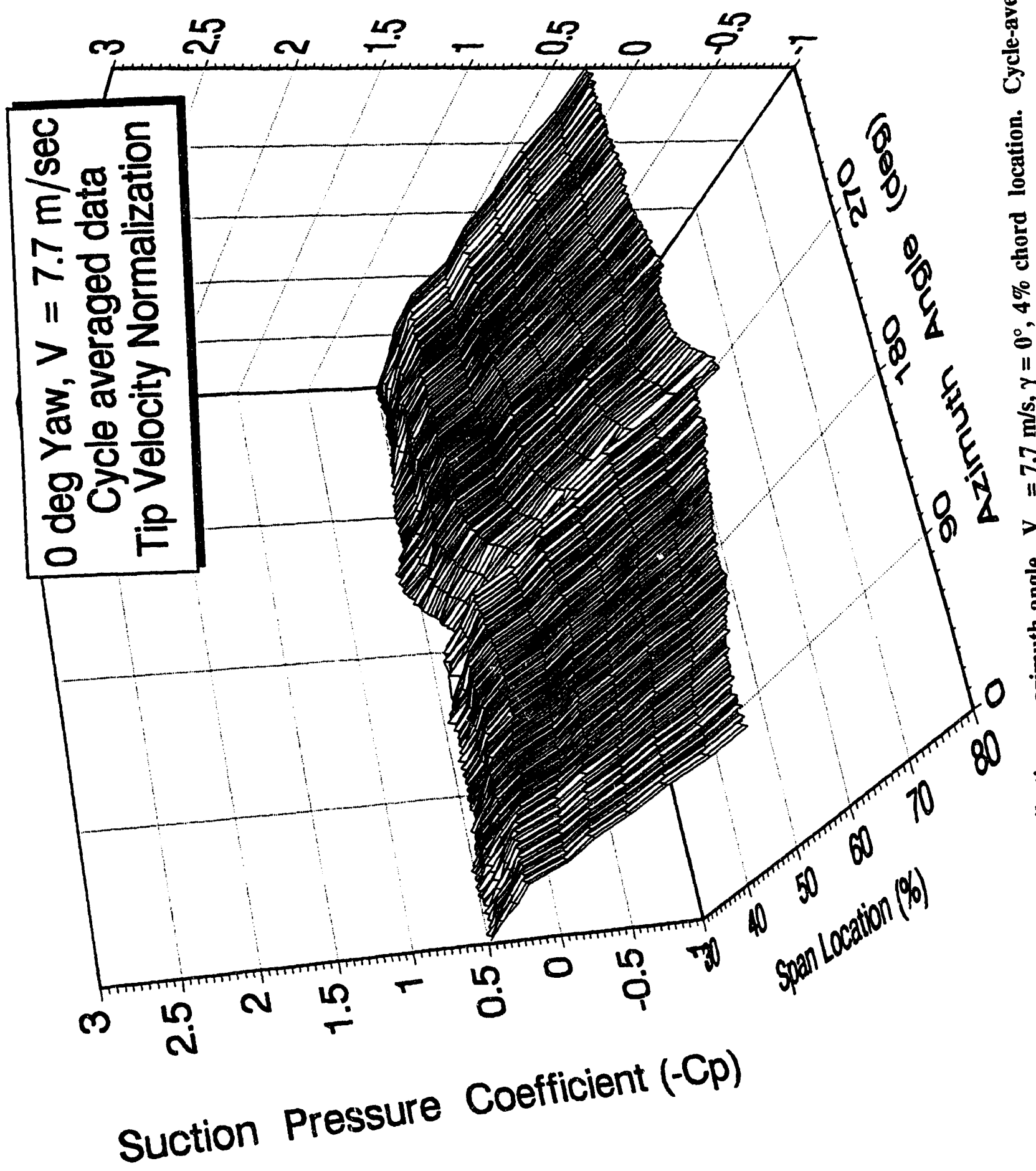

营

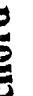




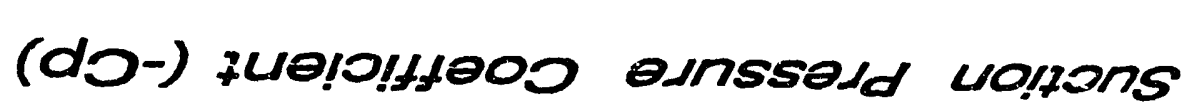

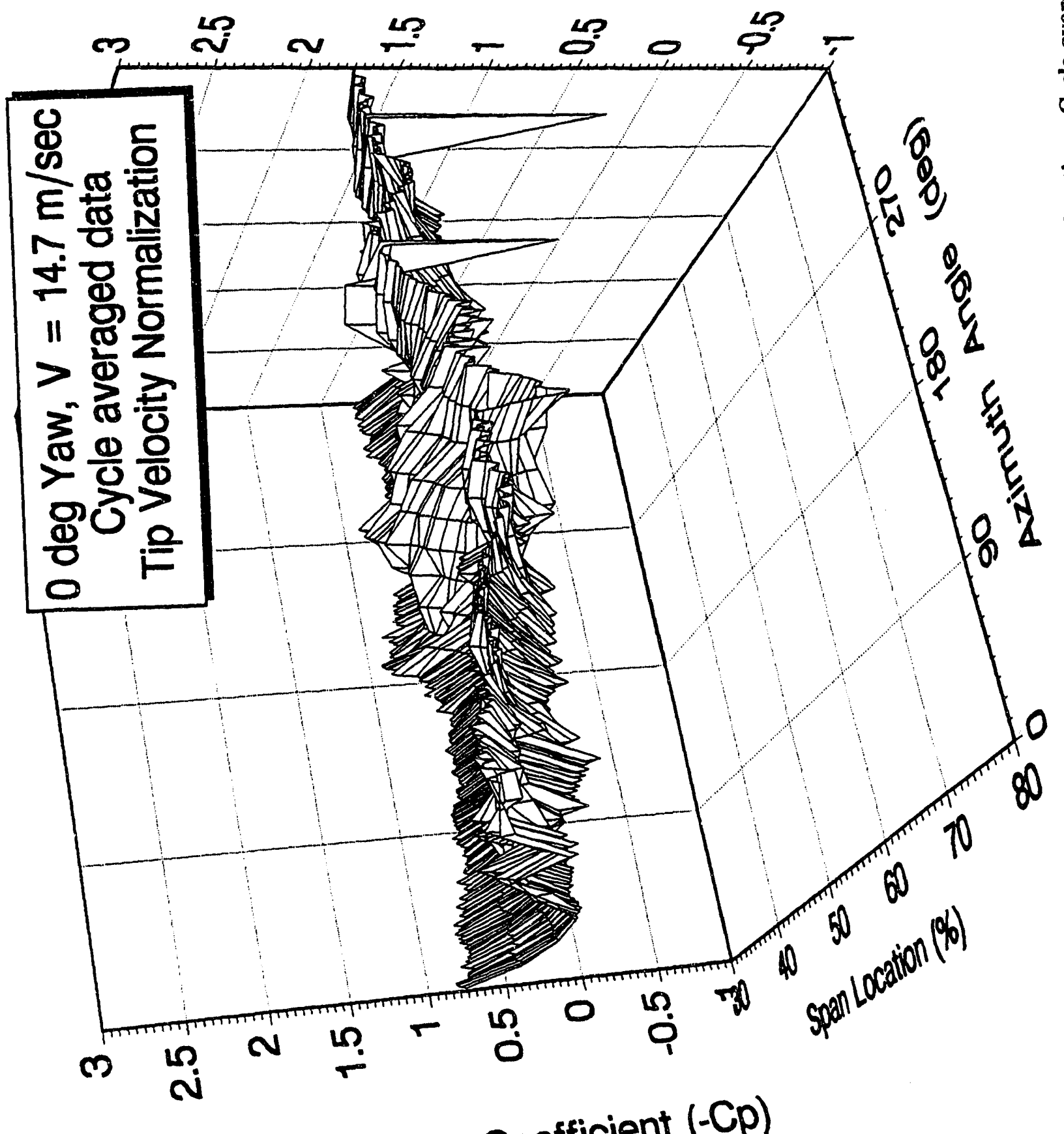

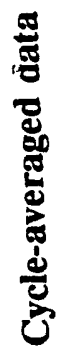

Suction Pressure Coefficient $(-C P)$ 


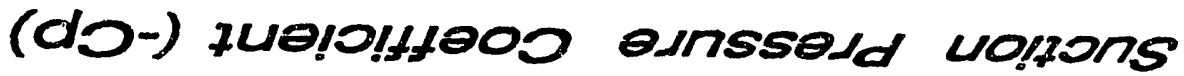

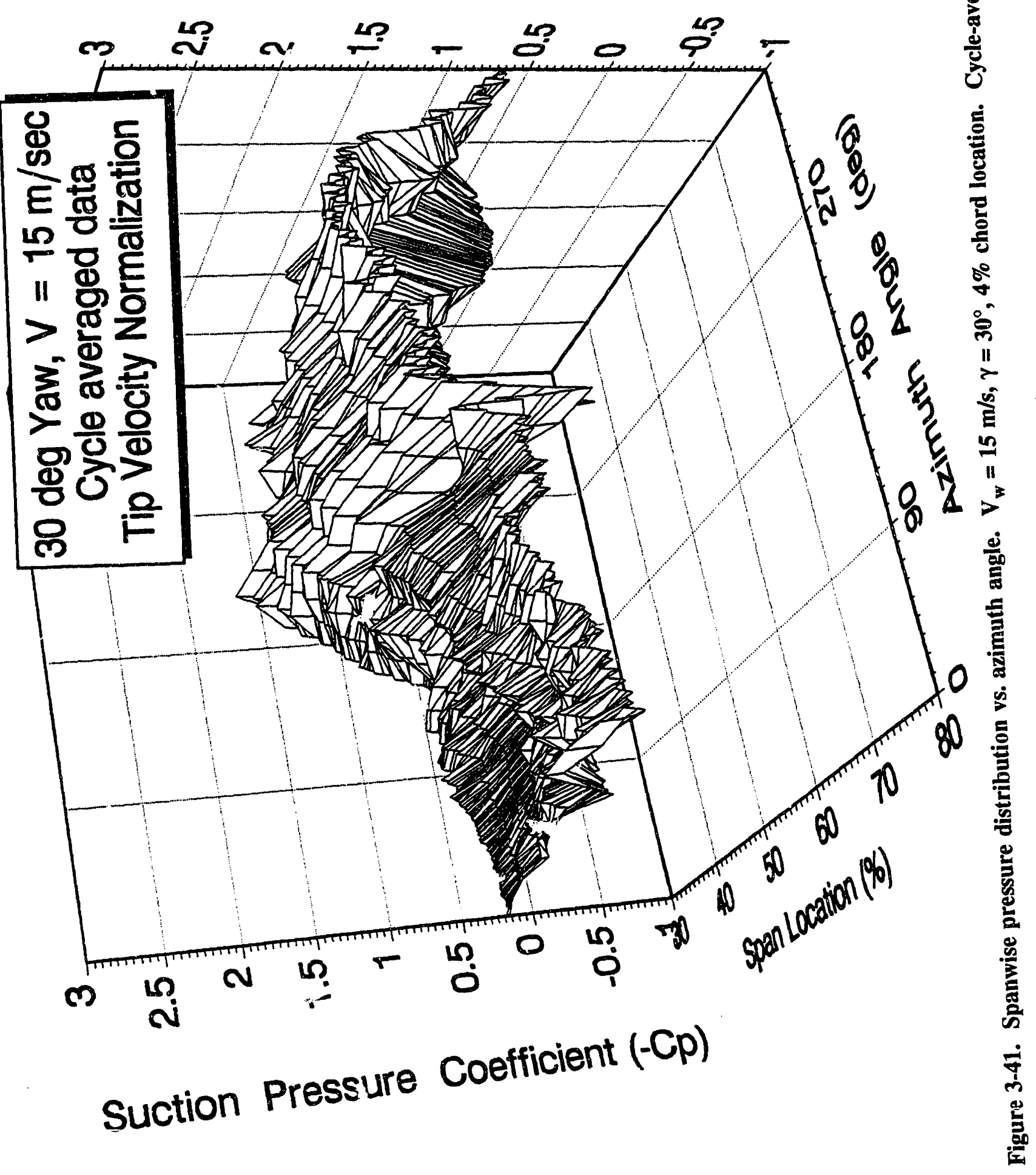



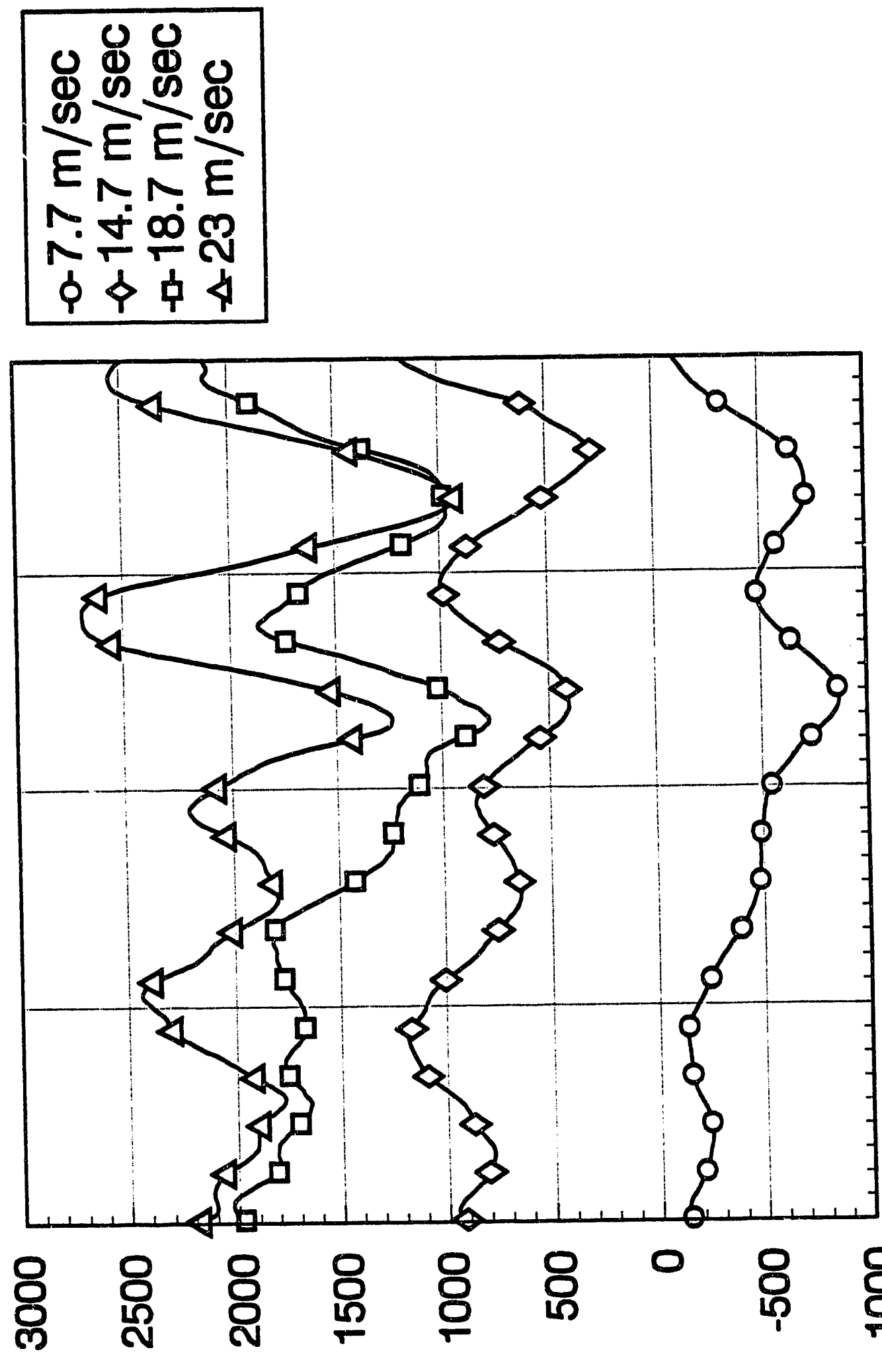

\&

苞 
appeared to be four large fluctuations in flap bending moment; these fluctuations were a direct result of the natural resonant frequency of the blade, which also corresponded to four per rev. An increase in wind velocity, however, resulted in the generation of larger amplitude fluctuations in bending moment. For $\mathrm{V}_{\mathrm{w}}=23 \mathrm{~m} / \mathrm{s}$, fluctuations as large as $1500 \mathrm{~N}-\mathrm{m}$ were observed. The largest fluctuations were also seen just after the blade passed through the tower wake.

Low-speed shaft torque was plotted as a function of azimuth angle in Figure 3-43. An increase in wind velocity resulted in a corresponding increase in torque. A fourfold increase in torque was seen as $V_{w}$ was doubled from 7.7 to $14.7 \mathrm{~m} / \mathrm{s}$. Increases in $V_{w}$ to $23 \mathrm{~m} / \mathrm{s}$ resulted in only a $25 \%$ increase in torque. There appeared to be a variation in torque over the rotation cycle, with three distinct peaks in torque observed corresponding to the number of blades used on this turbine. Generator power is shown in Figure 3-44 and, as expected, correlates directly with low-speed shaft torque with a few exceptions. As Vw was doubled from $7.7 \mathrm{~m} / \mathrm{s}$ to $14.7 \mathrm{~m} / \mathrm{s}$, a sixfold increase in power was seen. Increases in $\mathrm{Vw}$ to $23 \mathrm{~m} / \mathrm{s}$ resulted in a $25 \%$ increase in power, which was also seen in the torque. There were also three distinct peaks in power output that corresponded directly with the jeaks seen in low-speed shaft torque with one important exception. Peak power was approximately $20^{\circ}$ out of phase with low-speed shaft torque and was consistently delayed until later in the rotation cycle.

Root flap bending moment (where bending occurs in the plane of the rotor disk) was plotted as a function of azimuth angle for the four yaw cases examined and is shown in Figure 3-45. Flap bending moment varies throughout the rotation cycle, with peak bending moments occurring at approximately $\mathrm{w}=240^{\circ}$ for the positive yaw cases and at approximately $90^{\circ}$ for the negative yaw cases. Positive and negative yaw cases demonstrated an approximate $150^{\circ}$ phase difference in terms of maximum flap bending moment. For each test case, a four per rev variation in flap bending moment was also seen, corresponding to the resonant frequency of the blade. The amplitude of the four per rev resonance appeared less for the yawed cases than for the zero yaw case of $14.7 \mathrm{~m} / \mathrm{s}$, especially for $15^{\circ}$ yaw error.

Low-speed shaft torque was also examined for the four yaw test cases and is shown in Figure 3-46. Torque varied approximately sinusoidally over the rotation cycle for each yaw case, and each of the traces appeared similar, with some exceptions. The $15^{\circ}$ yaw case generated the greatest average torque of approximately $2000 \mathrm{~N}-\mathrm{m}$. The $-15^{\circ}$ yaw case generated approximately $1750 \mathrm{~N}-\mathrm{m}$ of torque, and the $30^{\circ}$ and $-30^{\circ}$ yaw cases generated approximately $1700 \mathrm{~N}-\mathrm{m}$ of torque. Also, the positive yaw cases were approximately in phase with each other, and the negative yaw cases were in phase with each other, but were approximately $45^{\circ}$ out of phase with the positive yaw cases and were delayed until later in the cycle. Finally, an additional peak in torque was seen at $\psi=200^{\circ}$ for the $-30^{\circ}$ yaw case.

The corresponding generator power is plotted in Figure 3-47. Consistent with the zero yaw cases, generator power was approximately $20^{\circ}$ out of phase with corresponding torque and was delayed until later in the cycle. Approximately $12 \mathrm{~kW}$ of power was generated for the zero yaw case and $14.7 \mathrm{~m} / \mathrm{s}$. A $15^{\circ}$ yaw resulted in the generation of approximately $8 \%$ more power than that of the zero yaw case. Decreases in average power output were seen for the other yaw cases, with the largest decrease, approximately $12.5 \%$, seen for $-30^{\circ}$ yaw.

Yaw moment was plotted as a function of azimuth angle and may be seen in Figure 3-48. As expected, positive yaw moment was produced for negative yaw, and negative yaw moments were produced for positive yaw. Significant variation in yaw moment was observed over the rotation cycle for each of the yaw cases examined. Approximately three peaks in negative moment were seen for the positive yaw cases at $\psi=70^{\circ}, 190^{\circ}$, and $310^{\circ}$. The negative peak seen at $190^{\circ}$ was significantly smaller than peaks seen at the other two points in the rotation cycle. The peaks in positive moment seen for the negative yaw cases occurred at $\psi=20^{\circ}, 140^{\circ}$, and $260^{\circ}$, with the smallest relative peak occurring at $20^{\circ}$. These peak yaw 

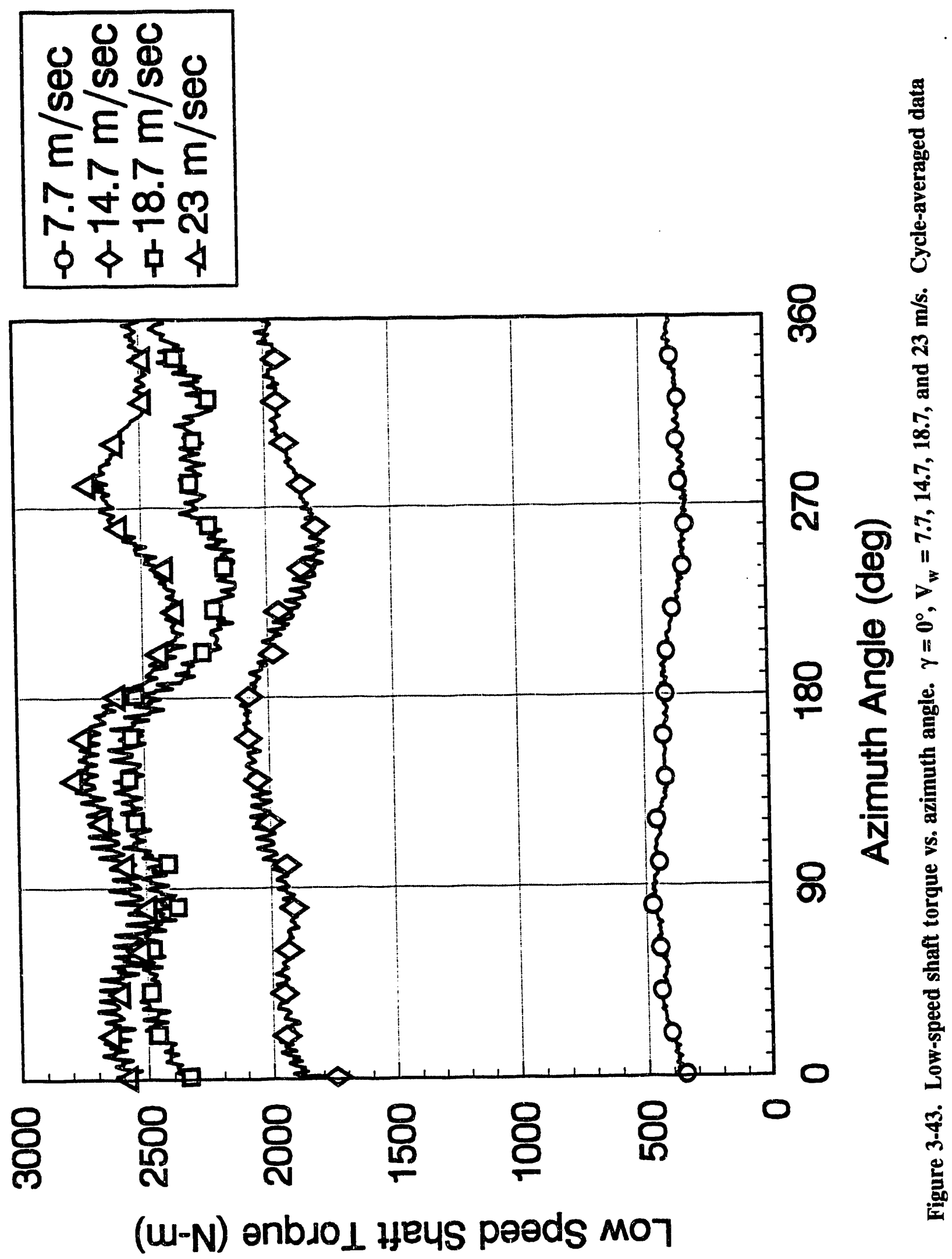


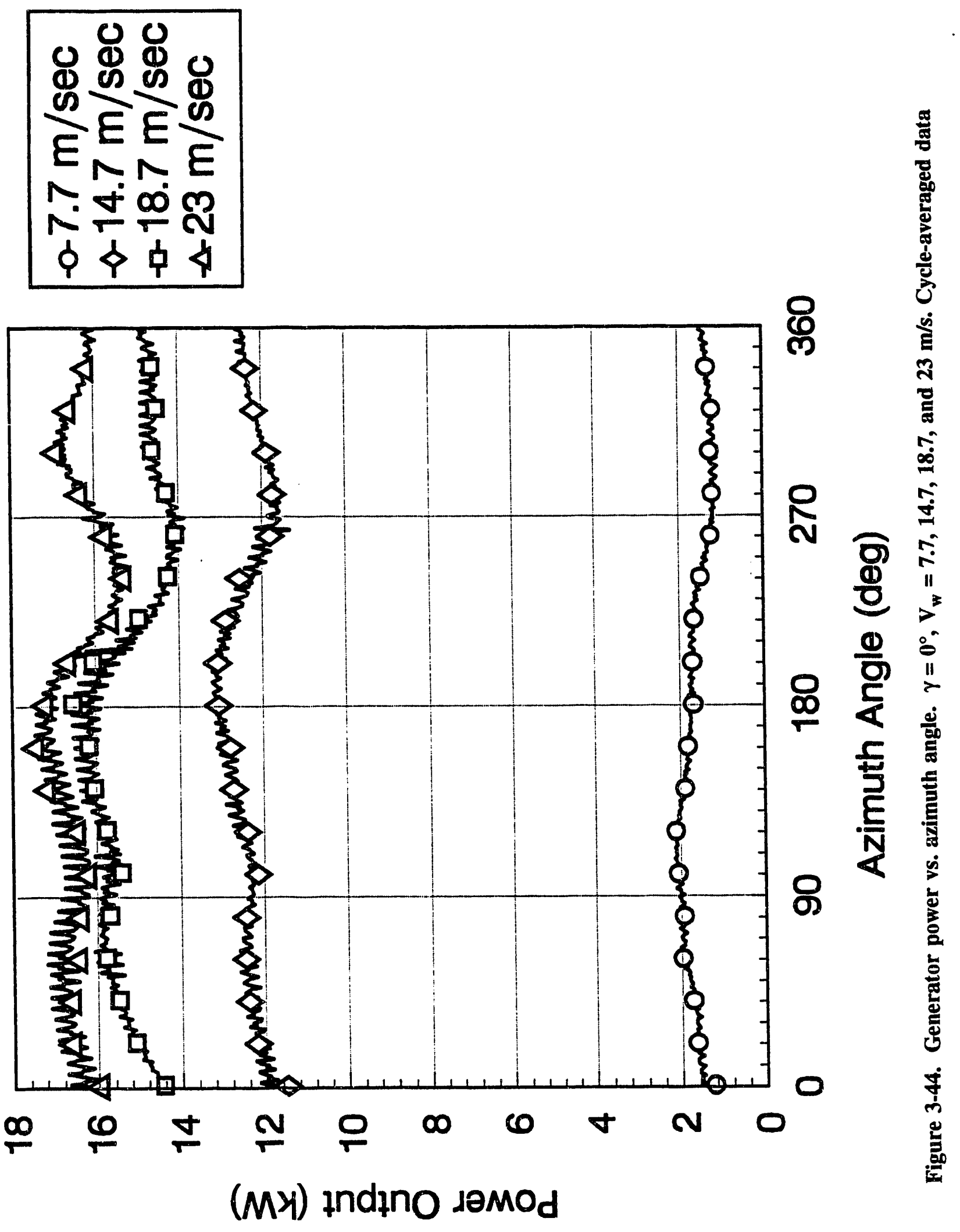




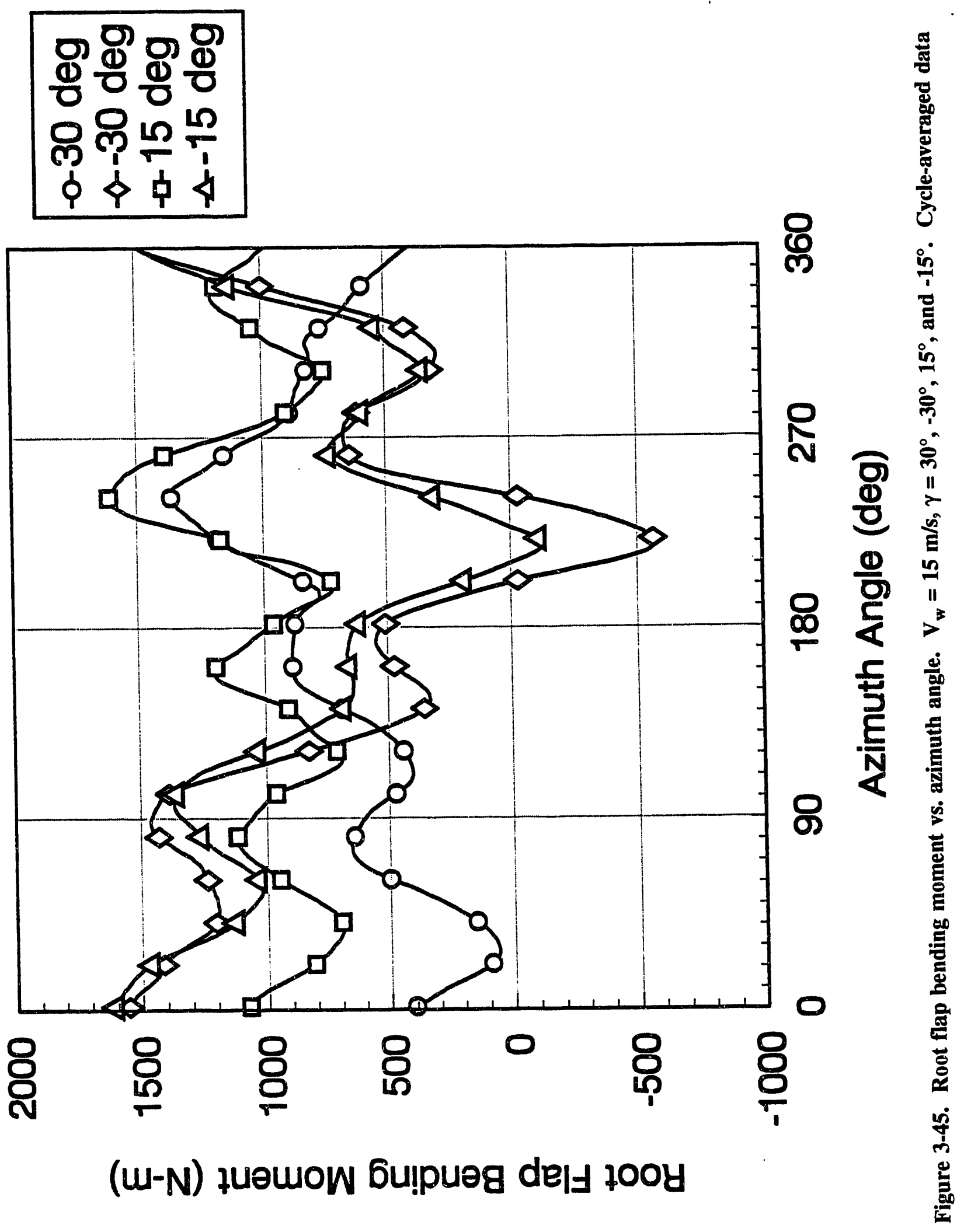




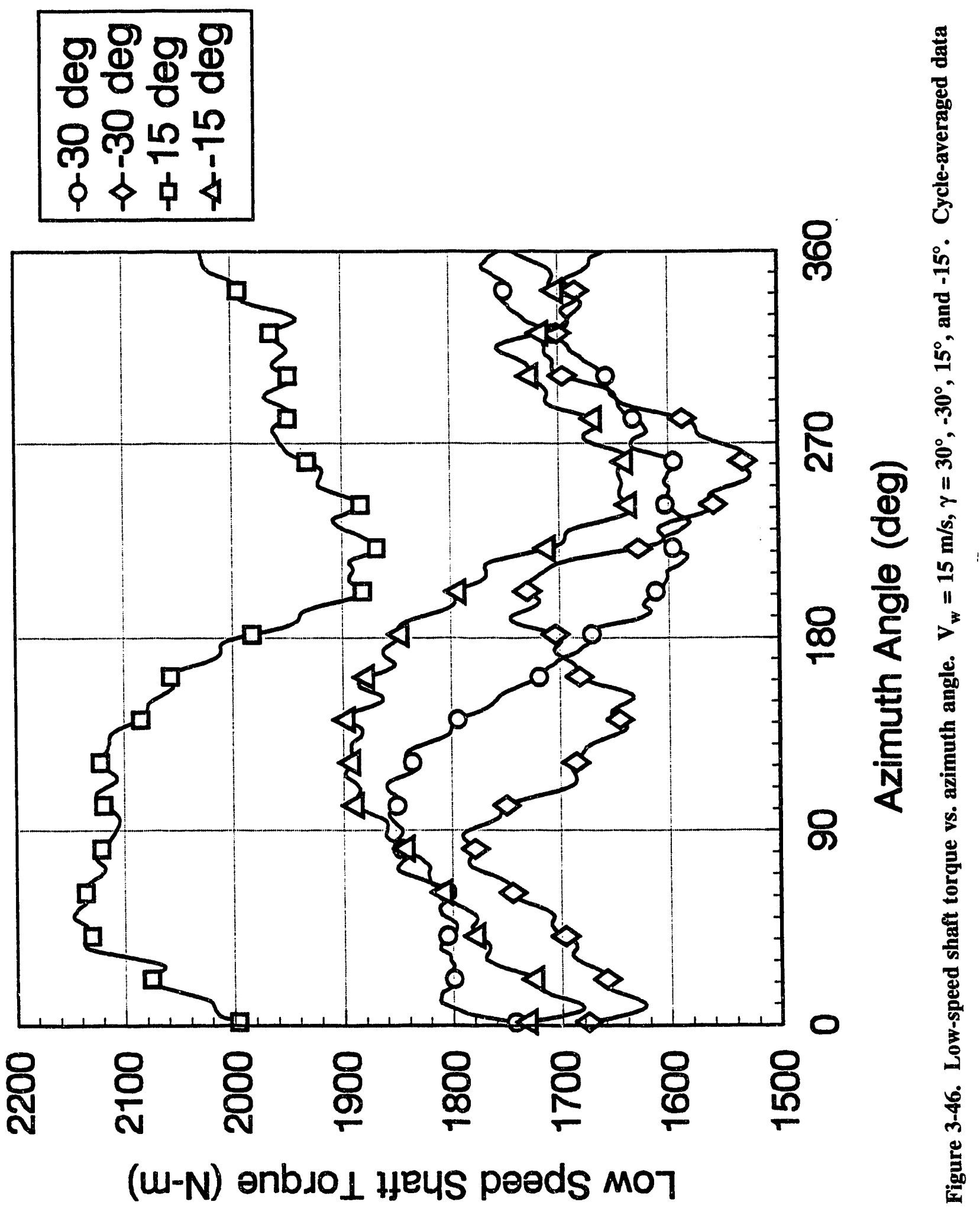




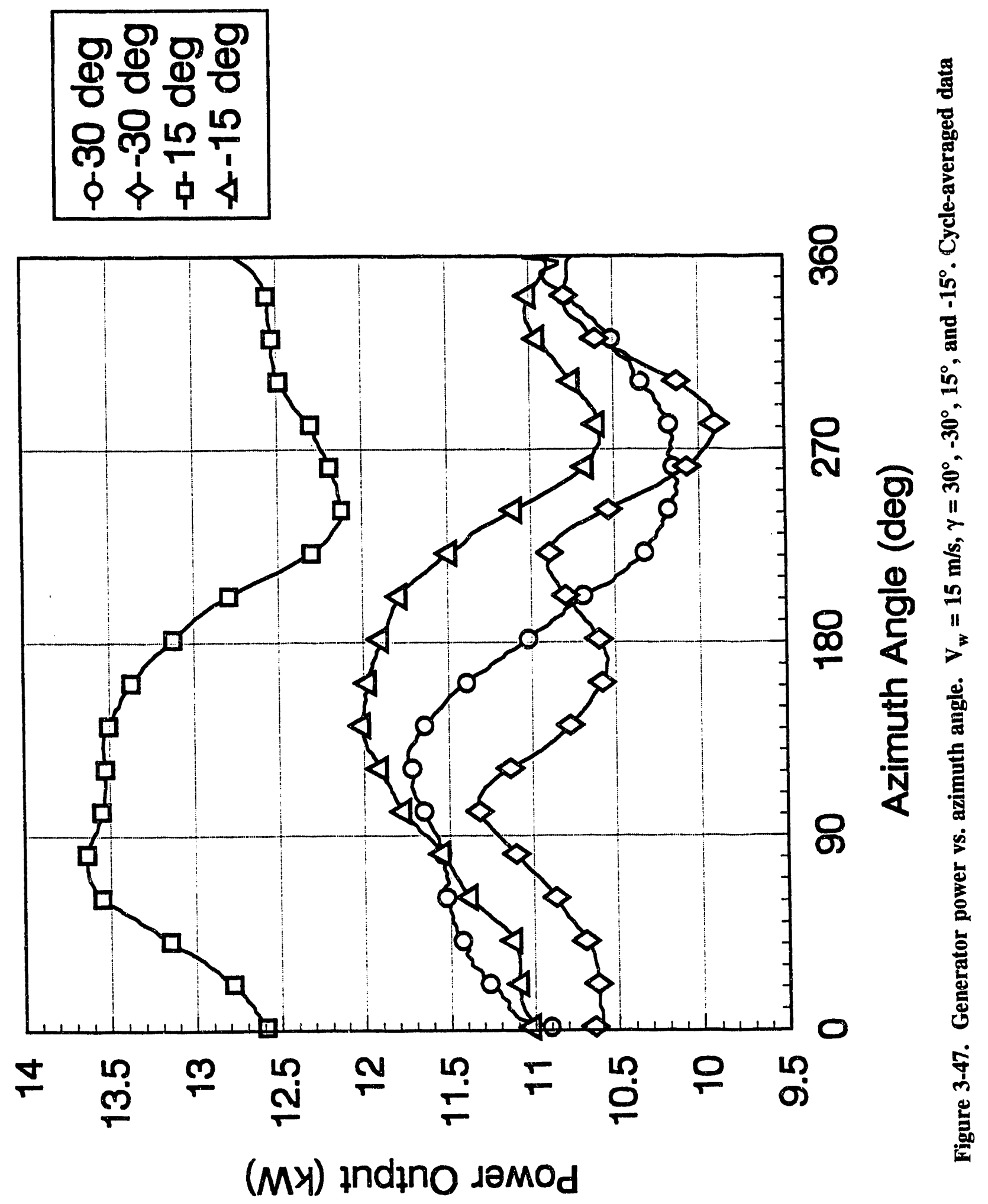




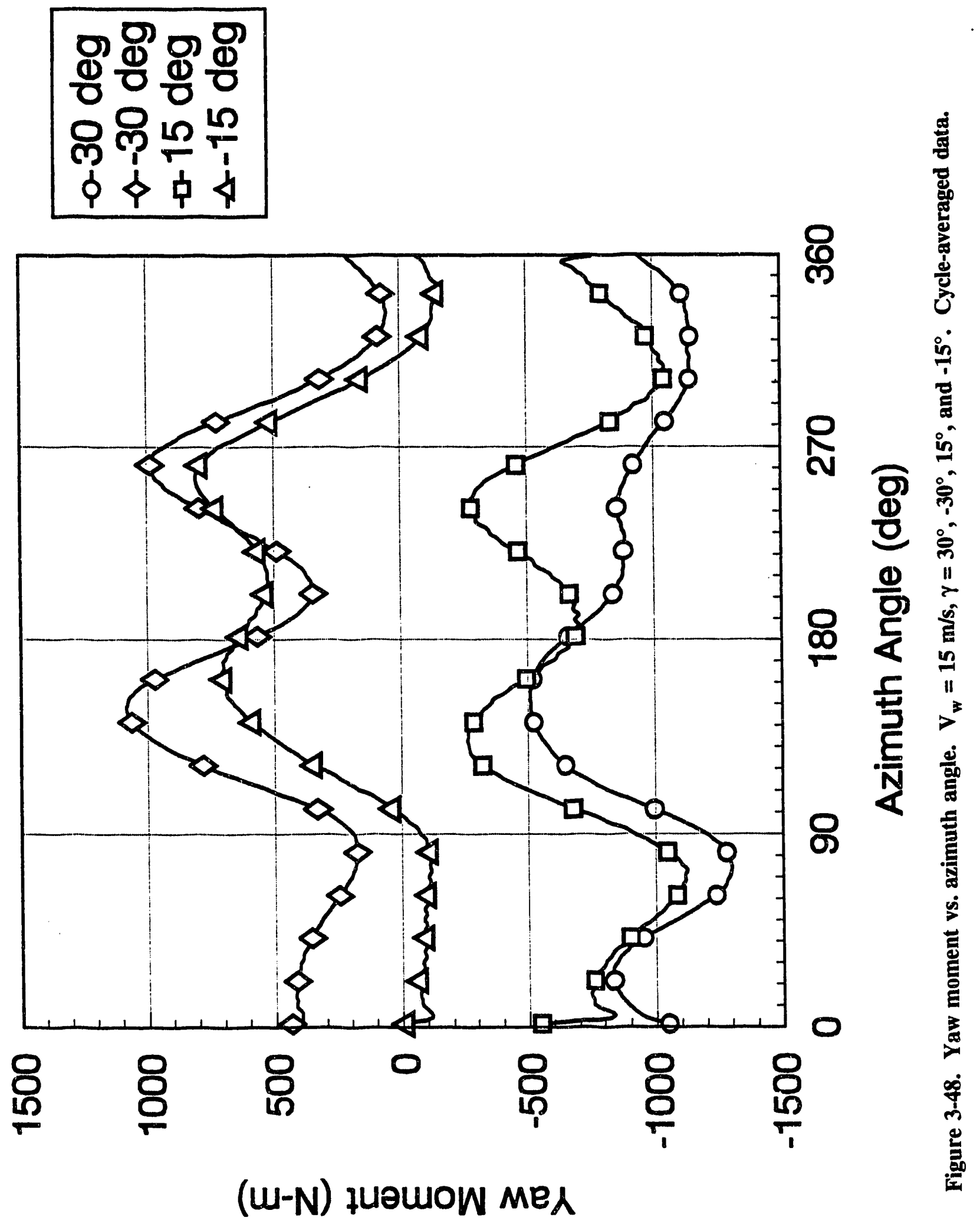


moments were approximately $160^{\circ}$ out of phase with respect to the peak negative yaw moments seen for the positive yaw cases. For the $-15^{\circ}$ yaw case, negative yaw moments were observed between $310^{\circ}$ and $100^{\circ}$ azimuth. This unique behavior was not seen for the other yaw cases. Finally, fluctuations in yaw moment of approximately $800 \mathrm{~N}-\mathrm{m}$ were seen for each yaw case examined. 


\subsection{Discussion}

Experimental data were reduced with the goal of first obtaining the most basic testcases, specifically, constant wind speed and selected yaw error. By maintaining constant wind velocity with zero yaw, it was predicted that the aerodynamic behavior would be quasisteady, and important information regarding pressure distributions and integrated force coefficients could be approximated using static methods. This prediction turned out to be completely incorrect. Pressure distributions demonstrated highly unsteady and three-dimensional behavior. Integrated normal force data showed that the magnitude of the forces, in addition to unsteadiness, could not be accurately predicted using steady-state results. As the wind turbine was yawed, the problem became much more complex, and issues concerning unsteady and three-dimensional aerodynamics became imperative for understanding the flow field behavior associated with a rotating wind turbine.

A great deal of information regarding unsteady aerodynamics has been gained through research at the University of Colorado [13-19]. Wind tunnel tests have been conducted for pitching and oscillating wings in constant freestream velocity. These variations in AOA are also seen on wind turbine blades, where impulsive or cyclic variation in relative wind velocity will produce geometrically similar effects. Tests conducted by Helin [14] and Robinson et al. [16] have investigated the unsteady flow fields and resultant pressure distributions produced by a pitching rectangular wing. A flow visualization sequence is presented in Figure 4-1, and the corresponding upper surface pressure distribution is shown in Figure 4-2 for a single pitch motion from $0^{\circ}$ to $60^{\circ} \mathrm{AOA}$ at a nondimensional pitch rate, $\alpha+$, of 0.4 . Single pitch motion is presented here because it represents the most basic unsteady motion from a geometric point of view. The topographical suction pressure distribution was plotted in a fashion similar to that for earlier pressure distributions presented in this report. As can be seen in Figure 4-1, a single pitch of a rectangular wing produces a highly complex flow field. This flow field, shown at midspan, shows the generation of a cohesive vortex structure that has been termed a dynamic stall vortex. Robinson showed that this vortex will generally form if wing AOA dynamically exceeds its static stall angle. The vortex initially forms proximal to the leading edge, with the flow field downstream remaining attached. As the wing continues to pitch up, this vortex grows and begins to convect over the wing upper surface. This vortex is then shed into the wake behind the wing and is followed by the generation of a trailing edge vortex that rotates in a direction opposite that of the dynamic stall vortex.

The pressure distribution shown in Figure 4-2 was used to demonstrate the effects of the dynamic stall vortex on the upper surface pressure distribution. This pressure distribution is remarkably similar to some of the pressure distributions seen earlier in this report. A build up of LE suction pressure may be seen and was correlated with attached flow as the wing pitched up. Maximum LE suction pressure was seen to occur during nascent formation of the LE vortex. As the vortex convected downstream, there was direct correlation between the vortex convection and the formation of a suction pressure ridge in the topographical pressure distribution. Finally, as the vortex was shed into the wake, the pressure distribution was characterized by stalled flow conditions. Similar correlations have also been done by McCroskey et al. [10,11] and Carr et al. [12] for oscillating airfoils. Again, there was direct correlation between the formation of a LE suction pressure peak with nascent formation of a cohesive vortex and the formation of a suction pressure ridge and convection of this dynamic stall vortex structure.

Geometrically, wind velocity and rotational velocity determine the effective AOA of the turbine blade relative to the wind. An increase in wind velocity results in an increase in AOA along the blade. Conversely, an increase in rotational velocity results in a decrease in AOA. For this reason, the local $\mathrm{AOA}$ decreases at outboard span locations for this particular turbine blade. Impulsive variations in AOA occur as the blade passes through the tower wake. Studies conducted on the wake behind a circular cylinder [20] have shown that velocity deficits as great as $30 \%$ of freestream values may occur. 

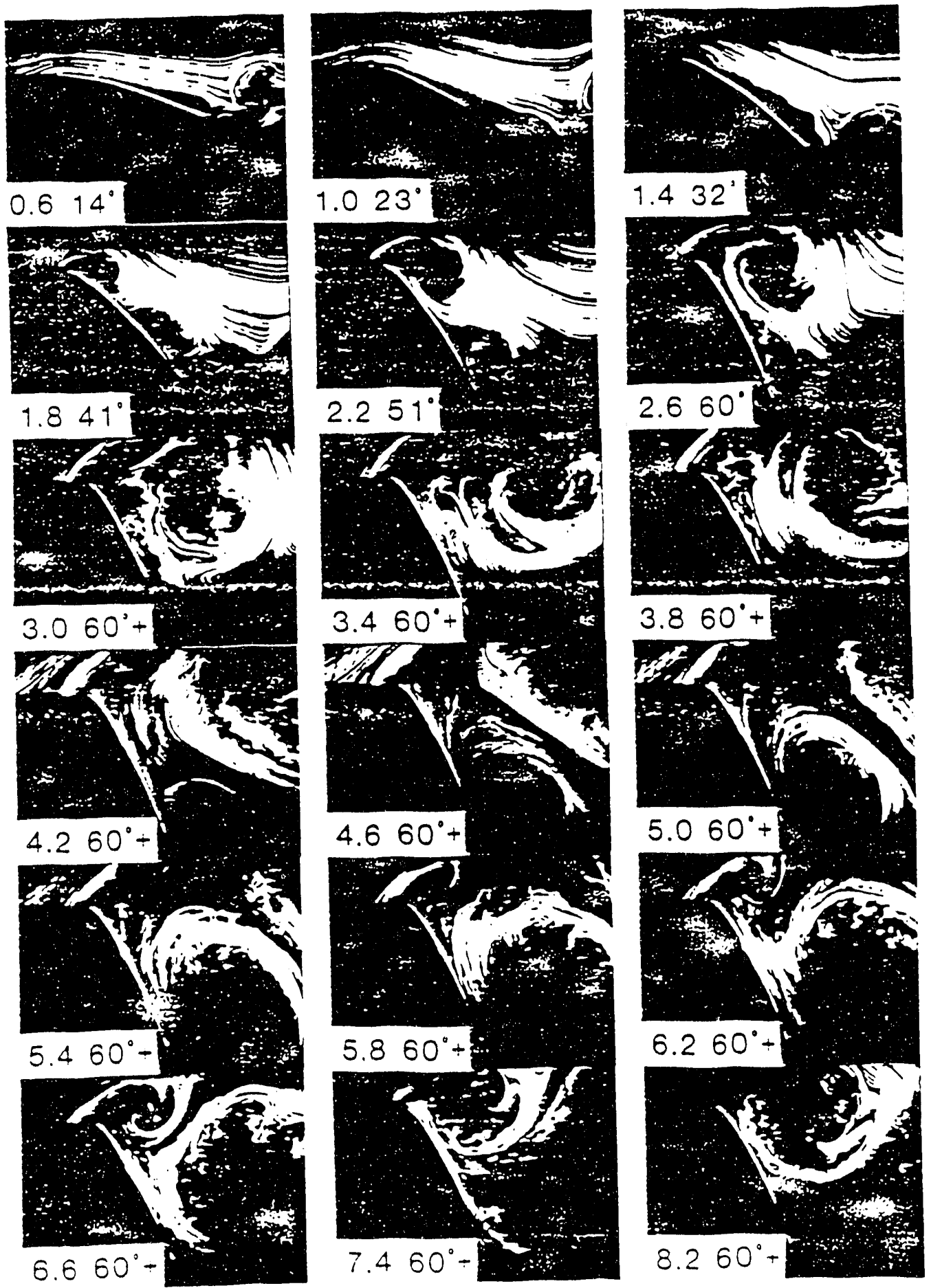

Figure 4-1. Flow visualization sequence depicting dynamic stall vortex formation and a pitching rectangular wing. $V=3.3 \mathrm{~m} / \mathrm{s}, \alpha+=0.4,0 \%-60^{\circ}$ single pitch. Taken from Helin [14] 


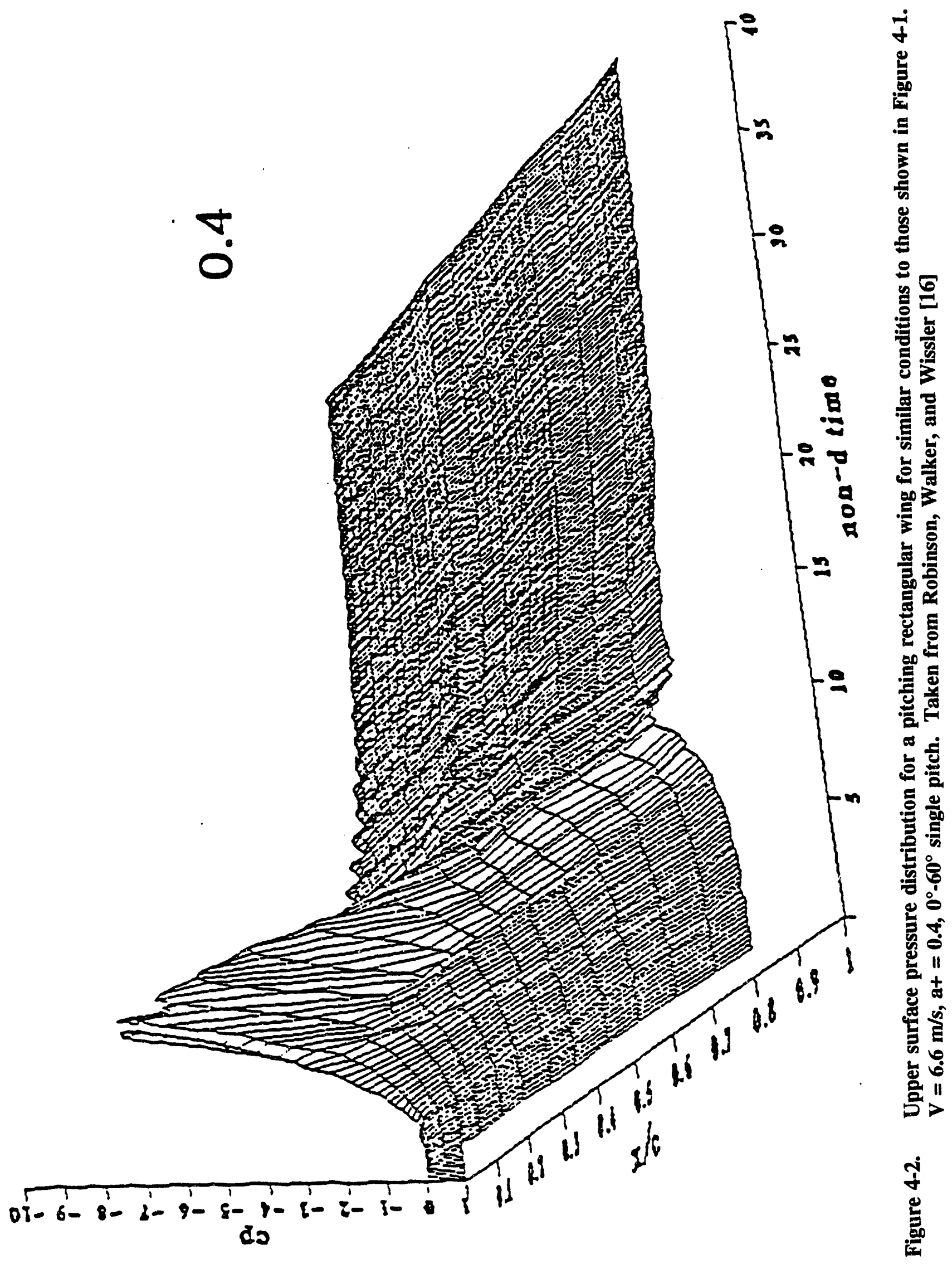


In addition, the velocity, profile through the wake may be approximated using a cosine function. As the blade passes through the tower wake, the blade experiences a decrease in local wind velocity, followed by an increase in wind velocity. This translates to a decrease, followed by an increase, in AOA. The tower wake is also confined to a very limited region compared with the total blade motion. Passage through the tower wake was found to be equivalent to a $50-\mathrm{Hz}$ impulsive motion. To allow for comparison, this effective impulsive motion was modeled as a single pitch motion of a rectangular wing. Nondimensionalized pitch rate, $\alpha+$, initial pitch angle, and pitch amplitude have been used by many researchers to relate both quantitative and qualitative data with effective wing motion. For zero yaw test cases, minimum and maximum AOAs were presented in Table 1, in which minimum angle occurs directly behind the tower, and maximum angle is the theoretical value under static conditions. Local a+ was shown on each pressure distribution to provide a reference to wind tunneì data and was found to vary between 0.15 and 0.3 , depending on local wind velocity. Physically, $\alpha+$ is an indication of the amount of fluid passing over the blade during a single pitch-up sequence. For example, an $\alpha+=0.5$ corresponds to approximately 2 chordlengths of fluid passing over the blade section during the pitch-up sequence.

Yaw error produces a cyclic variation in AOA. This variation arises because of a cross-flow velocity relative to the rotor disk. Depending on the position of the turbine blade in the rotation cycle, it may be "heading" into the cross-flow (resulting in decreased effective AOA) or "heading" with the wind (resulting in increased effective AOA). A sinusoidal variation in AOA results from and is dependent on yaw error for relative amplitude, wind velocity for mean $A O A$, and blade rotation frequency for the temporal disturbance. Because yaw error results in a cyclic variation in AOA, it is modeled using an oscillating wing. Reduced frequency, $\mathrm{K}$, mean AOA, and oscillation amplitude have been used in wind tunnel tests to describe differing flow phenomena in terms of these independent variables. Effective AOA variation was presented in Figure 3-34. Local reduced frequency is shown on each pressure distribution to provide a reference with wind tunnel data and was found to vary between 0.05 and 0.09 , depending on local wind velocity. Physically, reduced frequency is used as an indication of the amount of fluid passing over the blade section over a single cycle and is analogous to nondimensional pitch rate. For example, a reduced frequency of 0.1 suggests that 10 chordlengths of fluid pass over the blade during a single cycle.

It was demonstrated earlier that changes in velocity affect not only local AOA but also local instantaneous dynamic pressure. For cases of yaw, q will vary sinusoidally, but maximum $q$ values will be $180^{\circ}$ out of phase with respect to maximum AOA. Sinusoidal variation in total velocity, Vtot, results in an additional unsteady effect, namely, accelerated flow. It can be shown that this acceleration is for cases of yaw:

$$
\begin{aligned}
\mathrm{a}= & \mathrm{dV}_{\mathrm{tot}} / \mathrm{dt}=\mathrm{d} \psi / \mathrm{dt}\left(\mathrm{dV}_{\mathrm{tor}} / \mathrm{d} \psi\right) \\
& =-\left(7.54 \mathrm{~V}_{\mathrm{r}} \mathrm{V}_{\mathrm{c}} \sin \psi\right) / \mathrm{V}_{\mathrm{tot}}
\end{aligned}
$$

For a $30^{\circ}$ yaw error, relative flow acceleration may be significant. At $30 \%$ span, maximum acceleration is $34.26 \mathrm{~m} / \mathrm{s}^{2}$, or $3.5 \mathrm{~g}$, where $\mathrm{g}$ is the sea level gravitational force. At $80 \%$ span, maximum flow acceleration increases to $5.2 \mathrm{~g}$. Minimum flow acceleration is $-3.5 \mathrm{~g}$ and $-5.2 \mathrm{~g}$ at $30 \%$ and $80 \%$ span, respectively.

According to Rosenhead [20], flow acceleration alters the pressure gradients over the airfoil. Positive flow acceleration imposes a favorable pressure gradient that can translate to delayed stall effects. Negative acceleration, conversely, imposes an adverse pressure gradient that can translate to earlier stall effects. It was shown in AOA and dynamic pressure data that positive flow acceleration occurs as the effective AOA of the blade is decreasing, and negative flow acceleration occurs as the effective AOA is increasing. This may result in earlier flow separation and earlier flow reattachment with respect to the rotation cycle. 


\subsection{Tower Wake Effects}

As the blade passes through the tower wake, a decrease in AOA is followed by an increase in AOA caused by the velocity defect in the wake. This phenomenon may be modeled as a single pitch motion, with typical non-dimensional pitch rates of 0.2 encountered. As can be seen from Table 1, initial pitch angle and pitch amplitude vary with span location and wind velocity. It has been shown by Helin and Robinson et al. [14-16] that the static stall angle must be exceeded in order to form a dynamic stall vortex. For $\mathrm{V}_{\mathrm{w}}=7.7 \mathrm{~m} / \mathrm{s}$, there did not appear do be any sign of dynamic stall vortex formation at any span location except for a modest overshoot in LE suction pressure at $30 \%$ span. This would be expected, because only at $30 \%$ sf an did effective AOA exceed the static stall angle. A maximum AOA of $22^{\circ}$ was probably insufficient to generate a strong, well-defined vortex. Spanwise pressure distributions indicated quasisteady flow, which could also be verified upon examination of $C_{n}$ data.

As wind velocity was doubled to $14.7 \mathrm{~m} / \mathrm{s}$, conditions were favorable for generation of vortex structures at in'Joard span locations of $30 \%$ and $47 \%$ span. Pressure distributions demonstrated a buildup of LE suction pressure and formation of a suction pressure ridge consistent with formation and convection of a vortex structure. At $63 \%$ span, a buildup in LE suction pressure was seen, followed by a gradual decline. Als', there was no formation of a suction pressure ridge. It is likely that a maximum AOA of $19.5^{\circ}$ was insufficient to form a defined vortex structure. At $80 \%$ span, the flow also appeared quasisteady.

For high wind speeds of $18.7 \mathrm{~m} / \mathrm{s}$ and $23 \mathrm{~m} / \mathrm{s}$, it appears that dynamic stall vortex formation progressed to outboard span locations. At inboard locations of $30 \%$ and $47 \%$ span, there was little evidence of a LE suction pressure peak. This seems reasonable, because the flow would be characterized by deep stall and the boundary layer would be separated throughout the rotation cycle. Reattachment of the boundary layer has also been shown to be a necessary condition for the generation of a cohesive vortex structure [13-19]. AOA variations in the post-stall regime, however, did result in weak, diffuse vortex formations, and additional lift compared with static values was observed. At $63 \%$ and $80 \%$ span, LE suction pressure peaks and suction pressure ridges were seen, indicating the formation of a vortex. The suction pressure ridge would typically end at approximately $30 \%$ chord, however. This implies that the vortex did not convect over the entire upper surface. Rather, vortex convection occurred up to $30 \%$ chord and then completely separated from the blade upper surface. This resulted in loss of lift and boundary layer vorticity.

An increase in wind velocity resulted in the progression of dynamic stall vortex formation to outboard span locations. The formation of this vortex had tremendous ramifications in terms of the normal forces pro'juced. Lift overshoots as large as $40 \%$ over maximum static values werc observed at $80 \%$ span. This did not appear to greatly affect low-speed shaft torque or power output except for small fluctuations in piwer output. Significant effects were observed in root flap bending moment, however. Blade passage through the tower wake appeared to excite the natural resonant frequency of the blade, witich was $4.8 \mathrm{~Hz}$, or 4 per rev. Passage through the tower wake was found to be equivalent to imparting a $50 \mathrm{Jz}$ load on the blade-effectively a large impulsive load relative to the resonant frequency. With increascd wind velocity, the magnitude of this impulse increased, and fluctuations as large as $1500 \mathrm{~N}-\mathrm{m}$ in root flap bending moment were observed during high wind cases. It is possible that excessive fluctuations in bending moment could be transmitted to the tower itself. If this did occur, vibration of the tower could load to vortex formation that matches the blades' natural resonant frequency, and aeroelastic coupling between vortex shedding and blade vibration could result. 


\subsection{Yaw Error Effects}

When a wind turbine becomes yawed relative to the oncoming wind, cyclic variation in AOA and instantaneous dynamic pressure occur. Because of the geometry, these cyclic variations are $180^{\circ}$ out of phase with each other. These factors, including passage through the tower wake, resulted in the formation of a highly complex flow field. A simple physical model is proposed, based on a wing sinusoidally oscillating in pitch. It was shown earlier in this report that this effective motion approximates the actual motion that the blade experiences.

It has been demonstrated by McCroskey [11], Huyer et al. [13], and Robinson [15] that complex vortical flow fields are produced by sinusoidally oscillating wings. These vortices leave identifiable signatures in the pressure distributions that allow them to be tracked. It was shown earlier for the $30^{\circ}$ yaw case that clearly defined LE suction pressure peaks and suction pressure ridges are produced at inboard span locations. These LE pressure peaks and pressure ridges were most clear at $30 \%$ span. It was then possible to track the movement of this suction pressure ridge with respect to azimuth angle (effectively time). Upon examination of suction pressure peaks along the chord, chordwise pressure distributions positively suggested the presence of a defined vortex structure. The location of the chordwise pressure peak with respect to azimuth angle allowed for identification of vortex position with respect to time. The slope of this plot then could provide an indication of vortex convection velocity. Similar chordwise pressure distributions taken at $47 \%$ span demonstrated the formation and convection of a defined vortex structure. Initial appearance and convection of this vortex was delayed until later in the cycle, however.

At outboard span locations, vortex formation was less defined. At $63 \%$ span, a gradual buildup in LE suction pressure was observed, and constant $L E C_{p}$ values persisted over a large portion of the cycle. Vortex convection could only be implied up to $14 \%$ chord. At $80 \%$ span, no vortex convection could be implied. There was a precipitous decrease in LE suction pressure, however, that was preceded by a gradual buildup. At $80 \%$ span, recall that the AOA variation was consistently below static stall. Again, Robinson [15] showed that the static stall angle must be exceeded for a dynamic stall vortex to form.

The spanwise pressure distribution demonstrated approximately constant $C_{p}$ values at $4 \%$ chord along the entire span during initial buildup of LE suctiol pressure. Equivalent suction pressures were seen at $\psi=190^{\circ}$, but the pressure continued to increase modestly at outboard span locations. In addition, peak LE suction pressures persisted for a greater portion of the cycle at outboarc spanwise locations. This and the experimental evidence mentioned above suggest an interesting physical phenomenon. According to Helmhotz' Theorem, in three-dimensional space, the vortex lines that encompass a three-dimensional vortex structure must remain connected. This phenomenon has been dernonstratec' by Fievmuth $[17,18]$ and Huyer and Luttges [19! for oscillating three-dimensional wings. This theorem suggests that the vortex structures that form at each span location must be connected in some fashion. Another consequence of Helmholtz' Theorem is that the circulation must also remzin constant along a vortex. The spanwise pressure distribution showed that actual pressure remains approximateiy constant along the span at $w=190^{\circ}$. This implies uniform vortex initiation and/or buildup of LE vorticity. Because of differing AOAs at the various span locations, one would expect that a cohesive, well-defined vortex would form first at inboard span locations. This did occur, but the LE suction pressure must also correspcndingly increase along the span, according to Helmholtz. This was evidenced in the pressure distributions at the differing span locations. A LE st tion pressure spike was seen at $30 \%$ span, whereas the I.E suction pressure persisted over a quarter of the cycle at $63 \%$ span. Therefore, it appears that the vortex must form uniformly along the leading edge. It then begins to grow and convect first at inboard span locations. At $30 \%$ span, this vortex appeared to be shed into the wake. At the same time, this vortex was convecting over the upper surface at $47 \%$ span. The remaining section of the vortex was still attached or pinned near the LE at $63 \%$ span and outboard locations, however. At $47 \%$ span, the suction pressure ridge persisted to only $56 \%$ chord. This suggests vortex convection to only to $56 \%$ chord before separating from the 
surface. Immediately after the vortex separated at $47 \%$ span, it was seen to convect to $14 \%$ chord at $63 \%$ span before separating from the surface. The LE suction pressure peak remained at $80 \%$ span, but pressure distributions implied no vortex formation. There was a precipitous decrease in LE suction pressure at $80 \%$ span. This may be indicative of separating boundary layer vorticity as the rest of the vortex was shed into the wake.

Integrated normal force coefficients were consistent with the generation of cohesive vortex structures at inboard span locations. Significant overshoots in normal force were observed at all span locations. $C_{n}$ data showed that dynamic $C_{n}$ values twice maximum static values could be generated even at outboard span locations. Lift reduction was also seen, however, and was exemplified by the formation of hysteresis loops in the $C_{n}$ versus $\alpha$ curves.

The behavior of the pressure distributions and $C_{n}$ at $30 \%$ span was atypical compared with behavior seen at the other span locations. For $V_{w}=14.7 \mathrm{~m} / \mathrm{s}$ and zero yaw, the pressure distribution at $47 \%$ span indicated stalled flow conditions throughout most of the cycle. At $30 \%$ span, the pressure distribution did not indicate this, even though the local AOA would be much higher. In addition, for a given $A O A, C_{n}$ values at $30 \%$ span were consistently $50 \%$ or more greater than at other span locations for all test cases examined. A possible explanation of this phenomenon may be a result of nonuniform incoming wind. It is likely that relative incoming velocity is greater at $30 \%$ span than at outboard locations. This could be a direct result of the presence of the nacelle. Since the nacelle was not streamlined, it is possible that the flow accelerated around the nacelle, resulting in higher flow velocities in its proximity. An additional explanation for the atypical behavior seen at 30\% span may be spanwise flow and spanwise pressure gradients resulting from centrifugal forces in the viscous boundary layer. Regardless, additional evidence is required to sufficiently explain this phenomena.

A major consequence of the formation of these vortex structures is the highly transient effects produced in terms of structural loading. This can be seen especially well in the root flap bendin: moment. Yaw error imposes a cyclic variation in bending moment. The amplitude of this one per rev variation is approximately $1700 \mathrm{~N}-\mathrm{m}$ for the $30^{\circ}$ yau case and almost $2200 \mathrm{~N}-\mathrm{m}$ for the $-30^{\circ}$ yaw case. Also, blade resonance of 4-per-rev could be seen in the flap bending moment. The amplitude of the 4 per rev variation appears to be approximately $50 \%$ liss for the yawed test cases compared with zero yaw cases, however. This suggests that a cyclic variation in aerodynamic loads results in dampening of the blades' natural resonant frequency.

Low-speed shaft torque and generator power varied sinusoidally over the rotation cycle, with fluctuations of one-per-rev observed. The $-30^{\circ}$ yaw case also demonstrated a three-per-rev fluctuation superimposed on the one-per-rev variation. A pronounced effect was also seen in the yaw moment data. Three distinct peaks in yaw moment were observed and weie separated by $120^{\circ}$. This was expected because the turbine consisted of three blades that were separated hy $120^{\circ}$ azimuth. Large variation in yaw moment of approximately $800 \mathrm{~N}-\mathrm{m}$ was observed for all yaw cases examined. This large variation could adversely affect machines that are designed with yaw drives. 


\subsection{Conclusions}

The joint research effort between the National Renewable Energy Laboratory and the Experimental Fluids program at the University of Colorado has resulted in the systematic analysis of select data sets from a rotating wind turbine. These data clearly demonstrate the complexities of the aerodynamics associated with a wind turbine blade. Definite unsteady and three-dimensional behavior has been presented and discussed. It is also emphasized that these complex flow fields exist for even the most basic test cases. During normal operating conditions, it is likely that many of the issues regarding three-dimensionality and the generation of forced unsteady separated flow fields will become even more vital.

During conditions in which wind velocity and relative yaw were constant, significant lift enhancement was observed even at outboard span locations. Normal force values as great as $100 \%$ times maximum values were routinely observed. These enhanced lift values were likely caused by the formation of cohesive vortex structures. These vortices were shown to occur, especially at inboard span locations. Even if vortex formation was not seen at outboard locations, the formation of vortex structures inboard directly affected the nature of the flow field outboard. A physical model incorporating Helmholtz' Theorem was also proposed, based on this experimental evidence.

The unsteadiness was seen also in terms of structural loads, namely, root flap bending moment, low-speed shaft torque, and yaw moment. Passage through the tower wake appeared to excite the blades' natural resonance. By yawing the turbine, it was possible to decrease this effect, however. Cyclic variation in low-speed shaft torque directly resulted in cyclic fluctuations in generator power for cases of yaw. Three per rev variations in torque and power were observed for zero yaw cases. Finally, cyclic variations were observed in yaw moment for each of the yaw cases examined.

Analytical models of local AOA and instantaneous dynamic pressure were formulated, based on the geometry of the wind turbine and incoming flow field. Experimental data confirmed the validity of the geometric model used in this report. Improvement of a tower wake model must still be done. This work is being continued, and an improved model should be forthcoming. 


\subsection{Recommendations}

Studies must still be conducted to more accurately determine the characteristics of the incoming wind. This may provide further understanding of the seemingly atypical behavior in terms of pressure and normal force distributions seen at $30 \%$ span. This may easily be accomplished by using current instrumentation and obtaining data for nonrotating blade cases. Total pressure probes may be used to determine the flow velocity and correlate this with the velocities obtained from the vertical plane array. Surface pressure measurements will also be extremely valuable to determine the relative three-dimensionality of the inflow and possible effects of the nacelle.

While the cycle-averaged data provided the most statistically accurate results concerning temporal dependence, it should be possible to improve the cycle selection process even further. Just as bin-averaged data selected random points in a cycle, cycle-averaged data selected single cycles of data at different points in the test run. Additional selection criteria are recommended. First, examine three or more cycles of data consecutively that meet the desired input values of wind velocity and yaw error. Then, choose the cycles located in the middle of this set of consecutive cycles. These core cycles should provide an even more statistically accurate representation of the flow fields under the desired input conditions.

Additional work regarding vortex shedding off the tower needs to be accomplished. By using the total pressure probes with nonrotating blade cases, vortex shedding off the tower may more systematically be defined and incorporated into the dynamic pressure and AOA models presented in this report. Information from these probes may also be used to help determine possible aeroelastic coupling between blade vibration and vortex shedding off the tower for rotating blade test cases. Spectral analyses of the raw dynamic pressure data will allow for identification of possible vortex shedding frequencies and allow for comparisons with four-per-rev blade resonant frequencies.

It is suggested that additional wind tunnel tests be conducted to provide a data base for comparison between two-dimensional wind tunnel tests and the current field experiments. Resultant motion histories of a yawed wind turbine have been documented, as well as effective motion histories for blade passage through the tower wake. Wind tunnel tests identically matching these motion histories should provide an excellent comparative data base. Additional tests regarding accelerated flow and turbulence should also be conducted to determine their respective effects.

It is hoped that the research summarized in this report may provide a basis for future research regarding wind turbine aerodynamics. While I have attempted to summarize many of the important unsteady and three-dimensional effects, it is stressed that the test cases presented are of the most basic nature. It is obvious that additional dynamic effects such as accelerating wind, dynamic rotation of the disk as it adjusts to the wind direction, high levels of wind shear, and blade vibrational effects were not even considered. The Combined Experiment data provide a wealth of relevant experimental information that can be used to identify current and potential problems to the wind turbine industry. The wind site may also be used as a testbed for proposed solutions to these problems. The potential of this research also extends to areas outside the wind turbine industry. Recognition of this potential may assist in the development of future cooperative research projects. 


\subsection{References}

1. Butterfield, C.P., W.P. Musial, and D.A Simms, Combined Experiment Phase I, Final Report, NREL/TP-257-4655, National Renewable Energy Laboratory, Golden, CO, October 1992.

2. Butterfield, C.P., W.P. Musial, G.N. Scott, and D.A. Simms, Combined Experiment Phase II, Final Report, NREL/TP-442-4807, National Renewable Energy Laboratory, Golden, CO, In Press.

3. Butterfield, C.P., Three-dimensional Airfoil Performance Measurements on a Rotating Wing, SERI/TP-217-3505, Solar Energy Research Institute, Golden, CO, June 1989.

4. Butterfield, C.P., M. Jenks, D. Simms, and W. Musial, Aerodynamic Pressure Measuremerits on a Rotating Wind Turbine Blade, SERITP-257-3695, Solar Energy Research Institute, Golden, CO, May 1990.

5. Butterfield, C.P., G. Scott, and W. Musial, Comparison of Wind Tunnel Airfoil Performance Data with Wind Turbine Blade Data, SERI/TP-254-3799, Solar Energy Research Institute, Golden, CO, July 1990.

6. Hansen, A., and C. Xudong, Yaw Dynamics of Horizontal Axis Wind Turbines, SERI/STR-217-3476, Solar Energy Research Institute, Golden, CO, March 1989.

7. Butterfield, C.P., A.C. Hansen, D. Simms, and G. Scott, "Dynamic Stall on Wind Turbine Blades," AWEA Windpower '91 Conference Proceedings, Palm Springs, CA, September 1991.

8. Butterfield, C.P., S. Huyer, and D. Simms, "Recent Results from Data Analysis of Dynamic Stall on Wind Turbine Blades," presented at the International Energy Agency Experts Meeting on Wind Turbine Aerodynamics, Stuttgart, Germany, December 3-4, 1991.

9. Huyer, S.A., C.P. Butterfield, and D. Simms, "Characterization of Dynamic Stall Phenomena on Wind Turbine Blades Using Surface Pressure Measurements," presented at 11th ASME Wind Energy Symposium, Houston, TX, January 27-30, 1992.

10. McCroskey, W.J., L.W. Carr, and K.W. McAlister, Dynamic Stall Experiments on Oscillating Airfoils, AIAA paper no. 75-125, January 1975.

11. McCroskey, W.J., "Unsteady Airfoils," Annual Review of Fluid Mechanics, pp. 285-311, 1982.

12. Carr, L.W., K.W. McAlister, and W.J. McCroskey, Analysis of the Development of Dynamic Stall Based on Oscillating Airfoil Experiments, NASA Technical Note TN D-8382, 1977.

13. Huyer, S.A., M.A. Reavis, and M.W. Luttges, A Comparative Study of Differing Vortex Structures Arising in Unsteady Separated Flows, AIAA paper no. 88-2582-C , Williamsburg, VA, June 1988.

14. Helin, H.E., Experimental Studies on the Dynamic Development and Control of Unsteady Separated Flows, Doctoral Thesis, University of Colorado, Boulder, CO, 1986.

15. Robinson, M.C., Development of Vorticity and Vortices from Forced Unsteady Flow Separation, Doctoral Thesis, University of Colorado, Boulder, CO, 1985. 
16. Robinson. M.C., J. Walker, and J. Wissler, "Unsteady Surface Pressure Measurements on a Pitching Rectangular Wing," Workshop II on Unsteady Separated Flows, U.S. Air Force Academy, FJSRL-TR-88-0004, pp. 225-237, July 1987.

17. Freymuth, P., "Visualizing the Connectivity of Vortex Systems for Pitching Wings," 1st National Congress of Fluid Dynamics, Cincinnati, OH, July 1988.

18. Freymuth, P., Vortex Topology for Rectangular Wings in Pictures, Sketches and Conjectures, AIAA paper no. 91-1824, Honolulu, HI, June 1988.

19. Huyer, S.A., and M.W. Luttges, The Vortex Kinematics Associated with an Oscillating Delta Wing, AIAA paper no. 91-1797, Honolulu, HI, June 1991.

20. Rosenhead, L., Laminar Boundary Layers, Dover Publications, Inc., New York, 1963. 


\section{Appendix A}

\section{Computer Programs}

A-1 
This program reads 400 -byte, 101 -datum records

containing 1 pressure profile. When the new data with 5 profiles is available, a new version will have to be written. The changes will be in the areas:

- expand 'datain[]'

- modify dv_index[] to point to the right data

- check read_in chanls to see if it will hasc.le new file (See pcbins.c for further detail).

This version reads BRE format files - binary 4-byte reals, engineering units. It checks each cycle of data according to user-input specifications for mean and standard deviation (one cycle consists of one set of jata with azimuthal angle from 0 to 360 degrees). Any cycle that meets these criteria has the data from specified channels written to an ASCII file. This file may be used as-is to plot out data from each cycle.

WRITTEN: M. Querijero SERI/WIND

/* Wind Direction Modifications by Steve Huyer on $10 / 30 / 91$ */

/ DEcIAaration of gLobaI variables */

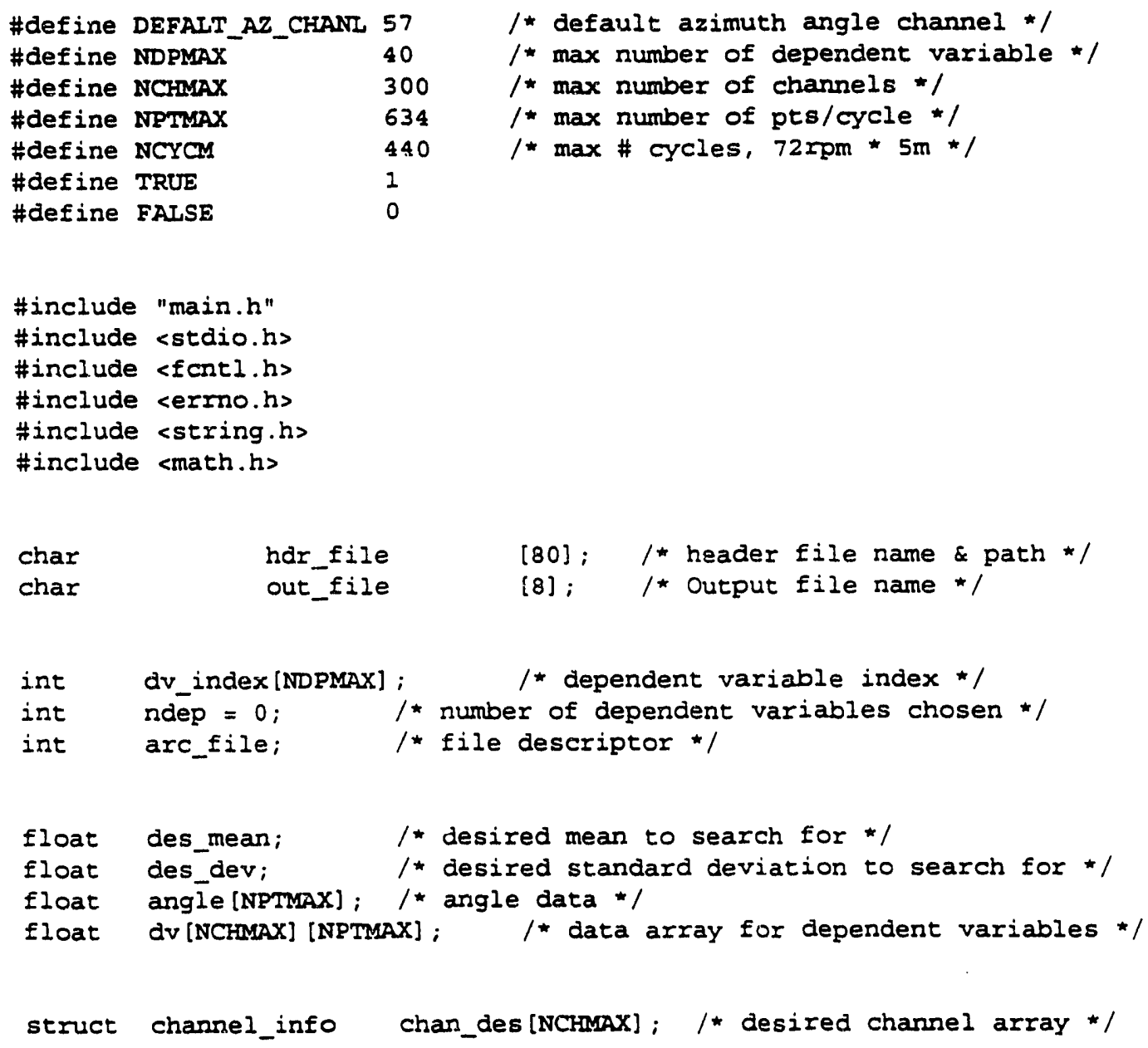




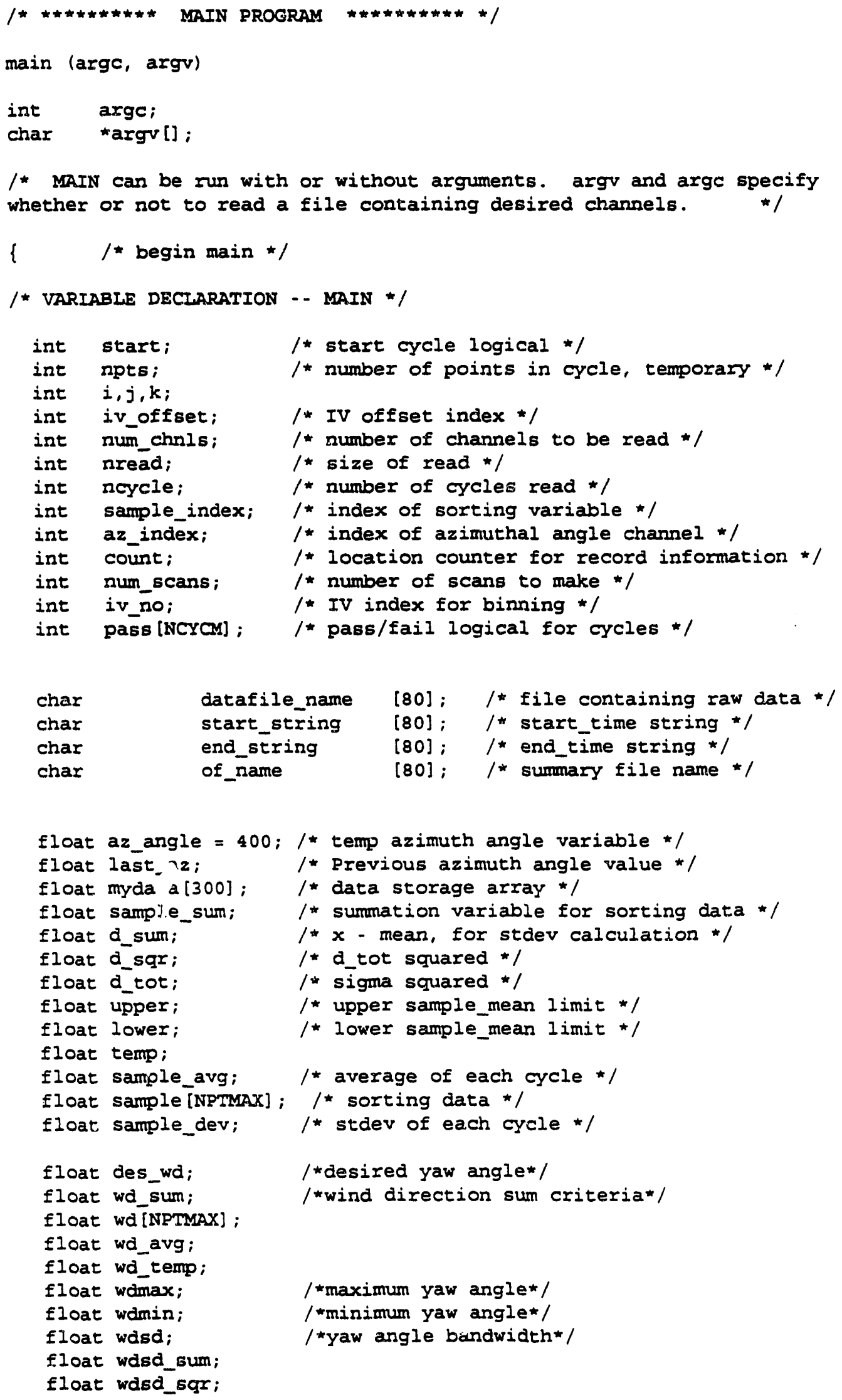




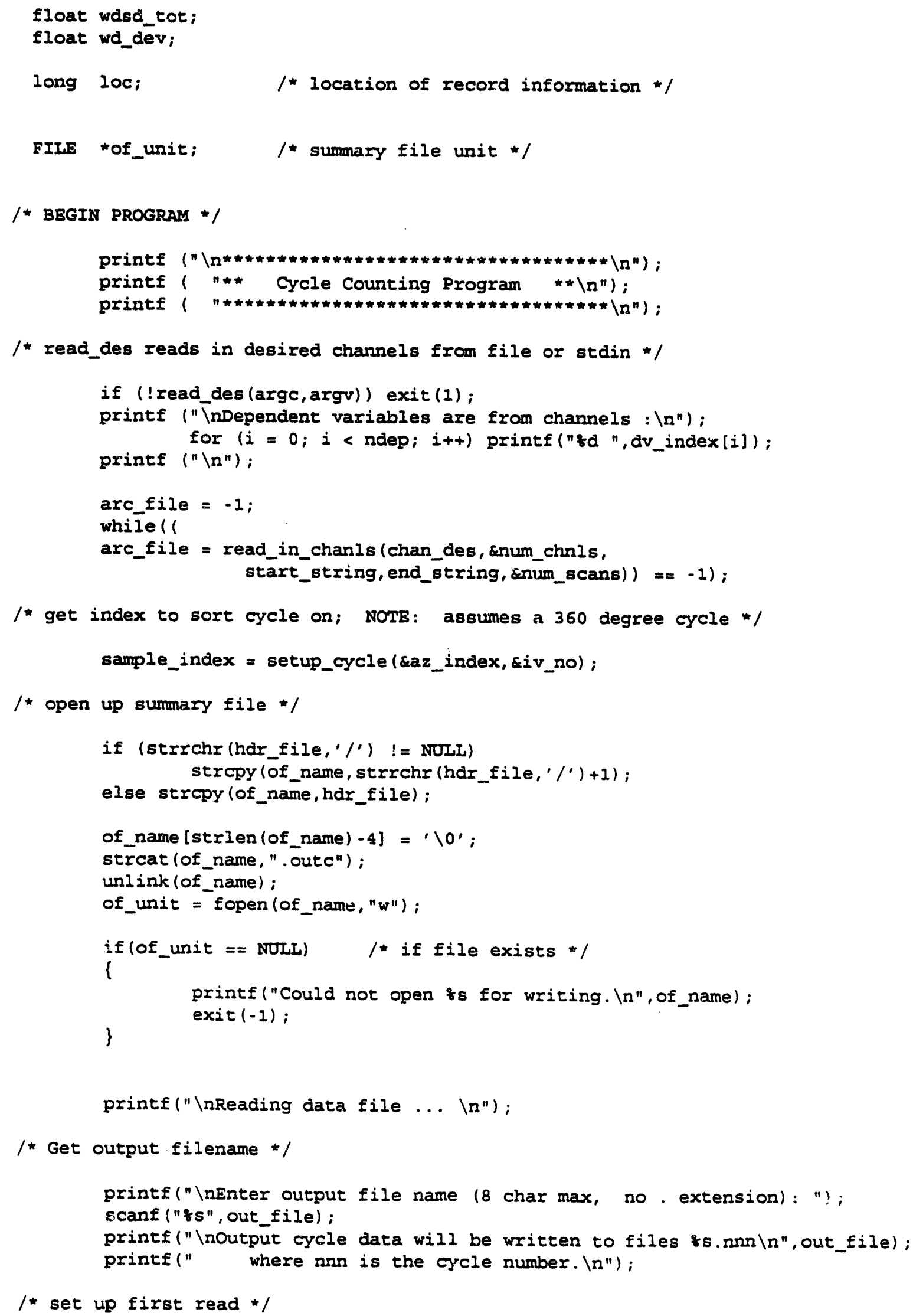




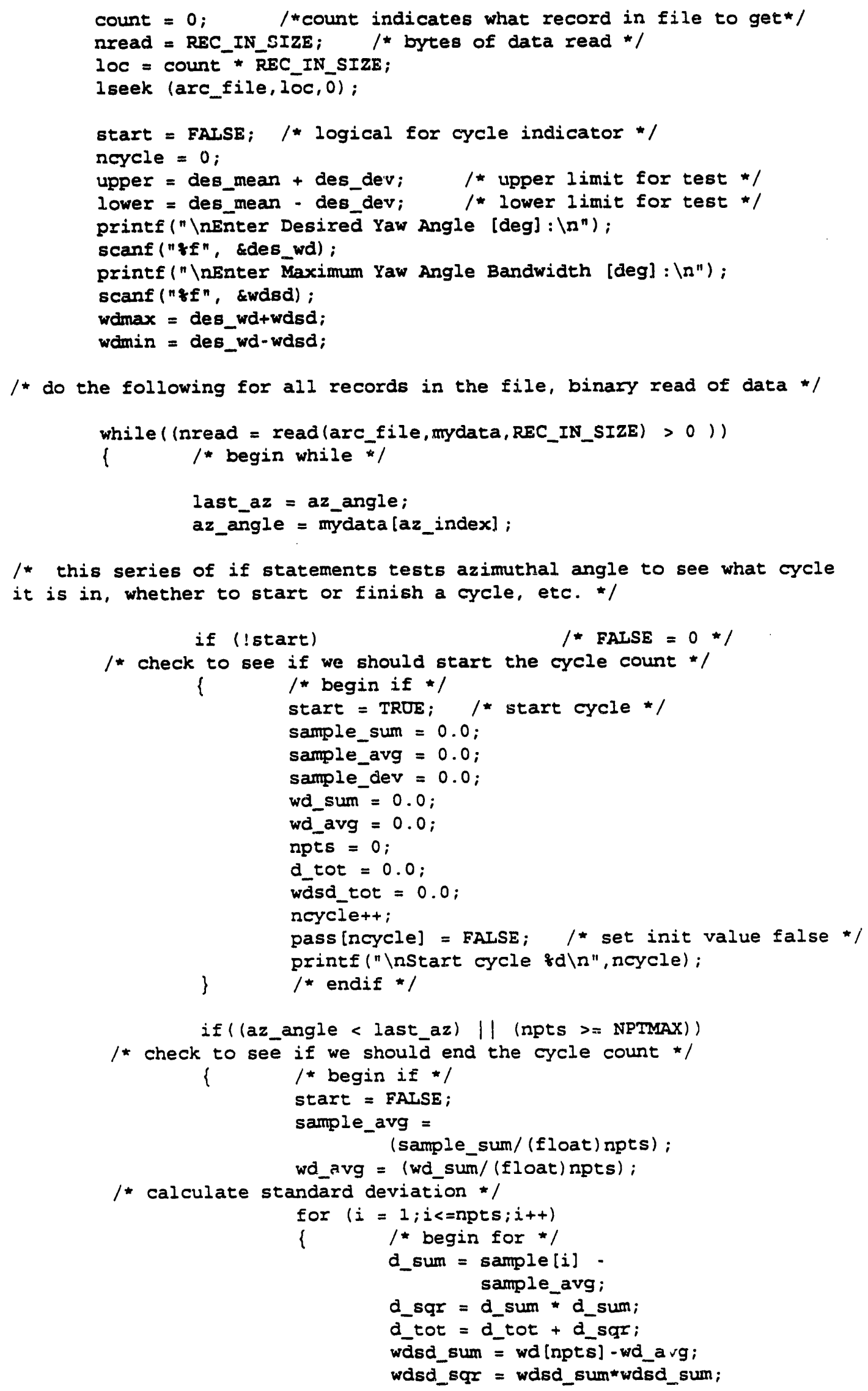




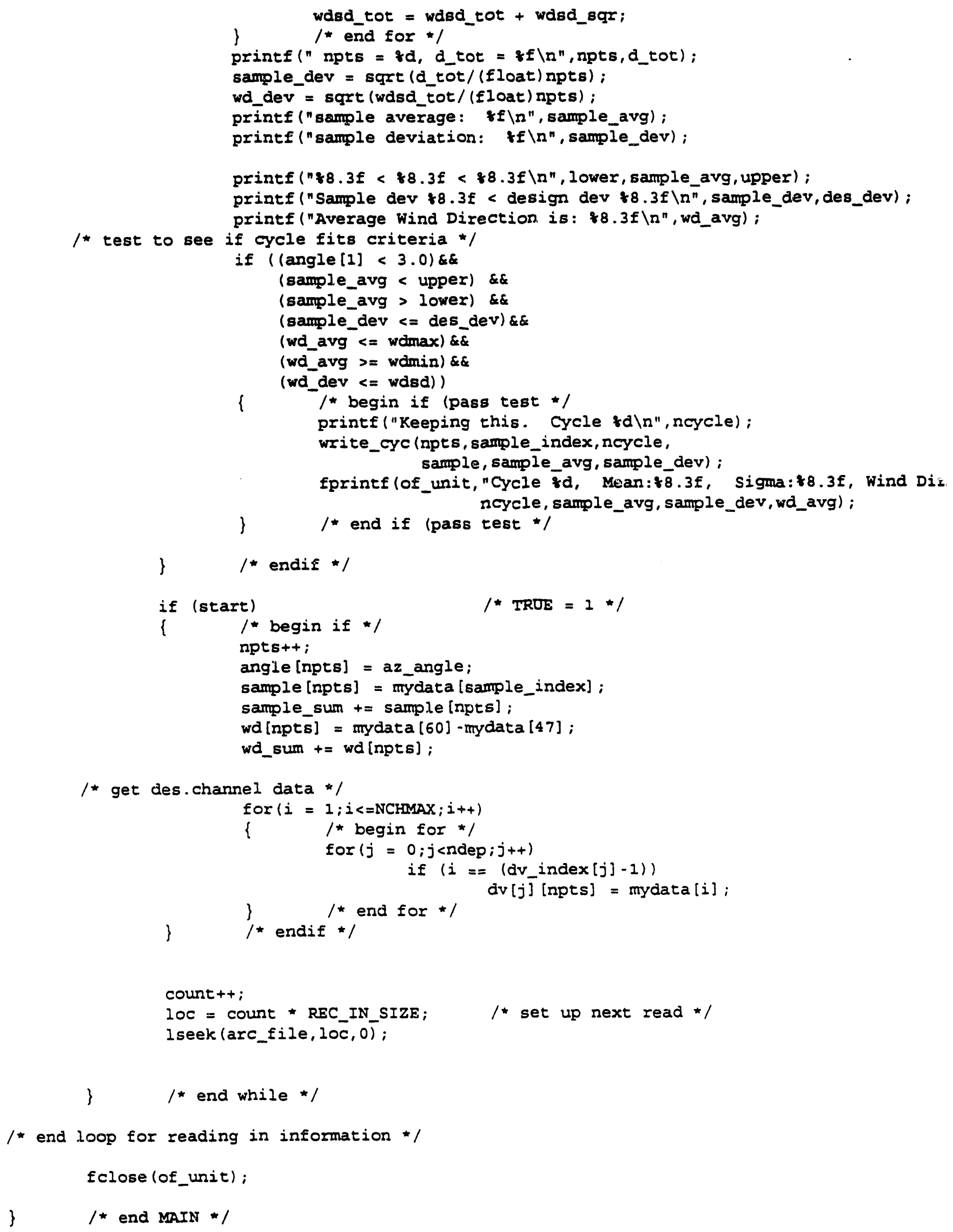




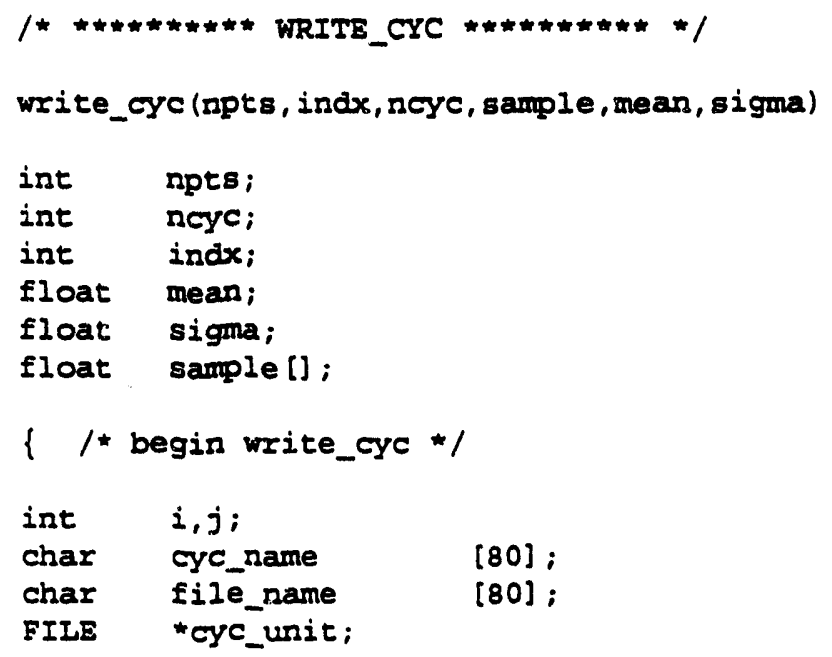



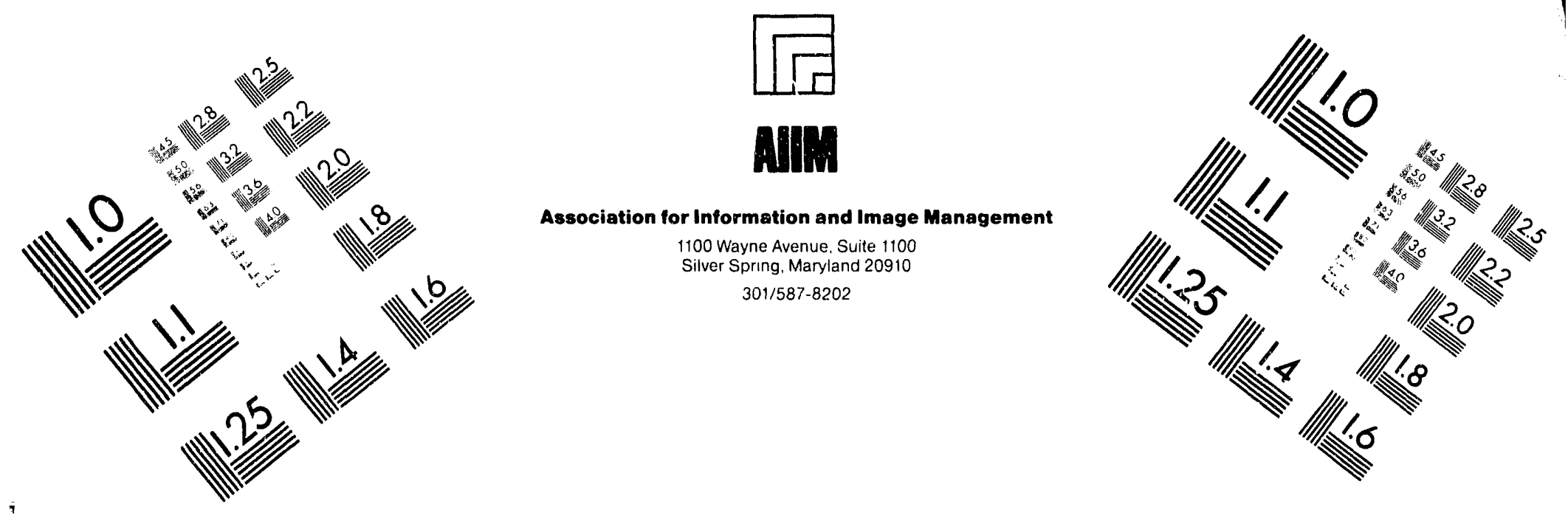

\section{Centimeter}

$\begin{array}{llllllllllllllll}1 & 2 & 3 & 4 & 5 & 6 & 7 & 8 & 9 & 10 & 11 & 12 & 13 & 14 & 15 & \mathrm{~mm}\end{array}$ Lulud Inches
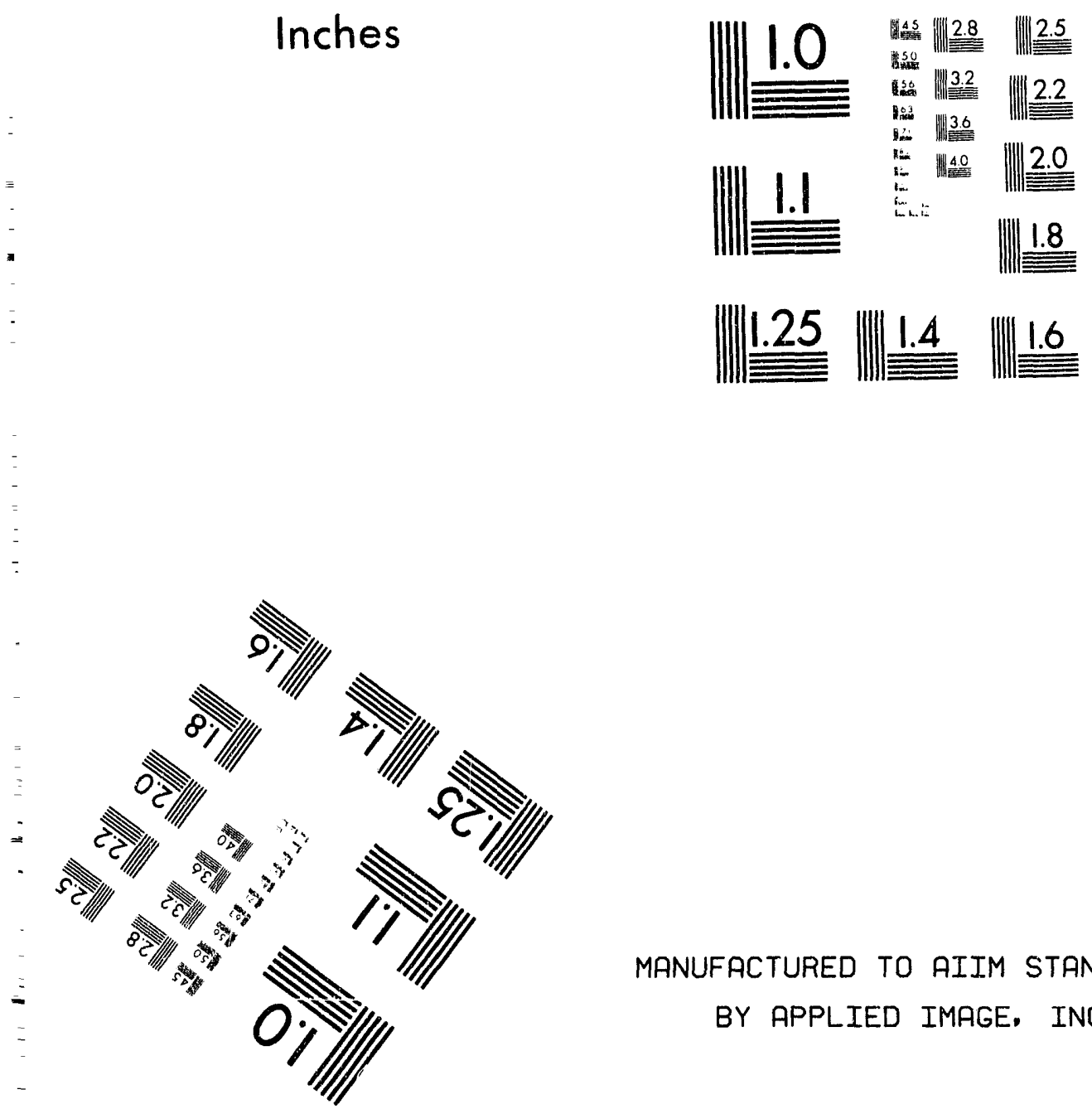

MANUFACTURED TO AIIM STANDARDS

BY APPLIED IMAGE, INC.

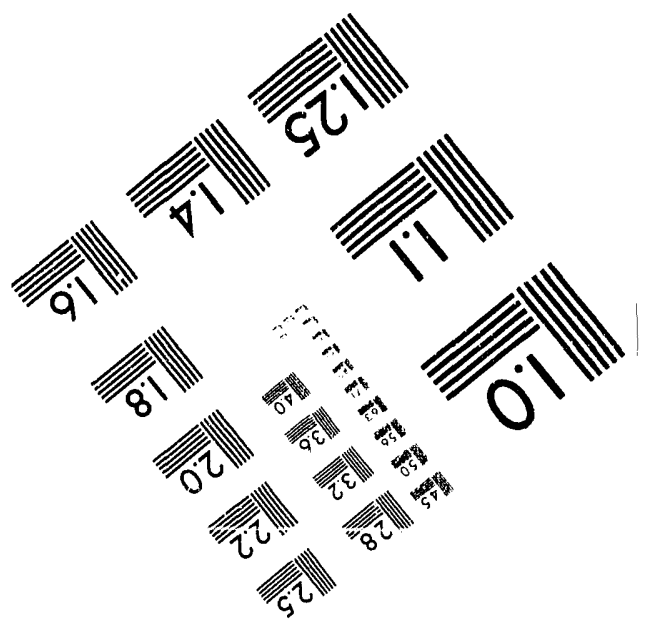



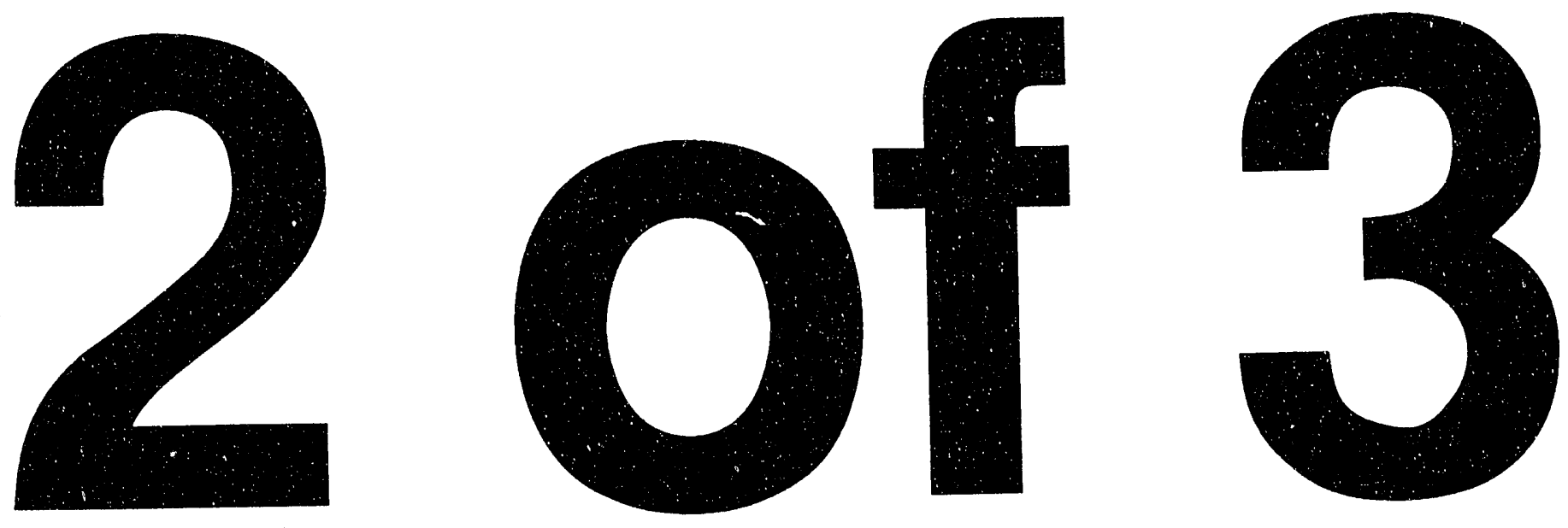


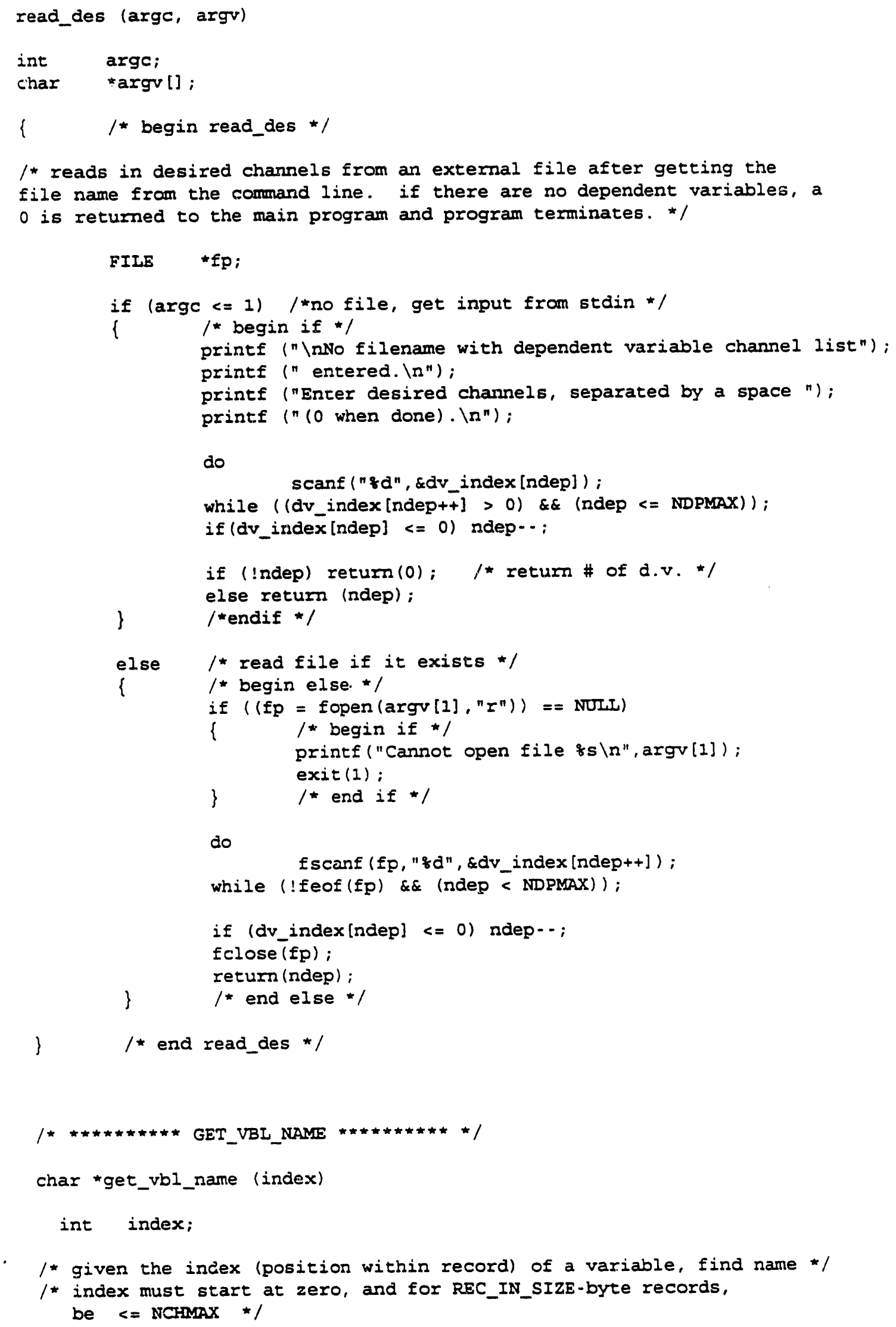




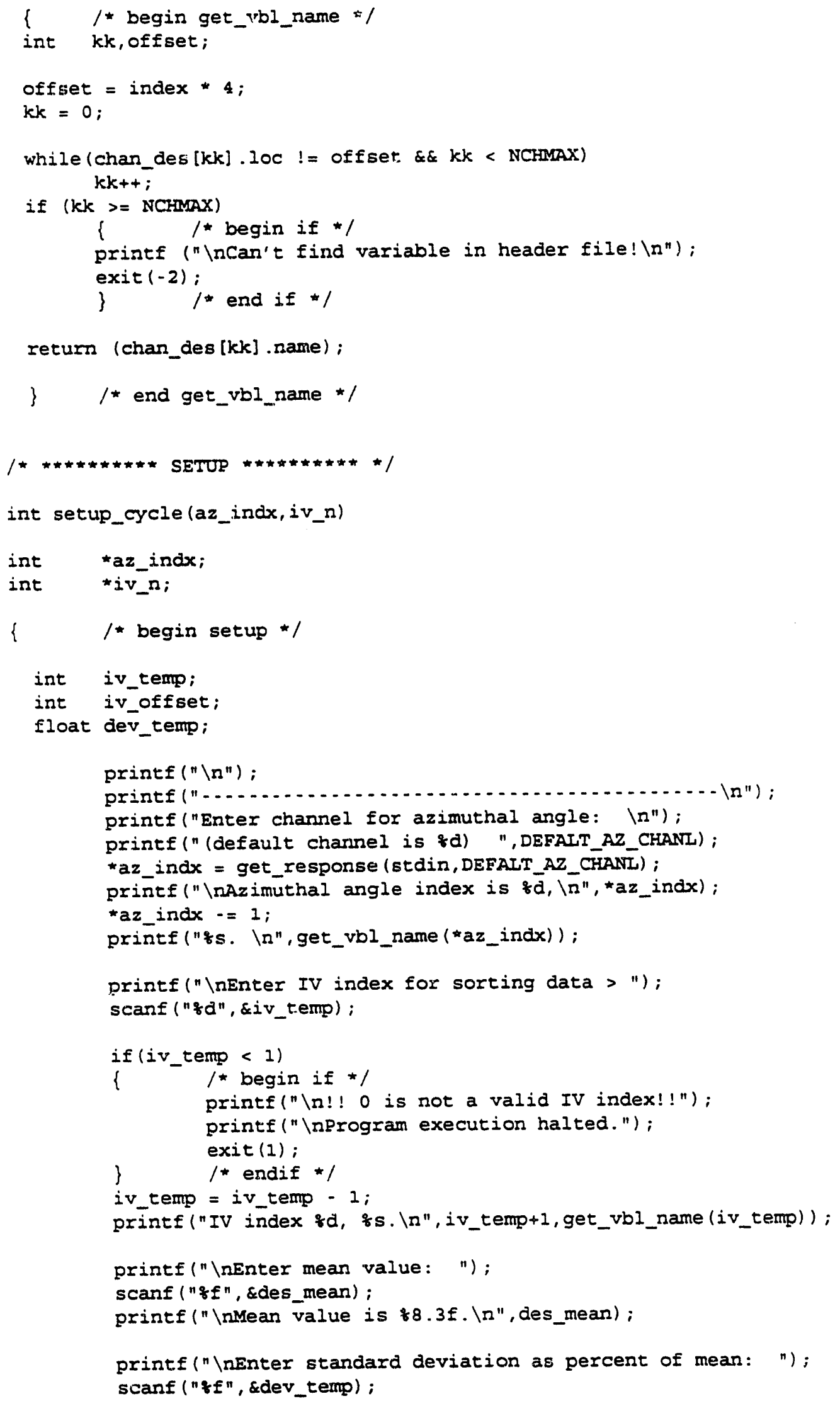


printf (" \Standard deviation is $88.3 f .(n "$, dev_temp);

des_dev = des_mean * (dev_temp/100);

return (iv_temp);

\} $\quad /$ end setup */ 


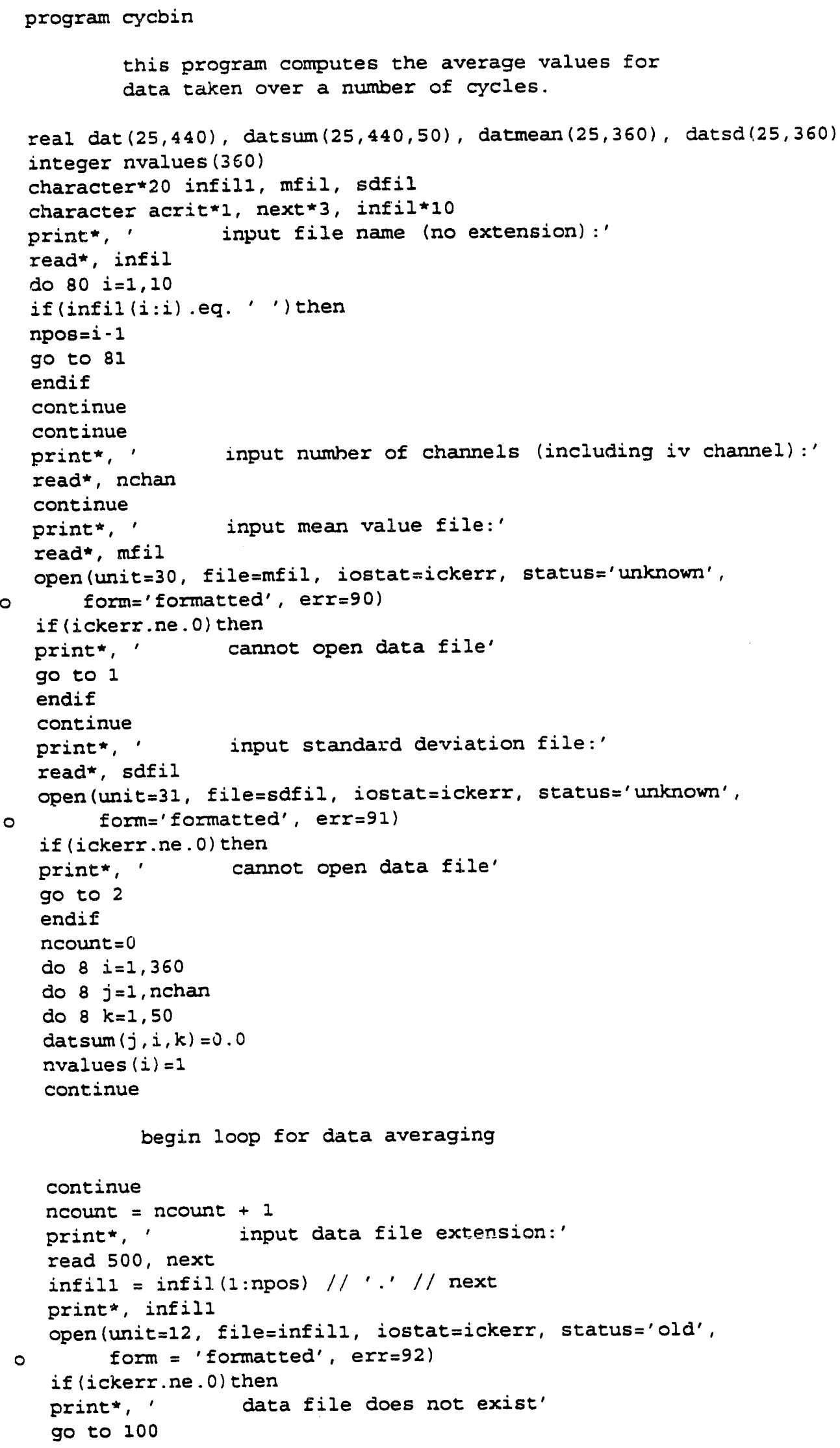




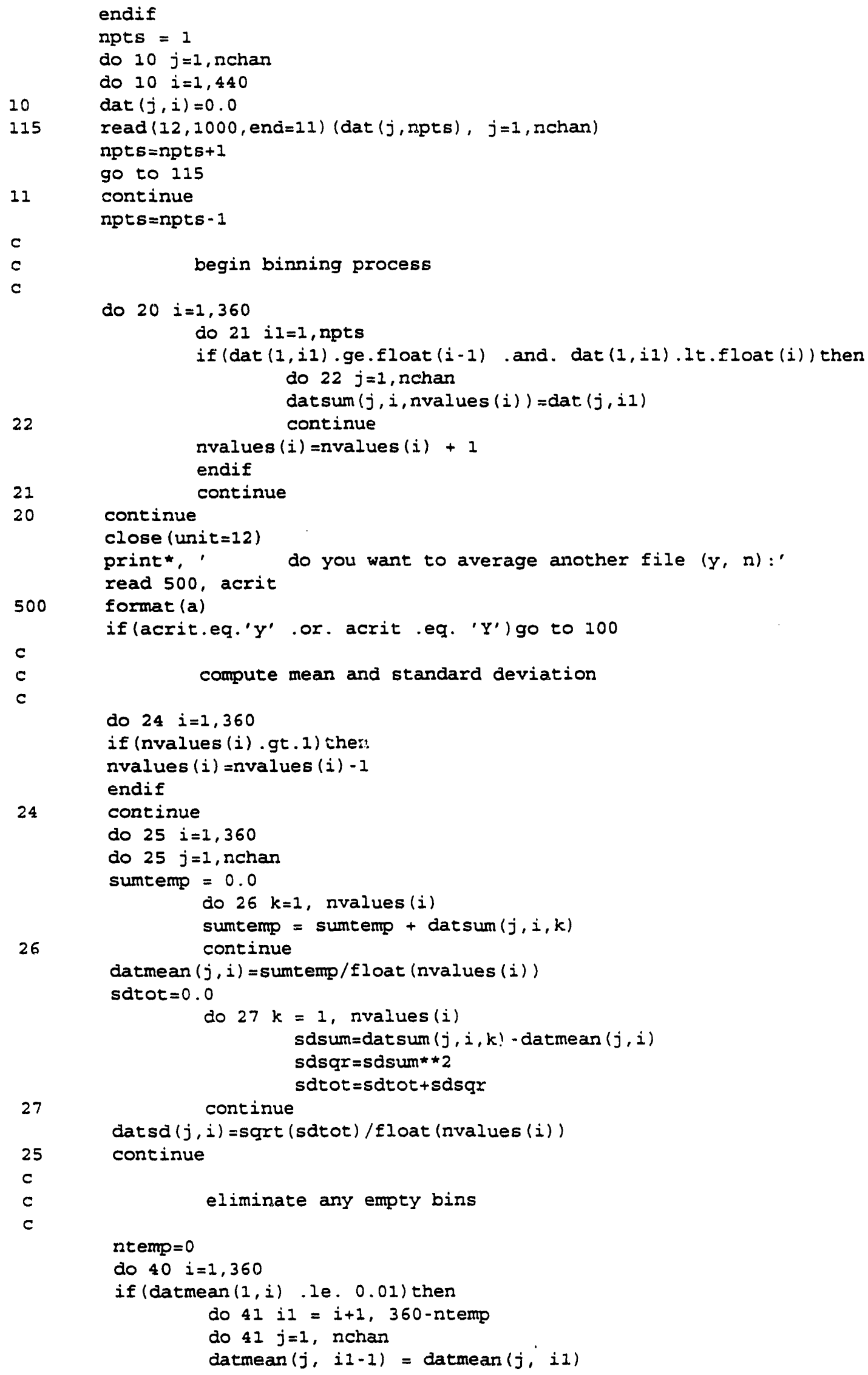




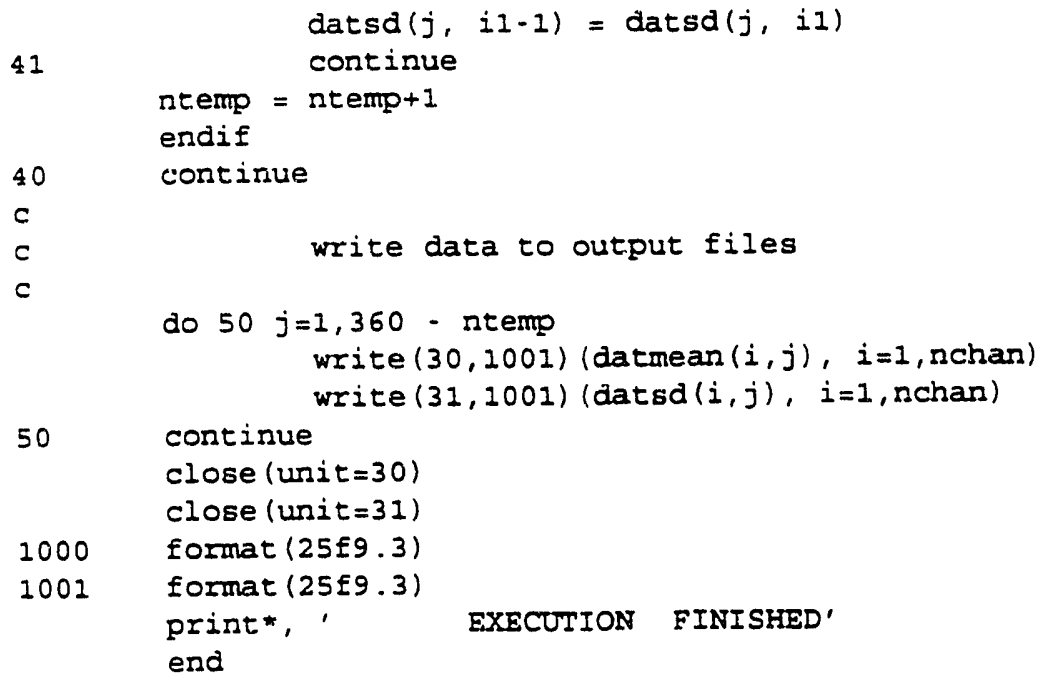




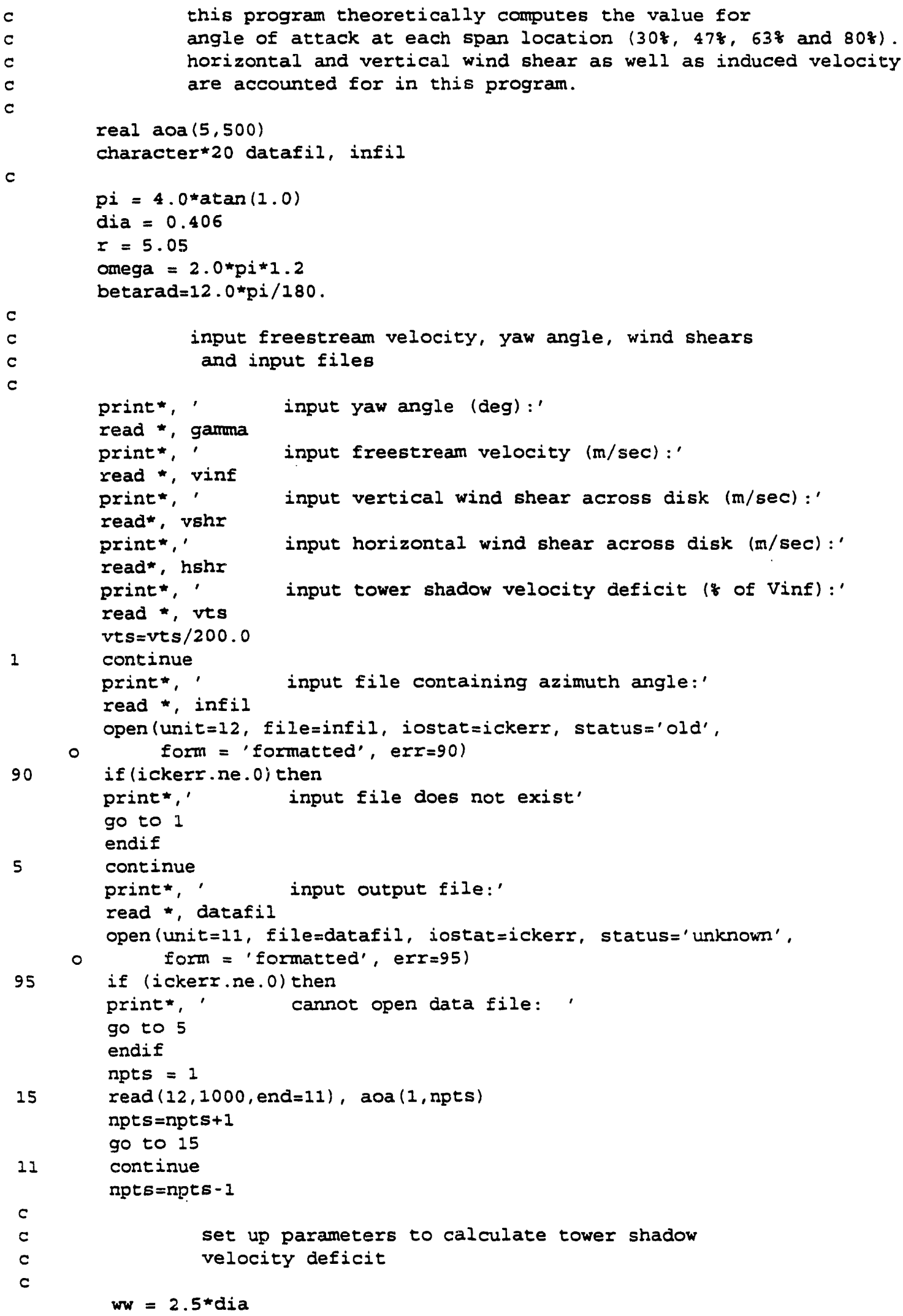




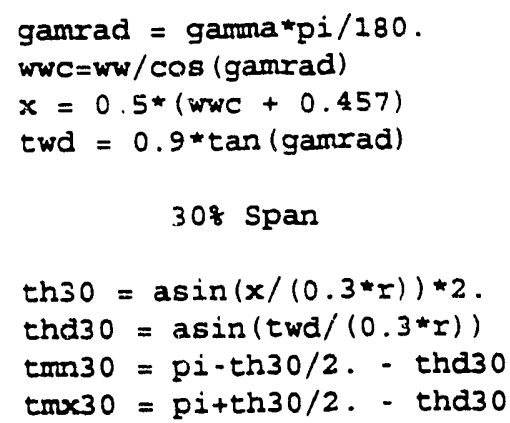

c 


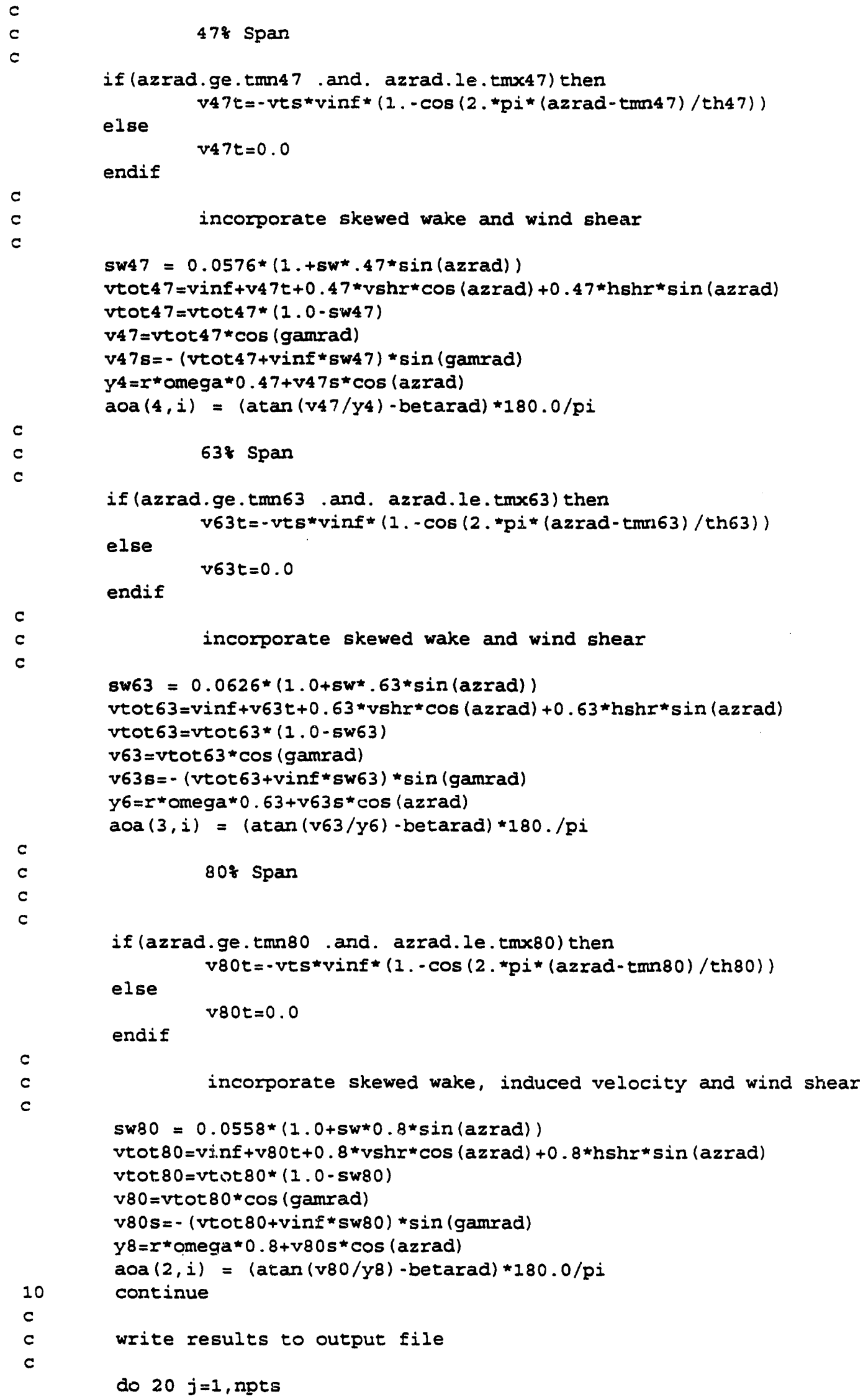

$47 \%$ Span

if (azrad.ge.tmn47 .and. azrad.le.tmx47) then v4 $7 t=-v t s * v i n f *\left(1 .-\cos \left(2 .{ }^{*} p *(\operatorname{azrad}-\operatorname{tmn} 47) / \operatorname{th} 47\right)\right)$

else

endif $\mathbf{v 4} 7 t=0.0$

incorporate skewed wake and wind shear 
write $(11,201)$ (aoa $(i, j), i=1,5)$

20

continue

close (unit=11)

close (unit $=12$ )

201 format $(5 \neq 10.5)$

1000 format ( $\$ 9.3$ )

print*,

end

EXBCUTION FINISHED' 
c

1

90

5

C

program qocalc

this program theoretically computes the value for qo at each span localtion.

real $q(5,500)$

character $2^{20}$ datafil, infil

$p i=4.0 * \operatorname{atan}(1.0)$

dia $=0.406$

$x=5.05$

omega $=2.0 \star p i * 1.2$

input freestream velocity, yaw angle and input files

print*, '

read *, gamma

print*,

read *, vinf

print*, '

read *, tempc

print*, '

read *, pmbar

input yaw angle (deg):'

input freestream velocity (m/sec) :'

input temperature $(\operatorname{deg} C): '$

input atmospheric pressure (mbar):'

continue

print*,

read *, infil

input file containing azimuth angle:'

open (unit=12, file=infil, iostat=ickerr, status='old', form $=$ 'formatted', err $=90$ )

\section{if (ickerr.ne. 0 ) then}

print*.'

input file does not exist'

go to 1

endif

continue

print*, ' input output file:'

read *, datafil

open (unit=11, file=datafil, iostat=ickerr, status='unknown' ,

$\circ$ form = 'formatted', err $=95$ )

if (ickerr.ne.0) then

print*,

cannot open data file:

go to 5

endif

npts $=1$

$\operatorname{read}(12,1000$, end $=11), q(1$, npts $)$

npts $=$ npts +1

go to 15

continue

npts $=$ npts -1

compute aix density

$r g c=287.0$

tempk $=$ tempc +273.0

$\mathrm{pnm}=$ pmbar $/ 1000.0 * 0.9869 * I 4.696 * 6895.0$

rho $=\mathrm{pmm} /(\mathrm{rgc}$ tempk $)$

set up parameters to calculate tower shadow

velocity deficit

$w w=2.5 \star d i a$ 
gamrad $=$ gama*pi/180.

$w w C=n w / \cos$ (gamrad)

$x=0.5 *(w w c+0.457)$

twd $=0.9 * \tan ($ gamrad)

c

304 span

$\operatorname{th} 30=\operatorname{asin}(x /(0.3 * x)) * 2$.

$\operatorname{thd} 30=\operatorname{asin}\left(t w d /\left(0.3^{*} x\right)\right)$

$\tan 30=p i-\operatorname{th} 30 / 2 .-\operatorname{thd} 30$

$\operatorname{tm} \times 30=p i+\operatorname{th} 30 / 2 .-\operatorname{thd} 30$

478 Span

$\operatorname{ch} 47=\operatorname{asin}(x /(0.47 * x)) \div 2$.

$\operatorname{thd} 47=\operatorname{asin}(t w d /(0.47 \star x))$

$\operatorname{tm} 47=p i-\operatorname{th} 47 / 2 .-\operatorname{thd} 47$

$\operatorname{tmx} 47=p i+\operatorname{th} 47 / 2 .-[$ thd 47

63* Span

$\operatorname{th} 63=\operatorname{asin}(x /(0.63 * x)) \star 2$.

thd63 $=\operatorname{asin}($ twd $/(0.63 * x))$

$\operatorname{tmn} 63=p i-\operatorname{th} 63 / 2 .-\operatorname{thd} 63$

$\operatorname{tm} \times 63=p i+t h 63 / 2 .-t h d 63$

80t Span

$\operatorname{th} 80=\operatorname{asin}(x /(0.8 * x)) * 2$.

thd80 $=\operatorname{asin}\left(t w d /\left(0.8^{*} \tau\right)\right)$

$\operatorname{tm} 80=p i-t h 80 / 2 .-t h d 80$

tm $\times 80=p i+t h 80 / 2 .-t h d 80$

compute corrected $q$ from yaw motion and assuming

tower wake velocity deficit modeled by cosine function

vnorm $=$ vinf*cos (gamrad)

vspan $=-v i n f \star \sin ($ gamrad)

do $10 i=1$, npts

azrad $=q(1, i) * p i / 280$.

$308 \mathrm{span}$

$y=r \star$ omega $0.3+v \operatorname{vpan} \star \cos ($ azrad)

$z=\operatorname{vspan}{ }^{*} \sin ($ azrad$)$

if (azrad.ge. $\operatorname{tmn} 30$.and. azrad.le.tmx30) then

$-30=v$ morm $-0.1 *$ vnorm* $\left(1 .-\cos \left(2 .{ }^{*} i *(\operatorname{azrad}-\operatorname{tmn} 30) / \operatorname{th} 30\right)\right)$

else

v30 =vnorm

endif

$q(5, i)=0.5 *$ xho* $(y \star 2+v 30 \star \star 2+z \star \star 2) / 6895.0$

47\% Span

$y=r *$ omega*0.47+vspan*cos (azrad)

if (azrad.ge.tmn47 and. azrad.le.tmx47) then

$\mathrm{v} 47=\operatorname{vnorm}-0.1 * \operatorname{vnorm} *\left(1 .-\cos \left(2 .{ }^{*} \mathrm{pi} *(\operatorname{azrad}-\operatorname{tmn} 47) / \operatorname{th} 47\right)\right)$

else

v4 $7=$ vnorm

endif 


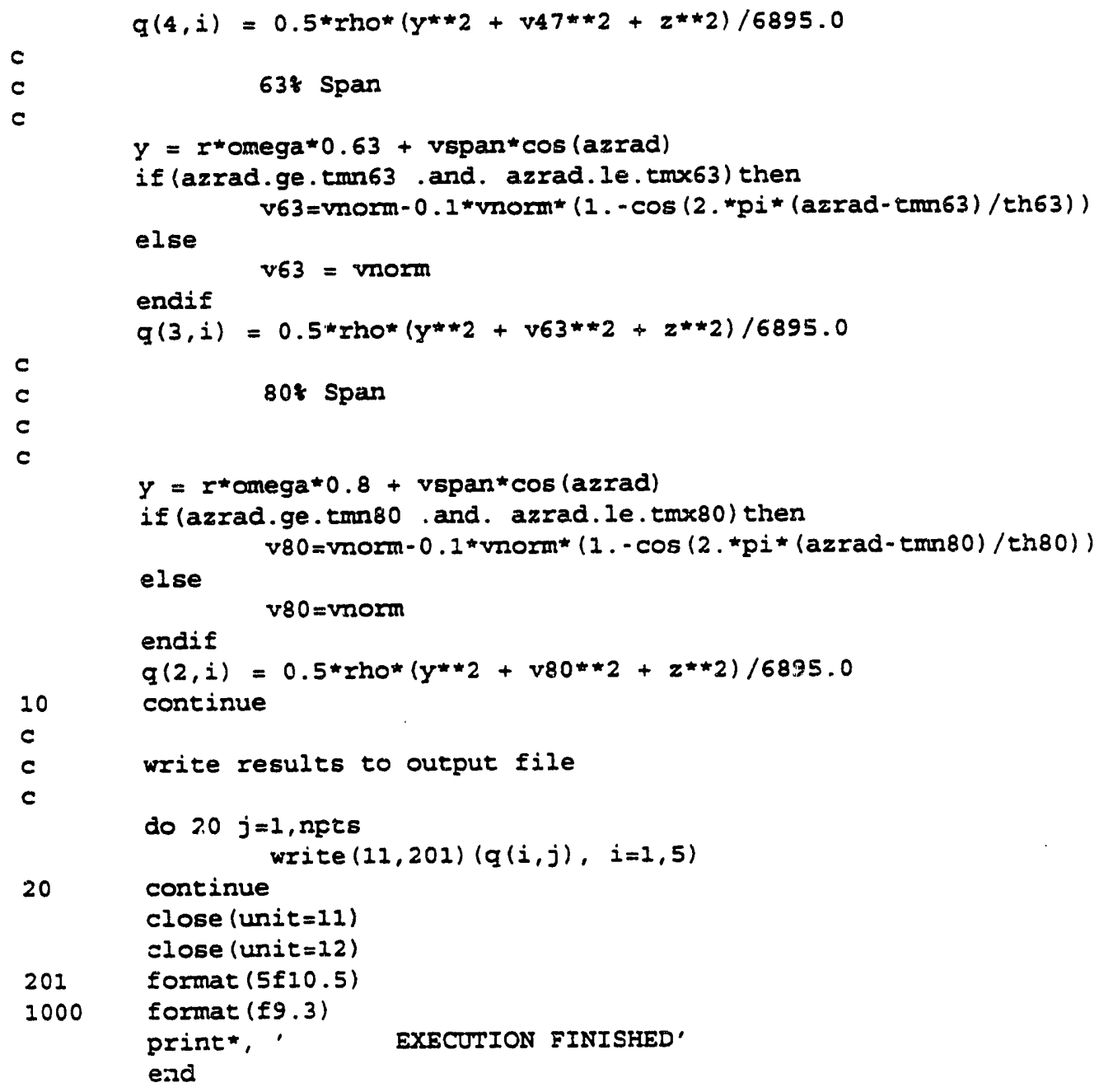


c

c

c

c

c

c

c

c

$c$

c

1

this program theoretically computes the value for go at each span location (30\%, 47\%, 63\% and 80\%). effects of induced velocity, skewed wake and vertical and/or horizontal wind shear is included.

real $q(5,500)$

character 20 datafil, infil

$p i=4.0 \star a t a n(1.0)$

di. $=0.406$

$r=5.05$

omega $=2.0 * p i \star 1.2$

input freestream velocity, yaw angle and input files

print*, ,

read *, gamma

print*,

read *, vinf

print*, ,

read*, vshr

print*, '

read*, hshr

print*, '

read *, ves

vts $=v t s / 200.0$

print*,

read *, tempc

print*, ,

read *, pmbar

input yaw angle (deg):'

input freestream velocity $(\mathrm{m} / \mathrm{sec}):^{\prime}$

input vertical wind shear across disk (m/sec):'

input horizontal wind shear across disk (m/sec):'

input tower shadow velocity deficit (t of Vinf):'

input temperature $(\operatorname{deg} C): '$

input atmospheric pressure (mbar):'

continue

print*, ,

read *, infil

input file containing azimuth angle:'

open (unit=12, file=infil, iostat=ickerr, status='old',

if (ickerr.ne.0) then

print*,'

input file does not exist'

go to 1

endif

5

continue

print*, ' input output file:'

read *, datafil

open (unit=11, file=datafil, iostat=ickerr, status='unknown',

$\circ$ form = 'formatted', err $=95$ )

95

if (ickerr.ne.0) then

print*,

cannot open data file: ,

go to 5

endif

npts $=1$

$\operatorname{read}(12,1000$, end $=11), q(1$, npts $)$

npts $=$ npts +1

go to 15

11

continue

npts $=$ npts -1

c

compute air density 


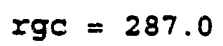

C

c

$c$

c

c

308 Span

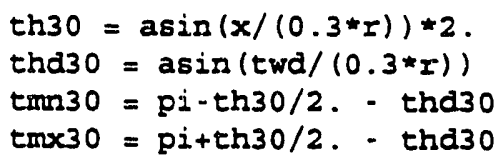


c

C

C

c

c

$c$

c

c

c

c

c

c

incorporate skewed wake and wind shear

$\operatorname{sw3} 3=0.1172 *(1.0+5 w * 3 * \sin ($ azrad $))$

vtot $30=v i n f+v 30 t+0.3 * v s h r * \cos ($ azrad) $+0.3 *$ hshr*sin (azrad)

vtot $30=v \operatorname{tot} 30 *(1.0-8 w 30)$

v30 $=$ vtot $30 * \cos$ (gamrad)

v30s $=-($ vtot $30+$ vinf*sw30)*sin (gamrad)

$y=r \star$ omega $\star 0.3+v 30 s \star \cos (a z r a d)$

$z=v 30 g^{*} \sin (\mathrm{azrad})$

$q(5, i)=0.5 *$ rho* $(y * 2+v 30 * \approx 2+z * \star 2) / 6895.0$

478 Span

if (azrad.ge.tmn47 and. azrad.le.tmx47) then $v 47 t=-v t 8 * v i n f *(1 .-\cos (2 . * p i *(\operatorname{arrad}-t m a 47) / t h 47))$

else

endif

$v 47 t=0.0$

incorporate skewed wake and wind shear

sw47 $=0.0576 *(1 .+8 w * .47 * \sin (\operatorname{azrad}))$

vtot $47=$ vinf $+v 47 t+0.47 *$ vshr* $\cos ($ azrad $)+0.47 \star h s h r * \sin ($ azrad)

vtot $47=\operatorname{vtot} 47 *(1.0-5 w 47)$

v4 $7=v t o t 47 * \cos$ (gamrad)

v $48=-($ vtot $47+$ vinf*gw47) *sin (gamrad)

$y=r *$ anega $0.47+v 478 * \cos (a z r a d)$

$2=v 47 \mathrm{~s} * \sin$ (azrad)

$q(4, i)=0.5 *$ rho* $(y * 2+v 47 * 2+z * \star 2) / 6895.0$

63\% Span

if (azrad.ge.tmn63 and. azrad.le.tmx63) then v63t $=-v t s \star v i n f *(1 .-\cos (2 . * p i \star(\operatorname{azrad}-\operatorname{tmn} 63) / \operatorname{th} 63))$

else

$v 63 t=0.0$

endif

incorporate skewed wake and wind shear

sw63 $=0.0626 *\left(1.0+5 w^{\star} .63 * \sin (\right.$ azrad $\left.)\right)$

vtot $63=$ vinf $+v 63 t+0.63 *$ vshr* $\cos ($ azrad $)+0.63 *$ hshr*sin (azrad)

vtot $63=v \operatorname{tot} 63 *(1.0-5 w 30)$

$\mathrm{v} 63=\mathrm{vtot} 63 * \cos ($ gamrad)

v63s $=-($ vtot $63+$ vinf*sw63) *sin (gamrad)

$y=r *$ omega*0.63+v63s*cos (azrad)

$z=v 63 s^{\star} \sin ($ azrad)

$q(3, i)=0.5 \star$ rho* $(y \star \star 2+v 63 \star \star 2+z \star \star 2) / 6895.0$

80 Span

if (azrad.ge.tmn30 and. azrad. le.tmx80) then

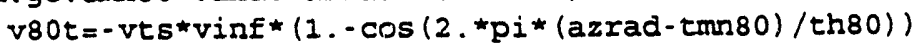

else $v 80 t=0.0$

endif

incorporate skewed wake, induced velocity and wind shear 


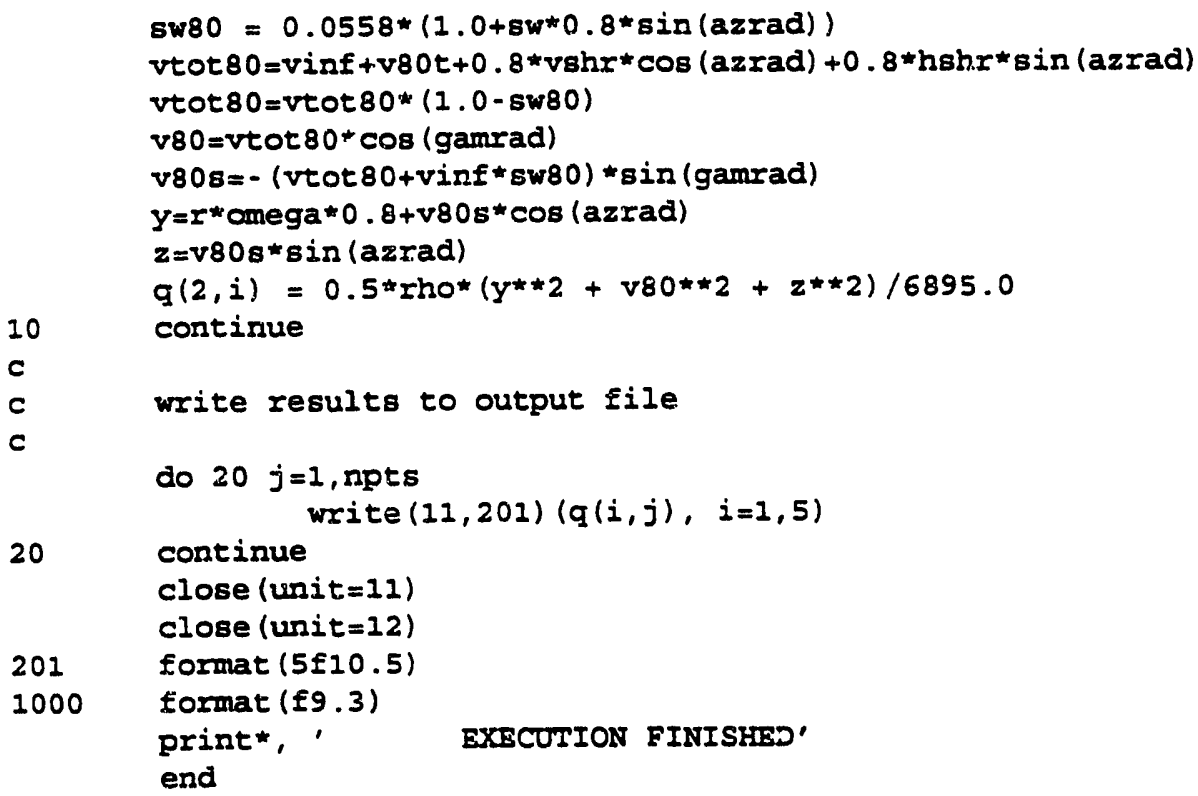


c

2

program qonorm

This program reads in $c$ and computed dynamic pressure files and then recomputes the normal force coefficients based on time varying dynamic pressure.

real en $(5,500), q(5,500)$

character 20 datafil, infil, qfil

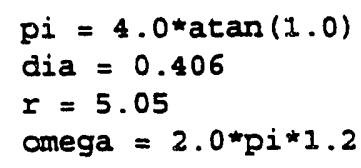


c

$\mathrm{rgc}=\mathbf{2 8 7 . 0}$

tempk $=t+273.0$

$\mathrm{pnm}=\mathrm{p} / 1000.0 * 0.9869 * 14.696 * 6895.0$

rho $=\mathrm{pnm} /($ Igc* tempk)

renormalize normal force coefficients

c

do $10 i=1$, npts

azrad $=\operatorname{cn}(1, i) \star p i / 180$.

c

c

c

$30 \%$ span

$\operatorname{cn}(5, i)=\operatorname{crs}(5, i) \star 0.5 *$ rho* $($ vinf $\star \star 2+(I \star$ onega $* 0.3) \star \star 2) /(6895.0 \star q(5, i))$

478 Span

$\operatorname{cn}(4, i)=\operatorname{cn}(4, i) \star 0.5 *$ rho* $($ vinf $* \star 2+(r *$ omega*0.47)**2)/(6895.0*q(4,i))

63\% Span

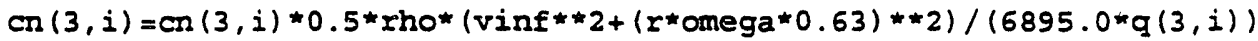

807 Span

$\operatorname{cn}(2, i)=\operatorname{cn}(2, i) \star 0.5 *$ rho* $($ vinf $* * 2+(r \star$ omega*0.8)**2)/(6895.0*q(2,i))

continue

write results to output file

do $20 j=1$, npes

write $(11,201)(\mathrm{cn}(i, j), i=1,5)$

20 continue

close (unit =11)

close (unit $=12$ )

close (unit $=13$ )

format ( $5 f 10.3$ )

format ( $5 f 9.3$ )

format ( $5 f 10.5)$

print*, '

EXECUTION FINISHED'

end 


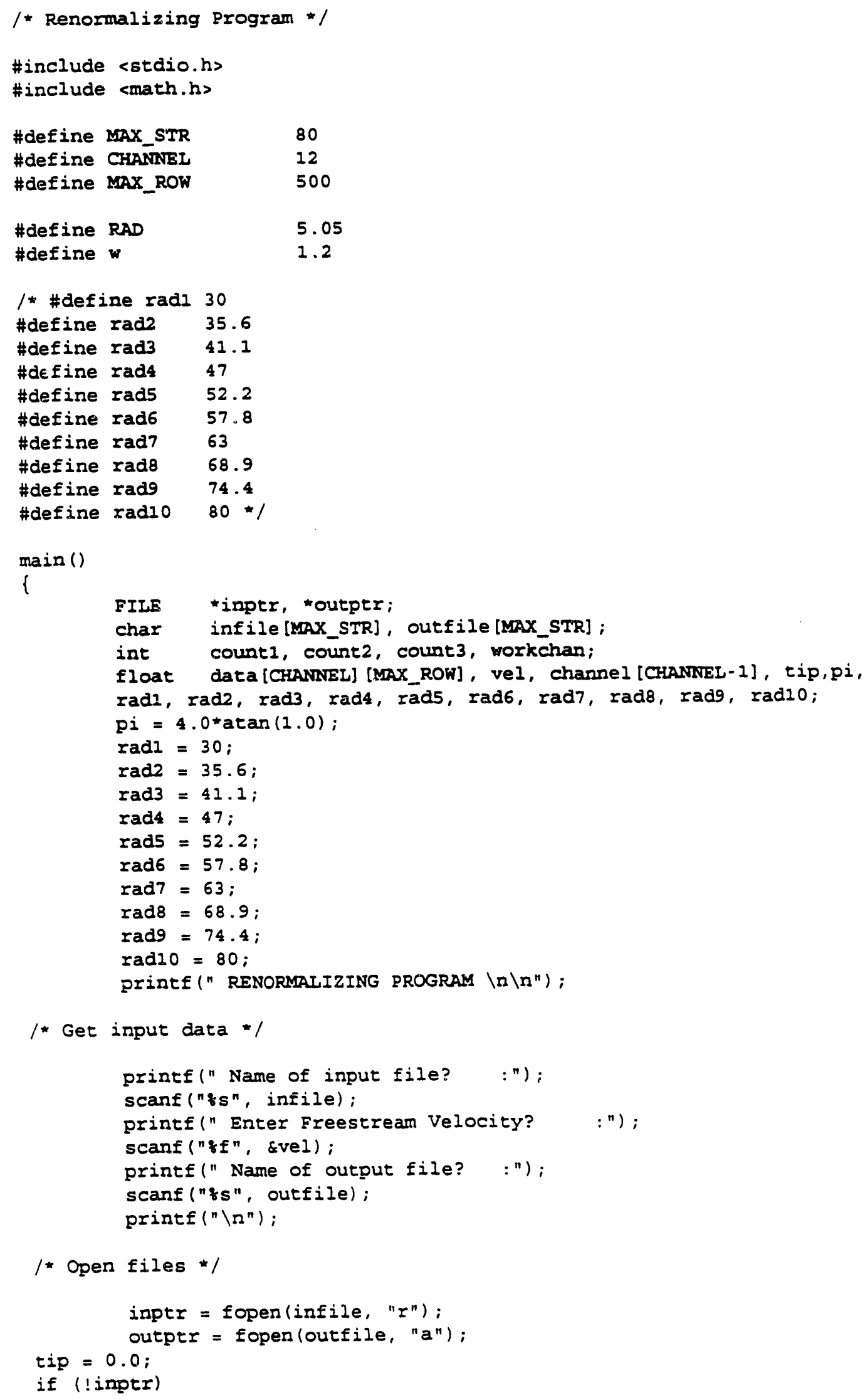




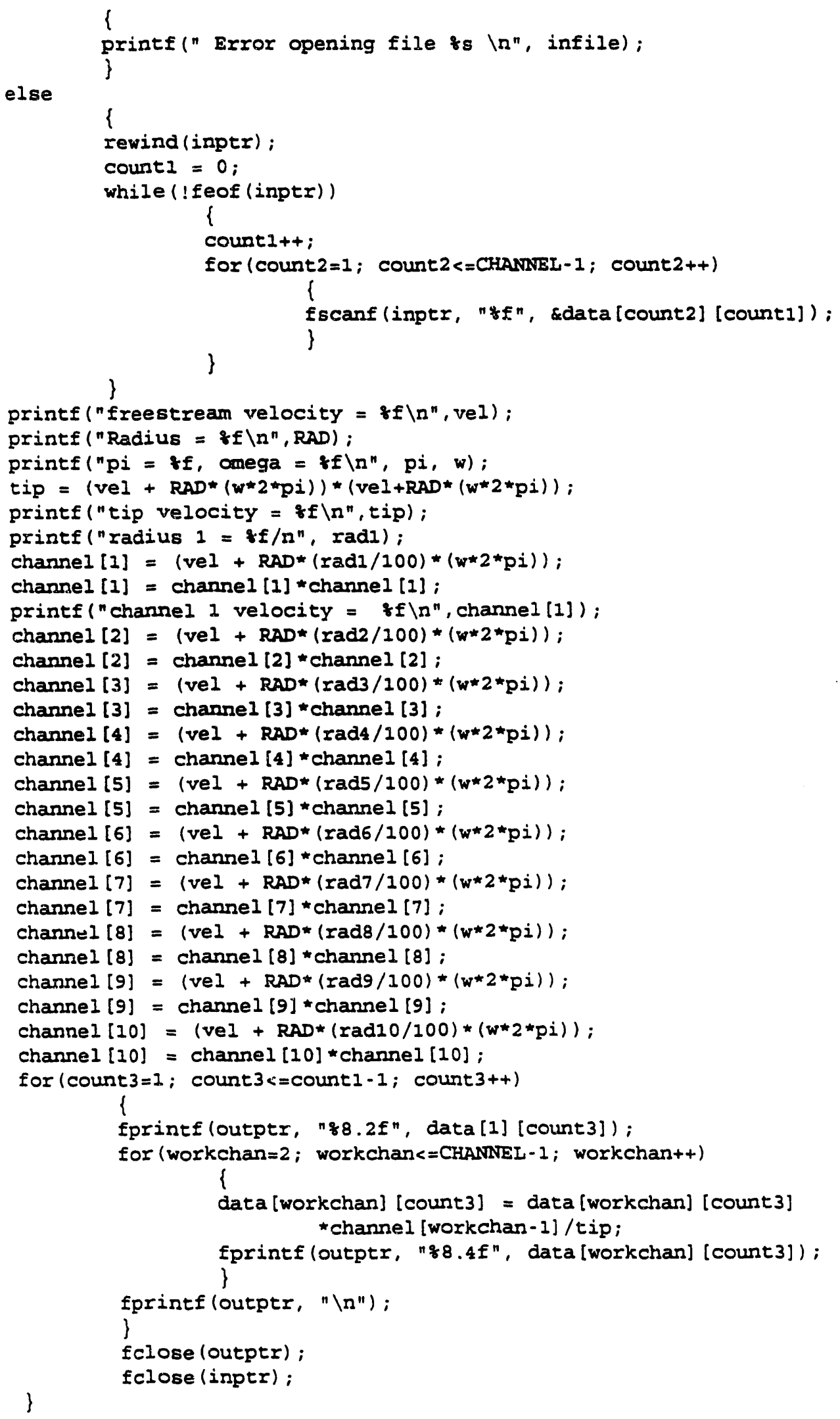




\section{Appendix B}

Experimental and Theoretical Motion Histories

B-1 


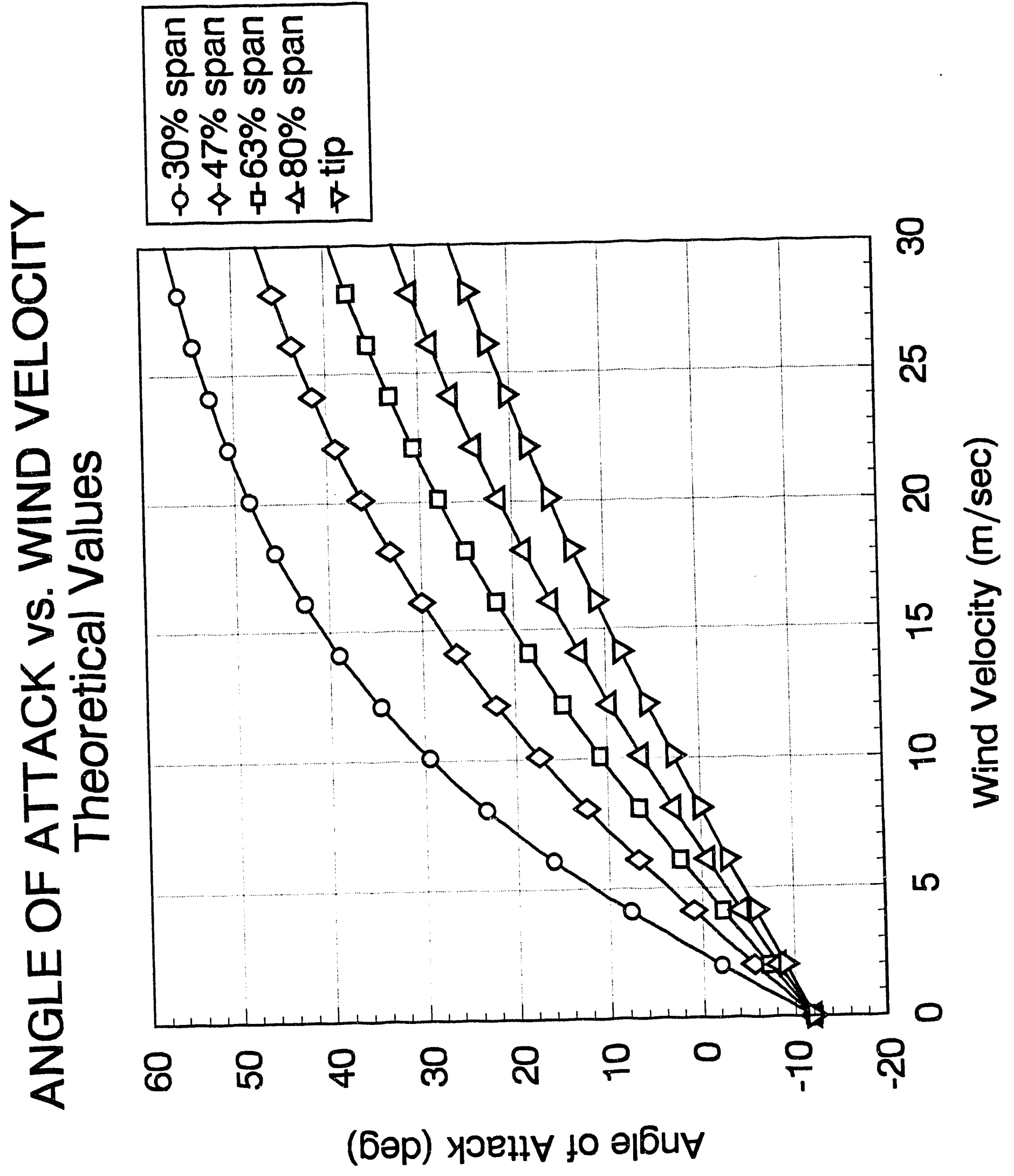




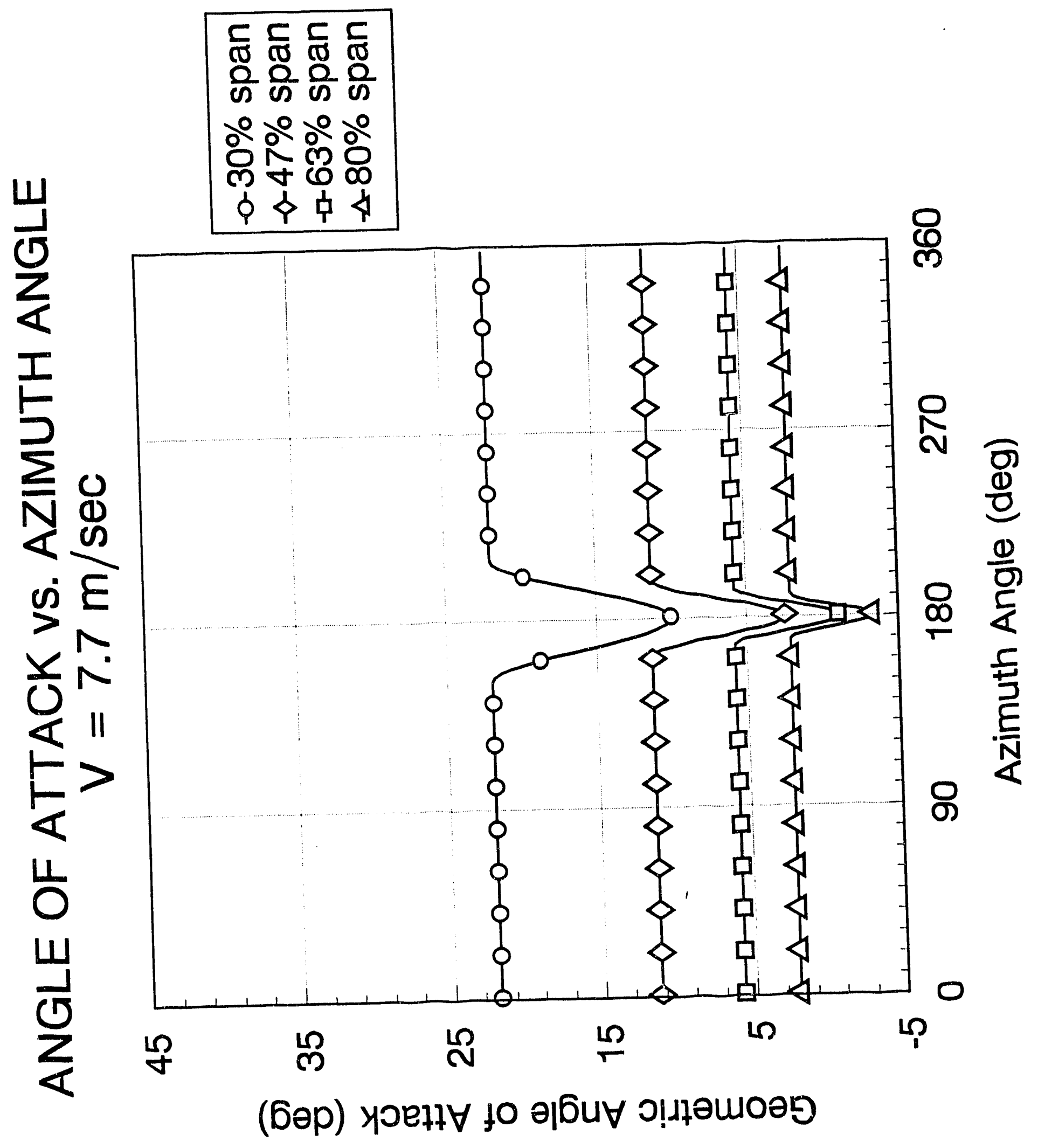




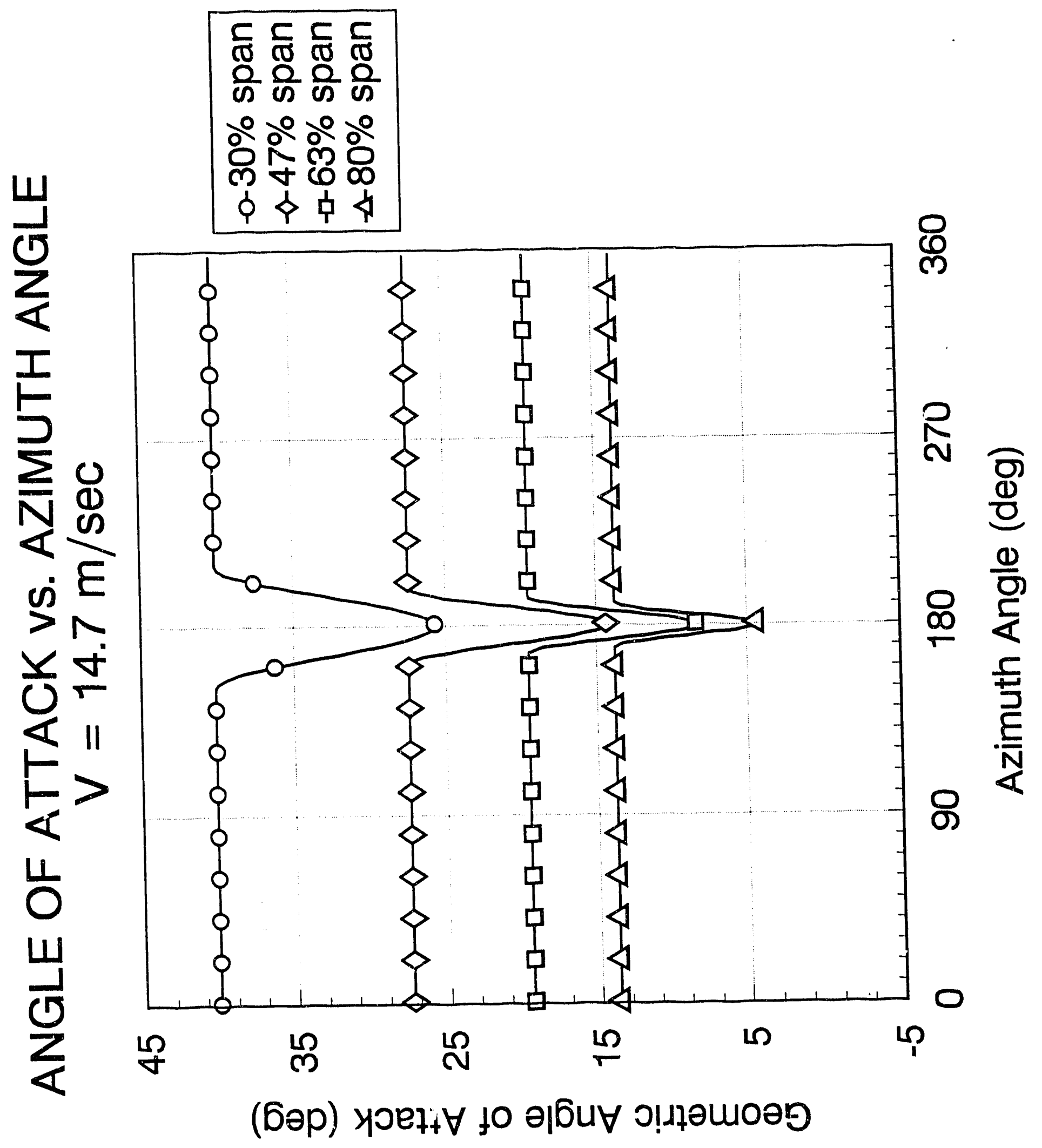



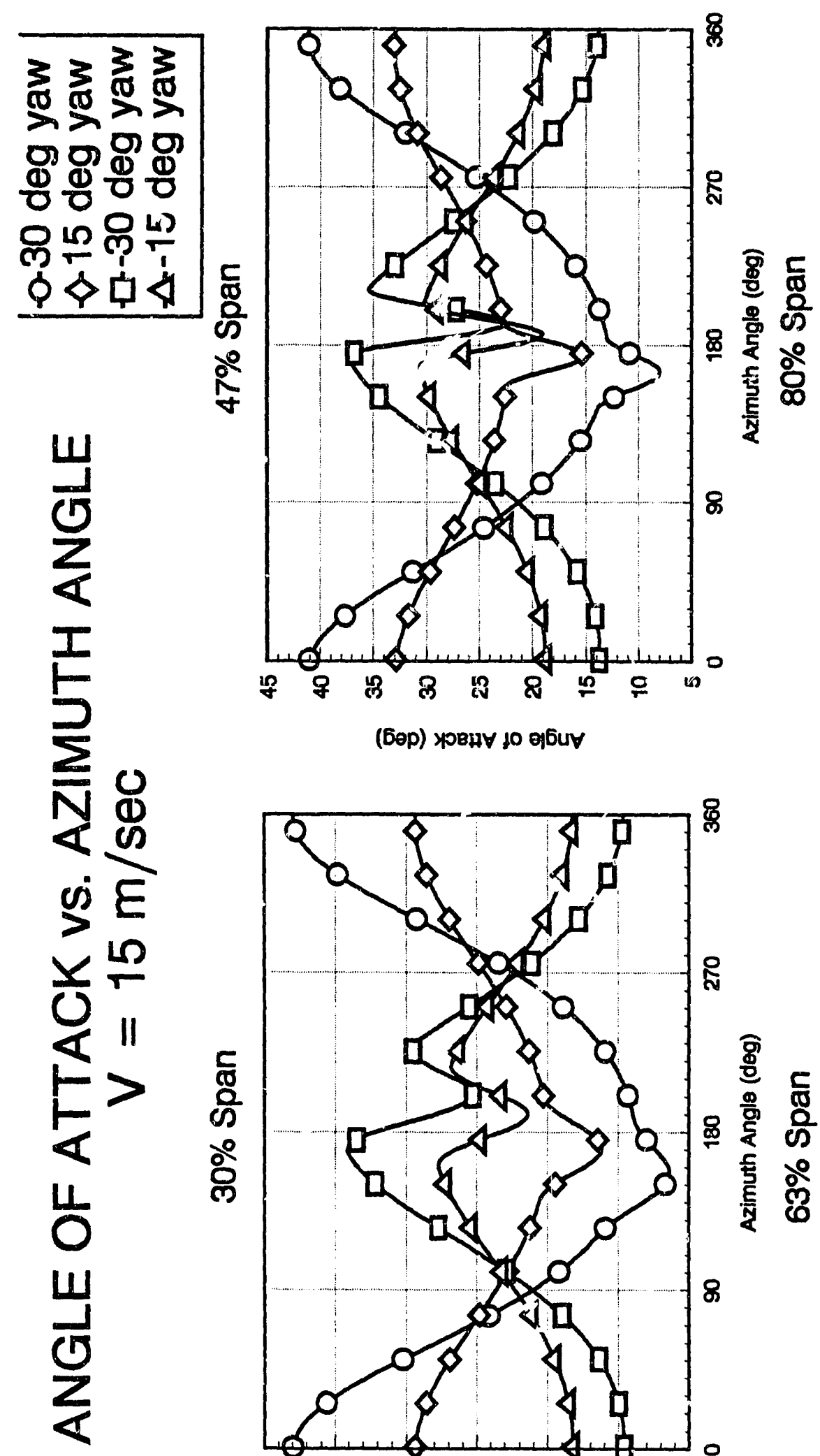

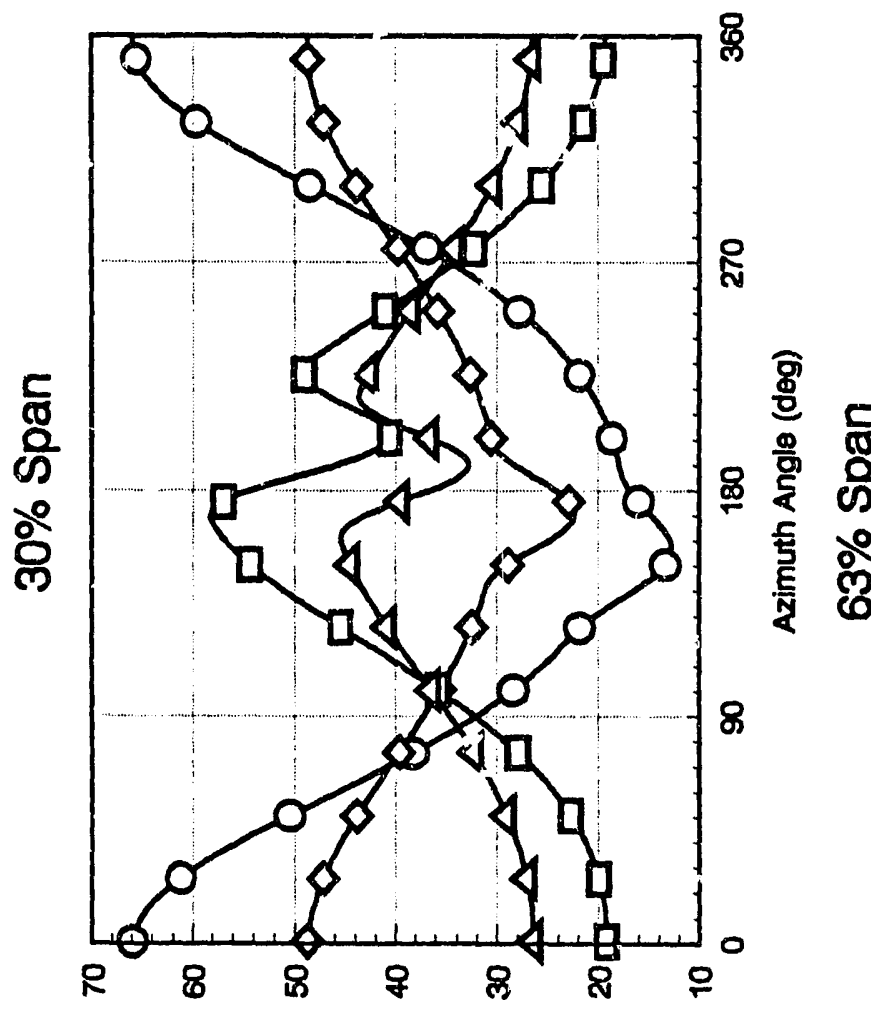

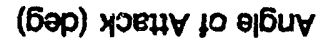

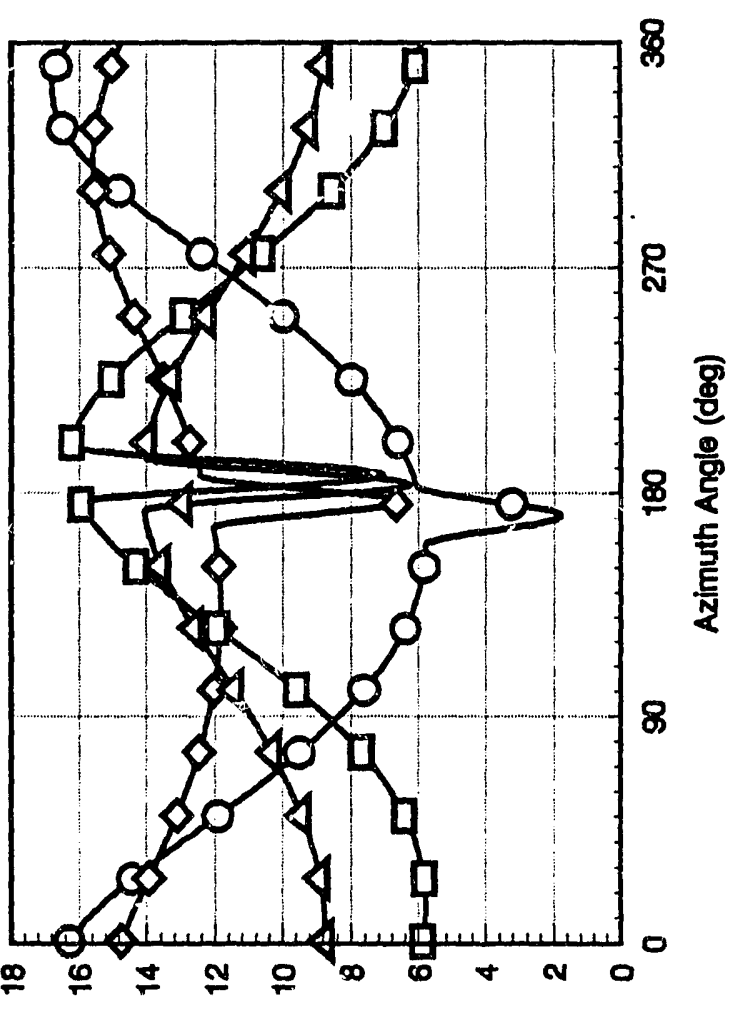

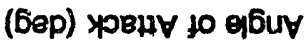

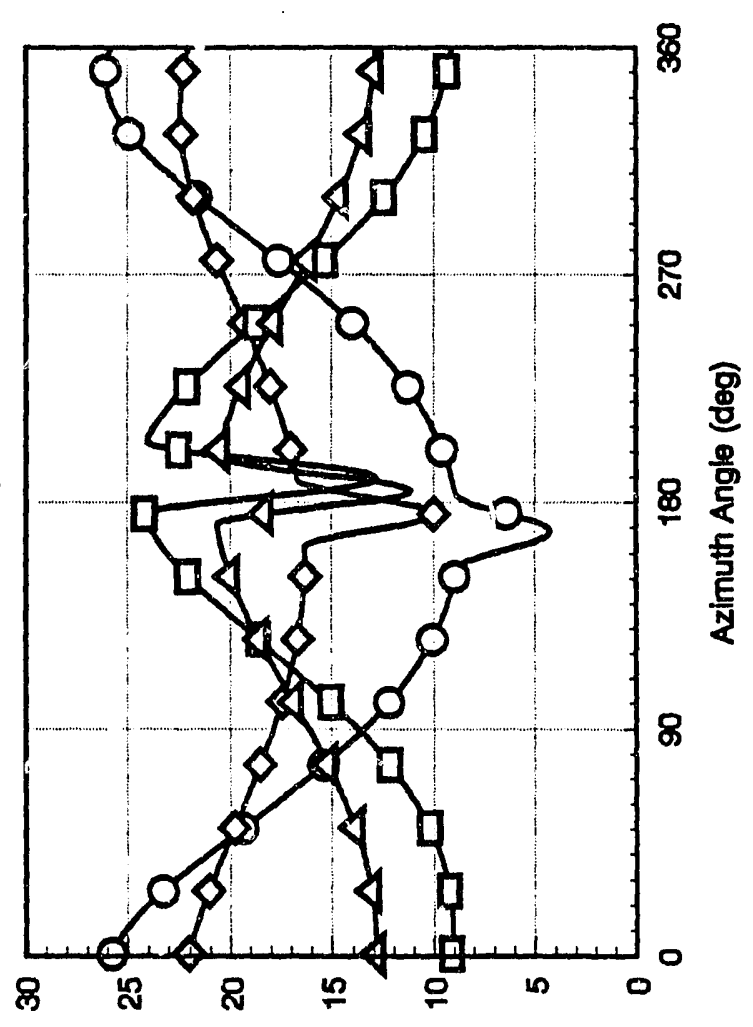

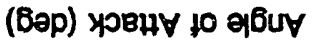



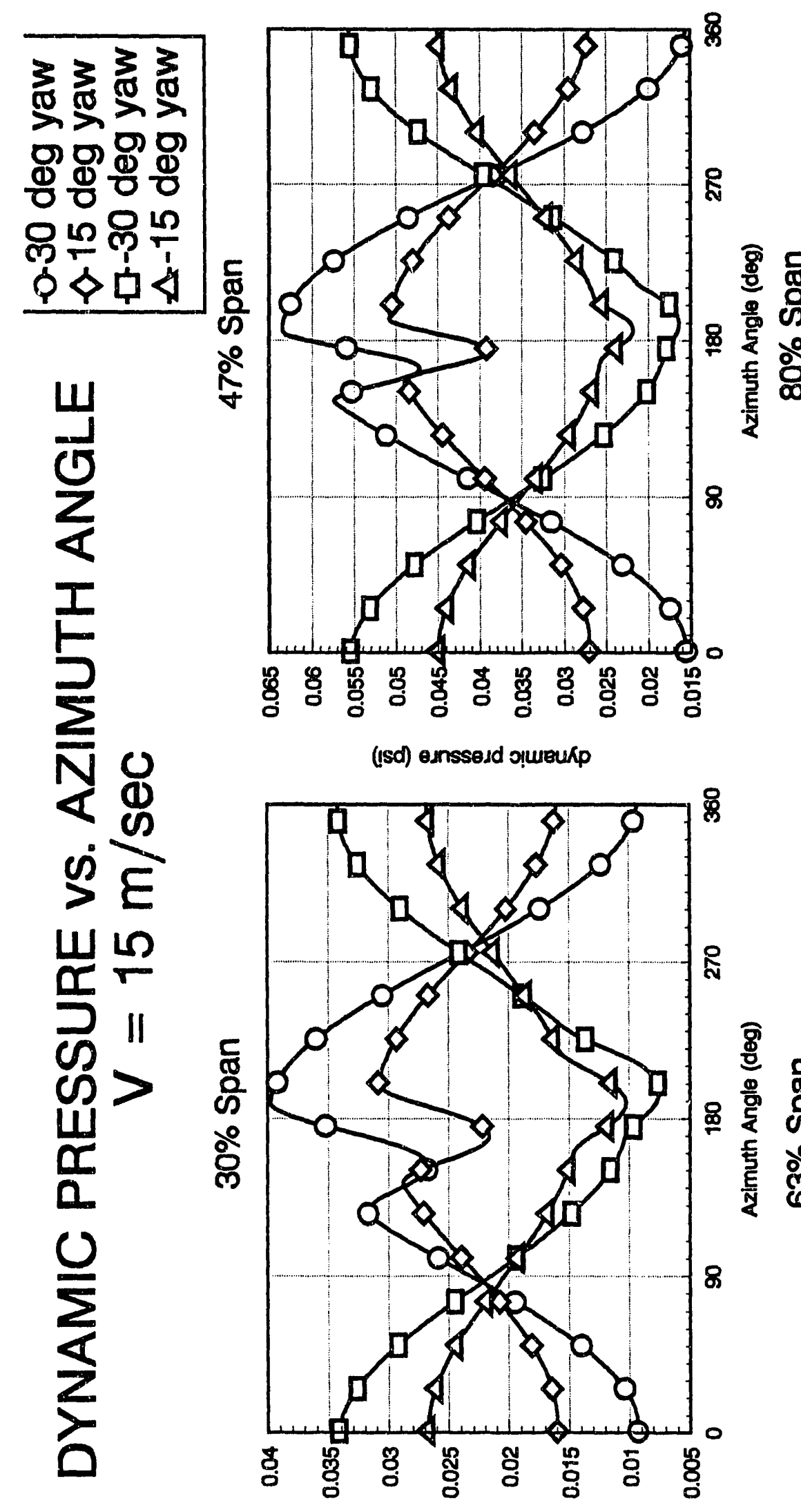

(Isd) ednssejd o!weuxp

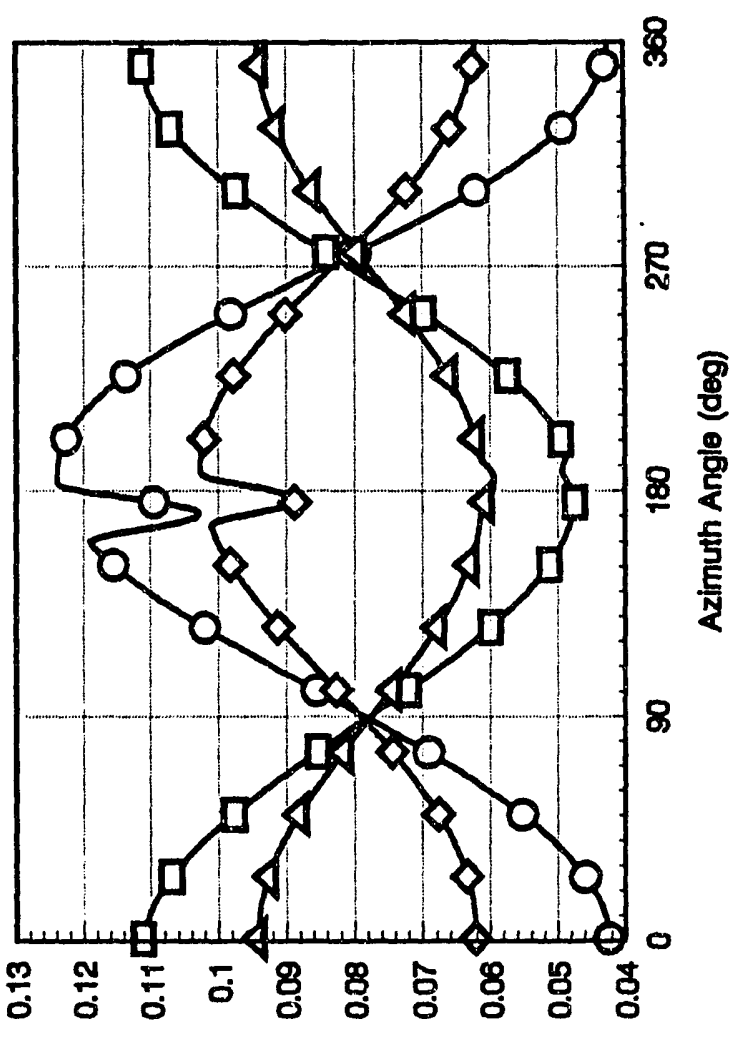

(Isd) emssesd פịreukp
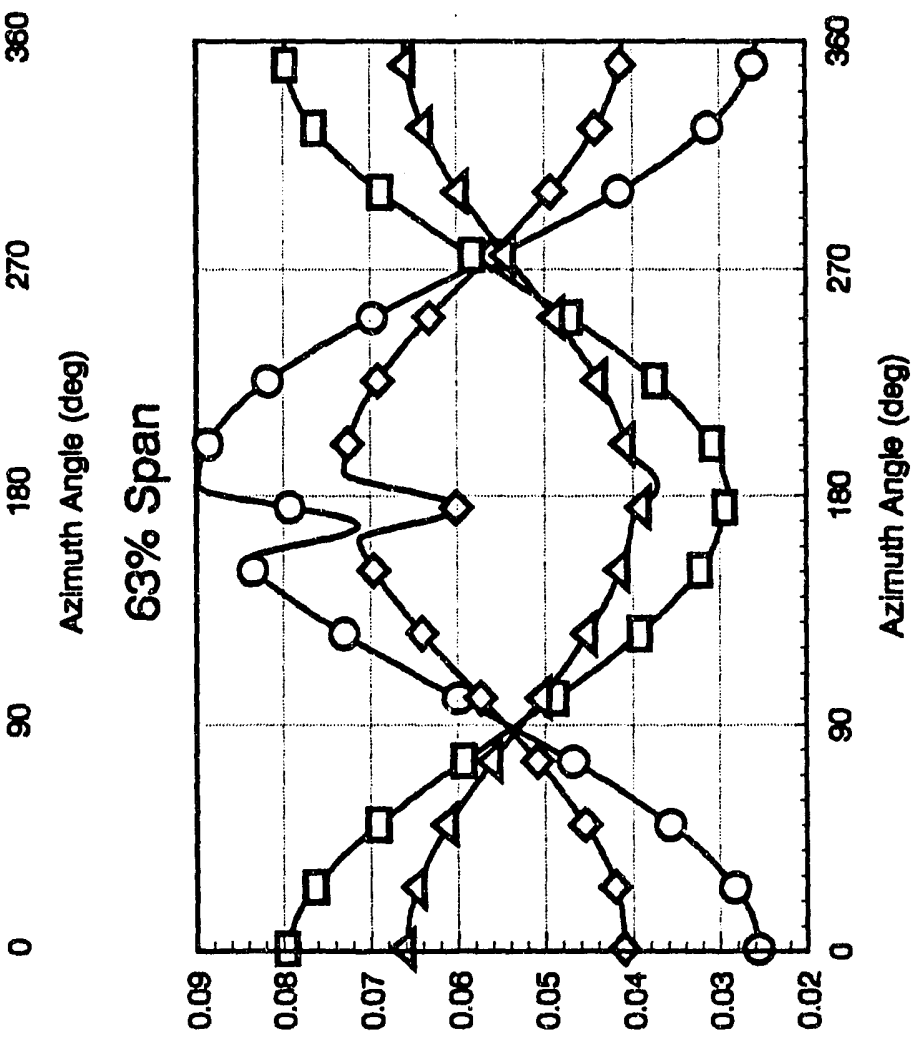

(Isd) eunssejd opweuKp 


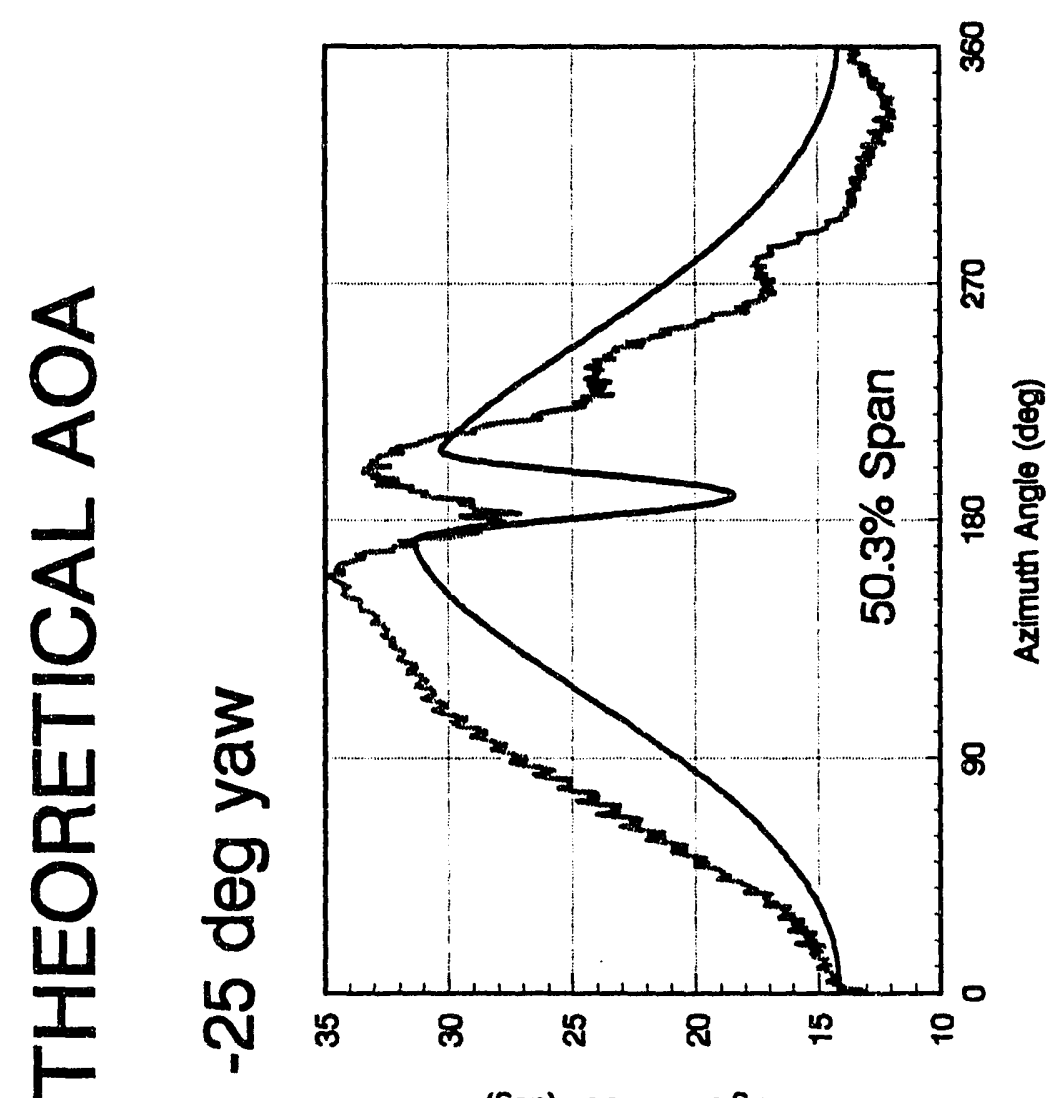

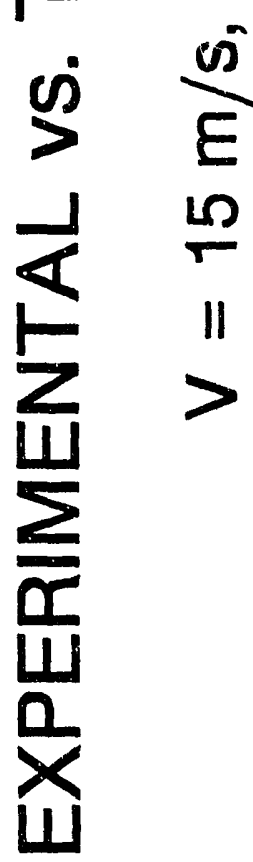

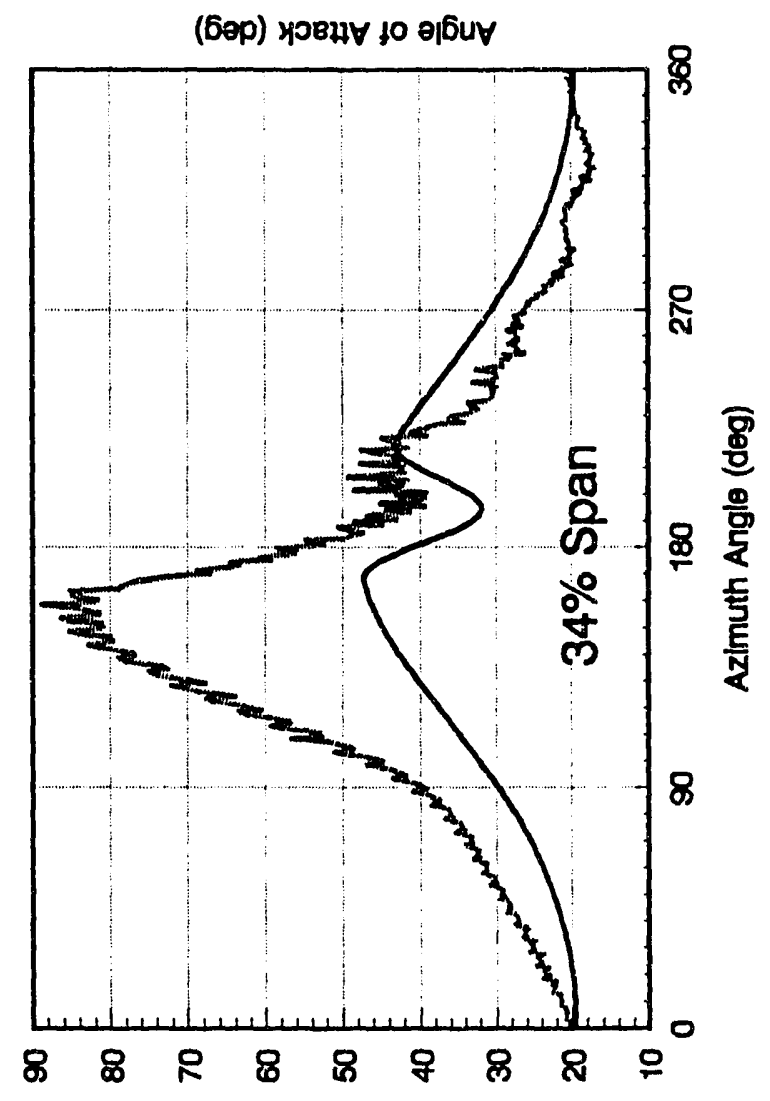

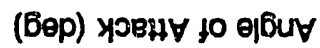
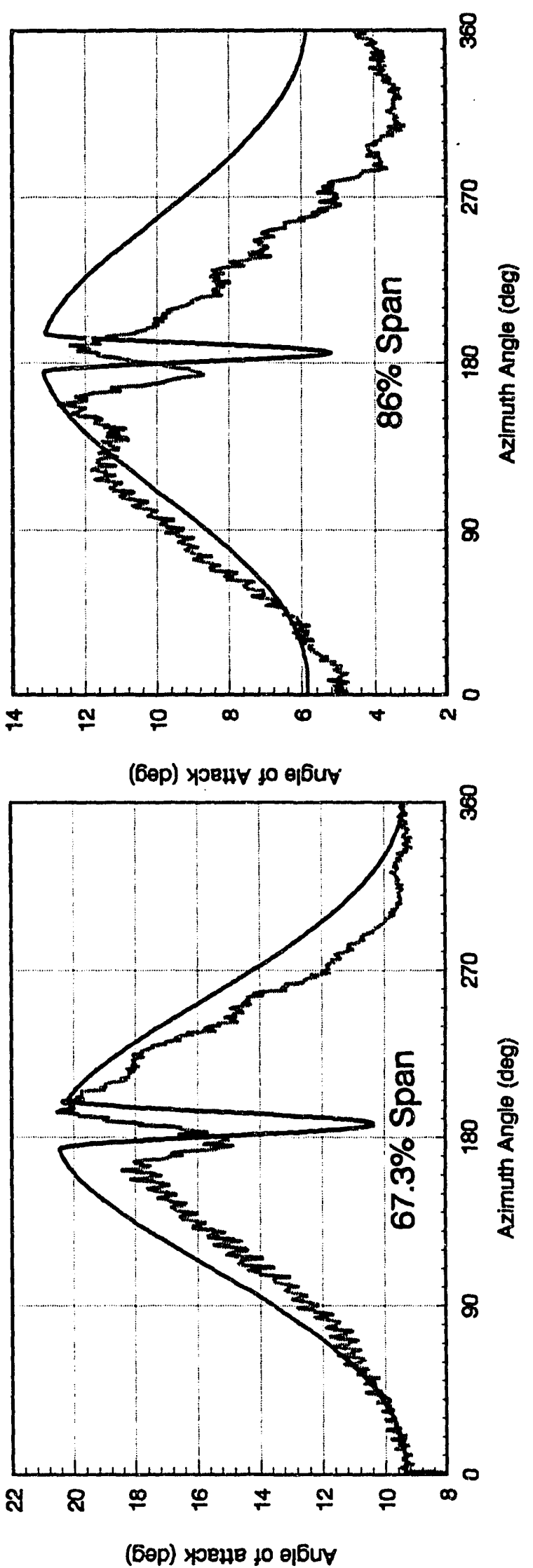

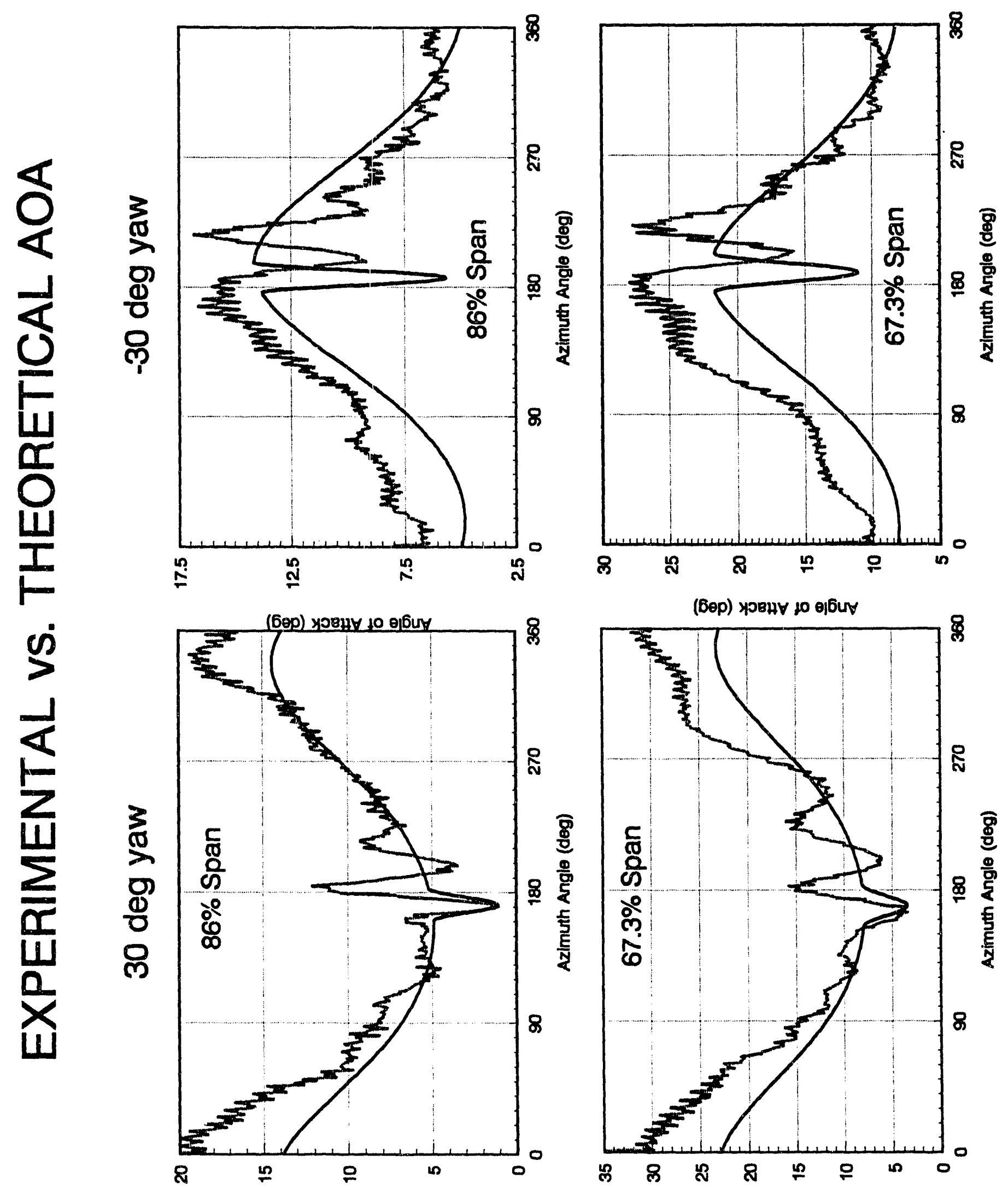

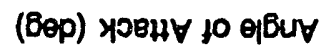

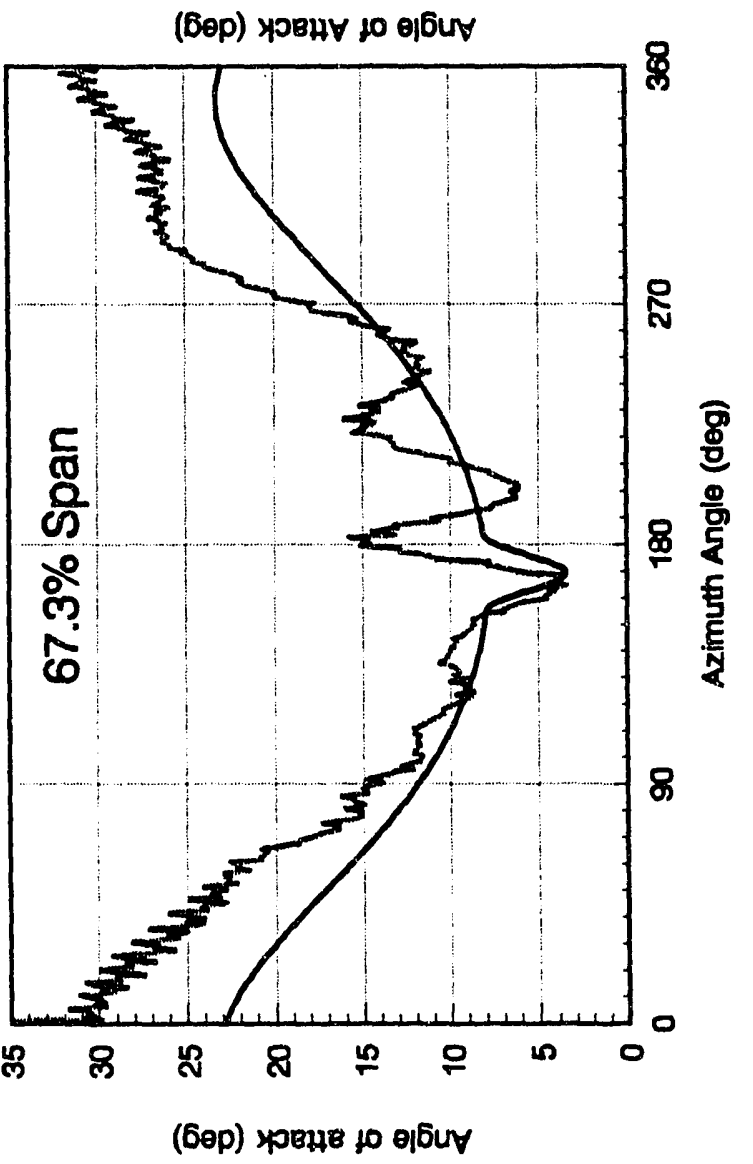



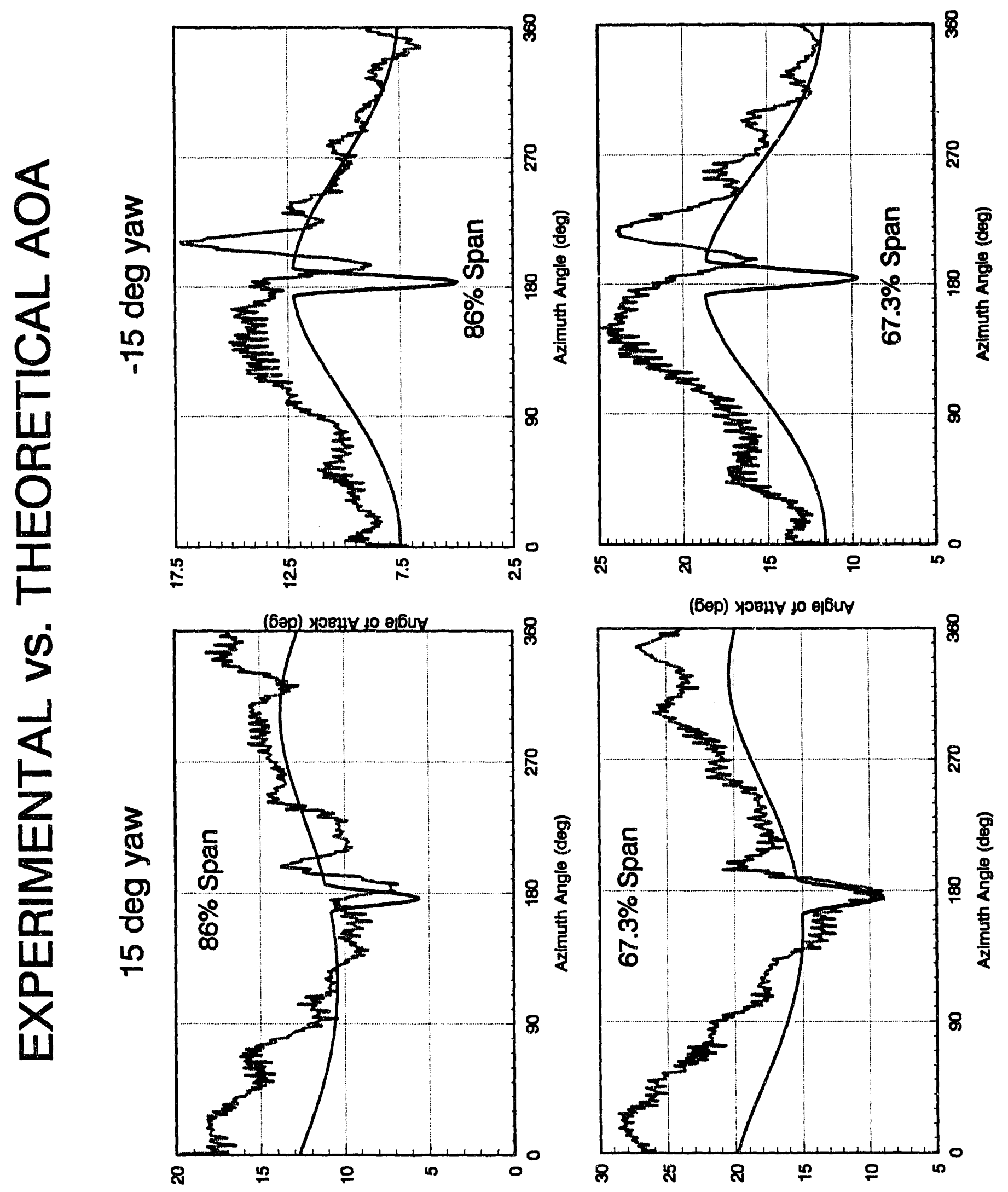

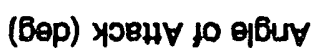

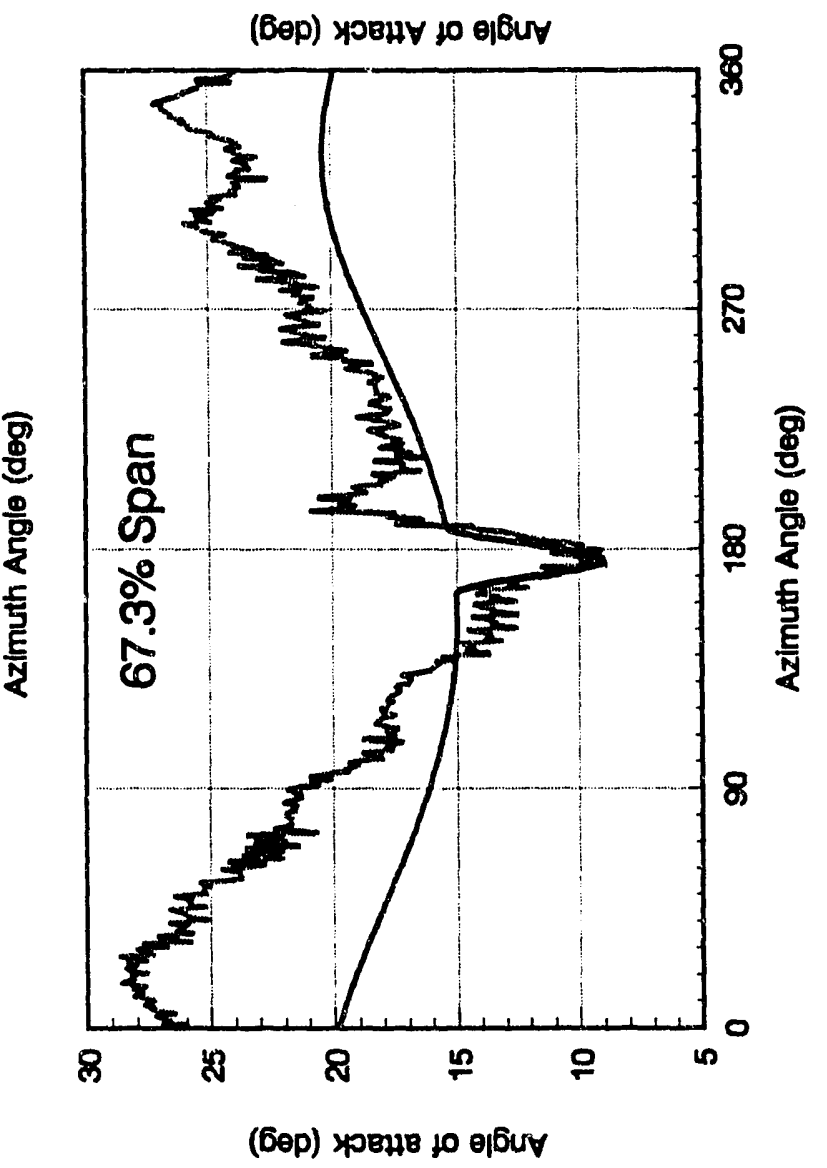




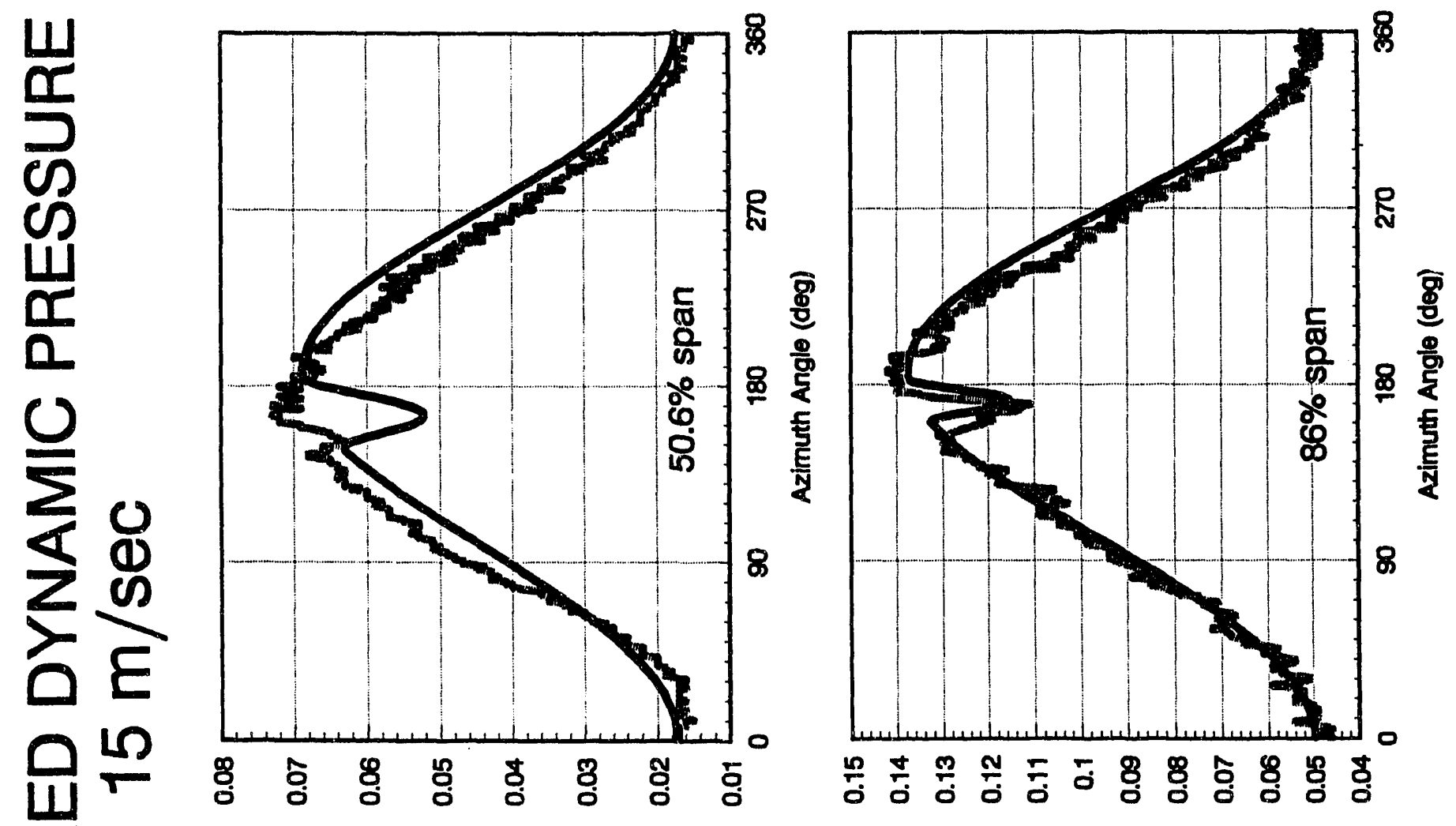

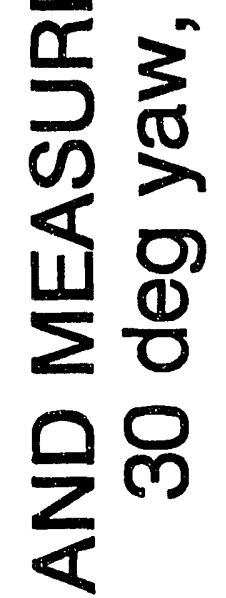

(!sd) e.snssedd ọmвuka

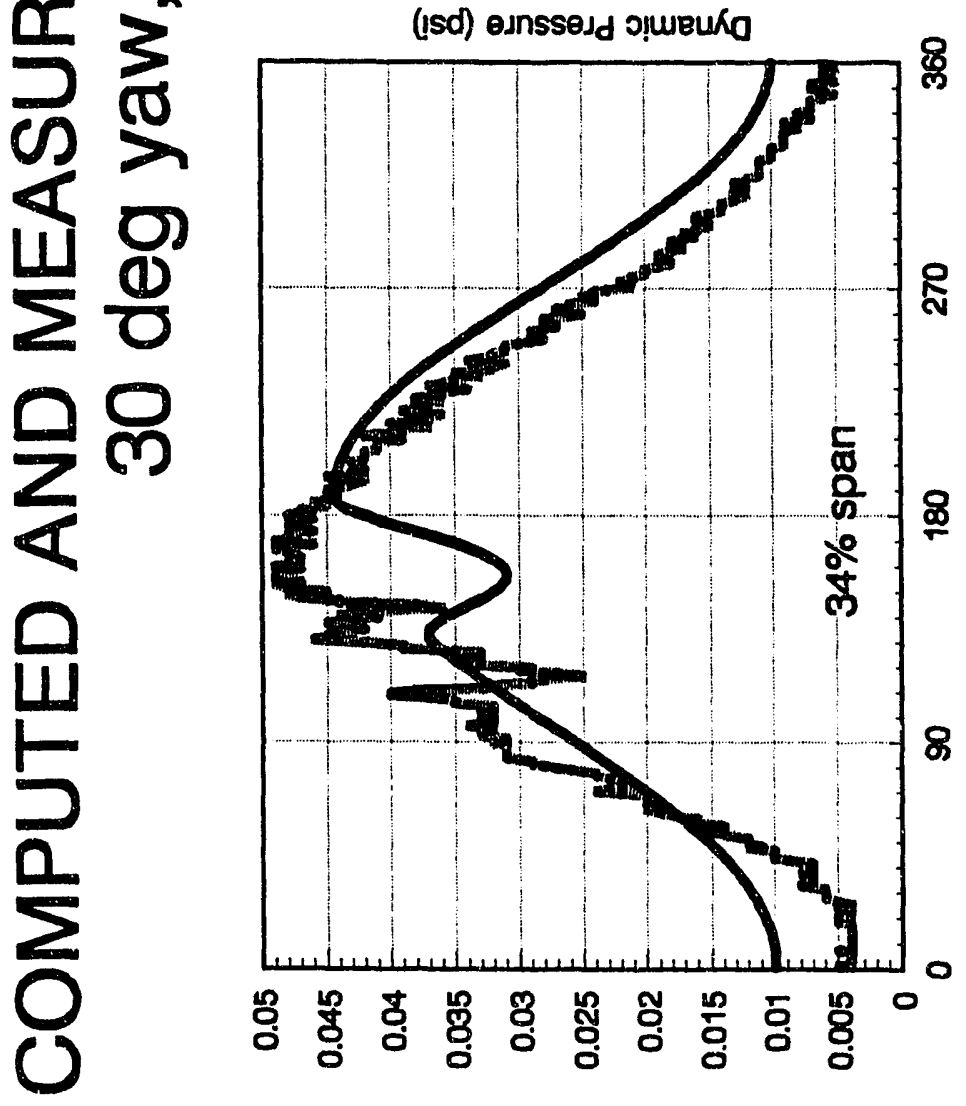

\&

ำ

8

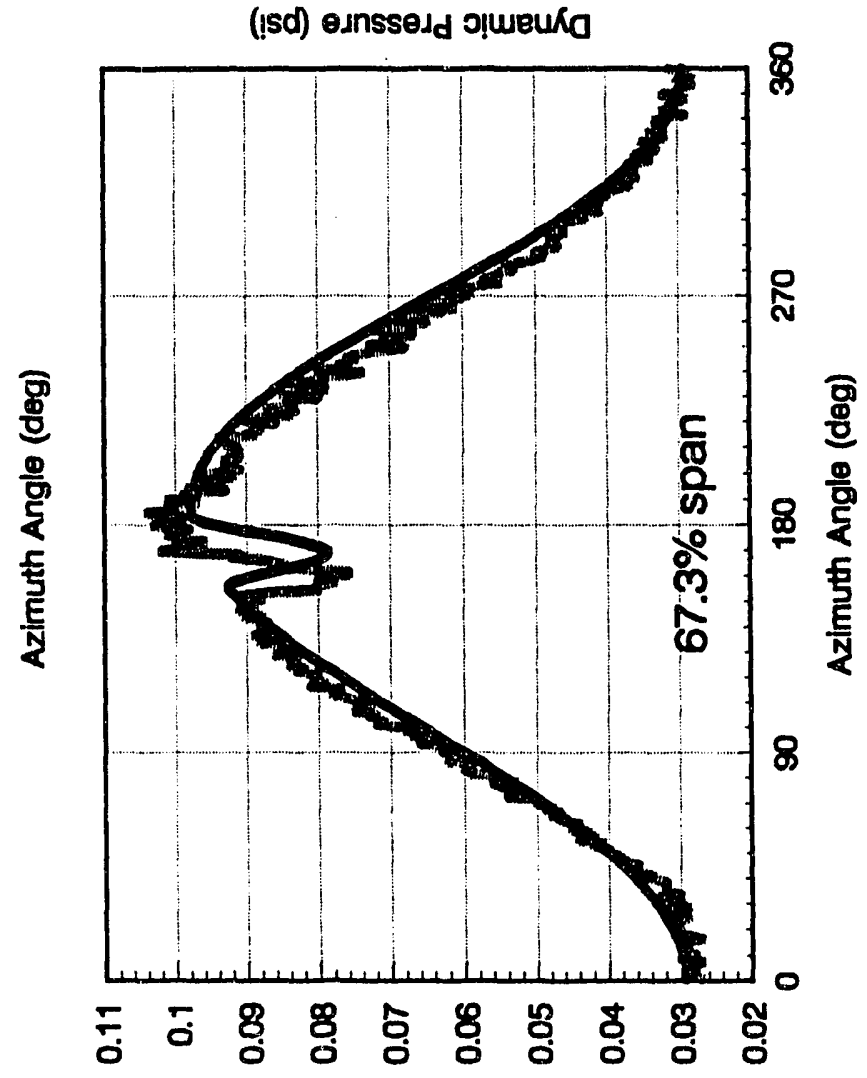

(isd) esnssedd गุursuxa 


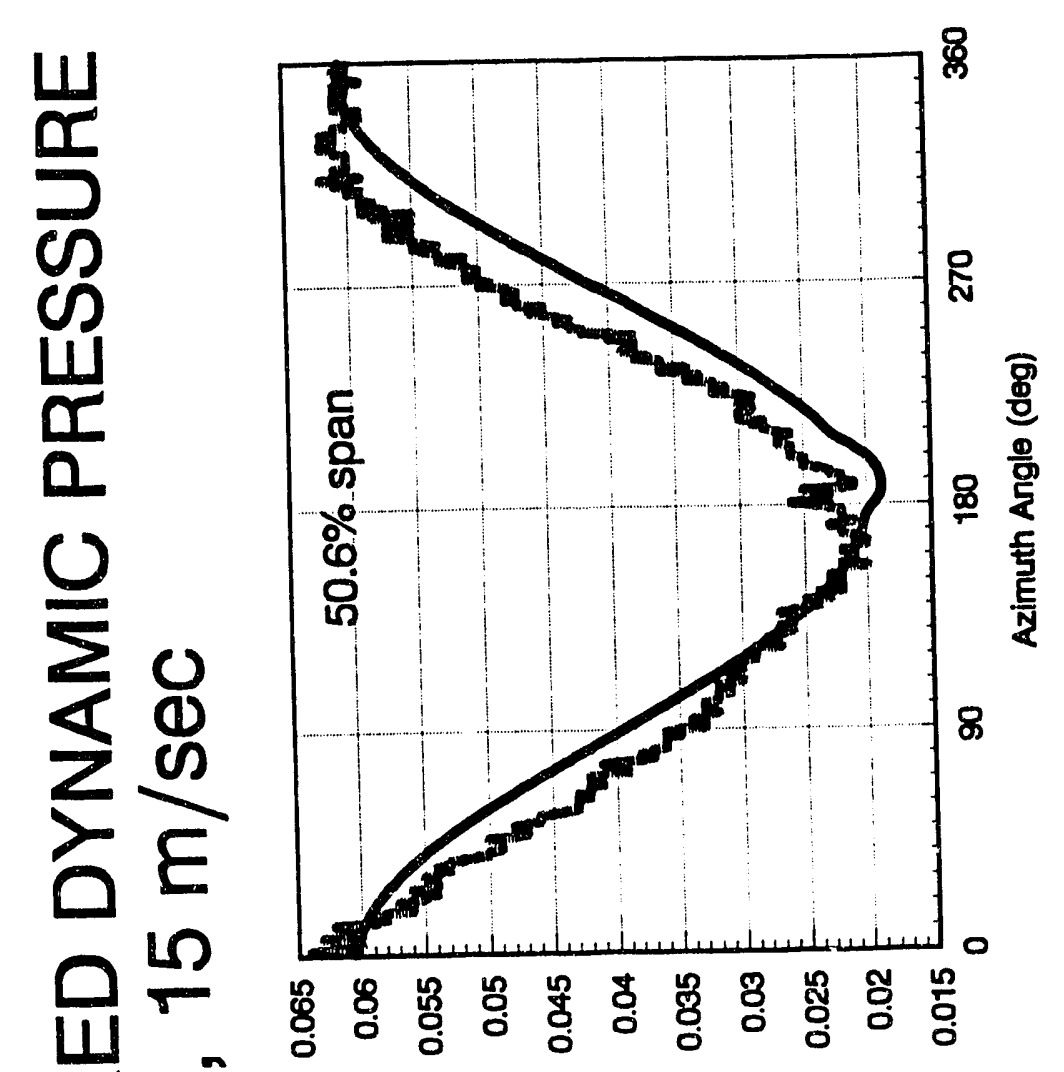

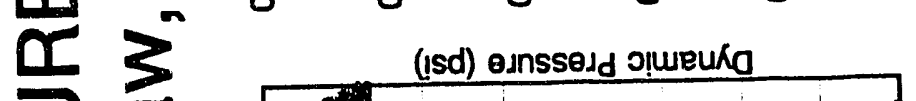

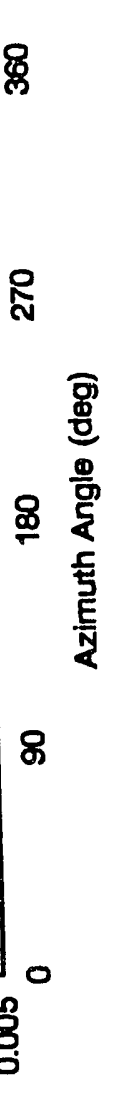

(!sc) esnssejd g̣weuka

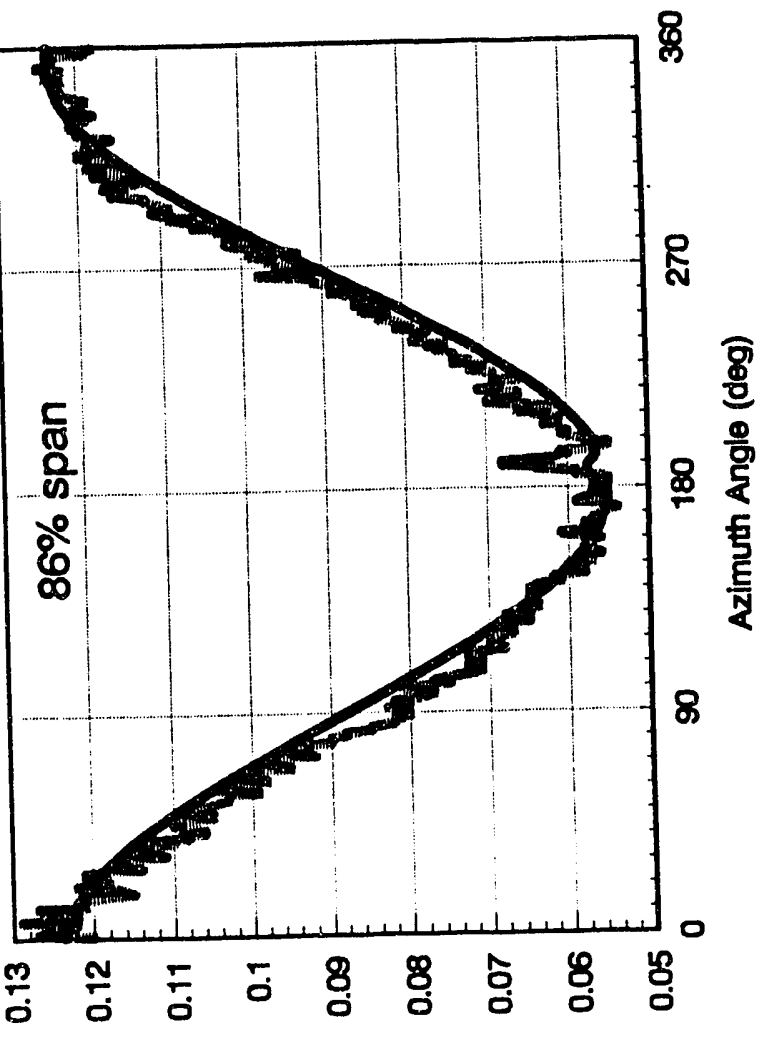

(Isd) esnssedd Oiweuka

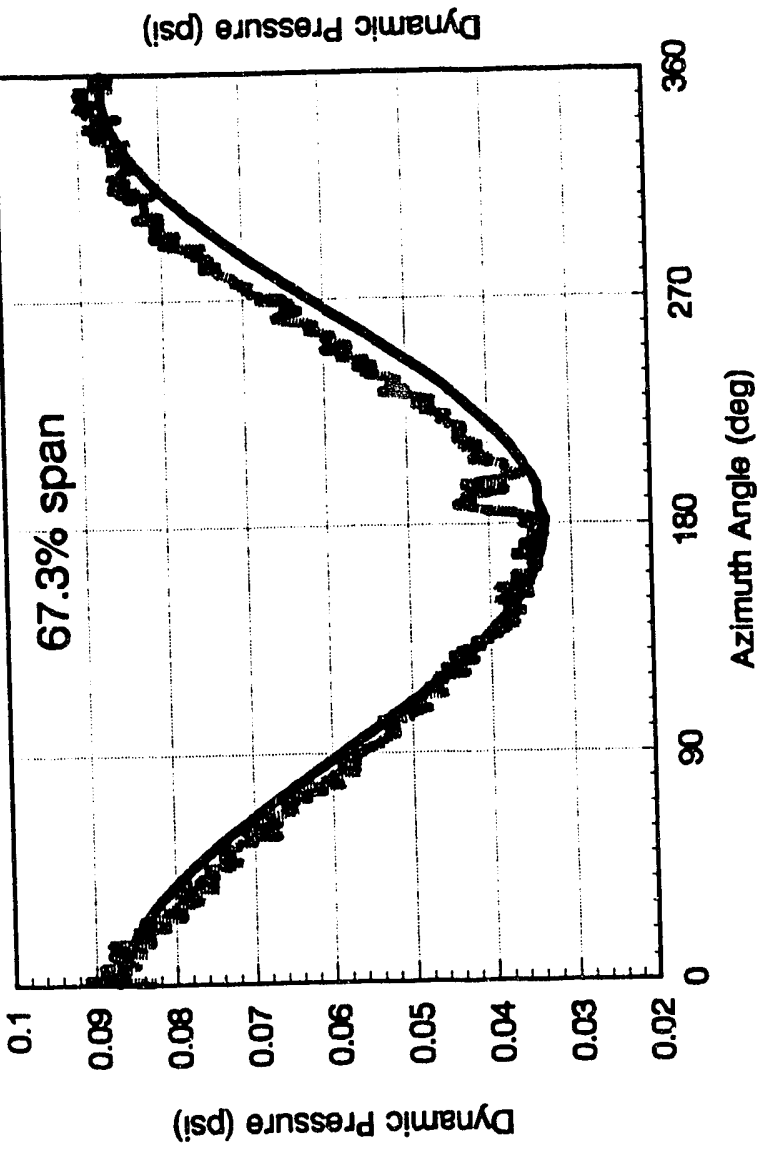




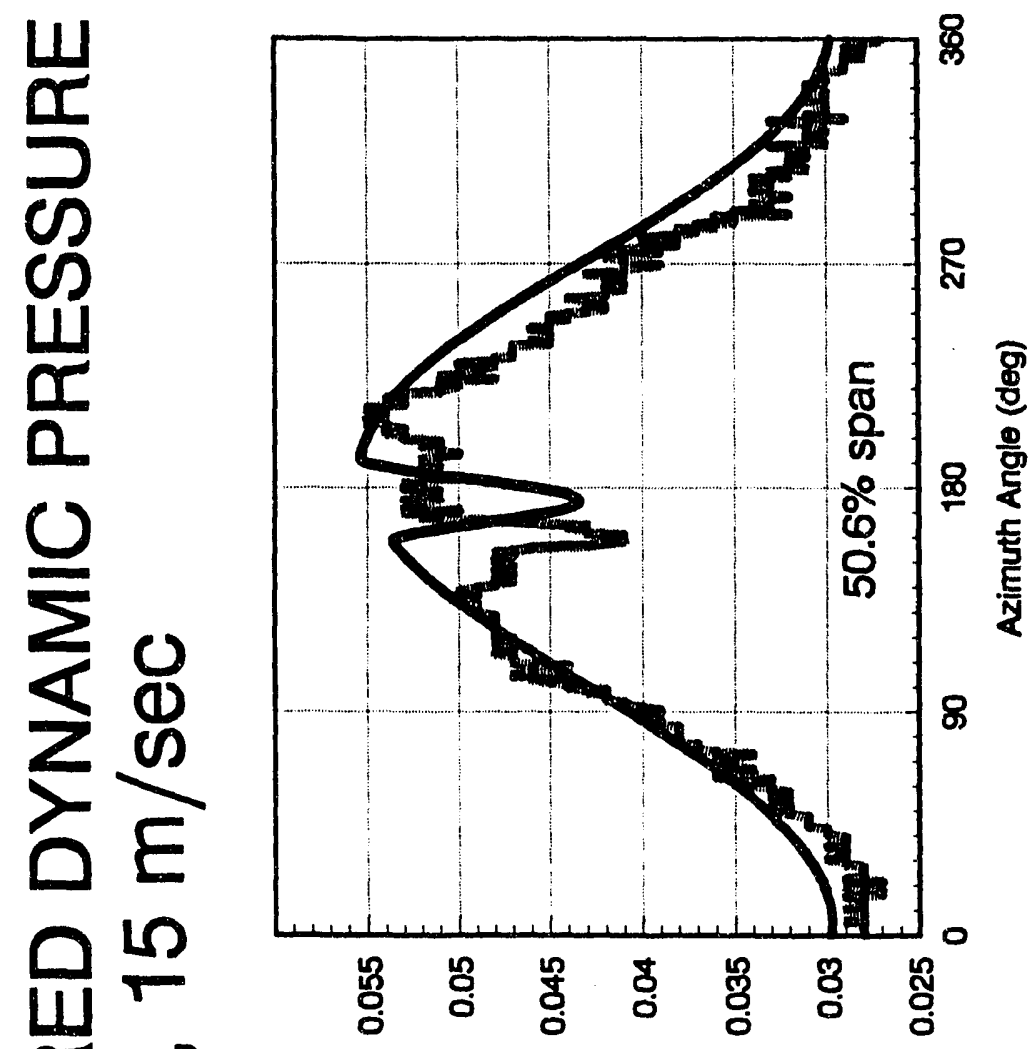

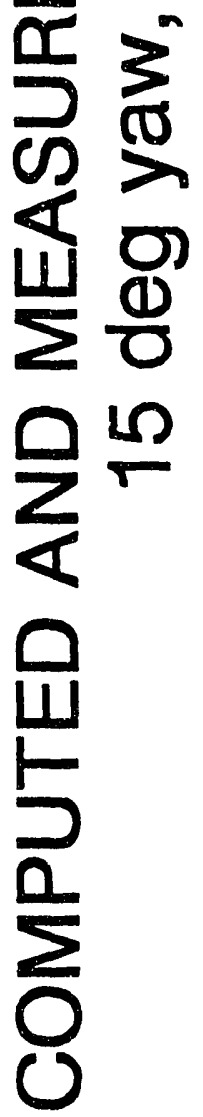

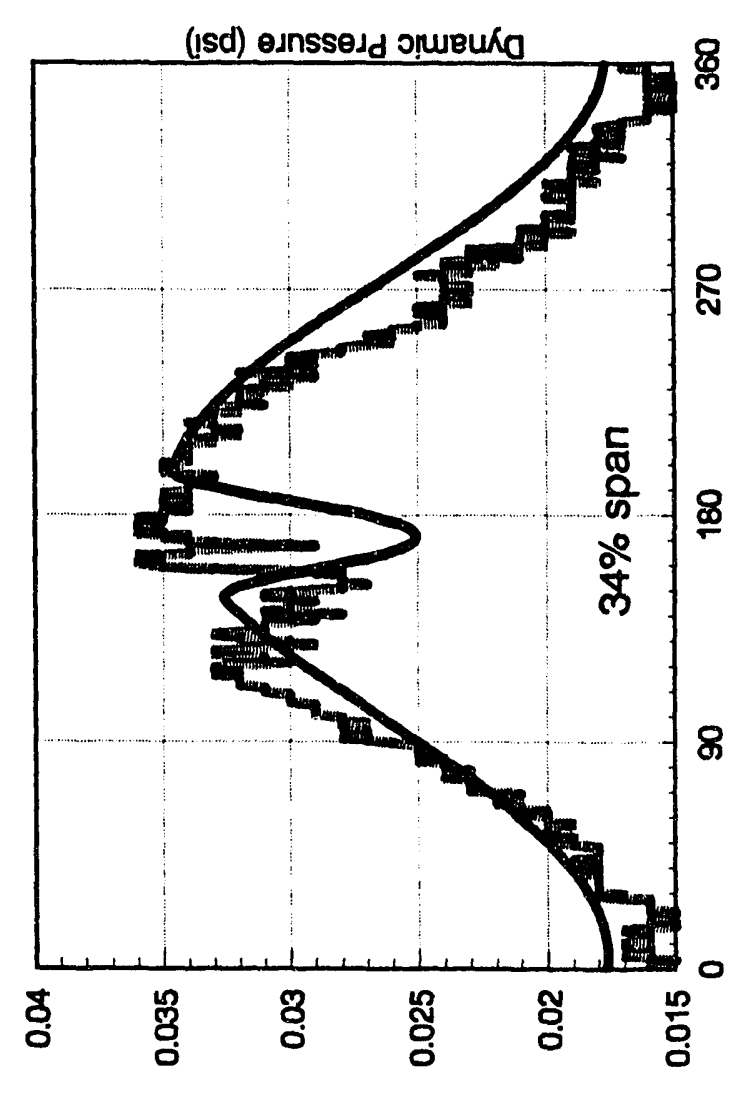

(Isd) ednssedd Ọயruka

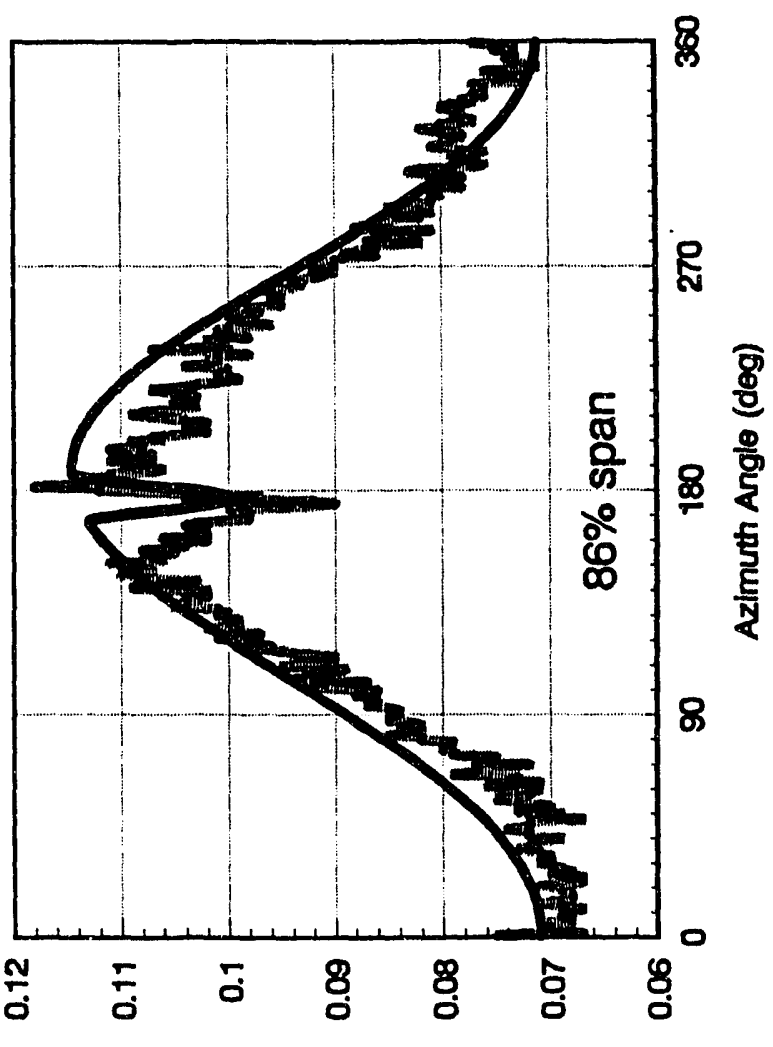

(!sd) ejnssedd ग!weuka

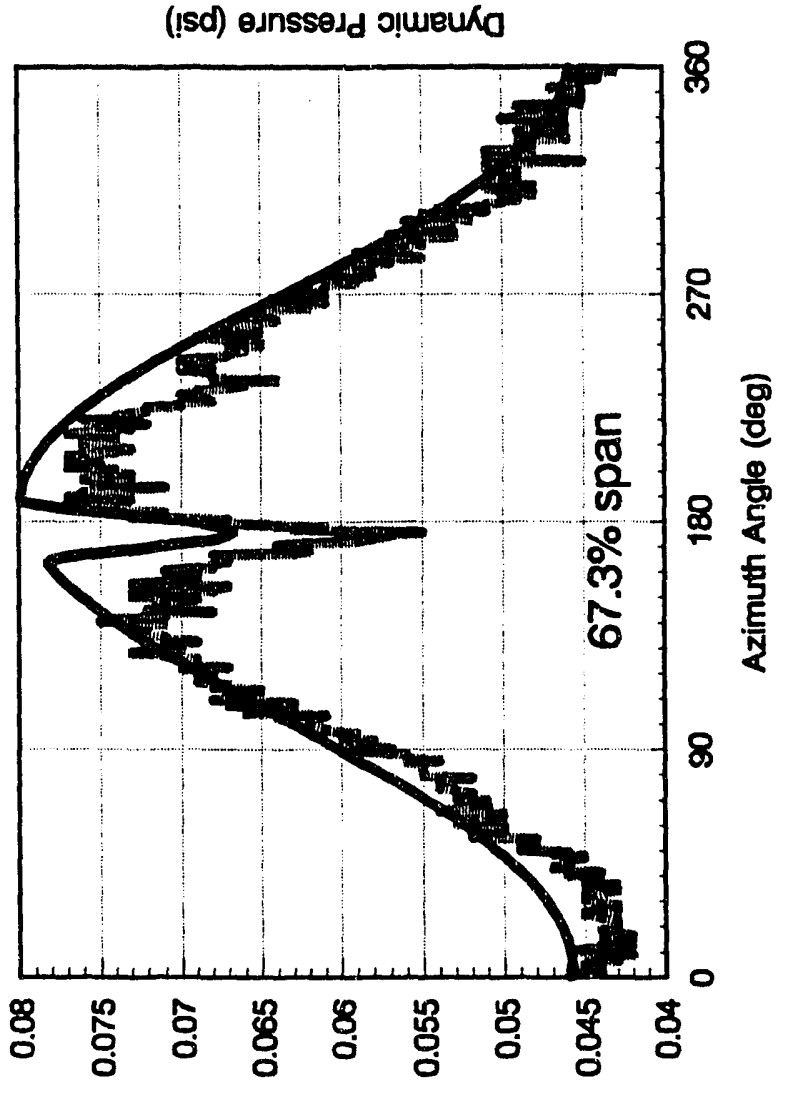

(isd) ejnssedd ọwreuka 

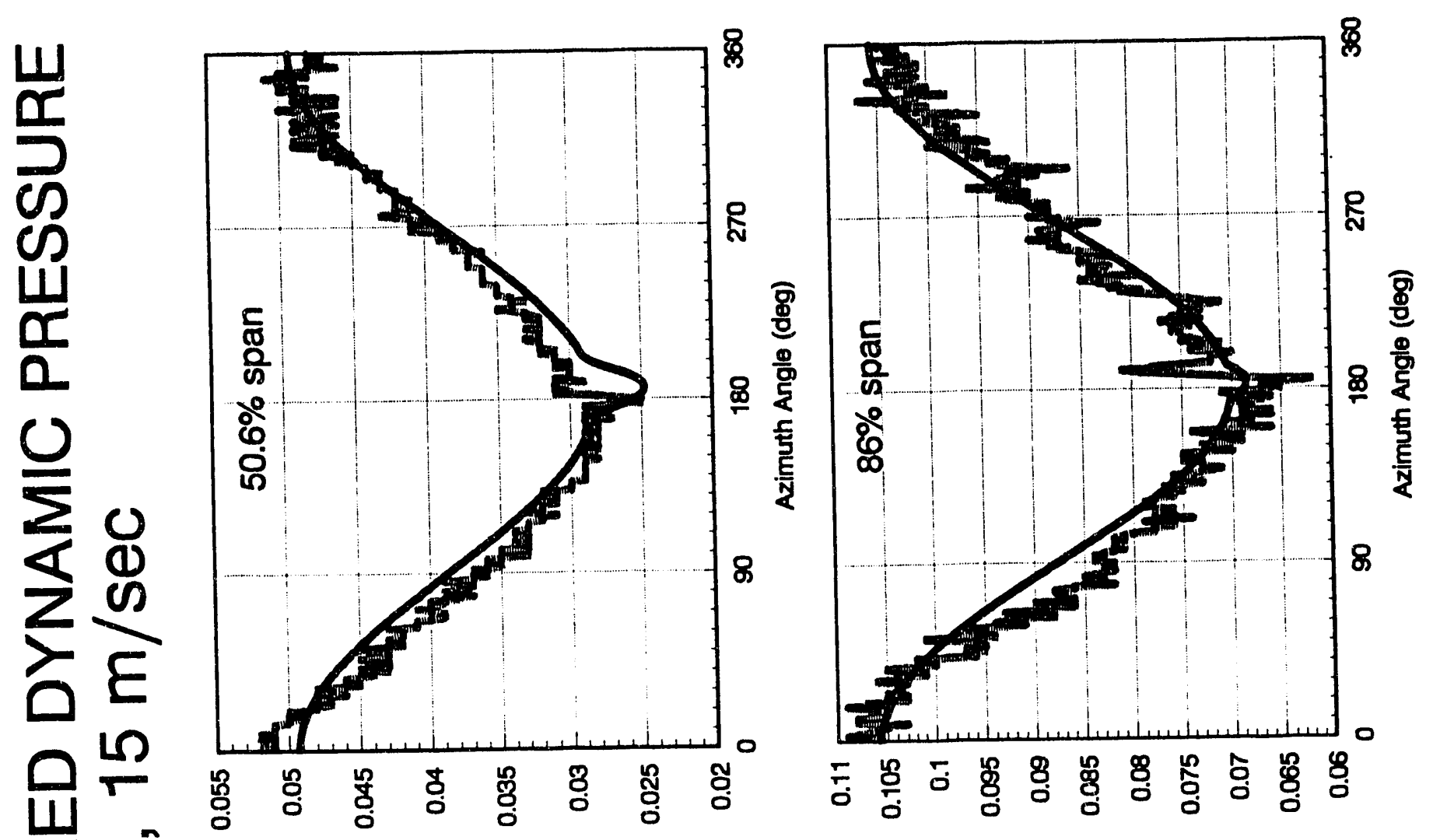

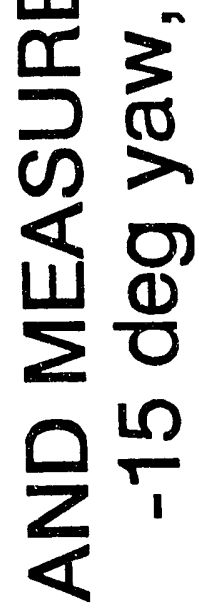

邑
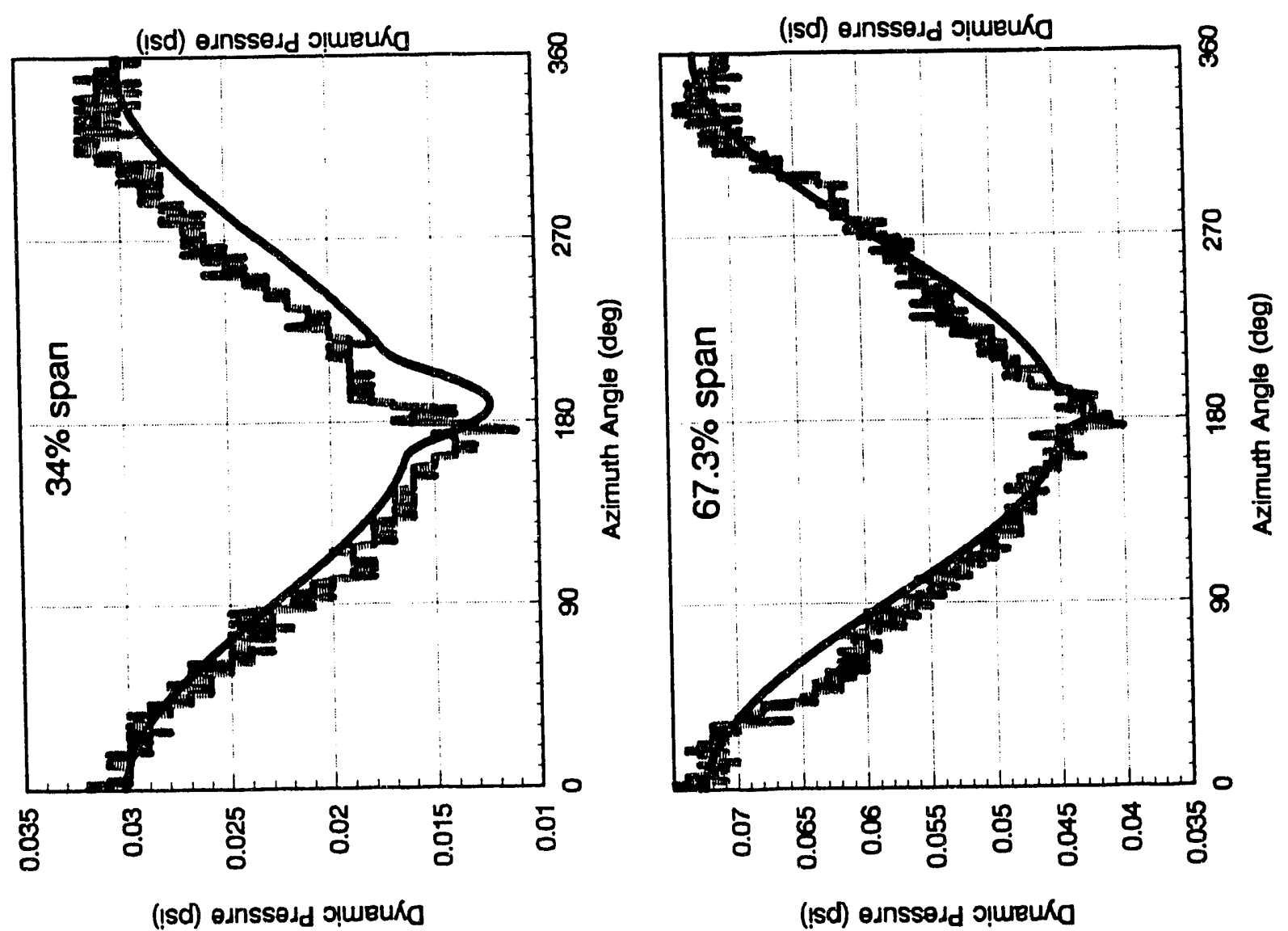


\section{Appendix C}

\section{Zero Yaw Test Cases, Cycle Averaged}




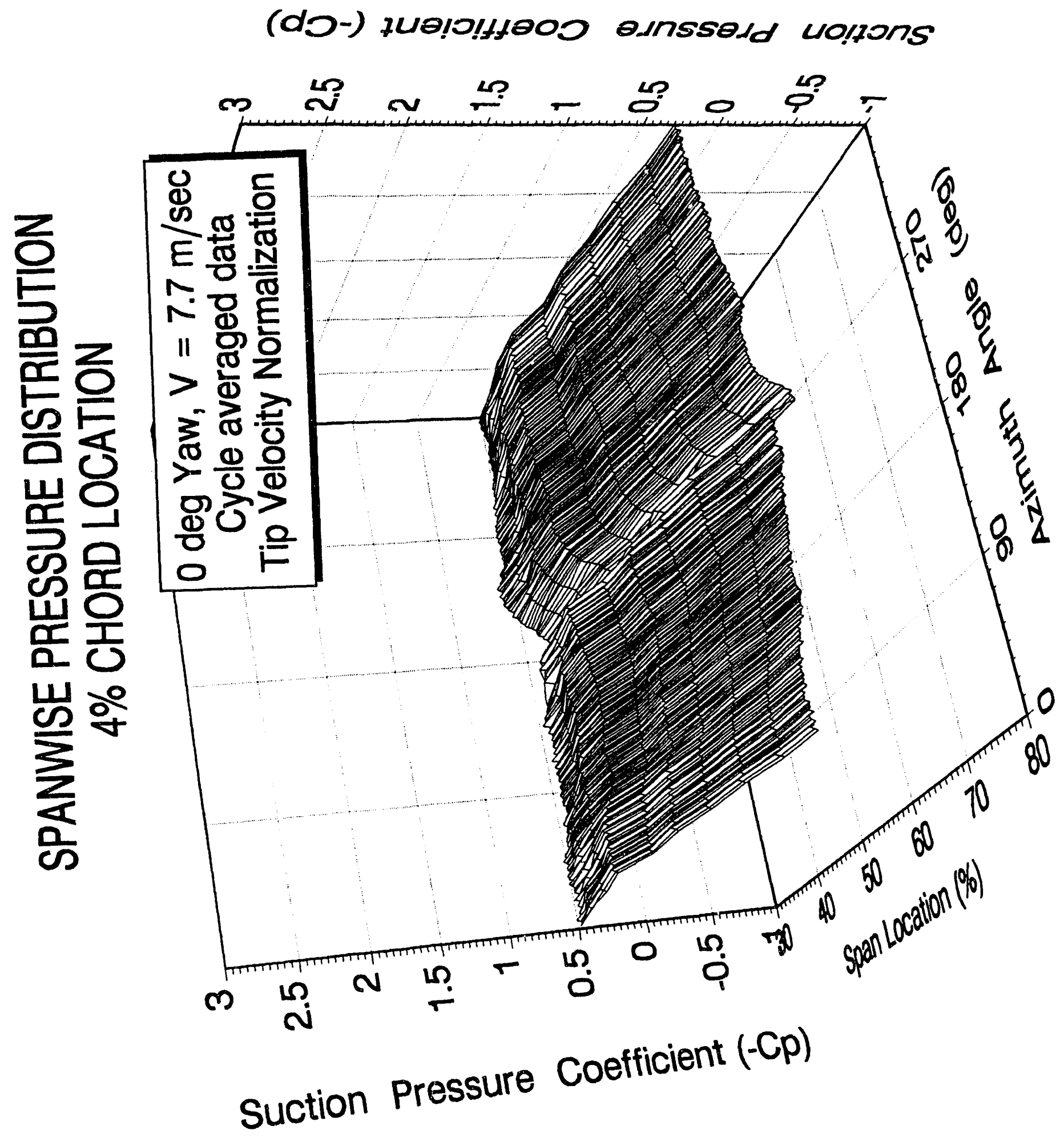




\section{(do-) zublolffeos esnssedd wolfons}

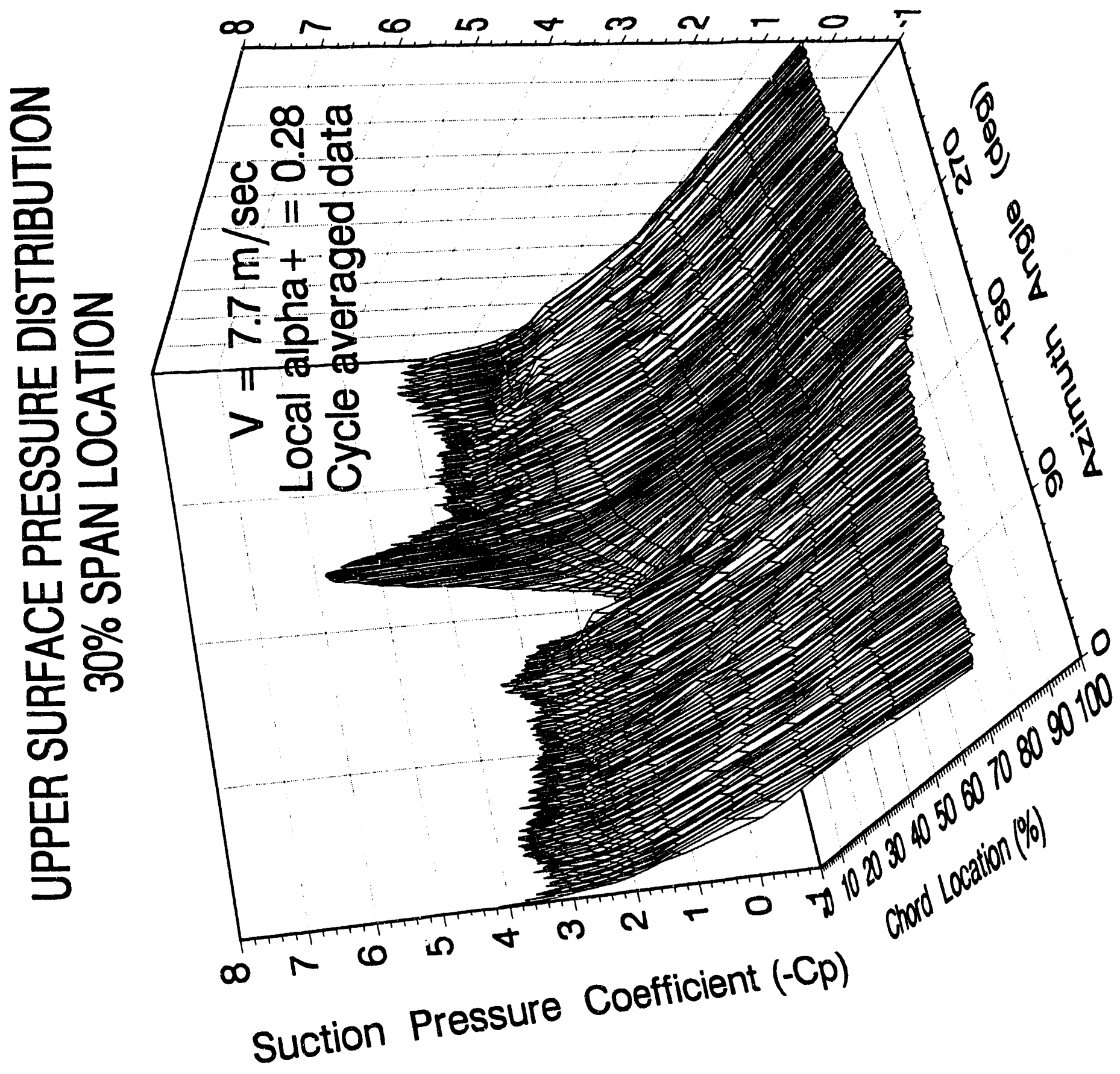




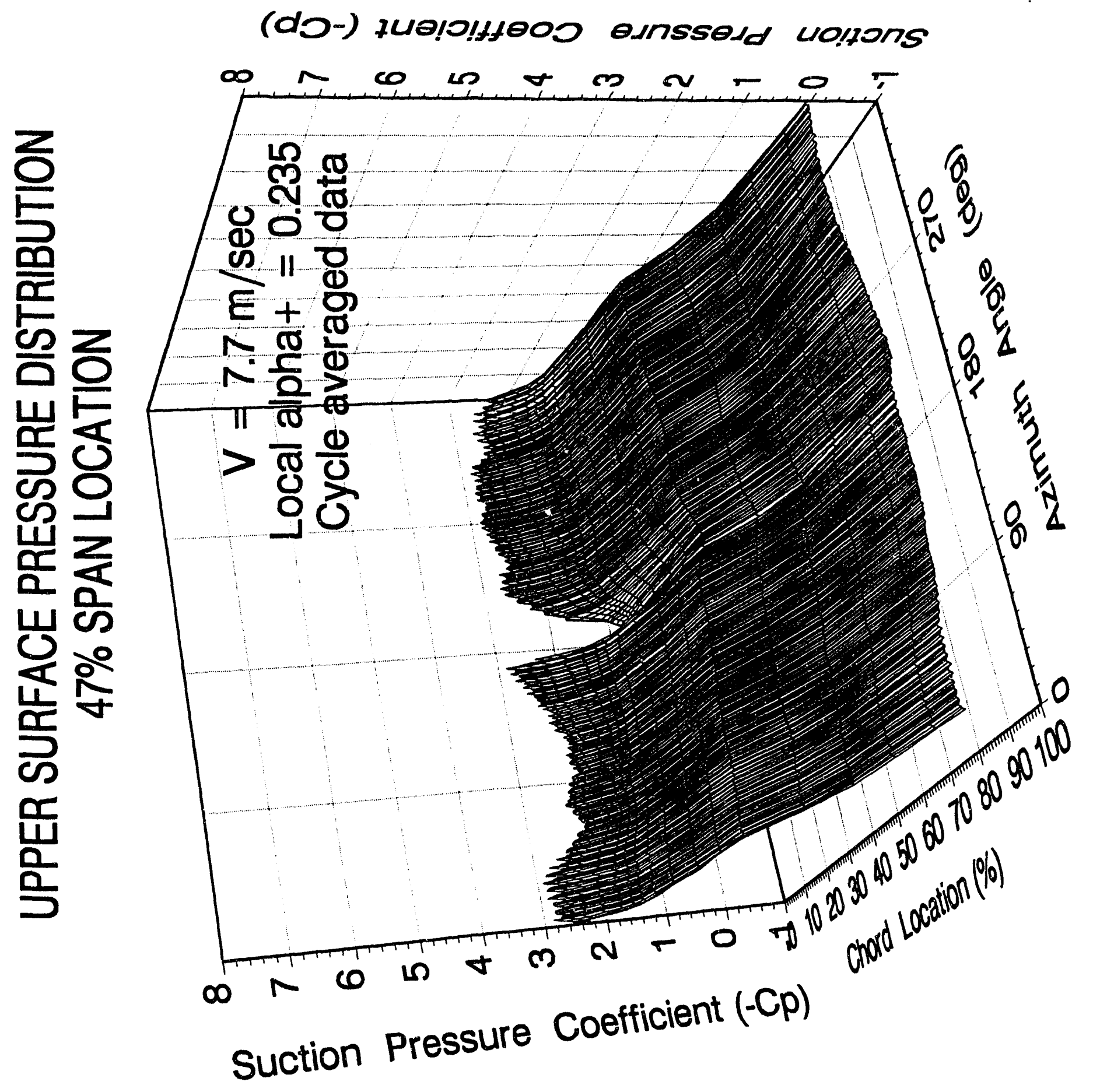




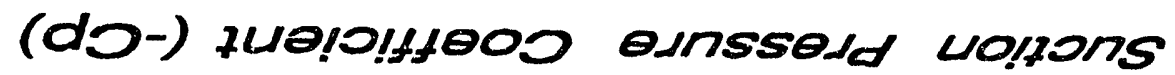

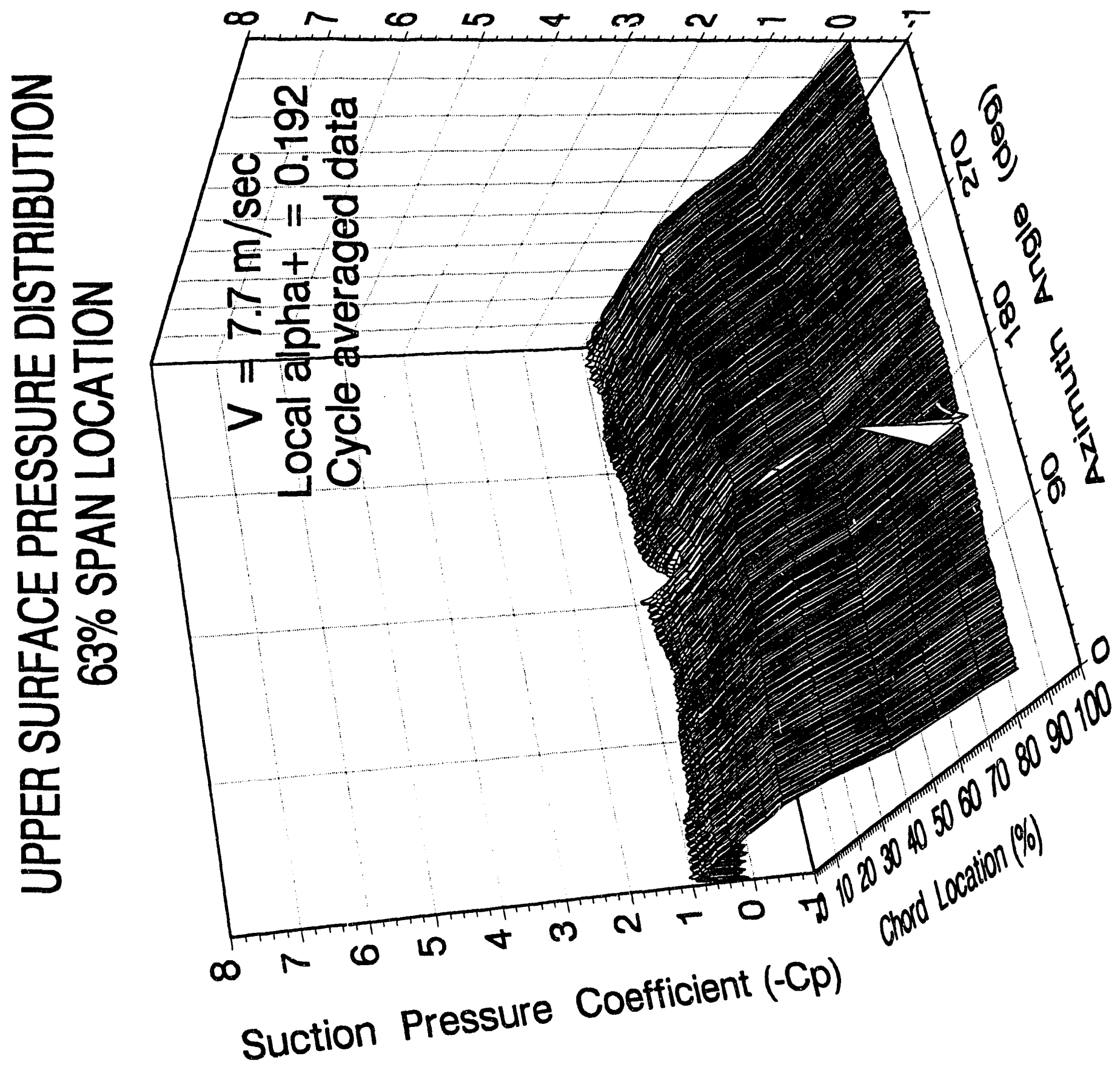




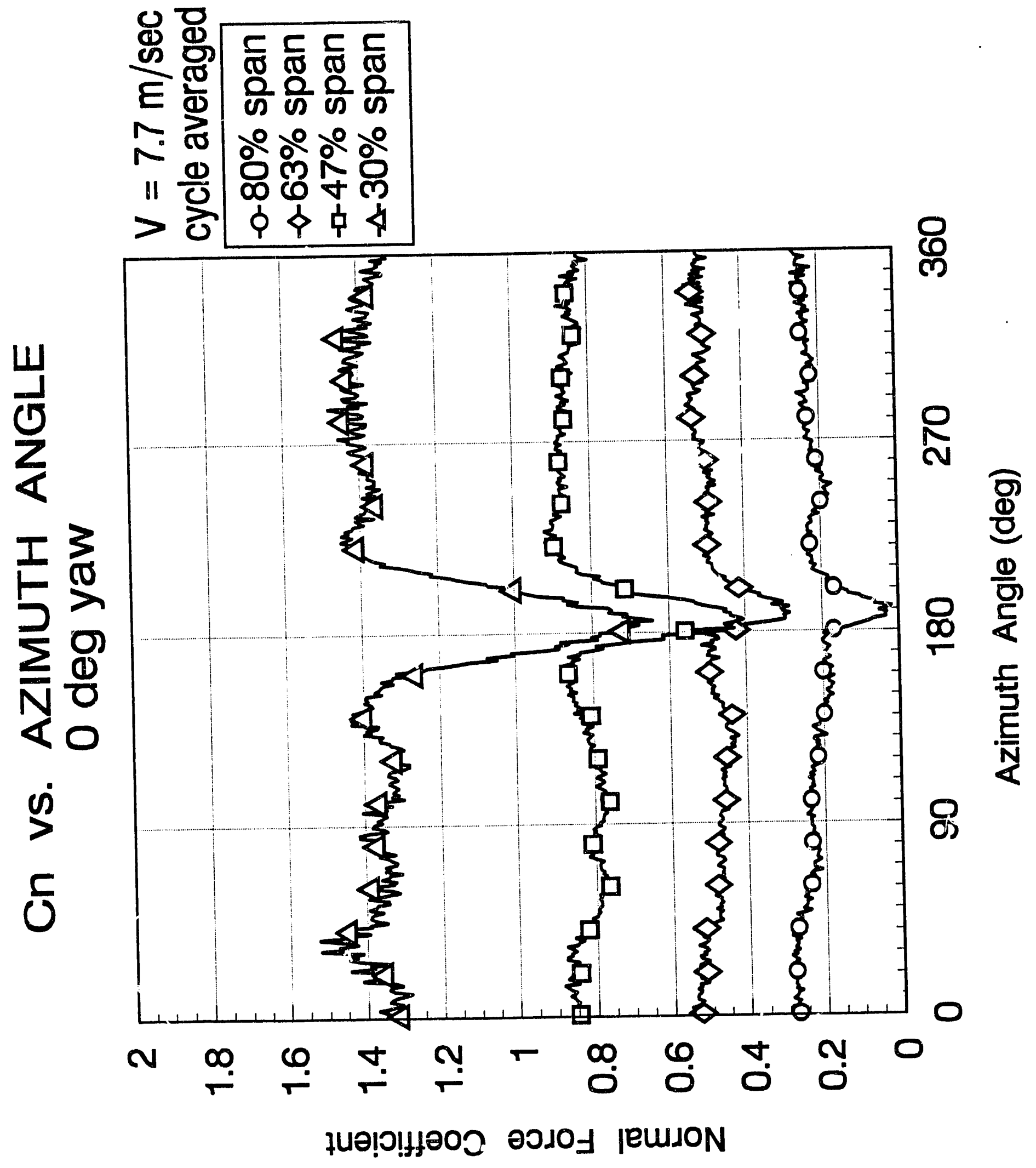




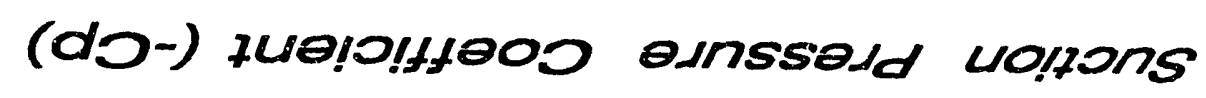

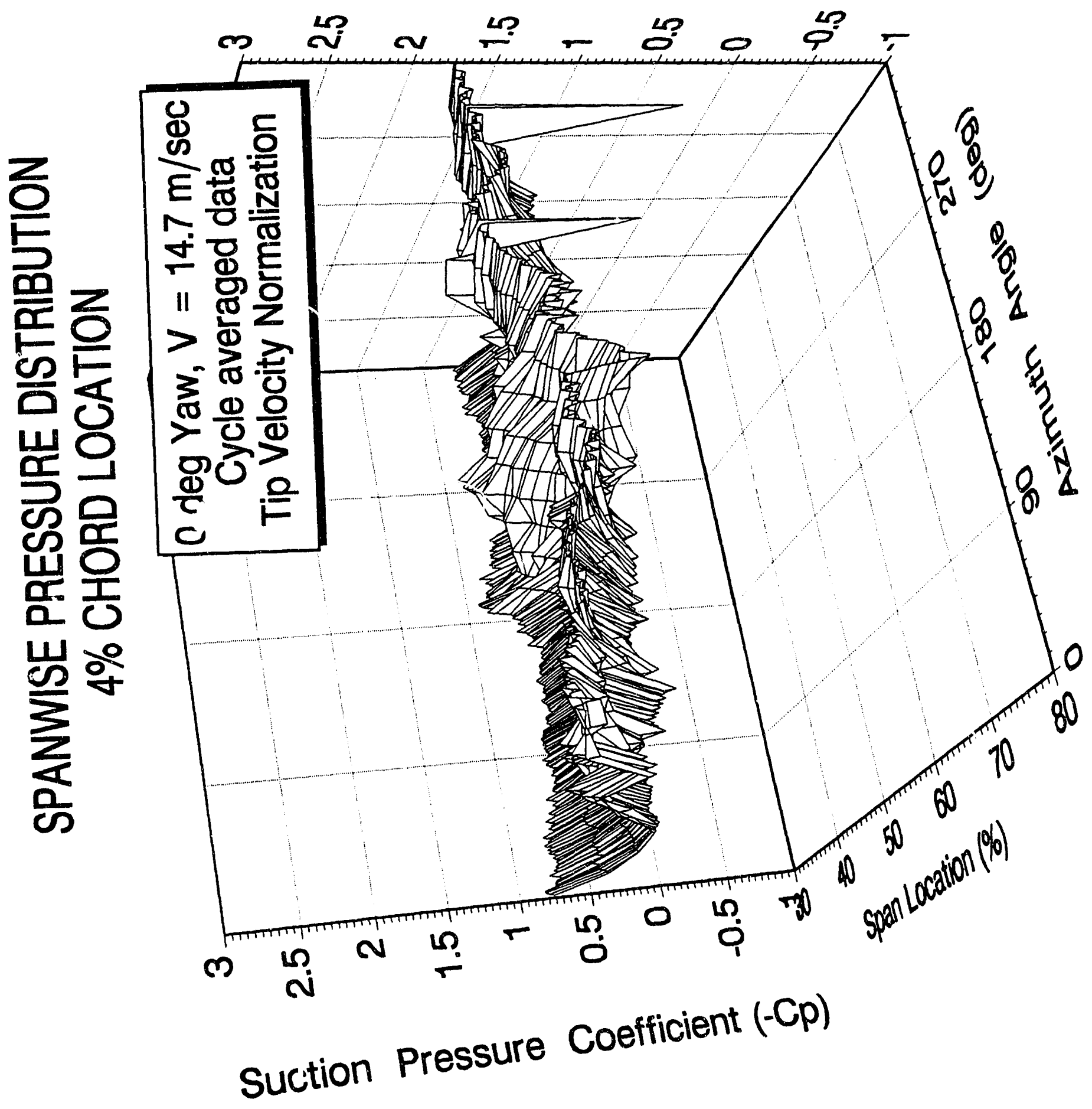




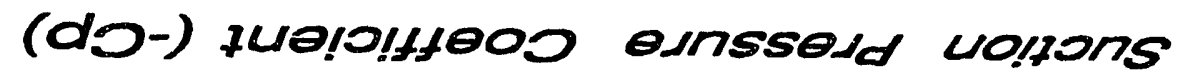

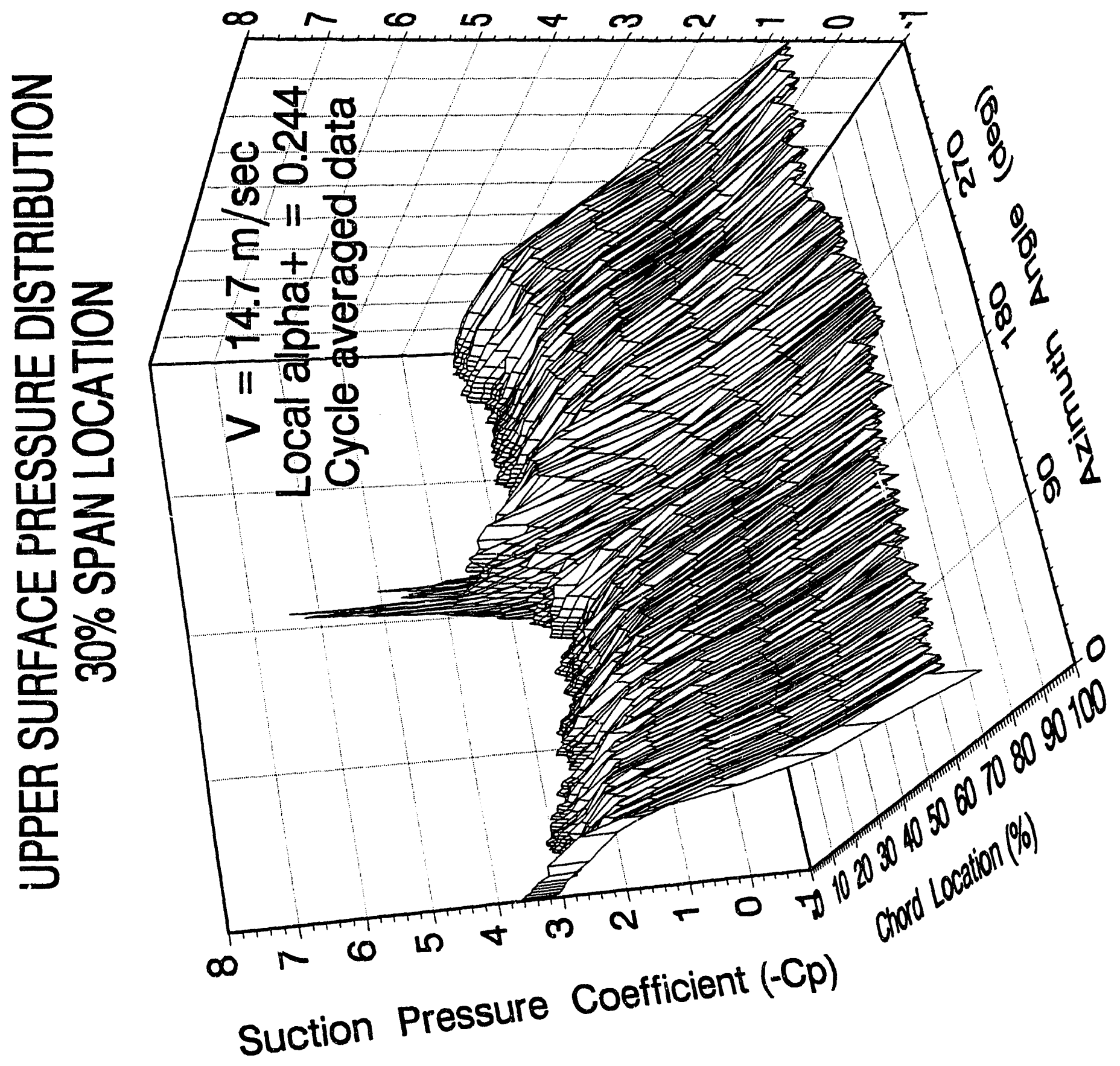




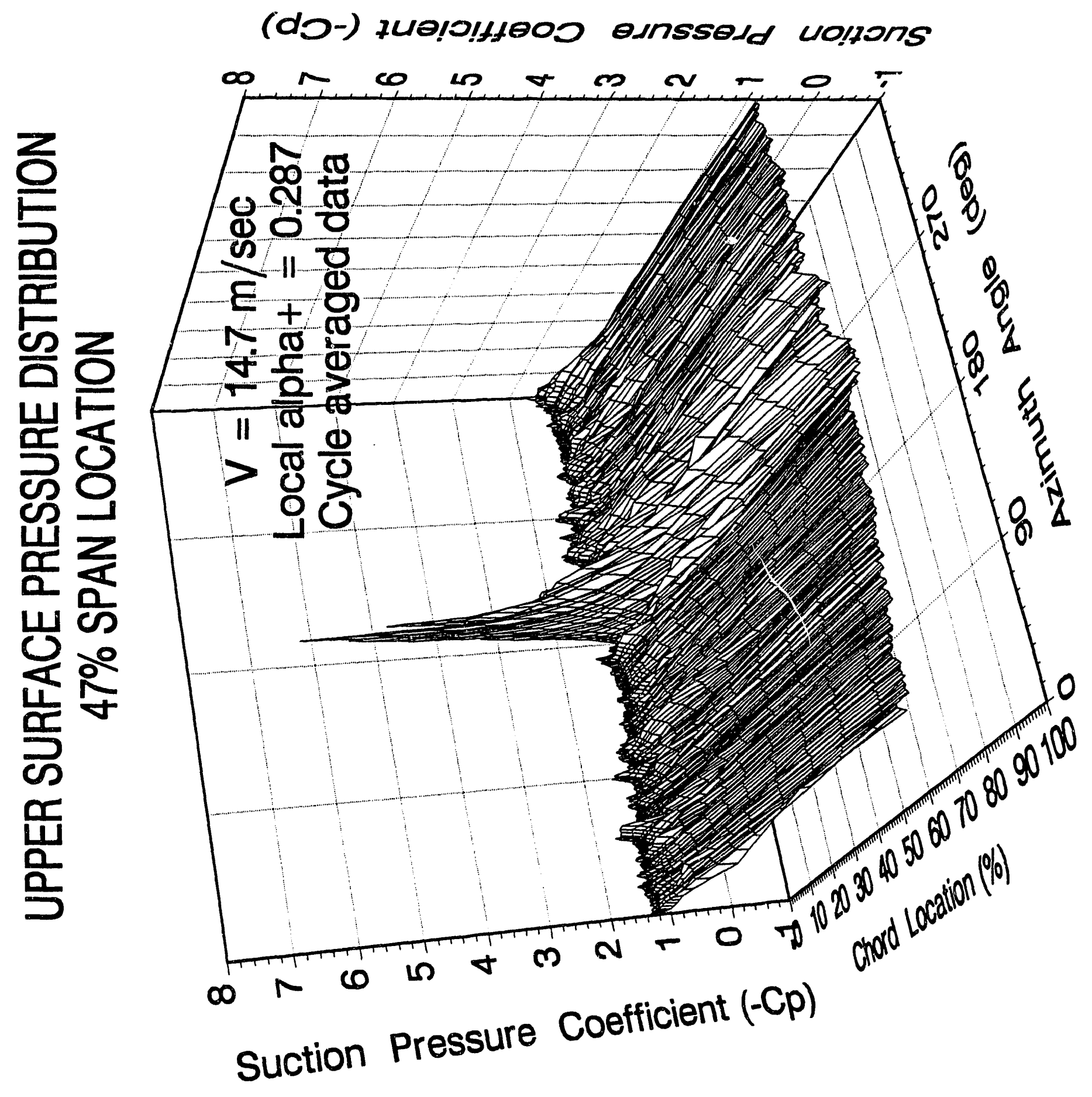




\section{(d)-) 7uolo!fle00 exnssedd wolpons}

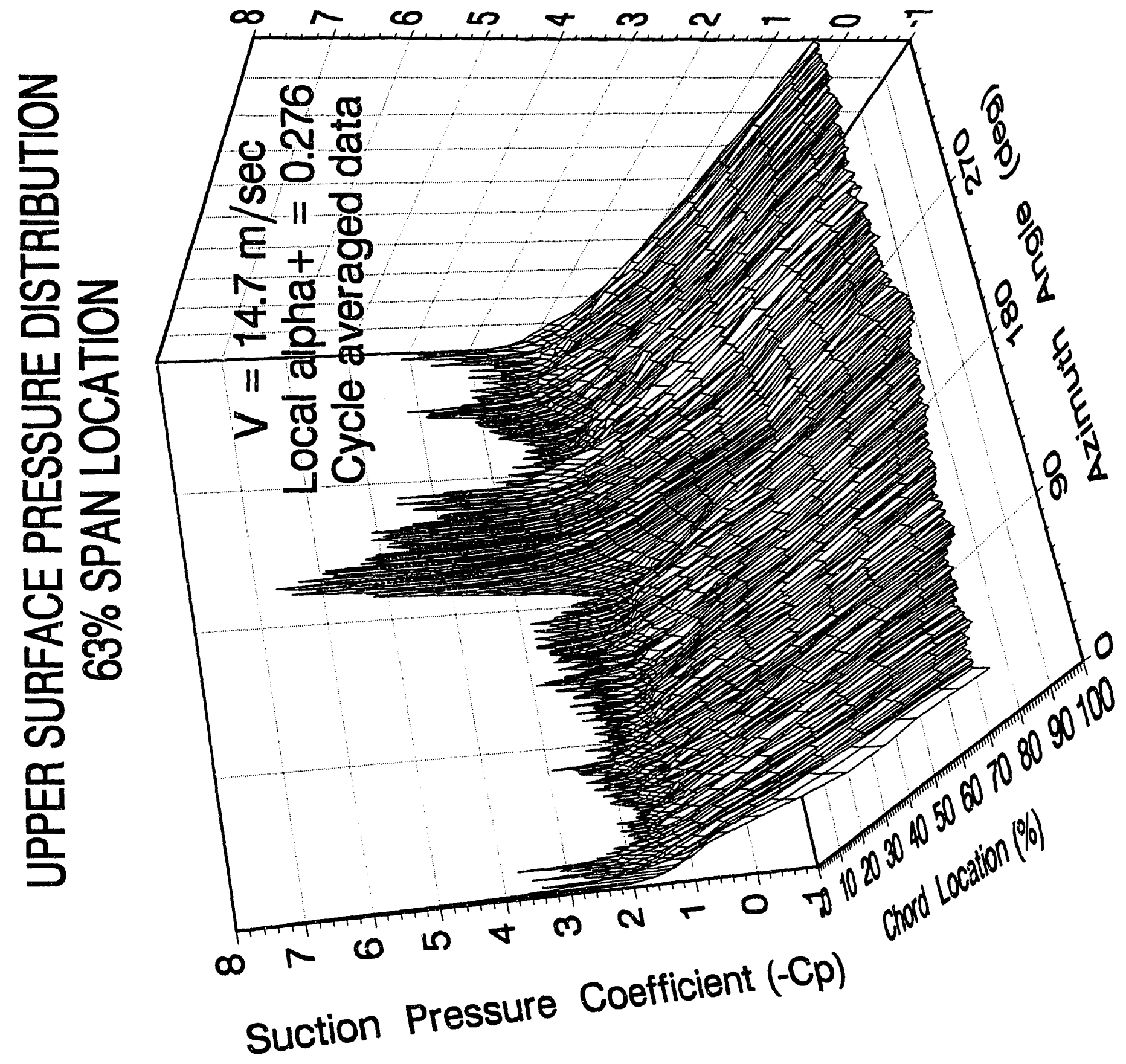


(dD-) 7w0!o!fle00 ejnsseld wolfons

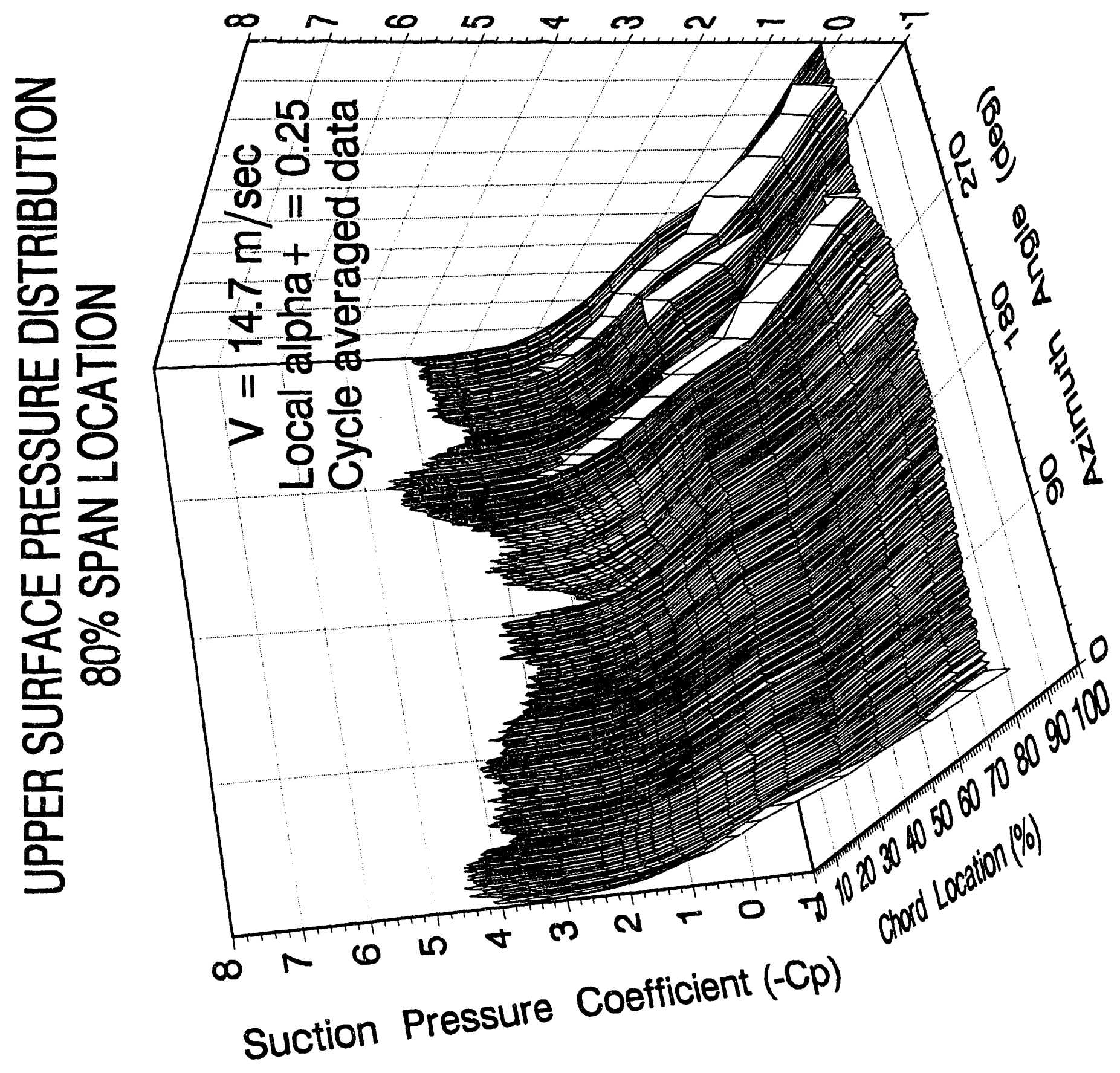




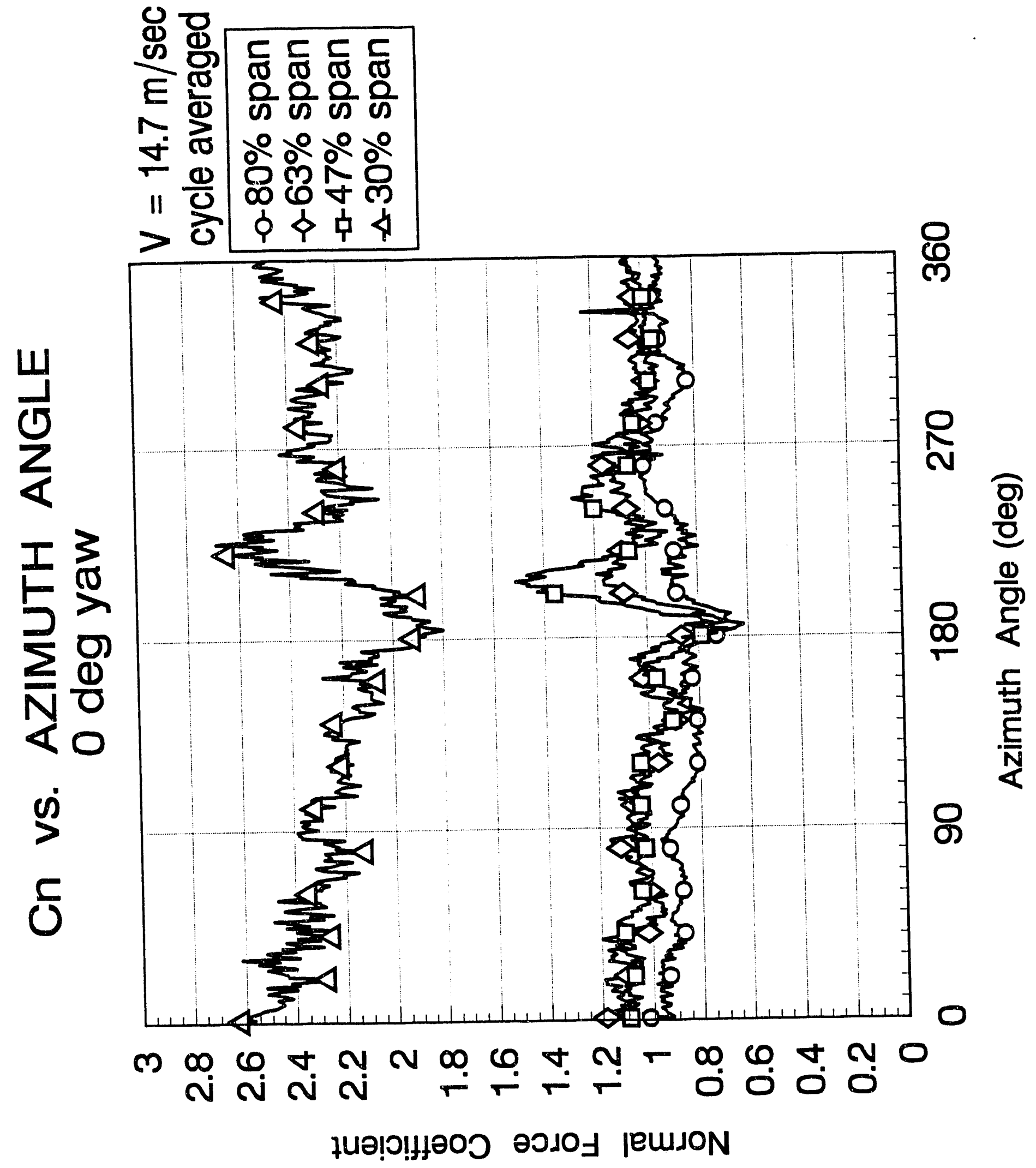


(d)-) zualo!ffe00 asnssald wolpons

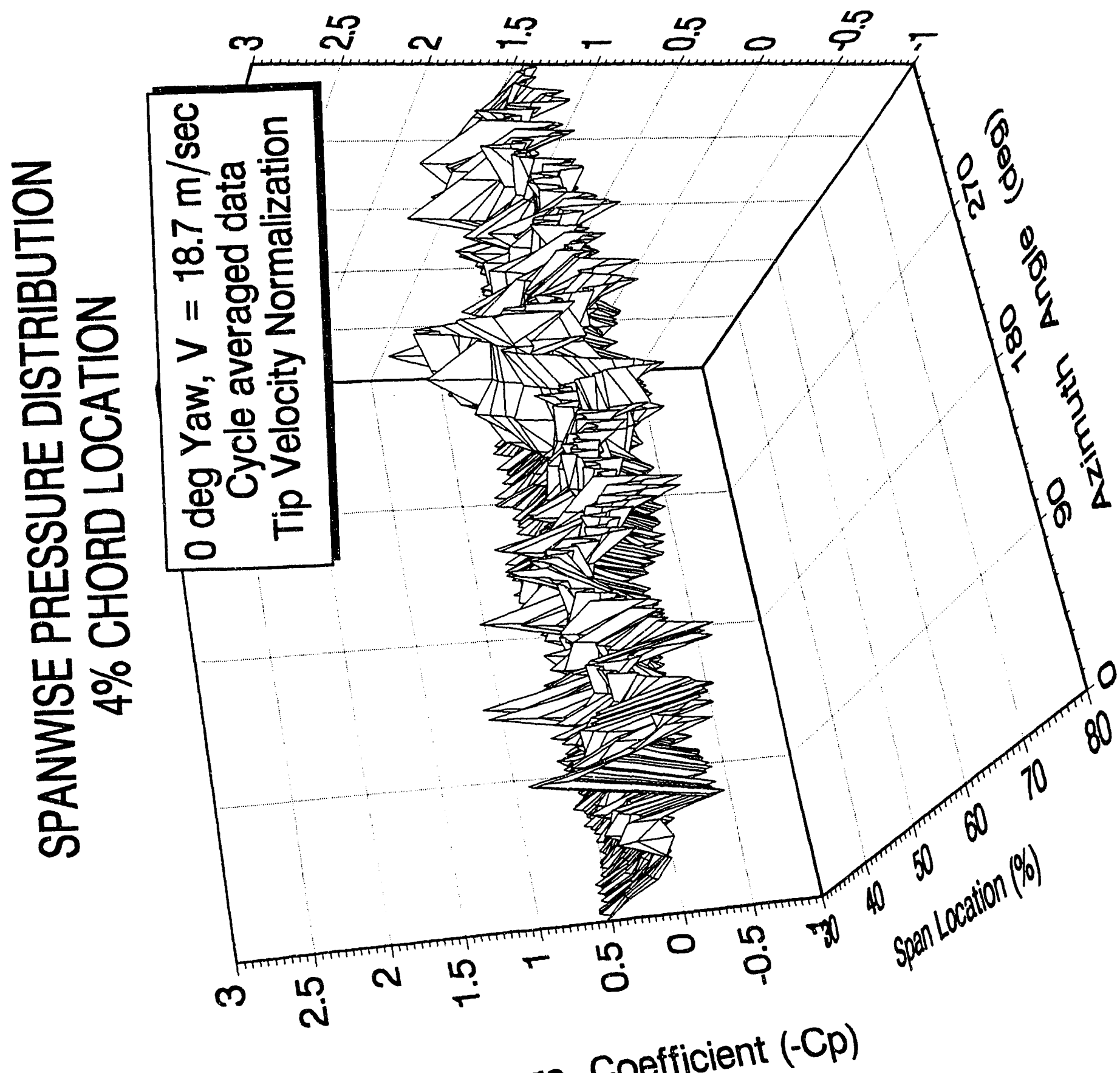

Suction Pressure Coefficient (-Cp) 


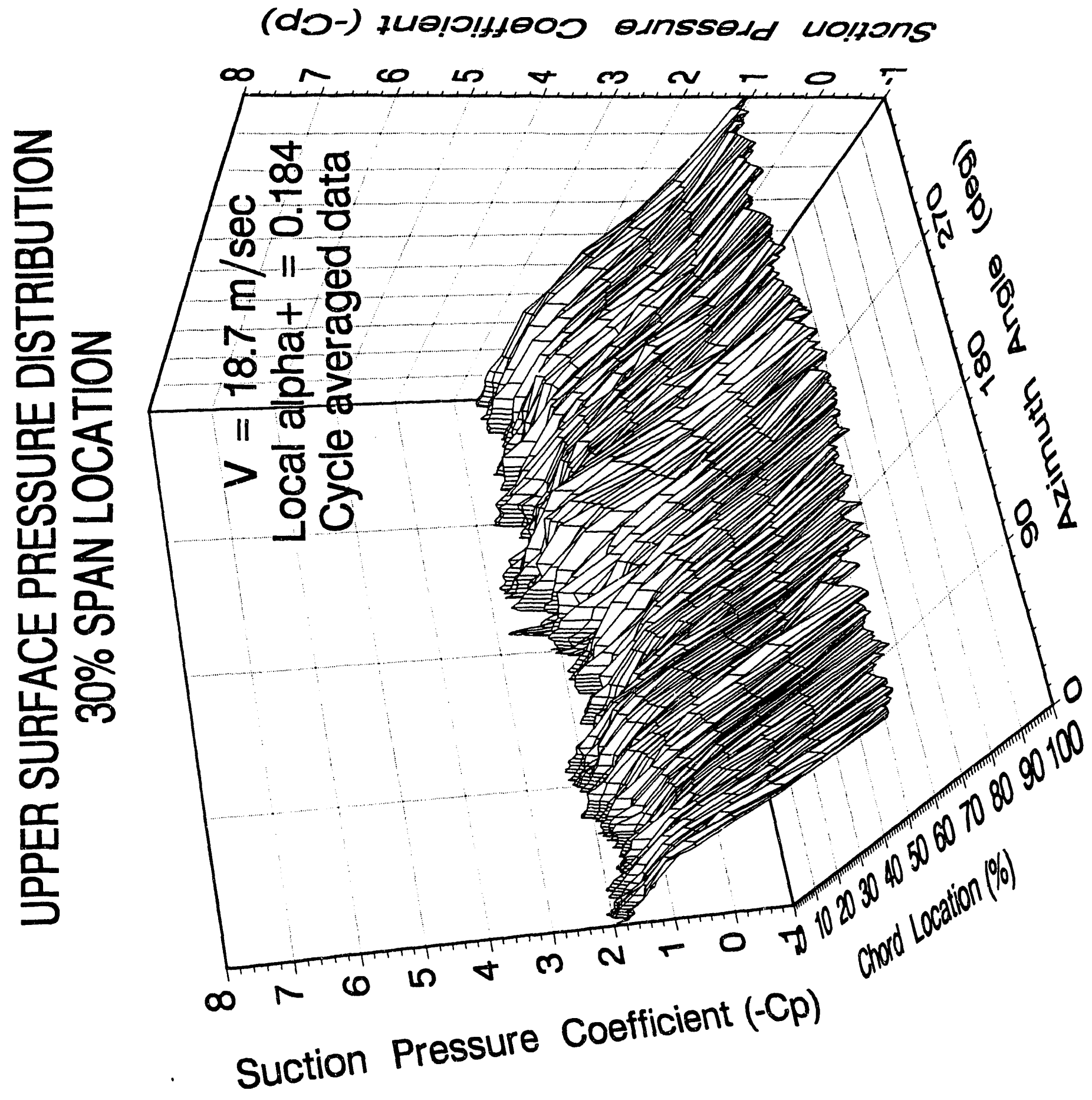




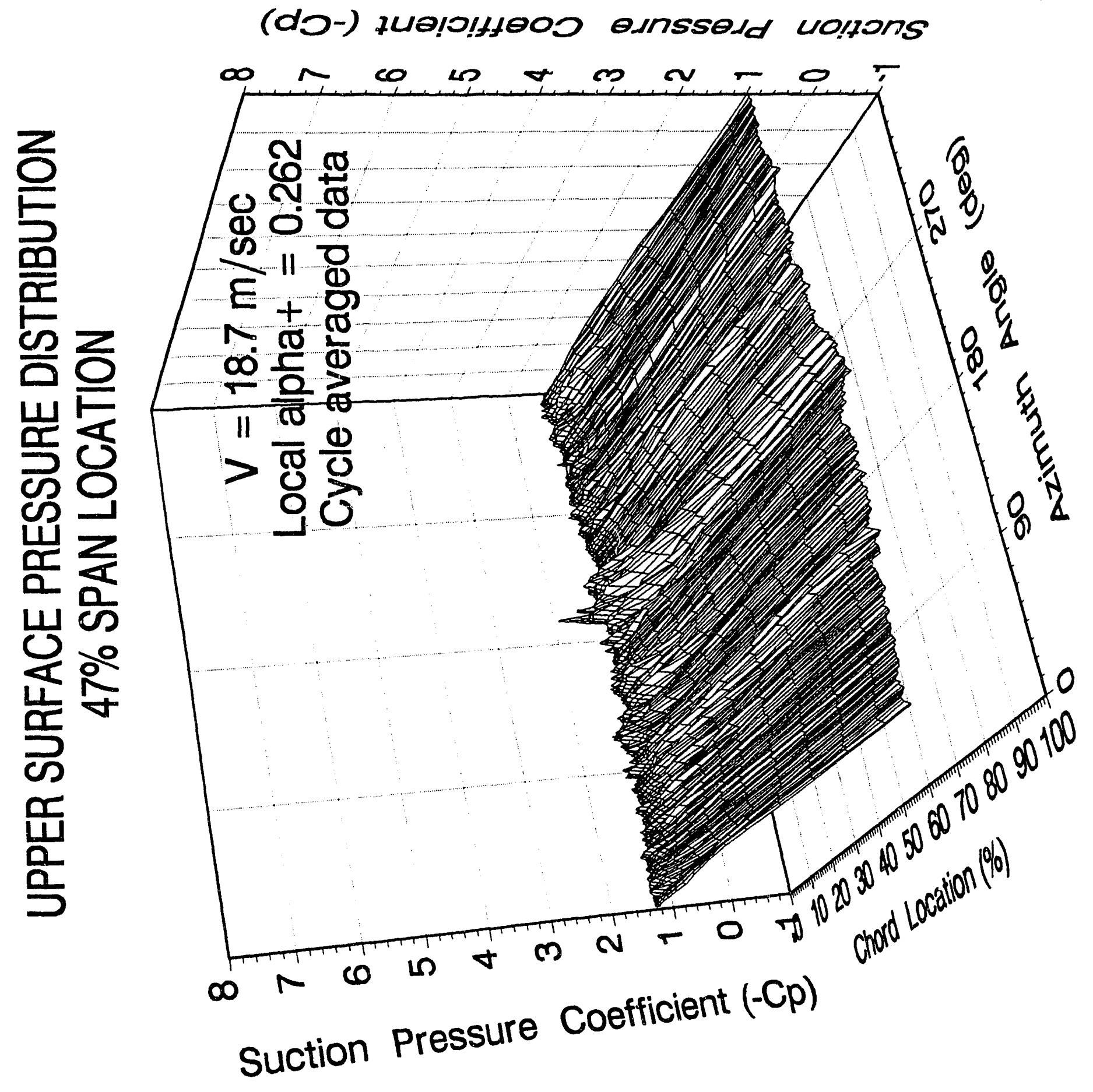




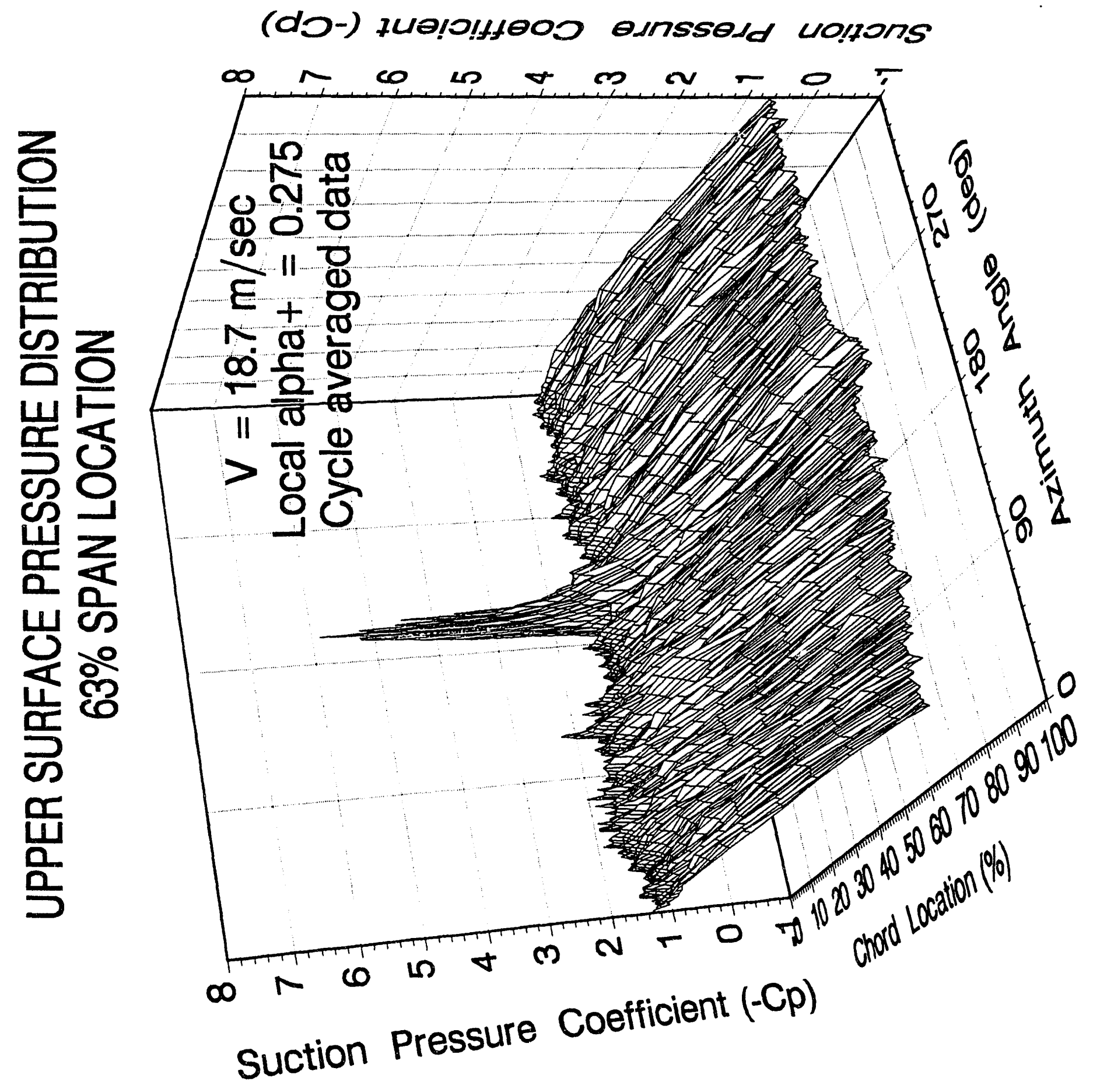




\section{(d)-) 7w0!o!ffe00 esnssedd wolfons}

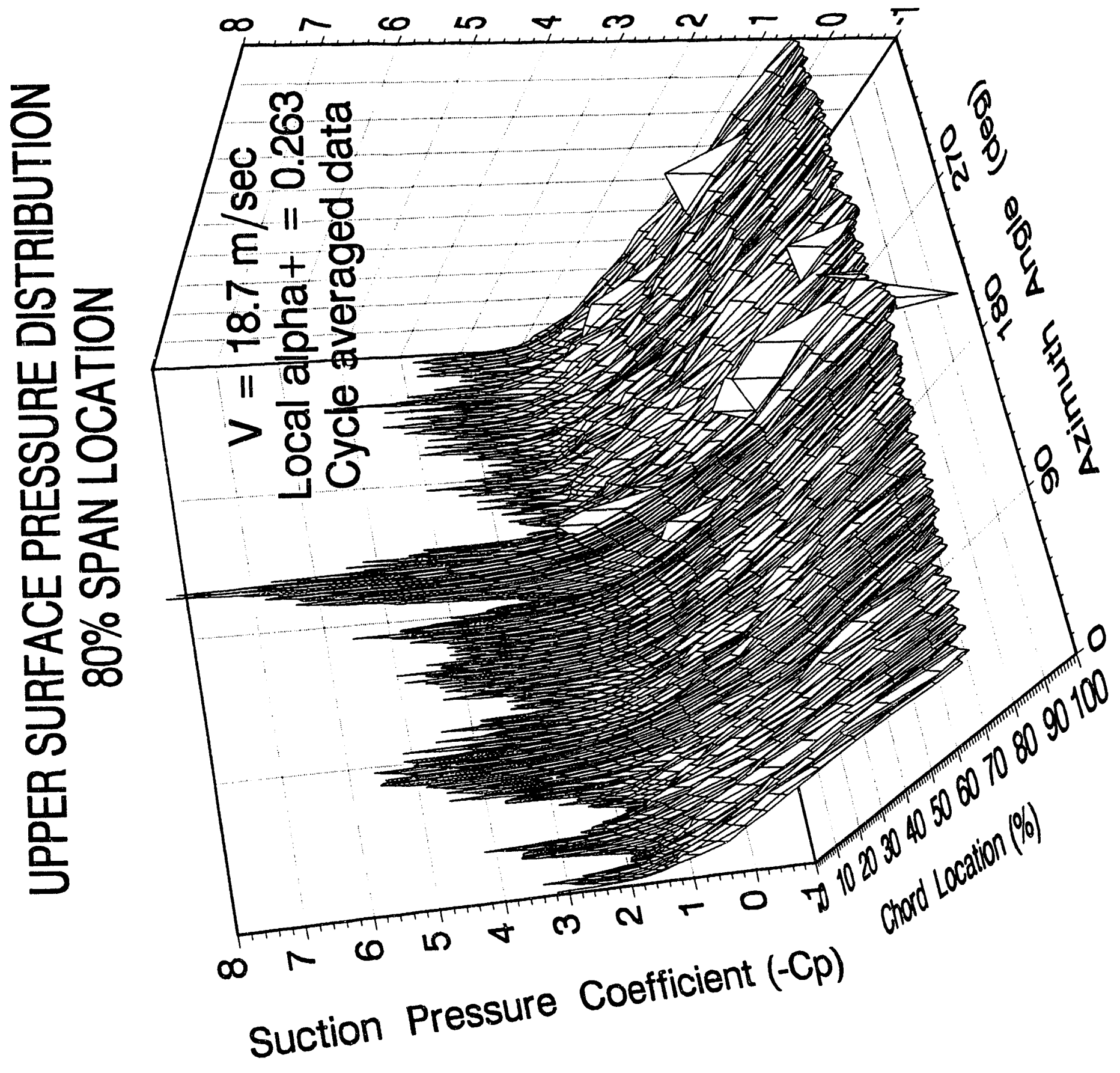




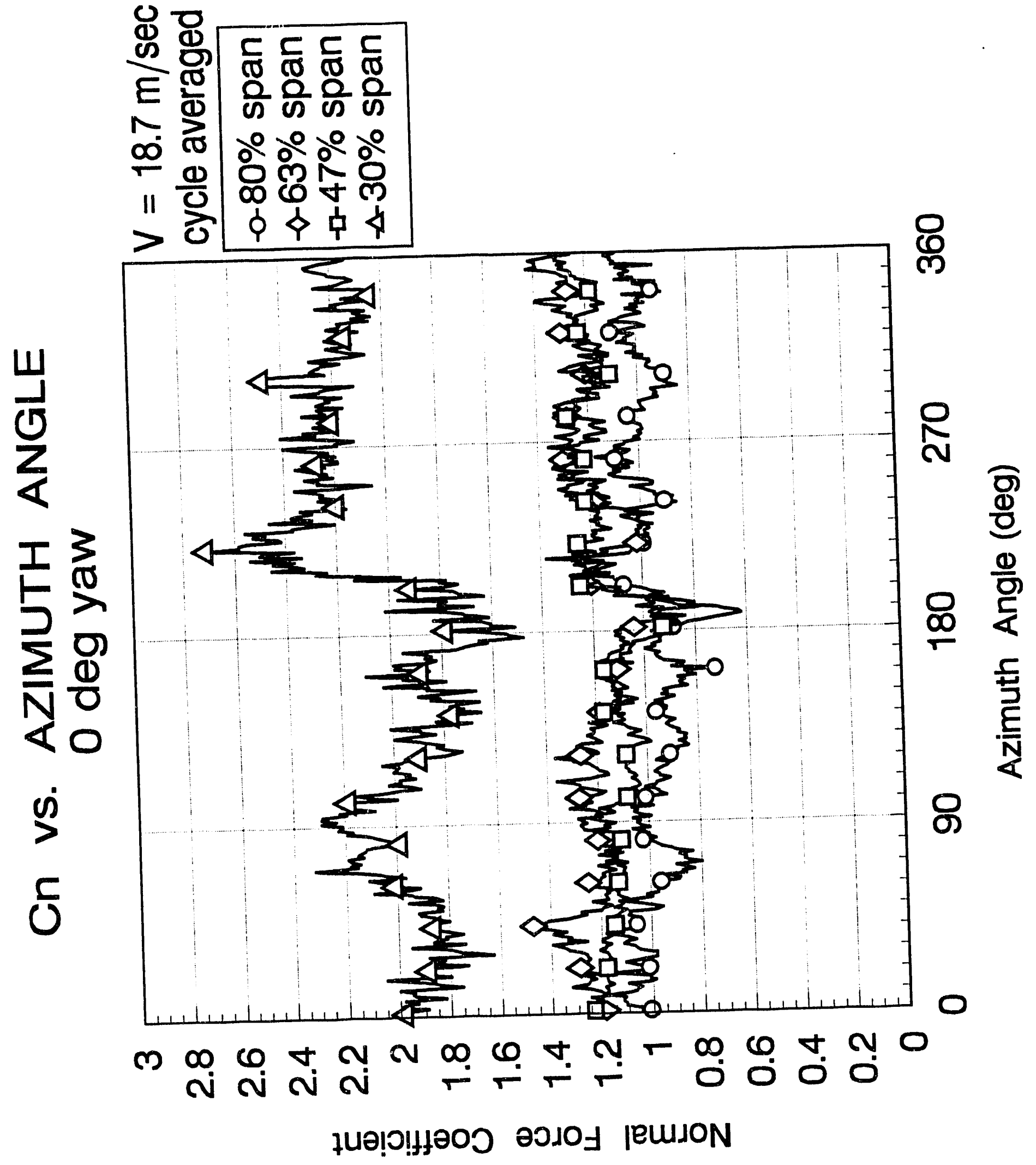




\section{(d)-) zublolffe00 asnssald wolfons}

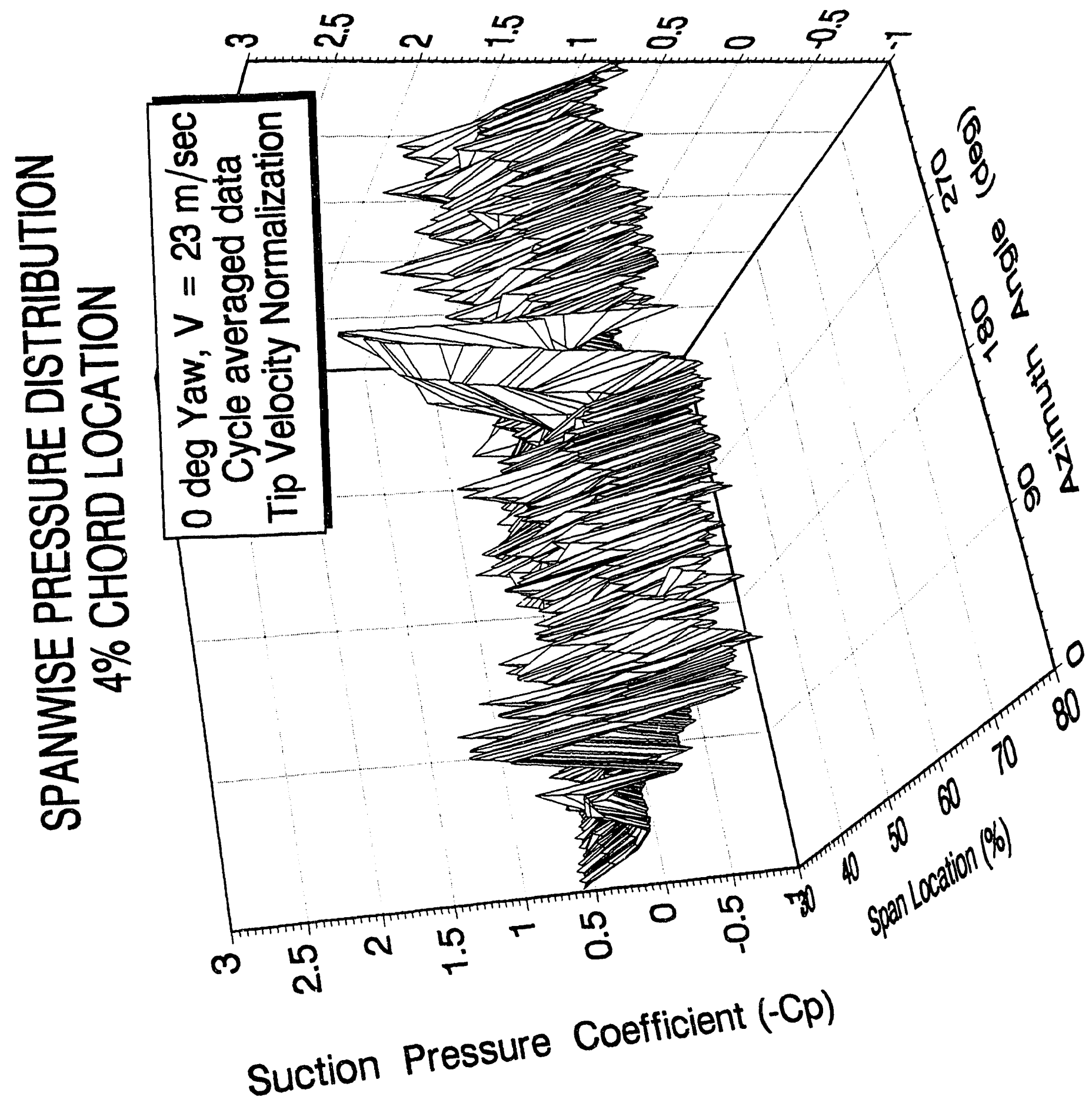




\section{(d)-) zualoiffeos esnssedd wolfons}

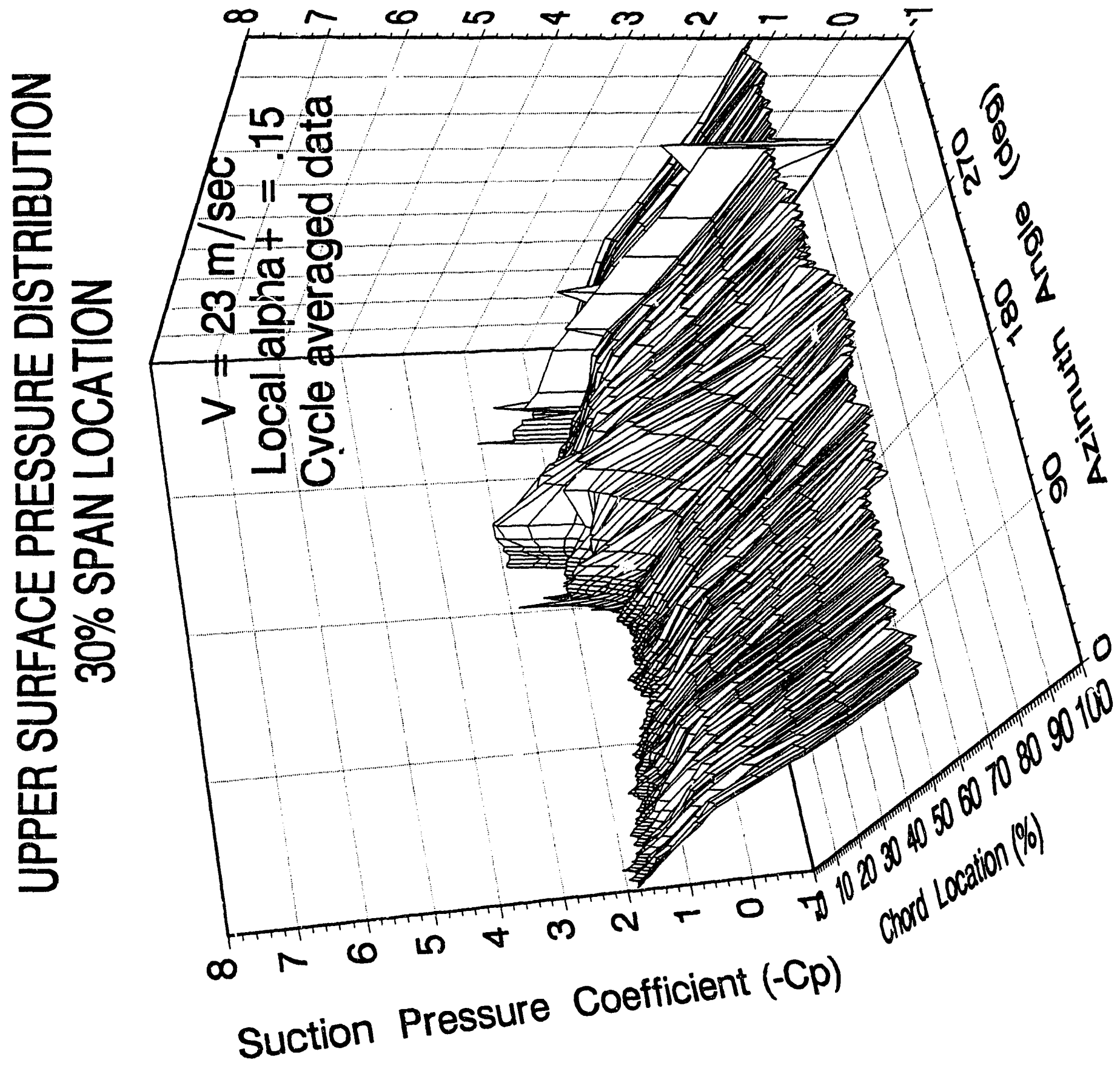




\section{(d)-) 7w0iolffeos esnssejd wolfons}

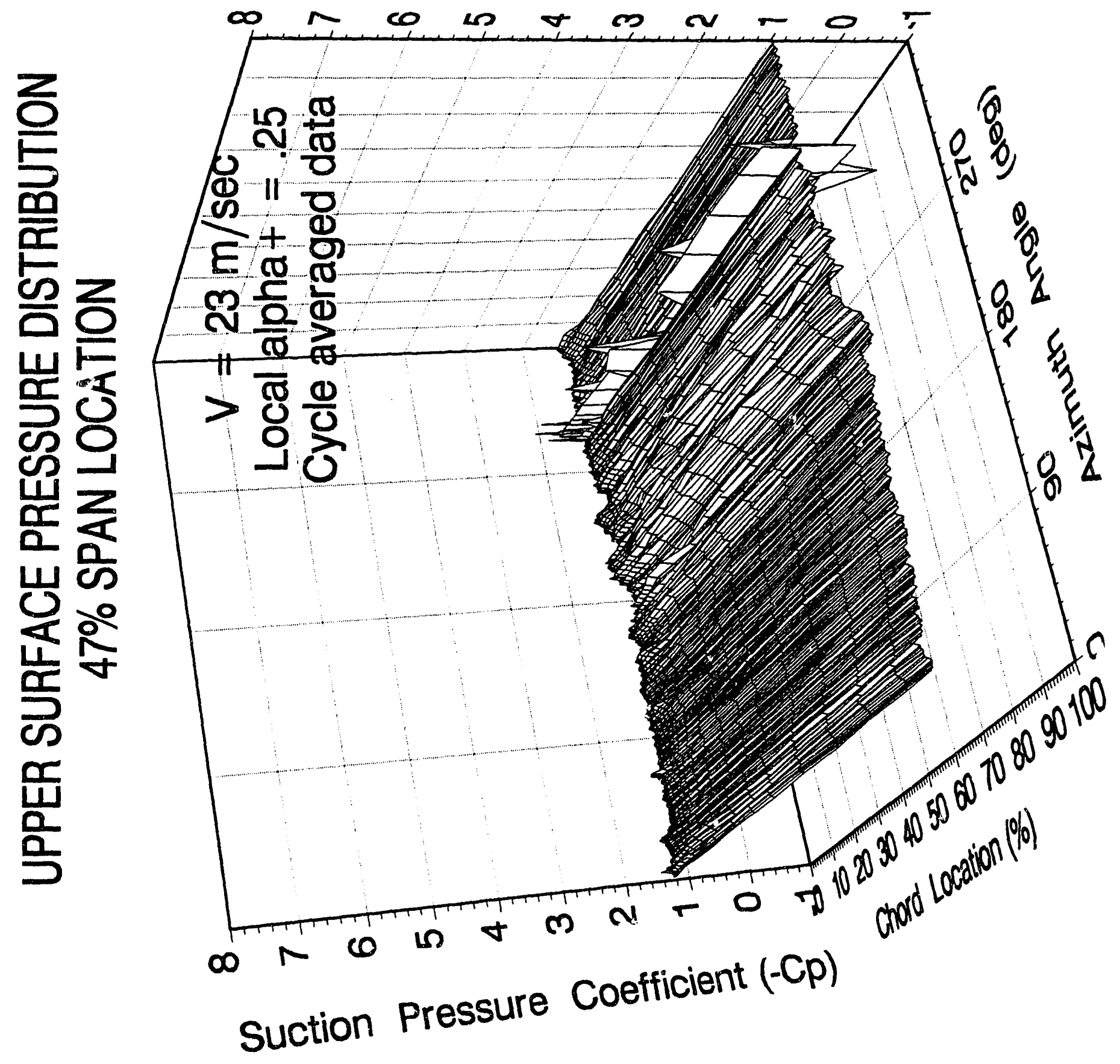




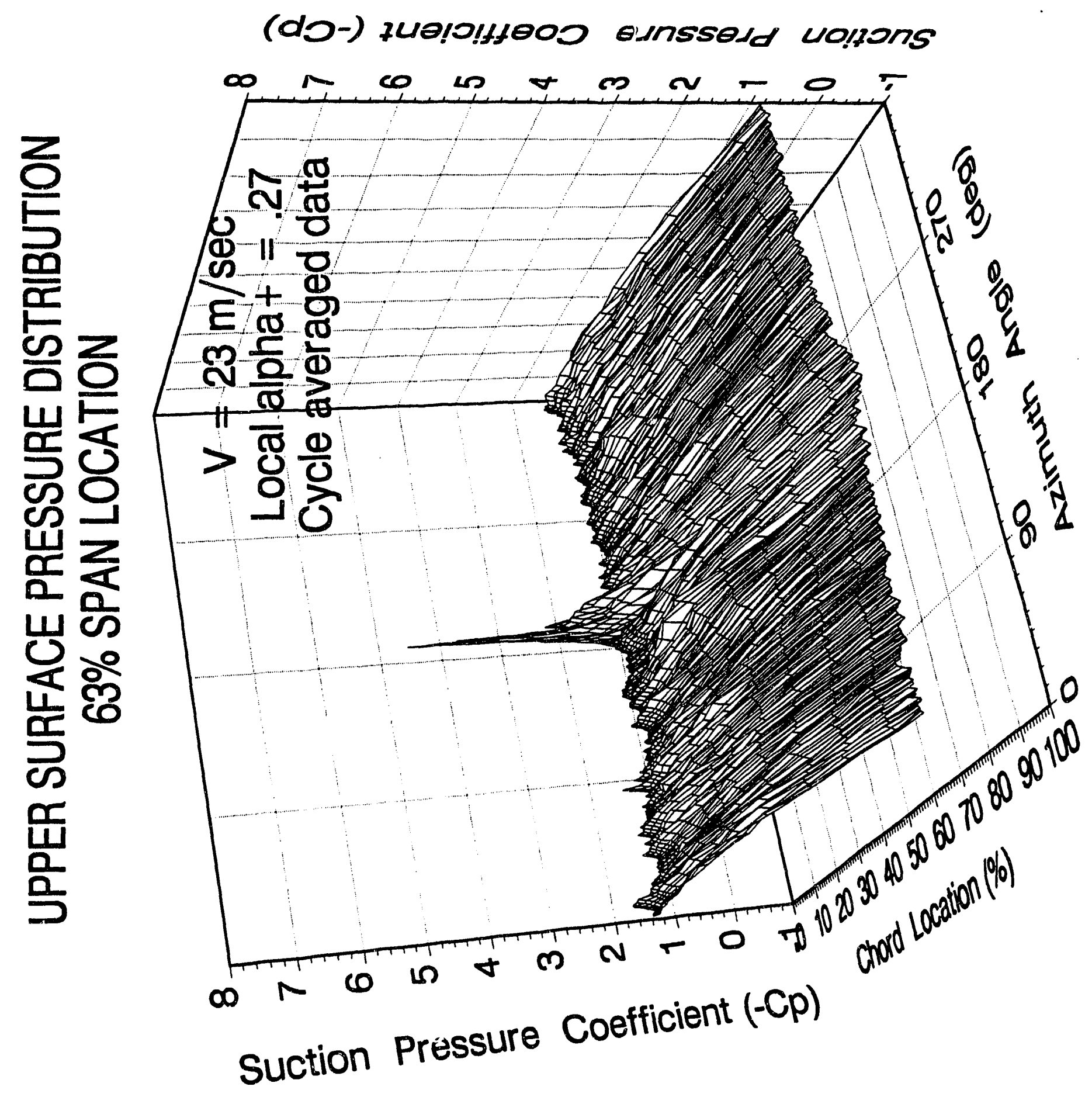




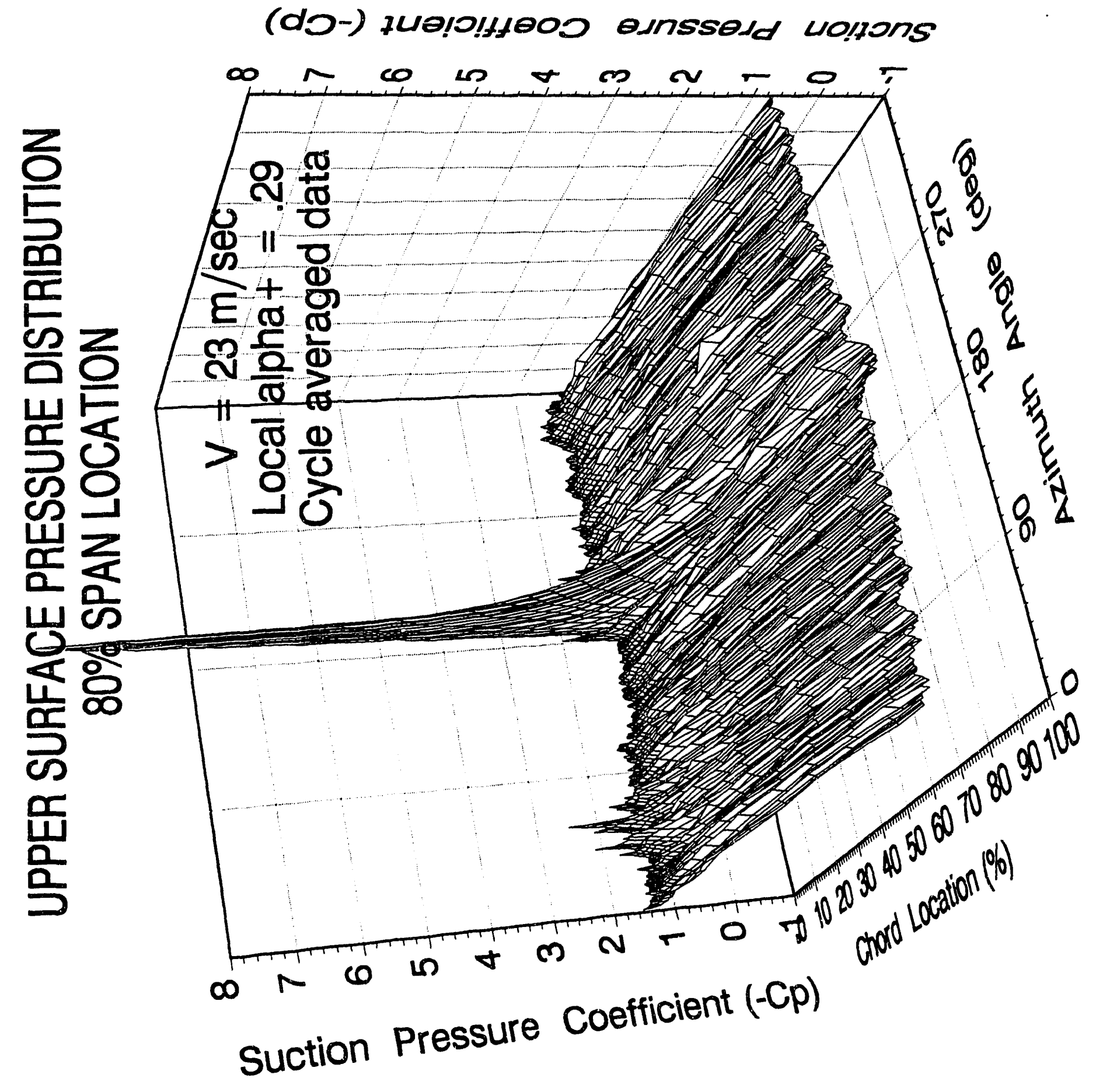




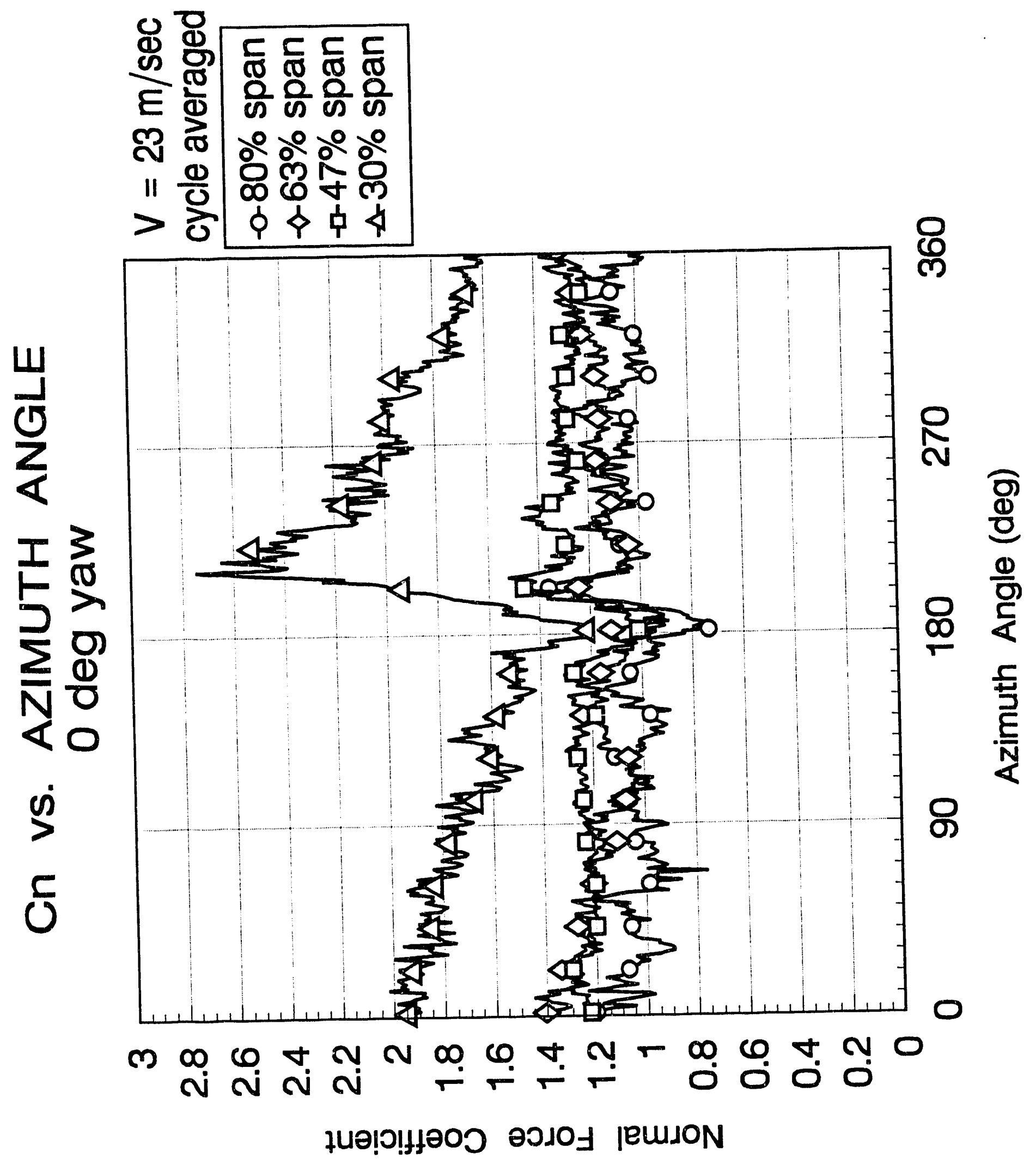




\section{Appendix D \\ Yaw Error Test Cases, Cycle Averaged}

D-1 
(d)-) 7ualo!fteos asnssald wolfons

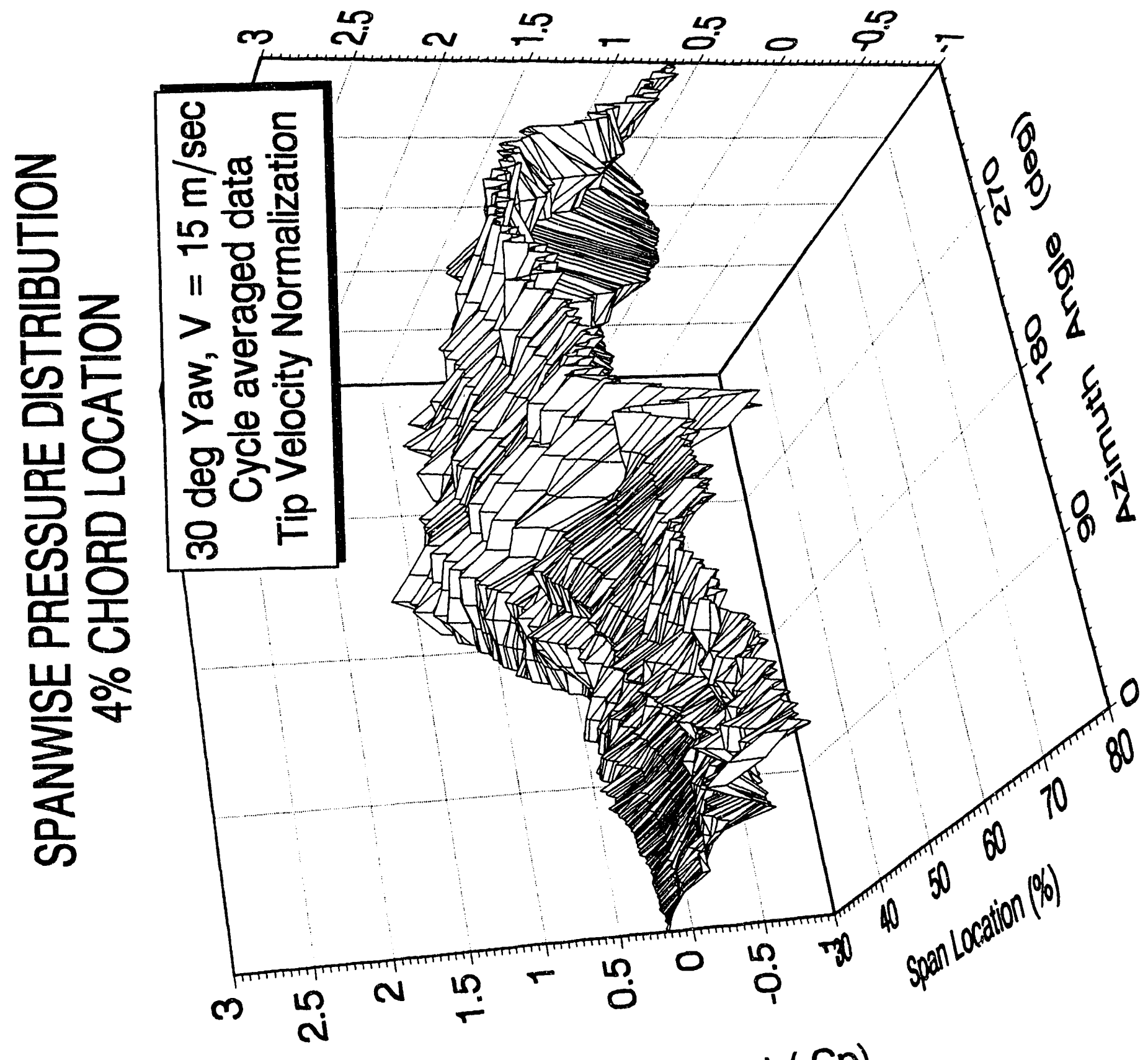

Suction Pressure Coefficient $(-C p)$ 


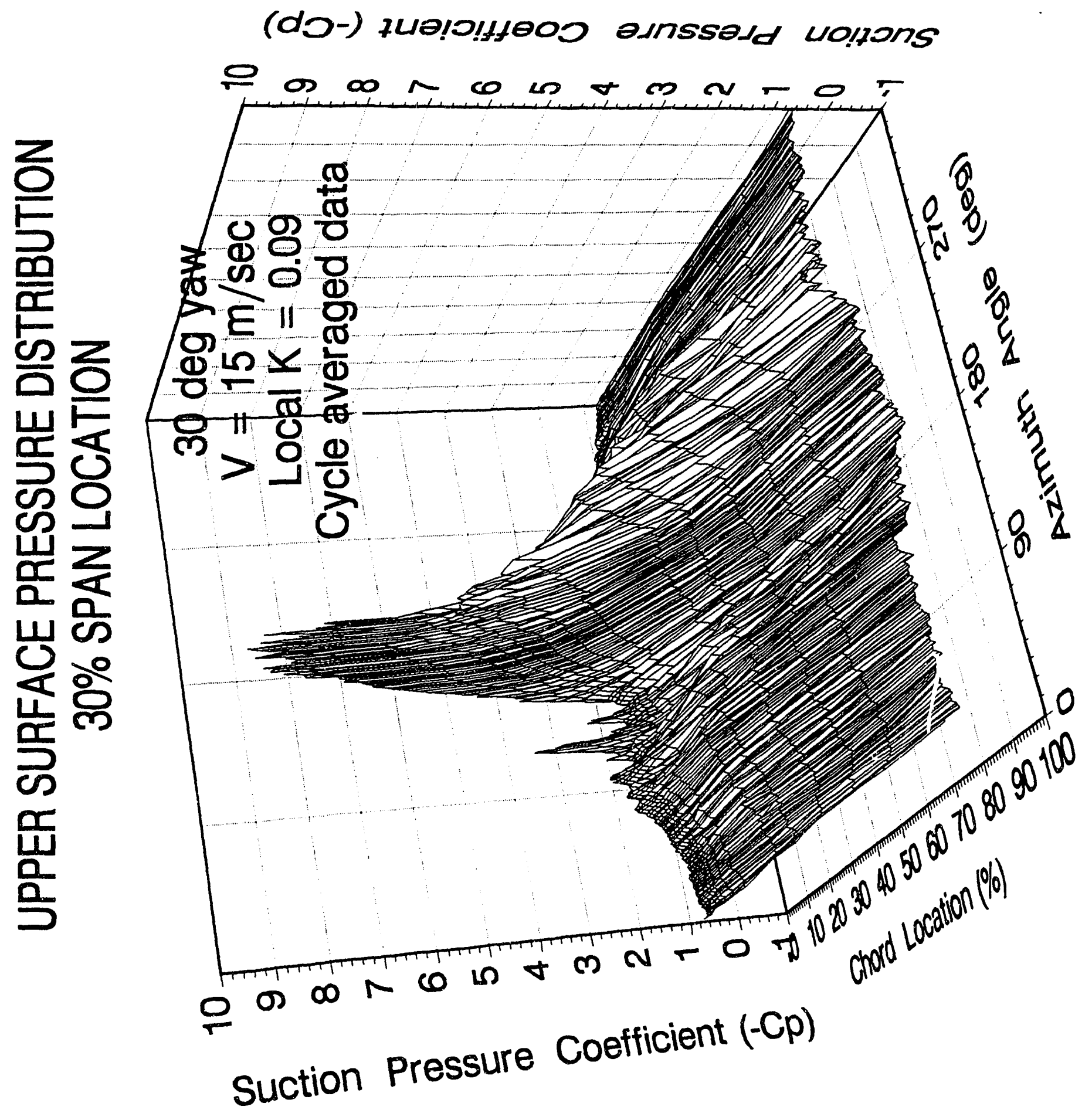




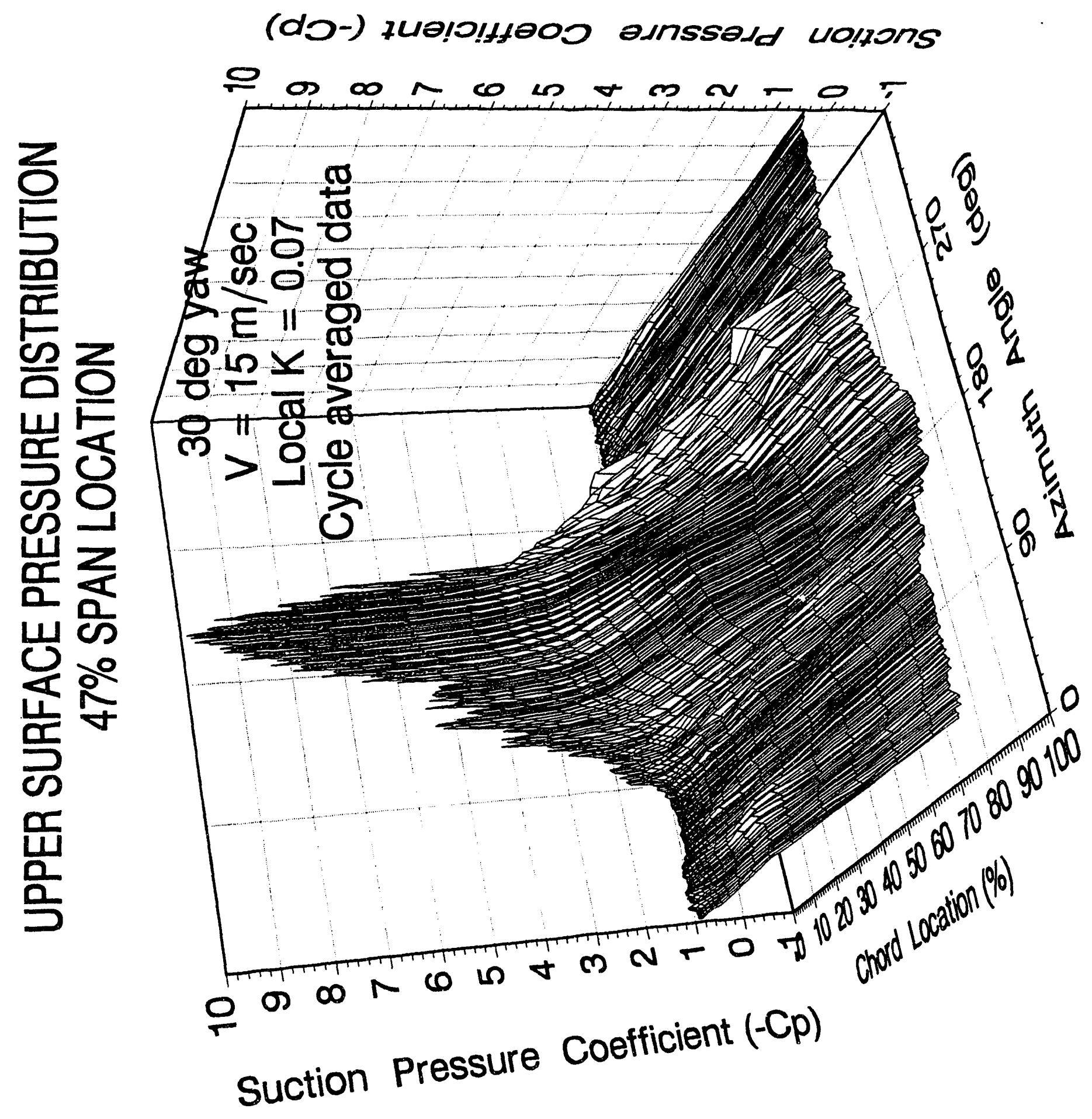




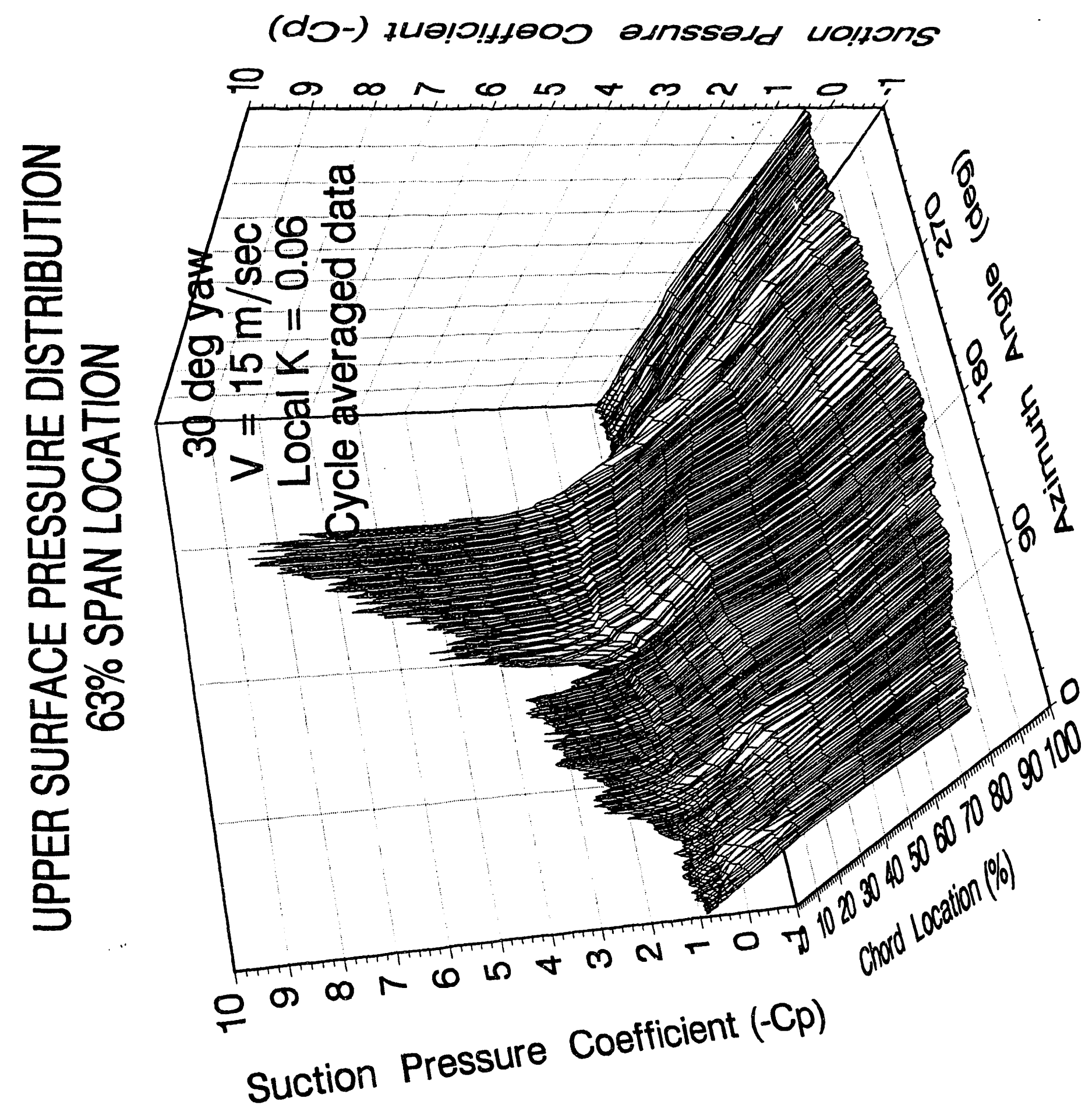




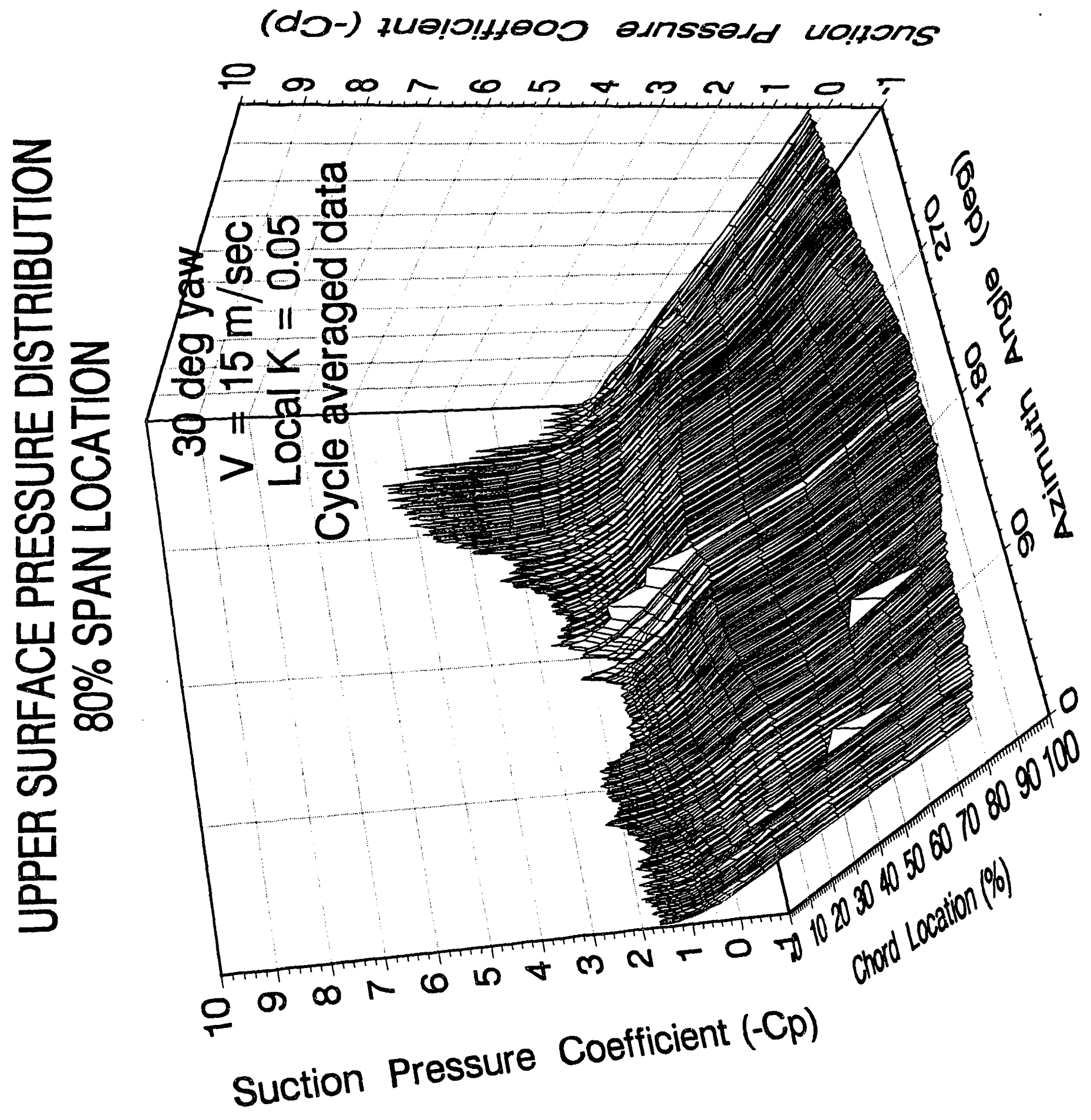




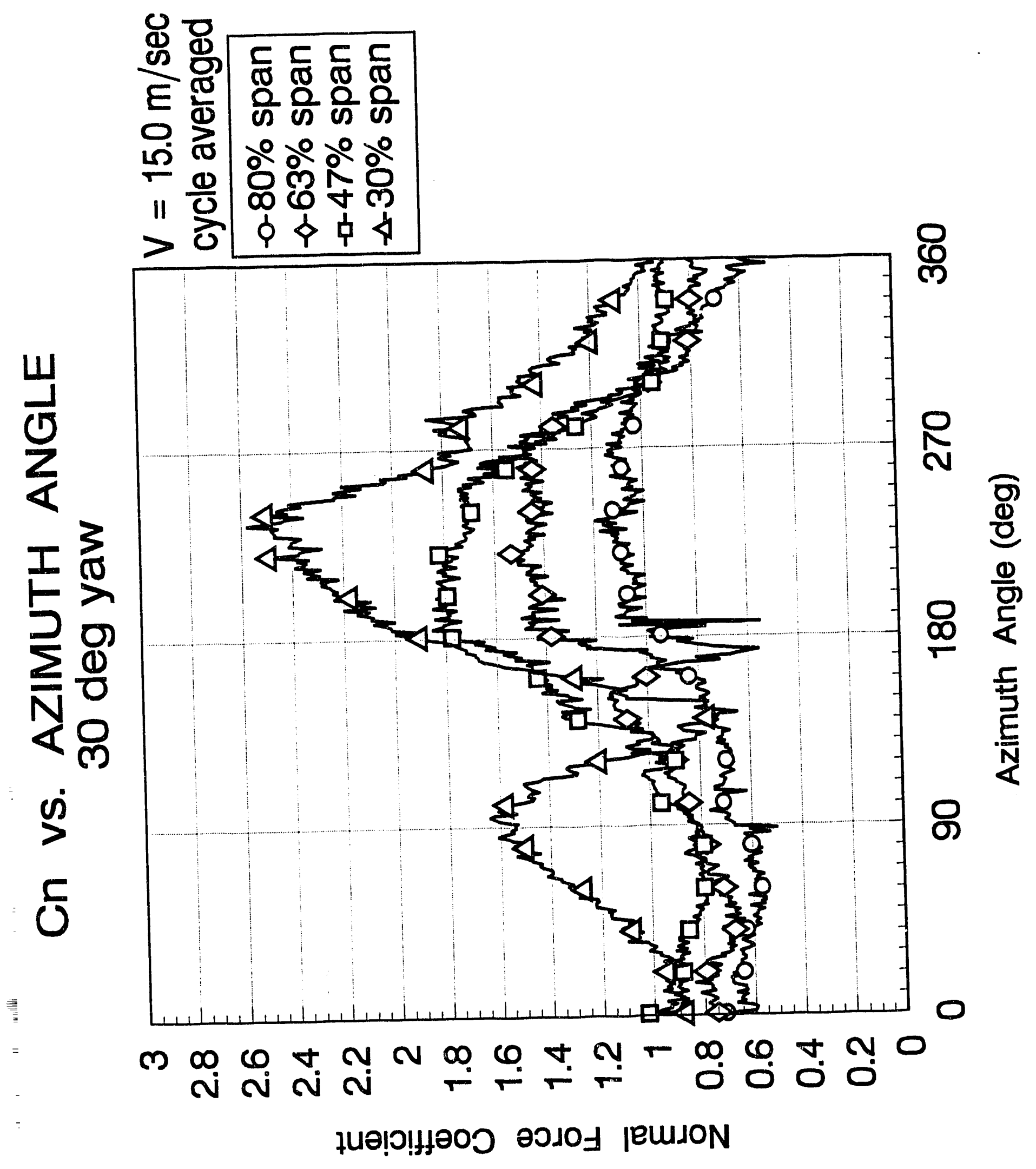

D-7 


\section{(d)- 7ublolfyeod asnssald wolfons}

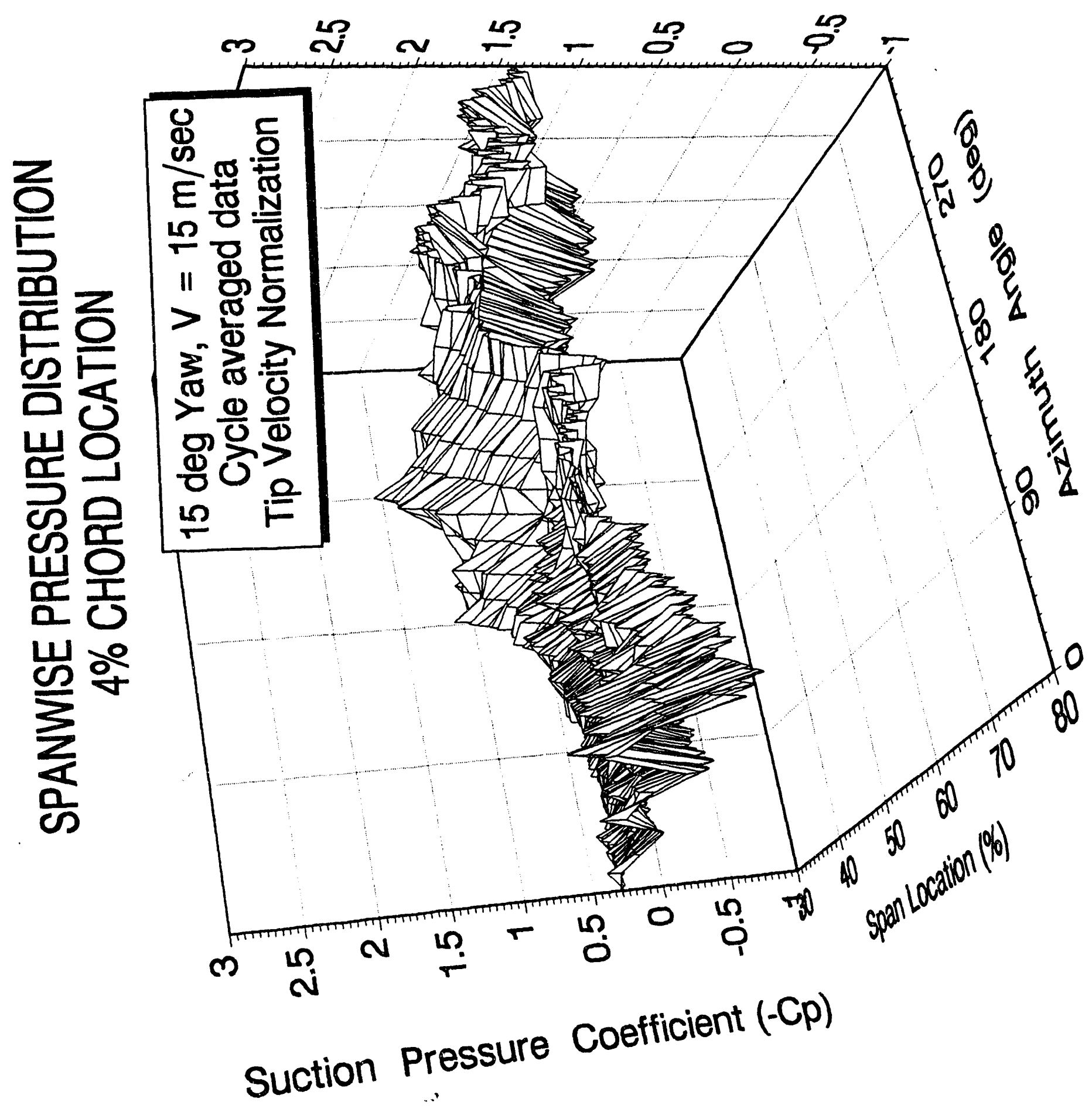




\section{(d)-) 7ualo!kf000 asnssajd wolpons}

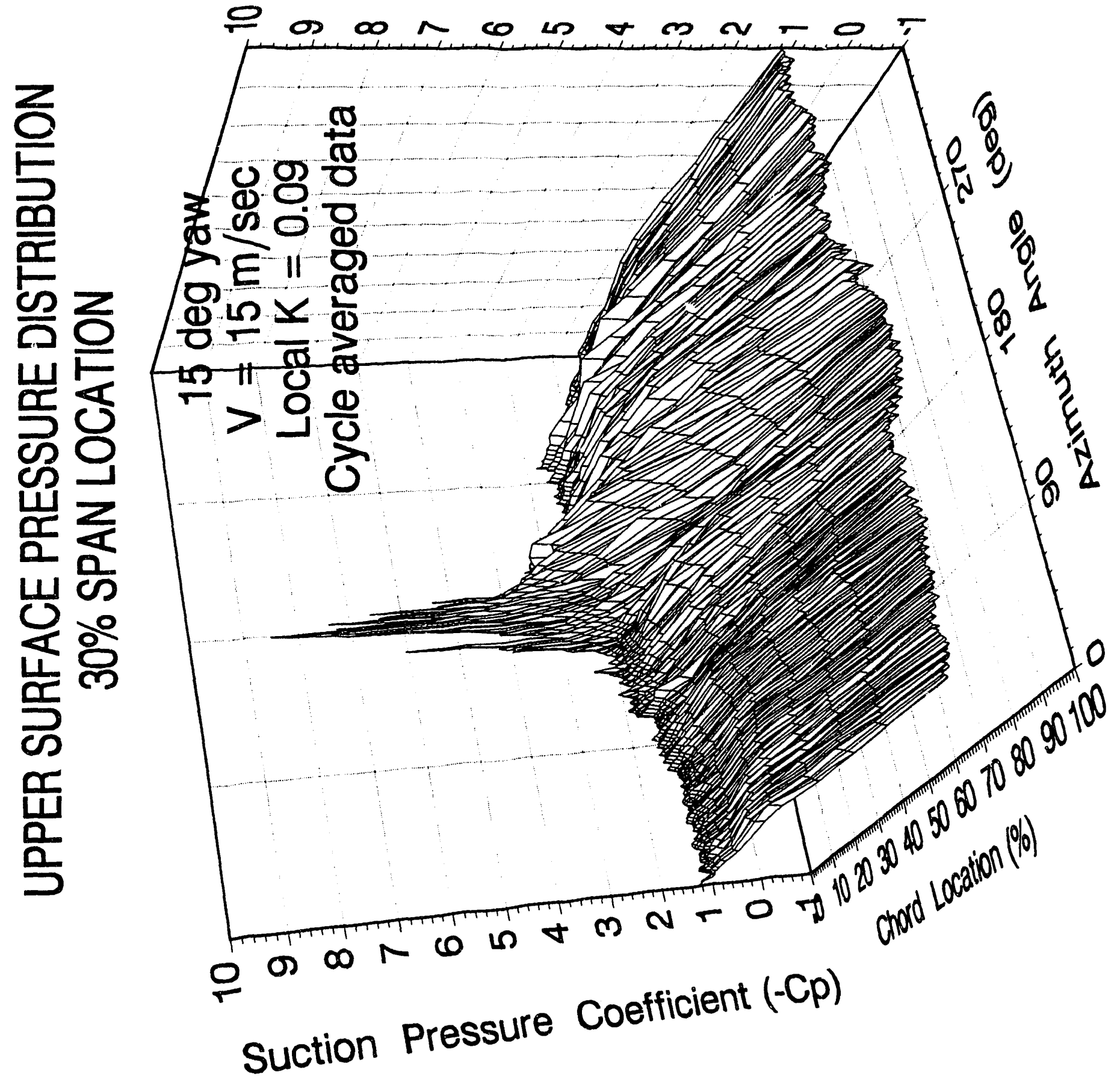




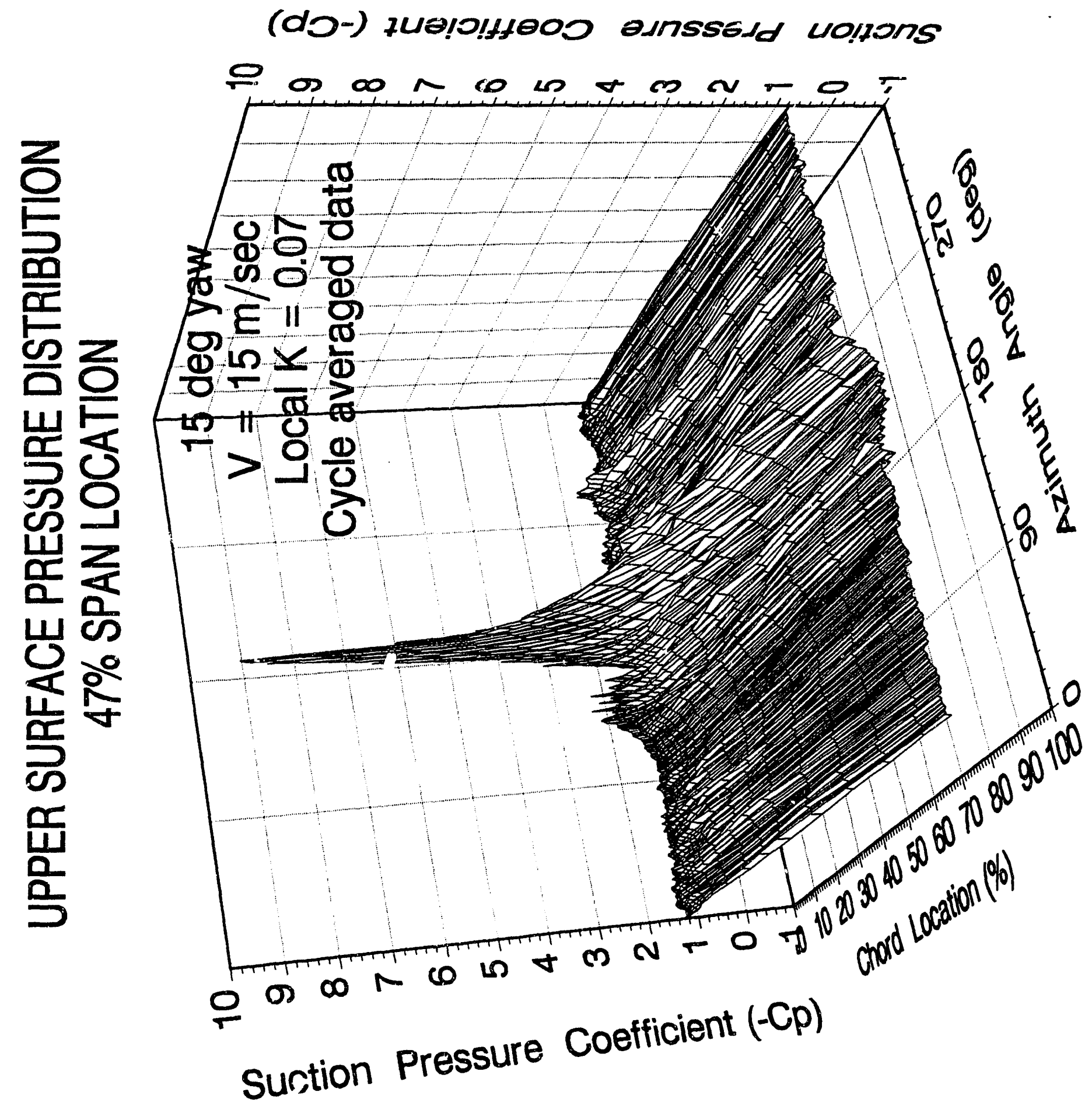




\section{(do-) 7uolo!ffe00 asnssald Lulpans}

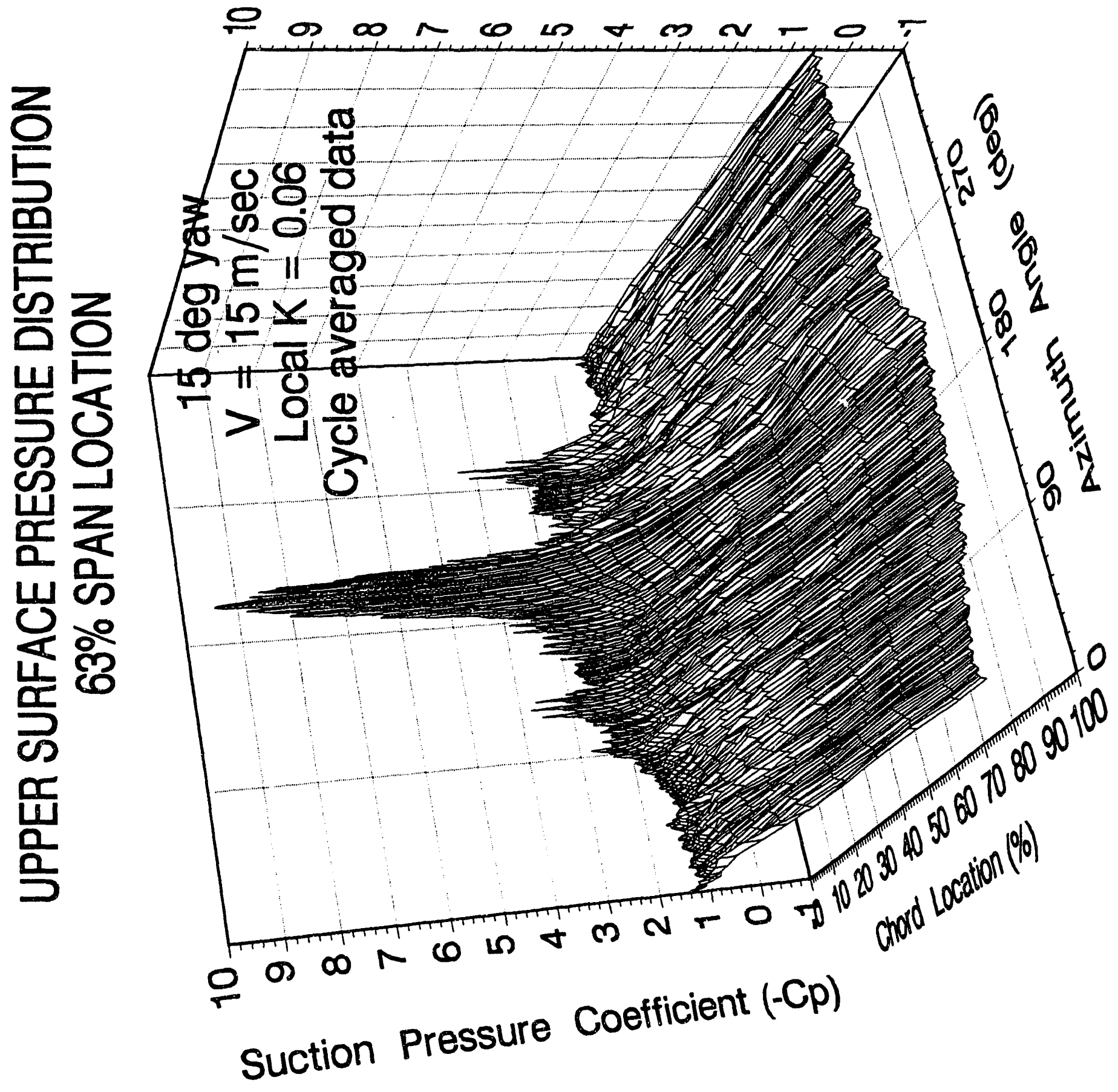




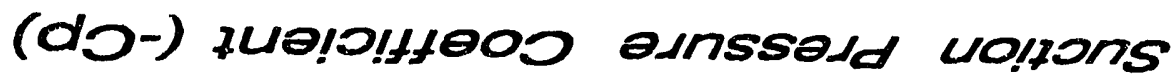

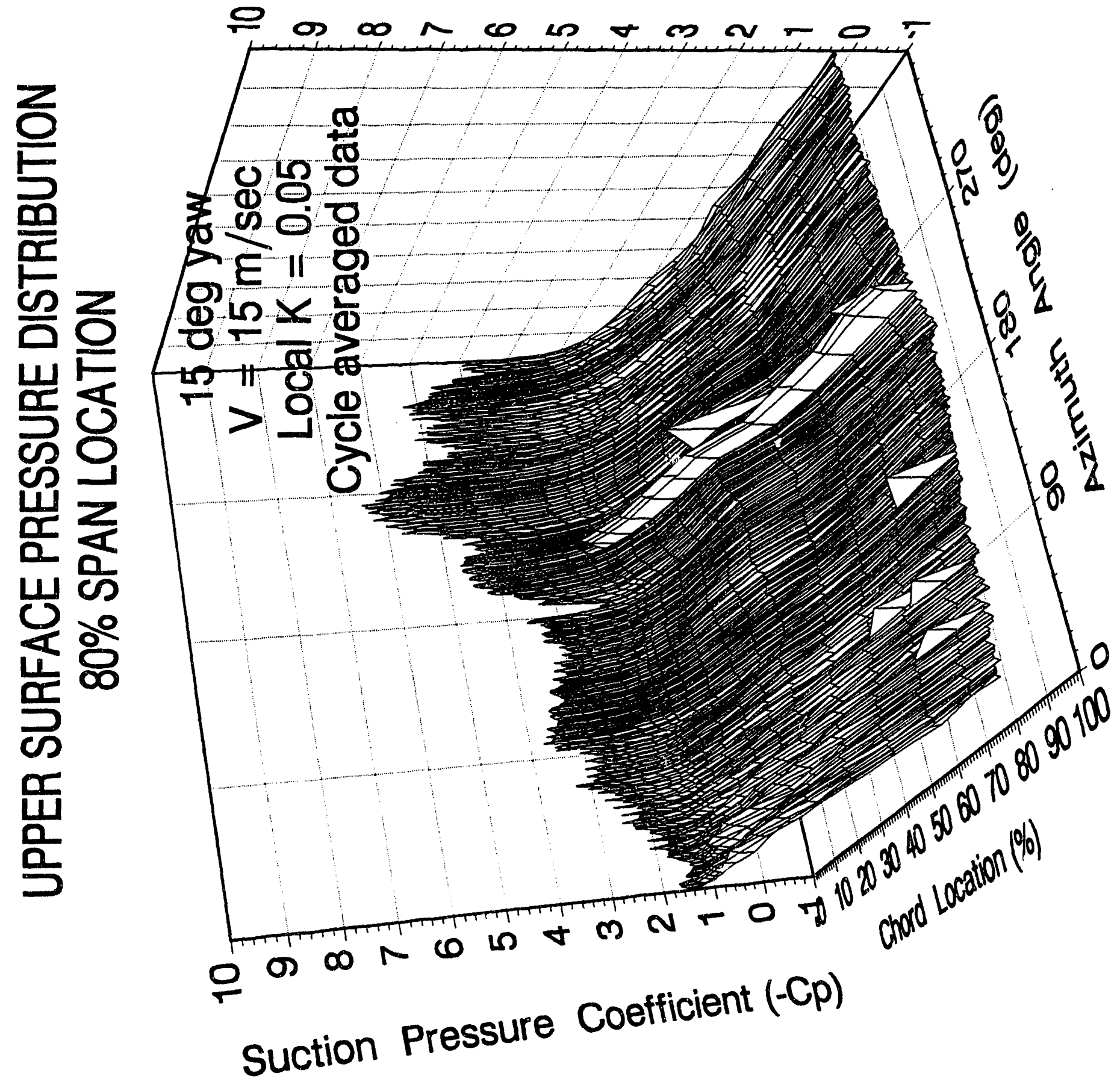




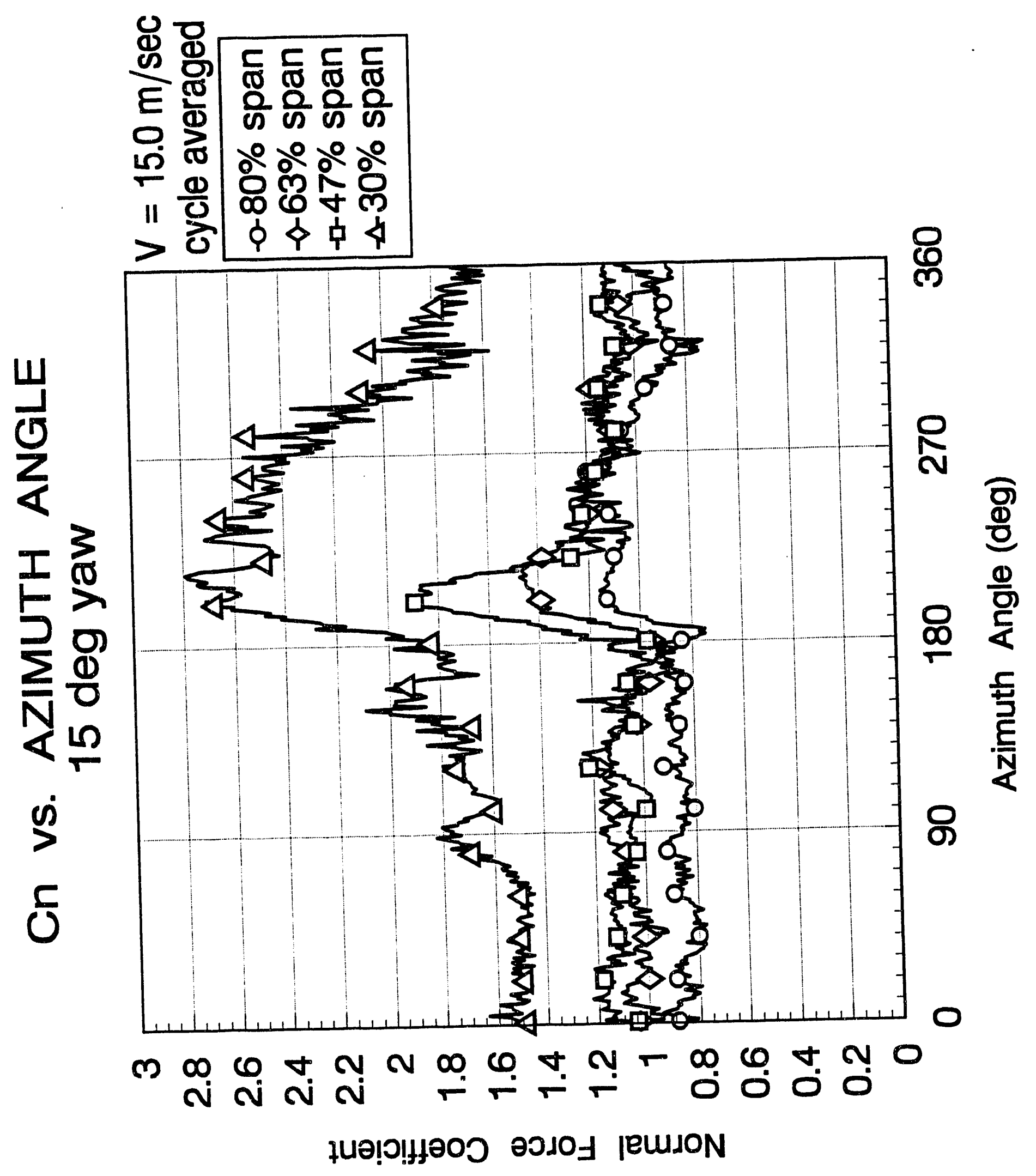




\section{(do-) 7wololffeod esnssedd wolfons}

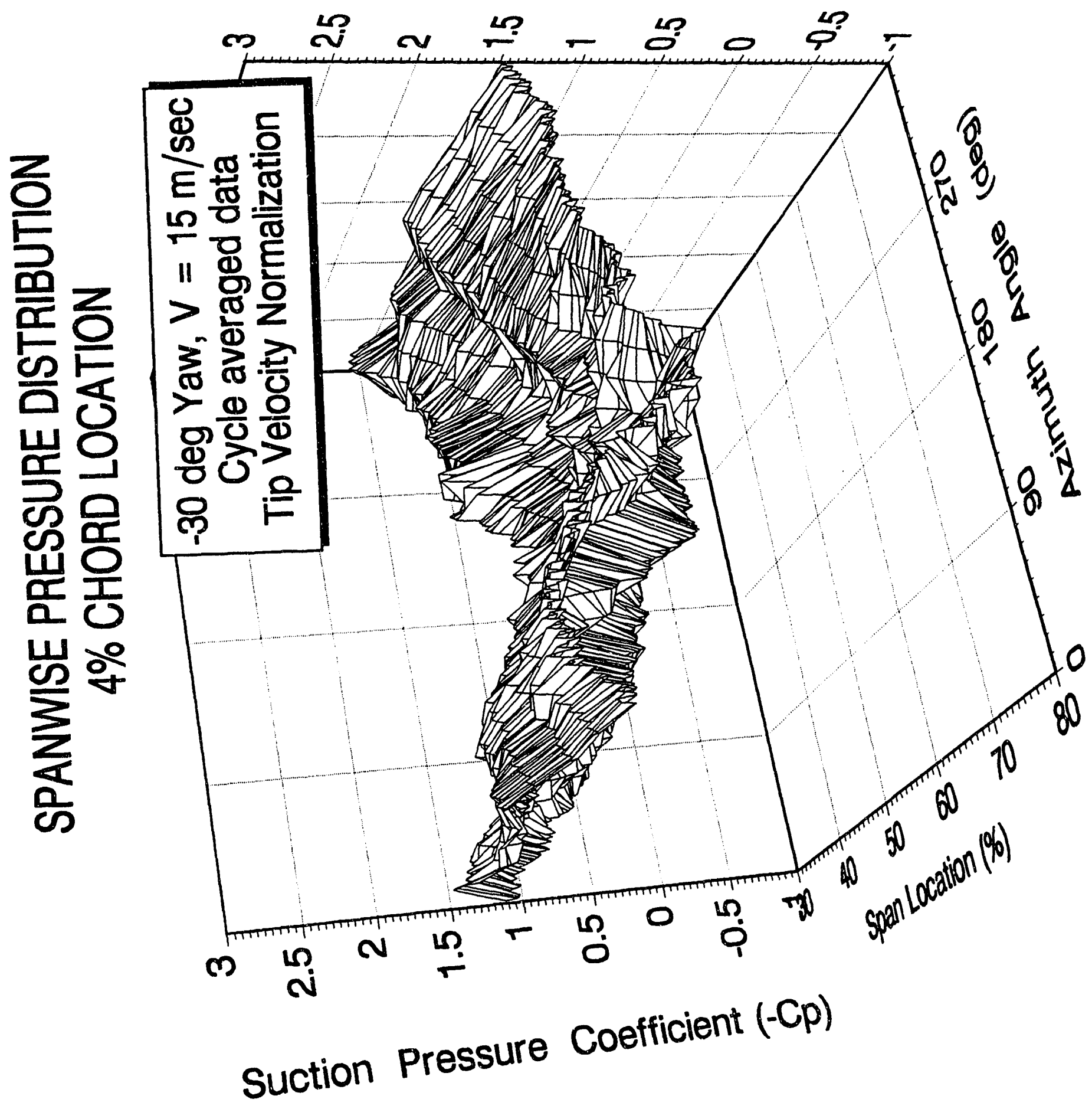




\section{(do-) zuolo!ffe00 asnssald wolpons}

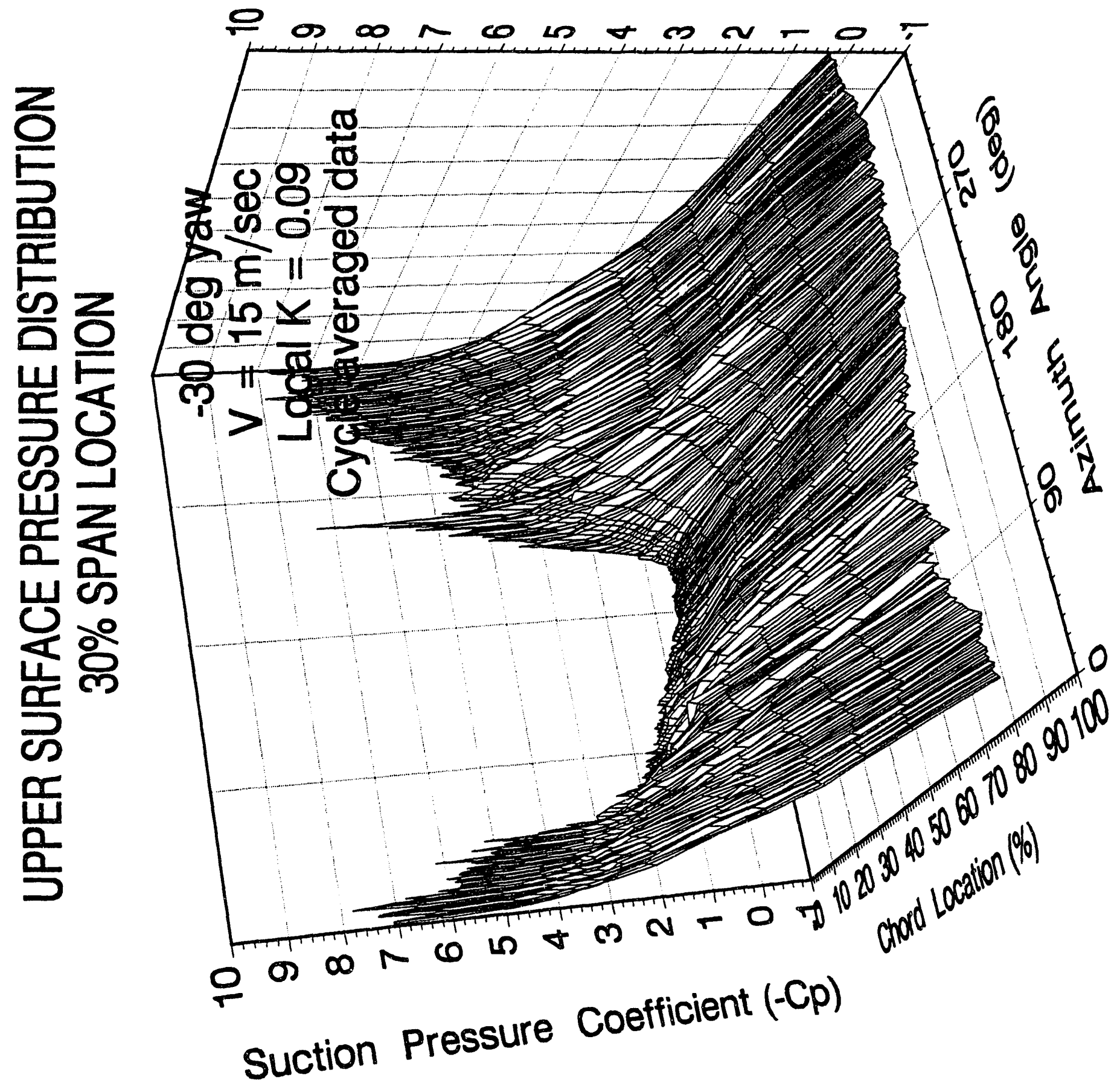




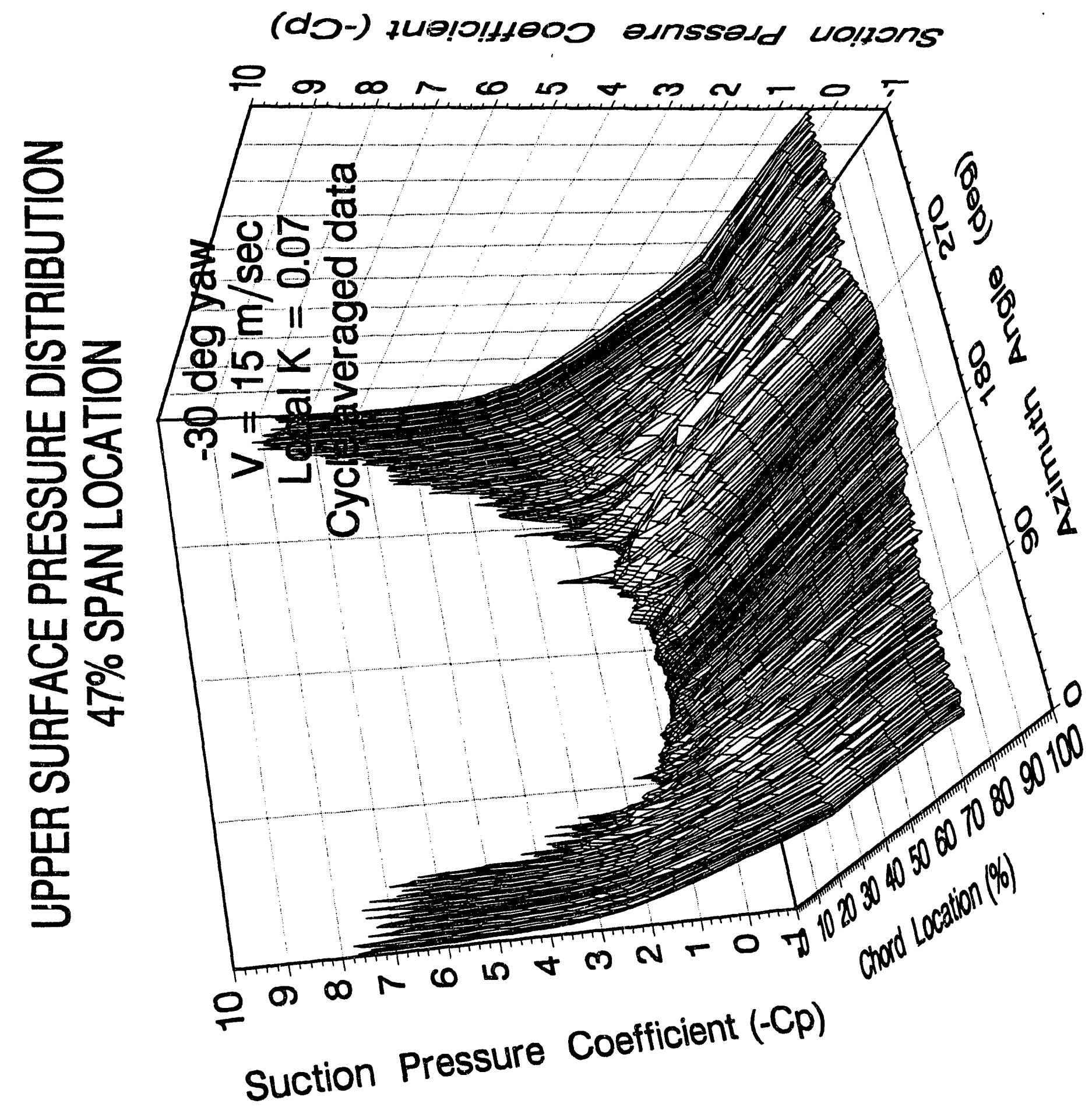




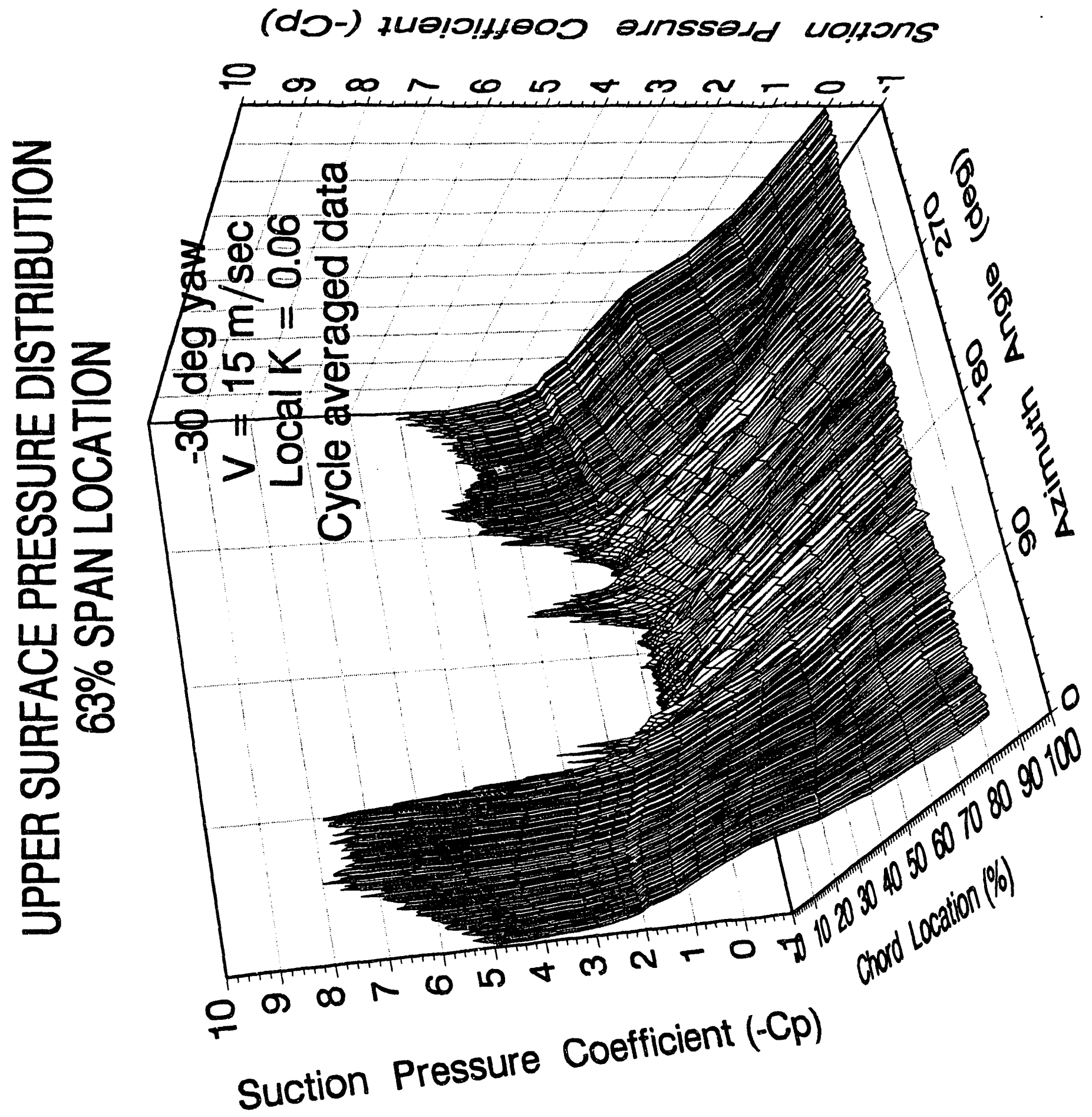




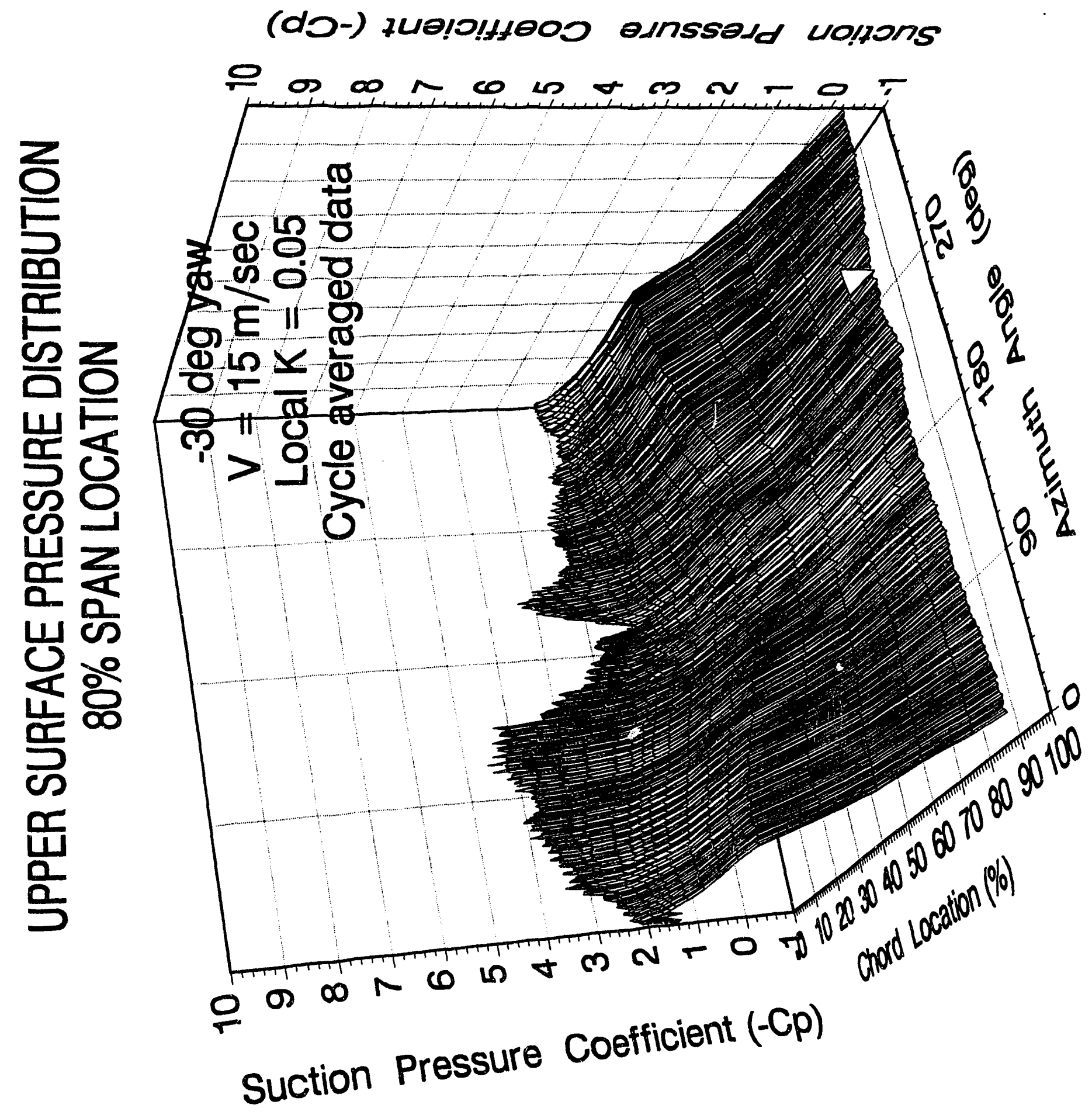




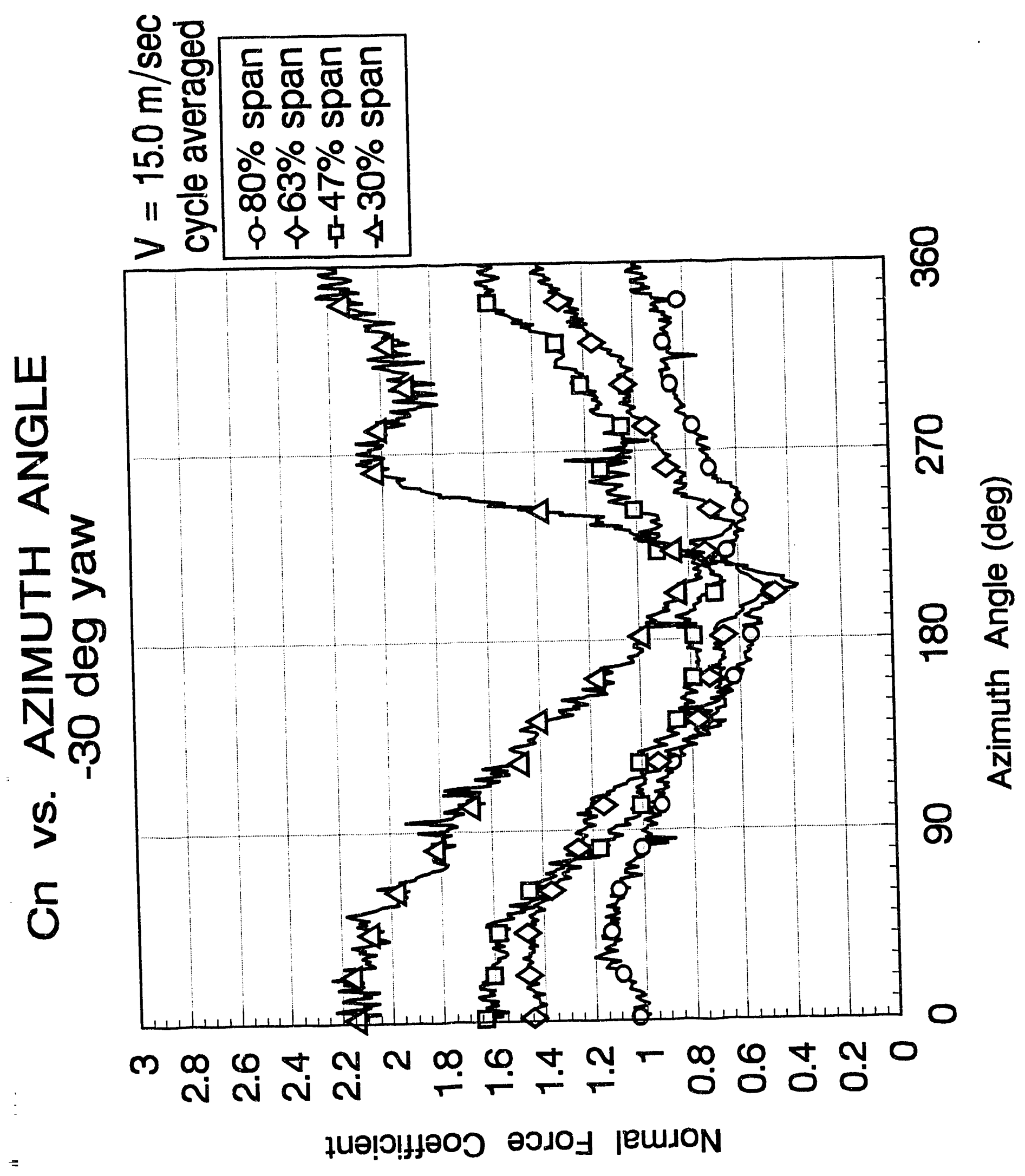




\section{(d)-) zuelolfye00 asnssedd wolfons}

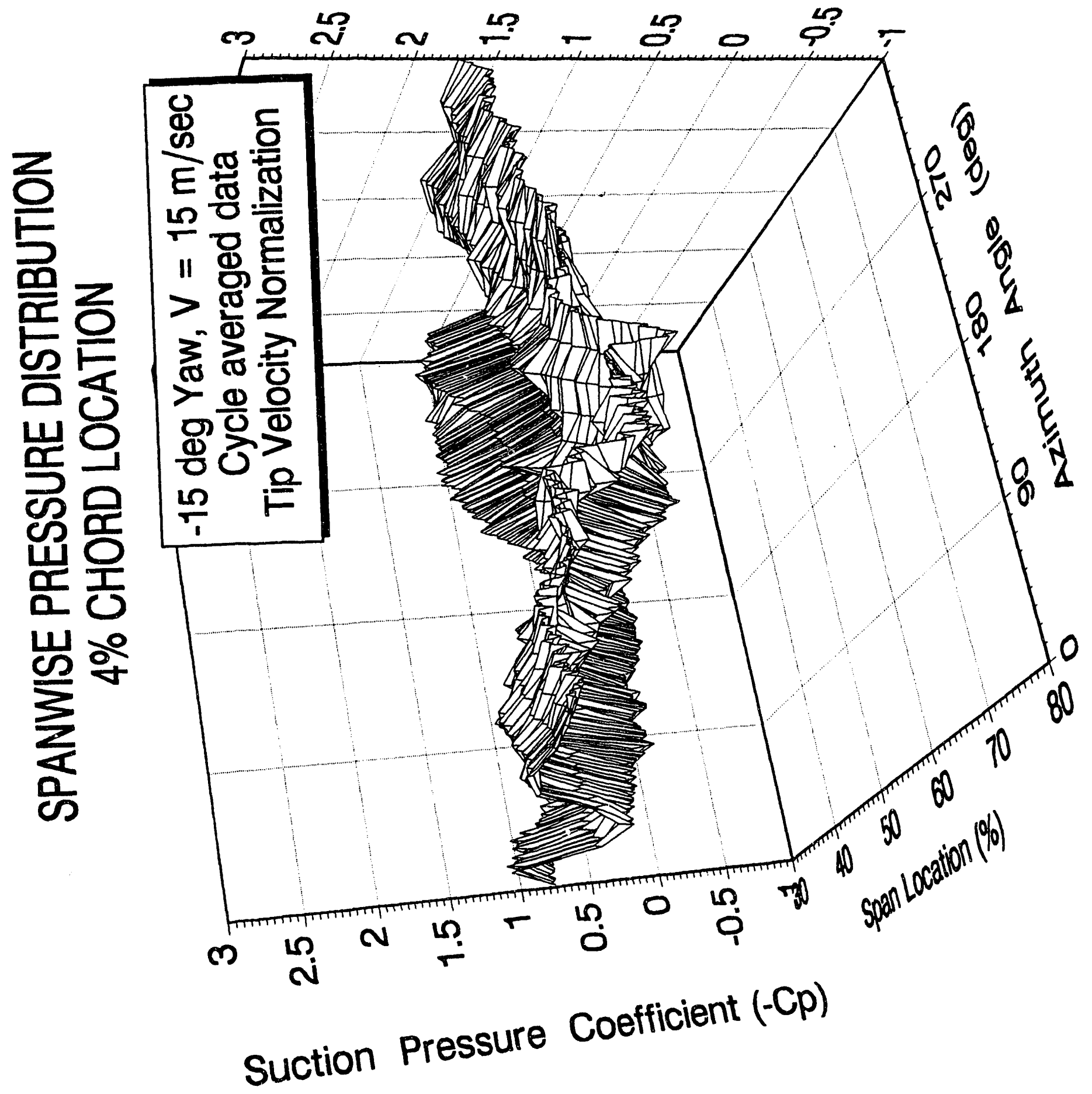




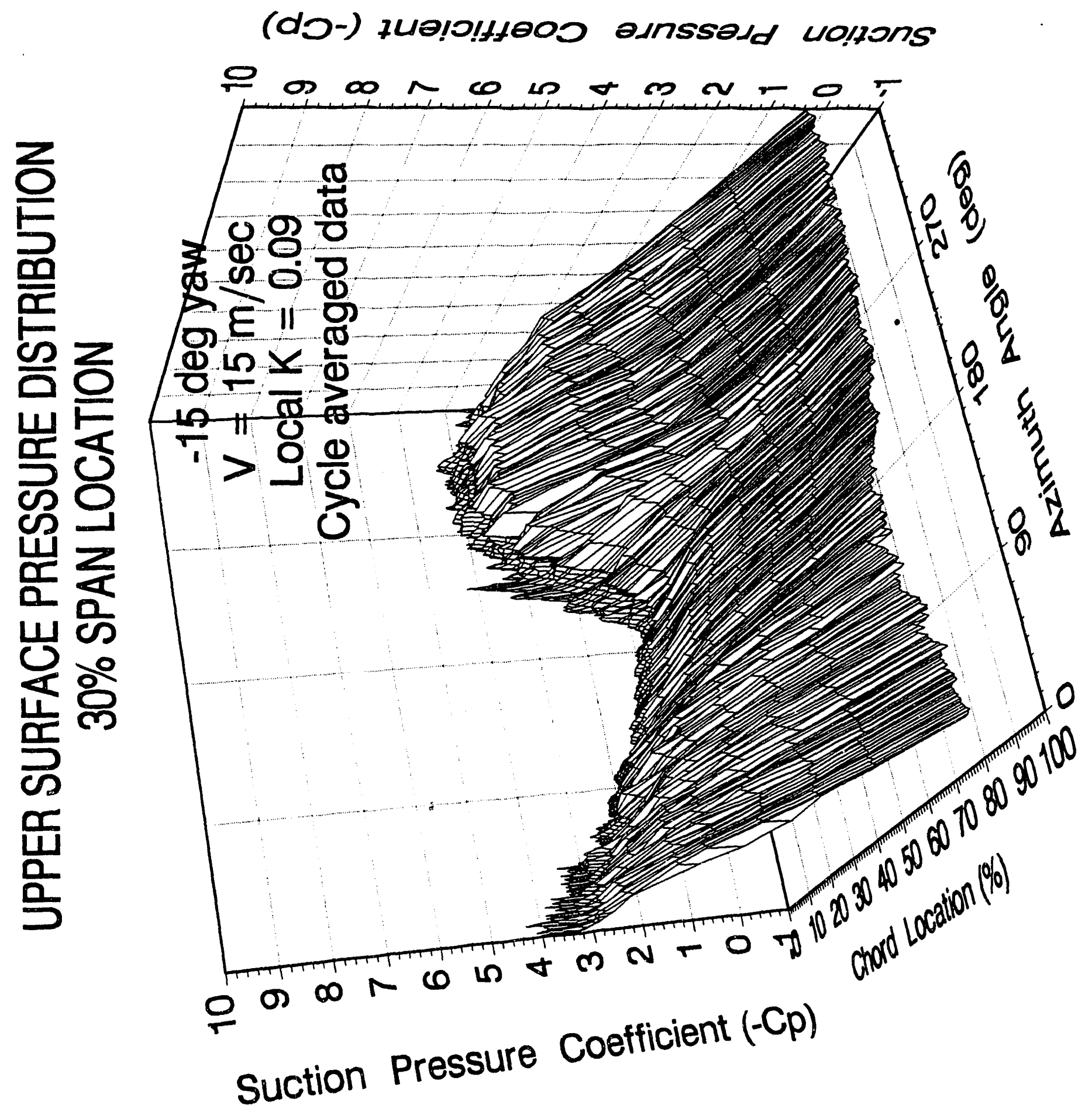




\section{(do-) zuololfte00 asnssald volpons}

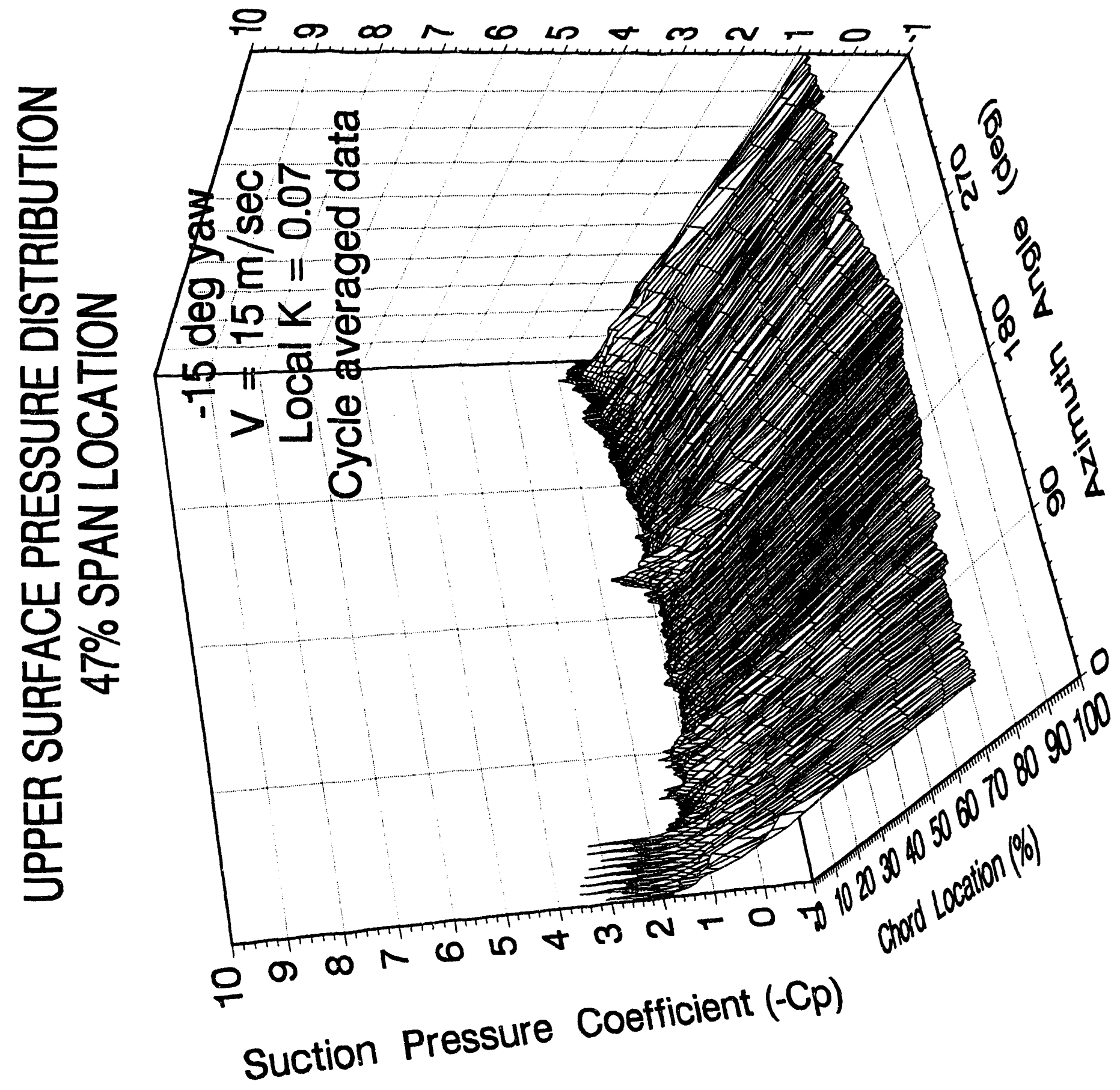




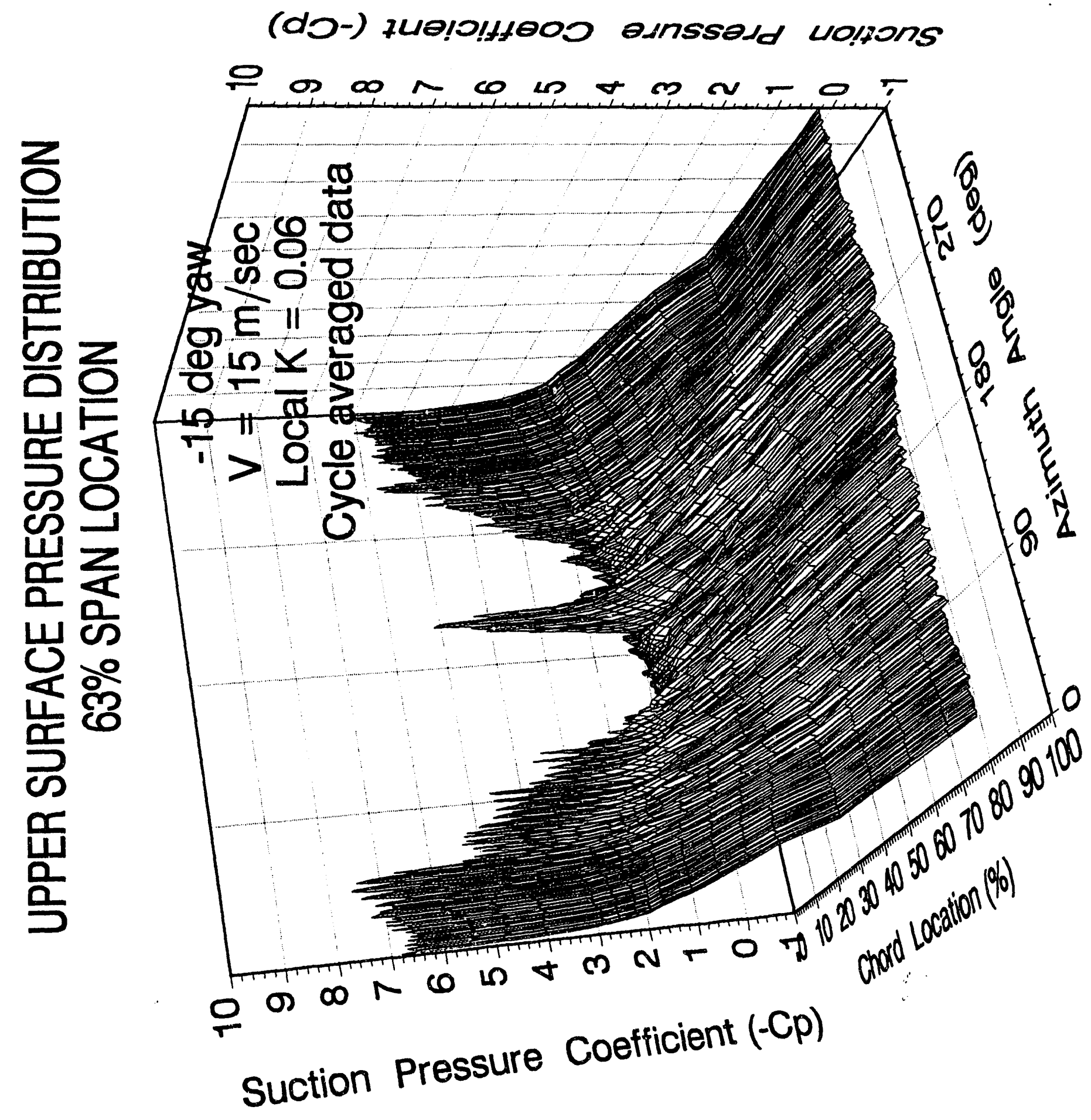




\section{(d)-) 7wolo!ffe00 asnssedd wolpons}

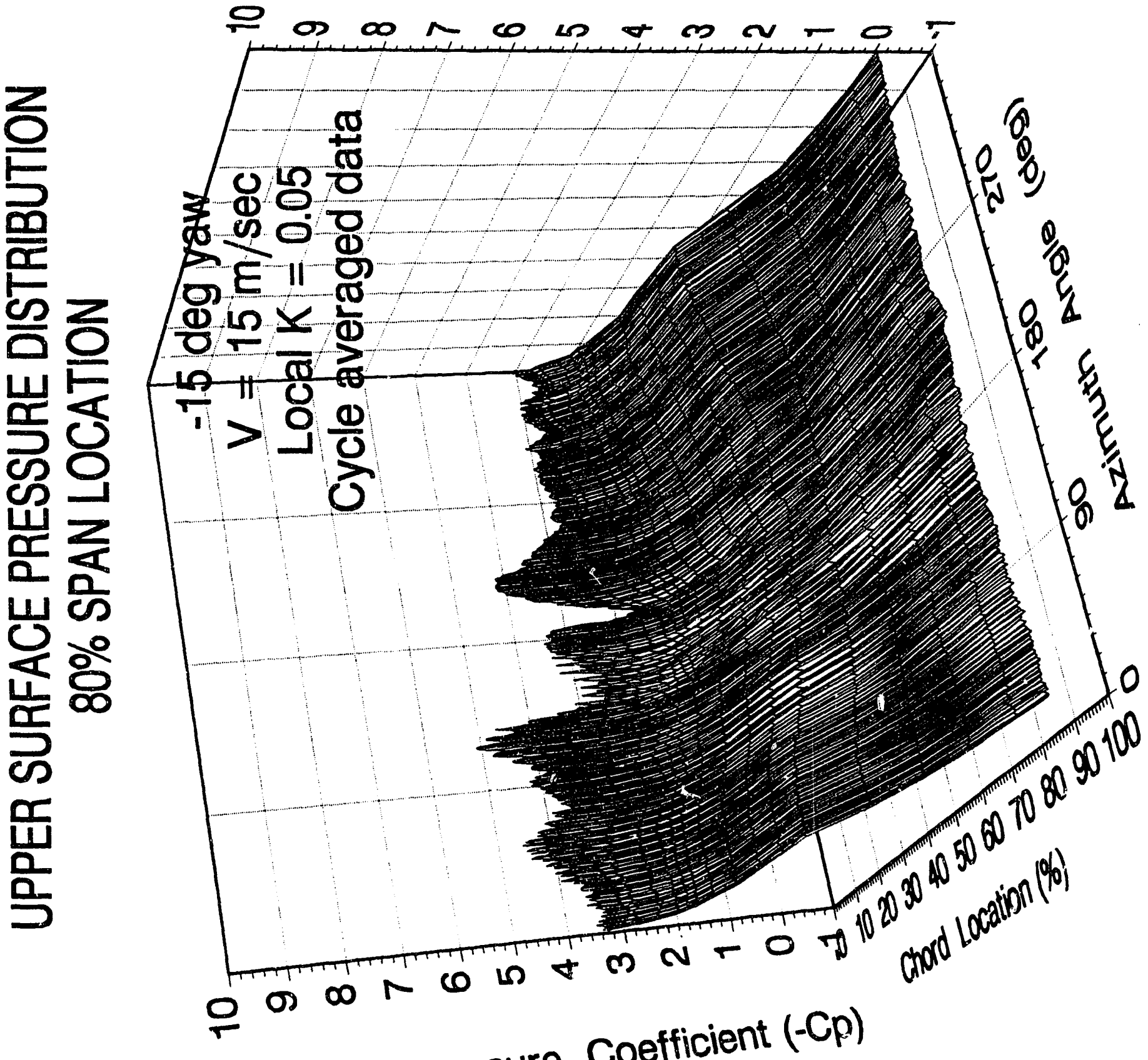

Suction Pressure Coefficient (-Cp) 


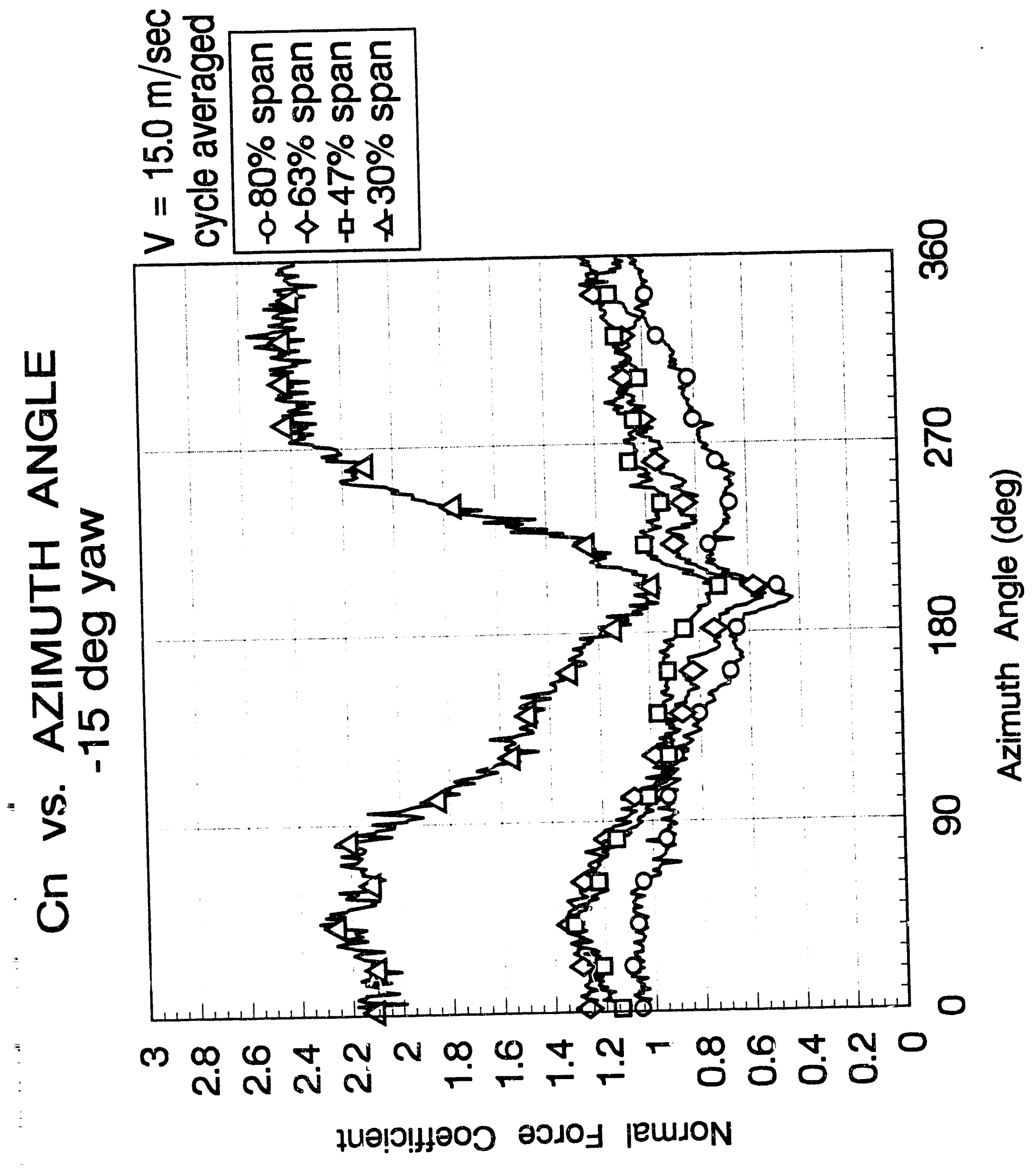




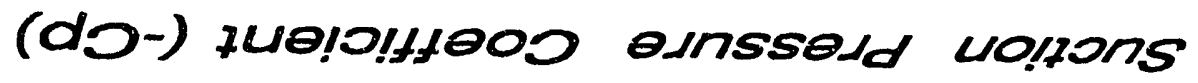

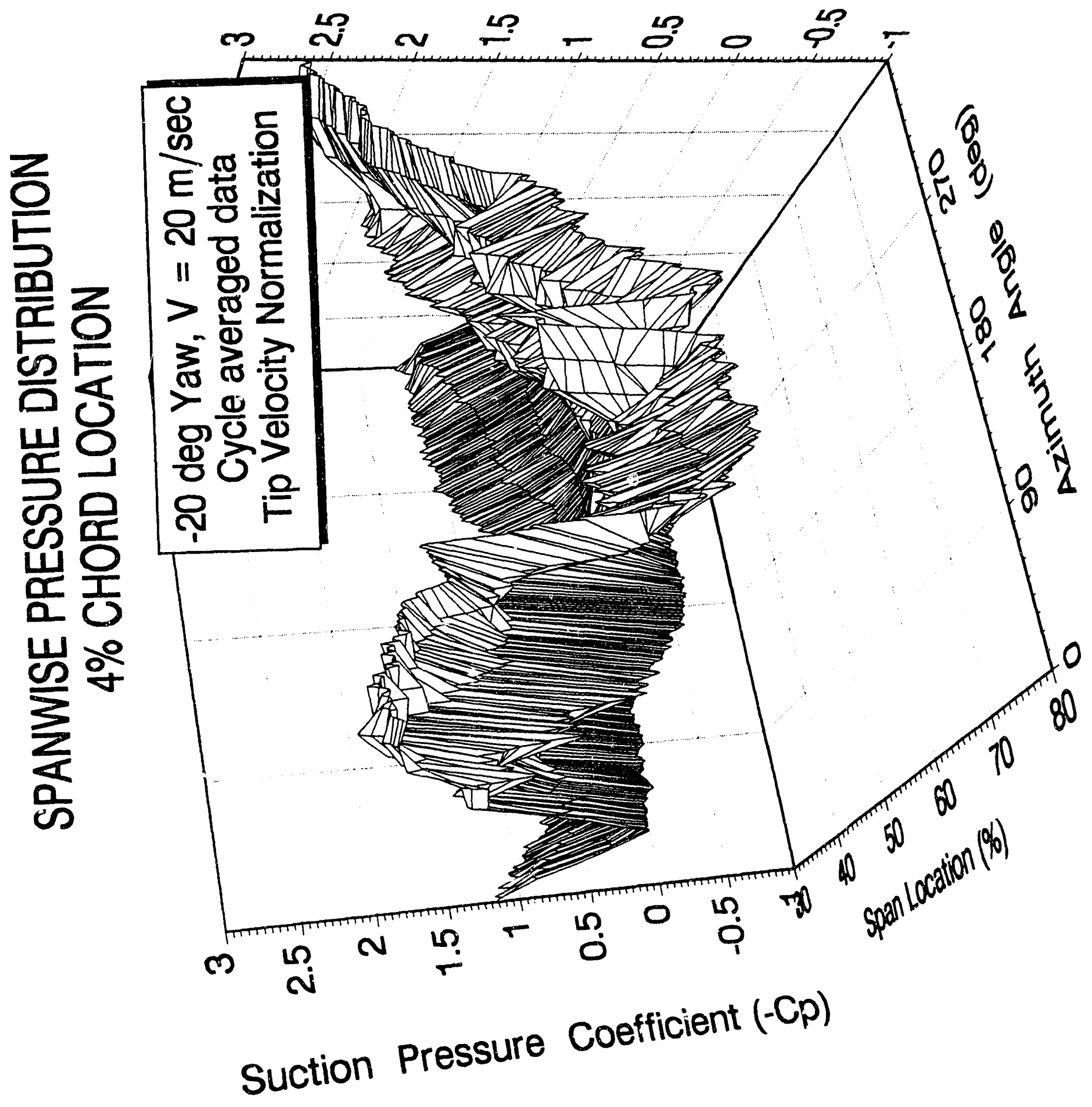


(d)-) 7Lololffe00 asnssadd wolpons

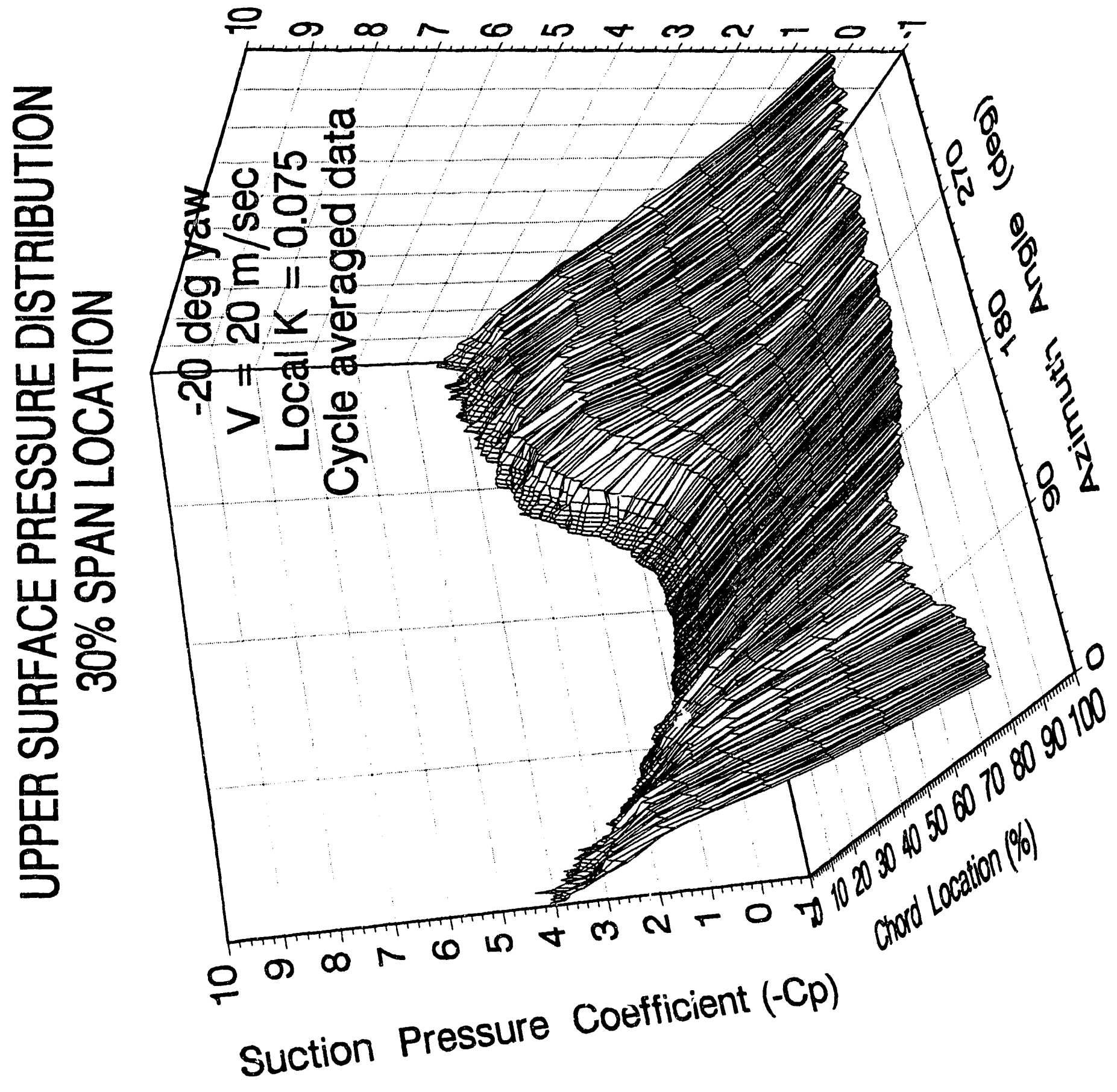




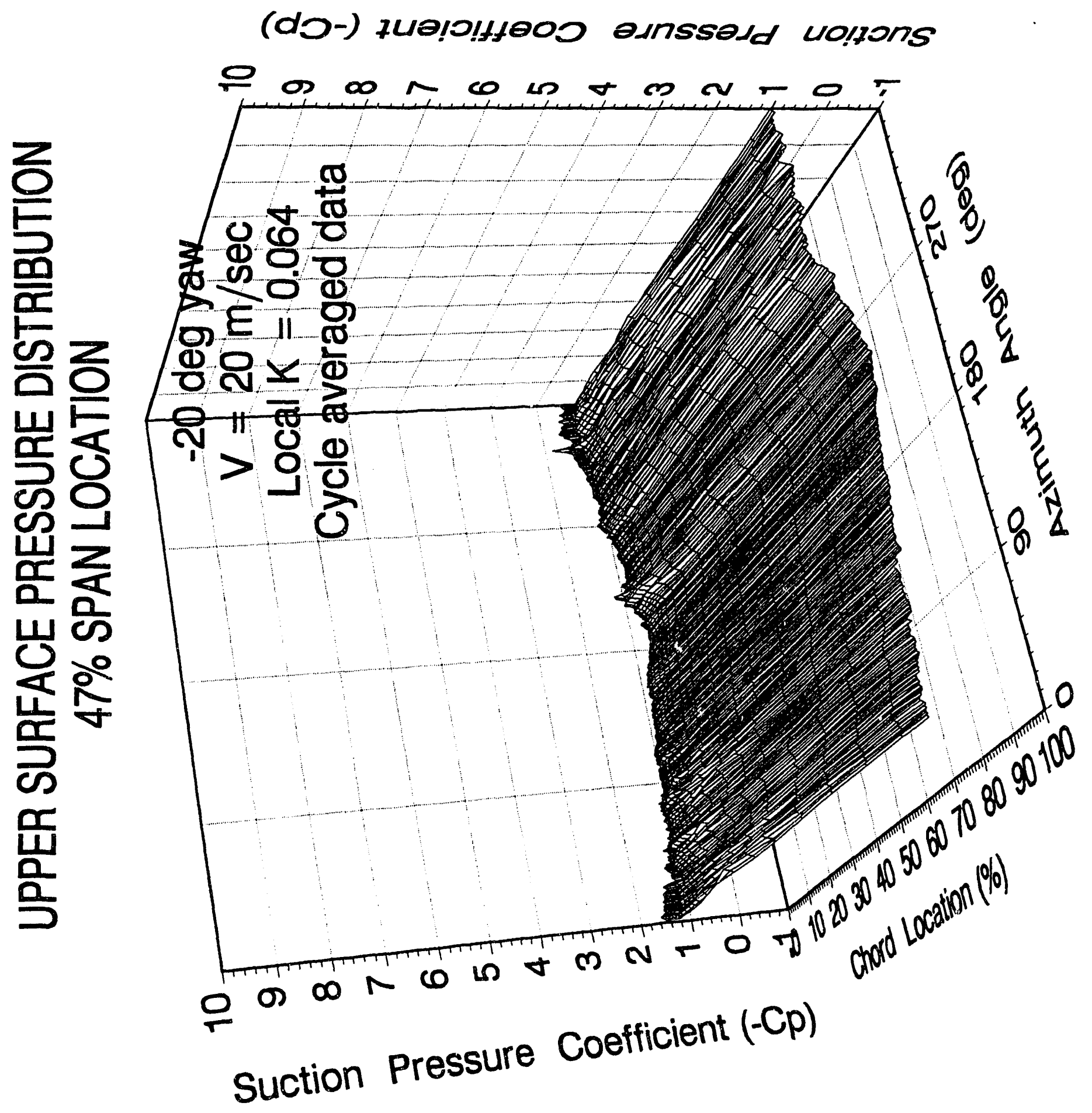


(d)-) 7wololfHe00 asnssejd wolfons

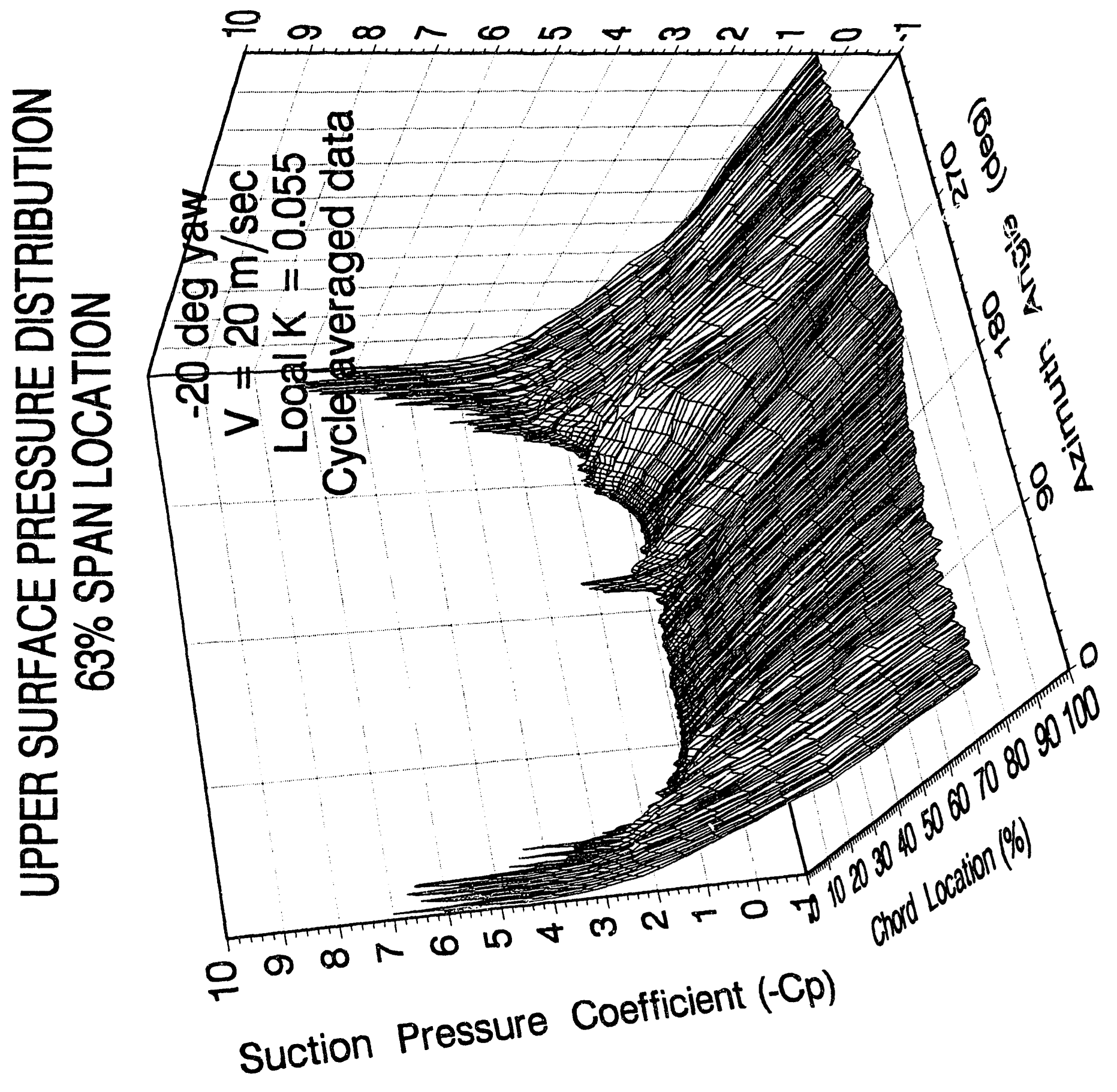




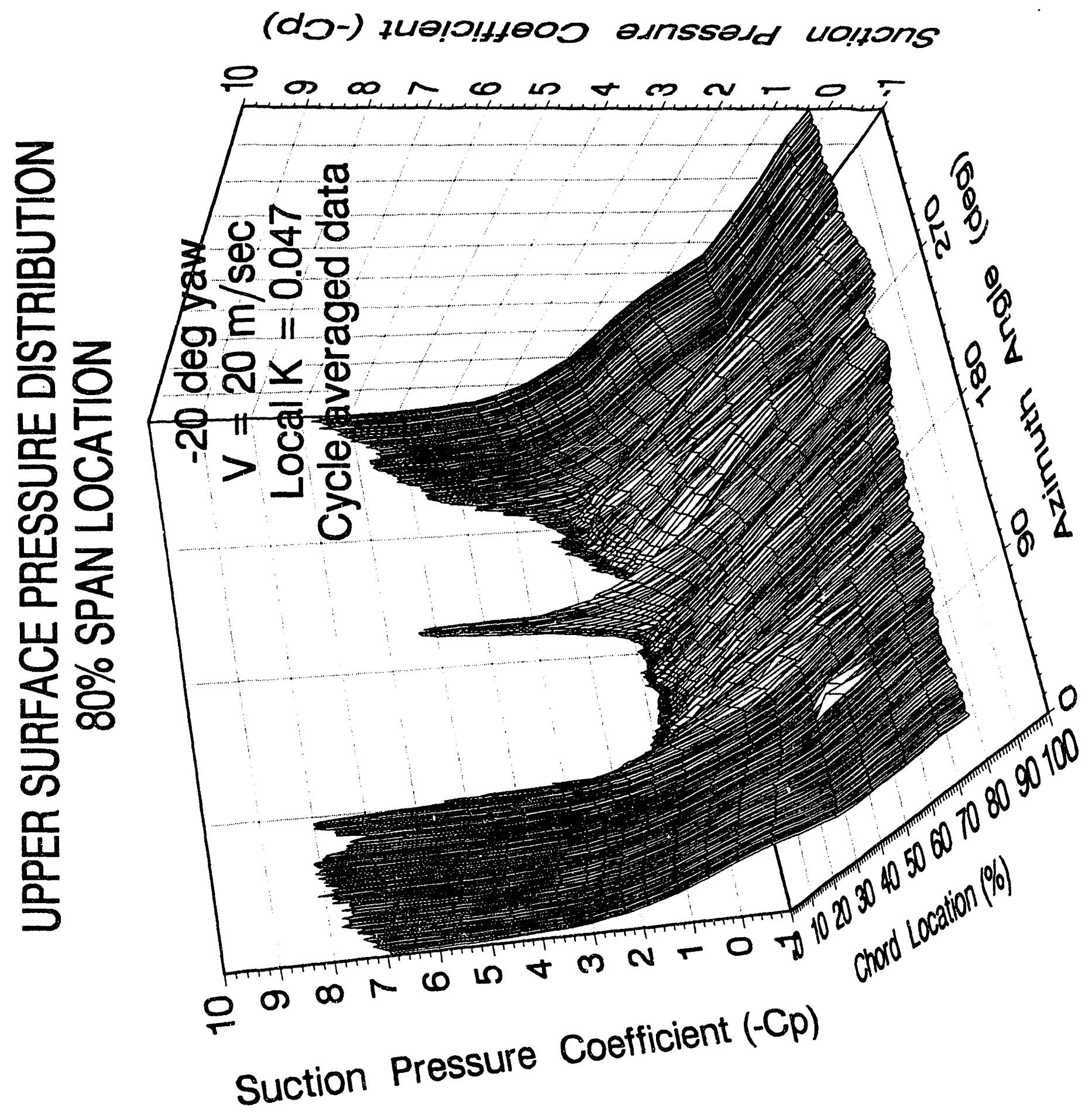




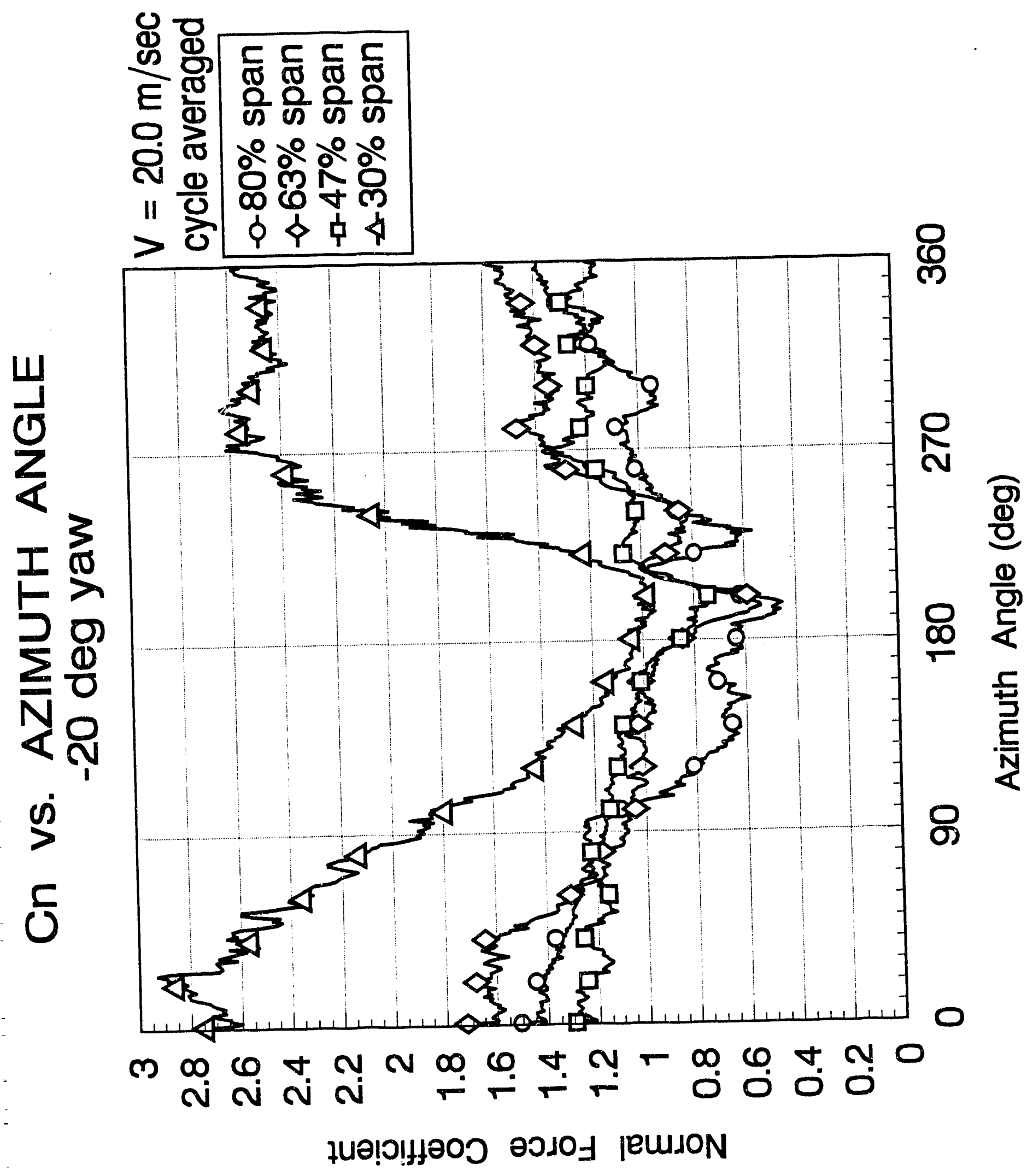



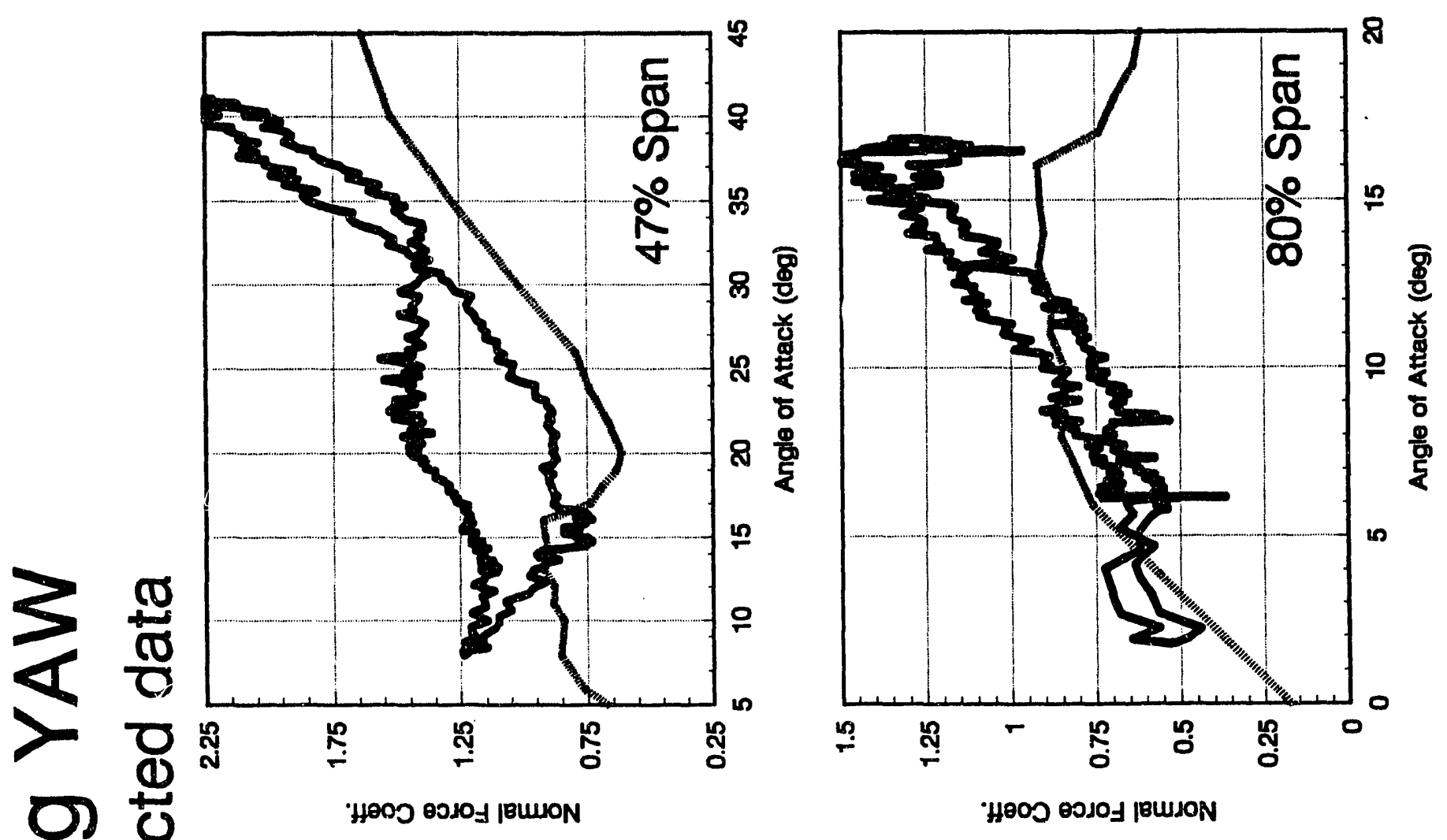

$\begin{array}{ll}\text { 1) } & 0 \\ 0 & 0\end{array}$
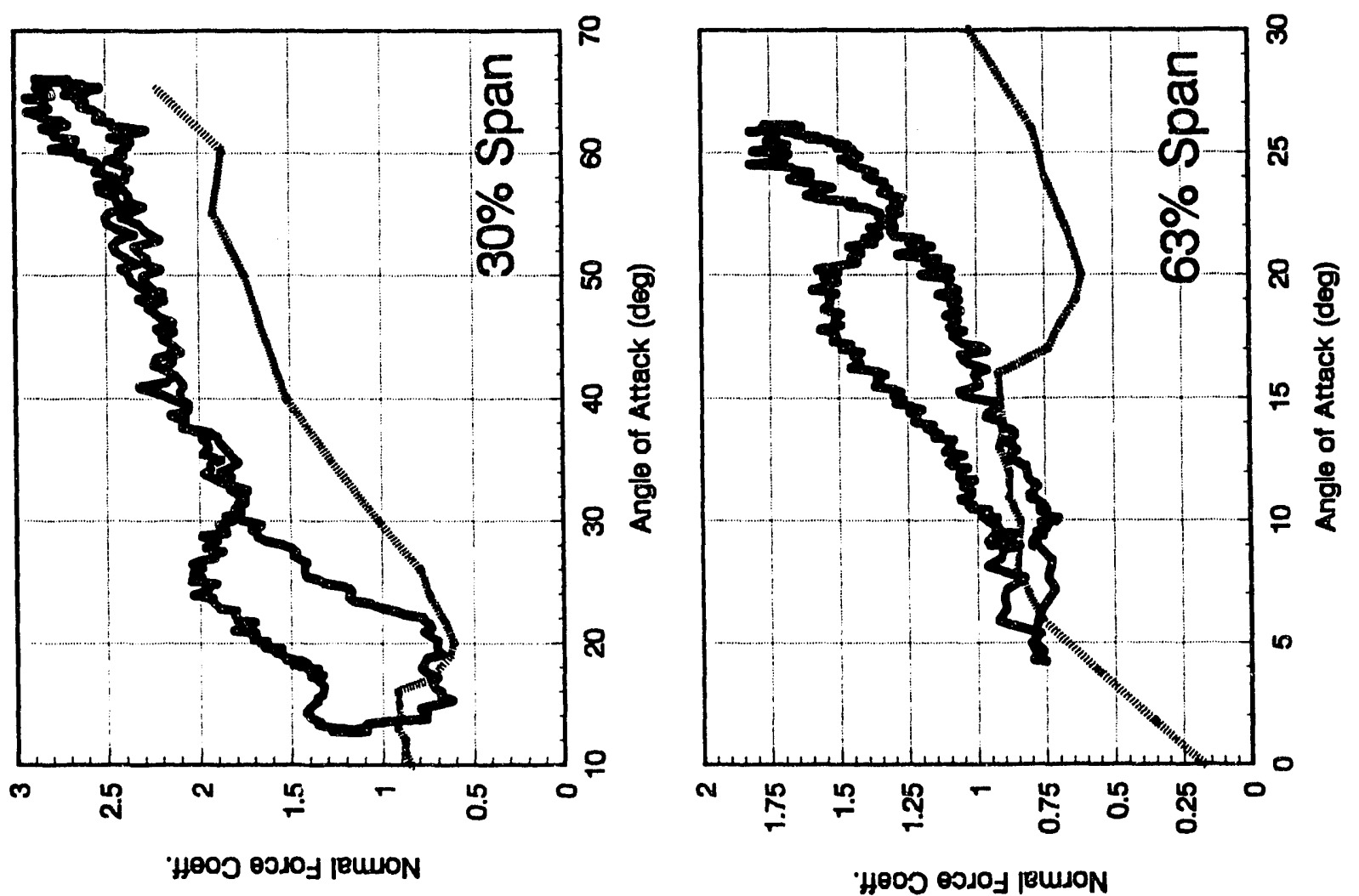

D-32 

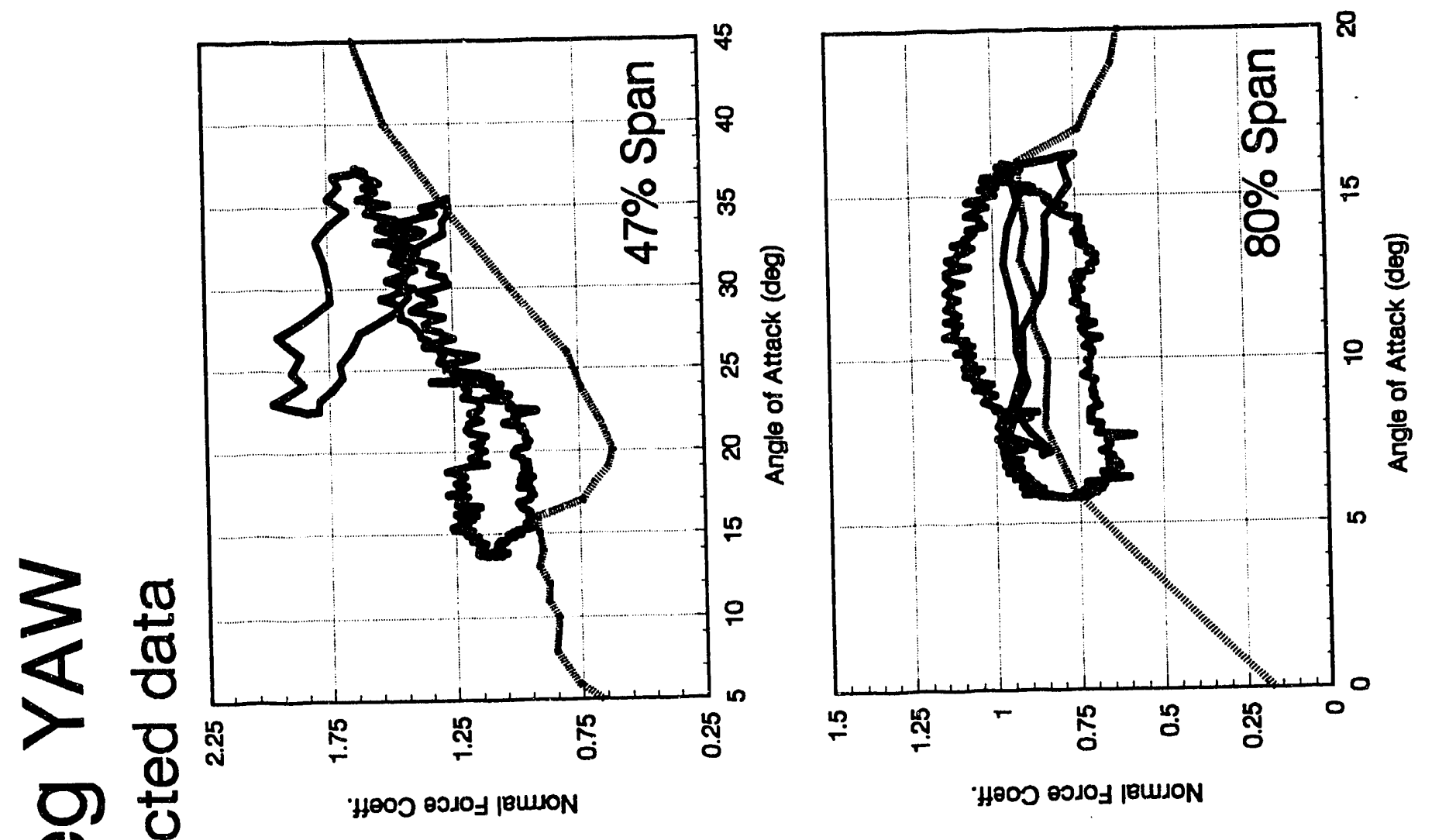

\begin{tabular}{ll}
$\Phi$ & 0 \\
0 & 0 \\
\hline & 0
\end{tabular}

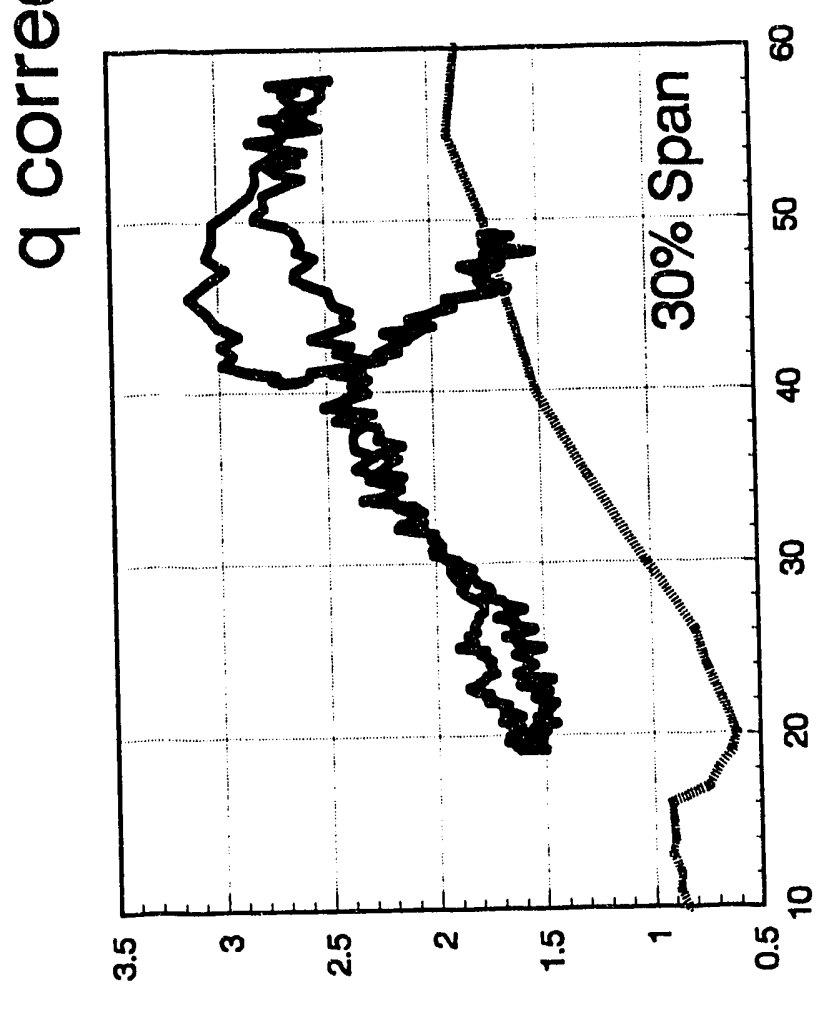

HOOJ OOSOJ IBULON
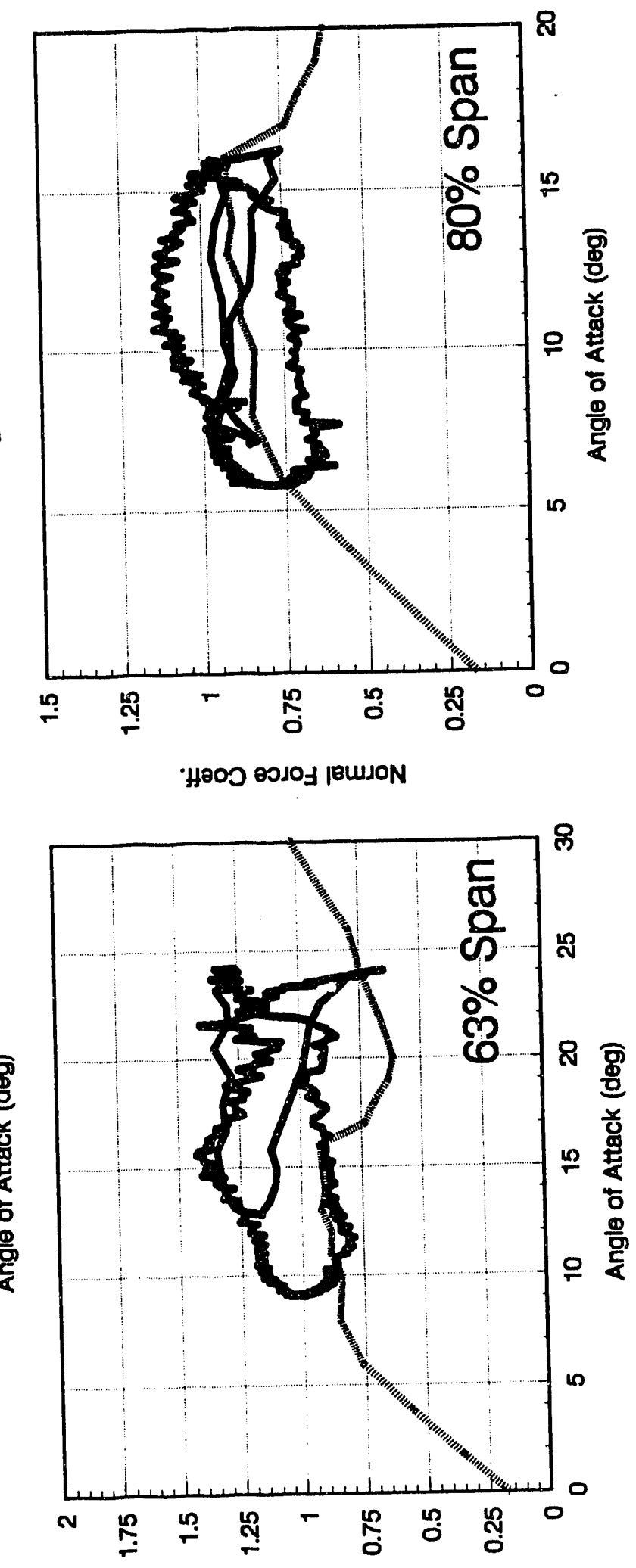

HOOJ ODJOJ IEULON 


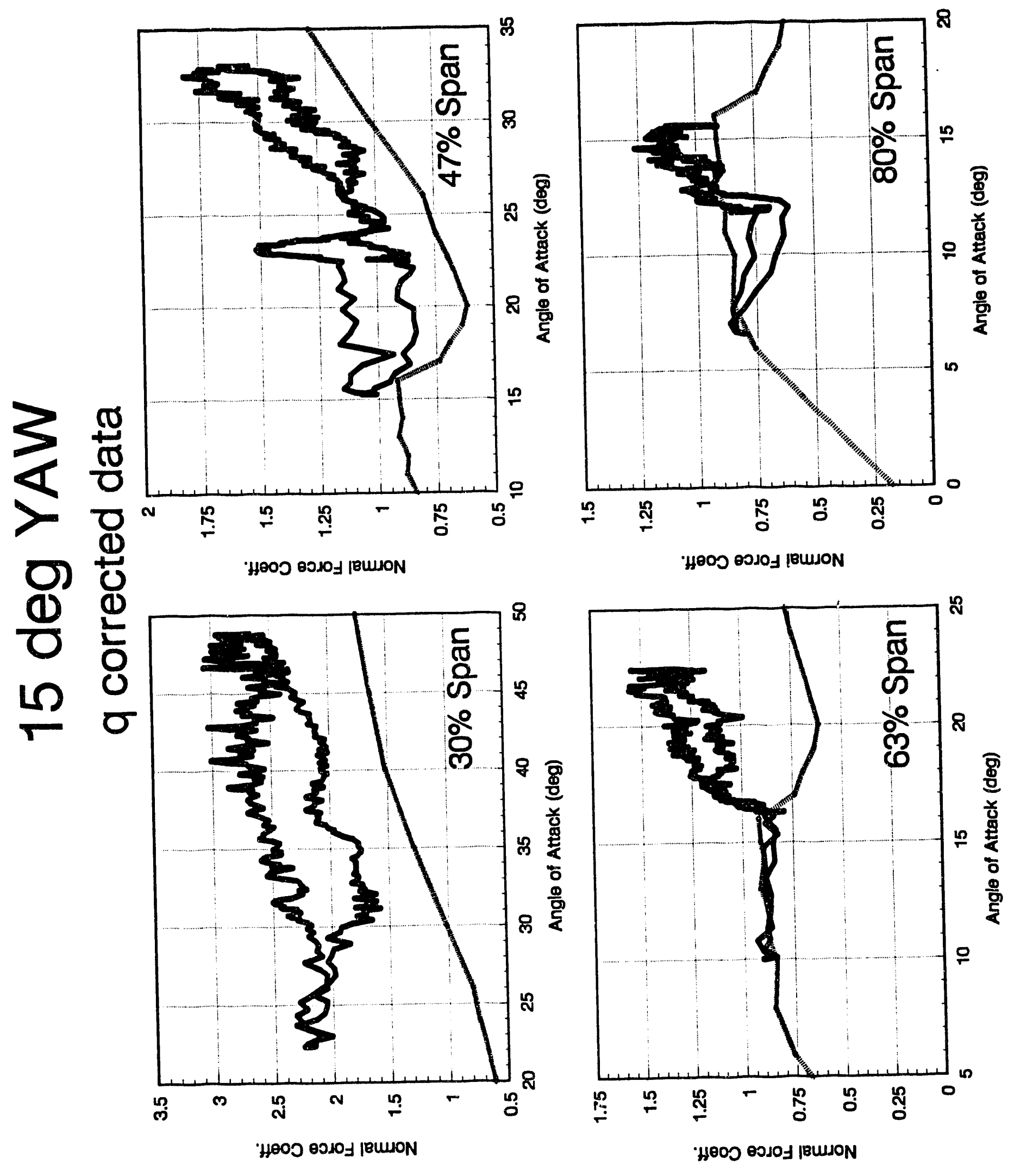



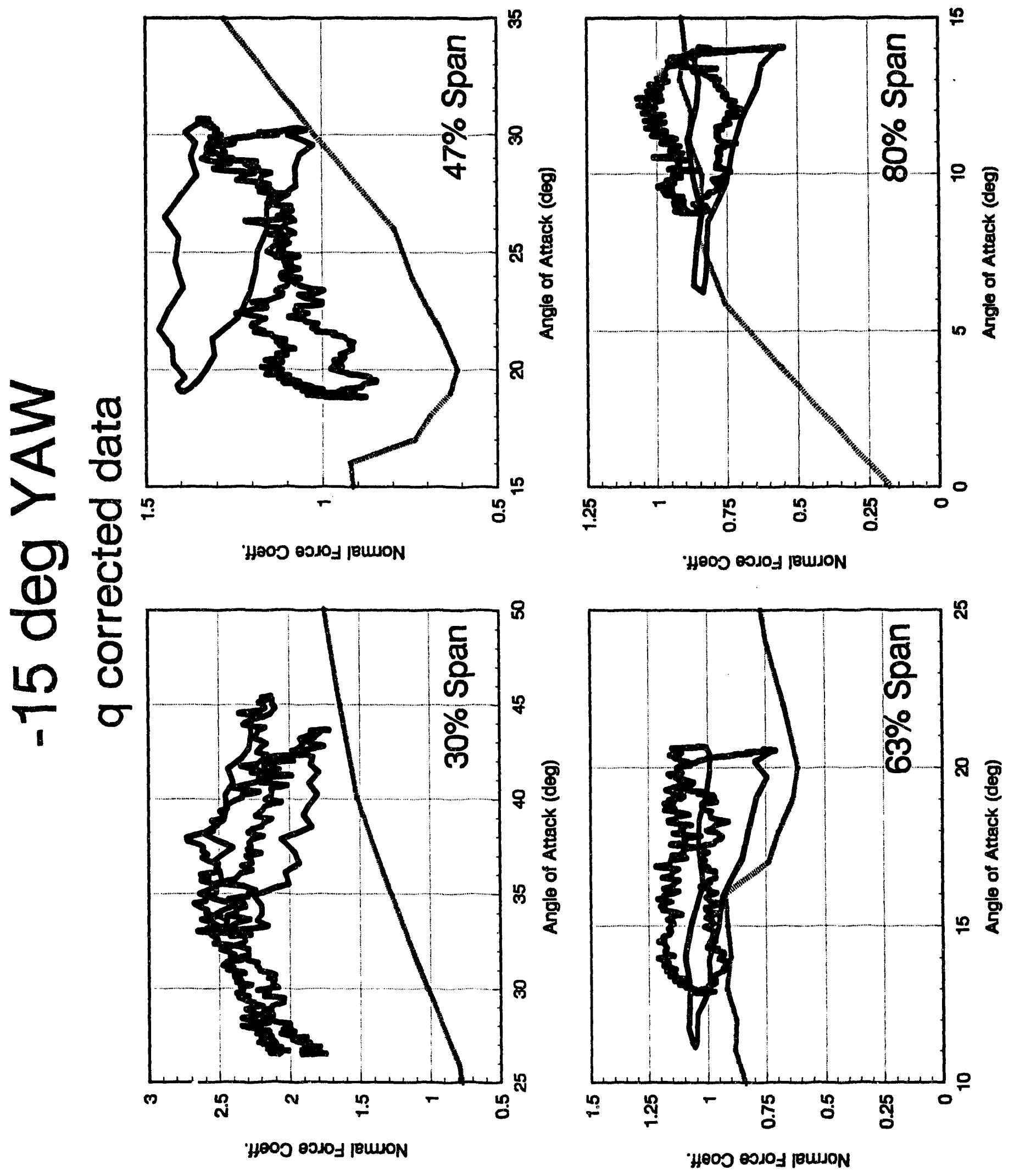

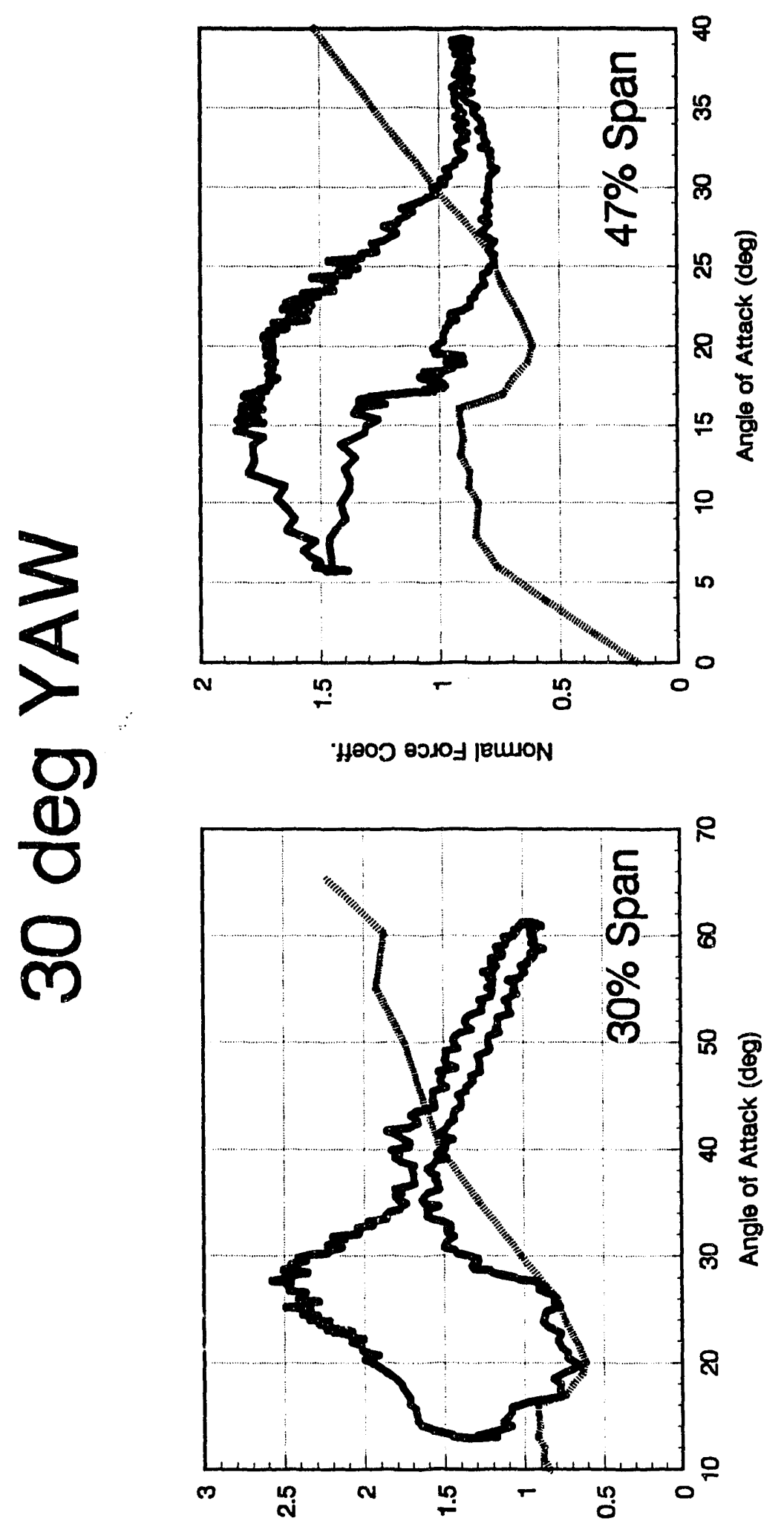

HOOJ OONOA IEULON

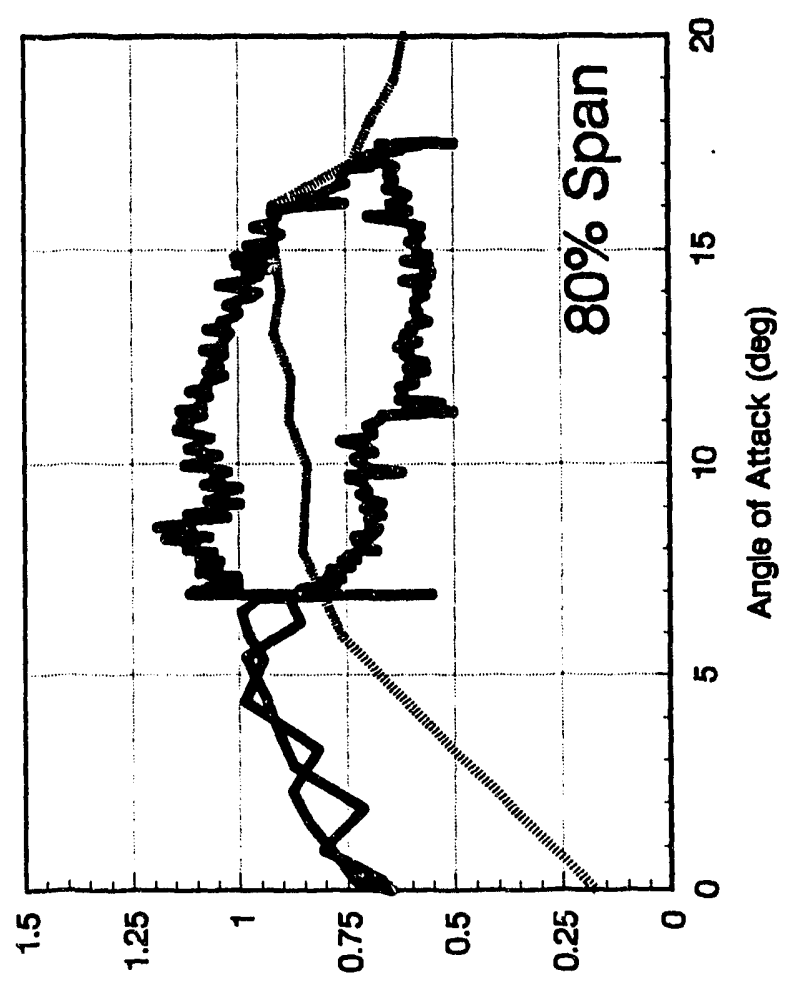

HOOJ อDJOA IBUION

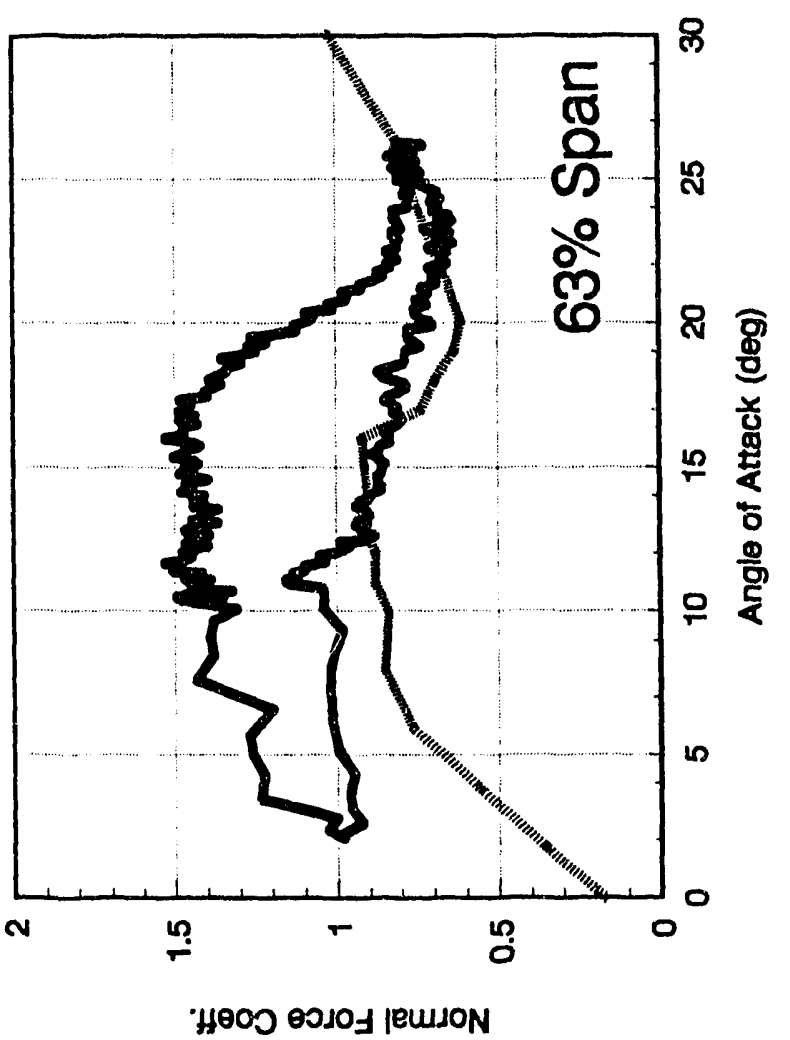



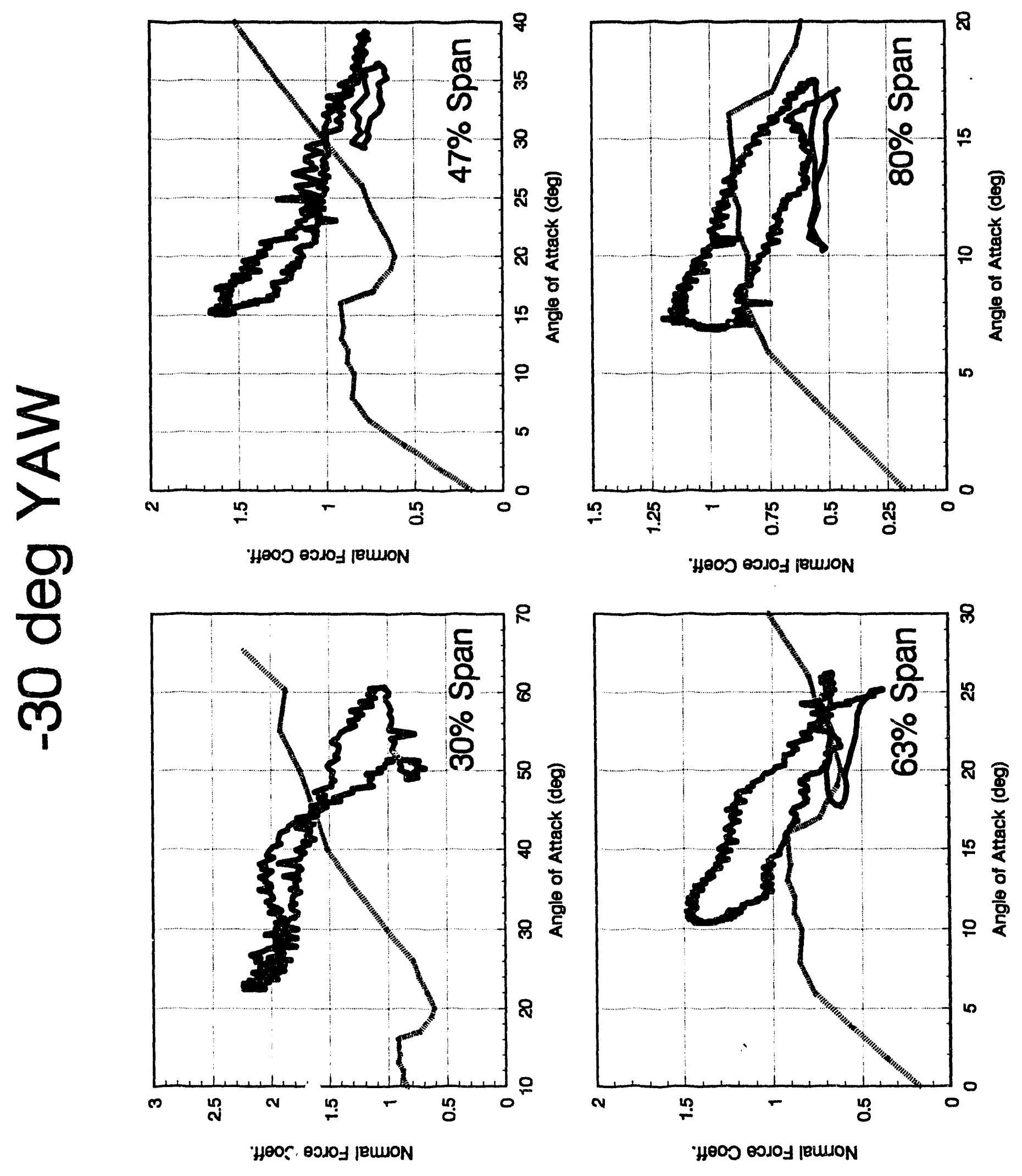

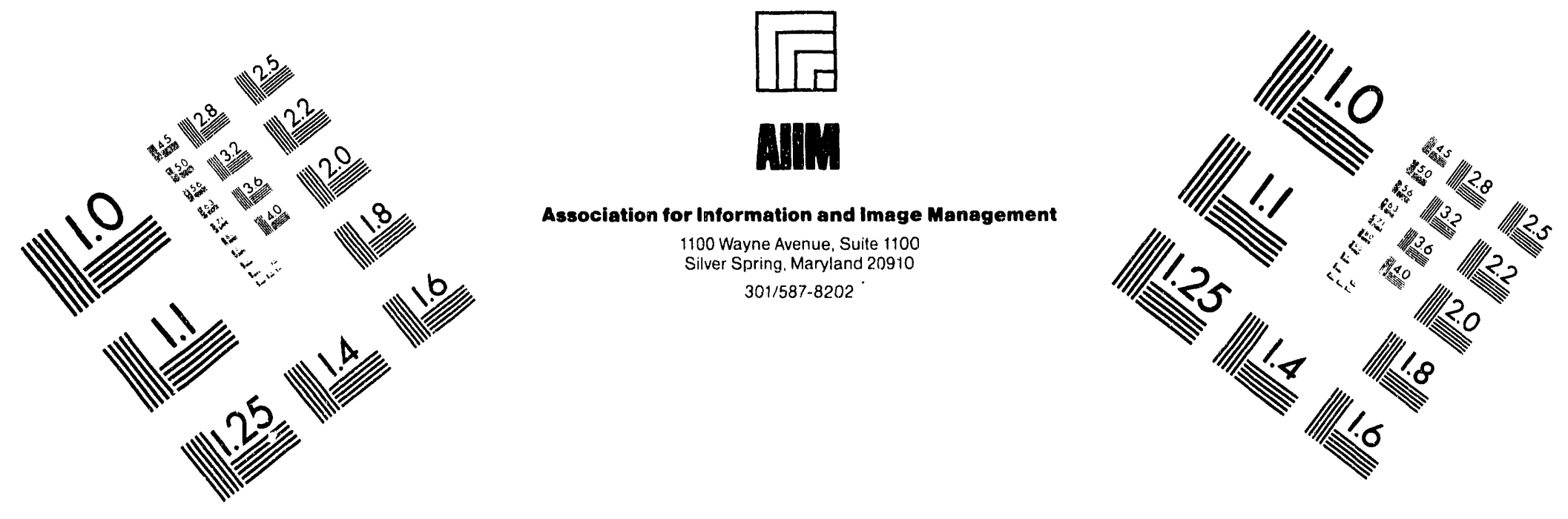

\section{Centimeter}

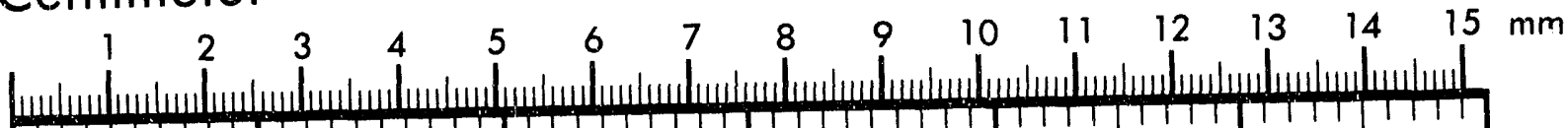

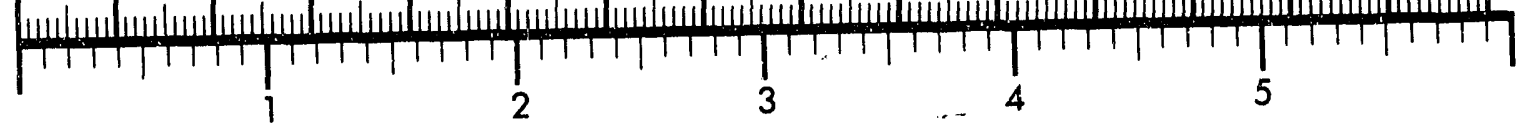
Inches
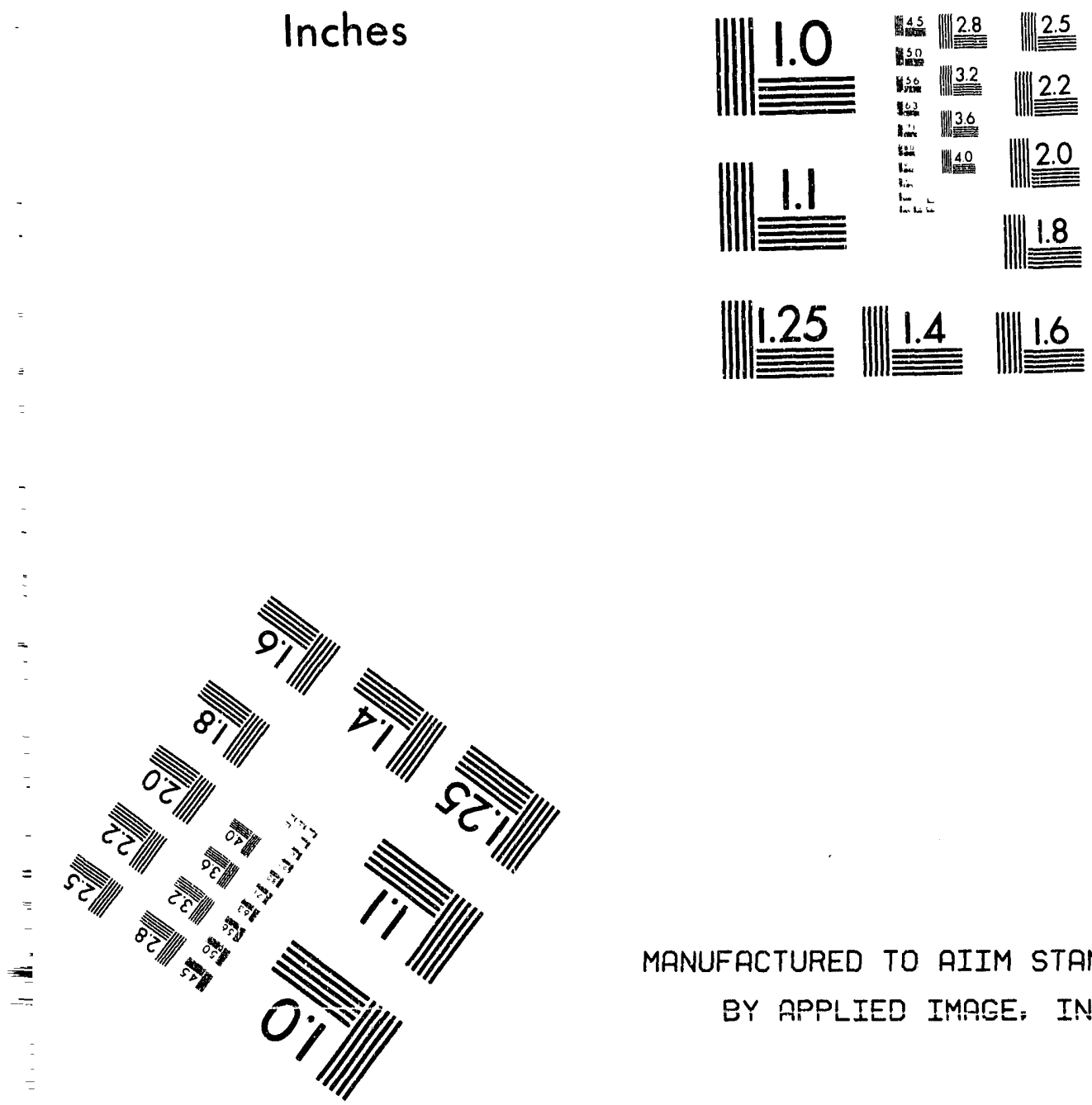

MANUFACTURED TO AIIM STANDARDS

BY APPLIED IMPGE; INC.

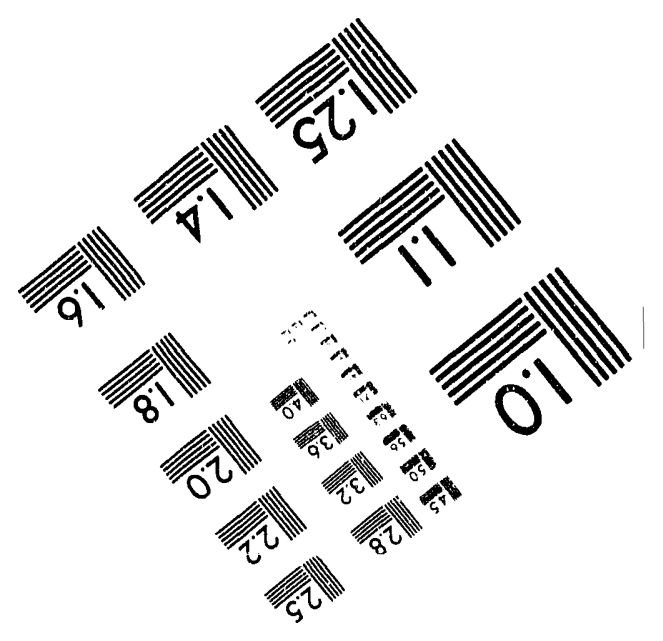



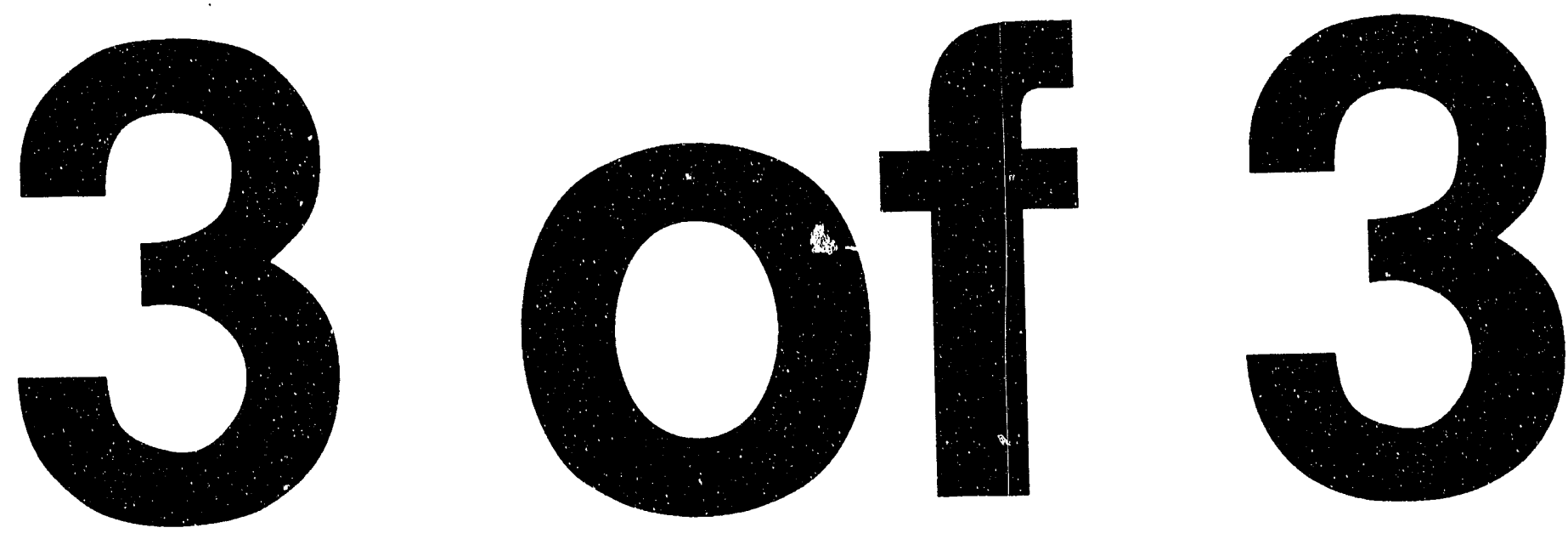

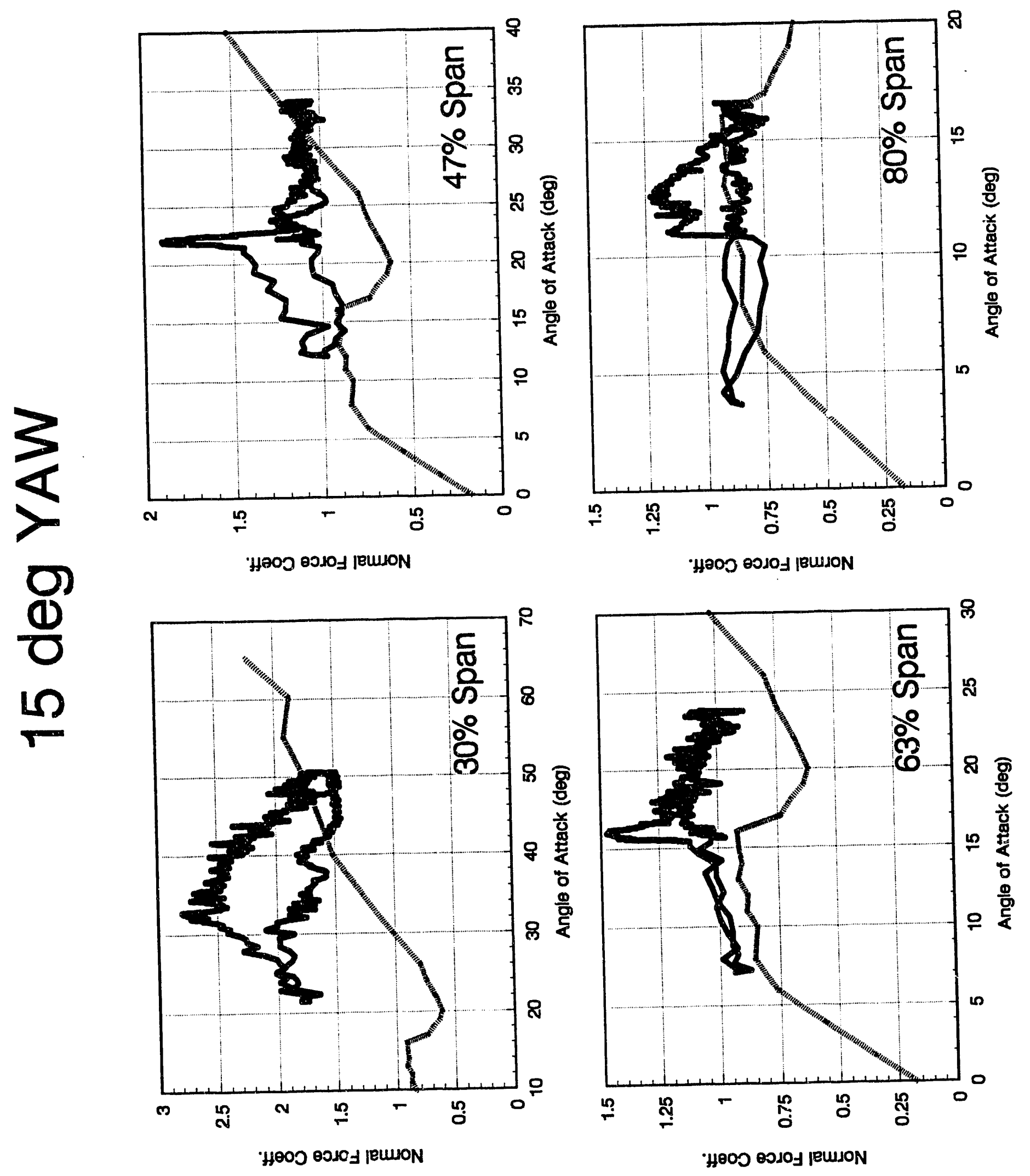

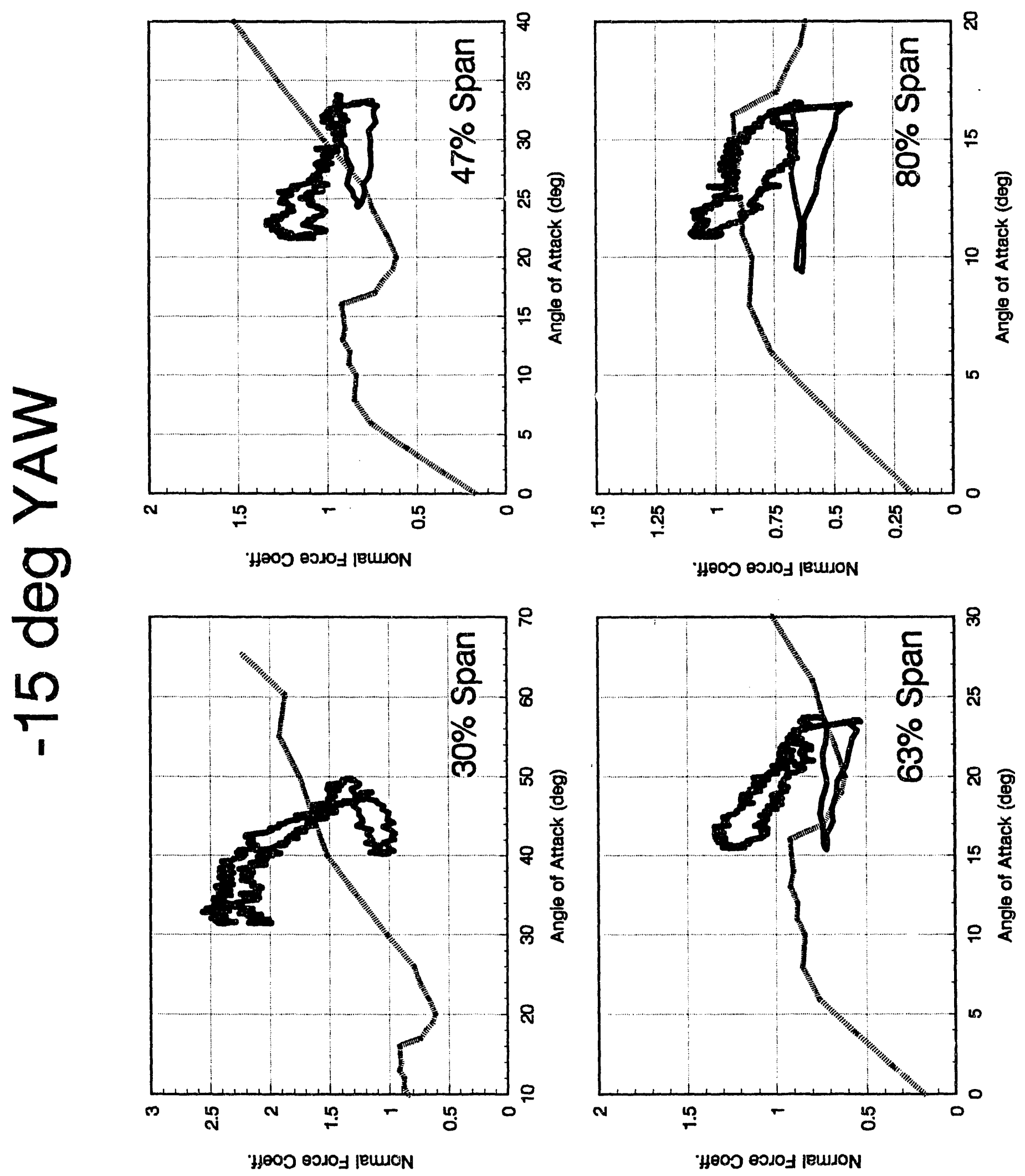

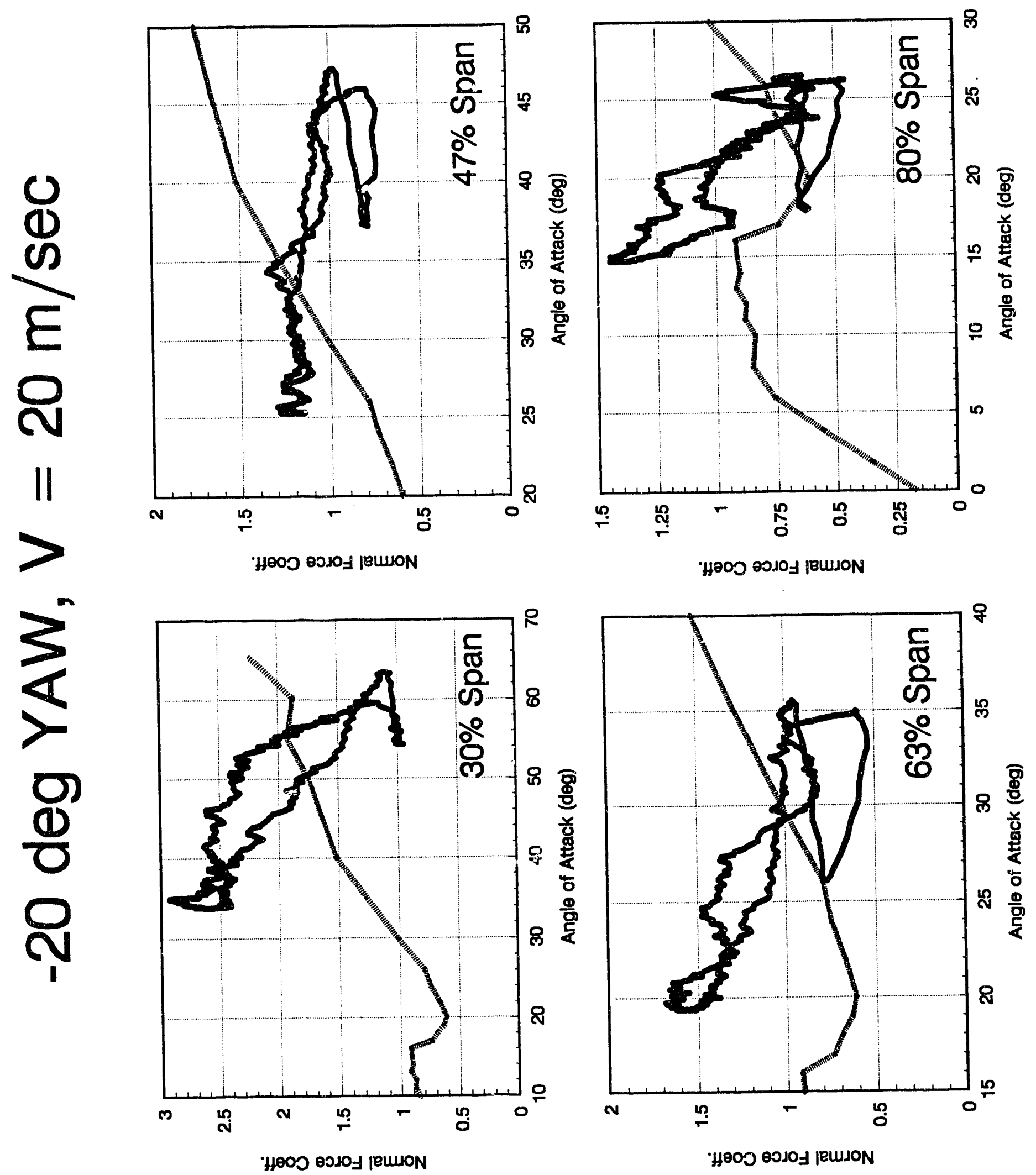


\section{Appendix E}

\section{Yaw Error Test Cases, Single Cycle Data}




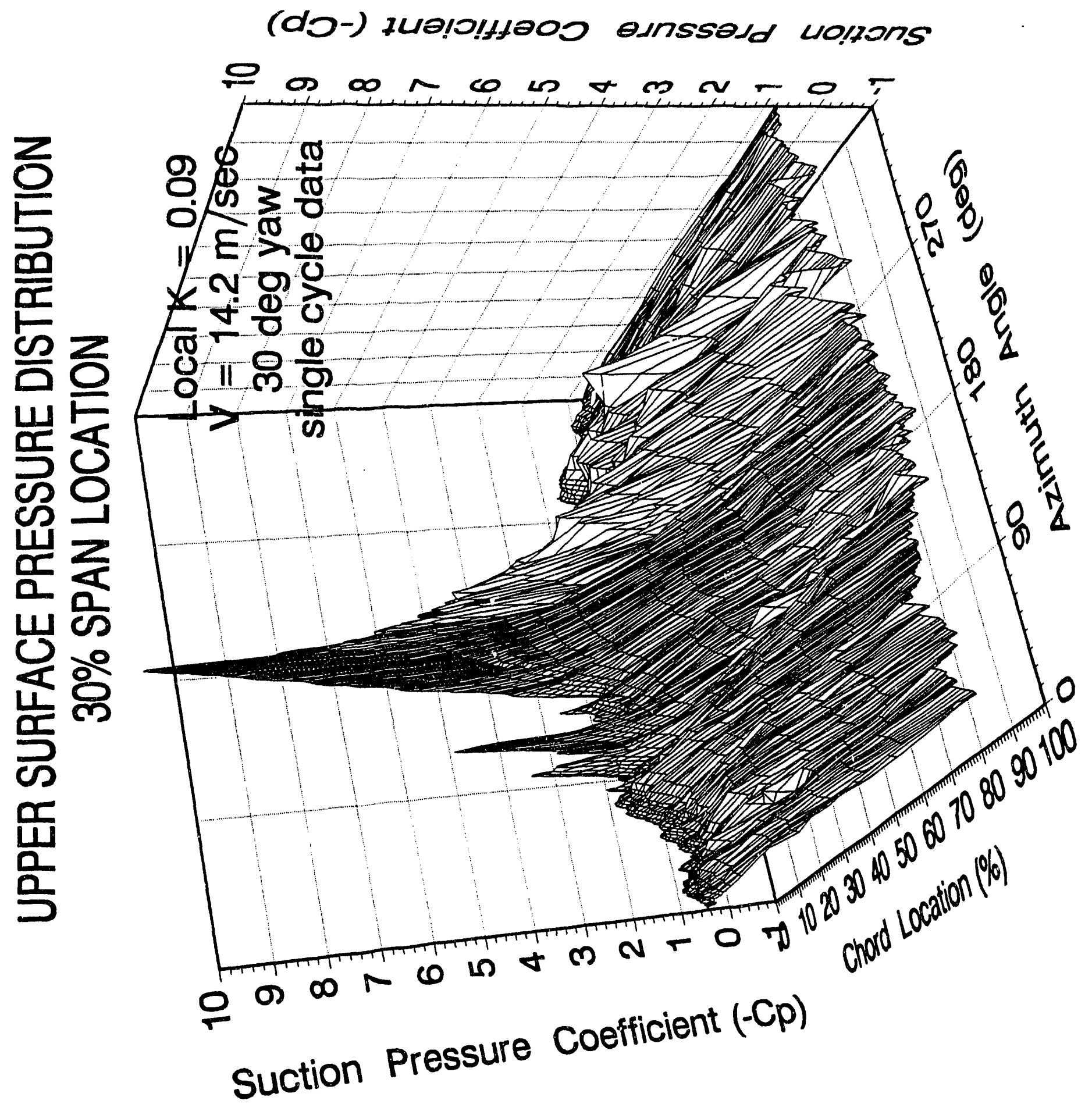




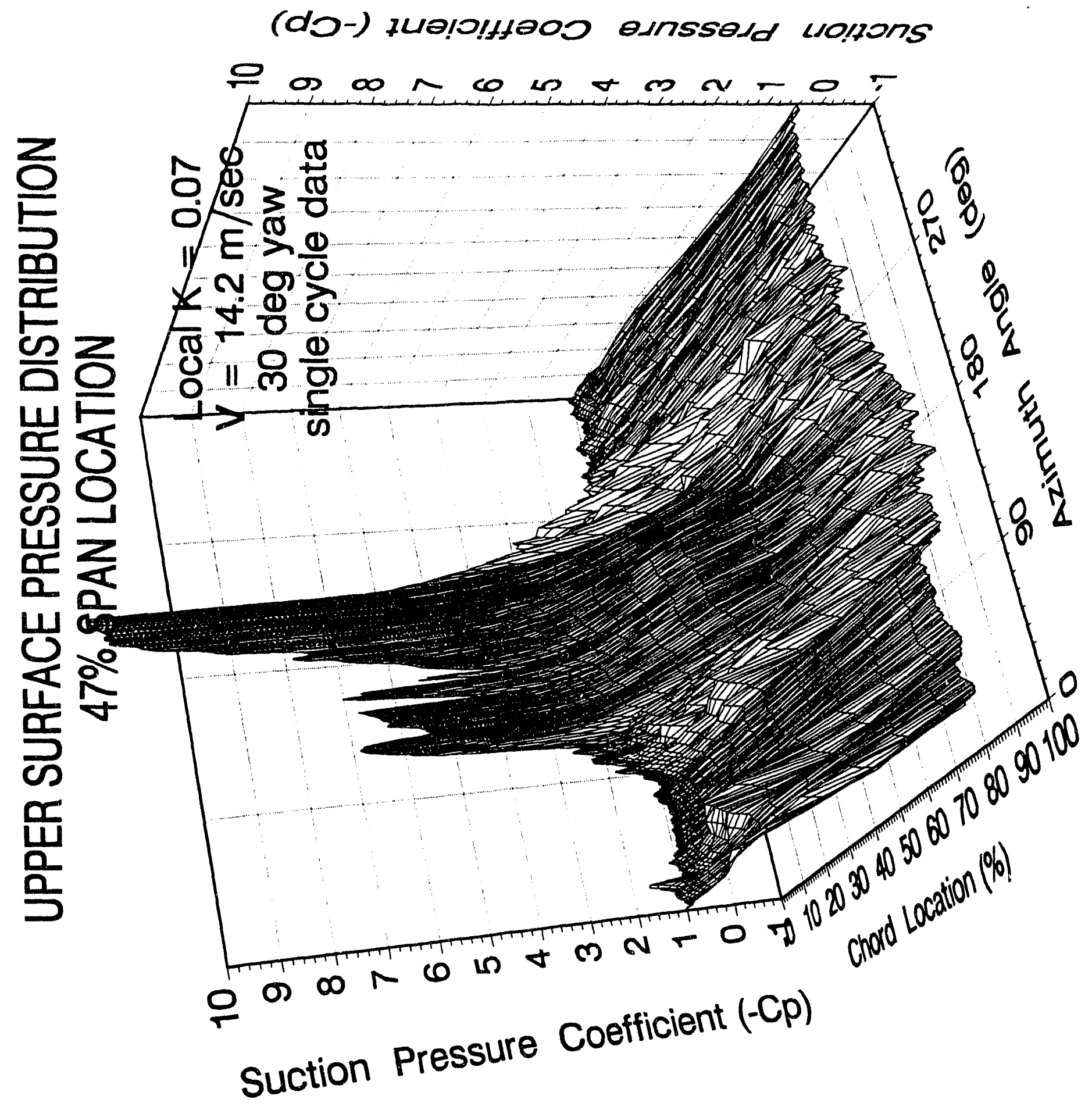




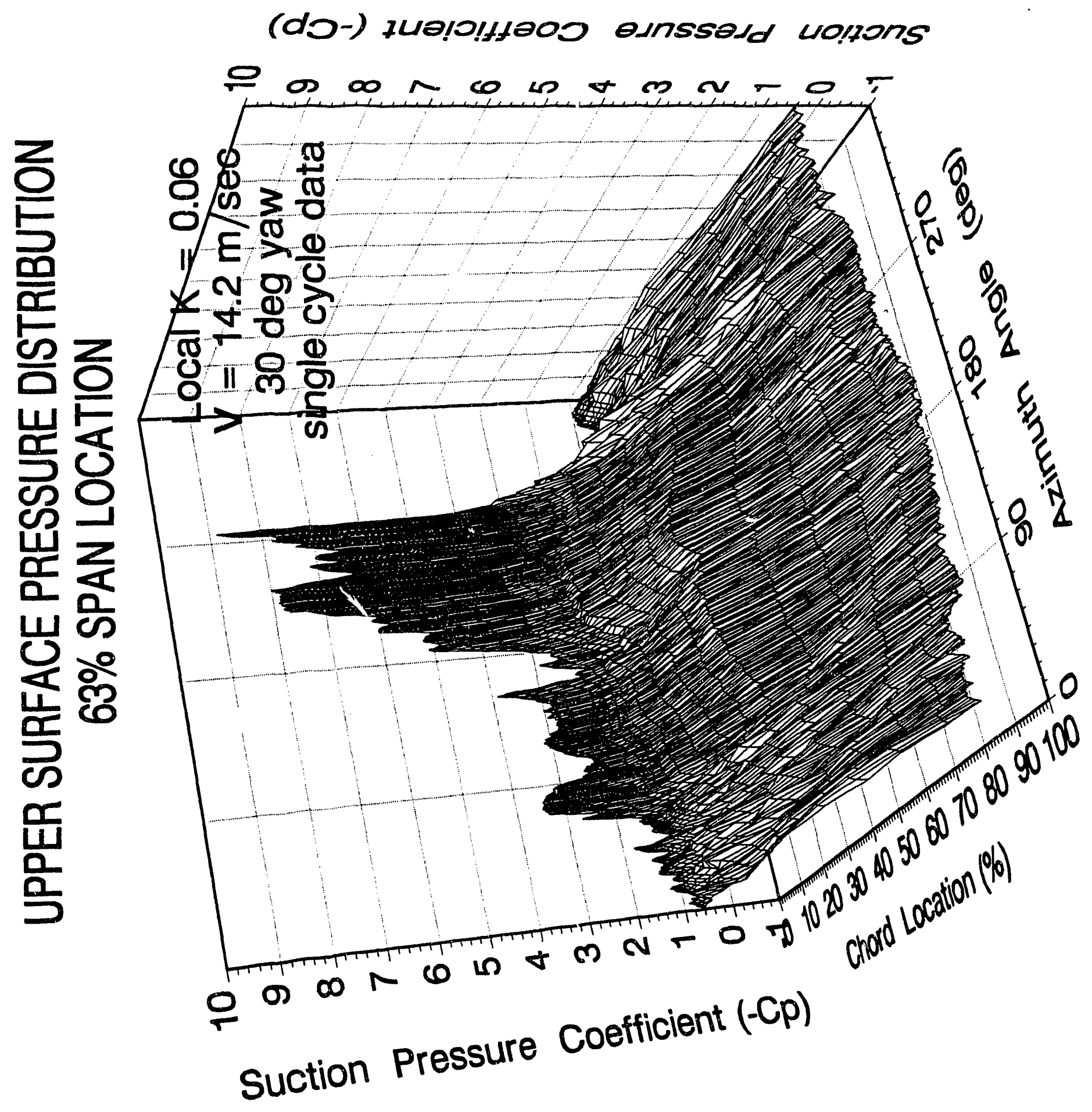




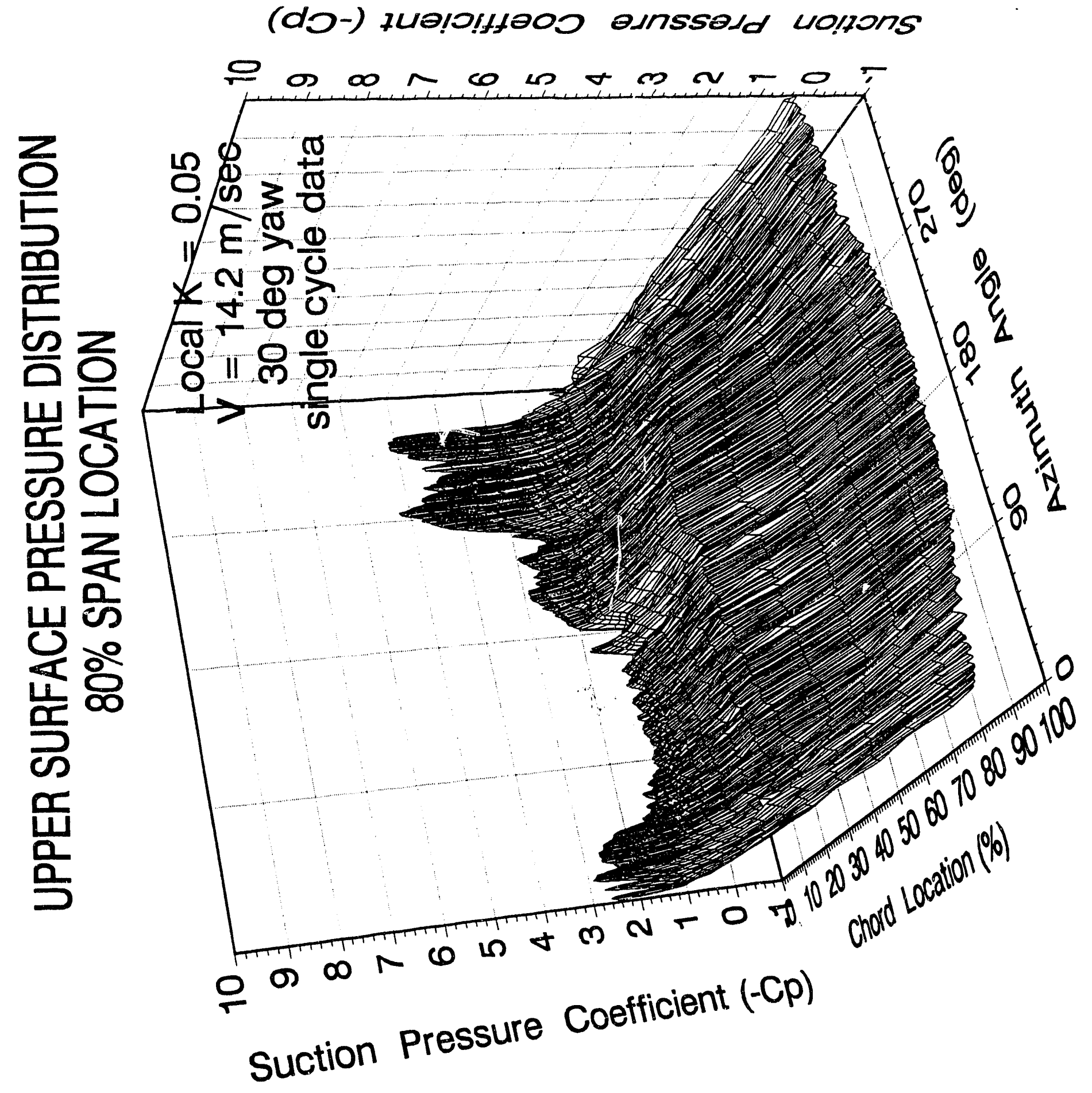




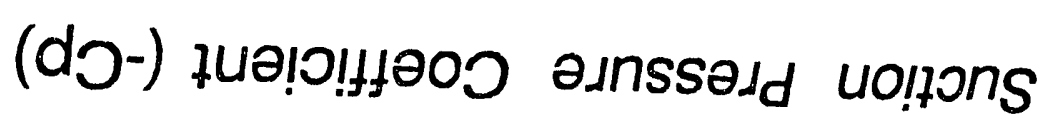

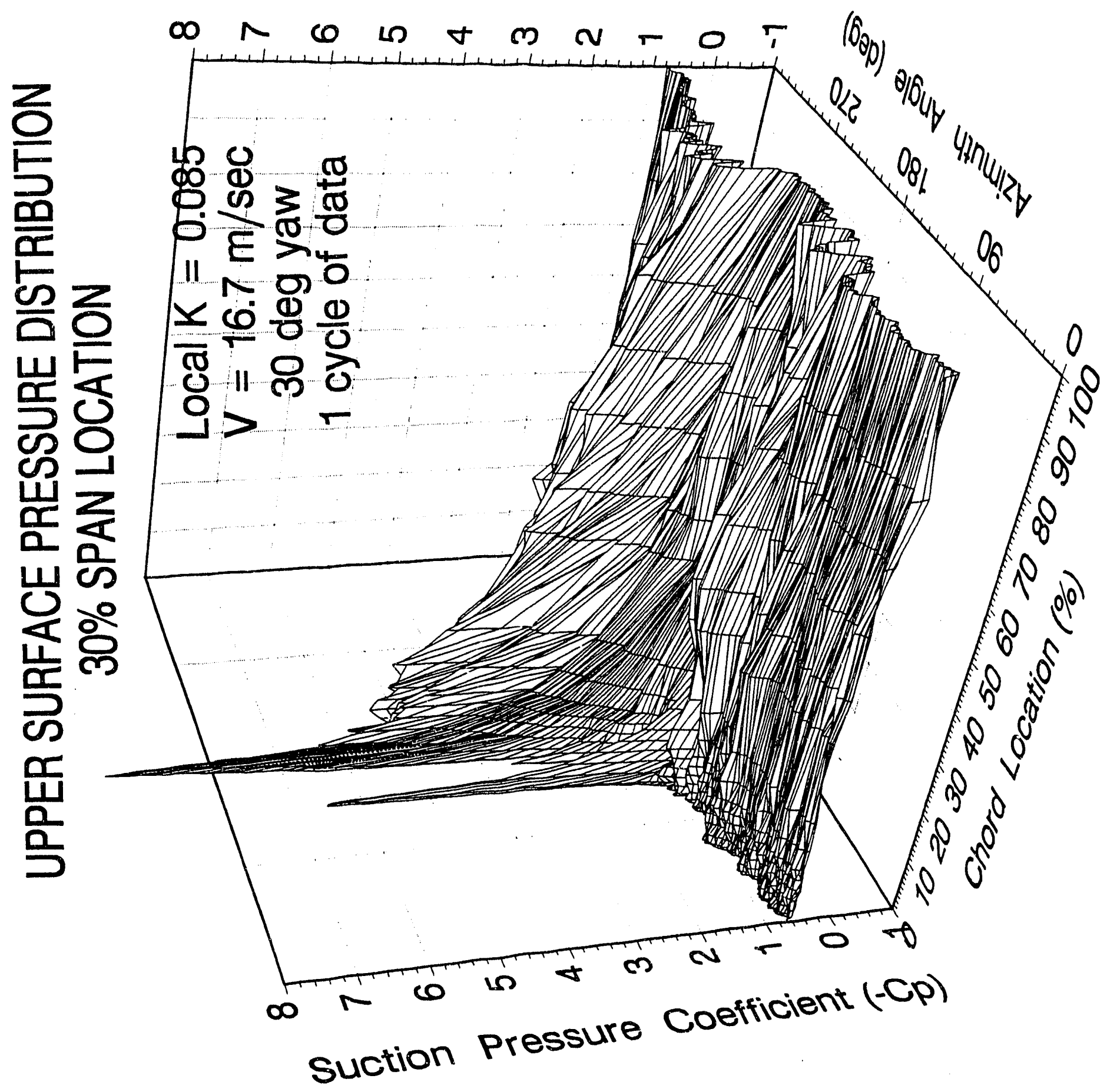




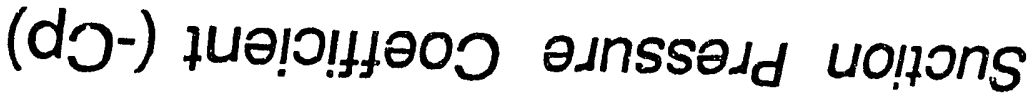

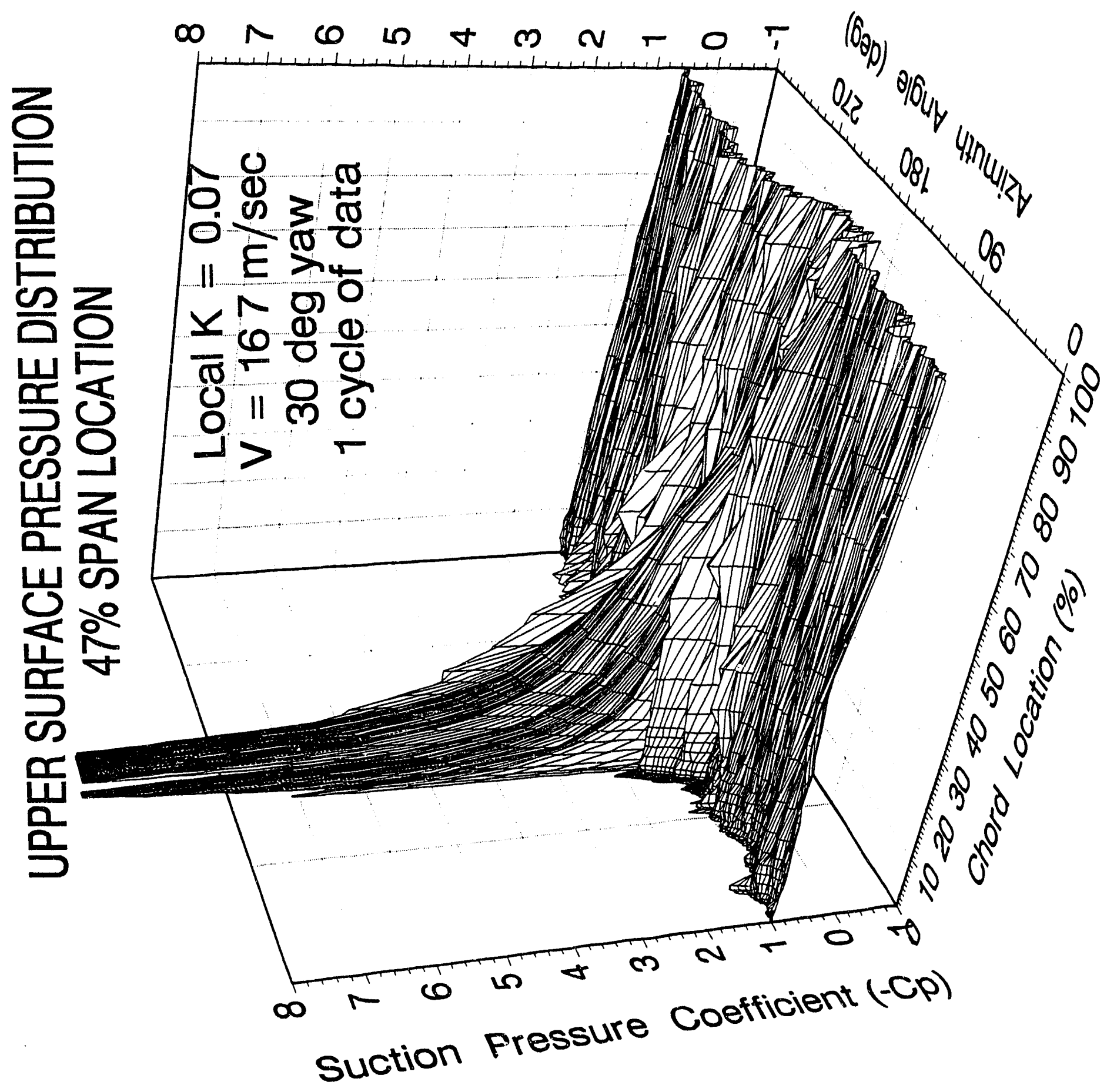




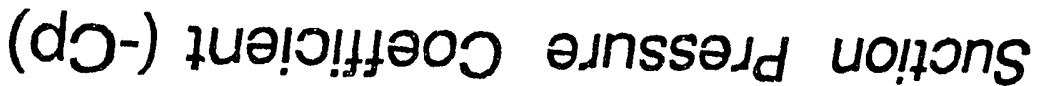

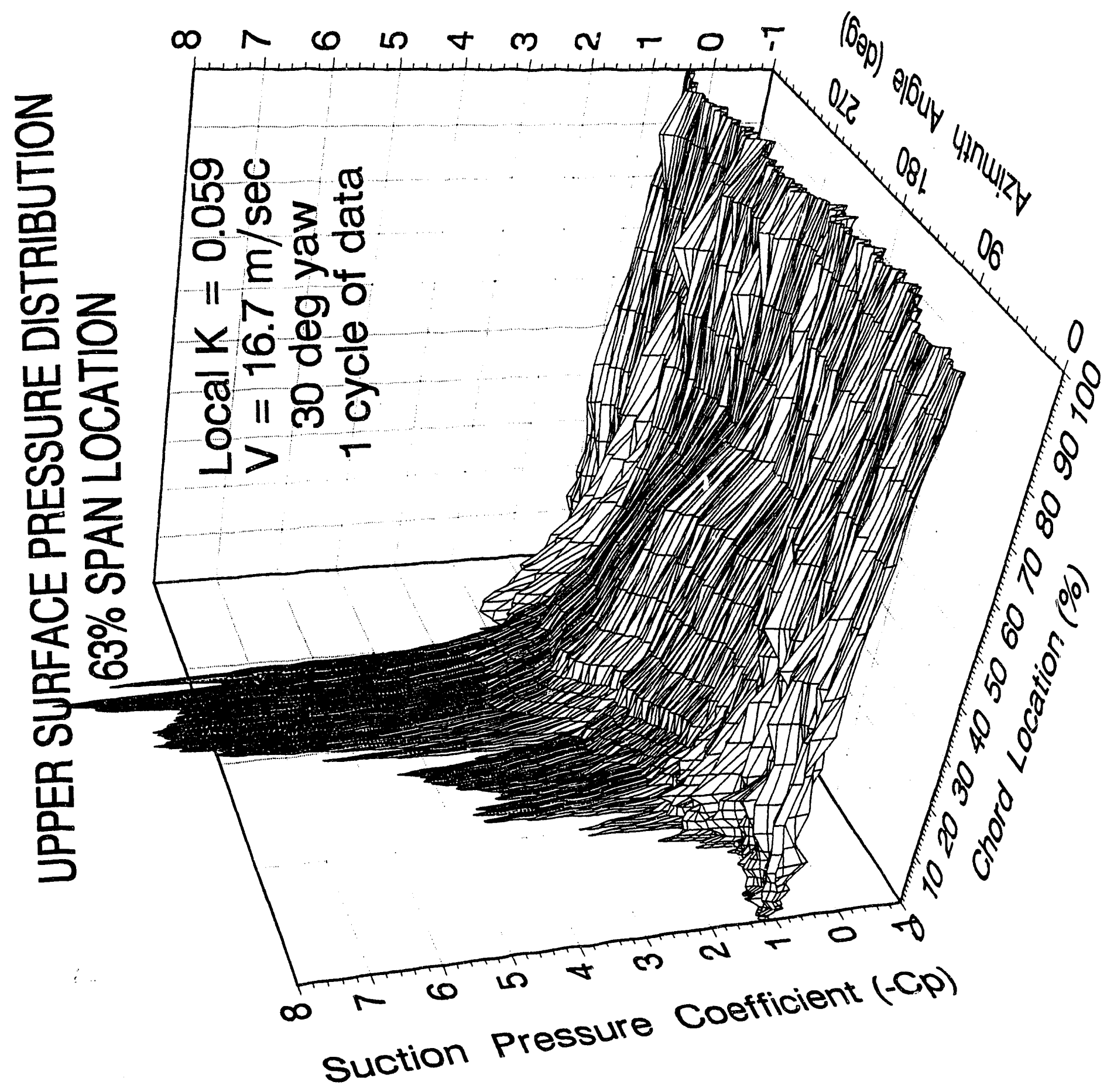




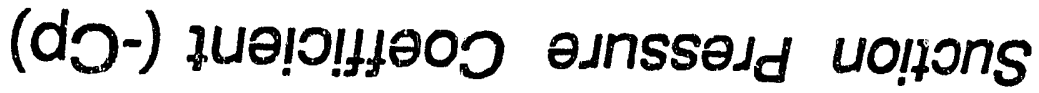

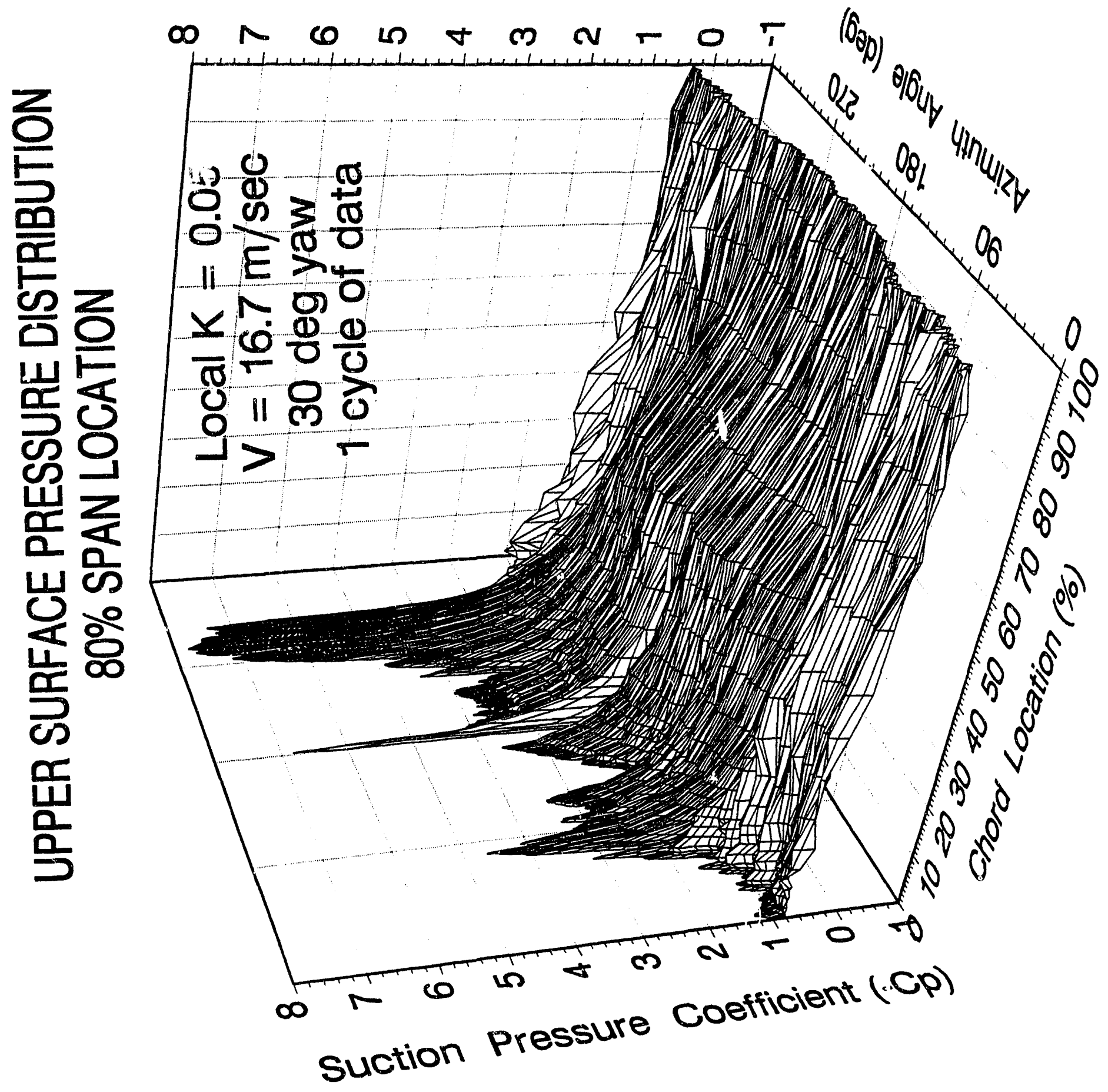




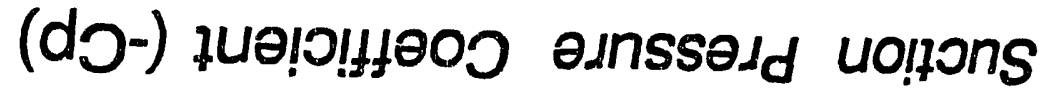

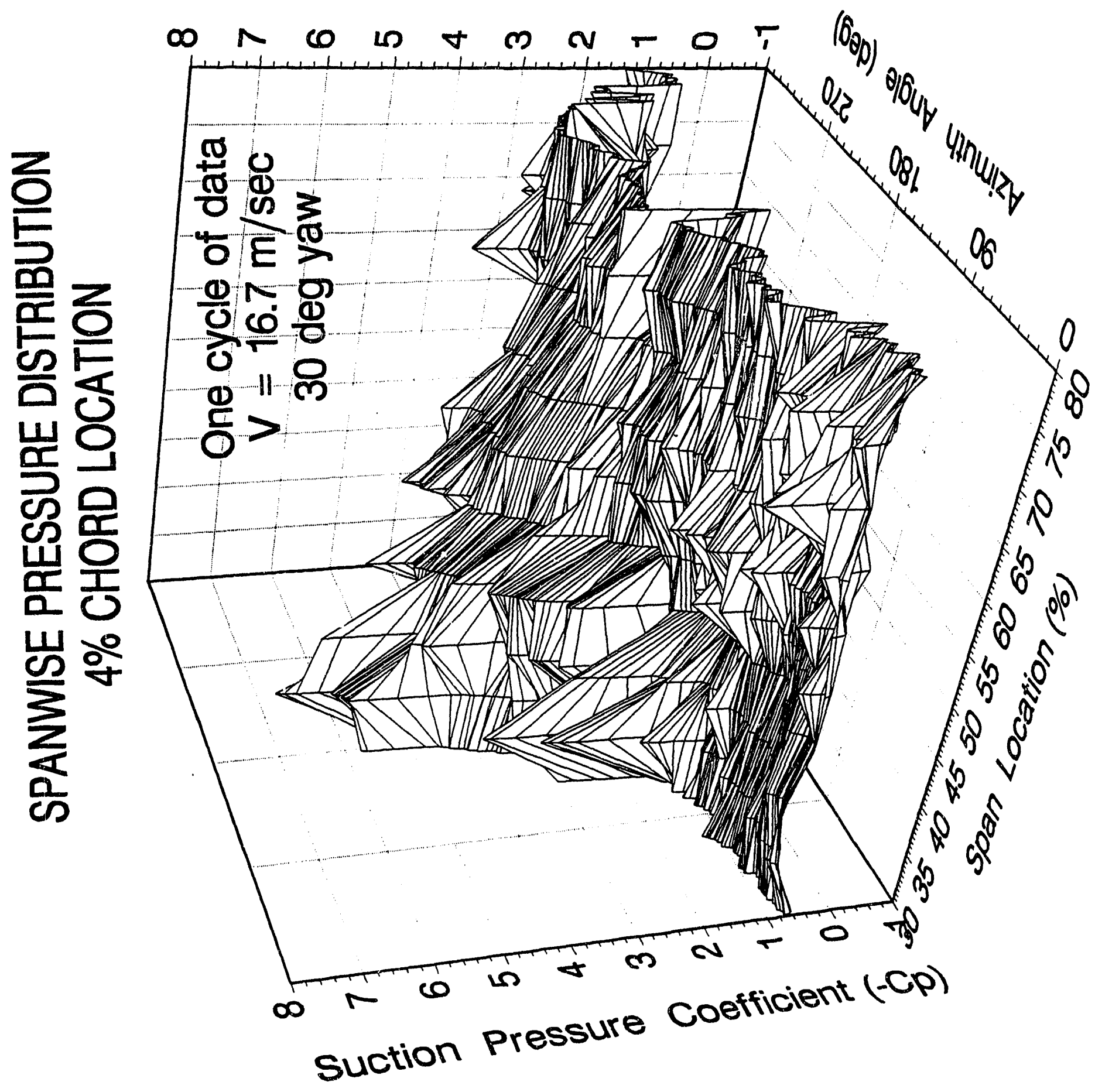




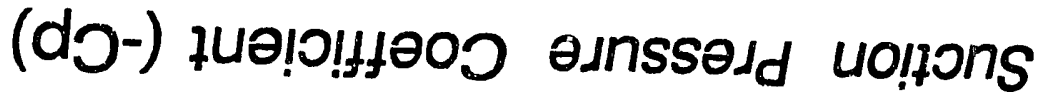

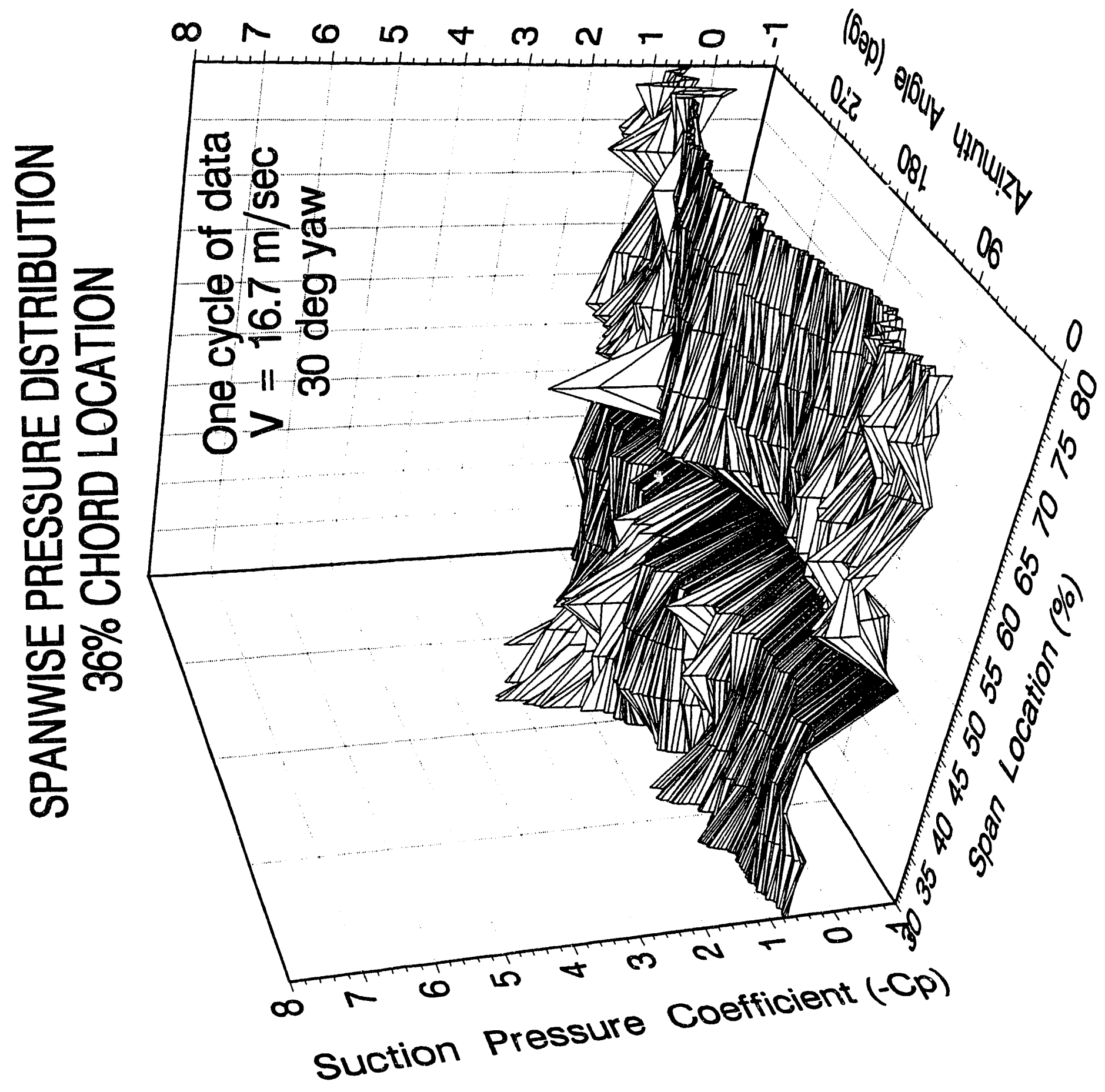




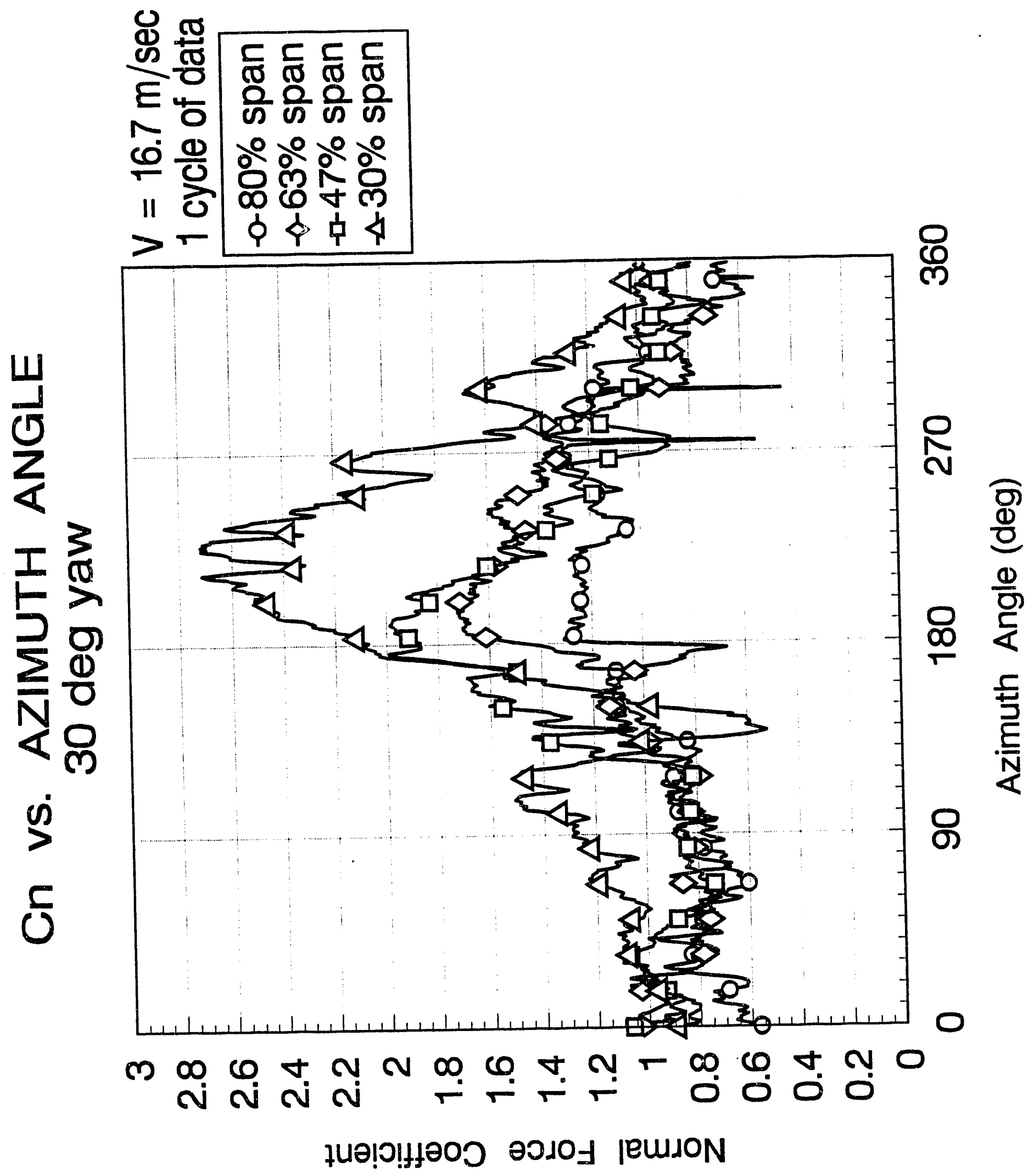




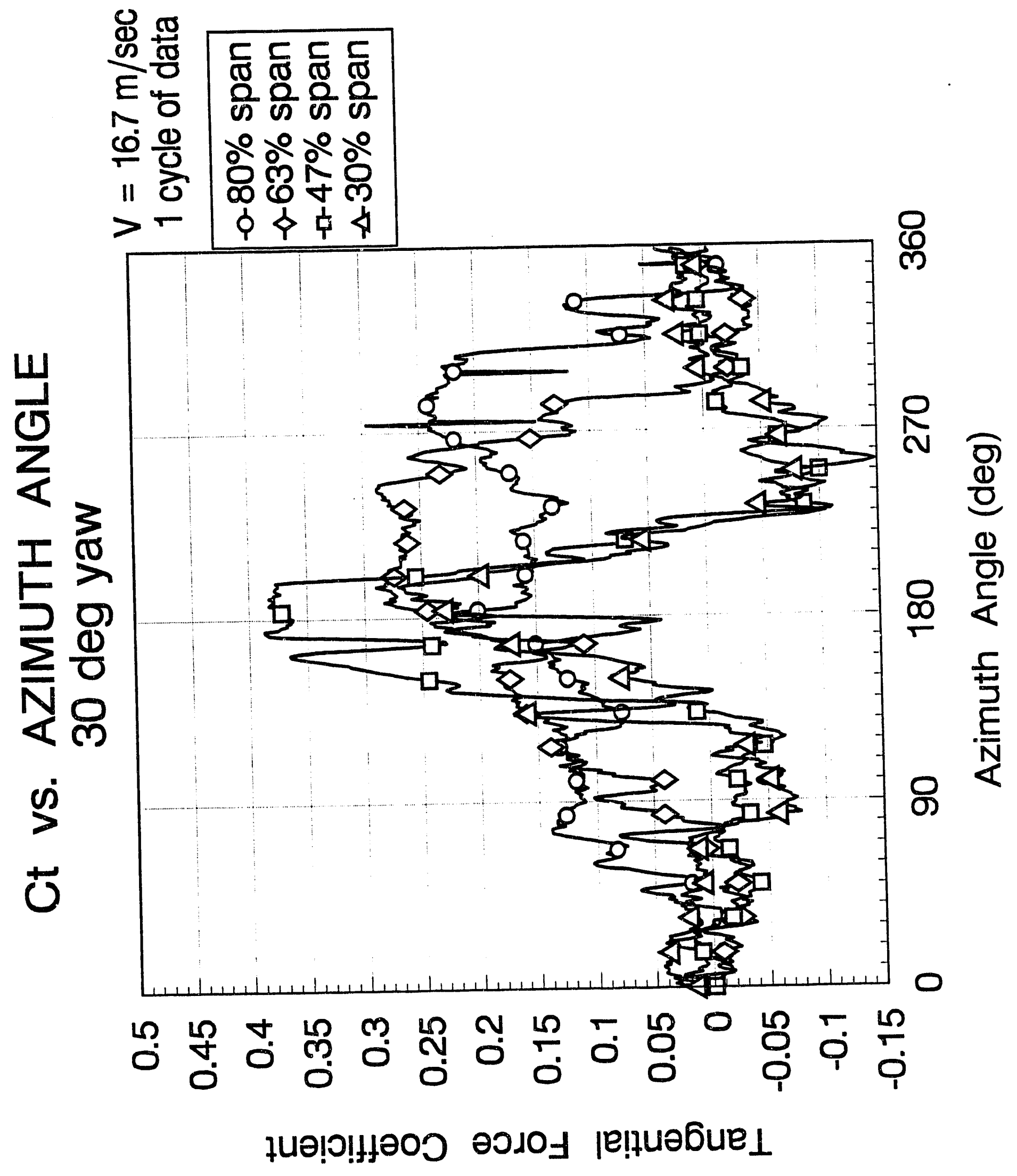




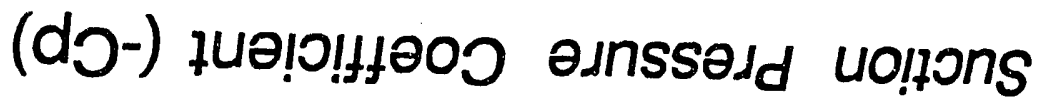

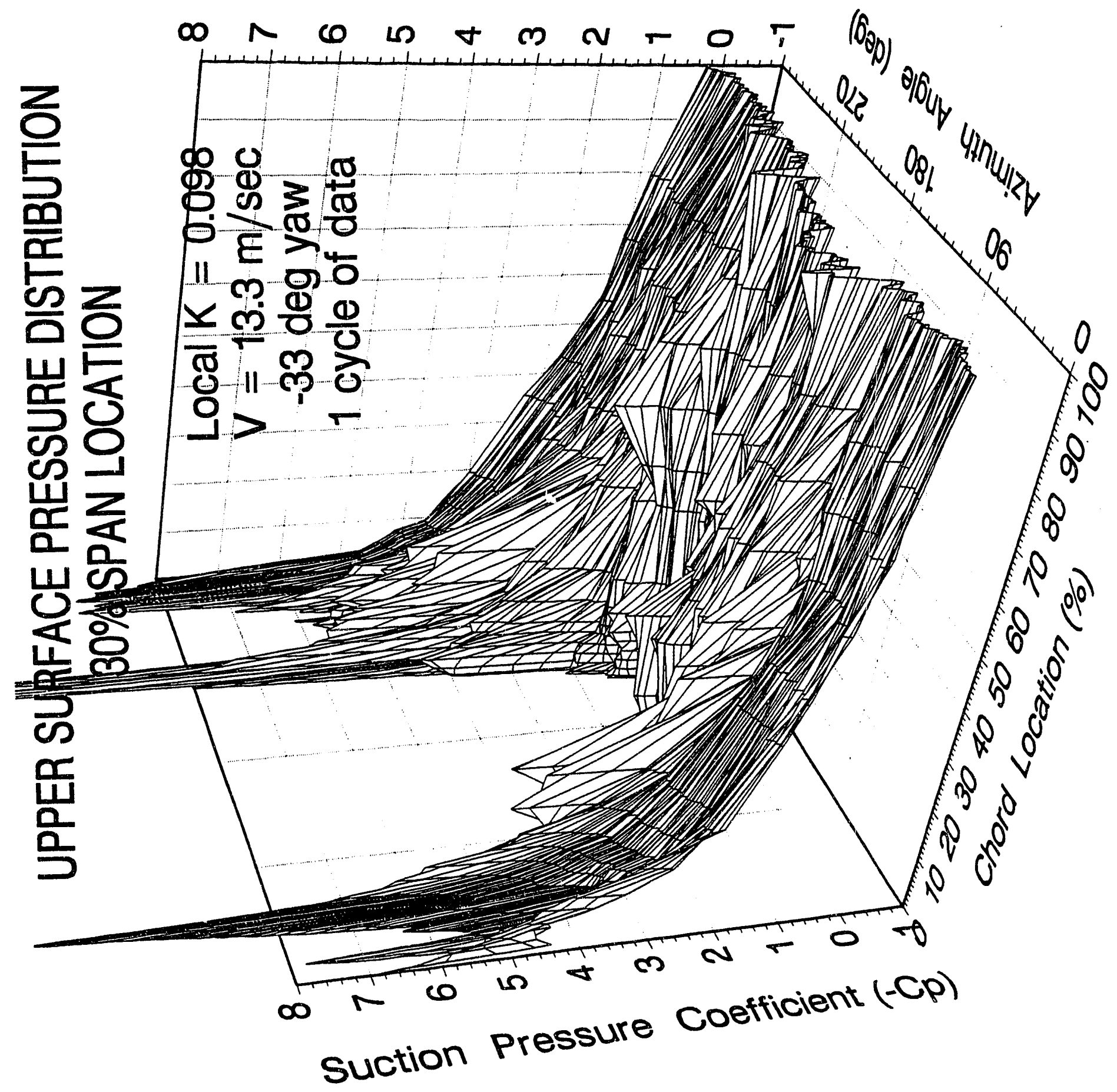




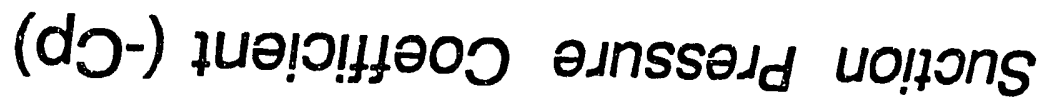

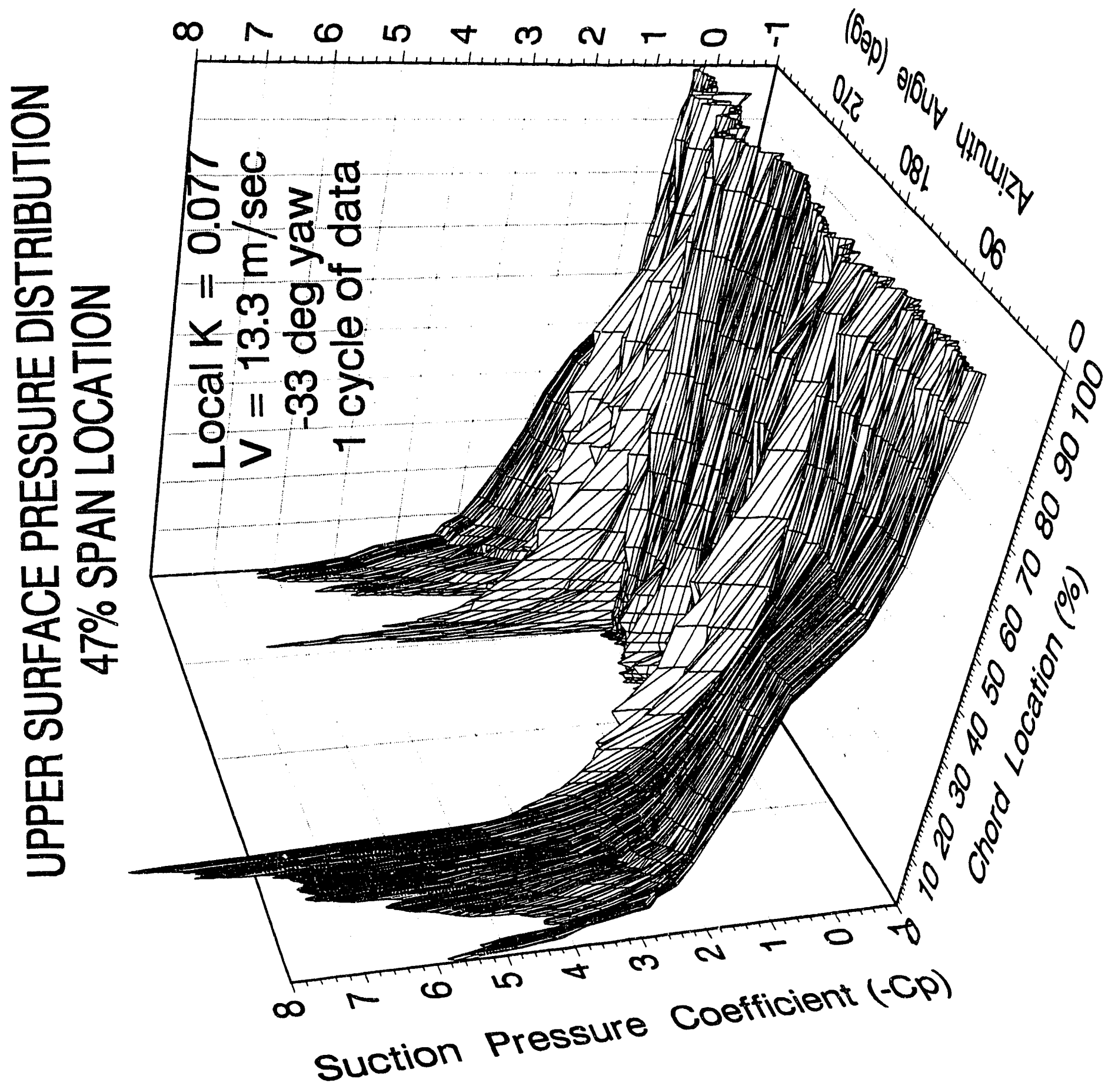




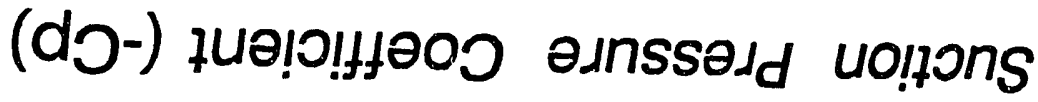

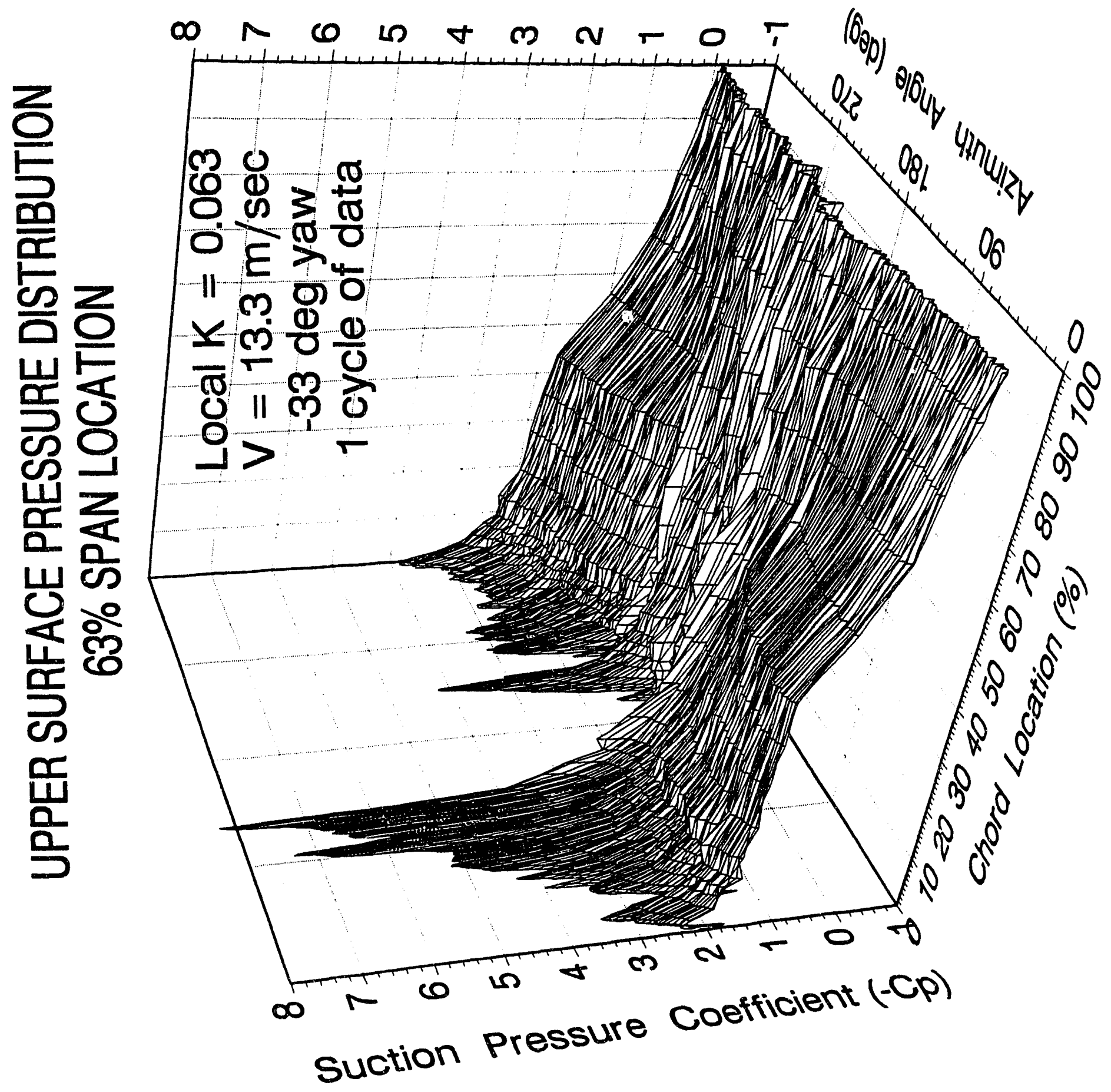




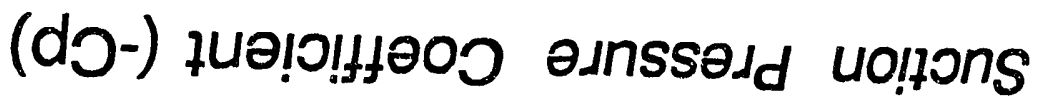

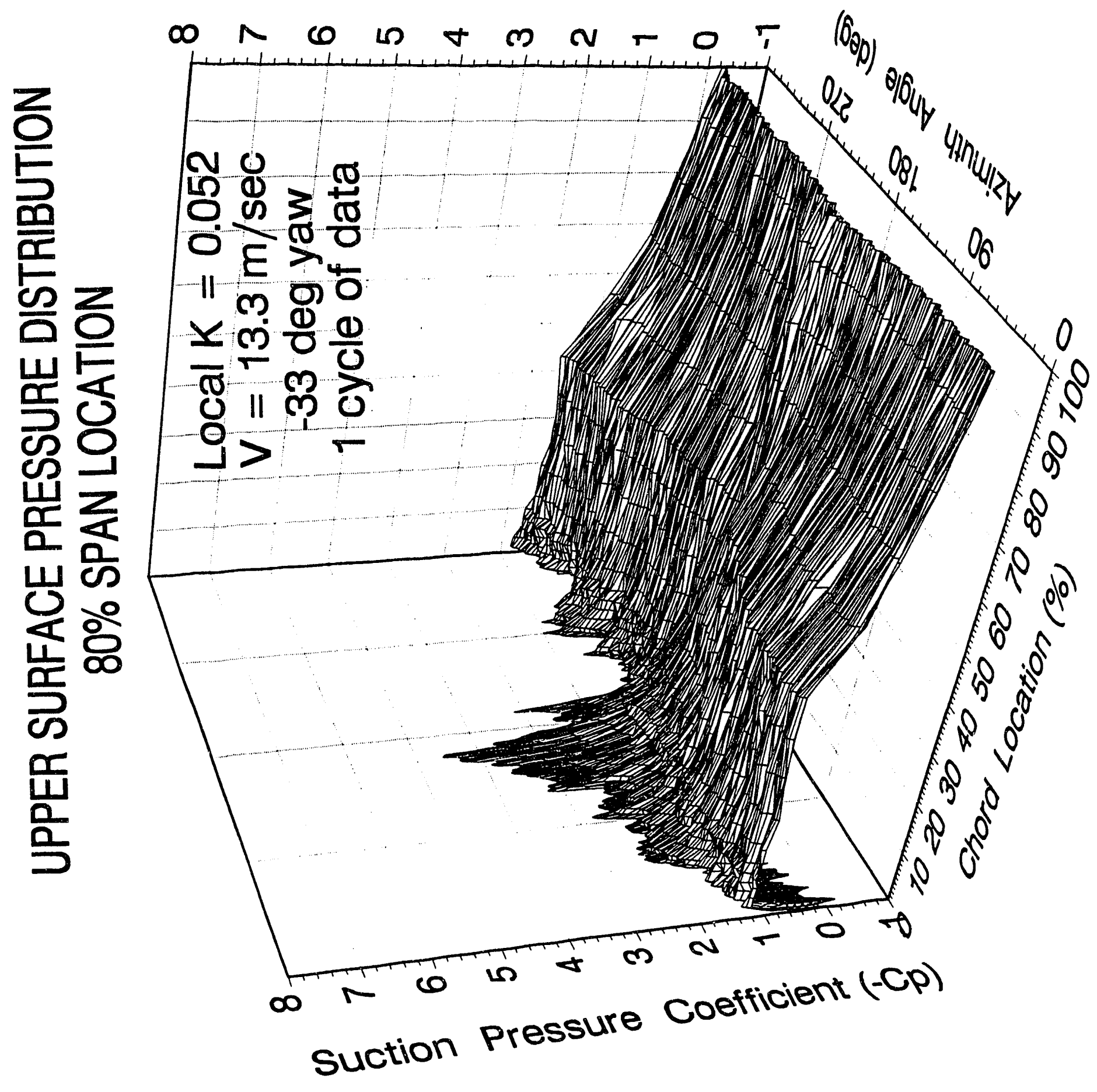




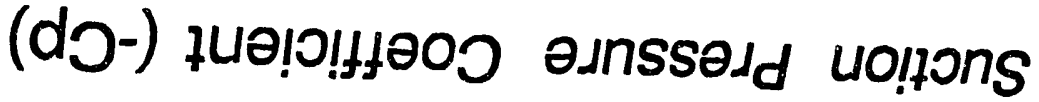

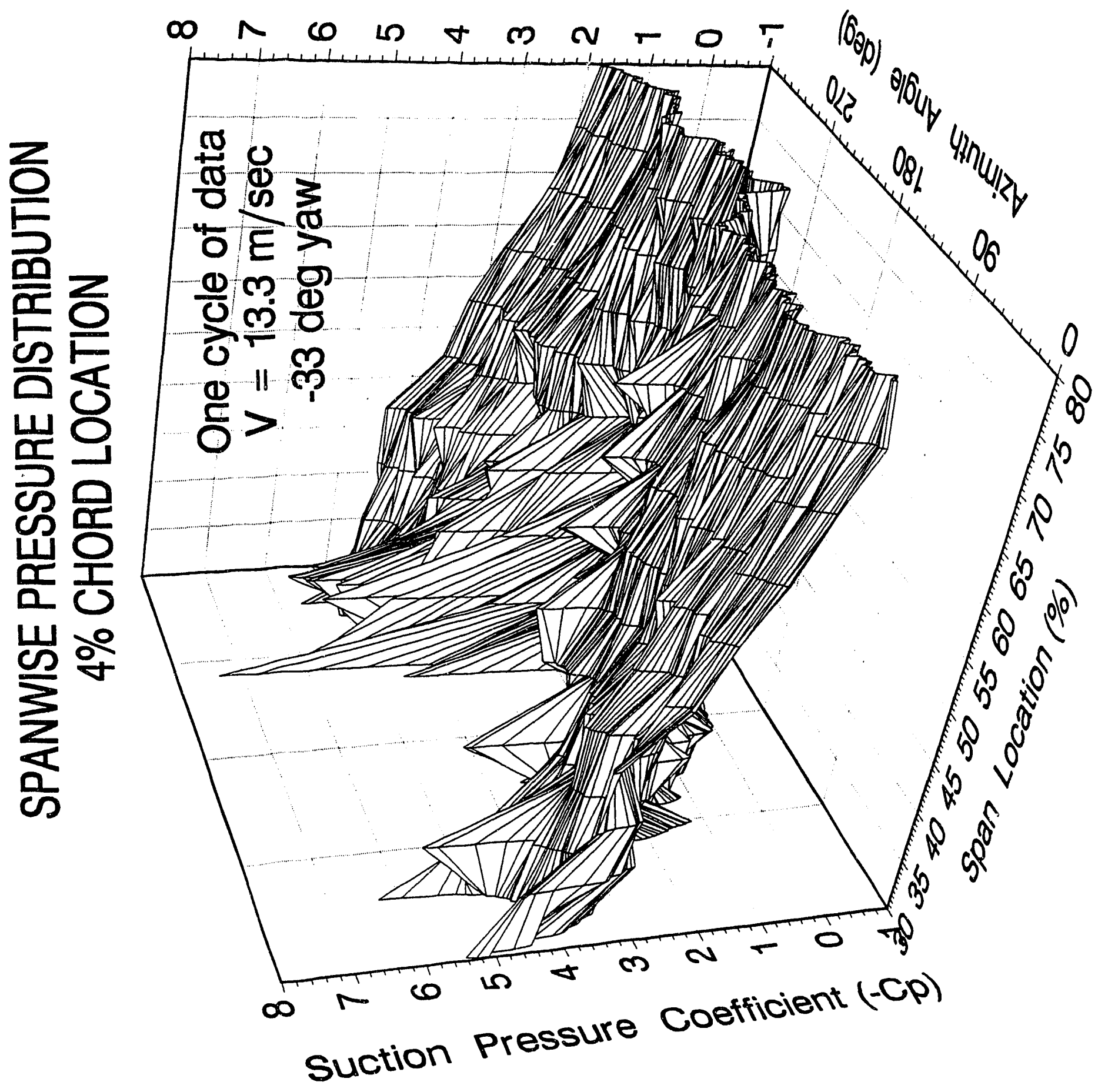




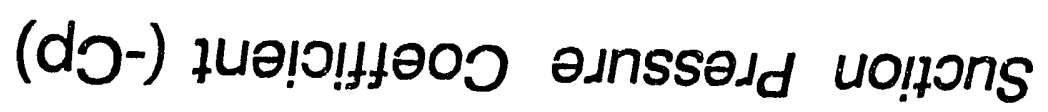

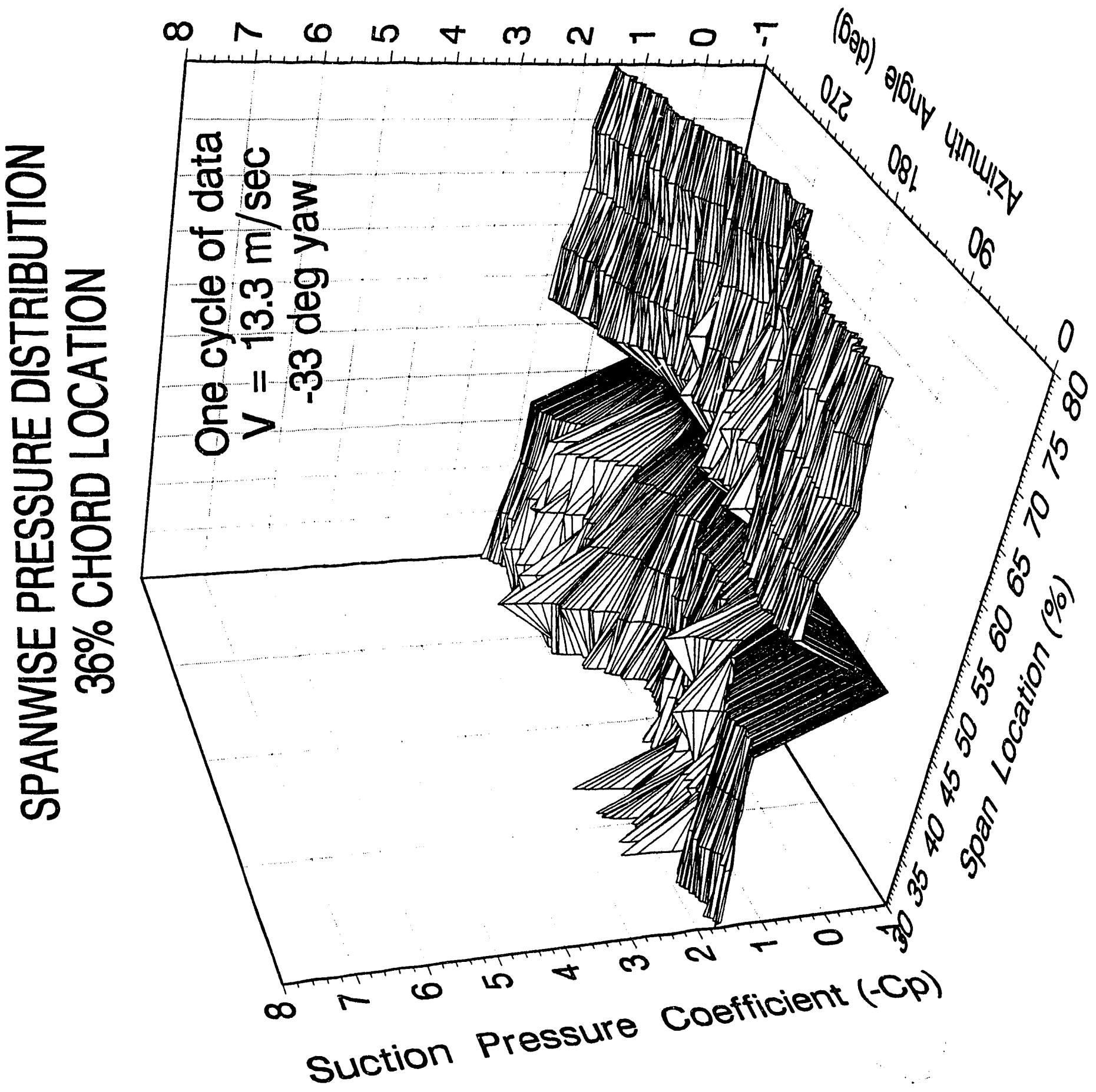




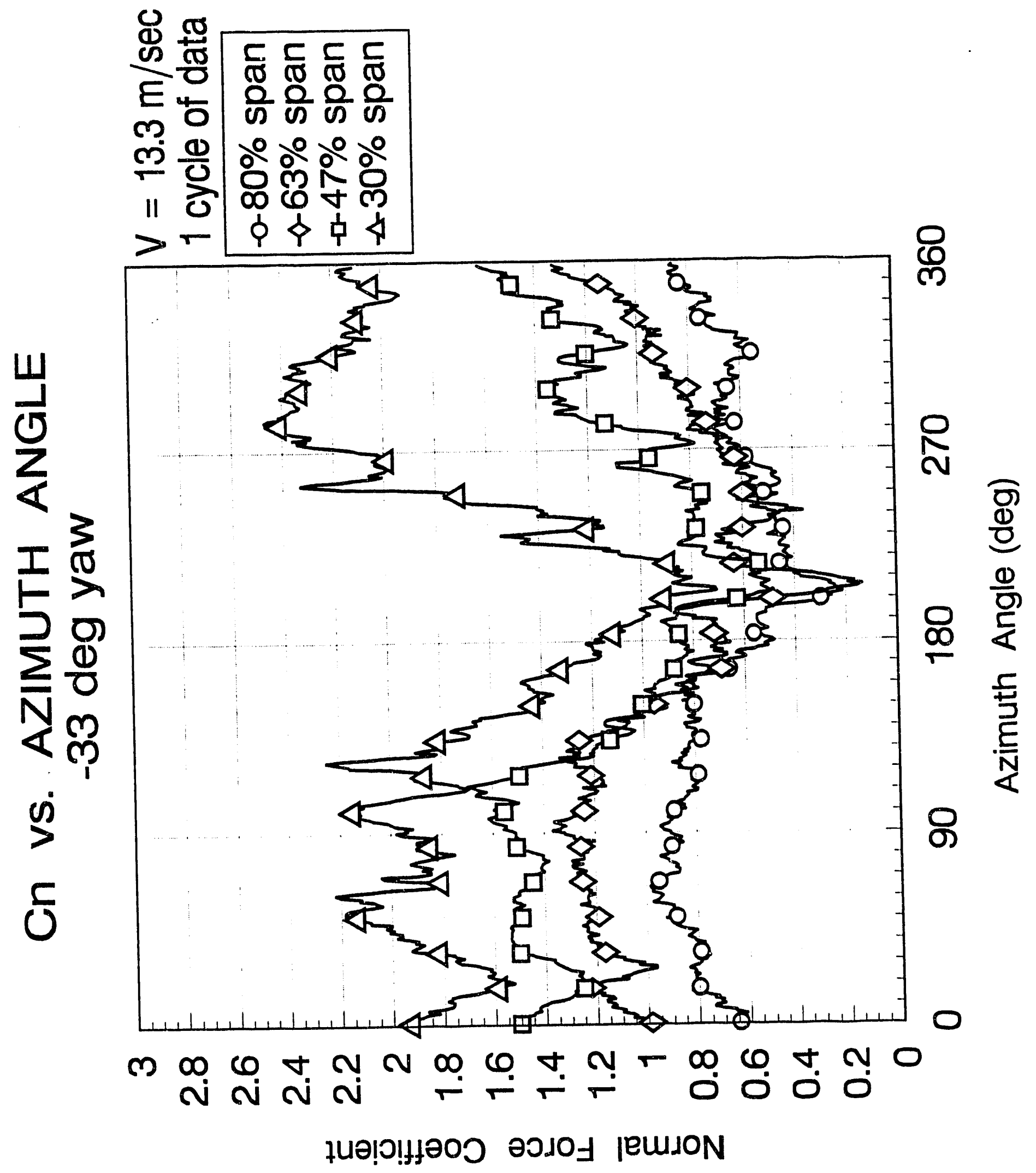




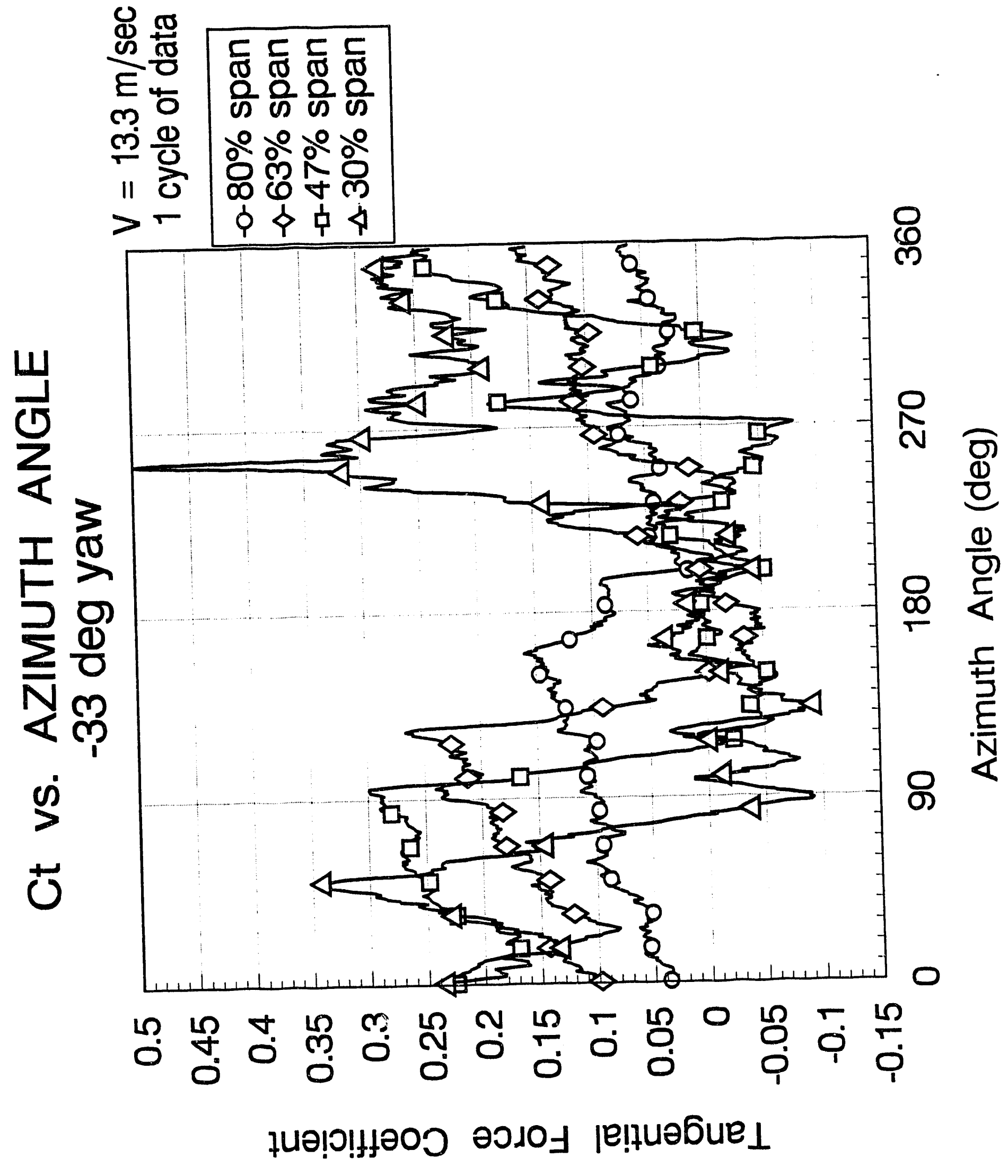




\section{Appendix F}

\section{Chordwise Pressure Distributions, From Single Cycle Data}



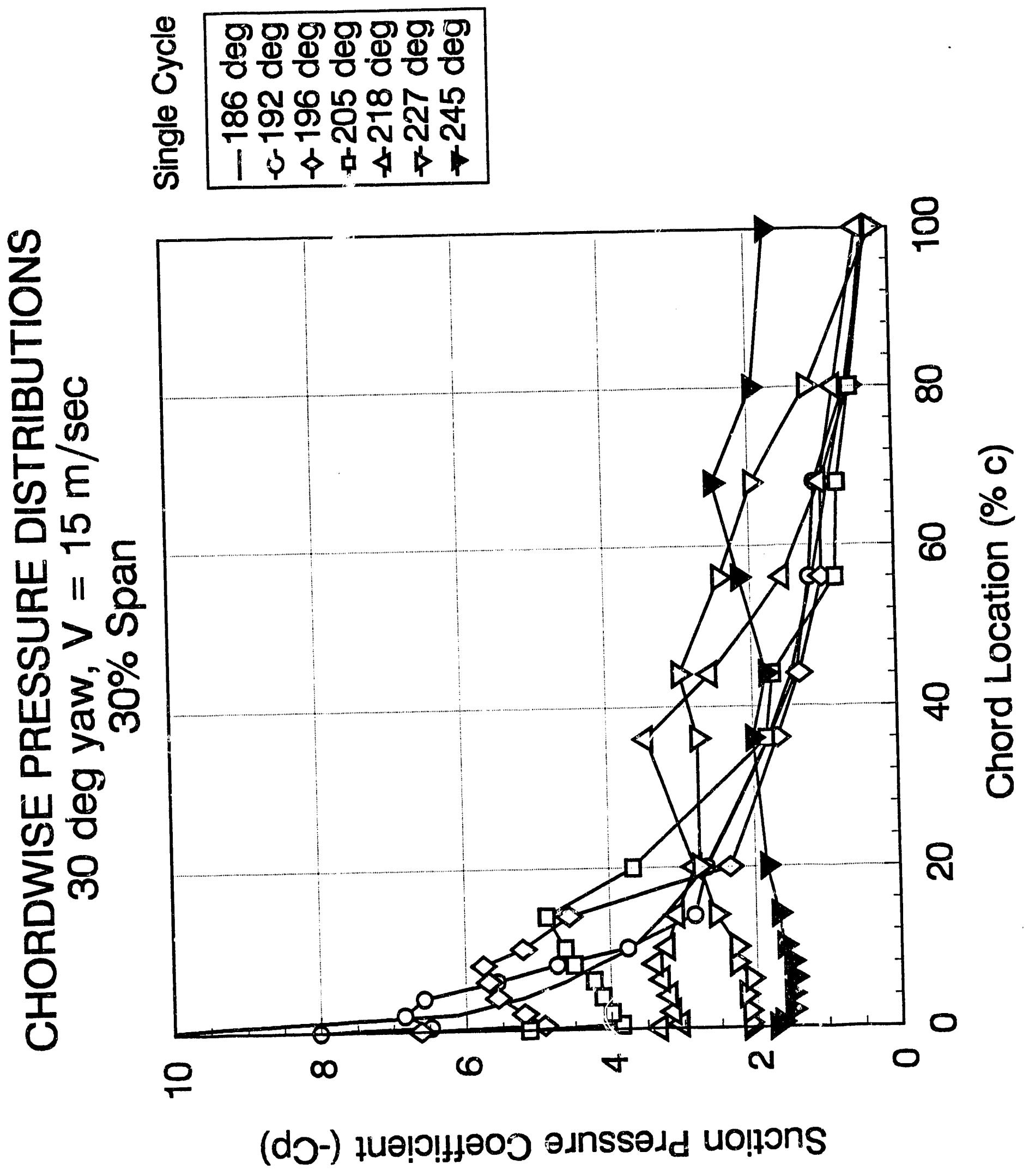


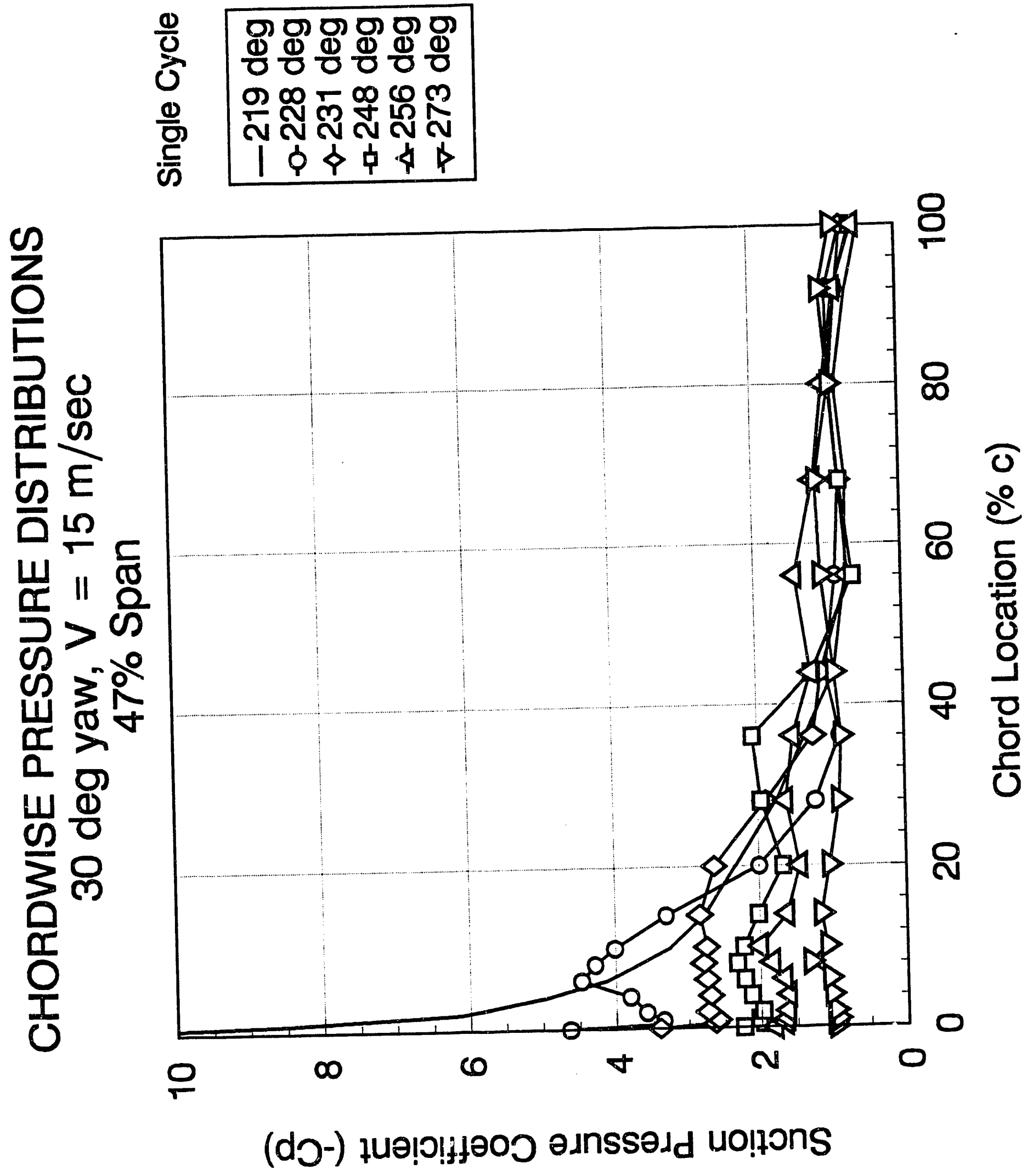




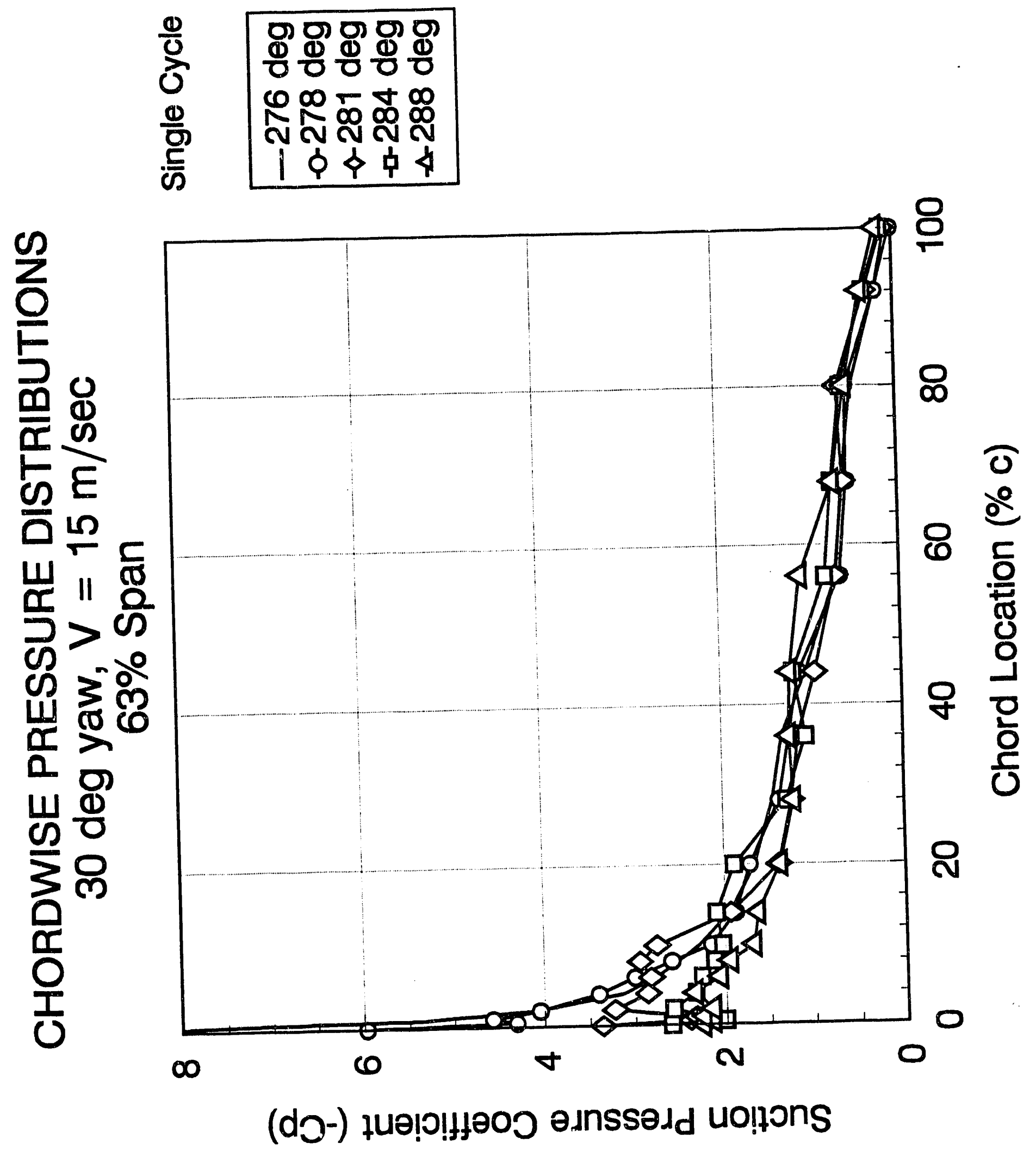



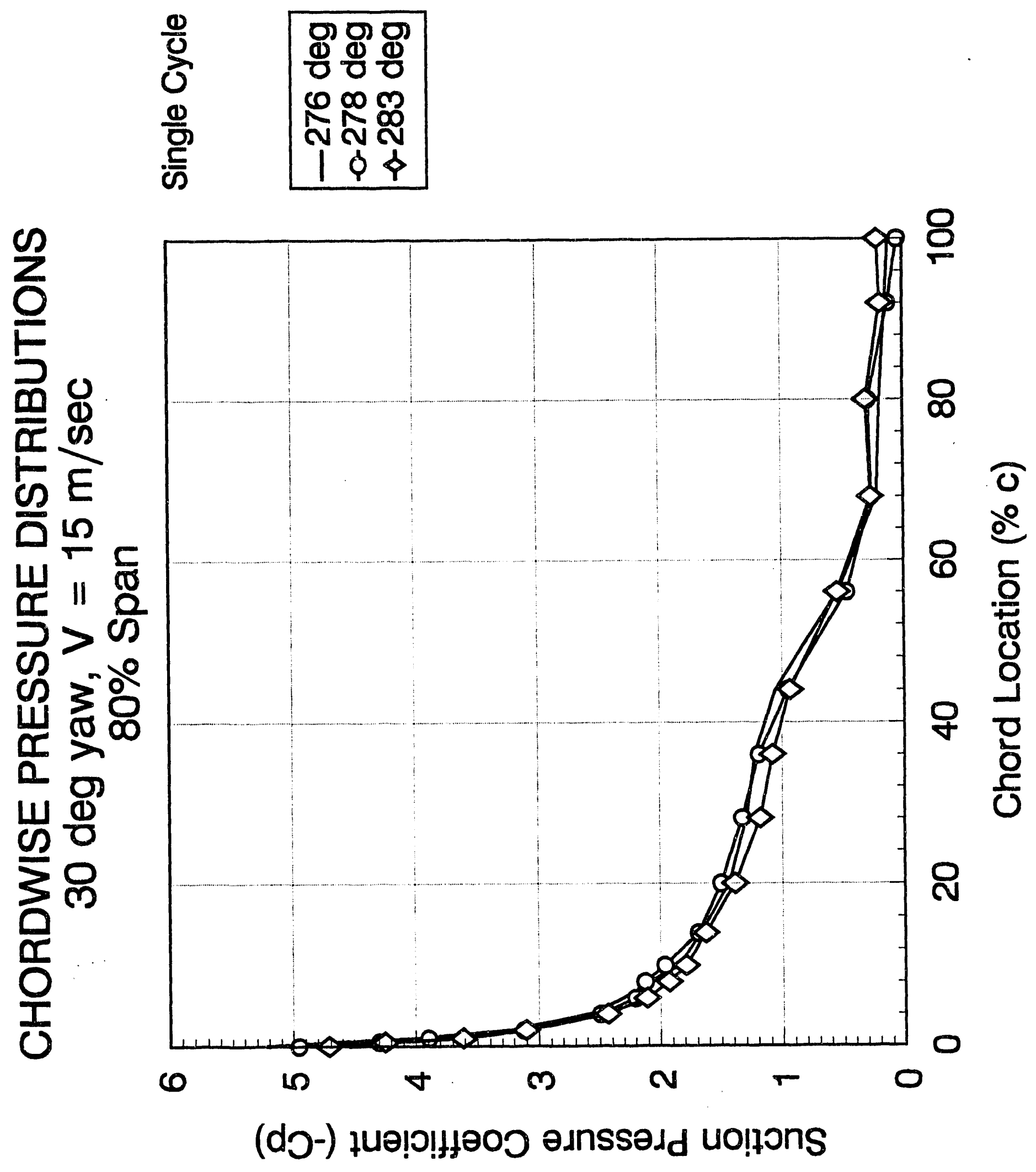


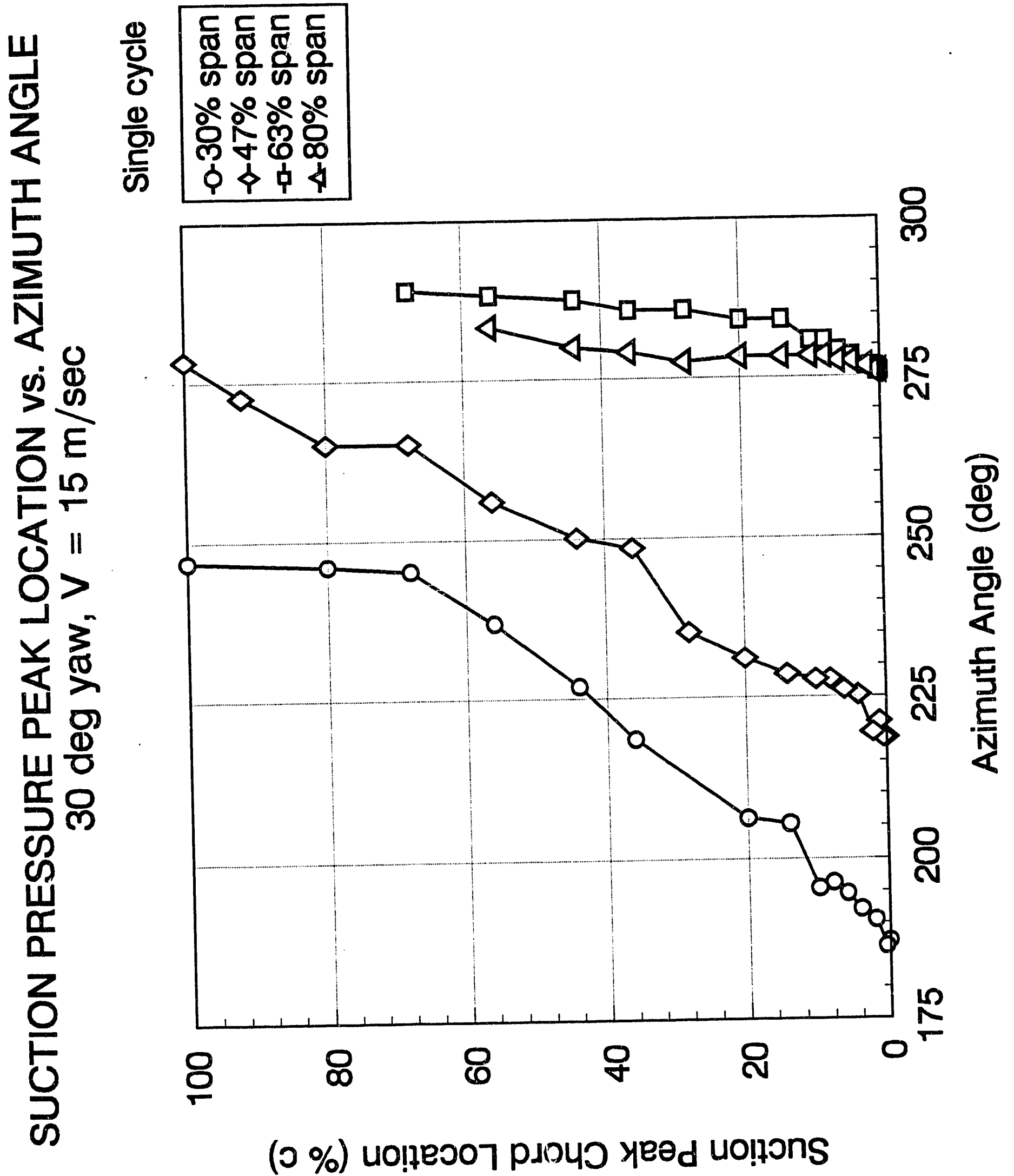




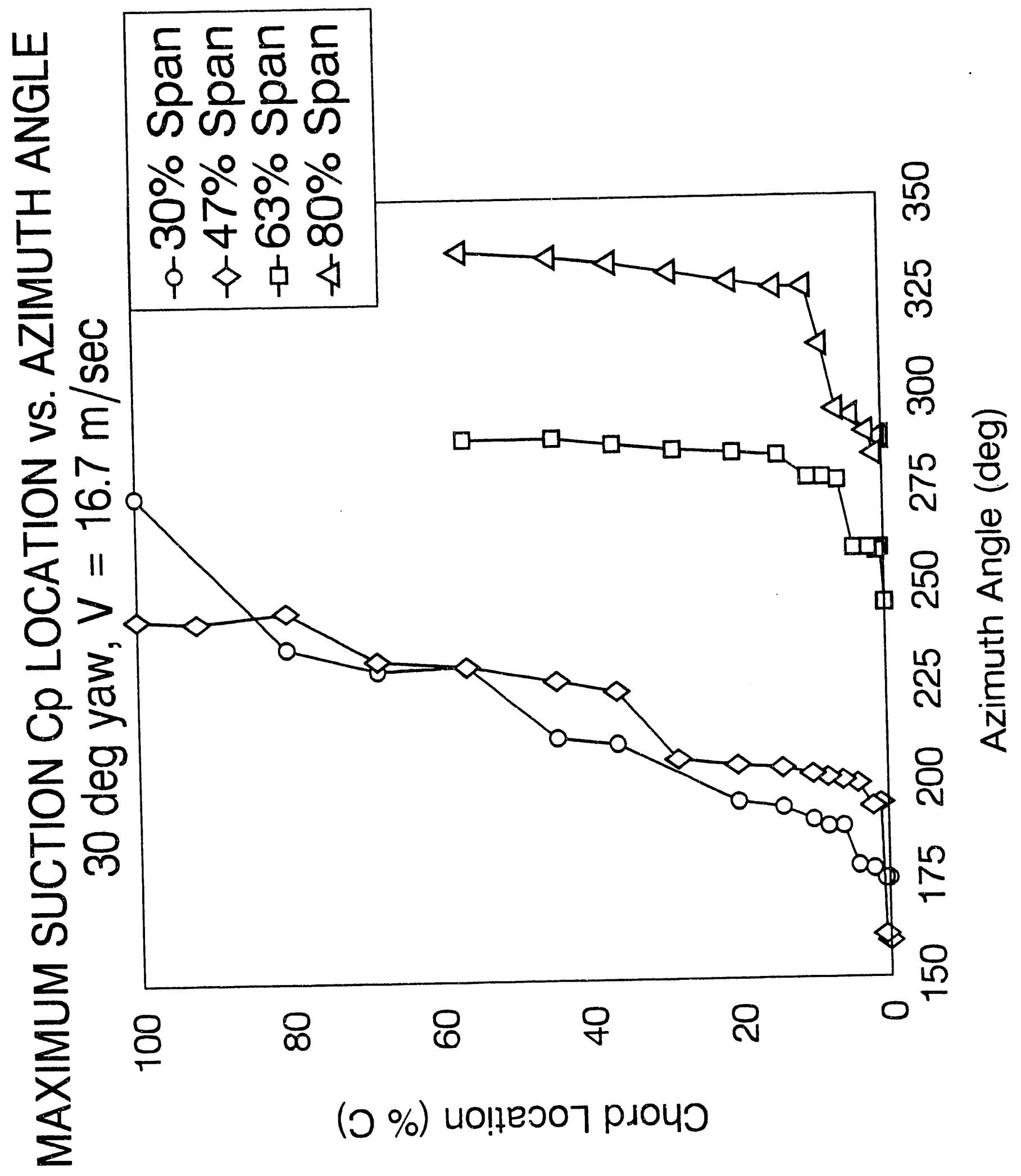




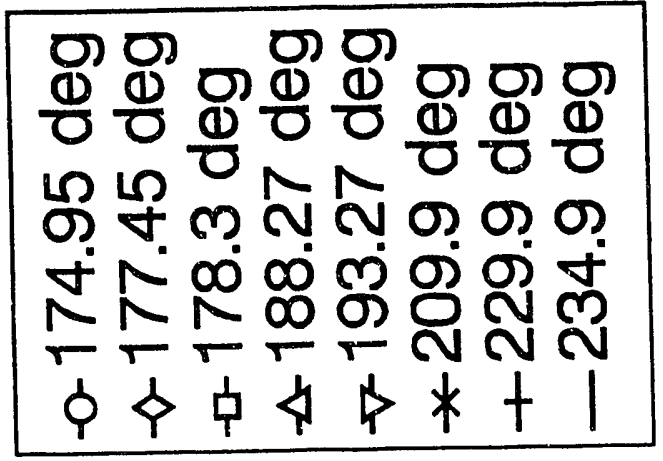

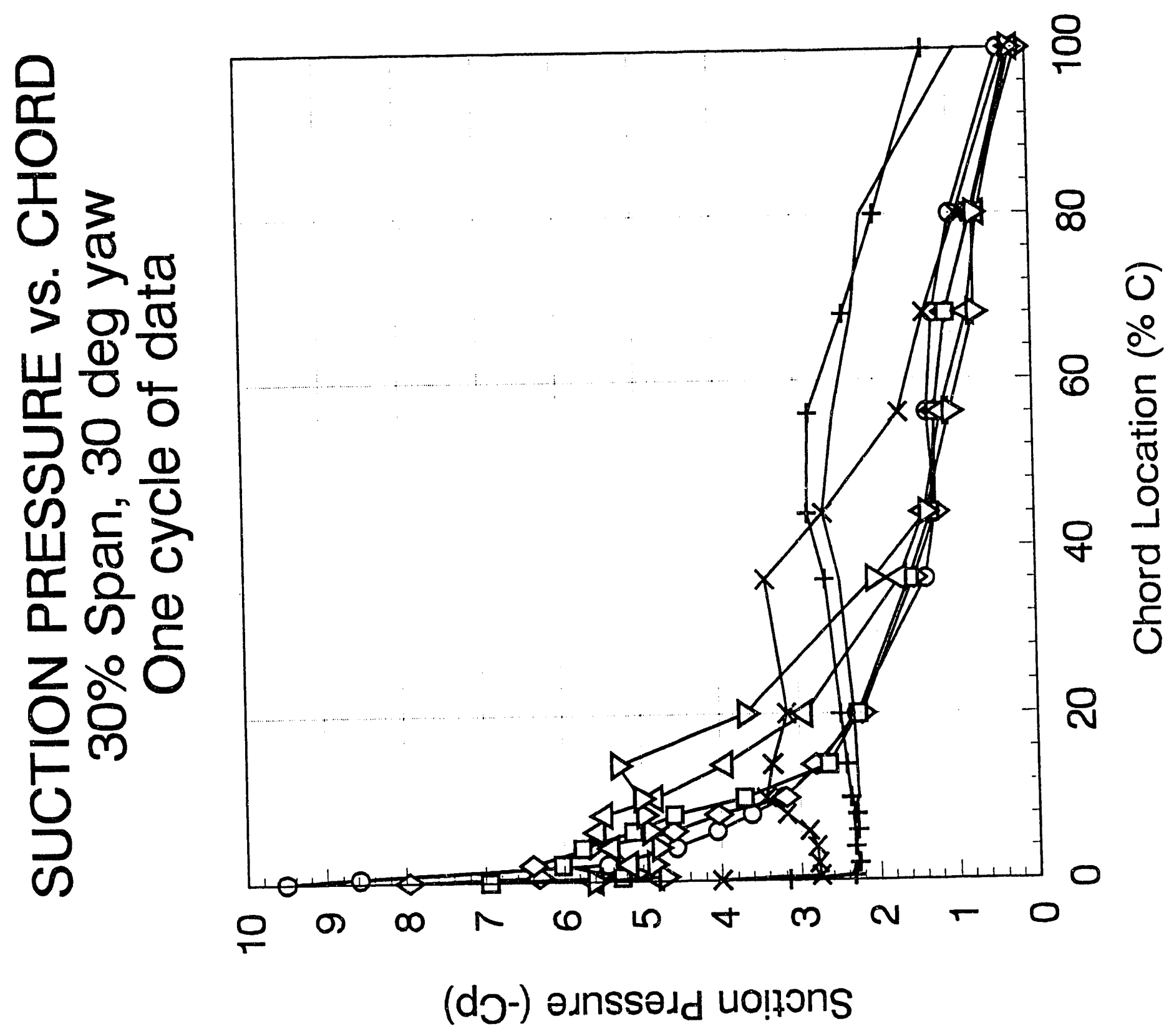




\begin{tabular}{|c|}
\hline 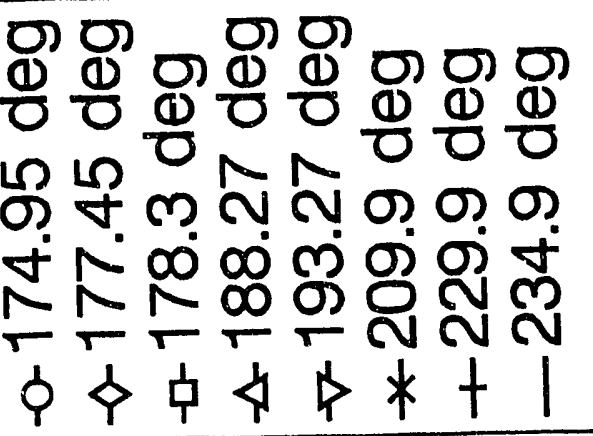 \\
\hline
\end{tabular}

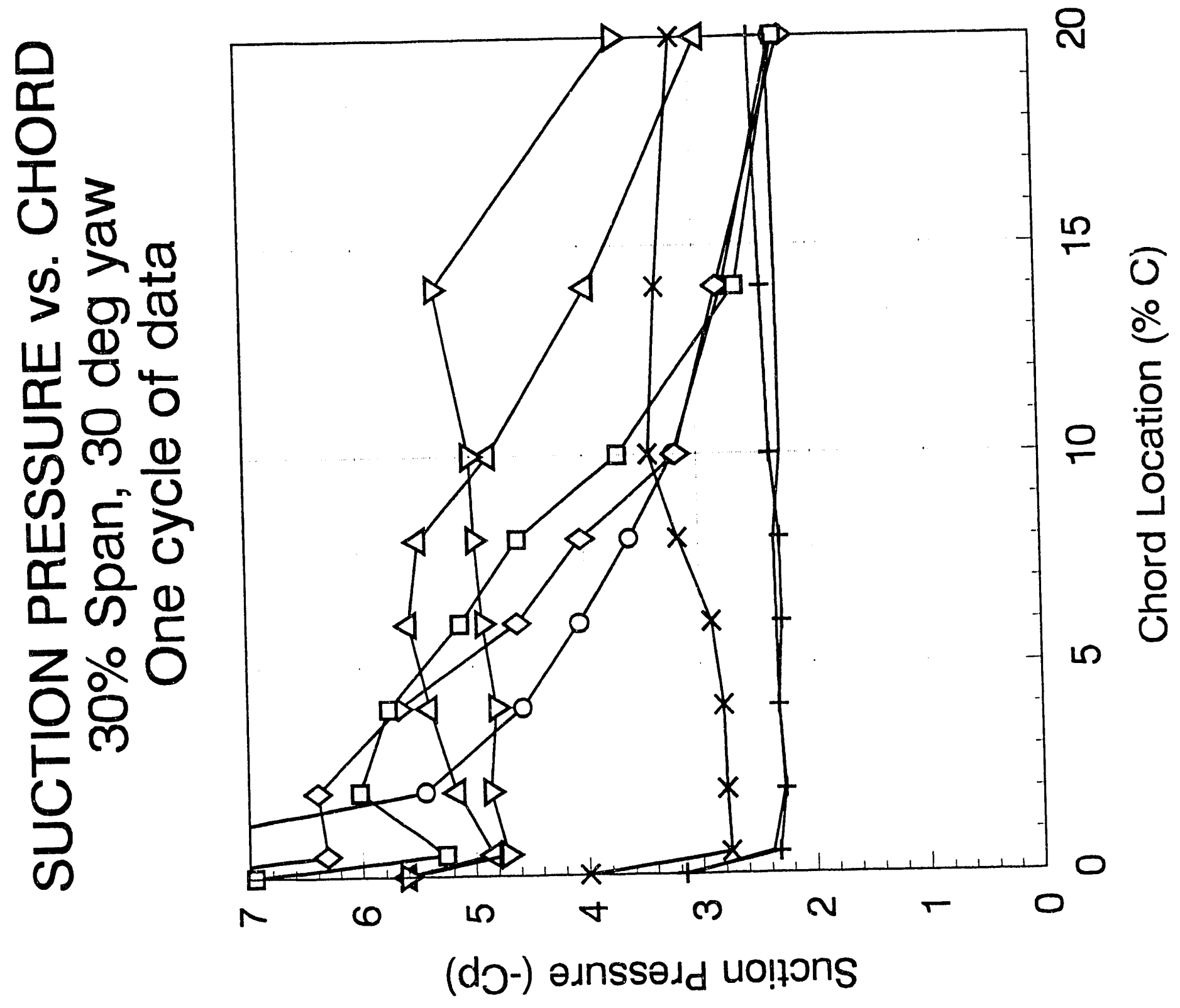



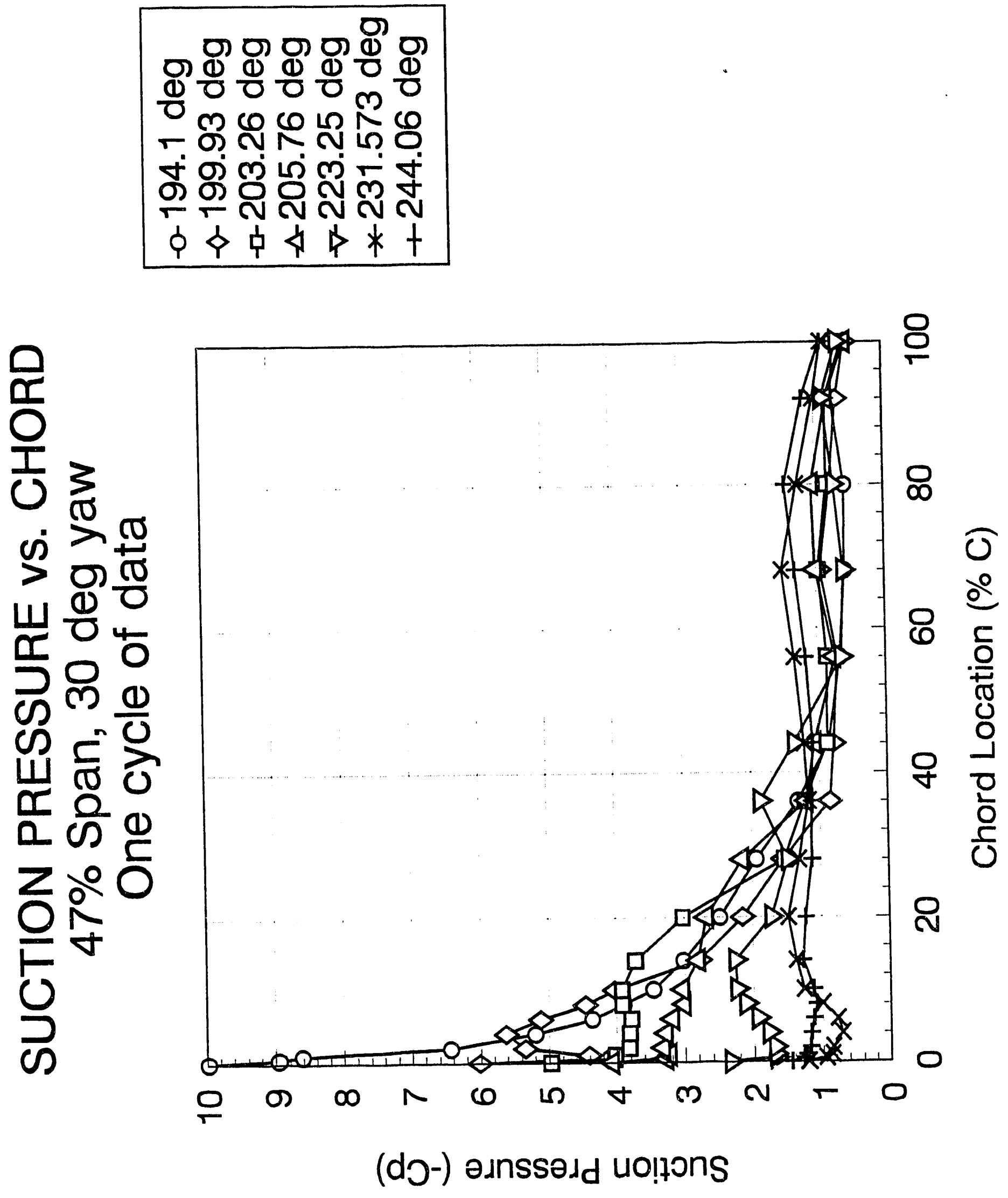

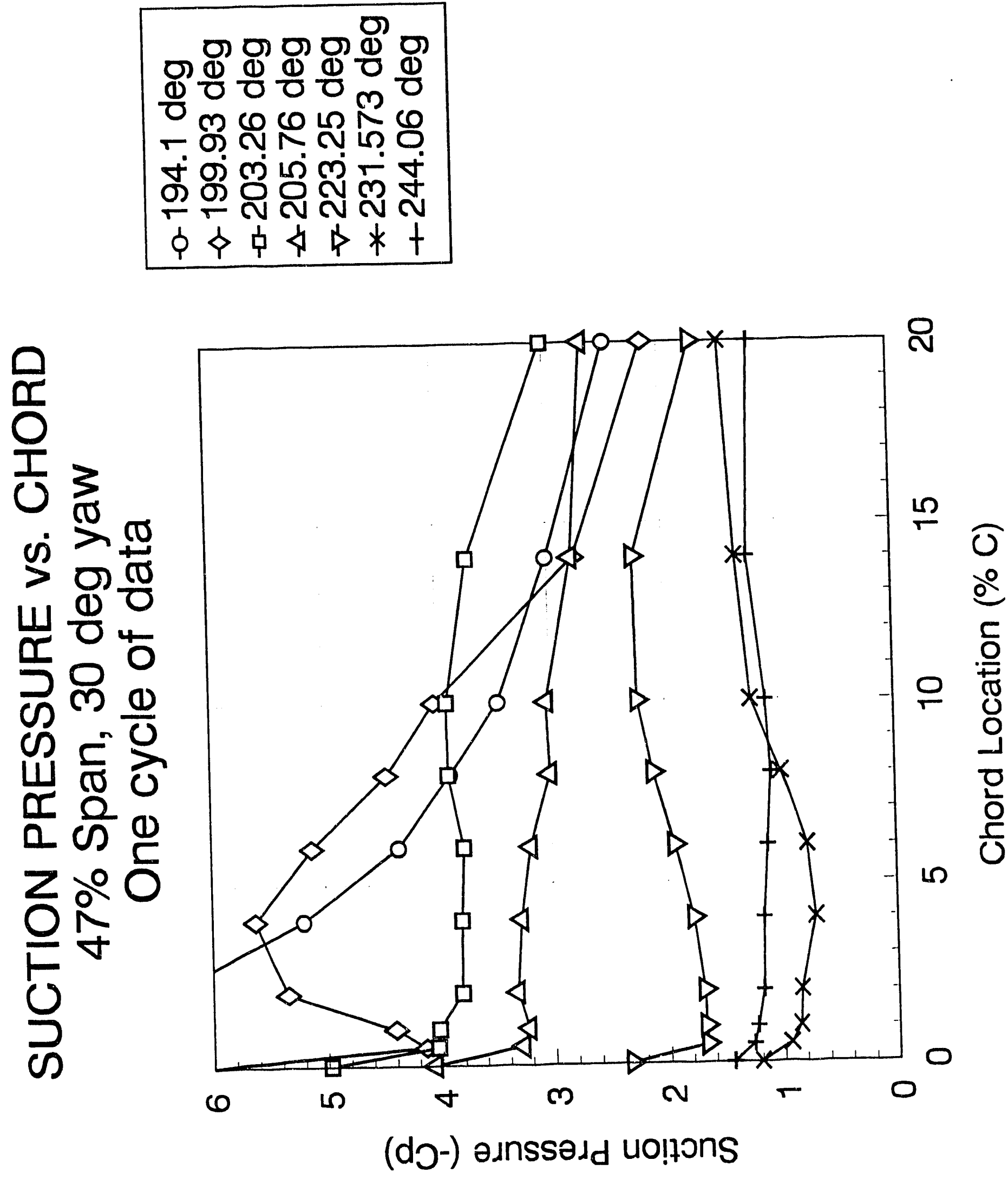

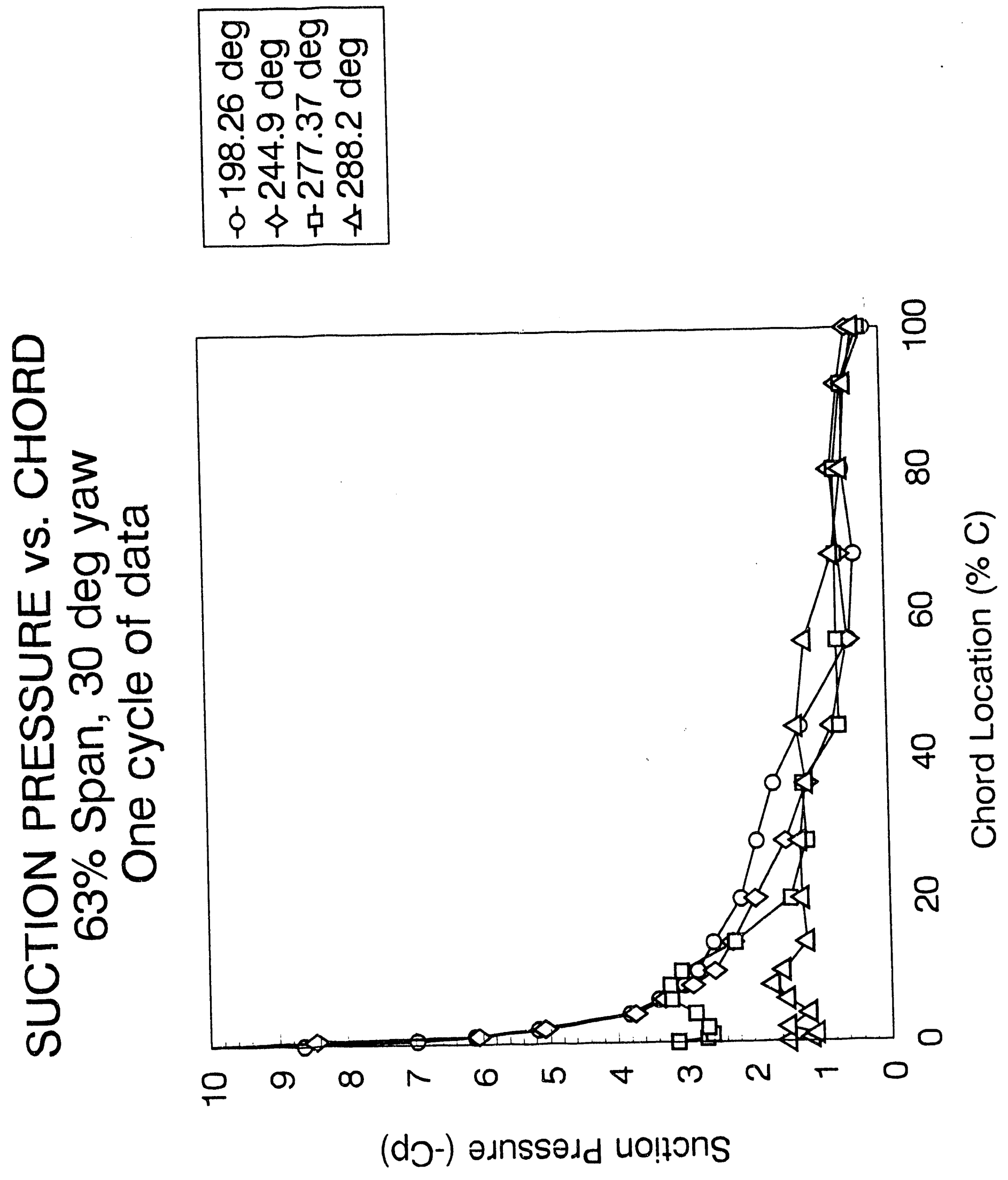

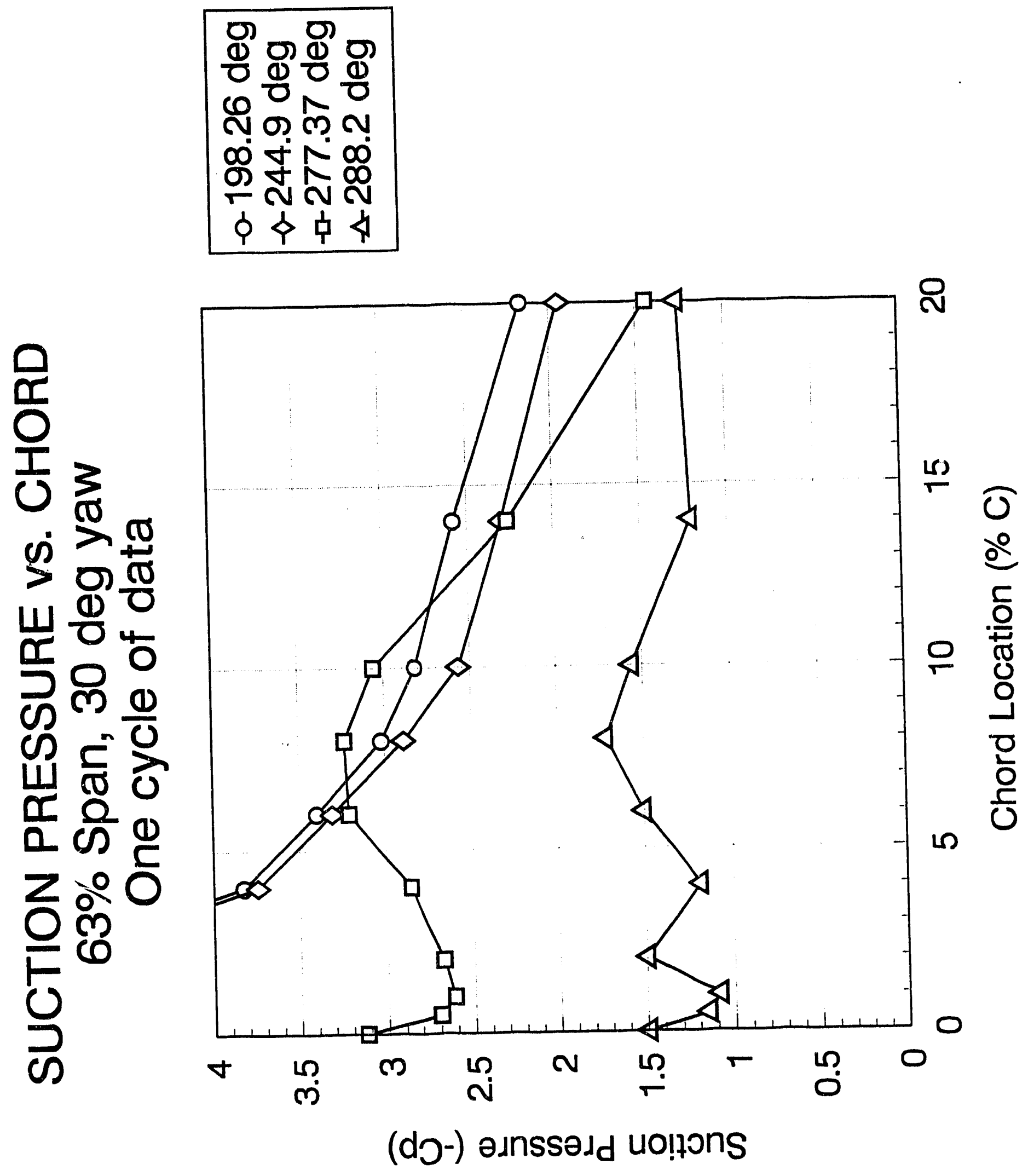


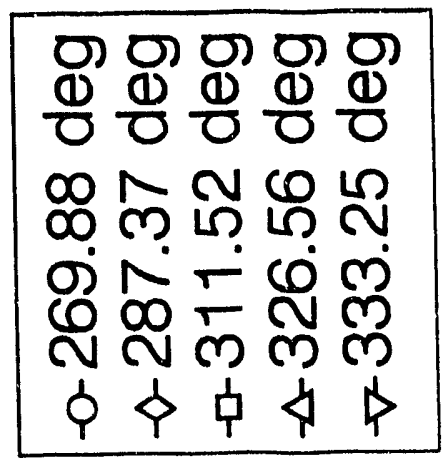

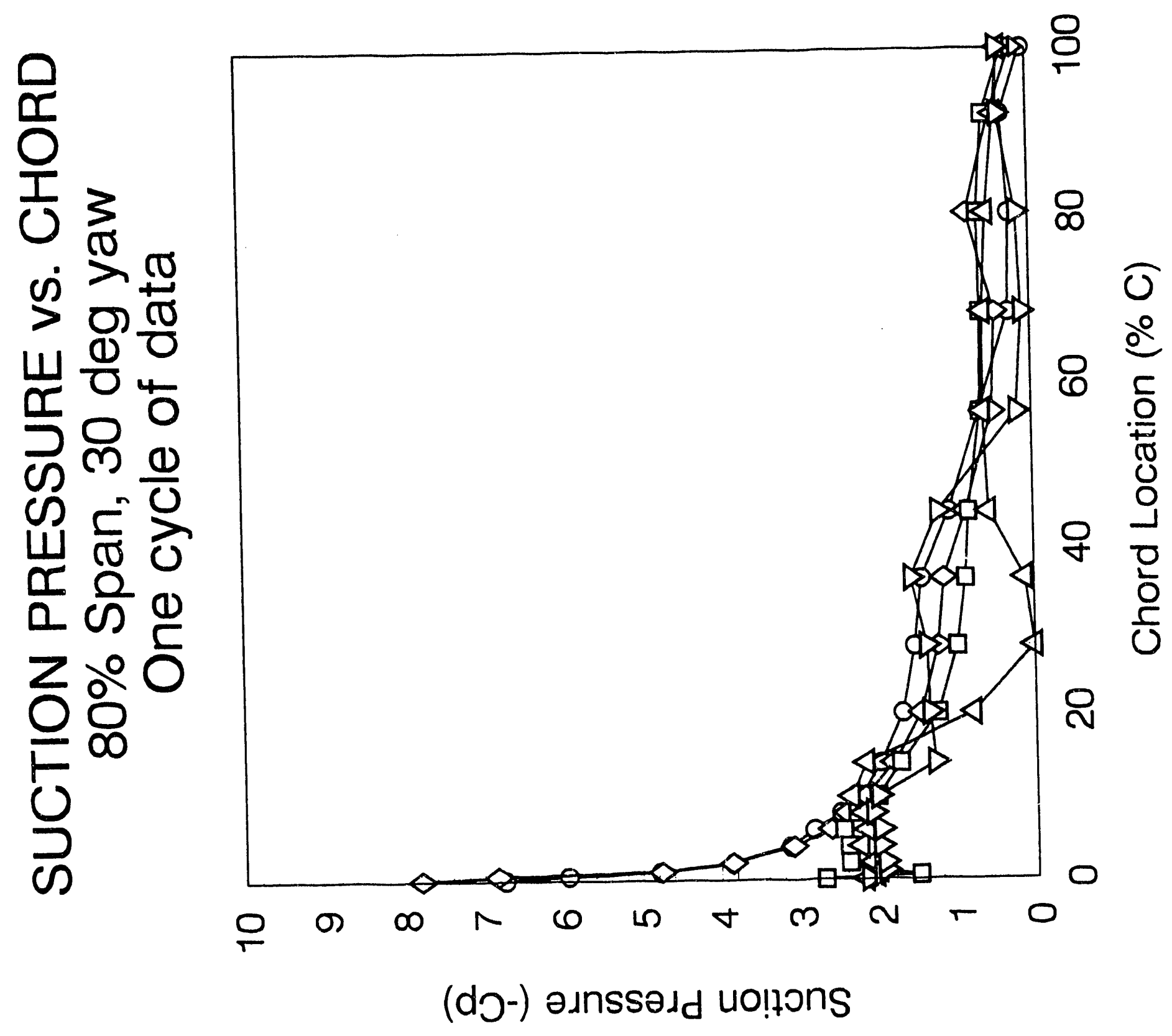



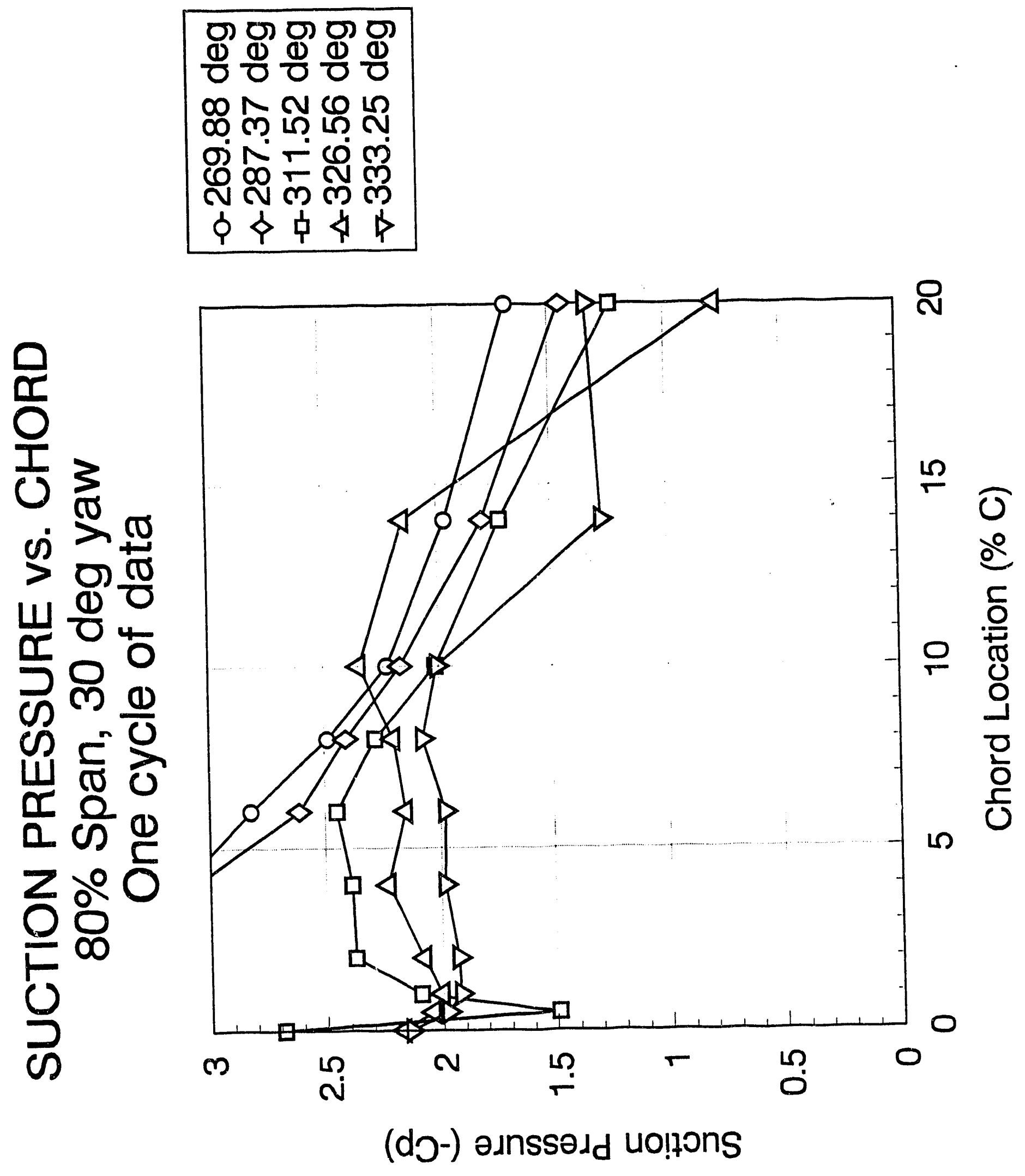

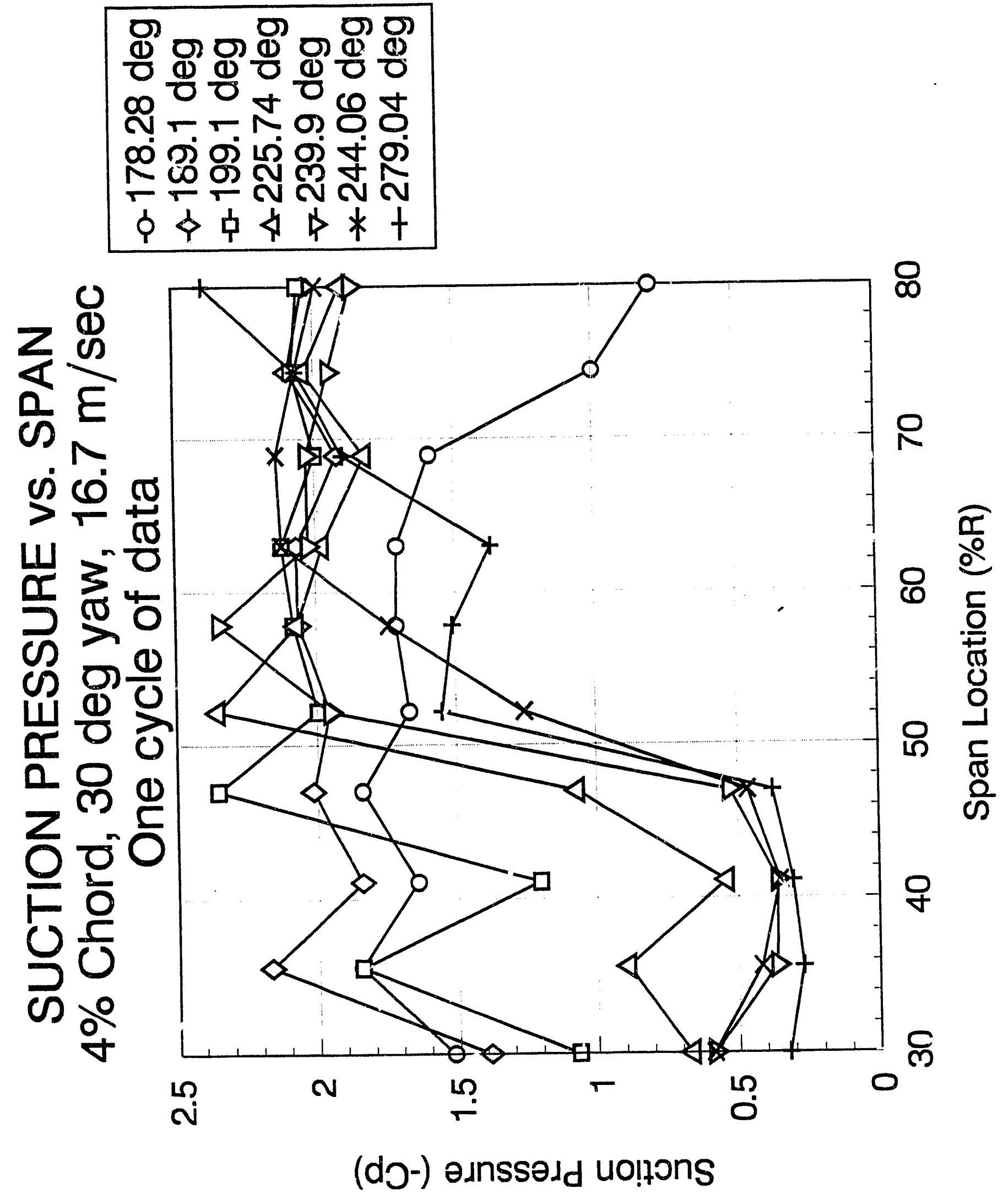

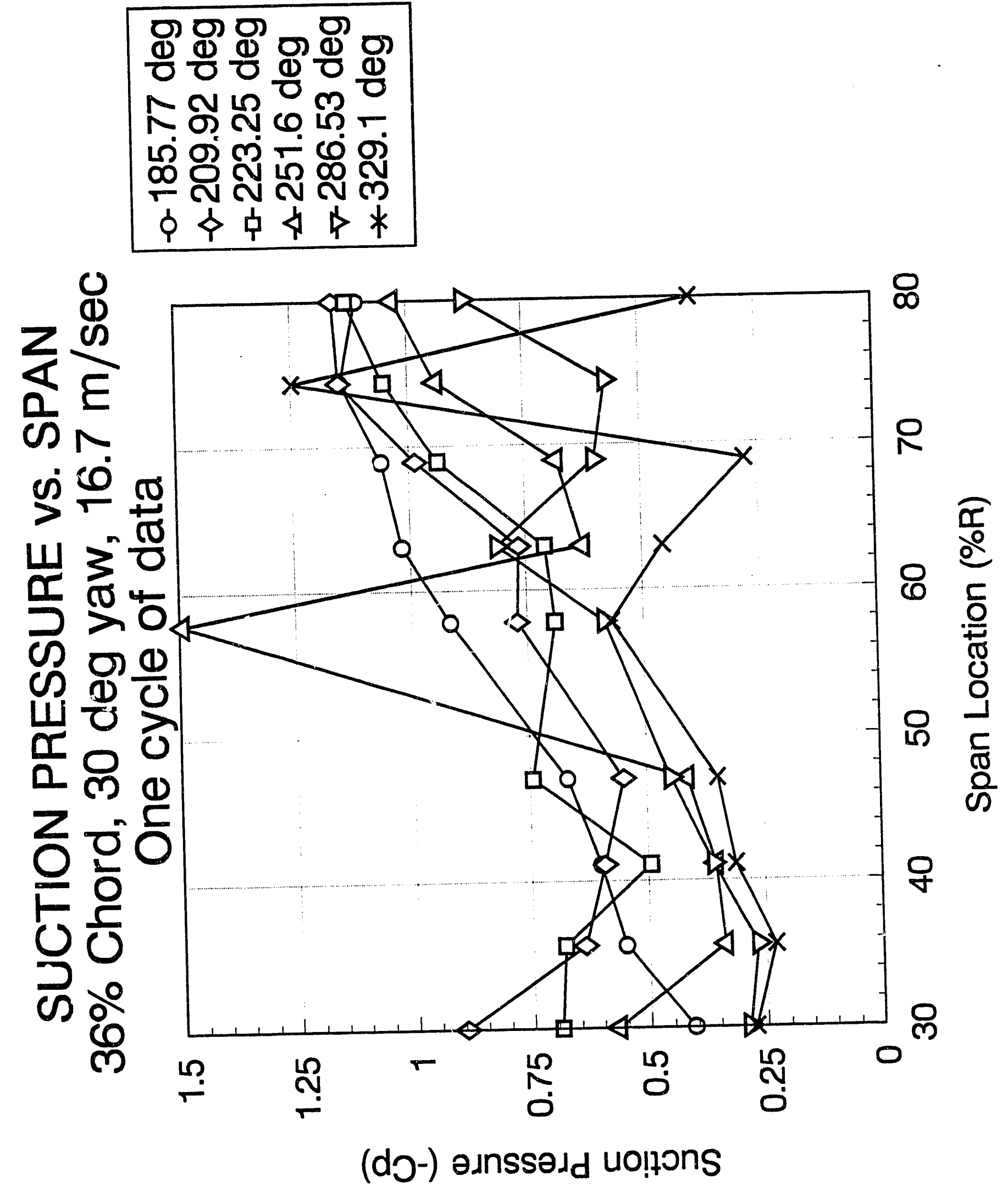


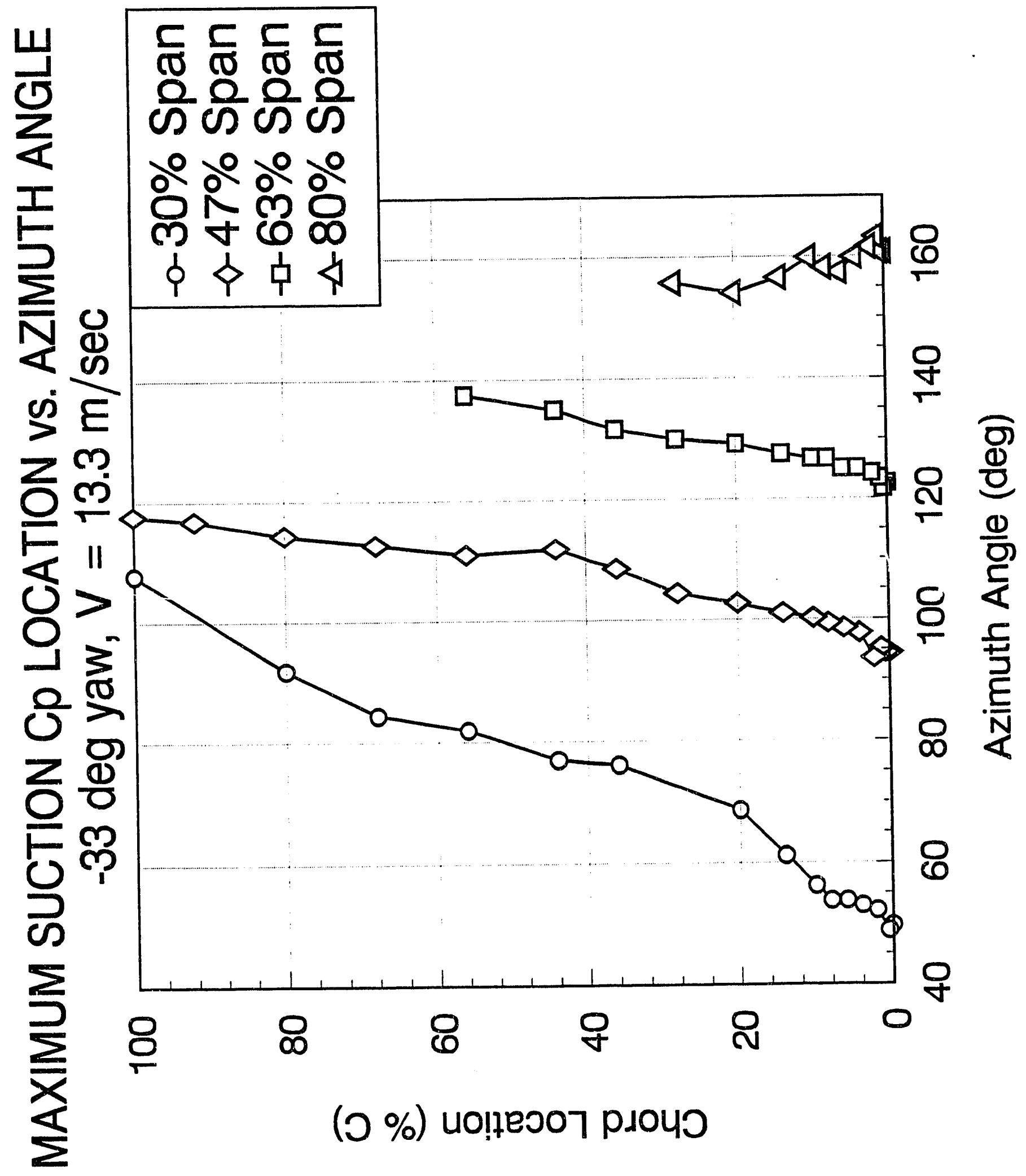




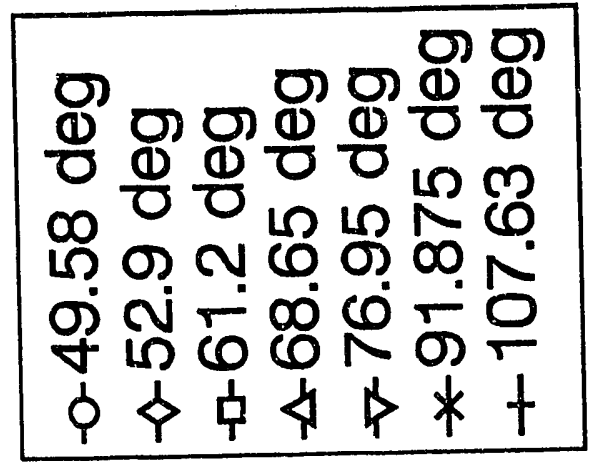

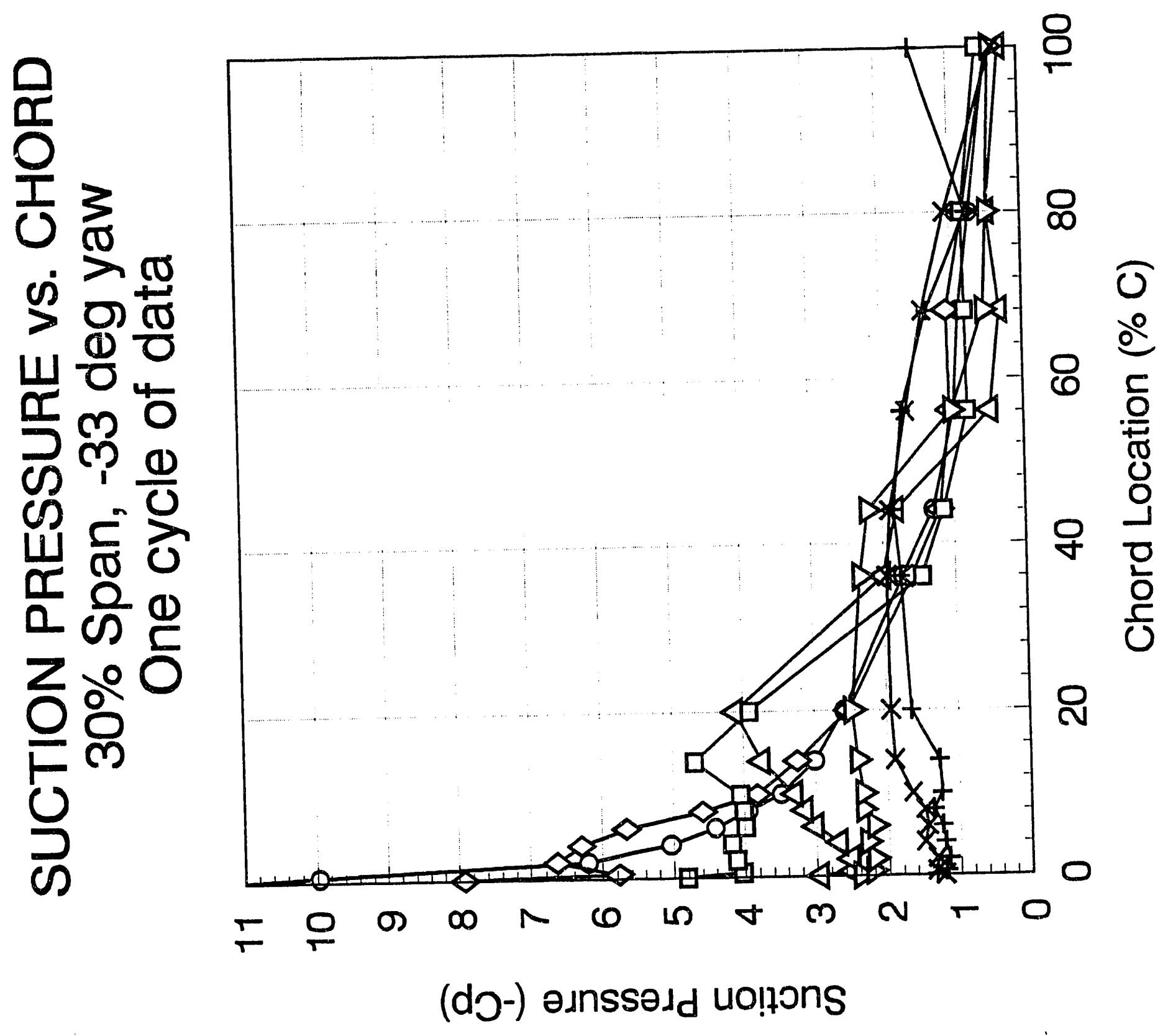




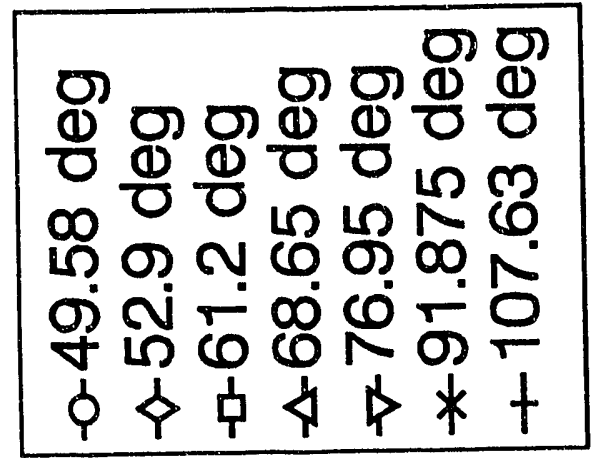

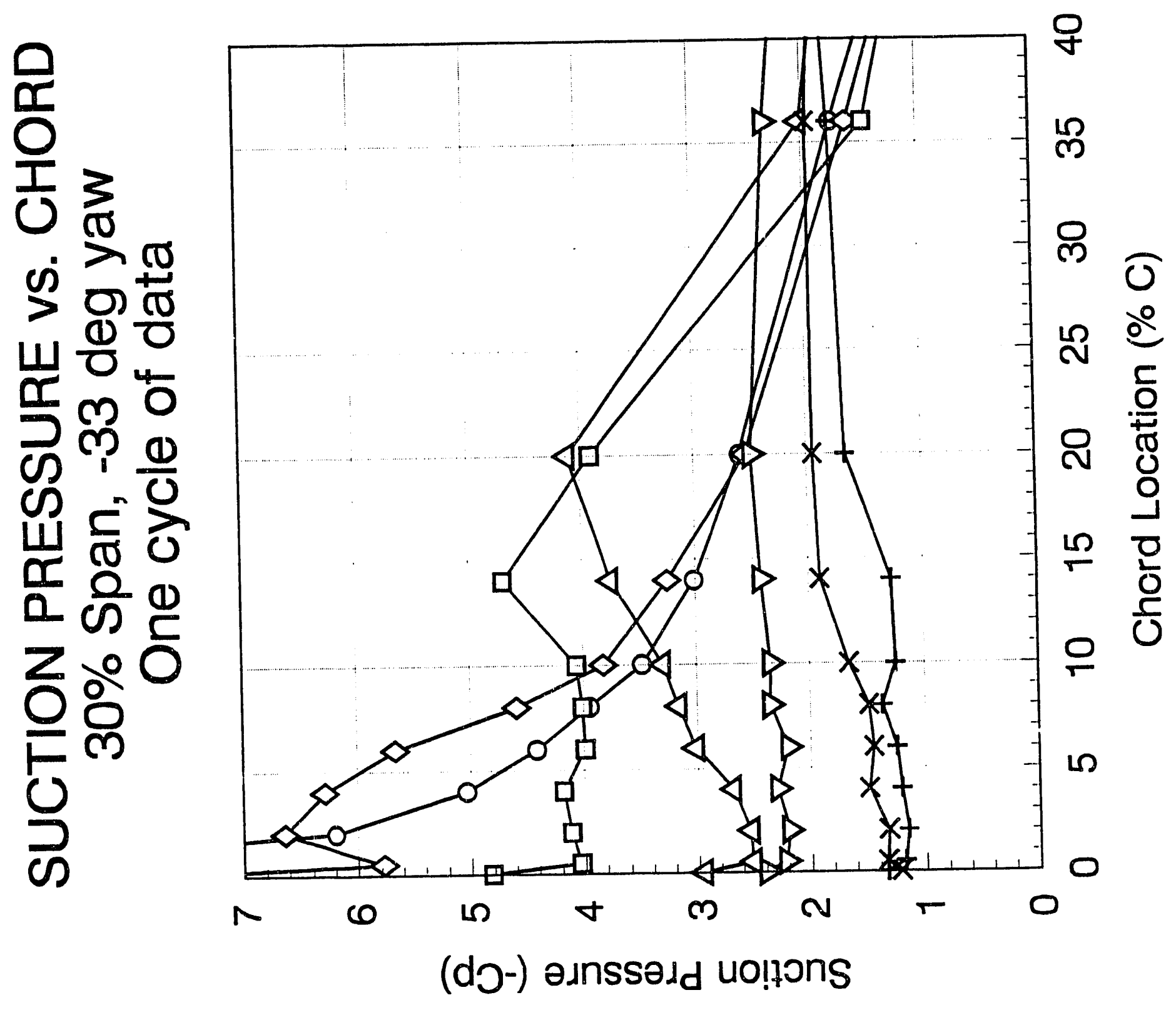




\begin{tabular}{|c|}
\hline 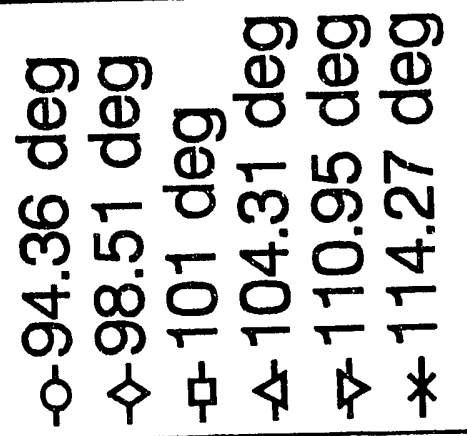 \\
\hline
\end{tabular}

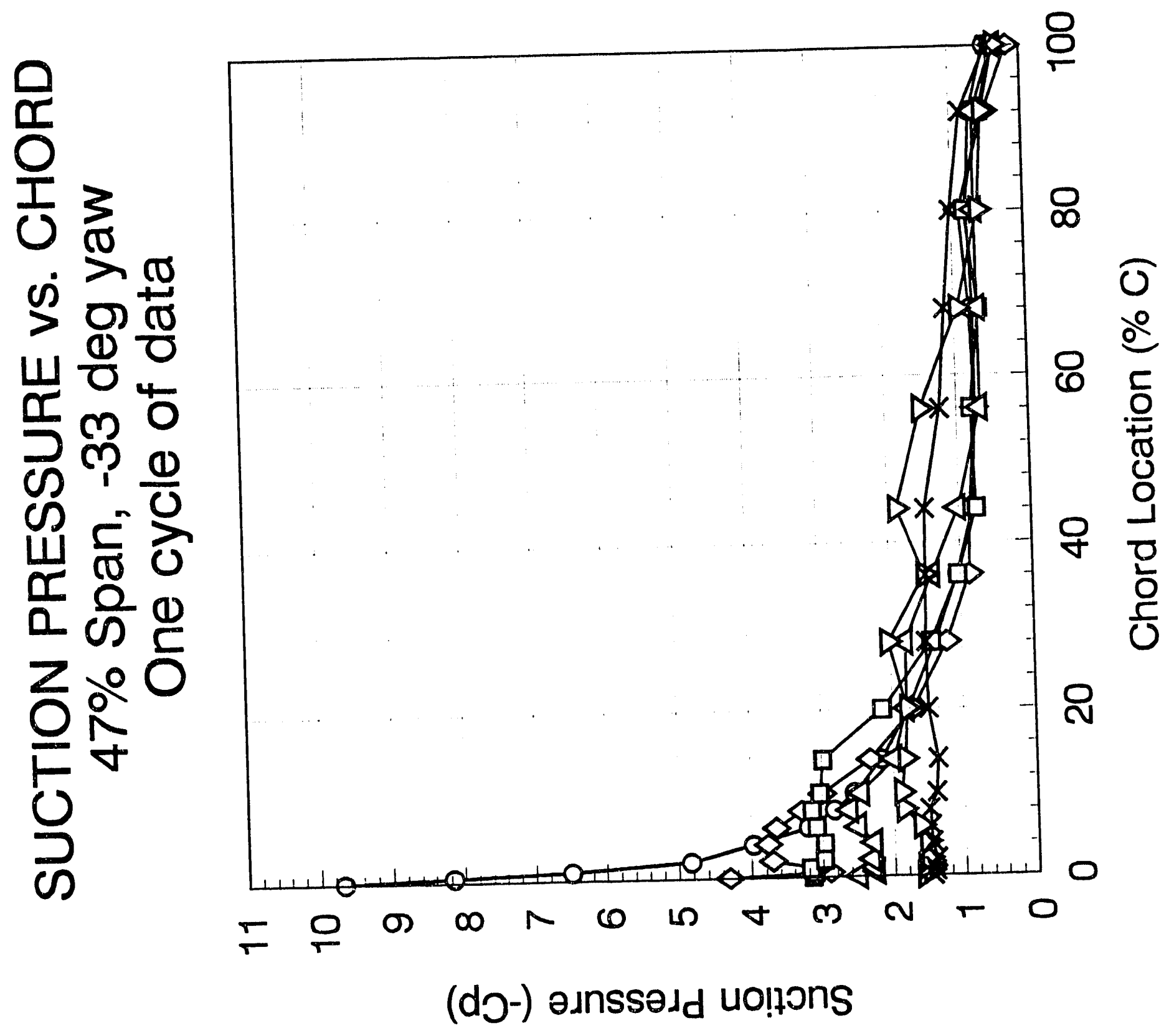



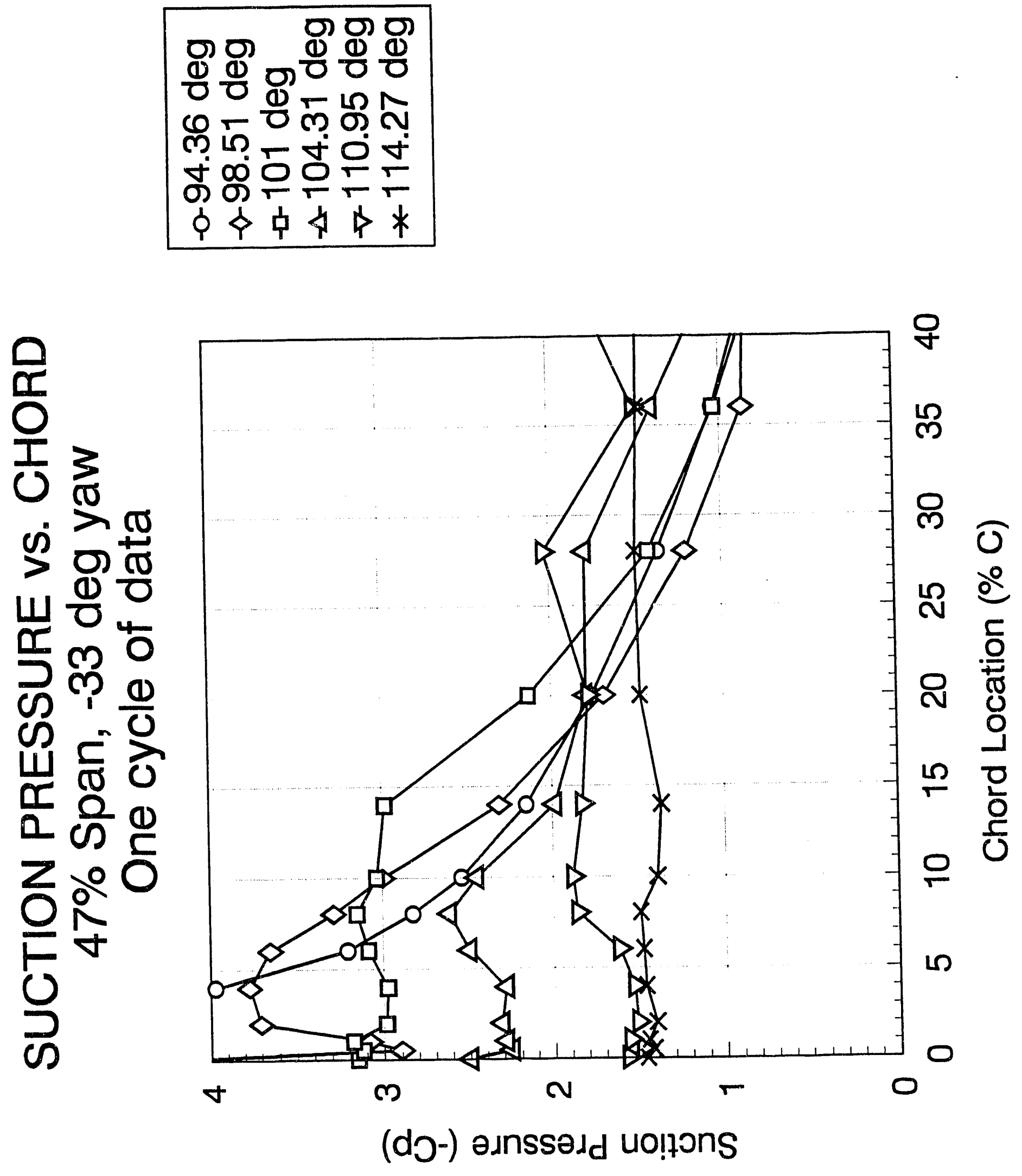


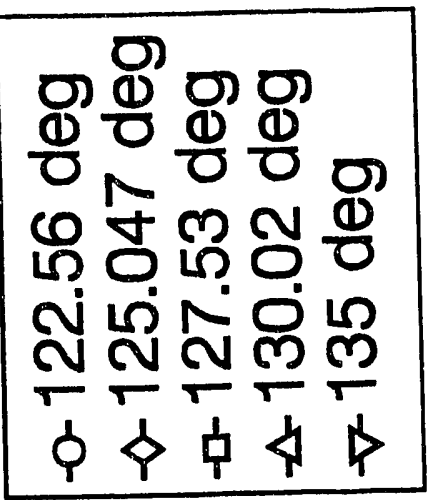

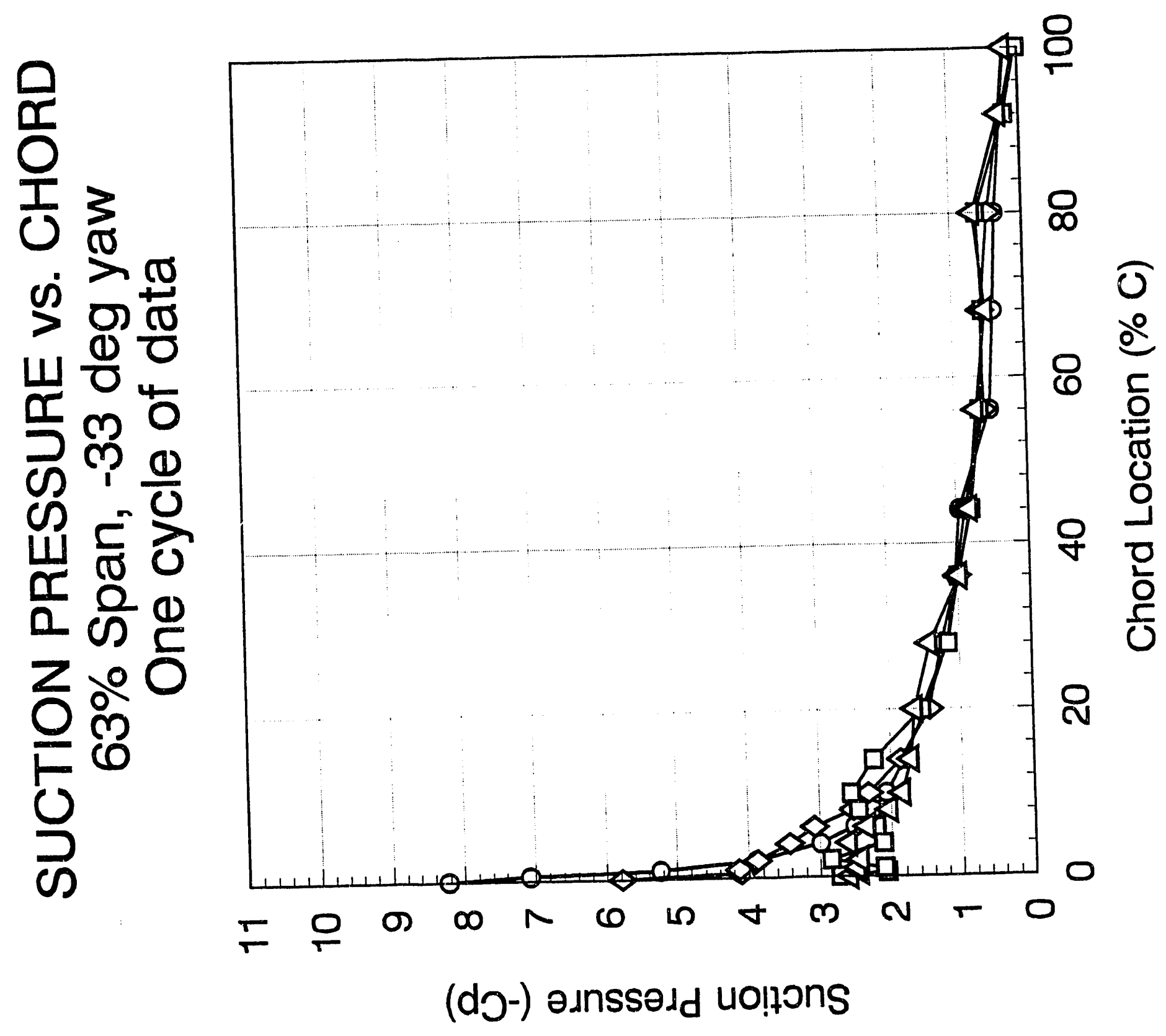




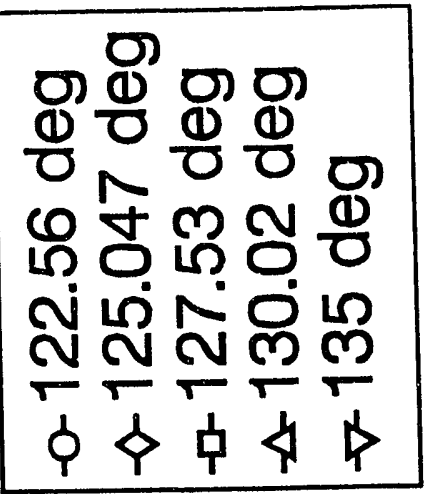

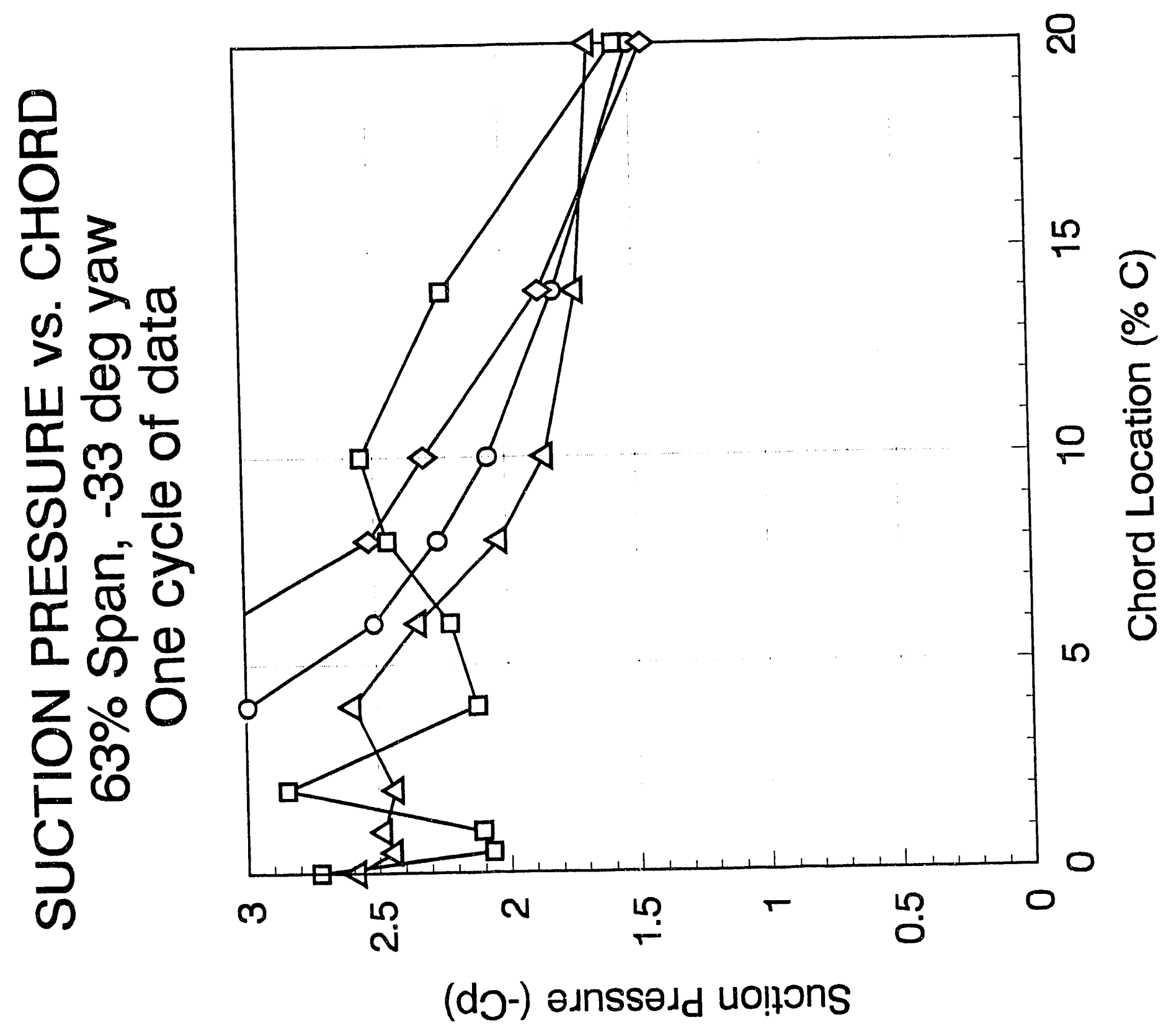



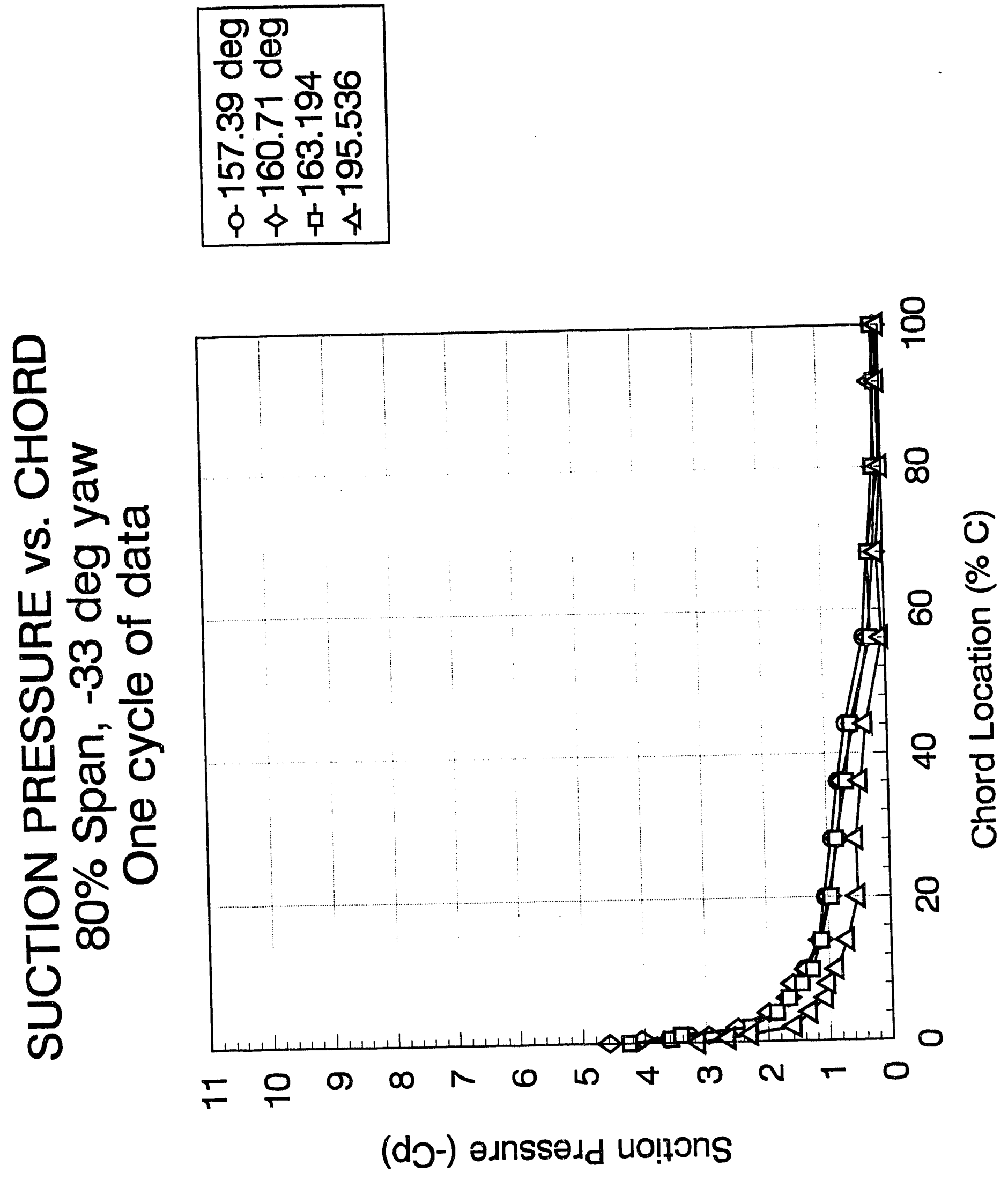

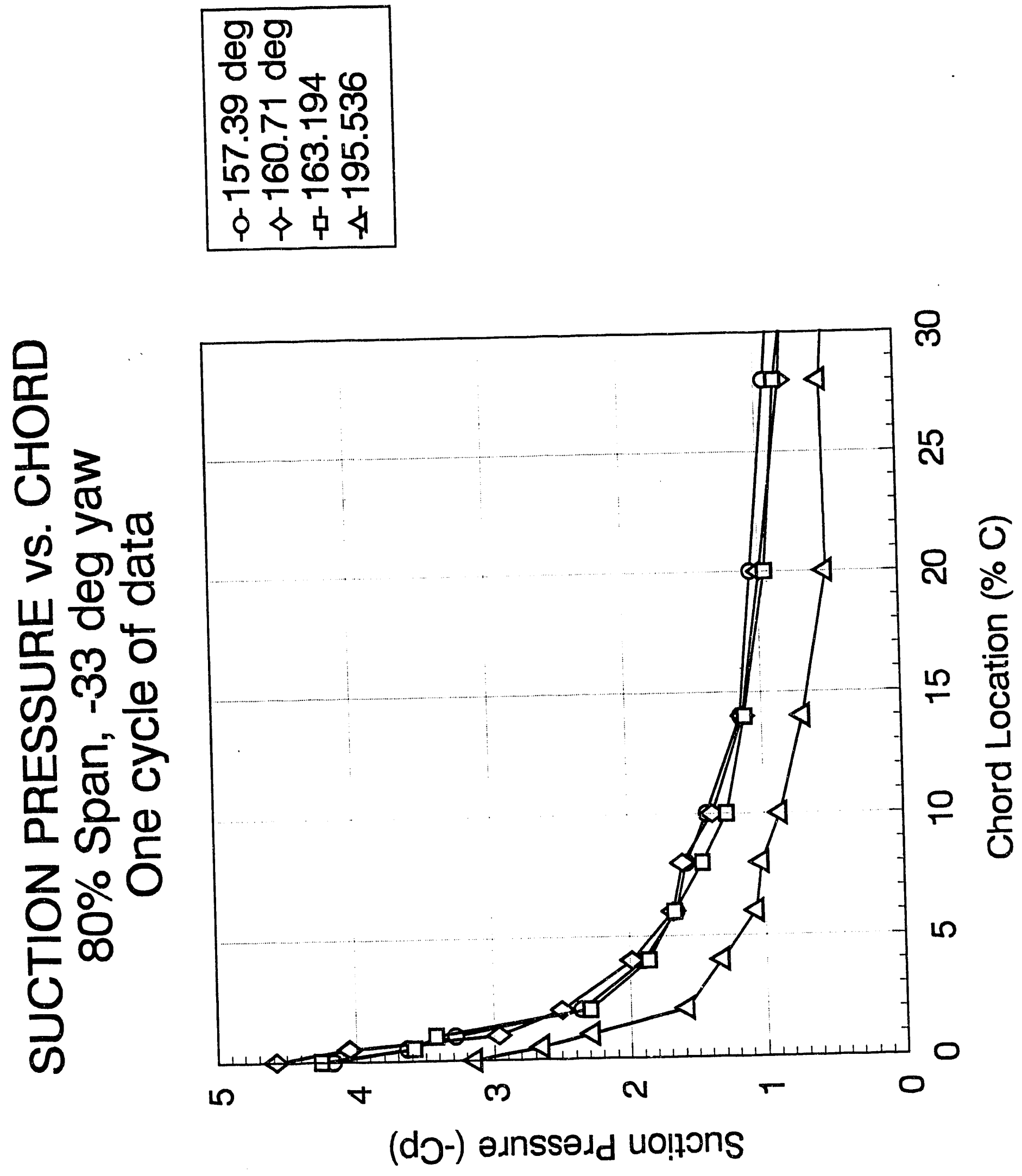


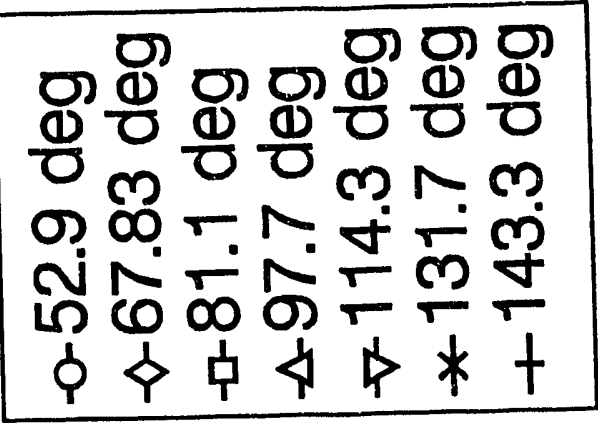

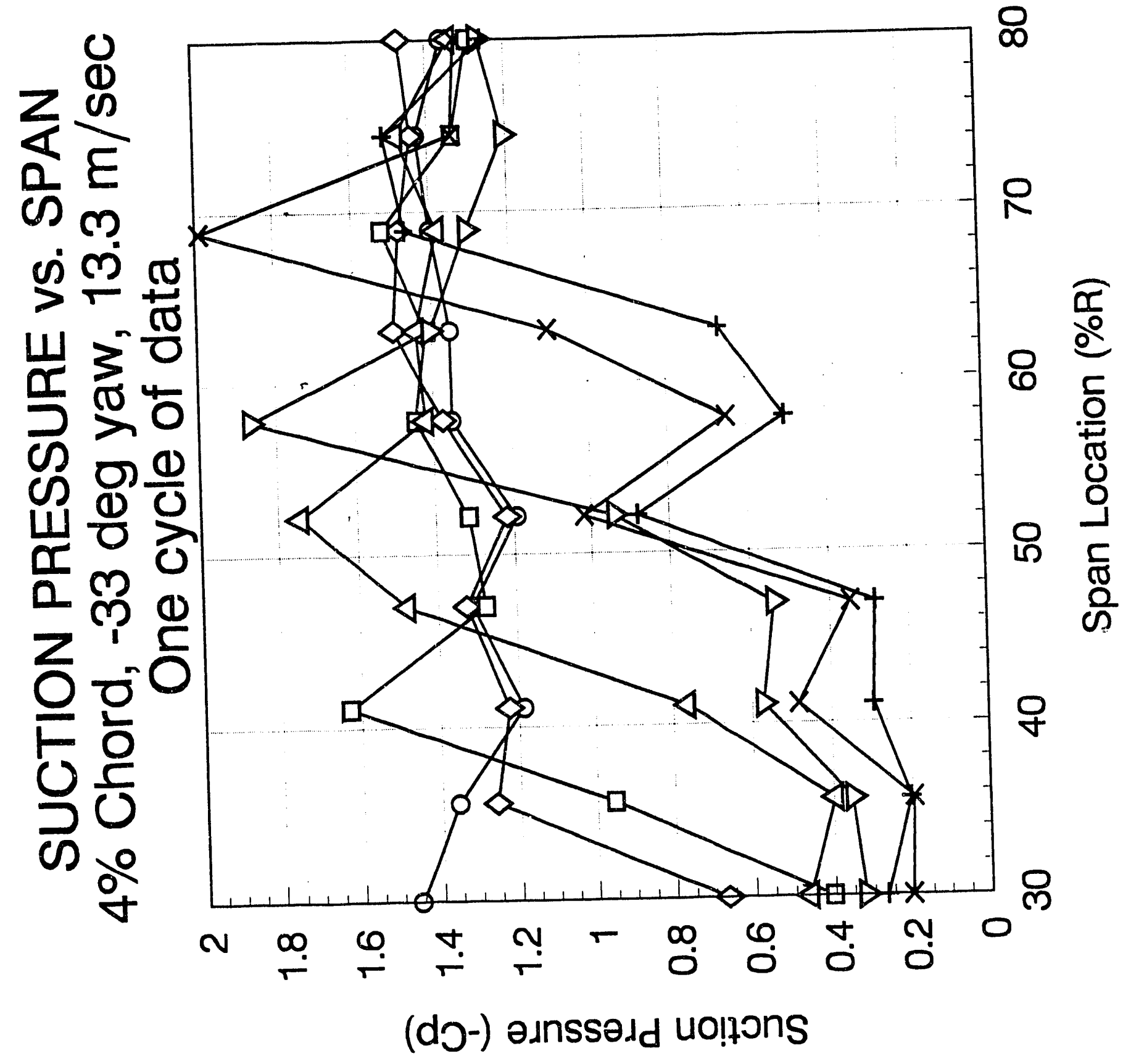



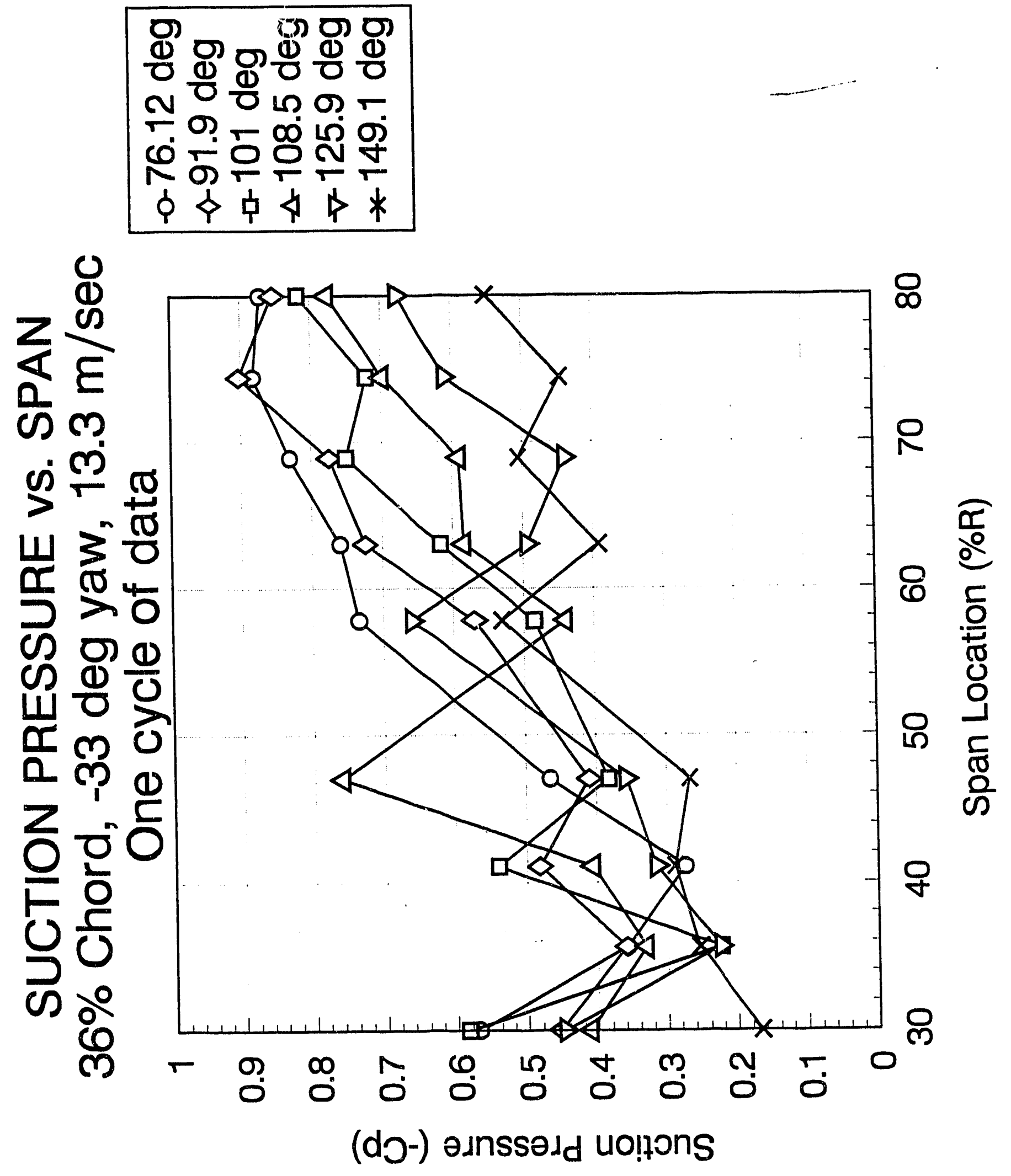

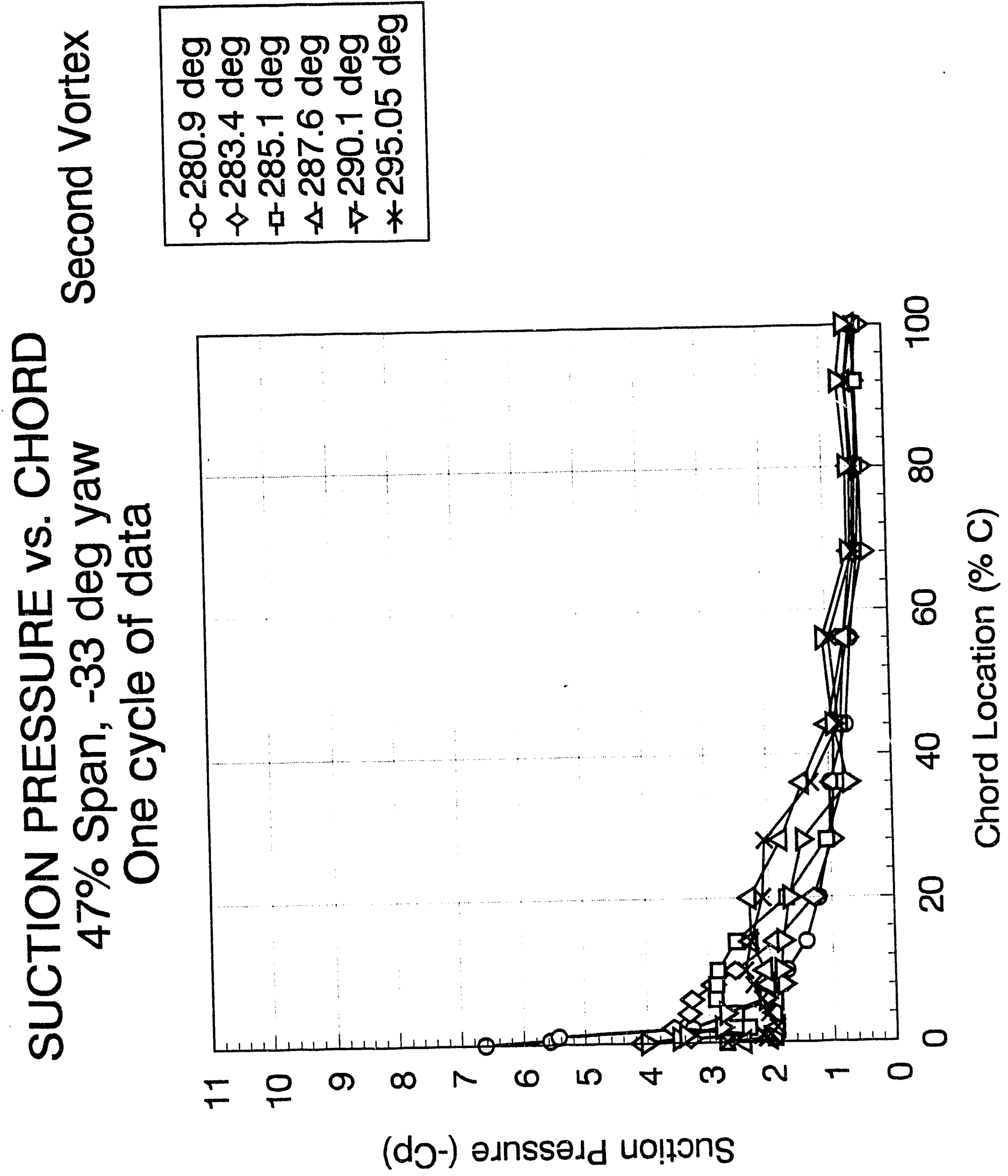

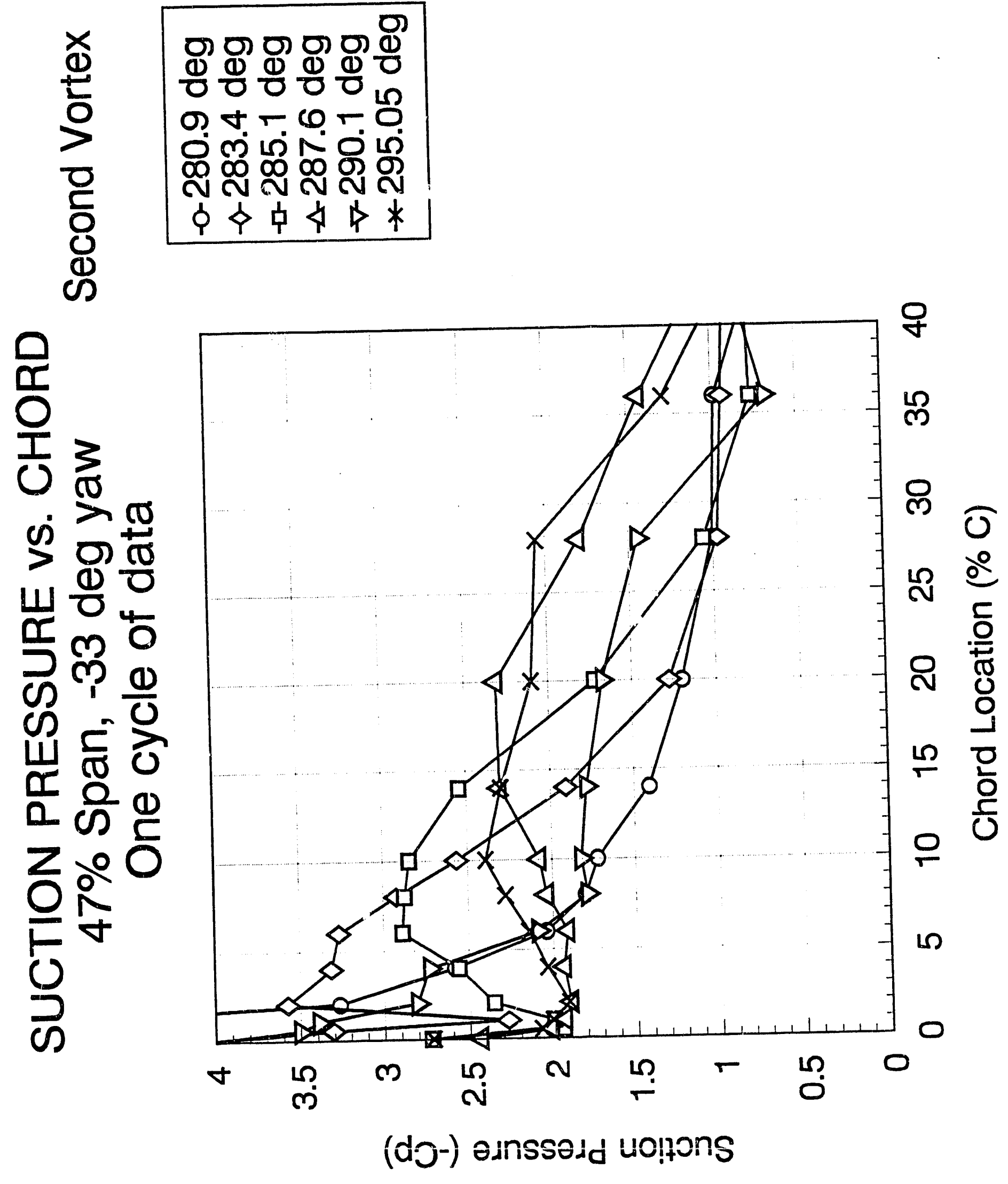


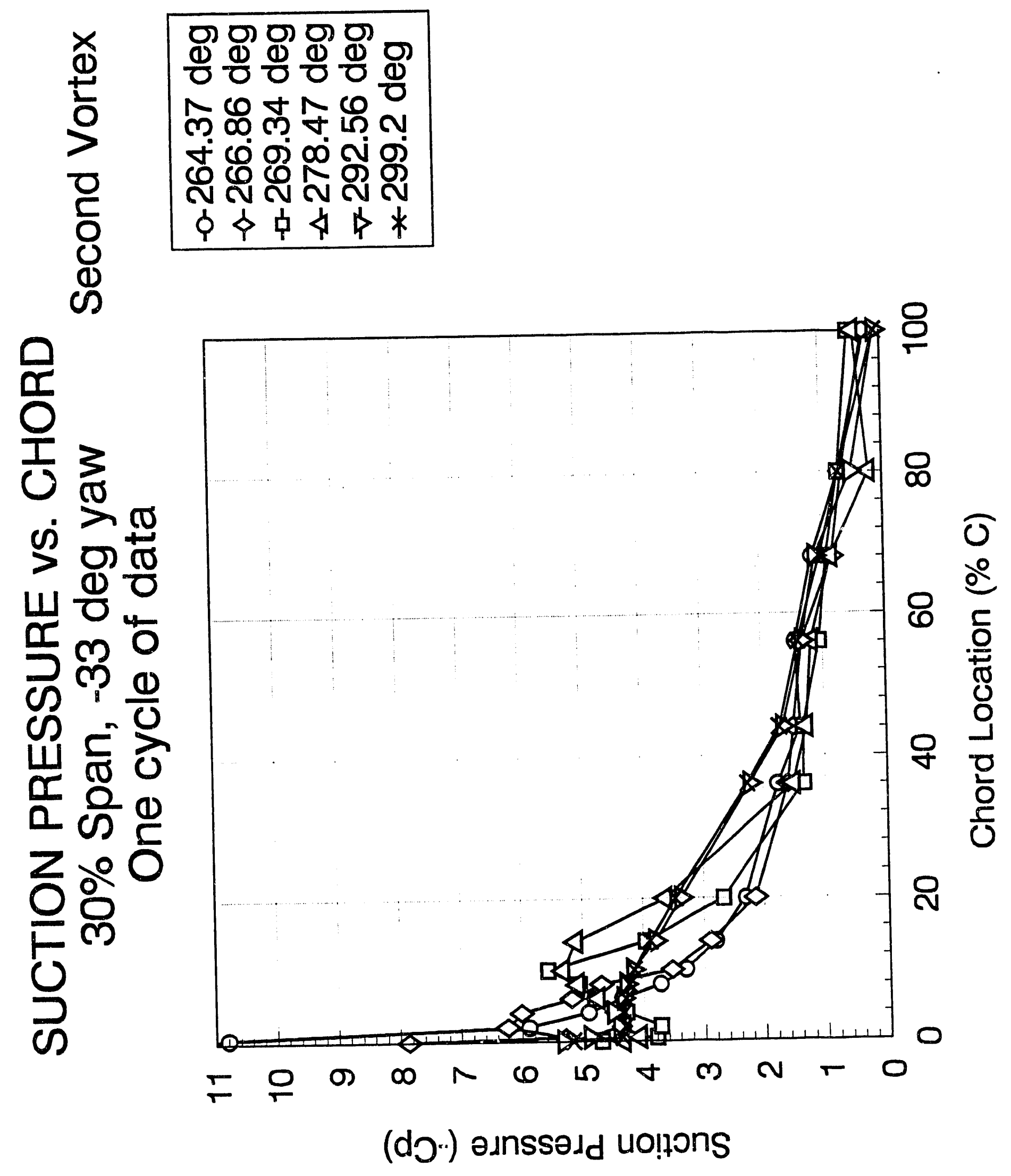




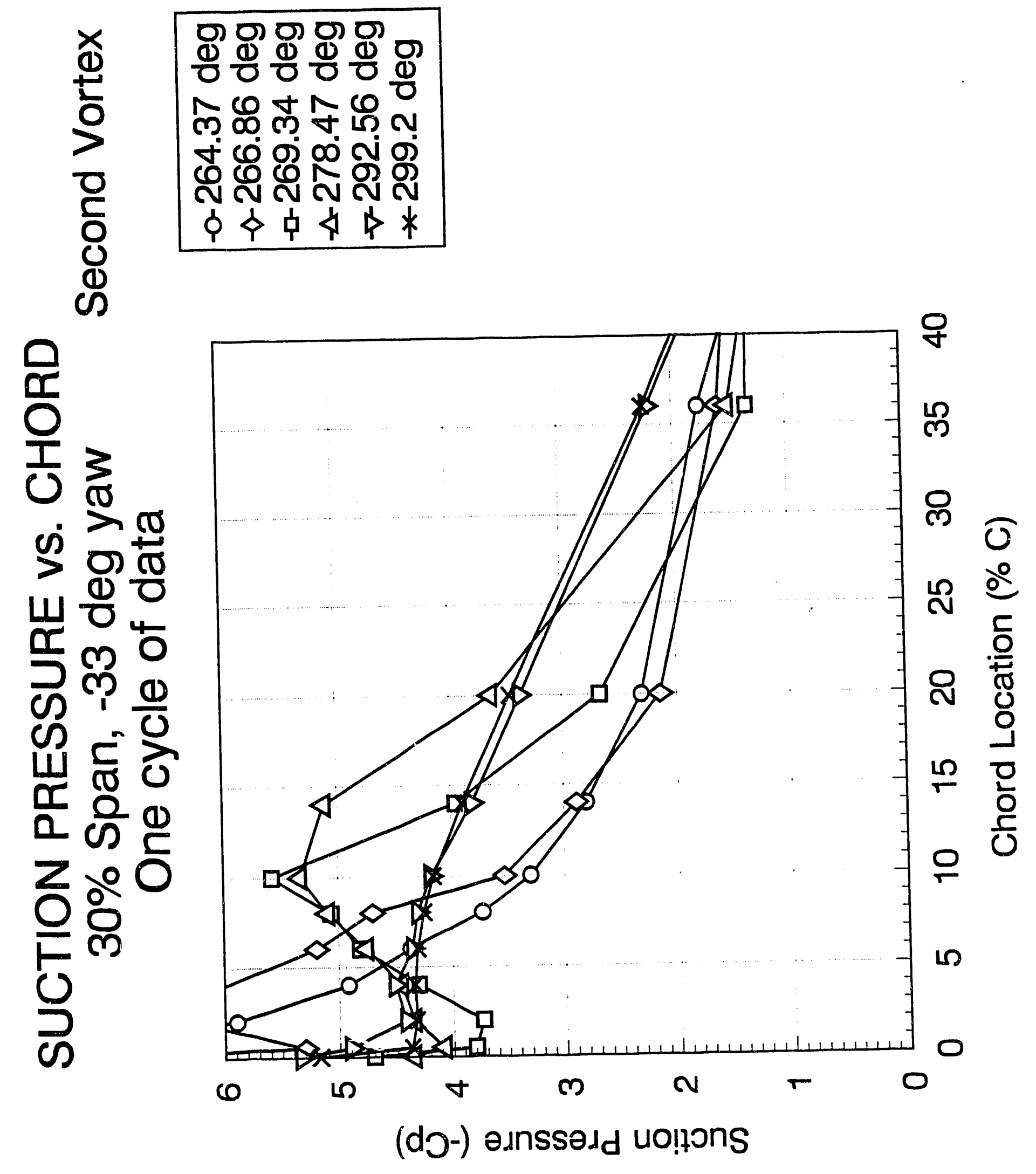



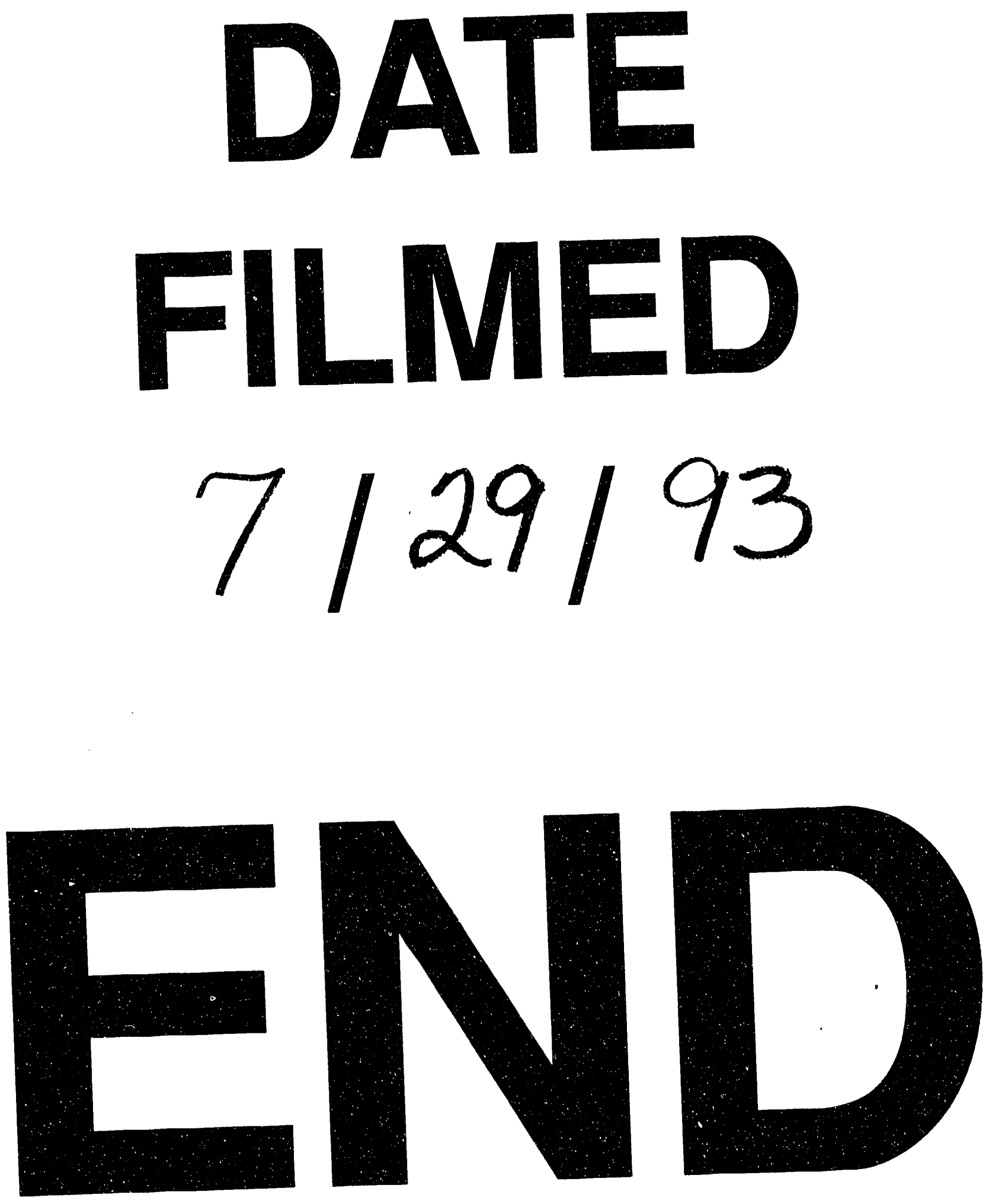
